2809392642
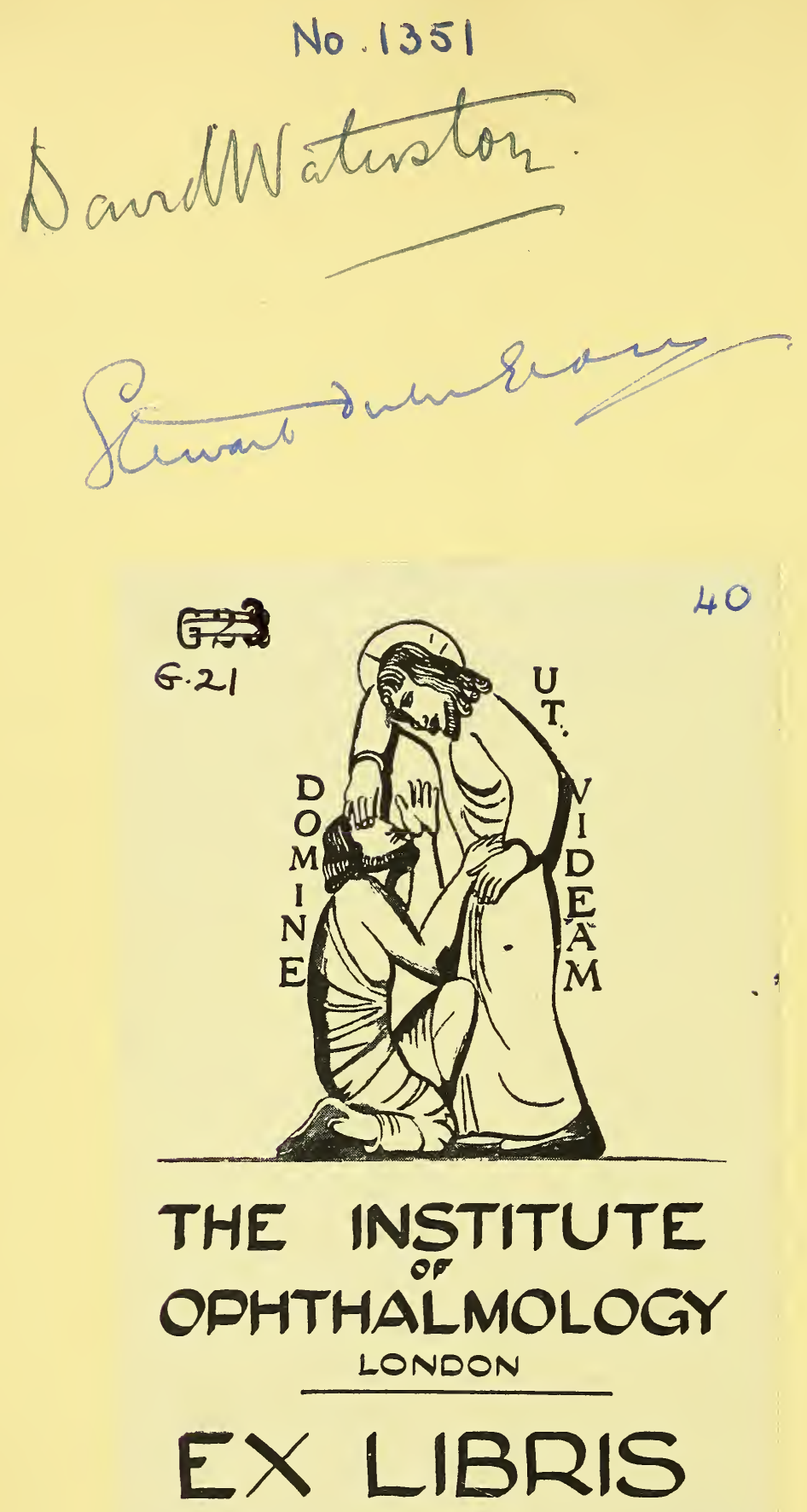
Institute of Ophthalmology Library

This volume is due to be returned on the last date shown below.

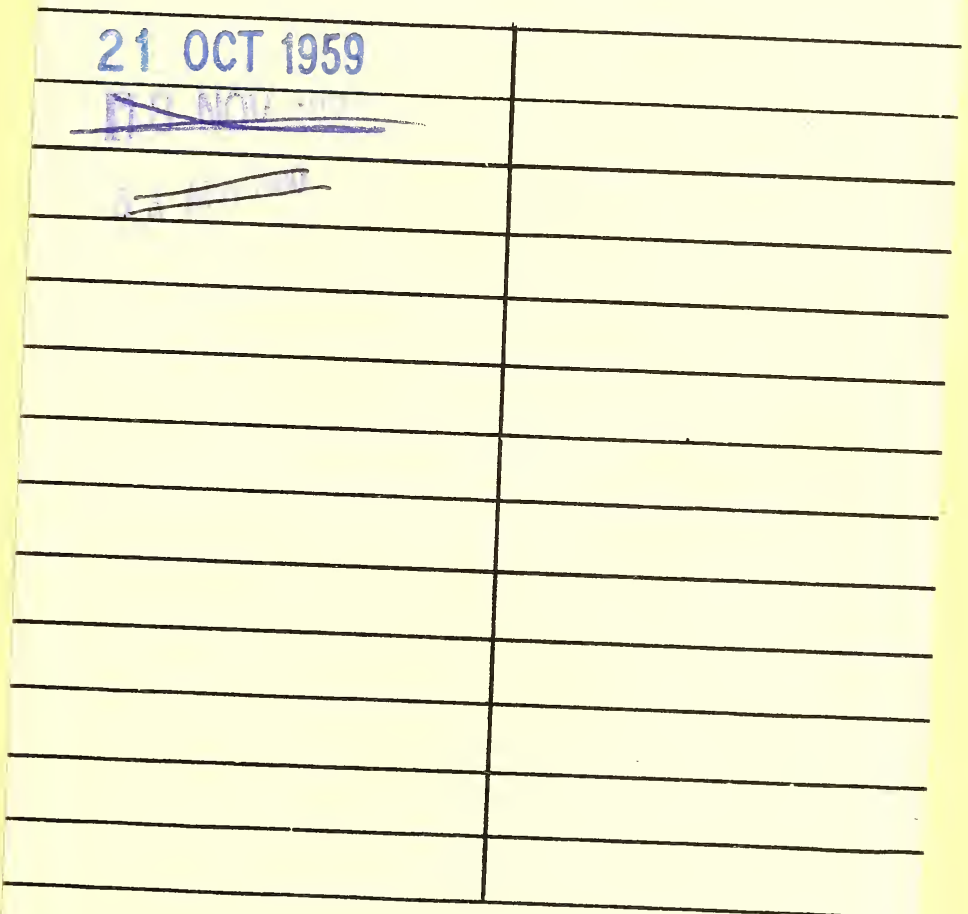




Digitized by the Internet Archive in 2014 




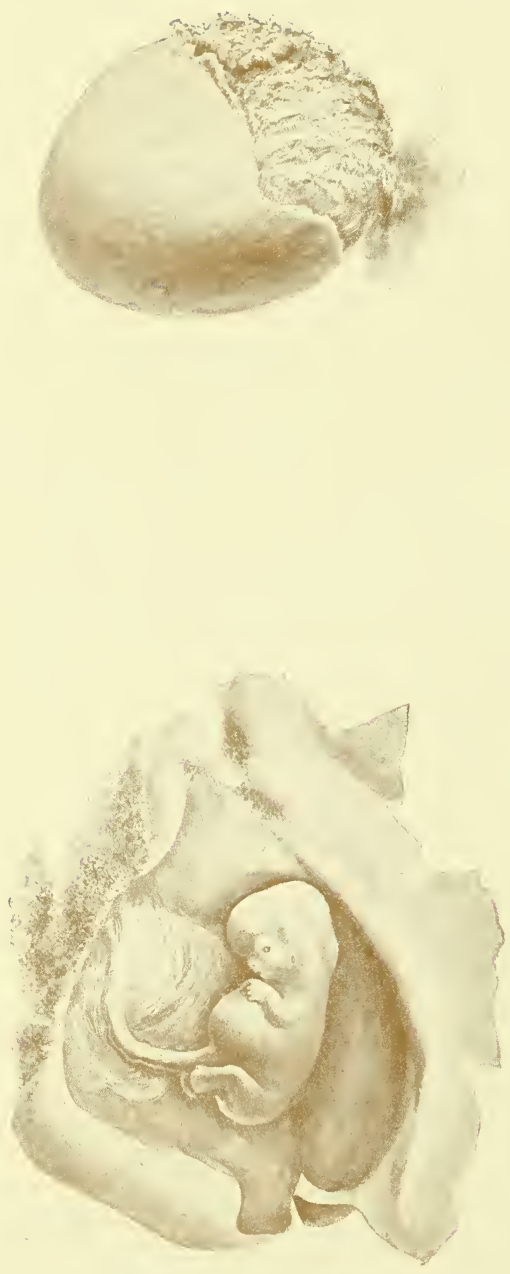

Ovum of about eight weeks, with membranes. In the upper figure (actual size), in the upper right-hand area, the chorion frondosum is apparent. In the lower figure (slightly larger than actual size) the membranes are laid open. 

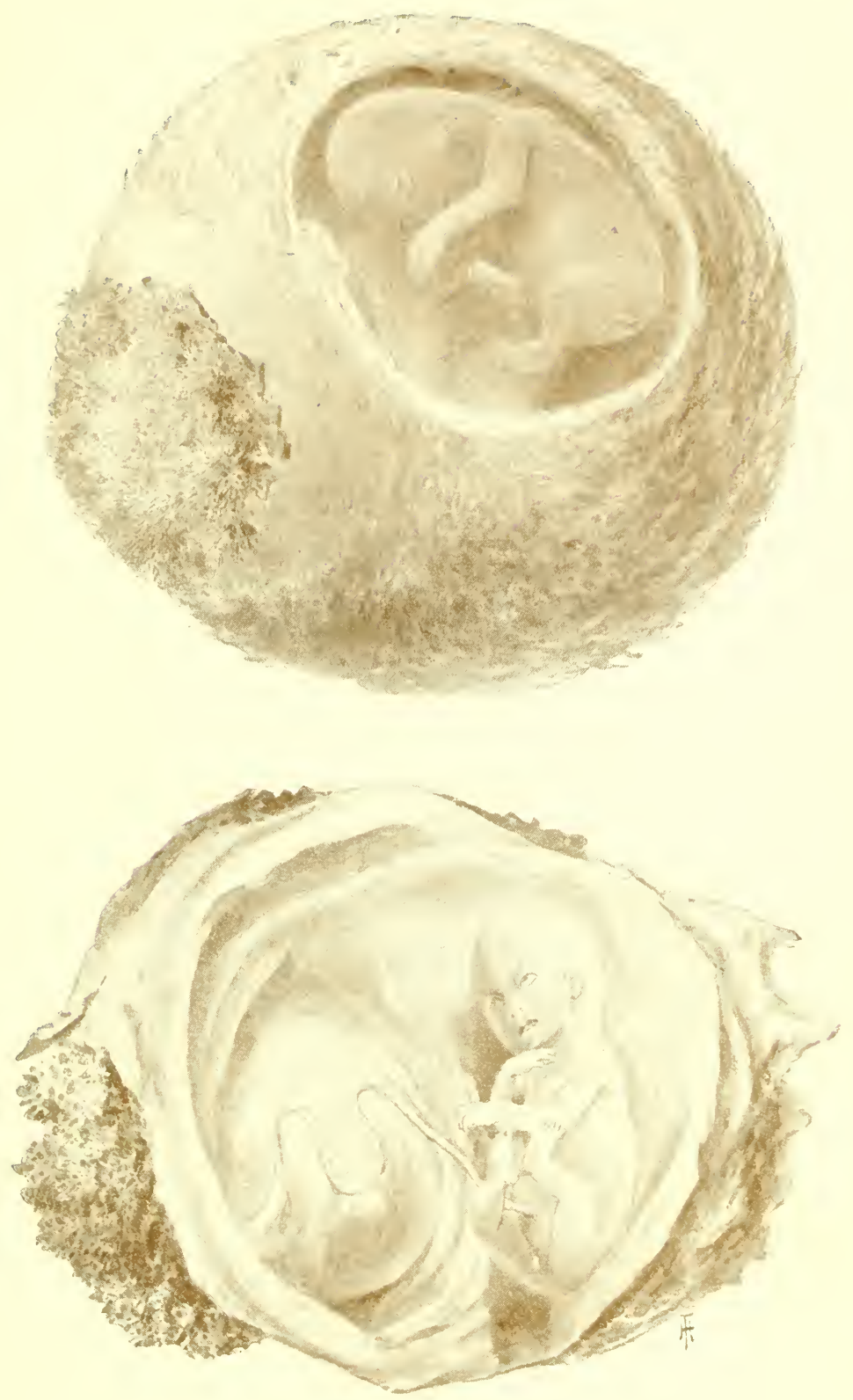

Fetus of third month, with fetal membranes and newly-formed placenta (natural size). In the upper figure the chorion is cut, showing the fetus through the amnion; in the lower figure the membranes are laid open. 



\section{A TEXT-BOOK}

OF

\section{E M B R Y O LO G Y}

FOR STUDENTS OF MEDICINE

BY

JOHN CLEMENT HEISLER, M.D.

Professor of Anatomy in the Medico-Chirurgical College, Philadelphia

WITH 212 ILLUSTRATIONS, 32 OF THEM IN COLORS

Tbiro Edition, Tborongbly TRevised

PHILADELPHIA AND LONDON

W. B. SA U N ERS COM PA N Y 
Set up, electrotyped, printed, and copyrighted October, r899. Revised, reprinted, and recopyrighted September, rgor. Reprinted September, 1902, and September, 1905. Revised, reprinted, and recopyrighted March, I907.

Copyright, 1907,

BY W. B. SAUNDERS COMPANY.

ELECTROTYPED BY WESTCOTT \& THOMSON, PHILADA.
PRESS OF W. B. SAUNDERS COMPANY 


\section{PREFACE TO THIRD EDITION}

The great activity along the lines of embryological research during the past half-dozen years has brought forth much literature and some new facts. In some cases existing views have thereby been modified or set aside; in other's, they have been more firmly established. In presenting the third edition of this book the effort has been made so to revise the text as to harmonize it with the results of recent researches. To this end, certain of the sections have been practically rewritten, while others have been slightly altered, and still others have been merely somewhat amplified. Where changes have been made the authorities therefor have usually been cited. The revised portions of the book include the sections dealing with the ovum, the spermatozoön, the blastodermic vesicle, the amnion, the vascular system, the pancreas, the spleen, the larynx, the thymus, the thyroid, the parathyroid, the adrenal, the kidney, the spinal cord, the vitreous, the musculature, and the vertebral column.

$$
\text { J. C. H. }
$$

3829 Walnut St., Philadelphia,

March, 1907. 



\section{PREFACE}

THE facts of embryology having acquired in recent years such great interest in connection with the teaching and with the proper comprehension of human anatomy, it is of first importance to the student of medicine that a concise and yet sufficiently full text-book upon the subject be available. It was with the aim of presenting such a book that this volume was written, the author, in his experience as a teacher of anatomy, having been impressed with the fact that students were seriously handicapped in their study of the subject of embryology by the lack of a text-book full enough to be intelligible, and yet without that minuteness of detail which characterizes the larger treatises, and which so often serves only to confuse and discourage the beginner.

In the arrangement of the subject-matter of the book, it has been the aim not only to present a connected story of human development, but also to make each chapter as nearly as possible complete in itself, for the sake of convenience of reference. It is for this reason that some repetitions occur in the text. The frequent allusions to certain facts of comparative embryology are rendered necessary by the very nature of the subject, but it has been the writer's aina to make these allusions as simple and as easily intelligible as possible.

In the selection of the illustrations, great care has been exercised to employ those of the greatest teaching value, and to arrange them, with reference to any one chapter, as nearly 
as possible in proper chronological sequence. Due acknowledgement is made in each case for every illustration borrowed from other works.

With few exceptions, no attempt has been made to cite authorities in the text, and the author would here express his obligations to the writings of His, O. Hertwig, Kölliker, Schultze, Bonnet, Balfour, Marshall, Piersol, Minot, Tourneux, and many others.

3829 Walnut St.,

J. C. H.

Philadelphia. 


\section{CONTENTS.}

CHAPTER I.

The Male and Female Sexual Elements; Maturation; Ovu= lation; Menstruation; Fertilization . . . . . . . . 17

The Spermatozoon . . . . . . . . . . . . . 20

The OvuM . . . . . . . . . . . . . . . . . 24

^The Hen's Egg . . . . . . . . . . . . . . . . . 27

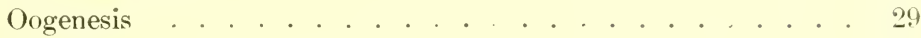

Maturation of the Ovum . . . . . . . . . . . . 32

Ovulation . . . . . . . . . . . . . . . . 36

Menstruation . . . . . . . . . . . . . . . . . . . . . .

The Relation of Menstruation to Ovulation and Conception . . 40

Fertilization . . . . . . . . . . . . . . . 41

Artificial Fertilization . . . . . . . . . . . 44

CHAPTER II.

The Segmentation of the Ovum and Formation of the Blas= todermic Vesicle . . . . . . . . . . . . 45

Segmentation . . . . . . . . . . . . . . . . 45

The Stage of the Blastula . . . . . . . . . . . 49

CHAPTER III.

The Germ=layers and the Primitive Streak . . . . . . 52

The Two-layered Stage of the Blastodermic Vesicle . . . 52

The Embryonal Area . . . . . . . . . . . . . . . 58

The Primitive Streak . . . . . . . . . . . . . . . 59

The Development of the Mesoderm . . . . . . . . 62

The Derivatives of the Germ-lavers . . . . . . . . . . 67

CHAPTER IV.

The Beginning Differentiation of the Embryo; the Neural Canal; The Chorda Dorsalis ; the Mesoblastic Somites The Neural or Medullary Canal . . . . . . . . . . . . . . 70

The Notochord or Chorda Dorsalis . . . . . . . . 78

The Neurenteric Canal . . . . . . . . . . . . 74

The Somites or Primitive Segments . . . . . . . . . . . To 
The Formation of the Body=wall, of the Intestinal Canal, and of the Fetal Membranes

The Formation of the Body-Wall and of the Intestinal Canal of the Embryo . . . . . . . . . . . . . . . 79

The Amnion . . . . . . . . . . . . . . . . . . 82

The Yolk-SAC . . . . . . . . . . . . . . . . 87

The Allantois . . . . . . . . . . . . . . . . . . 89

The Chorion . . . . . . . . . . . . . . . . . 92

CHAPTER VI.

The Decidur and the Embedding of the Ovum; the Placenta; the Umbilical Cord . . . . . . . . . . 95

The Decidum and the Embedding of the Ovum . . . . 95

The Placenta. . . . . . . . . . . . . . . . . . . . 98

The Umbilical Cord . . . . . . . . . . . . . . 102

Relations of the Fetal Membranes at Birth ..... 194

CHAPTER VII.

The Further Development of the External Form of the Body 105

The Stage of the Ovum . . . . . . . . . . . . 106

The Stage of the Embryo . . . . . . . . . . . . . 107

The Visceral Arches and Clefts . . . . . . . . . . . 111

The Stage of the Fetus . . . . . . . . . . . . . 118

CHAPTER VIII.

The Development of the Connective Tissues of the Body, and of the Lymphatic System . . . . . . . . . . 124

The Connective Tissues . . . . . . . . . . . . . 124

The Development of the Lymphatic System . . . . . . 127

CHAPTER IX.

The Development of the Face and the Mouth Cavity . . . . 130

The Evolution of the Face . . . . . . . . . . . . . . 130

Тне Мостн . . . . . . . . . . . . . . . . . . 134

The Teeth . . . . . . . . . . . . . . . . . 137

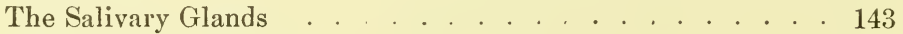

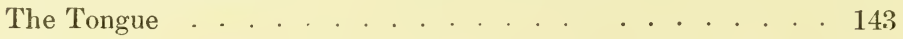

The Nose . . . . . . . . . . . . . . . . . 145

CHAPTER X.

The Development of the Vascular System . . . . . . . . 147

The Vitelline Circulation and the Origin of the Blood 147

The Development of the Heart . . . . . . . . . . 152

The Metamorphosis of the Single into the Double Heart . . . . 156

The Valves of the Heart . . . . . . . . . . . . . 161

The Allantoic and the Placental Circulation . . . . 163 
The Fetal Arterial System

The Fetal Venous System . . . . . . . . . . . 169

The Formation of the Pericardium, the Pleura, and the

Diaphragi . . . . . . . . . . . . . . . . . . 174

The Portal Circulation . . . . . . . . . . . . 177

The Final Stage of the Fetal Vascular system . . . 181

CHAPTER XI.

The Development of the Digestive System . . . . . . 185

The Мouth . . . . . . . . . . . . . . . . . . 192

The Pharyex . . . . . . . . . . . . . 193

The Tongue . . . . . . . . . . . . . . . . 194

The Tonsil . . . . . . . . . . . . . . 194

The Anus . . . . . . . . . . . . . . . . . 195

The Differentiation of the Mlimentary Canal into SepÁrate Regions. . . . . . . . . . . . . 197

Increase in Length and Further Subdivision . . . . . . . . 201

Alteration in the Relative Position of Parts, and Further Devel-

opment . . . . . . . . . . . . . . . 202

Histological Alterations . . . . . . . . . . . 205

Meckel's Diverticulum . . . . . . . . . . . . . 207

The Denelopment of the Liver . . . . . . . . . . 207

The Gall-bladder . . . . . . . . . . . . . . 209

The Ligaments of the Liver . . . . . . . . . . . . . . . 209

The Development of the Pancreas. . . . . . . . 211

The Development of the Spleen . . . . . . . . . 212

The Evolution of the Peritonelis . . . . . . . . 214

CHAPTER XII.

The Development of the Respiratory System . . . . . . 2.22

The Thyroid, the Parathyroid, and the Thymes Bodies . . 226

CHAPTER XIII.

The Development of the Genito=urinary System . . . . . . 232

The Development of the Kinney and the Ureter . . 29:2

The Mesonephros or Wolffian Body . . . . . . . . . . . . . 234

The Metanephros or Permanent Kidney . . . . . . . . . . . 237

The Suprarenal Bodies . . . . . . . . . . . . 241

The Development of the Internat, Generative Organs . 243

The Indifferent Type . . . . . . . . . . . . . . . . . 243

The Male Type . . . . . . . . . . . . . . . . . . . . 245

The Female Type . . . . . . . . . . . . . . . . . 249

The Bladder and the Prostate Gland . . . . . . . 25.5

The External Organs of Generation . . . . . . . 25s

The Female External Genitals . . . . . . . . . . . . . . . 259

The Male External Genitals . . . . . . . . . . . . . . 261

Summary . . . . . . . . . . . . . . . 264 
The Development of the Skin and its Appendages . . . . . 268

THE SkIN . . . . . . . . . . . . . . . . 268

The Appendages of the Skin . . . . . . . . . 270

The Nails . . . . . . . . . . . . . . 270

The Hair . . . . . . . . . . . . . . . . . 271

The Sebaceous and Sweat Glands . . . . . . . . . 273

The Mammary Gland . . . . . . . . . . . . 274

CHAPTER XV.

The Development of the Nervous System . . . . . . 278

The Development of the Spinal Cord . . . . . . . . . 281

The Development of the Brain . . . . . . . . . . 286

The Fifth Brain-vesicle . . . . . . . . . . . . . . . . 289

The Hind-brain Vesicle . . . . . . . . . . . . . . . 292

The Mid-brain Vesicle . . . . . . . . . . . . . . . . 294

The Inter-brain Vesicle . . . . . . . . . . . . . . 296

The Fore-brain Vesicle . . . . . . . . . . . . . 302

The Development of the Peripheral Nervous System • 316 The Development of the Sympathetic System . . . . . 324

The Carotid Body, the Coccygeal Body, and the Organs of Zuckerkandl . . . . . . . . . . . . . . 325

\section{CHAP'TER XVI.}

The Development of the Sense Organs . . . . . . . . . . 326

The Development of the Eye . . . . . . . . . 326

The Retina and Optic Nerve . . . . . . . . . . . . . . . . . 328

The Crystalline Lens . . . . . . . . . . . . . . . . 336

The Vitreous Body . . . . . . . . . . . . . . 338

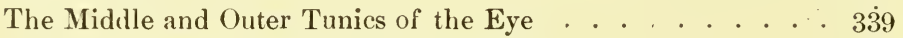

The Eyelids and the Lacrimal Apparatus . . . . . . . . 343

The Development of the Organ of Hearing . . . . 345

The Internal Ear . . . . . . . . . . . . . . . . . 346

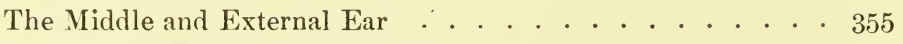

The Development of the Nose. . . . . . . . 358

CHAPTER XVII.

The Development of the Muscular System . . . . . . 363

The Striated or Voluntary Muscles . . . . . . . 363

The Muscles of the Trunk Proper . . . . . . . . . . . 363

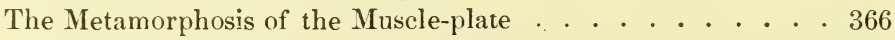

The Branchial Muscles . . . . . . . . . . . . . 369

The Muscles of the Extremities . . . . . . . . . . . 370

The Involuntary or Unstriated Muscular Tissue . . . 371

The Cardiac Muscle . . . . . . . . . . . . . . . 371 
The Development of the Trunk . . . . . . . . . . . 373

The Stage of the Chorda . . . . . . . . . . 373

The Membranous Stage . . . . . . . . . . . . . . . 374

The Cartilaginous Stage . . . . . . . . . . . . 377

The Osseous Stage . . . . . . . . . . . . . . . . . 379

The Development of the Ribs and Sternum . . . . . 382

The Development of the Head Skeleton . . . . . . . . 384

The Membranous Cranium . . . . . . . . . . . . 385

The Cartilaginous Cranium . . . . . . . . . . . 386

The Osseous Stage . . . . . . . . . . . . . . . . 389

The Appendicular Skeleton . . . . . . . . . 402

The Pectoral and Pelvic Girdles . . . . . . . . . 403

The Bones of the Extremities . . . . . . . . . . . . 404

The Development of the Limbs . . . . . . . 406

The Position of the Limbs . . . . . . . . . 407

Tabulated Chronology of Development . . . . . . . . 409

Index . . . . . . . . . . . . . 417 



\section{CHA PTE R I.}

\section{THE MALE AND FEMALE SEXUAL ELEMENTS; MATURATION ; OVULATION ; MENSTRUATION ; FERTILIZATION.}

Embryology is that department of biology which treats of the generation and development of organisms. It may refer to the development of the race or stock-Phylogeny-or to that of the individual-Ontogeny; again, it may treat of animal or of vegetable development.

Since no observations have been made upon embryos of an age less than four or five days, and but few, indeed, upon those younger than sixteen or eighteen days, we cannot be said to possess definite knowledge of the very earliest processes of development in man. There is, however, sufficient analogy between the known facts of human development and those of corresponding stages in allied groups of animals, as well as between the various groups of animals themselves, to establish certain broad general principles of agreement in essential features. In tracing the history of human development, therefore, frequent recourse must be had to the development of animals, since in this way only is it possible at present to fill up the gaps in our knowledge of human embryology.

That a new individual may be called into existence, the union of the male element, or spermatozoon, with the female element, or ovum, is necessary. Such union is variously called fertilization, fecundation, and impregnation.

Prior to the beginning of the present century, little or nothing was definitely known concerning reproduction and development. The opinions of the biologists of early times found expression in a theory which was then called the theory of unfoiding or of evolution, but which more recently has 
been designated the preformation theory. According to this doctrine, the egg or germ contained all the parts of the adult organism in an exceedingly minute condition, and development consisted in the simple growth or unfolding of already formed parts. As the theory of unfolding implied the preformation not only of the immediate but of all subsequent offspring, its votaries were able to compute that the ovary of Eve contained 200,000 millions of human germs.

With the discovery of the spermatozoon in 1677 by Hanm, a pupil of Leuwenhoeck, a controversy arose as to whether it was the spermatic filament or the ovum that contained the germ. Those who maintained the former view were known as animalculists; those who held the latter, as ovists. According to the opinions of the animalculists, the spermatozoon was the complete organism in miniature, and it required for its growth the soil or environment which the ovum alone could furnish.

The enunciation by Wolff, in 1759, of his doctrine of epigenesis completely overturned the preformation theory. Wolff maintained that the germ was unorganized matter, and that the union of male and female material was essential to reproduction. While Wolff's theory was in the main correct, it remained for later investigators to show that the ovum did not consist of unorganized matter, as he thought, but that it possessed definite structural characteristics. Thus, the germinal vesicle of the hen's egg was discovered in 1825 by Purkinje, and the germinal spot in 1826 by Wagner. Soon after the enunciation of the cell-doctrine by Schleiden and Schwann, it was seen that the ovum was in reality a typical cell, possessing all the parts of such a structure.

It was not, however, until about the year 1840 that it was shown, by Kölliker, Reichert, and others, that the spermatozoa are the active agents in fecundation. Previously it had been held, since the refutation of the preformation theory, that the seminal fluid performed this function, and that the spermatozoa were parasitic organisms.

The length of time necessary for the development of the new individual varies according to the species; in man it 
occupies nine calendar months or about ten lunar monthsthat is, from 273 to 280 days. The period of human gestation is arbitrarily divided by His into three stages: (1) The
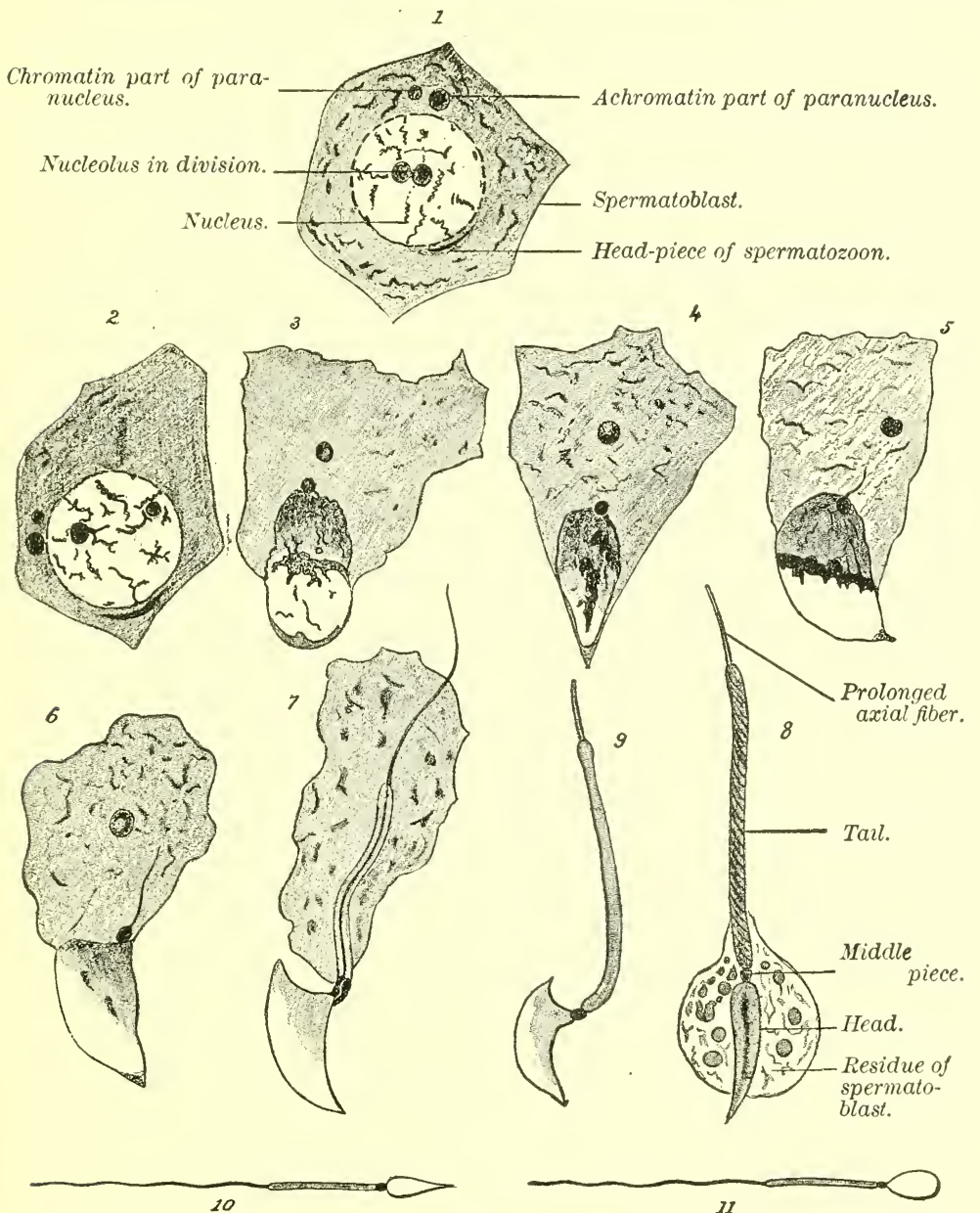

FIG. 1. -1 to 8 , Various stages of the development of the spermatozoon of the mouse; 9, the spermatozoon of the mouse (after F. Hermann); 10 and 11, spermatozoa of the $\operatorname{dog} ; 10$, as seen from the side; 11 , as seen from the broader surface (after Bonnet).

stage of the ovum, comprising the first two weeks of development; (2) The stage of the embryo, extending from the end of the second week to the fifth week, during which time the germ begins to assume definite form; and (3) The stage of 
the fetus, which includes the remainder of the term of intrauterine existence.

It may be pointed out that the term ovum, as employed in embryology, has three different significations: it designates the female sexual cell prior to its impregnation; it is used in the sense noted above to designate the fertilized egg; and it is somewhat loosely applied to the product of conception during various stages of development.

\section{THE SPERMATOZOON.}

It is noteworthy that both spermatozoa and ova-that is, both sexual cells-are products of metamorphoses taking place in epithelial structures, the former being derived from the spermatogenic cells found in the seminiferous tubules of

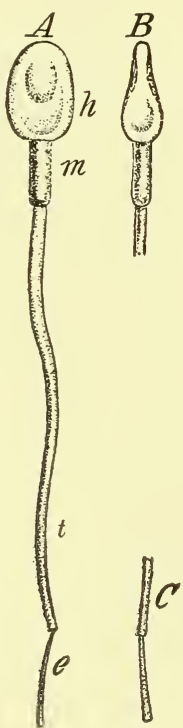

Fig. 2.-Human spermatozoa (after Retzius): $A$, spermatozoon seen en face; $h$, head; $m$, middlepiece; $t$, tail ; $e$, end-piece; $B, C$, seen from the side. the testicle, while the latter come from the germinal epithelium of the ovary.

The form of the seminal filament varies greatly in different species (Fig. 1), being usually an elongated flagellate cell. The human spermatozoon (Fig. 2) is about $0.05 \mathrm{~mm}$. $\left(\frac{1}{500}\right.$ inch $)$ in length, consisting of a head, a middle piece, a tail or flagellum, and an end-piece.

The head is much thickened as compared with the other segments, appearing egg shaped as seen upon its broader surface, the smaller extremity being connected with the middle piece; seen in profile, it is convex on one side and concave on the other. The middle piece is somewhat longer and much thinner than the head, while the tail is a slender filament slightly more than four-fifths of the entire length of the spermatozoon. Lying in the center of the spermatozoon, and extending throughout its entire length, is the slender axial fiber, which is prolonged slightly beyond the tail as the end-piece or ter. 
minal filament. At the anterior end of the axial fiber is a small body, the end-knob (not shown in the figure).

The power of locomotion which the spermatozoon exhibits is conferred by the vibratile movement of its tail, accompanied by a rotation about its long axis through an arc of 90 degrees. The rate of progression is about 0.05 or $0.06 \mathrm{~mm}$, or its own length, per second.

Spermatozoa possess remarkable vitality, remaining active in the male genital tract for several days after death. In the genital passages of the female, they may retain their activity for several wecks, and when mounted and protected from evaporation they have been known to show vibratile motion after the lapse of nine days (Piersol). Weak alkaline solutions render them more active, while acids, even quite dilute, destroy them. The spermatozoa of the bat, being deposited in the female genital passages in the autumn, retain their power of fecundating ova until the following spring.

Spermatogenesis. - The details of spermatozoon-formation, or spermatogenesis, vary in different animals. A cross section of a seminiferous tubule of a mammal (Fig. 3) shows a layer of cuboidal cells called parietal cells, lying in contact

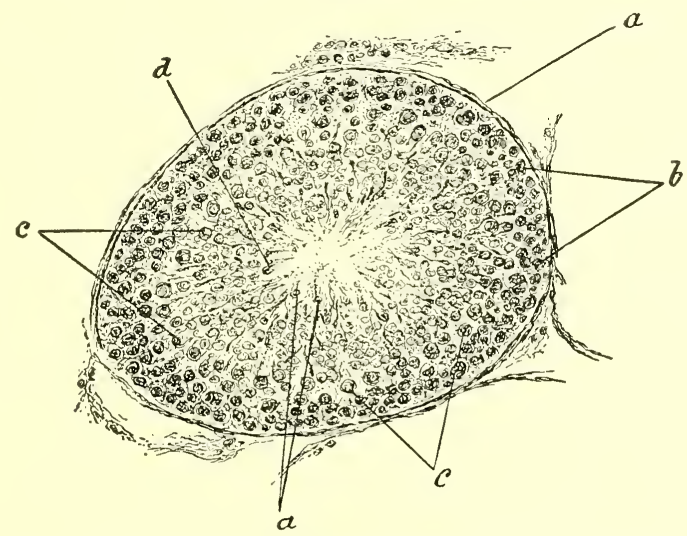

FIG. 3.-Section of testicle of musk-rat; seminiferous tubule seen in cross section : $a$, wall of tubule; $b$, parietal cells ; $c$, mother-cells ; $d$, spermatoblasts.

with the basement membrane of the tubule wall. This layer consists of the so-called Sertolli's columns, or sustentacular cells, and of the spermatogenic cells or spermatogonia. The sustentacular cells are merely supporting; the spermatogenic cells give rise to the spermatozoa. 
The spermatogonia undergo repeated mitotic division with a concomitant decrease in size. The last generation of the spermatogonia, after an intervening period of growth, give rise, also by mitotic division, to the mother-cells or primary spermatocytes, which lie nearer the lumen of the tubule than do their predecessors. The primary spermatocytes now divide to form the daughter-cells or secondary spermatocytes, and these in turn undergo division to form the spermatoblasts or spermatids. From the spermatids, by rearrangement of their constituent elements and certain special modifications in form, are produced the spermatozoa.

Not all the details of the differentiation of the spermatozoon from the spermatid are as yet clear; moreover, these details vary somewhat in different species. As observed in mammals, the nucleus of the spermatid becomes somewhat flattened and elongated to become finally the head (nucleus) of the spermatozoon. The centrosome migrates to that side of the nucleus which is toward the lumen of the tubule, becoming attached here to the nuclear membrane, while the attraction sphere (archoplasm) goes to the opposite side. The attraction sphere produces the head-cap and lance which are present in the spermatozoa of some mammals. From the centrosome a delicate filament grows through the cytoplasm, toward the lumen of the tubule, the centrosome itself-or centrosomes, as there may be two-giving rise to at least the neck of the middle piece, that is, the part adjoining the head (Meves and Lenhossék) or, according to others, persisting as the end-knob. Although the axial filament seems to grow forth from the centrosome it is believed by Meves that it is differentiated from the cytoplasm of the spermatid, which latter also gives rise to the remaining part of the middle piece and its sheath as well as to the tail and its sheath. Meanwhile the cytoplasm in relation with the nucleus is reduced to an exceedingly thin layer, a portion of it being cut off in some cases.

During the metamorphosis of the spermatids the Sertolli cells increase in size, elongating toward the lumen of the tubule. To each such Sertolli column a number of spermatids become attached, the Sertolli cell being apparently used up in yielding nourishment to the developing sperm-cells. 
The descent of the spermatozoa from the spermatogonia is accompanied by a peculiar modification of ordinary mitosis known as the reduction of the chromosomes, or reduction-division. The spireme, or chromatin thread, of the ordinary cells of the body, known as body-cells or somatic cells in contradistinction to the reproductive or germ-cells, breaks up at the beginning of mitosis into a definite number of segments or chromosomes, which number is constant and characteristic for the species. Thus in man, the guinea-pig, and the ox, there are sixteen chromosomes; in the mouse, the salamander, and the trout, twenty-four; in some sharks, thirty-six; in the grasshopper, twelve. After the division of the chromatin into the characteristic number of chromosomes, in the case of a somatic cell, each chromosome splits longitudinally, so that each daughter-nucleus receives as many chromosomes as there were in the parent cell. Thus the number of chromosomes remains constant notwithstanding repeated cell-divisons. In the division of the germ-cells, however, an important modification of this process has been observed, resulting in the reduction of the number of chromosomes. This reduction-division occurs both in the development of the spermatozoon and in the "maturation" of the ovum. The essence of reduction-division is that in the germ-cell the chromatin divides into half as many chromosomes as in the case of the somatic cell, but these chromosomes are tetrad and hence are equivalent to double the number of the somatic chromosomes; and that two subsequent cell-divisions occur without intervening reconstruction of the nucleus, so that one element of each tetrad passes to each one of the four descendent cells. Thus each of the four cells descended from any one germ-cell contains half as many chromosomes as a somatic cell, and when, during fertilization, such a (male) germ-cell unites with a similar (female) germcell the normal number of chromosomes is restored.

During spermatogenesis the multiplication of the spermatogonia is effected by the usual method of mitosis, as is also the formation of the primary spermatocytes from the last generation of the spermatogonia, but when the primary spermatocyte enters upon the process of mitosis its chromatin 
divides (in the case of the Ascaris megalocephala whose somatic chromosomes number four) into two chromosomes, each chromosome being tetrad. Without reconstruction of the nucleus the primary spermatocyte divides into two secondary spermatocytes (Fig. 4), each tetrad chromosome dividing into two dyads, one for each new nucleus. Again, without reconstruction of the nucleus, each secondary spermatocyte divides into two spermatids, each dyad breaking

Primordzal sexual cell.

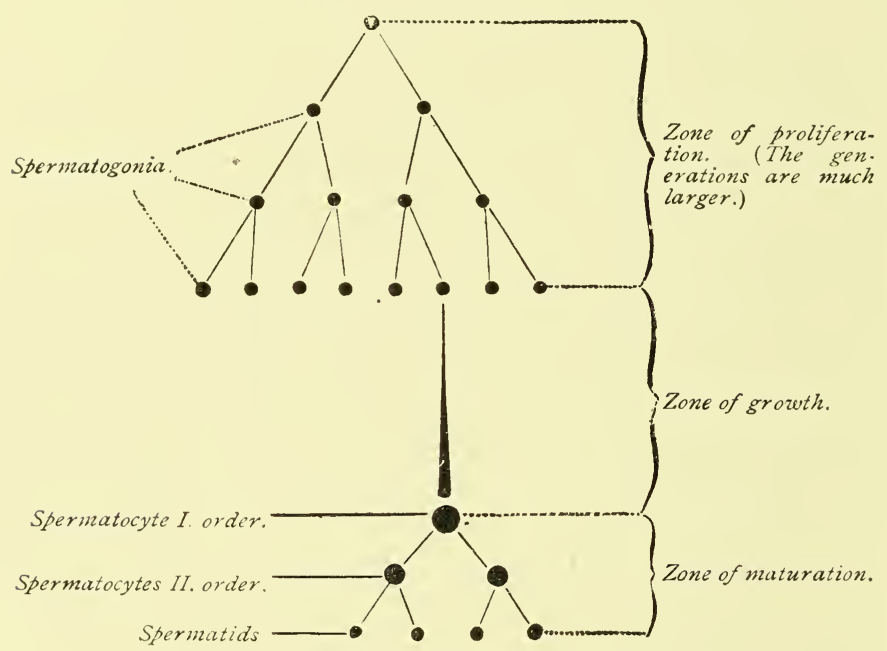

Fig. 4.-Schematic diagram of spermatogenesis as it occurs in ascaris (after Boveri). ("Ergebn. d. Anat. u. Entw.," Bd. I.)

up into two single chromosomes, one for each spermatid. Thus each spermatid contains half as many chromosomes as the somatic number characteristic for the species, and each primary spermatocyte is the parent of four spermatids, which subsequently become functional spermatozoa.

\section{THE OVUM.}

The female sexual cell or ovum is remarkable among animal cells for its size, it being a rule, to which there are no known exceptions, that it is much larger than any other cell in the body of the parent. The human ovum measures, in the mature state, $0.2 \mathrm{~mm}$. in diameter.

In structure, the ovum presents the parts of a typical cell ; 
namely, a cell-wall, here called the vitelline membrane, the cell-contents, or vitellus or yolk, a nucleus or germinal vesicle, and a nucleolus or germinal spot.

Surrounding the ovum is a somewhat loosely-fitting transparent, elastic envelope, the zona pellucida, and outside of this is the corona radiata. These two layers are often re-

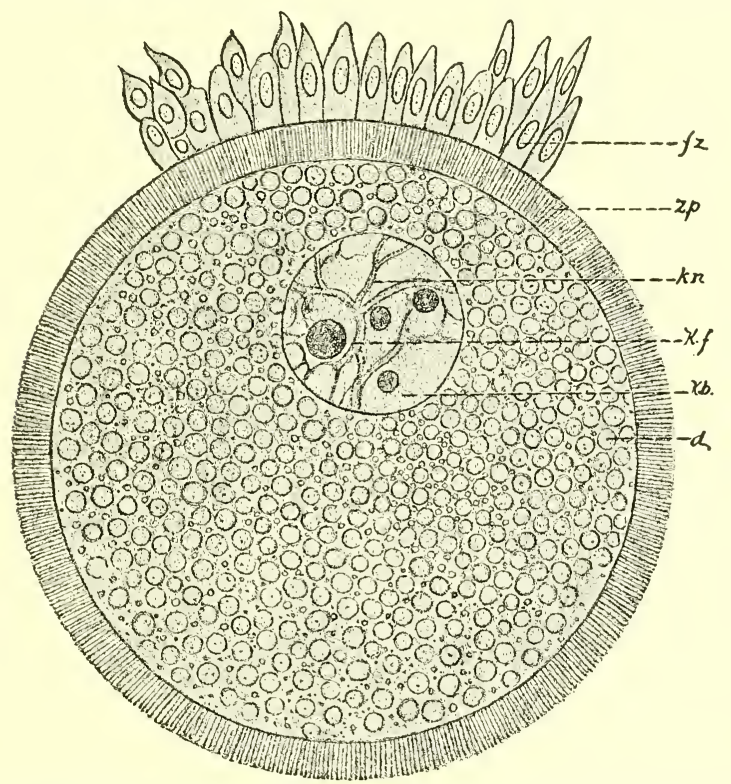

FIG. 5.-Egg from a rabbit's follicle which was $0.2 \mathrm{~mm}$. ( $\frac{1}{12} 5$ inch) in diameter (after Waldeyer). It is surrounded by the zona pellucida $(z \cdot p$.), on which there rest at one place follicular cells $(f, z)$. The yolk contains deutoplasmic granules $(d$.$) . In the germinative vesicle (k, b$. ) the nuclear network $(k, n$. $)$ is especially marked, and contains a large germinative dot $(k \cdot f$.).

ferred to as the egg-envelopes; but since they are contributed by the discus proligerus of the Graafian follicle, it must be remembered that they are not, properly speaking, a part of the orum. Between the zona pellucida and the ovum is the small perivitelline space. The radial striation of the zona is generally regarded as due to the presence of minute canals opening into this space. The canals are thought by some to facilitate the ingress of spermatozoa, thus corresponding in function to the micropyle, a small aperture found in the less easily penetrable egg-envelopes of many invertebrates and of some fishes. 
The vitelline membrane does not call for extended description. It may be regarded as a slightly specialized condensation of the peripheral part of the cell-contents.

The vitellus, or cell-contents, here, as in other cells, is essentially protoplasm or cytoplasm, to which is added material called deutoplasm, designed for the nutrition of the ovum at the beginning of development. The protoplasm is also called the formative yolk and the egg-plasm, while the deutoplasm is known as the nutritive yolk. In the human ovum these elements are more or less uniformly distributed; there is, however, a differentiation into an inner, slightly less clear region, containing more yolk-granules (deutoplasm) and a peripheral, clearer zone. The characteristic transparency of the human egg-cell is due to the fact that the deutoplasmic particles found in it are not cloudy as in the ova of other mammals. The following classification of ova by Balfour is based upon the arrangement of these constituents:

1. Alecithal ova are those in which the protoplasm and

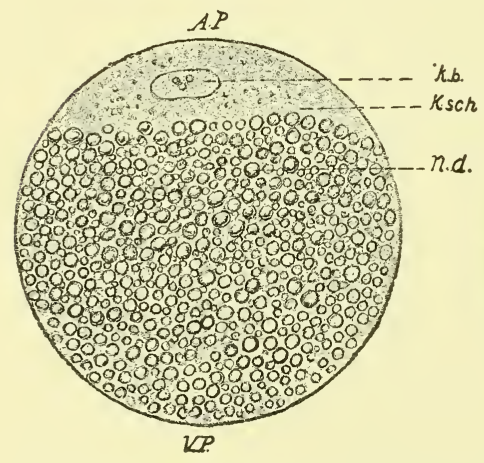

FIG. 6.-Diagram of an egg with the nutritive yolk in a polar position. The formative yolk constitutes at the animal pole (A.P.), a germ-disk (K.sch.) in which the germinative vesicle $(k, b$.$) is en-$ closed. The nutritive yolk $(n . d$.) fills the rest of the egg up to the vegetative pole ( $V . P$.) (Hertwig).

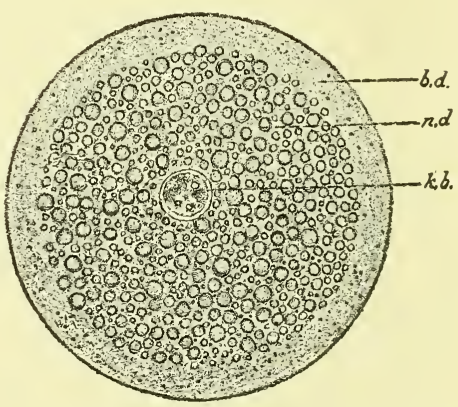

Fig. 7.-Diagram of an egg with the nutritive yolk in the center. The germinative vesicle $(k . b$.) occupies the middle of the nutritive yolk (n.d.), which is enveloped in a mantle of formative yolk (b.d.) (Hertwig).

dentoplasm are uniformly distributed, as in the ova of Mammalia, including man), and of amphioxus (Fig. 5).

2. Telolecithal ova are those in which the relatively abun- 
dant deutoplasm is accumulated at one side of the ovum, called the vegetative pole, while the protoplasm appears as a flat germ-disk at the animal pole on the opposite side. Here belong the eggs of birds, reptiles, and bony fishes (see Fig. 6).

3. Centrolecithal ova are those in which the deutoplasm is central, the protoplasm completely surrounding it, as in the eggs of arthropods (Fig. 7).

Ova are classified also according to their method of segmentation. This will be described later.

The germinal vesicle or nucleus is the most important part of the cell, since, as will be seen hereafter, it is essentially by the conjugation, or more accurately by the fusion, of the nuclei of the male and female parent-cells that generation is effected. As a rule, there is but one nucleus, though there may be two. Its position is usually - if not universallyeccentric, this being more marked where there is a distinct differentiation into animal and vegetative poles, in which ease it is found always near the animal pole. It is nearly spherical in shape, and like the nueleus of any other typical cell, it is composed of the nuclear network consisting of linin and chromatin, and nuclear juice or achromatin, the former containing the latter within its meshes. Surrounding the nucleus is the well-marked nuclear membrane, while within it is the nucleolus or germinal spot. The latter may be single or multiple, according to the species, though the number is fairly constant for each species. Nagel ascribes ameboid movement to the germinal spot.

Polarity.- The polarity of the egg has been incidentally referred to. Apparently it owes its existence to the eccentric position of the nucleus, the animal pole being that point on the surface to which the nucleus is nearest. Polarity bears a significant relation to the specific gravity of the ovum, since the nucleus reaches the surface of the latter at the animal pole and there extrudes the polar globules; and it is also related to the segmentation of the fertilized egg.

The Hen's Egg. - As the hen's egg is so largely utilized for the study of development, it will be profitable to consider briefly its structure. The ovum or egg-cell is represented by 
the yolk or yellow of the egg, the albumen or white, as well as the shell and shell-membrane, being egg-envelopes contributed by the oviduct. As in other ova, the egg proper is a single cell, having a vitelline membrane and a germinal vesicle. The enormous size of the cell is due to the large quantity of nutritive material or deutoplasm present, this contributing by far the greater part of the bulk, while the much smaller formative yolk or protoplasm, containing the germinal vesicle, is so eccentrically placed that it seems to float upon the surface of the deutoplasm. The little whitish spot on the surface of the yolk, known as the cicatricula or germinative disk, consists of the germinal vesicle with the surrounding formative yolk. It is in the germinative disk alone that segmentation takes place, and it is for this reason that eggs of this class are designated meroblastic, or partiallydividing eggs.

The deutoplasm is made up of white and of yellow yolk

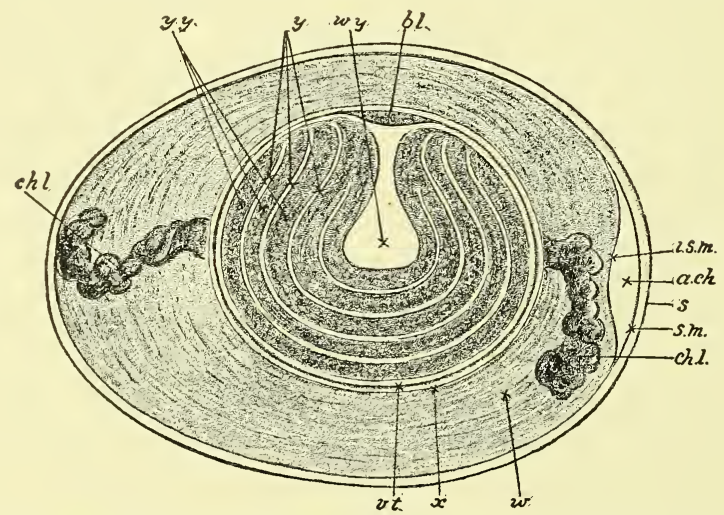

FIG. 8.-Diagrammatic longitudinal section of an unincubated hen's egg (after Allen Thomson). (Somewhat altered): $b, l$, germ-disk; $w . y$, white yolk, which consists of a central flask-shaped mass, and a number of concentric layers surrounding the yellow yolk (y.y.); v.t, vitelline membrane; $x$, a somewhat fluid albuminous layer which immediately envelopes the yolk; $w$, albumen, composed of alternating layers of more and less fluid portions; ch.l, chalazæ; a.ch, airchamber at the blunt end of the egg-simply a space between the two layers of the shell-membrane; $i . s . m$, inner, $s . m$, outer layer of the shell-membrane; $s$, shell.

(Fig. 8). The former consists of a thin layer spread over the surface of the latter; of a small mass, known as Pander's 
nucleus, situated under the germinative disk; of a larger mass, the latebra, more deeply placed; and of several concentric layers separated from each other by the yellow yolk.

Such is the egg as it leaves the hen's ovary. In the beginning of the oviduct it is fertilized by the spermatozoa already there. After fertilization it passes into the longitudinally furrowed second part of the tube, where it receives a copious coating of albuminous material, the white of the egg; thence it goes into the villous third part of the oviduct, where it acquires a calcareous coating, the shell; finally, passing through the fourth part of the canal, it is "laid."

The layer of albumen immediately surrounding the yolk is relatively dense; it is prolonged to either extremity of the egg, somewhat spirally twisted, as the chalazæ. Enclosing the albumen is the thin tough shell-membrane. This consists of two layers, which separate at the blunt pole of the egg soon after it is laid, giving rise to the air-chamber. The shell, composed largely of lime salts, is very porous and thus readily permits of the necessary gas-interchange between the contents of the egg and the external air during incubation.

Ova do not possess the remarkable vitality which is characteristic of spermatozoa. An unimpregnated ovum perishes in from seven to nine.days.

Oogenesis.-The formation of ova takes place throughout the greater part of fetal life and continues for a short time (two years, according to Waldeyer, Bischoff, and others) after birth. Their number is estimated to be about serenty thousand.

The ovum, the direct derivative of the germinal epithelium covering the free surface of the ovary, is situated in the cortical part of the latter organ, being enclosed in the Graafian follicle. As a rule, each Graafian follicle or ovisac contains but one ovum, though sometimes two, and more rarely three are present.

The Graafian follicle, in its mature condition, is a vesicle from 4 to $8 \mathrm{~mm}$. in diameter, which is surrounded by a sheath, the theca folliculi or tunica vasculosa, consisting of a condensation of the ovarian stroma. The outer, more fibrous 


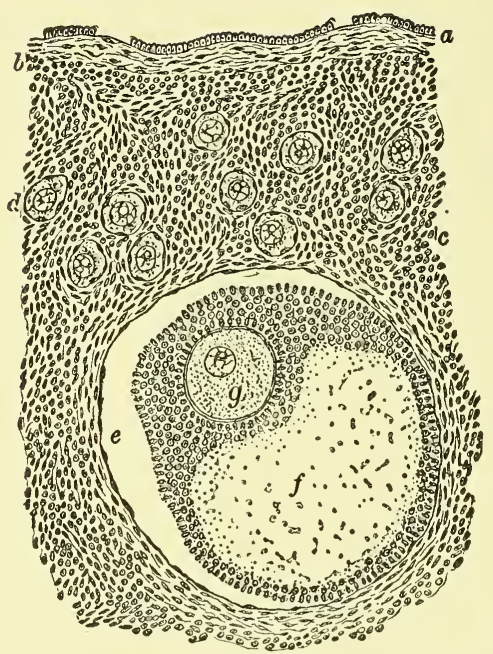

FIG. 9.-Section of human ovary, including cortex : $a$, germinal epithelium of free surface; $b$, tunica albuginea; $c$, peripheral stroma containing immature Graafian follicles $(d)$; $e$, well-advanced follicle from whose wall membrana granulosa has partially separated; $f$, cavity of liquor folliculi; $g$, ovum surrounded by cell-mass constituting discus proligerus (Piersol).

zone of the theca, containing large blood-vessels, is distinguished as the tunica fibrosa; the inner more cellular layer, rich in small vessels and capillaries, as the tunica propria.

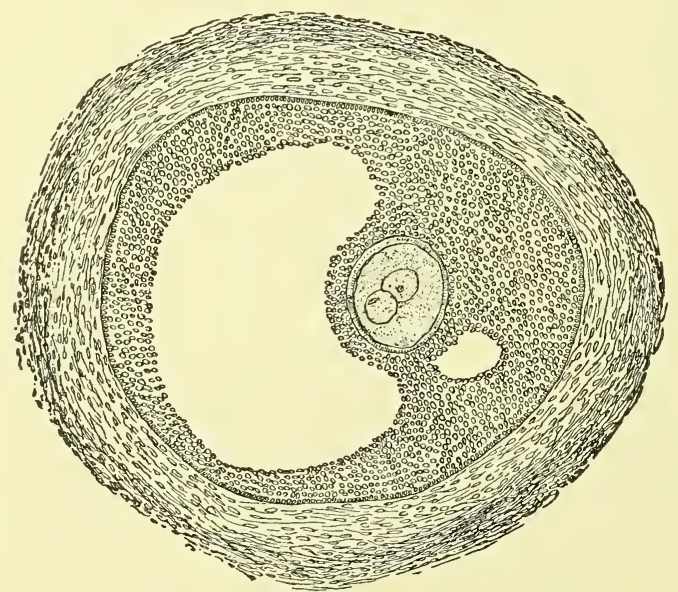

FIG. 10.-Section of well-developed Graafian follicle from human embryo (Von. Herff); the enclosed ovum contains two nuclei. 
The fibrous wall of the follicle is lined by the membrana granulosa, which consists of many layers of epithelial cells; these, at the point of contact with the ovum, project in such a manner as to surround it completely, the cellular envelope thus formed constituting the discus proligerus. The inner cells of the discus are arranged in two layers, the individual elements having their long axes radially directed. From the appearance of radial striation, conferred partly by this circumstance, the inner zone has been called the zona radiata or zona pellucida, and the outer the corona radiata. The cavity of the Graafian follicle is filled with fluid, the liquor folliculi.

The stigma, or hilum folliculi, a yellowish-white spot devoid of blood-vessels on the free surface of the Graafian follicle, indicates the point at which rupture will take place. After this event, which occurs when the ovum is "ripe," the latter passes into the Fallopian tube.

The ultimate origin of the $\operatorname{egg}^{1}$ is to be sought in that important group of cells on the surface of the ovary to which Waldeyer gave the name germinal epithelium. This first appears at about the fifth week of intra-uterine life, as a localized thickening of the cells of the structure that subsequently becomes the peritoneum. The thickened areas comprise two longitudinal elevations on the dorsal side of the future abdominal cavity, one on each side of the median plane of the body; these are the genital ridges. Owing to the development of connective tissue beneath the epithelium, the ridges increase in thickness, and, with the progress of other changes, finally become, in the female, the ovaries. At about the sixth or seventh week-the germinal epithelium now consisting of several layers of cells instead of being a single stratum thick, as at first-cord-like processes, the sexual cords, or primary egg-tubes, or egg-columns, grow from the surface into the underlying connective tissue, carrying with them certain of the surface-cells (see Fig. 128). Conspicuous among these are the large sexual cells, or primitive ova; while smaller cells, likewise from the germinal epithelium, are

\footnotetext{
${ }^{1}$ See p. 250.
} 
also present. The sexual cords become divided into groups of cells, each group containing one or more primitive ova and many of the smaller cells. Gradually, the small cells of the group surround the primitive ovum, at first as a single layer of flattened cells, which are succeeded by several layers of polygonal cells. From these enveloping cells come the membrana granulosa and the theca of the Graafian follicle.

The primitive ova or oögonia-analogous to the spermatogonia-having undergone repeated mitotic division, cease to divide at a certain period of their history and enter upon a period of rest and growth. They thus increase in size and become fully formed ovarian eggs or oöcytes, the nucleus enlarging and the cytoplasm becoming more or less laden with deutoplasmic material or food-stuffs.

The youngest ova are found nearest the surface of the ovary, the eggs as they develop advancing toward, but never entering, the medulla of the organ. Finally, in the fullydeveloped condition of the ovum and the follicle, the size of the latter is such that its diameter equals or exceeds the thickness of the ovarian cortex, its position being usually indicated by a small prominence on the surface of the ovary.

\section{MATURATION OF THE OVUM.}

By maturation or ripening is meant that series of changes by which the ovum is prepared for fertilization and without which the latter process is impossible. In nearly all mammals, including man, it occurs while the ovum is still in the Graafian follicle; in some other groups it takes place after the egg has reached the oviduct.

Briefly, maturation may be said to consist in the extrusion from the cell of a part of its nucleus and of a small part of its cytoplasm. The nucleus undergoes changes practically identical with those of ordinary cell-division. First, the nuclear membrane disappears, the nucleolus disintegrates, the nuclear juice becomes mingled with the surrounding protoplasm, and the nucleus moves toward the periphery of the egg (Fig. 11). There is now formed a nuclear spindle from the achromatin substance of the nucleus. The long axis of 


\section{PLATE I.}
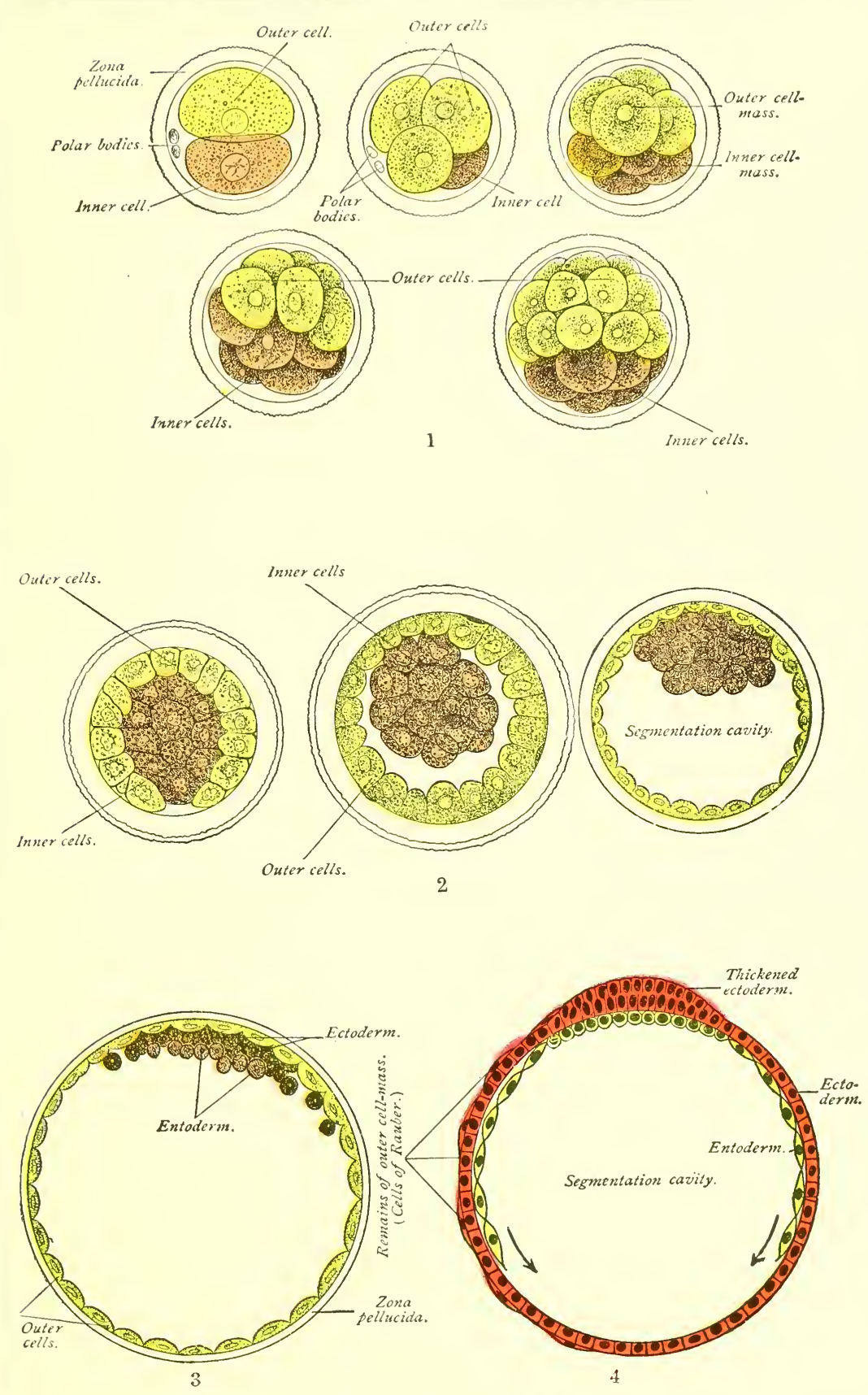

1, 2,3. Diagrams illustrating the segmentation of the mammalian ovum (Allen Thomson, after van Beneden). 4. Diagram illustrating the relation of the primary layers of the blastoderm, the segmentation-cavity of this stage corresponding with the archenteron of amphioxus (Bonnet). 

the spindle lies parallel with one of the radii, and its direction is determined by the position of the pole-corpuscles. Each pole-corpuscle is surrounded by a radiation, the attraction-sphere or polar striation. These bodies exercise a controlling influence upon the nuclear spindle, so that it assumes
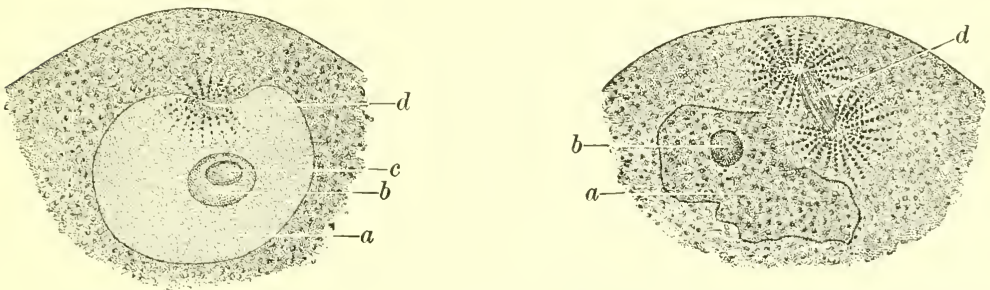

FIG. 11.-Portions of the ova of Asterias glacialis, showing changes affecting the germinal vesicle at the beginning of maturation (Hertwig): $a$, germinal vesjele; $b$, germinal spot, composed of nuclein and paranuclein $(c) ; d$, nuclear spindle in process of formation.

such a position that each of its apices points toward a polecorpuscle.

The outer extremity of the nuclear spindle, being made to protrude by the continued onward movement of the nucleus, becomes detached (Fig. 12); this separated piece, with the
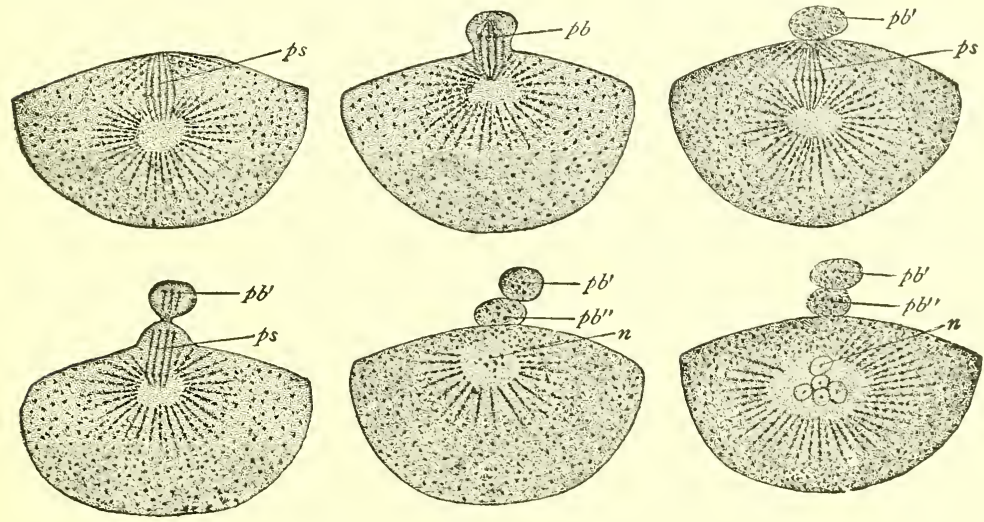

FIG. 12.-Formation of the polar bodies in the ova of Asterias glacialis (Hertwig): $p s$, polar spindle; $p b^{\prime}$, first polar body ; $p b^{\prime \prime}$, second polar body ; $n$, nucleus returning to condition of rest.

small surrounding constricted-off mass of protoplasm, constitutes the first polar body. From the remnant of the first 
nnclear spindle, a second one is formed, which in the same manner extrudes the second polar body. What remains of the nucleus now moves toward the center of the cell and is known as the female pronucleus. The position of the female pronucleus is nearly or absolutely central. The egg is now ready for fertilization.
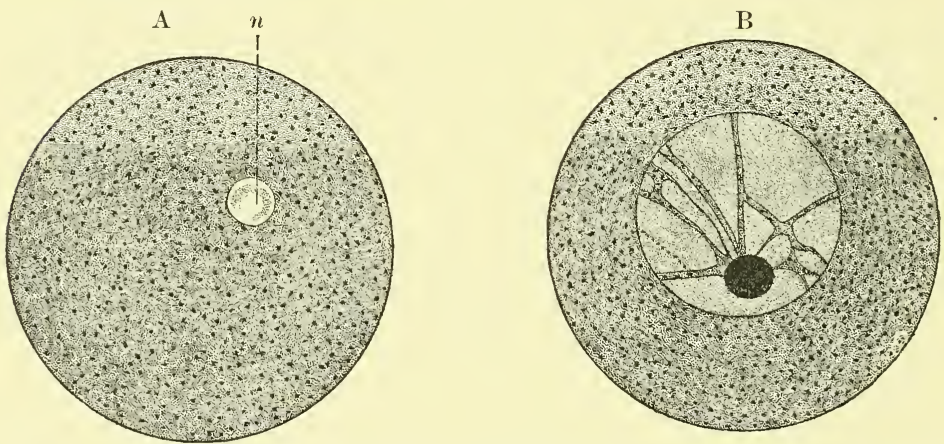

FIG. 13.-A, mature ovum of echinus; $n$, female pronucleus; $B$, immature ovarian ovum of echinus (Hertwig).

For some time after their extrusion, and pending their final disappearance and disintegration, the polar bodies are to be seen lying in the perivitelline space. The formation of polar globules is probably almost universal throughout the animal world. It is of interest to note that in some parthenogenetic eggs - that is, eggs capable of developing into a new individual without contact with the male element, as, for example, the summer eggs of plant lice and of some other arthropods-only one polar globule is said to be formed, and it has recently been shown (Sobotta) that in the maturation of the ovum of the mouse only one polar body was formed in the majority of cases.

The maturation of the ovum is essentially a reduction of the chromosomes precisely analogous to the reduction-division seen in the descent of the spermatozoon from the primary spermatocyte. The last generation of ooggonia having increased in size after their stage of rest, and having thus become the ovarian eggs or primary oöcytes, now undergo mitosis, but in a manner differing from that of their predecessors. The chromatin thread of the nucleus, instead of 
dividing into the number of chromosomes characteristic for the somatic cells, divides into half that number. These chromosomes are tetrads, that is, each one consists of four more or less loosely associated element: conceived to result from a primary longitudinal splitting of the chromosome, followed possibly by a transverse division of the two halves. In the division of the primary oöcyte (Fig. 14) to form two secondary oöcytes (one of which is the first polar body) each tetrad is halved so that the same number of dyads goes to

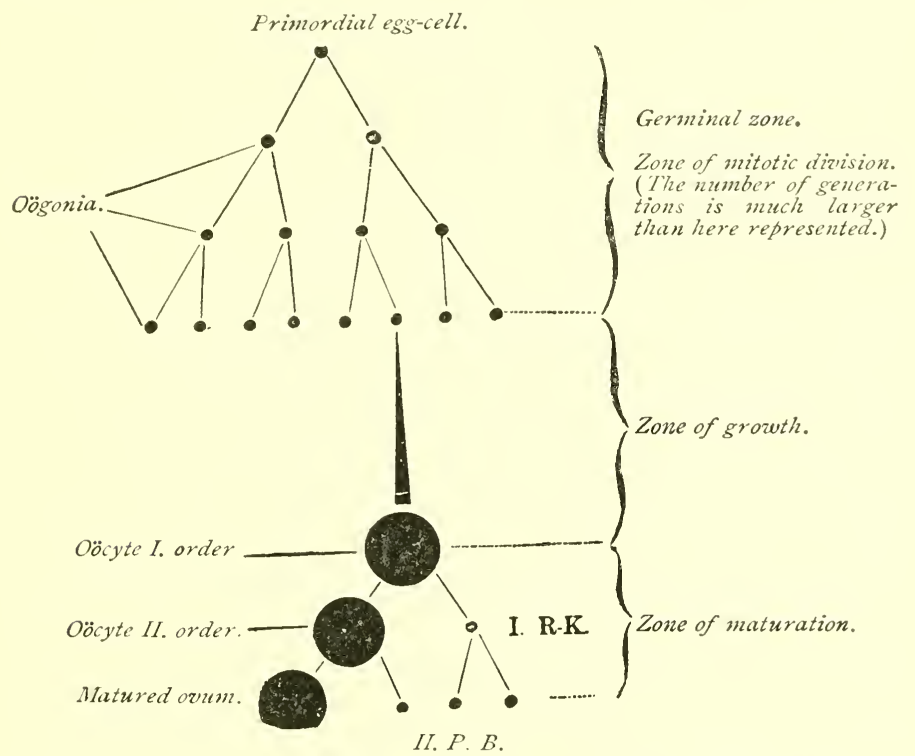

FIG. 14.-Scheme of the development and maturation of an ascaris ovum (after Boveri): P. B., Polar bodies. (From “Ergebn. d. Anat. u. Entw.," Bd. I.)

each new nucleus. The secondary oöcytes now undergo mitosis, but without reconstruction of the nucleus, each dyad chromosome giving one of its elements to each of the new cells, that is, to the now mature orum and the second polar body.

It will be apparent, therefore, that the casting off of the polar bodies is a cell-division, but one which results in the production of cells of very unequal size. It is noteworthy, as pointed out by E. B. Wilson, that the chromatin of the nucleus is exactly halved at each division, notwithstanding the disproportion in the division of the eytoplasm. 
In comparing the phenomena of maturation with those of spermatogenesis it is to be noted that in the latter case all four progeny of the primary spermatocyte become functional spermatozoa, while in the former case three of the progeny come to naught, only one of the number, the mature ovum, being functionally important. E. B. Wilson points out that the reduction of the chromosomes in the germ-cells is for the purpose of maintaining the constancy of the number of chromosomes which is peculiar to the species, since, if reduction did not occur, the number would be doubled at each generation; he further points out, however, that "the real problem is why the number of chromosomes should be held constant."

\section{OVULATION.}

Extrusion of the ovum from the Graafian follicle, or ovulation, occurs upon the completion of the process of maturation. As the time for this event approaches, the wall of the follicle at the site of the stigma becomes much thinned and finally ruptures, and the ovum passes into the Fallopian tube (Fig. 15). If, instead of passing into the tube, the

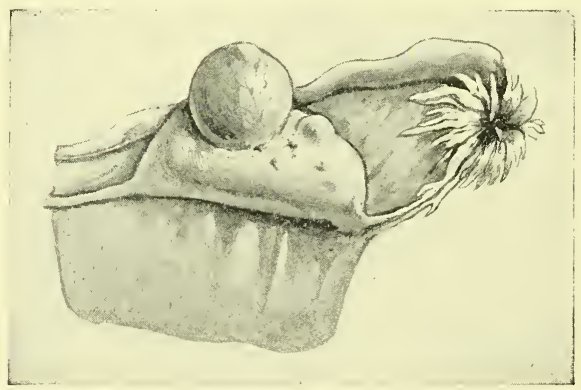

FIG. 15.-Ovary with mature Graatian follicle about ready to burst (RibemontDessaignes).

ovum maintains its connection with the ovary and is fertilized there, it may undergo partial development in siiu; such a condition constitutes one variety of extra-uterine pregnancy or ectopic gestation. ${ }^{1}$

Ova are extruded from the ovary, one or more at a time,

1 Other varieties of ectopic gestation are abdominal and tubal, the names of which are sufficiently descriptive. 
at regular, generally monthly, intervals, from puberty to the climacteric.

After the escape of the ovum, hemorrhage into the empty follicle oceurs, the resulting clot being the corpus hemorrhagicum. According to Leopold, if rupture occurs during the intermenstrual period instead of at the time of menstruation, hemorrhage will be small or entirely wanting, the resulting corpus luteum being called then atypical, to distinguish it from the typical body formed in the ordinary manner.

The blood-clot is soon permeated by cells originating in the wall of the follicle, some of which are fusiform connective-tissue cells, while others are large cells containing the yellow pigment, lutein. Meanwhile, the follicular wall thickens and becomes plicated. Later, upon the replacement of the mass of clot and cells by fibrous tissue and the development of eapillaries within it, the body assumes a yellowish cicatricial appearance and is known as the corpus luteum. (Fig. 16). The color of the corpus varies considerably in
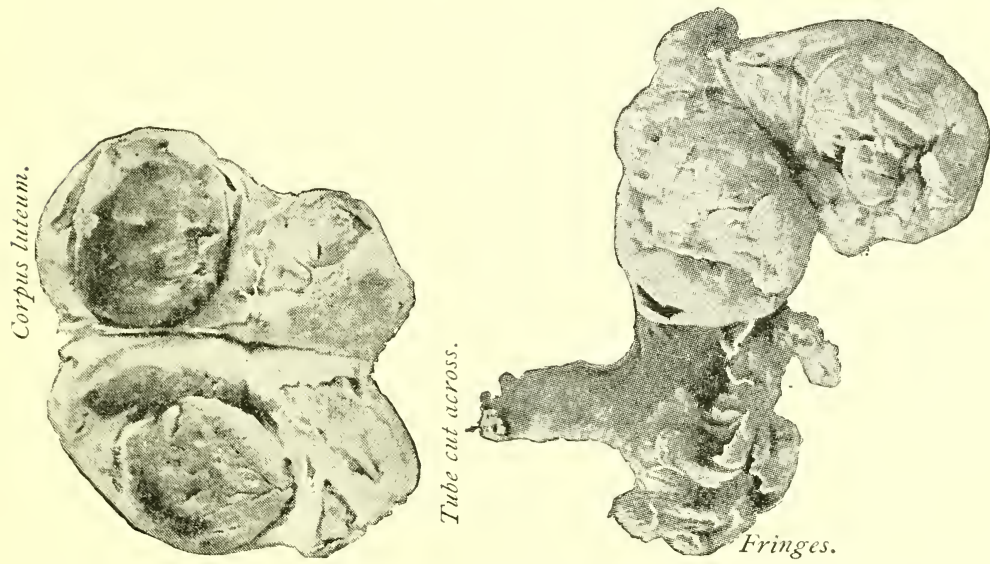

FIG. 16.-Ovaries of two virgins, showing large corpora lutea, resembling those of pregnaney (Hirst).

different species of animals, the yellow color being characteristic for the human subject.

If the ovum is not fertilized, the corpus luteum attains its maximum development in less than a week and begins to shrink at about the twelfth day, becoming completely ab- 
sorbed in a few weeks. If fertilization occurs, it continues to grow for two or three months and acquires a size onefourth or one-third that of the entire ovary ; persisting till toward the end of gestation, it finally shrinks to a small white scar, which may not totally disappear until a month or more after labor.

It has been customary to designate the larger, better developed yellow body, the true corpus luteum, or the corpus luteum of pregnancy, in contradistinction to the so-called false corpus luteum of menstruation, and to regard the presence of the former as absolute proof of previous impregnation. This view is no longer tenable, since bodies identical in appearance with true corpora lutea have been found in virgin ovaries (Hirst).

The relation of ovulation to the menstrual function has been much discussed. While the two processes usually occur at the same time, they are not to be regarded as dependent one upon the other. It has been shown by Coste, whose observations have been confirmed by Leopold, that as a rule Graafian follicles burst during menstruation, though they may rupture before or after this event. It has also been shown that in the rabbit sexual intercourse hastens the rupture of the follicle.

\section{MENSTRUATION.}

Menstruation, or the catamenial flow, is considered here because of its natural association with the function of ovulation.

Menstruation may be defined as a periodical discharge of blood and disintegrated epithelium and other structural elements of the mucous membrane of the body of the uterus, mixed with mucus from the uterine glands and the vagina, occurring normally about every twenty-eight days, and associated with more or less disturbance of the entire sexual system. The inauguration of the function marks the age of puberty, the beginning of the sexual life of woman; its cessation, known as the climacteric, or menopause, indicates the termination of the child-bearing period.

In temperate climates, the menses are established between 
the thirteenth and seventeentl years and cease between the ages of forty and fifty. In the tropics, they appear somewhat earlier; in cold climates, somewhat later. 'The function is suspended during pregnancy and, usually, during lactation.

The quantity of the discharge, though subject to considerable variation, is usually from 4 to 6 fluidounces. The blood is venous in character, and, owing to armixture of alkaline mueus, does not coagulate unless present in excessive amount.

The menstrual cycle of twenty-eight days may be divided into four periods: the constructive stage, comprising from five to seven days; the destructive stage, lasting about five days; the stage of repair, covering a period of three or four days; and the stage of quiescence, including the remaining twelve to fourteen days.

In the constructive stage, which occupies the six to seren days preceding the discharge, the mucous membrane of the uterus becomes markedly swollen, the normal thickness of from 1 to 2 millimeters being more than doubled. The uterine glands become wider and longer and also more branched. The blood-ressels, especially the capillaries and veins, undergo great increase in size, and the connective-tissue cells are increased in number. The thickened mucous membrane resulting from these alterations is the decidua menstrualis. The term "constructive" is applied to this series of changes for the reason that their apparent purpose is the preparation of the womb for the reception of a fertilized ovum.

The destructive stage, corresponding to menstruation proper, lasts from three to five days. It consists essentially in the partial destruction of the hypertrophied mucous membrane, the menstrual decidua, acompanied by hemorrhage. The initial step is the infiltration of blood into the subepithelial tissue; according to Overlach, this takes place, not by rupture of eapillaries, but by diapedesis. In a day or two the superficial layers of the mucous membrane disintegrate and are cast off, those portions of the enlarged uterine glands included within this stratum sharing the same fate. By the loss of the epithelium and the subjacent strata, the blood- 
vessels are exposed. Subsequently these rupture, giving rise to the characteristic hemorrhage. Fatty degeneration accompanies the death of the cast-off tissue, and was thought by Kundrat and Engelman to be the direct cause of the hemorrhage; it is probable, however, that fatty degeneration is not present until after the flow of blood has begun. ${ }^{1}$

The stage of repair, comprising the three or four days following the period of the discharge, witnesses the return of the uterine mucosa to its usual condition. With the gradual subsidence of the swelling, the superficial layers, which were lost, are replaced by the growth of new tissue from the deeper layers, which persisted. The formation of the new epithelium begins at the mouths of the uterine glands.

The stage of quiescence extends from the close of the preceding stage to the end of the cycle, or, in other words, to the beginning of the next constructive stage.

Other parts of the sexual apparatus, including the ovaries, the Fallopian tubes, and the mammary glands, show more or less sympathy with the uterus during menstruation, the changes in them consisting chiefly in swelling, hyperemia, and tenderness.

\section{The Relation of Menstruation to Ovulation and} Conception.--The function of menstruation and the extrusion of ova from the Graafian follicles, though closely associated, are not dependent upon each other. Ovulation occurs perhaps most commonly during the time of the menstrual discharge, but it may take place before or after this event. While it is now generally accepted that the two functions are not mutually interdependent in the sense that one is a necessary part of the other, yet, since the turgescence incident to sexual intercourse has been shown to hasten the rupture of the follicles, it seems reasonable to suppose that the ovarian hyperemia attendant upon the menstrual epoch would exert a like influence.

Since the function of menstruation is normally suspended during pregnancy, the relation between menstruation and

1 Marshall's "Vertebrate Embryology;" Minot's "Human Embryology." 
ovulation, and of these to conception, are of practical interest in determining the date of labor. The duration of pregnancy is from 270 to 280 days, nine calendar, or ten lunar, months, and it dates from the moment of conception. But since the ovum retains its vitality for about a week after its extrusion from the Graafian follicle, and since the activity of the spermatozoa may continue for several weeks after their entrance into the female genital tract, it is impossible to fix accurately the date of eonception even in those cases in which there has been but one coitus. It is now believed by most embryologists that the orum is fertilizable only while it is in the Fallopian tube, a period probably of about seven days; if this be true, it follows that conception must occur within a week after ovulation, although it may be effected as late as two weeks after coitus. Since the orum is usually discharged from the ovary during the menstrual period, it is evident that the time most farorable for conception is the week following menstruation; and inasmuch as the latter function is suspended during pregnancy, it is obvious that the most reliable basis for ealculating the probable date of conception is the last menstruation. The method usually employed is to count nine months and seven days from the first day of the last menstruation. After what has been said it is perhaps needless to remind the reader that this can furnish only approximately the date of labor. In a case where conception occurred a few days prior to the first omitted period, there would be a discrepancy of several weeks between the actual, and the calculated, termination of pregnancy.

\section{FERTILIZATION.}

Fertilization is that peculiar union of spermatozoon and egg-cell which initiates the phenomena resulting in the formation of a new individual. As implied in a preceding section, impregnation is possible in the higher organisms only after the completion of maturation, while in others, as for example the maw-worm of the horse, spermatozoa enter the orum before the extrusion of the polar bodies, and thus one process overlaps the other. 
The more primitive method of fertilization is that effected without copulation of the parent organisms, or external fertilization; this occurs in osseous fishes, in some amphibians, and in many invertebrates. In these groups, both ova and semen are discharged into the water and there meet. In frogs, however, there is a quasi-copulation, the male embracing the female during the breeding season and depositing semen upon the eggs as they are evacuated. In all higher animals, internal fertilization occurs, this being effected by sexual congress.

In man, fertilization normally occurs in the outer third of the Fallopian tube. The semen having been deposited in the vagina, or the uterus, or even upon the vulva, the spermatazoa make their way into the oviduct by the vibratile motion of their tails. Meeting the ovum, they swarm around it, and some of them pass through the zona pellucida into the perivitelline space. It is believed by many investigators that the canals of the zona constitute the avenues of entrance for the spermatozoa. In the rather firm egg-envelopes of insects and some fishes, there is a small aperture, the micropyle, through which the spermatozoa gain entrance.

While many spermatozoa may pass through the zona, only one--that one whose head first impinges against the vitelline membrane-enters the ovum. Why others do not or cannot enter is unknown; possibly because the egg's power of attraction is annulled (Minot). Polyspermia, or the penetration of several spermatozoa, may occur, however, if the ovum is unhealthy; and in some lower types it is said to be normal.

As the spermatozoon is about to strike the vitelline membrane, the protoplasm swells up at the point of contact into the receptive prominence (Fig. 17). Through this the spermatozoon bores its way, its tail being absorbed by the eytoplasm of the ovum or being left outside in the case of the sea-urchin. The middle piece, or at least a part of it, including the end-knob, which latter represents the centrosome of the spermatid, enters the ovm with the head (nucleus) of the spermatozoon. This nucleus or head now enlarges, becoming thus the male pronucleus. The male and 
female pronuclei approach each other and finally meet in the center of the ovum, the two bodies apparently fusing to form the single segmentation-nucleus or cleavage-nucleus. It must not be understood, however, that an actual single membranate nucleus is formed. As the pronuclei approach each other the centrosome of the spermatozoon lies between them surrounded by its attraction-sphere, and gives rise to a nuclear spindle after the manner of ordinary mitosis, the chromatin threads of the two pronuclei lying in relation with its equator, but on opposite sides from each other. In other words, the chromatin contributed respectively by the two pronuclei
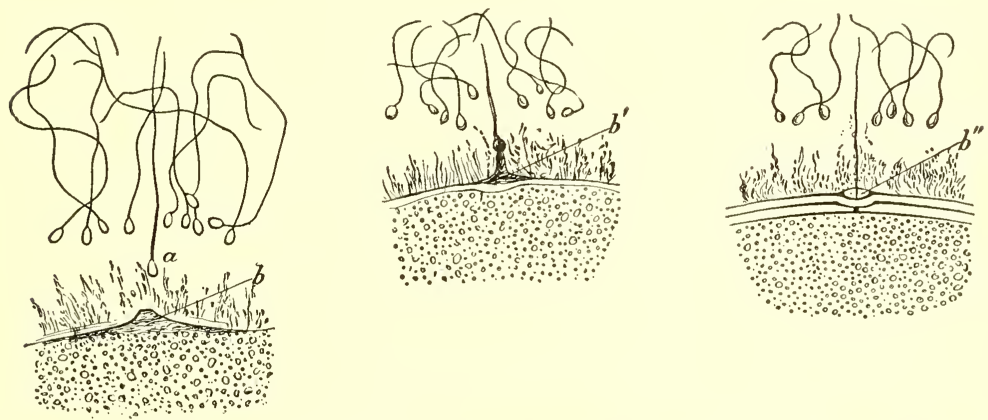

FIf. 17,-Portions of the ova of Asterias glacialis, showing the approach and fusion of the spermatozoon with the ovum (Hertwig): $a$, fertilizing male element; $b$, elevation of protoplasm of egg; $b^{\prime}, b^{\prime \prime}$, stages of fusion of the head of the spermatozoon with the ovum.

retains in each case its identity, the "segmentation-nucleus" entering upon the processes of mitotic division without previous intermingling of the chromatin of the ovum with that of the spermatozoon. As will be shown in Chapter II., one-half of each chromatin threar goes to one pole of the spindle, while the other half of each goes to the opposite pole, to give rise to the two daughter-nuclei resulting from this first segmentation of the segmentation-nucleus. It will be evident that the segmentation-nucleus consists of chromatin substance derived from each parent. As this fact has been thought to explain, anatomically, the offspring's inheritance of both paternal and maternal characteristics, it has been 
made the basis of a theory of heredity formulated by Hertwig and independently advanced by Strasburger.

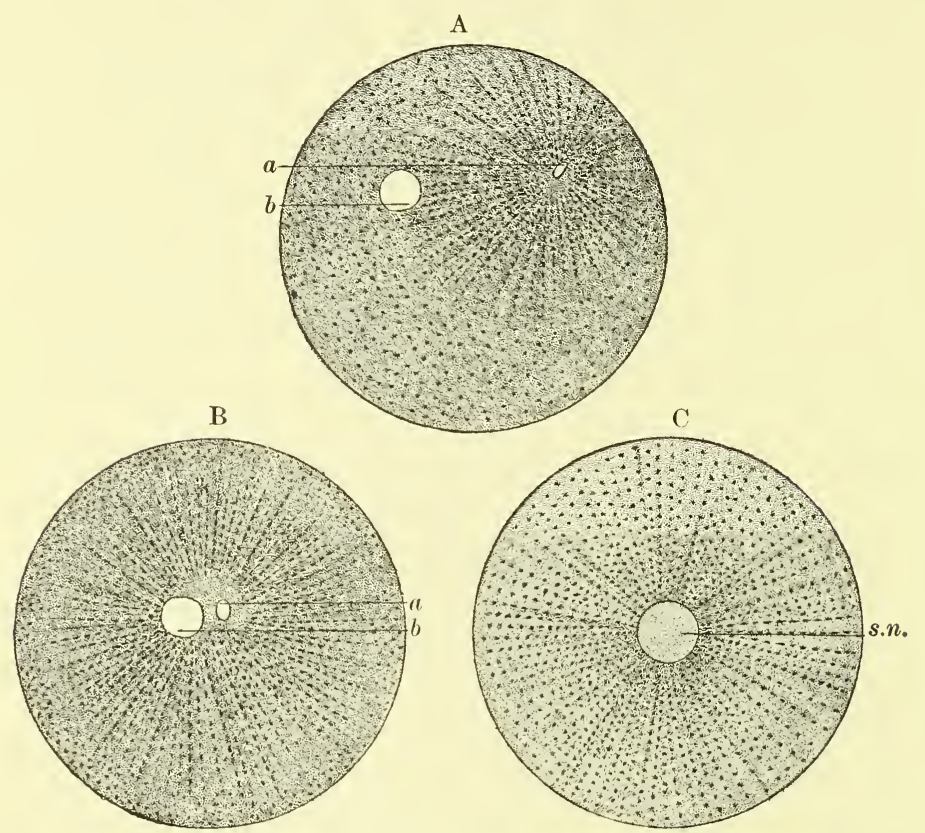

FIG. 18.-A, fertilized ovum of echinus (Hertwig): the male (a) and the female pronucleus (b) are approaching; in B they have almost fused; $\mathrm{C}$, ovum of echinus after completion of fertilization (Hertwig): s.n., segmentation-nucleus.

Artificial fertilization, or the bringing about of the derelopment of the ovum by artificial (chemical) means, without the participation of the male element, has been recently experimentally effected with the eggs of the sea-urchin by Loeb, of Chicago. These eggs, when first immersed for about two hours in a mixture of sea-water and a weak solution of magnesium chlorid, and then transferred to normal seawater, were found to undergo complete and normal development, producing perfect larvæ. This artificially induced development differed from that of the ordinary method only in being slower. 


\section{CHA P'TER II.}

\section{THE SEGMENTATION OF THE OVUM AND FORMA= TION OF THE BLASTODERMIC VESICLE.}

While the fertilized orum is passing along the Fallopian tube to the uterus-a journey believed to require seven or eight days in man ${ }^{1}$ - it undergoes repeated segmentation, or cleavage, becoming a more or less globular mass of cells or blastomeres. This mass is the mulberry-mass or morula.

The details of the proces of division correspond closely to those of ordinary indirect cell-division, or karyokinesis. The first indication of approaching cleavage is seen in the segmentation-nuclens, just as, in other cells, the sequence of changes leading to cell-division is inaugurated in the nueleus.

The achromatin-substance of the segmentation-nucleus forms a nuclear spindle in the ordinary manner, with a centrosome or pole-corpuscle at each apex. The centrosome is surrounded by the polar striation or attraction-sphere. After the usual preliminary changes, the chromatin-substance assumes the form of $V$-shaped loops arranged around the equator of the spindle in such a manner as to produce the wreath or aster. Each chromatin loop splits longitudinally, and the resulting halves of each move to opposite poles of the spindle, where they become grouped about the pole-corpuscle to constitute the daughter-wreaths of the new nuclei. The vitellus now begins to divide, the first step being the formation of an encireling groove on its surface; this groove deepens more and more until finally division of the cell is complete. In like manner, each daughter-cell divides into two, and each of these two into other two, the cell-division continuing until there results the mass of cells, or morula, already mentioned (Plate I., Fig. 1). The two cells or blastomeres resulting from the division of the segmentationnucleus do not al ways divide simul taneously; if the division of

${ }^{1}$ Recent investigations by Peters, of Vienna, upon an ovum of three or four days, already embedded in the uterine mucosa, would indicate that less time than this is occupied in traversing the oviduct. 
one cell precedes that of the other there will be a stage when three blastomeres are present. Further irregularity in division results in the production of five-celled and six-celled stages.

These processes have been followed the most accurately in the egg of the sea-urchin; in reptilian eggs, as well as in those of the rabbit and other mammals, they have been studied also and have been found to agree with the former in all essential respects. Certain modifications dependent upon the relations and proportions of formative-yolk and food-yolk will be pointed out hereafter.

While no one has seen the segmentation of the human ovum, there is no reason to suppose that it differs materially from that of other mammals.

An interesting and probably significant modification of the method of cleavage as just described has been observed by Van Beneden in the ova of the maw-worm of the horse. In this case male and female pronuclei do not fuse but merely lie close together. At the beginning of segmentation, the chromatin of each pronucleus assumes the form of a convoluted thread, which divides transversely into two sisterthreads. In this manner are produced four loops of chromatin, which become grouped around the equator of the nuclear spindle just formed, and each one of which then splits longitudinally into two threads. In the migration of the segments that now ensues, each pair of sister-threads separates, one thread going to one pole of the spindle, one to the other. Hence, at each pole, and taking part, therefore, in the formation of each new nucleus, are two male and two female threads of chromatin. Thus the male and female pronuclei contribute equal shares of chromatin to each daughter-nucleus.

Since the segmentation-nucleus of the ovum gives rise to all the cells of the body, every cell of the adult organism must consist of equal amounts of material from each parent.

Cleavage-planes. - The direction of the planes of cleavage is determined by certain laws. The direction of the plane of the first cleavage bears a definite relation to the long axis of the nuclear spindle, whose position, in turn, depends upon the manner of distribution of the egg's proto- 
plasm, its direction coinciding with the longest diameter of an oval egg, but lying in any diameter of a spherical one. The first cleavage-plane always cuts the axis of the nuclear spindle perpendicularly at its center; the second bisects the tirst, also perpendicularly; and the third is perpendicular to the two others and passes through the middle of their axis of intersection.

Kinds of Cleavage.-The mode of cleavage of the ovum is influenced by the relation of the protoplasm and the deutoplasm to each other, and by their relative proportions. The classification of ova according to their method of cleavage is as follows:

1. Holoblastic ova are those in which segmentation is total - that is, the entire ovum undergoes division. If the resulting cells are of equal size, there is said to be

(a) Total equal cleavage; to this class belong the alecithal ova of mammals (Fig. 5) and of amphioxus, to the segmentation of which the above description may be said to apply. Strictly speaking, the cells are not of exactly equal size, those in the region of the vegetative pole of the egg being slightly larger than those at the animal pole. Contrasted with this is

(b) The total unequal cleavage of amphibian ova, whose segments are of unequal size (Fig. 19). These eggs being
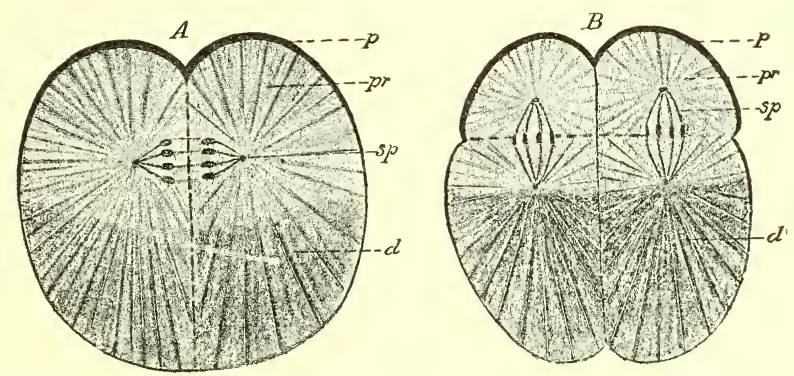

FIG. 19.-Diagram of the division of the frog's egg: $A$, stage of the first division. $B$, stage of the second division. The four segments of the second stage of division are beginning to be divided by an cquatorial furrow into eight segments; $p$, pigmented surface of the egg at the animal pole; $p r$, the part of the egg which is richer in protoplasm; $d$, the part which is richer in deutoplasm; $s p$, nuclear spindle (Hertwig).

telolecithal, the lignter protoplasmic animal pole is directed upward, while the deutoplasmic vegetative pole is under- 
neath. The inequality of the resulting segments, as well as the direction of the cleavage planes, may be appreciated by reference to Fig. 19, which represents a frog's ovum.

2. Meroblastic ova are those in which the segmentation is partial, division being limited to the formative yolk, or protoplasm.

(a) Partial discoidal cleavage is the variety of meroblastic cleavage that occurs in those telolecithal ova having a germdisk (Fig. 8), to which latter the segmentation is limited. This method of segmentation is seen in the eggs of birds, reptiles, and fishes. In the egg of the bird, which may be taken as a typical example, the germ-disk, in whatever position the egg may be placed, floats on the top of the yolk. The beginning of the first segmentation is indicated by a furrow in the center of the surface of the germ-disk (Fig. 20). This furrow deepens, eutting vertically from the
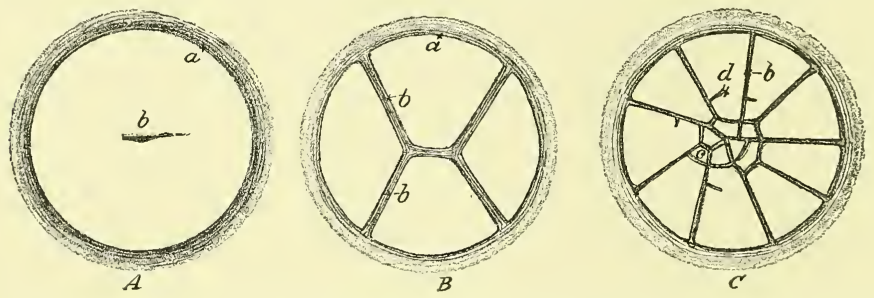

FIG. 20.-Surface view of the first stages of cleavage in the hen's egg (after Coste) : $a$, border of the germ-disk; $b$, vertical furrow ; $c$, small central segment; $d$, large peripheral segment.

upper to the lower surface of the germ-disk, dividing it into two equal parts. Another groove, crossing the first at a right angle, bisects each of the two segments, and each of these is in turn bisected by a radial furrow, so that the germ-disk now consists of eight sector-shaped cells. Cross furrows, appearing near the center of the disk, cut off the apices of the sectors, adding small central segments. Celldivision continues until the germ-disk consists of a flattened mass of cells, several strata thick, lying on the surface of the yolk.

The second method of meroblastic segmentation is

(b) Peripheral cleavage, which occurs in the centrolecithal ova of arthropods (Fig. 7). In these eggs, it will be remem- 
bered, the nutritive-yolk is centrally placed and is surrounded by the formative-yolk. The segmentation-nucleus lies in the center of the nutritive-yolk, and in this position undergoes division and subdivision. The new nuclei now migrate into the peripherally placed formative-yolk, when the latter divides into as many parts as there are nuclei, and thus the central unsegmented nutritive-yolk becomes enclosed in a sac composed of small cells.

\section{THE STAGE OF THE BLASTODERMIC VESICLE.}

The blastomeres of the morula soon show a differentiation into two groups of cells, a peripheral or outer and a central or inner group (Plate I., Fig. 2, left figure). 'This differentiation is indeed foreshadowed by the fact that of the first

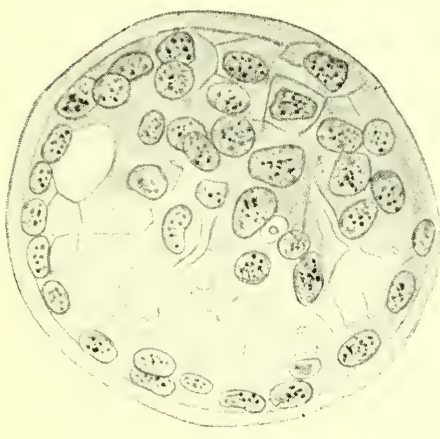

FIG. 21--Ovum of the bat, showing vacuolation of the segmented egg to form the blastodermic cavity. $\times 500$ (Van Beneden).

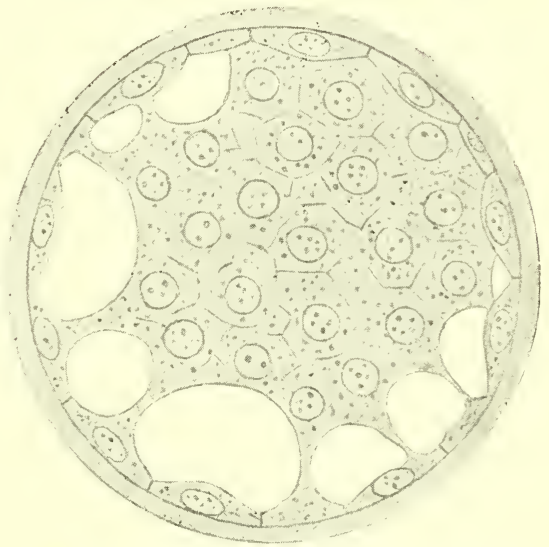

FIG. 2.2.-Ovum of the bat, showing vacuolation of the segmented egg to form the blastodermic cavity. $\times 600$ (Van Beneden).

two blastomeres, one is slightly larger than the other. Vacuoles now appear in some of the central cells, and these, becoming larger, finally coalesce to form a fissure-like space, the cleavage-cavity or segmentation-cavity, the lecithocele of Van Beneden (Figs. 21 and 22). The ovum is now in the stage of the blastodermic vesicle.

It will be profitable to compare this stage of the mammalian ovum with the corresponding blastula stage of the 
lancelet, or amphioxus lanceolatus, one of the lowest vertebrates, a fish-like animal several inches in length inhabiting the Mediterranean Sea. The blastula in this case is a simple sac composed of cells which surround the cleavage-cavity as a single layer (Fig. 26, A). The cells in the region of the vegetative pole are larger and more turbid, because more deutoplasmic, than those at the animal pole, as shown in the same figure.

The mammalian blastodermic vesicle, varying in shape in different species, consists of a layer of somewhat flattened cells, the enveloping or subzonal layer (the "outer cell-mass"), surrounding a space, the clearage-cavity or segmentationcavity, and of an irregular mass of more spherical cells, the

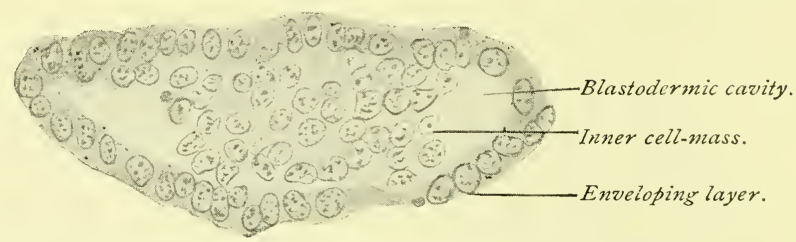

FIG. 23.--Ovum of the bat, showing inner cell-mass (Van Beneden).

inner cell-mass, which latter is attached at one point to the enveloping layer and encroaches upon the space (Fig. 23 and Plate I., Fig. 2). The cleavage-cavity contains an albuminous fluid. It is during this stage that the germ, in the case of mammals, reaches the uterus. As a peculiarity of mammalian development the blastodermic vesicle now rapidly increases in size, the cleavage-cavity becoming relatively much larger. The zona pellucida, which still surrounds the ovum, is by this time quite attenuated and is called the prochorion. ${ }^{1}$ The cells of the enveloping layer thin out to constitute what is known as Rauber's layer. ${ }^{2}$ In the rabbit embryo, Rauber's layer, being functionless, disappears at about the seventh day; in most mammals, however, it persists to play a part in further development

1 The term prochorion is also applied to a coating of albuminous material which the ovum receives as it passes along the oviduct.

2 Some authors define Rauber's layer as that part of the enveloping layer which covers the embryonic shield. 
(Van Beneden). The form of the blastula of amphibians and of the Sauropsida (birds and reptiles) is greatly modified by the relatively abundant nutritive-yolk with which their ova are endowed. An amphibian ovum in the blastula stage is shown in Fig. 24. It will be seen that its walls consist of several layers of cells, and the cleavageeavity is encroached upon to a considerable extent by the large and abundant cells of the vegetative pole, which are especially rich in deutoplasm. In the

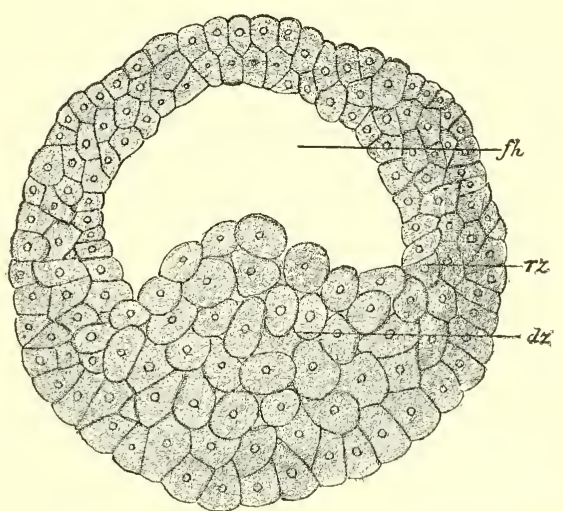

FIG. 24.-Blastula of triton tæniatus: $f h$, segmentation-cavity; $r z$, marginal zone; $d z$, cells with abundant yolk (Hertwig). eggs of birds and reptiles-that is, in the telolecithal eggs that undergo partial discoidal segmentation-the blastula form is so markedly modified as to be scarcely recognizable. In this case, as shown in Fig. 25, the cleavage-cavity is a narrow fissure

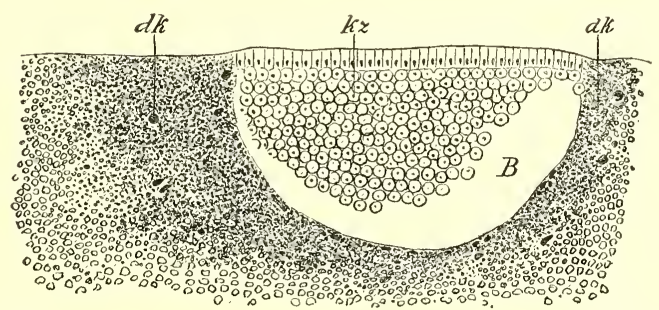

FIG. 25.-Median section through a germ-disk of pristiurus in the blastula stage (after Rickert): $B$, cavity of the blastula; $k z$, segmented germ; $d k$, finely granular yolk with yolk-nuclei.

whose roof is the germ-disk, and whose floor is the unsegmented nutritive-yolk, which latter corresponds therefore to the large vegetative cells forming the floor of the amphibian egg shown in Fig. 24. 


\section{CH A P'T E R I I I.}

\section{THE GERM=LAYERS AND THE PRIMITIVE STREAK.}

\section{THE TWO=LAYERED STAGE OF THE BLASTODERMIC VESICLE.}

Is studying the complicated and obscure phenomena of the formation of the germ-layers in mammals, comparison with what occurs in certain lower forms is helpful. In the case of the amphioxus, the one-layered stage, the blastula is succeeded by the sac-like double-layered gastrula stage. The gastrula, in its typical form, consists of two layers of cells surrounding a central cavity, which latter communicates with the exterior by means of a small aperture, the blastopore. The cavity is the archenteron or celenteron or intestino-body cavity. The outer layer of cells is the ectoderm or epiblast; the inner layer is the entoderm or hypoblast. This form of the germ is scen in holoblastic invertebrate, as well as in some vertebrate ova, and is typically exemplified in the development of the amphioxus. The blastula of this animal is a simple sac, the wall of which is a single layer of epithelial cells surrounding the cleavage-cavity (Fig. 26, A). By a pushing-in of the vegetative cells, the cleavage-cavity is encroached upon and finally is completely obliterated, being replaced by the archenteron (Fig. 26, C). From this it is obvious that gastrulation occurs here by a simple process of invagination. In ova with a large amount of food-yolk, as in those of frogs, birds, and fishes, the process is modified and complicated by this condition. Still further modifications of the process are seen in the development of mammals, since their ova again show a reduction in the amount of fool-yolk.

According to the so-called gastrula theory of Haeckel, all metazoa - that is, multicellular animals as distinguished from protozoa, or unicellular organisms-pass through a typical gastrula stage in the course of their development.

It has been held as a general principle that the higher 
animals during their development repeat, to a greater or less extent, the embryonic or the larval forms of the lower members of the group to which they belong. Huxley has pointed out the morphological identity of the adult form of the colenterata with the two-layered gastrula.

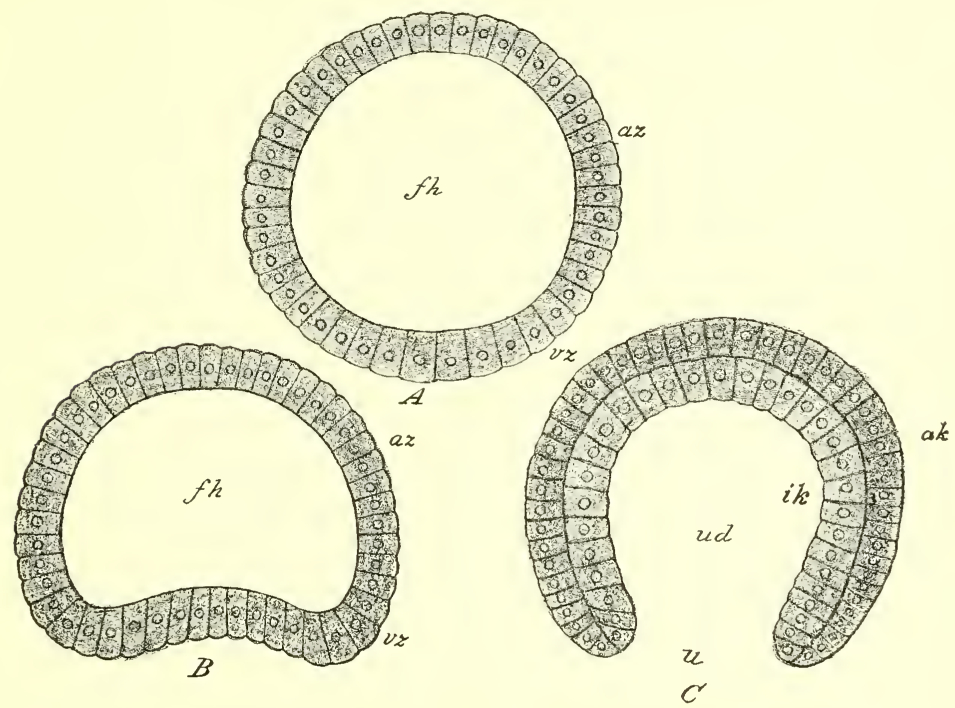

FIG. 26.-Gastrulation of amphioxus (modified from Hatschek). A. Blastula: $a z$, animal cells; $v z$, vegetative cells; $f h$, cleavage-cavity. $B$. Beginning invagination of vegetative pole. $C$. Gastrula stage, the invagination of the vegetative cells being complete: $a k$, outer germ-layer; $i k$, inner germ-layer; $u d$, archenteron; $u$, blastopore.

It must not be understood, however, that we find in mammals a gastrula stage such as that of amphioxus; we do find, indeed, that the single-layered blastodermic vesicle as described above acquires two and, still later, three cellular layers, but to what extent the layers of the didermic blastodermic vesicle correspond to the ectoderm and entoderm of the gastrula of lower types is not quite clear; nor are all the details of the growth of these layers as yet clear. The phenomena have been studied in the mole, the rabbit, the bat, the sheep, and the $\log$, as well as in some other mammals. Aceording to the investigations of Van Beneden upon the development of the rabbit and the bat, the inner cell-mass spreads out upon the inner surface of the enveloping layer and shows a differentiation into two groups of cells. One group, occupy- 
ing the center of the mass, Van Beneden's embryonic bud (Fig. 27) consists of cells that are at first globular, but later cuboidal; the second group, composed of flatter and darker cells, covers continuously that surface of the mass which looks toward the blastodermic cavity and soon extends beyond the limits of the mass to line the inner surface of the enveloping layer, thus constituting the entoderm (Fig. 27). Some of the cells of the lighter group now vacuolate, the several vacuoles later becoming confluent to form one cavity, which is the future amniotic cavity (Fig. 28). In the rabbit this vacuolation does not occur, at least not at this stage. The more or less globular mass of cells remaining after the vacuolation is known as the embryonic bud or

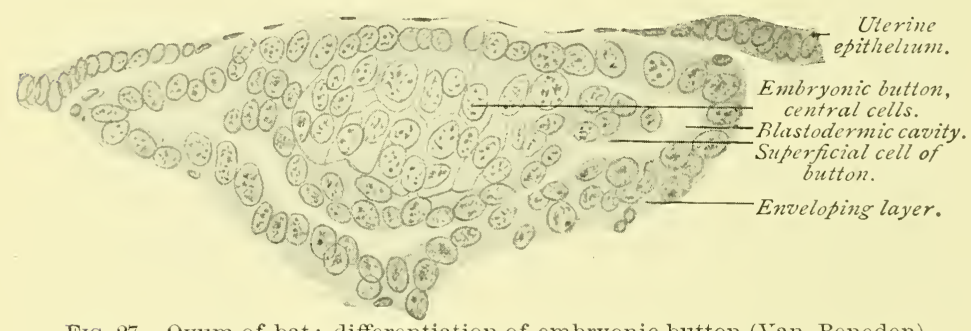

FIG. 27.-Ovum of bat; differentiation of embryonic button (Van Beneden).

embryonic button or embryonic disk (Fig. 29). It will be observed that the amniotic cavity lies between the embryonic bud and the enveloping layer. It is important to note that the embryonic bud is the anlage of the body of the embryo, that it is from this group of cells alone that the embryonic body develops.

The enveloping layer, which disappears in the rabbit at about the seventh day, but which persists in other mammals so far as known, resolves itself into two laminæ in the region overlying the embryonic bud and the amniotic cavity, an inner layer, the cystoblast, and an outer, the plasmodoblast or placentoblast, composed of flattened cells. The cystoblast now constitutes the immediate roof or vault of the amniotic cavity, while a layer of cells differentiated from the amniotic surface of the embryonic bud forms the floor of the cavity. This latter lamina becomes the outer layer of the didermic 
embryo, that is, it represents the embryonic ectoderm, while the enveloping layer would correspond to the extra-embry-

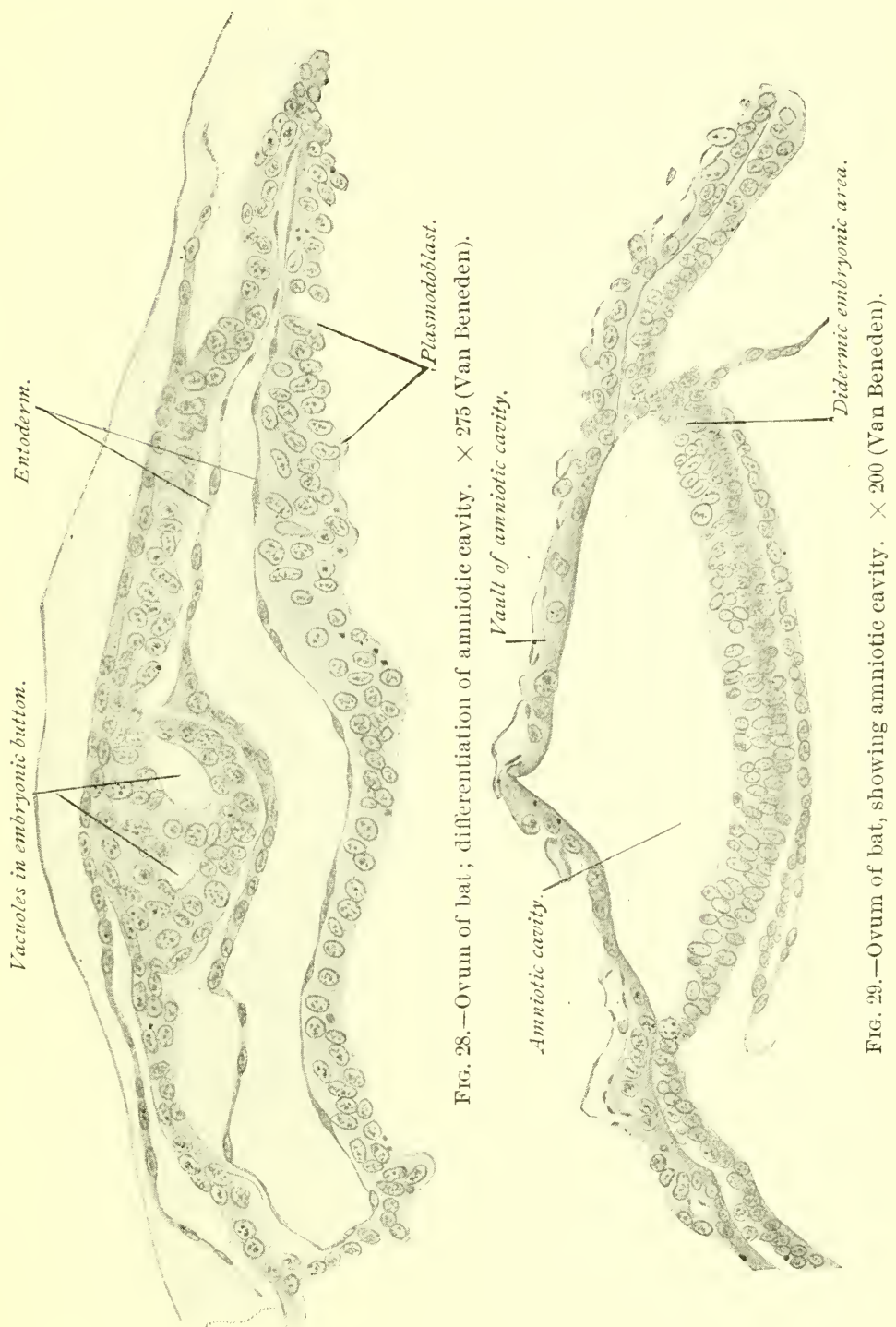

onic ectoderm. Somewhat later the eystoblast in the vault of the amniotic eavity disappears and the lamina of cells referred to above as forming the floor of the amniotic eavity 
becomes continuous at the periphery of the embryonic bud with the enveloping layer.

Comparison of bat and mole embryos with the orum of Peters (Fig. 30) shows that essentially the same phenomena occur in the development of the human ovum. The ovum of Peters was estimated by him to be between three and four days old and was the youngest human orum as yet studied. One may see here the embryonic bud or disk E. Sch., the

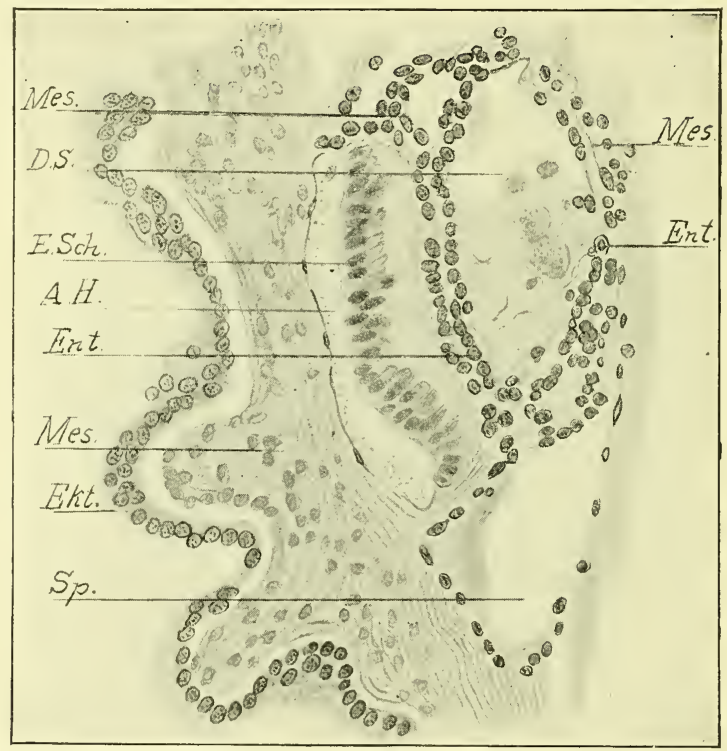

FIG. 30.-Section through embryonic region of ovum. First week of pregnancy (H. Peters): E. Sch, Embryonic epiblast; Ent., embryonic hypoblast; Mcs., embryonic mesoblast; D.S., umbilical vesicle ; Eht., chorionic epiblast; Sp., fold in exocolom; A.H., amniotic cavity lined by a single layer of flattened cells, which are in striking contrast with the layer of cylindric cells of the embryonic epiblast.

amniotic cavity $A . H$., the extra-embryonic ectoderm forming the vault of this cavity, the entoderm Ent., and likewise the yolk-sac $D$. S., since this ovum is some what more advanced in development than the stage now under consideration.

From the foregoing account one may see that the singlelayered blastodermic vesicle of mammals becomes converted into the two-layered or diploblastic vesicle, consisting of the entoderm and the ectoderm, not, as in the amphioxus, by a simple process of invagination, but largely by a rearrange- 
ment of the cells of the inner cell-mass; and that, partly as a consequence of this rearrangement of cells, the amniotic cavity is produced and a differentiation becomes manifest between a group of cells, the embryonic bud, which is to serve for the development of the embryonic body, and other cells which are destined to produce the accessory organs or envelopes of the developing embryo.

As previously stated, the process of gastrulation and the form of the gastrula are modified in the case of ova possessing a large proportion of deutoplasm. In the case of the frog, for example, as well as in other amphibians, the blastula has the form shown in Fig. 24. By an invagination of the blastula-wall at the place of transition from the animal cells to the vegetative cells, all of the latter and a part of the former are carried into the interior of the blastula to form the lining of the archenteron (Fig. 31). Compare this with the amphi-

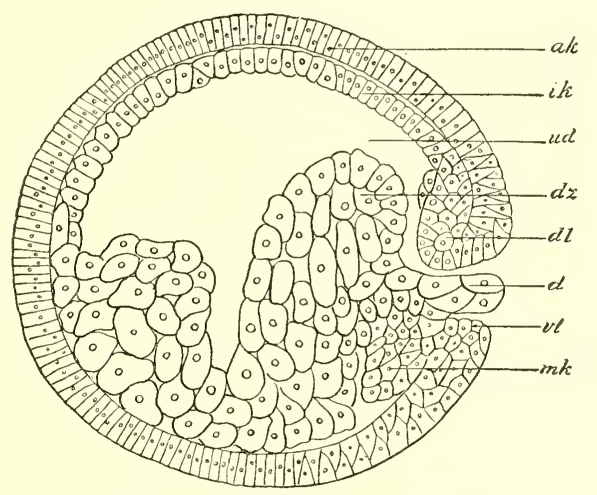

FIG. 31.-Sagittal section through an egg of triton (after the end of gastrulation): $a k$, outer germ-layer; $i k$, inner germ-layer; $d z$, yolk-cells; $d l$ and $v l$, dorsal and ventral lips of the cœlenteron; $u d$, cœlenteron; $d$, vitelline plug; $m k$, middle germ-layer (Hertwig).

oxus gastrula as shown in Fig. 26. In the bird's egg, the form of whose gastrula is shown in Fig. 32, an infolding or invagination occurs, as in the frog's egg, at the place of transition from the animal cells to the vegetative cells, or, in other words, at the margin of the germ-disk. The gastrula thus formed is represented in Fig. 32. Its archenteron is a 
narrow fissure, and its blastopore, situated at the posterior margin of the germ-disk, is exceedingly small.

The Embryonal Area.-Upon the surface of the germ at the beginning of gastrulation-that is, at about the fifth day of development in the case of the rabbit's germ-there is

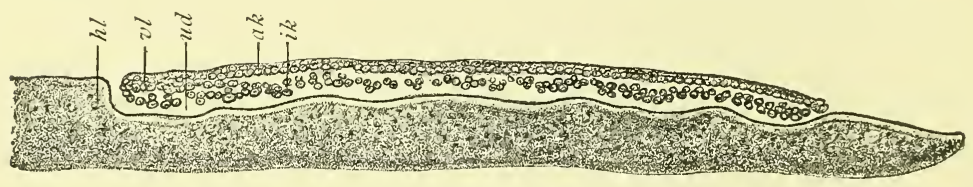

FIG. 32.-Longitudinal section through the germ-disk of a fertilized unincubated egg of the nightingale (after Duval): $a k$, outer, $i k$, inner germ-layer; $u d$, coelenteron; $v l$, anterior, $h l$, posterior lip of the blastopore (crescentic groove).

a round whitish spot, the embryonal area. Its position corresponds to that formerly held by the inner cell-mass of the blastodermic vesicle, as shown in Plate I., Fig. 2. It is only in this region that the wall of the vesicle is, at this particular stage, composed of more than a single layer of cells, the ectoderm and the entoderm not extending much, if at all, beyond its periphery.

The embryonal area, soon becoming oval (Fig. 33) and, later, pear-shaped, exhibits, at its posterior margin, a trans-

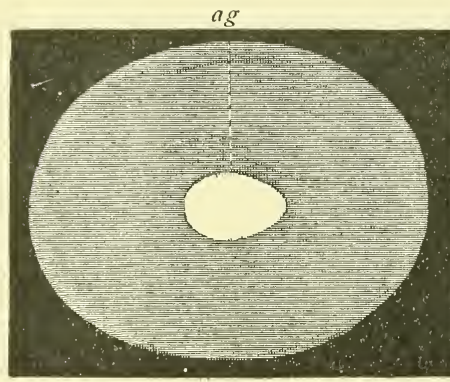

A

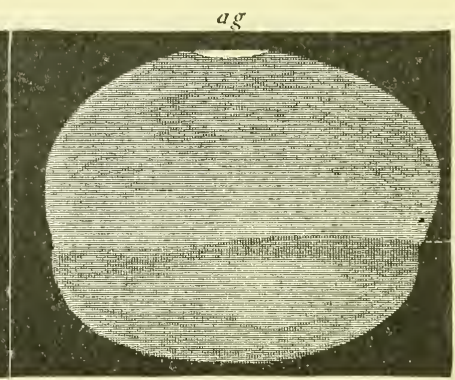

$B$

Fig. 33.-Blastula of the rabbit seven days old without the outer egg-membranes. Length $4.4 \mathrm{~mm}$. (after Kölliker). Magnified ten diameters. Seen in $A$ from above, in $B$ from the side: $a g$, embryonic spot (area embryonalis); ge, the line up to which the blastula is two-layered.

verse thickening called the terminal ridge, which is believed to be the anterior lip of the blastopore. It may be not amiss to say that the terms anterior and posterior are used with 
reference to the future body, the narrow end of the area embryonalis corresponding to the posterior pole or caudal extremity of the fetus.

In the chick's egg, the embryonic area (Fig. 34), or embryonic shield, appears while the egg is yet in the oviduct.
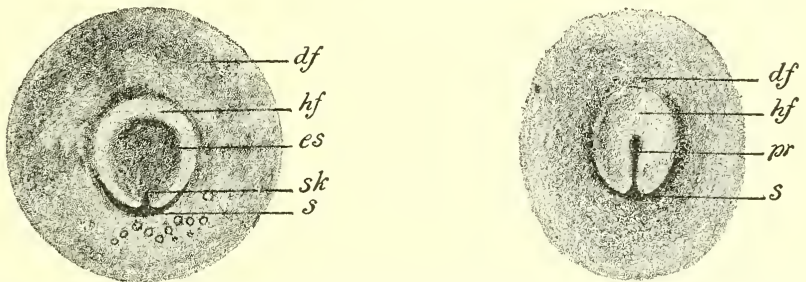

Fig. 34.-Two germ-disks of hen's egg in the first hours of incubation (after Koller) : $d f$, area opaca ; $h f$, area pellucida ; $s$, crescent; $s k$, crescent-knob; $e s$, embryonic shield; $p r$, primitive groove.

Its embryonic crescent corresponds to the mamalian terminal ridge. Segmentation being limited to the germ-disk in the chick's egg, the resulting blastorlerm, which is not a vesicle, but a flattened mass (Fig. 32) composed of several layers of cells, rests by its margin upon the partially liquefied yolk. The central region of the blastoderm, which overlies the liquefied portion of the yolk, from its translucence is known as the area pellucida (Fig. 34), while the dark opaque rim, resting upon the yolk is the area opaca. The inner rim of the area opaca is the area vasculosa. These regions are observed also in the mammalian egg.

It is in the embryonal area alone that the body of the embryo is developed; the other parts of the germ produce extra-embryonic structures, such as the amnion, the yolksac, ete.

Partial longitudinal division of the embryonic area during development results in the production of some form of double monster ; its complete cleavage gives rise to homologous or homogeneous twins, which are twins of the same sex and of almost absolutely identical structure. Ordinary twins are developed from separate ova, which may or may not have come from the same ovary.

The Primitive Streak.-The primitive streak is a linear 
median marking lying in the long axis of the embryonal area and containing a median furrow, the primitive groove (Fig. 35). A transverse section through the primitive streak

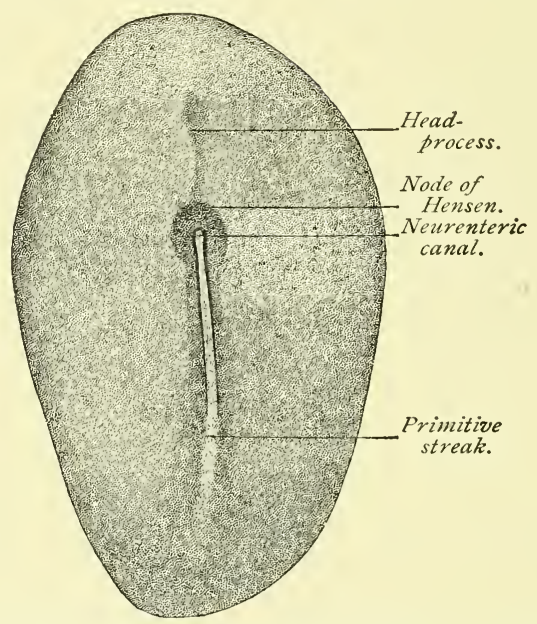

FIG. 35.-Embryonic area of rabbit-embryo (E. v. Beneden): primitive streak beginning in cell-proliferation known as the "node of Hensen."

(Figs. 36 and 37) shows that this surface-marking is produced by a thickening of the ectoderm along the median line, owing to a proliferation of cells from its under side. The length of the streak is about two-thirds of that of the embryonal area. In the rabbit's ovum it is seen at about the seventh day; in the human germ the time of its appear-

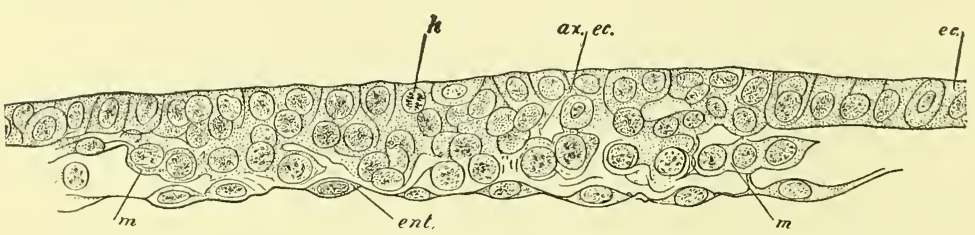

FIa. 35.-Section across the primitive streak of rabbit-embryo (Kölliker): $e c$, ectoderm; $a x$. ec, axial ectoderm undergoing proliferation, as shown by karyokinetic figures $(k)$; ent, entoderm; $m$, mesoderm.

ance is not known, but is probably about the third or fourth day. In the case of such a gastrula as that of the amphioxus (Fig. 26), the lips of the blastopore approach each 
other and fuse in a line corresponding to the median longitudinal axis of the future embryonic area, the fusion or concrescence beginning at the anterior extremity of this line and proceeding toward its caudal end. The surface-marking produced by the apposition and partial union of the blastoporic lips was called the primitive streak, and its median furrow was known as the primitive groove, long before their true significance was appreciated. Since the edge of the blastopore marks the place of transition from the entoderm to the ectoderm (Fig. 26), the two germ-layers after the

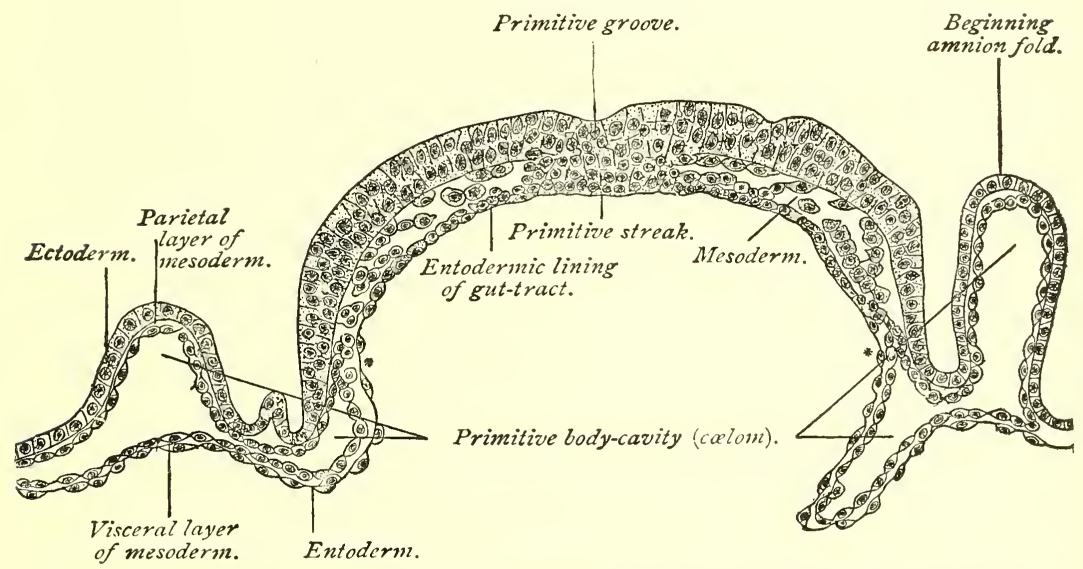

Fig. 37.-Transverse section of the embryonic area of a fourteen-and-a-half-day ovum of sheep (Bonnet).

union of the edges of this opening are in intimate association under the primitive streak, as shown in Fig. 37.

Morphologically the primitive streak of the higher vertebrates is regarded as the fused and extended blastopore of lower types. The terminal ridge of the mammalian embryonic area, as well as the crescent of the embryonic shield of avian and reptilian eggs, represents, as stated above, the anterior lip of the blastopore. Since the embryonal area is increasing in circumference while the lips of the blastopore are undergoing union or concrescence, the transversely directed terminal ridge, which lies at the posterior edge of the embryonal area, and which remains a fixed point, becomes a 
longitudinal marking, and this marking or primitive streak comes to lie, therefore, behind the site of the blastopore. Reference to Duval's diagram (Fig. 38) will make this clear.
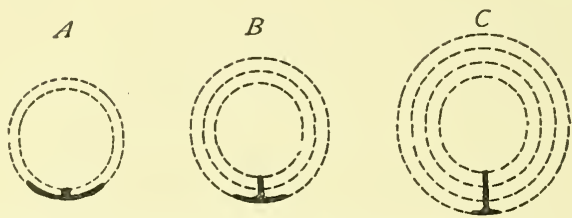

FIG. 38.-Diagram elucidating the formation of the primitive groove (after Duval). The increasing size of the germ-disk in the course of the development is indicated by dotted circular lines. The heavy lines represent the crescentic groove and the primitive groove which arises from it by the fusion of the edges of the crescent.

After the development of the primitive streak, there is seen, in the median line of the embryonal area, anterior to the streak, another marking, the head-process of the primitive streak. This is almost identical with the primitive axis of Minot, which that investigator describes as a median band of cells connected with the entoderm and extending forward from the blastopore.

Hensen's node is an accumulation of cells on the under surface of the ectoderm at the anterior end of the primitive streak. It is important because of its relation to the neurenteric canal, which will be described later.

Although the primitive streak and blastopore play no part in the later stages of development, it is worthy of note that the former lies in the line of the longitudinal axis of the future body, and that the position of the blastopore marks the posterior or caudal end of the embryo.

The Development of the Mesoderm.-The mesoderm or mesoblast is a structure composed of several layers of cells lying between the ectoderm and the entoderm. It is earliest formed in the vicinity of the front end of the primitive streak, the position formerly held by the blastopore. From this point it grows laterally and posteriorly and, later, anteriorly as well. It is not, however, until other important changes have taken place that it extends completely around the germ. 
The terms gastral mesoderm and peristomal mesoderm are used to designate respectively that portion developing from the region of the head-process of the primitive streak and that portion growing from the region of the blastopore.

Concerning the origin of the mesoderm much difference of opinion prevails. 'The simpler and more primitive method is seen in the amphioxus, in which it develops as two evaginations from the dorsal wall of the archenteron, one on each side of the mid-line. These entodermic folds, containing each a cavity, the enterocel, grow out laterally between the inner and the outer germ-layers. By transverse constriction, each fold divides into a series of segments, the somites, which lie on either side of the median line from the head-end to the tail-end of the embryo. Each somite divides into a dorsal part, the "protovertebra," and a ventral part, the lateral plate. By the fusion of the lateral plates of each side their several cavities become one, the body-cavity or cœlom.

The origin of the middle germ-layer in higher vertebrates is far less clearly made out. Some investigators hold that it arises in essentially the same manner as does that of amphioxus-that is, by evagination or outfolding of the entoderm bounding the colenteron; the investigations, however, of Bonnet and of Duval respectively upon sheep and chick embryos, point to a different conclusion. Bonnet's observations show that the mesodermic tissue, starting from Hensen's node, grows out laterally between the ectoderm and the entoderm, and that at some distance from the median line of the embryonic area there is a delamination or splitting-off of cells from the entoderm; and, further, that these two primitive areas grow toward each other and unite to form one continuous sheet of mesoderm. It may be said, therefore, that the mesoderm originates fiom a double source, chiefly from the entoderm, but also from the ectoderm, since the cells giving rise to the part that grows from the region of Hensen's node are cetodermic. A section of the germ transverse to the long axis of the embryonic area (Figs. 36 and 37) shows the mesoderm to be a distinct and independent layer, sharply defined from the other germ-layers everywhere except 
in the region of the mid-line, in which position the three layers are so closely related as to constitute one structure. The mesoderm does not extend completely around the germ at this stage, being deficient on the side opposite the embryonic area.

The mesoderm, after its formation, grows by the proliferation of its own cells, independently of the ectoderm and the entoderm.

If the expansion of the mesoderm, as indicated by the surface appearance of the germ (Fig. 39), be noted, it will

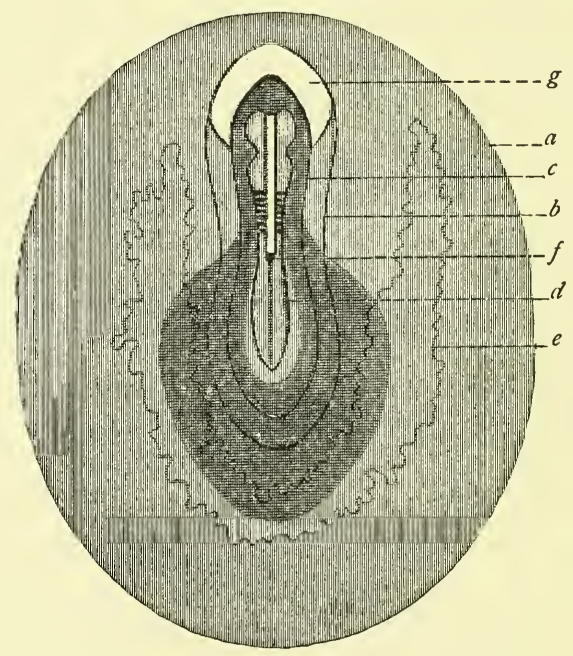

FIG. 39.-Diagrammatic surface view of rabbit's ovum of 205 hours (after Tourneux). The darkly shaded area indieates the extent of the mesoderm. $a$, Peripheral limit of area opaca; $b$, of area pellucida; $c$, of parietal zone; $d$, of stem-zone; $f$, Hensen's node; $g$, proamnion.

be seen that at first it is present throughout a pear-shaped area whose narrow end is directed forward. Somewhat later, two wing-like expansions grow forward from the front end of this area (Fig. 40); these wings, meeting at their tips, enclose a space, the proamnion, which is devoid of mesoderm. Referring again to the transverse section (Fig. 37), it is evident that the middle germ-layer in the vicinity of the median line is composed of a somewhat irregular mass of cells, while farther away it constitutes a lamina on each side. 
As development advances, these two portions become more differentiated from each other, although they are not entirely separated until much later. The thick mass adjacent to the median line is the vertebral plate, or primitive segment plate, or paraxial mesoderm; the more flattened lateral portion is the lateral plate. The mesoderm at this stage, therefore, consists of four parts-the two paraxial masses, lying one on each side of the median line, and extending from the head-

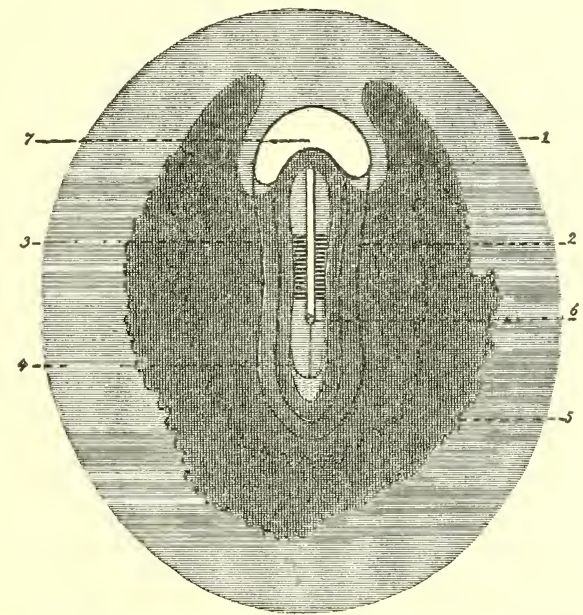

Fig. 40-Diagrammatic surface view of rabbil's ovum of 211 hours (after Tourneux). The darkly shaded area indicates the extent of the mesoderm. 1, Peripheral limit of area opaca; 2 , of area pellucida; 3 , of parietal zone; 4 , of stem-zone; 6 , Hensen's node; 7, proamnion.

end to the tail-end of the embryonal area, and the two lateral plates, situated upon the outer sides of the paraxial columns.

Each primitive segment plate undergoes transverse division into a number of irregularly cubical masses, the mesoblastic somites, or primitive segments, often improperly called the protovertebræ. 'The presence and position of the primitive segments are indicated by transverse parallel lines on the surface of the germ, which constitute a series on either side of the primitive streak and its head-process (Figs. 40 and 46). The formation of the somites begins at the cephalic end of the embryo and progresses tailward.

The lateral plate of the mesoderm splits into two lamellæ, 
of which the outer or parietal layer is the somatic mesoderm. and the inner or visceral layer is the splanchnic mesoderm. The somatic mesoderm unites with the ectoderm, forming the somatopleure; the splanchnic mesoderm unites with the entoderm, forming the splanchnopleure. 'The fissure-like cavity between the somatopleure and the splanchnopleure is the cœlom, or body-cavity, or pleuroperitoneal cavity (Fig. 45). The great serous cavities of the adult body-pleural, pericardial, and peritoneal-are later subdivisions of the cœlom.

The mesodermic cells bounding the body-cavity become flattened and endothelioid in character, and constitute the mesothelium; from them are descended the varions endothelial cells lining the serous cavities of the mature organism. According to some authorities, among whom Hertwig may be especially mentioned, there develop from the mesothelium at an early stage certain cells whose particular function is the formation of the different kinds of connective tissue, such as bone, eartilage, fibrous tissue, ete.; these elements are often distinguished as mesenchymal cells, or collectively, as mesenchyme. According to this classification, the importance of which is insisted upon by Minot, the mesenchyme includes all the mesodermic tissue except the flattened cells, the mesothelium, lining the body-cavity." "Mesenchyma consists of widely separated cells which form a continuous network of protoplasm, the meshes of which are originally filled by a homogeneous intercellular substance or matrix." -Minot.

His claims a double origin for the mesoderm. He maintains that the mesothelium and the smooth musculature of the body are of intra-embryonic origin, and these structures he terms the archiblast; while all other parts of the mesoderm, which he designates the parablast, have, in his opinion, an extra-embryonic soures, being derived possibly from the granulosa cells of the ovary. These views are not shared, however, by the majority of embryologists.

1 Minot holds with Goette that the mesenchymal cells are the product of the mesothelium. Hertwig maintains that the mesenchyma arises from all the other germ-layers by the emigration of isolated cells. 
The Derivatives of the Germ-1ayers.-From the three primary germ-layers are developed the various tissues and organs of the body by metamorphoses which may be referred to the two fundamental processes of specialization, or the adaptation of structure to function, and of unequal growth, which latter results in the formation of folds, ridges, and constrictions.

From the ectoderm are produced :-

The epidermis and its appendages, including the nails, the epithelium of the sebaceous and sweat-glands and their involuntary muscles, the hair, and the epithelium of the mammary glands.

The infoldings of the epidermis, including the epithelium of the mouth, with the enamel of the teeth, the epithelium of the salivary glands, and the anterior lobe of the pituitary body :

The epithelium of the nasal tract with its glands and communicating cavities:

The epithelial lining of the external auditory canal, including the outer stratum of the membrana tympani :

The lining of the anus and of the anterior part of the urethra :

The epithelium of the conjunctiva and of the anterior part of the cornea, the crystalline lens.

The spinal cord, the brain with its outgrowths, including the optic nerve, the retina, and the posterior lobe of the pituitary body.

The epithelium of the internal ear.

From the entoderm are produced:-

The epithelium of the respiratory tract.

The epithelium of the digestive tract, from the back part of the pharynx to the anus, including its associated glands, the liver, and the pancreas.

The epithelial parts of the middle ear and of the Eustachian tube. 
The epithelium of the thymus and thyroid bodies.

The epithelium of the bladder, and of the first part of the male urethra, and of the entire female urethra.

From the mesoderm are developed :-

Conrective tissue in all its modified forms, such as bone, dentine, cartilage, lymph, blood, fibrous and areolar tissue.

Muscular tissue.

All endothelial cells, as of joint-cavities, bursal sacs, lymph-sacs, blood-vessels, pericardium and endocardium, pleura, and peritoneum.

The spleen.

The kidney and the ureter.

The testicle and its system of excretory ducts.

The ovary, the Fallopian tube, the uterus, and the vagina.

From the foregoing tabulation it may be seen that, generally speaking, all epithelial structures originate from either the ectoderm or the entoderm, the notable exception to this rule being that the epithelium of the sexual glands and their ducts, and also that of the kidney and of the ureter, proceed from the mesoderm. 


\section{CHAPTER IV.}

\section{THE BEGINNING DIFFERENTIATION OF THE EM= BRYO; THE NEURAL CANAL; THE CHORDA DORSALIS; THE MESOBLASTIC SOMITES.}

THe germ, in the stages thus far considered, has the form of a hollow vesicle more or less irregularly spherical. It will be seen, in following the further history of development, that the layers of cells constituting the walls of the vesicle give rise to the alterations of external form and to the rudiments of the various organs of later stages by processes which, though seemingly complex, are referable to certain simple fundamental principles. It is, namely, in the mequal growth of different parts of the germ, in outfoldings and infoldings, and in the furrowing and constricting-off of parts, as well as in the adaptation of structure to function, that we find an explanation of the various developmental processes.

The first indication of the formation of the embryo and of its differentiation from the parts of the germ that are destined to produce, wholly or in part, the several extra-embryonic structures, is the marking out of the embryonic area by the thickening of the cells of the vesicle-wall in a definitely circumscribed region. The structures designated as extra-embryonic are the umbilical vesicle, the amnion, the allantois, and the fetal part of the placenta. The development of these and the production of the external form of the body of the embryo will be considered in the next chapter.

The primitive streak and its head-process have been already described. After their appearance the further evolution of the embryonic body is closely associated with three fundamentally important processes-namely, the formation of the neural canal, of the chorda dorsalis, and of the mesoblastic somites. 
The Neura1 or Medullary Cana1.-The neural canal is an elongated tube lying beneath the ectoderm in the median longitudinal axis of the embryonic body, its position corresponding to that of the future spinal canal. Its walls are composed of cylindrical epithelial cells.

To follow the development of the medullary canal, it is necessary to study the surface appearance of the ovum at the stage when the mesoderm is beginning to grow out from the region of the head-process of the primitive streak. Upon the surface of such a germ (Fig. 35), one may see the primitive streak and, in front of it, also in the median line of the embryonic area, the head-process of the primitive streak. The ectodermic cells overlying the head-process thicken so as to

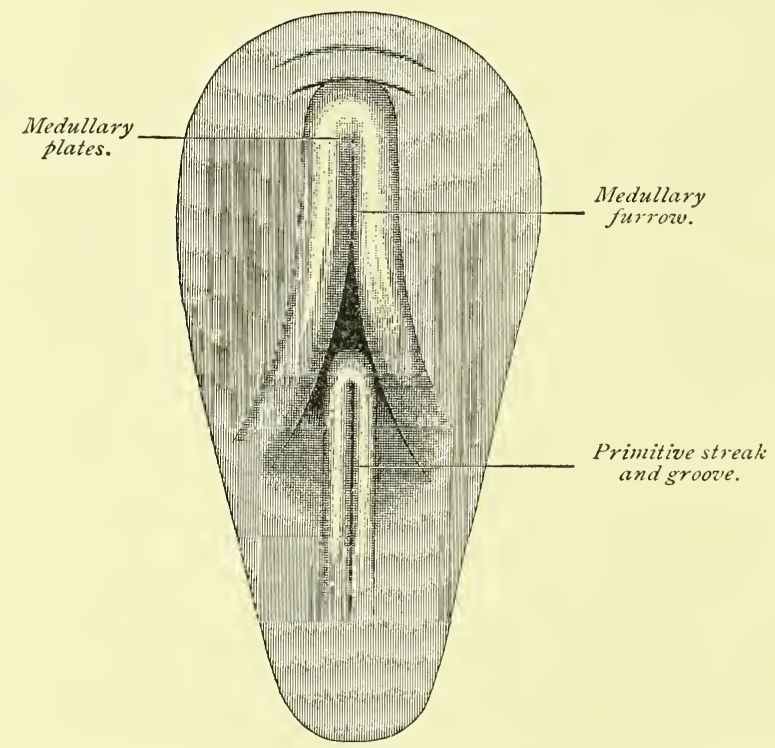

FIG. 41.-Surface view of area pellucida of an eighteen-hour chick-embryo (Balfour).

become columnar, while those on each side of it become flattened. This differentiation results in the production of a relatively thick axial plate of ectoderm, the medullary plate, which is present at the beginning of the eighth day in the rabbit's germ, and in the human germ at about the fourteenth 


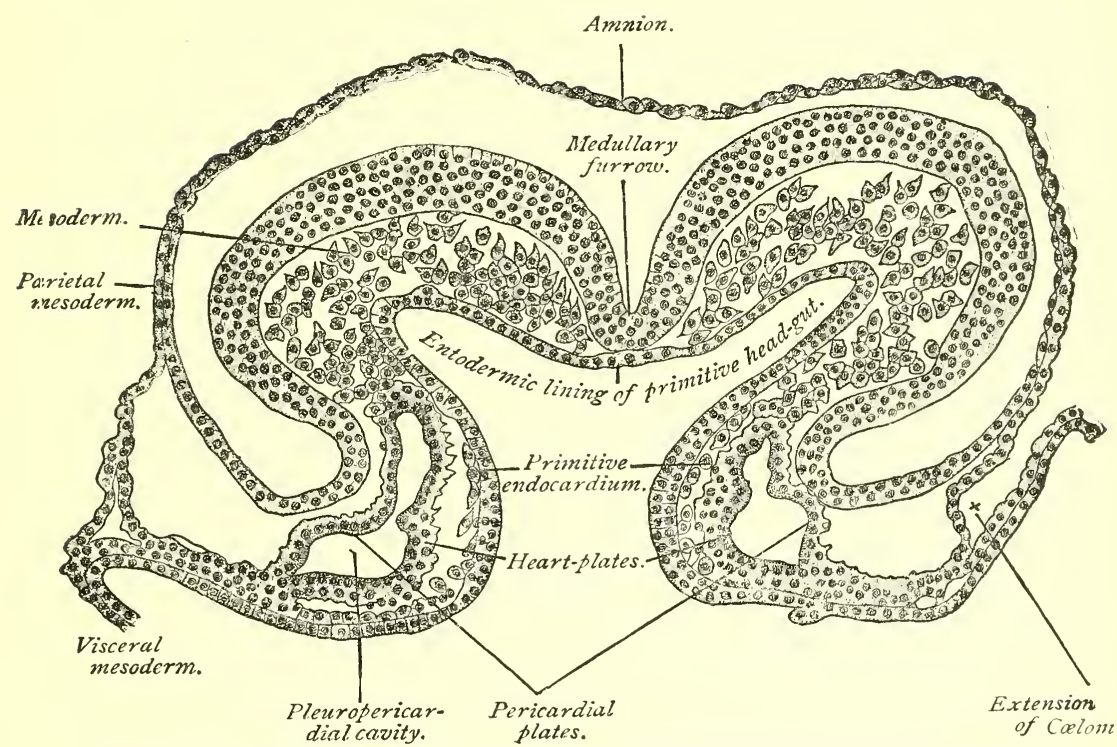

Fig. 42.-Transverse section of a sixteen-and-a-half-day sheep-embryo (Bonnet).

day. Almost as soon as the plate is formed, its lateral and anterior edges begin to curl up, producing the medullary fur-

Medullary

furrow.
Ectoderm. Uncleft

Amnion.

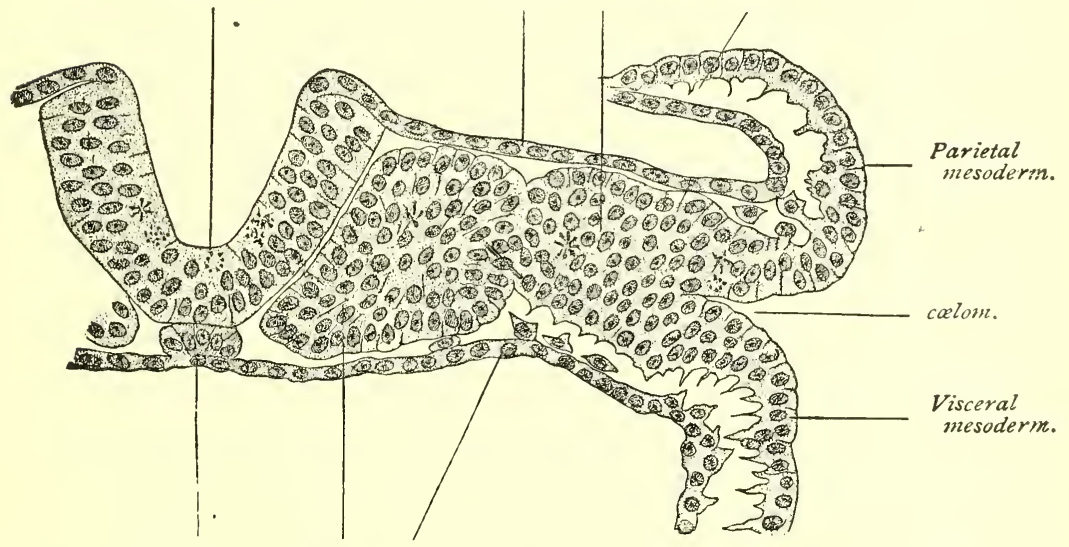

Notochord. Somite. Gut entoderm.

Fig. 43.-Transverse section of a sixteen-and-a-half-day sheep-embryo possessing six somites (Bonnet).

row or groove (Figs. 41, 42, and 43). The curling margins of the plate carry with them, as they rise, the adjacent 
thinner ectoderm; these projections constitute the medullary folds. A surface view shows the medullary folds to be continuous with each other in front, while their posterior ends are separated and embrace between them the front end of the primitive streak (Fig. 41). Since the formation of these structures is always more advanced in the anterior part of the embryonic area, their posterior extremities are not sharply defined but fade away (Fig. 41). The edges of the medullary plate continue to curl until they meet, when they unite, forming the medullary or neural canal (Figs. 44 and

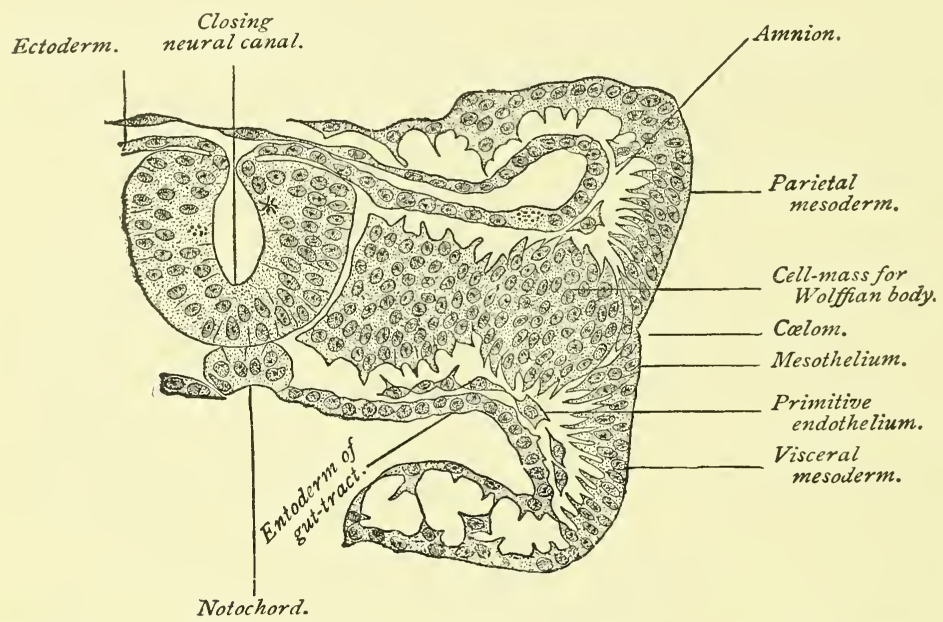

FIG. 44.-Transverse section of a fifteen-and-a-half-day sheep-embryo possessing seven somites (Bonnet).

45). The medullary folds and plate continuing to advance toward the tail-end of the embryonic area, and the closure of the tube taking place from before backward, the entire primitive streak is made to disappear by being included within the neural tube.

The medullary folds having grown toward each other a short time before the union of the edges of the medullary plate now unite over the partially formed neural tube. By the growth of the medullary folds and their subsequent coalescence, the completed neural tube comes to lie under the surface ectoderm, its connection with which is afterward lost. 
It is apparent, therefore, that the neural tube is a structure whose walls are composed of ectodermic cells, and that it has originated from the ectoderm by what may be called a process of infolding.

The medullary canal is the fundament of the entire adult nervous system. The first step in the conversion of a structure so simple into one so complex consists in the dilatation of the cephalic end of the neural tube and the subsequent division of this dilated extremity into three imperfectly separated compartments, named respectively the fore-brain, the mid-brain, and the hind-brain vesicles. It is by the multiplication and

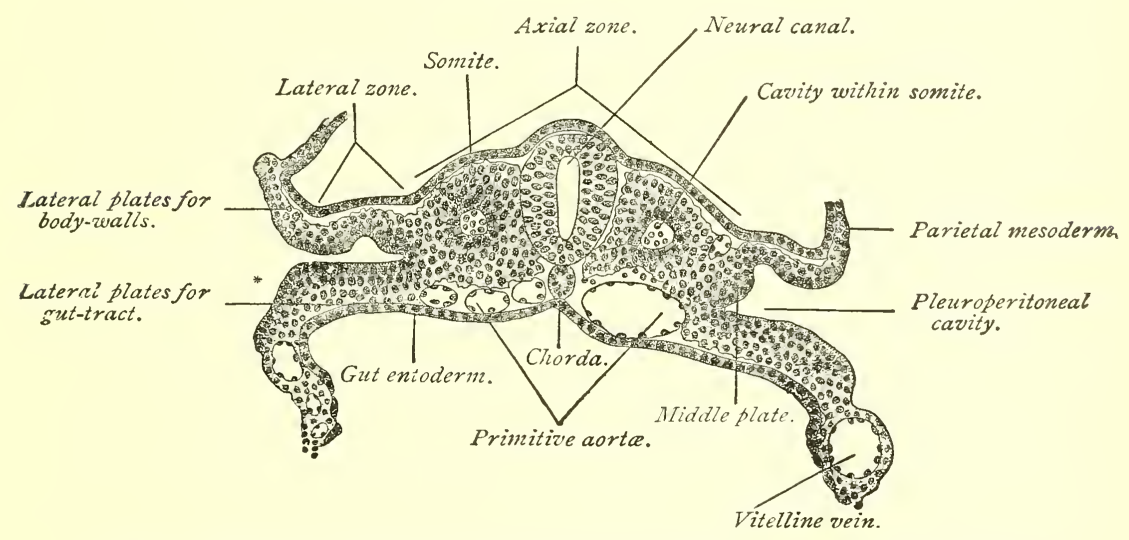

FIG. 45.-Transverse section of a seventeen-and-a-half-day sheep-embryo (Bonnet).

specialization of the cells composing the walls of the medullary tube that the cerebrospinal axis is produced, the brainvesicles giving rise to the brain-mass, while the remainder of the tube produces the spinal cord. Approximately one-half of the length of the tube is devoted to the formation of the brain, the other half forming the spinal cord.

The neural tube closes first in the future cervical region, the cephalic part of the canal remaining open for a time. From the neck region the closure of the tube progresses toward either end of the embryo.

The Notochord or Chorda Dorsalis.-The notochord is a solid cylindrical column of cells lying parallel with the medullary tube, on the dorsal side of the archenteric cavity. 
Its position is that of a line passing through the centers of the bodies of the future vertebræ. The derelopment of the chorda occurs at the same time as that of the neural tube, and in a very similar manner. A thickening of the cells of the entoderm in a longitudinal line extending along the dorsal aspect of the cœlenteron produces the chordal plate. Along either edge of the chordal plate a small fold of entoderm projects ventralward. By the curling around of the edges of the chordal plate, the latter becomes a solid cylinder of cells, which is separated from the entoderm proper by the union of the chordal folds, as shown in Figs. 44 and 45.

The appearance of the notochord is the first indication of the axis of the embryo, since around it the permanent spinal column is built up. The relative size of the chorda is less in the higher vertebrates than in the lower members of this group. It is one of the distinctive features of a vertebrated animal.

The chorda is essentially an embryonic structure, since it gives rise to no adult organ. Its only representative in postnatal life is the pulpy substance in the centers of the intervertebral disks. It is a permanent structure in one vertebrate only, the amphioxus. In this animal it is the representative of the spinal column of higher vertebrates. The notochord affords another illustration of the principle that higher organisms repeat, in their development, the structure of the lower members of the group to which they belong.

The Neurenteric Cana1.-The neurenteric canal is closely associated with the development of the medullary canal and with the disappearance of the primitive groove. We have learned that the blastopore is the orifice through which the colenteron opens to the exterior, and also that in birds and mammals the position of the blastopore, as indicated by the presence of the terminal ridge, corresponds to the anterior end of the primitive streak, and therefore of the primitive groove. Reference to Fig. 41 will show that the medullary folds have extended so far posteriorly that they embrace between them the primitive groove; therefore when 
they unite to form the neural canal, the primitive streak falls within its limits.

In a gastrula with an open blastopore, such as that of the amphioxus and those of amphibians, the blastopore is included between the medullary folds, and, after the completion of the neural canal, it constitutes an avenue of communication between the latter and the celenteron or primitive enteric cavity; this communication is the neurenteric canal. In mammals, as also in birds, reptiles, and selachians, classes in which the primitive streak is the representative of the closed blastopore, a small canal is found at the anterior end of the primitive groove, passing through Hensen's node, and opening into the colenteron. With the covering in of the primitive groove by the medullary folds, this canal becomes the neurenteric canal. According to Graf Spee, a neurenteric canal is found in the human embryo, as well as in the groups above mentioned. The canal is a temporary structure and gives rise to no organ of the adult.

The Somites or Primitive Segments.-The mesoblastic somites are cuboidal masses of cells, arranged in two parallel rows, one on each side of the notochord, extending the entire length of the body of the embryo. They are sometimes called protovertebre, but this term if used at all should be restricted to a subdivision of them that appears later.

The development of the somites was incidentally referred to in the description of the mesoderm. As mentioned in that connection, the paraxial plates of mesoderm, lying as parallel longitudinal columns, one on each side of the notochord, break up, each one into its corresponding series of primitive segments. The division throughout the entire length of the body takes place not simultaneously, but consecutively, beginning at the head-end.

The segmentation of the axial mesoderm is indicated by certain surface markings. The surface of the embryonal area, at the stage when the primitive streak and the medullary groove are present, shows a dark zone on either side of the median line, the so-called stem-zone, which marks the 
limits of the axial plate of mesodern (Fig. 46); the position of the lateral plates is indicated by the peripheral lighter parietal zone. The stem-zone soon exhibits, on each side of the primitive streak and medullary groove, a series of parallel transverse lines, produced by the transverse furrowing of the axial plates, preparatory to their division into the prinitive segments. The first pair of somites is formed in the fiture cervical region, before the medullary folds have united to form the neural tube, and when the primitive streak is yet present. After the appearance of the first pair, the forma-

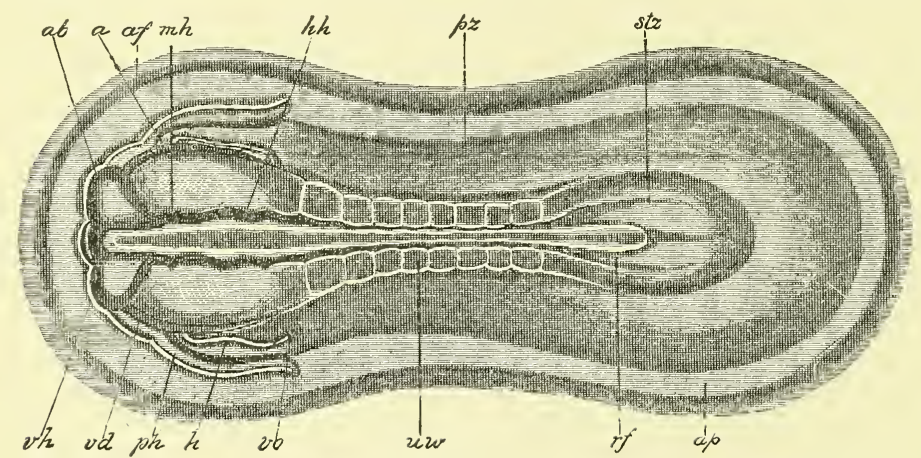

FIG. 46. - Rabbit embryo of the ninth day, seen from the dorsal side (after Kölliker). Magnified 21 diameters. The stem-zone $(s t z)$ and the parietal zone $(p z)$ are to be distinguished. In the former 8 pairs of primitive segments have been established at the side of the chorda and neutral tube; $a p$, area pellucida; $r f$, medullary groove; $v h$, fore-brain; $a b$, eve-vesicle; $m h$, mid-brain; $h b$, hind-brain; $u w$, primitive segment: stz, stem-zone; $p z$, parietal zone; $h$, heart : $p h$, pericardial part of the body-cavity; $v d$, margin of the entrance to the head-gut (vordere Darmpforte), seen through the overlying structures; af, amniotic fold; vo, vena omphalomesenterica.

tion of other segments proceeds headward and tailward. In selachians the number of head-segments has been shown to be nine; in higher vertebrates the number is possibly less. The trunk-segments are added in regular order from the neck-region to the tail-end of the embryo. In the human embryo there are thirty-eight pairs of neck and trunk somites and perhaps four pairs in the occipital region of the head.

The first somites appear on the eighth day in the rabbit, and between the twentieth and twenty-second hours in the chick. While they are forming the neural canal is closing, 
the notochord is differentiating from the entoderm, and the lateral plates of mesoderm are splitting to form the bodycavity or cœelom.

In structure the primitive segments of lower vertebrates consist of columnar cells arranged around a central cavity (Figs. 43 and 45). The eavity, in the amphioxus, communicates for a time with the colenteron, since the segments are in this case developed as entodermic evaginations; in selachians, the method of formation of whose primitive segments may be regarded as the primitive method for vertebrates, the cavity is for a time in communication with the body-cavity, since the segments in these animals develop as if by evagination from the dorsal side of the mesoderm after it has separated into its parietal and visceral layers and before it has divided into the axial and lateral plates. 'The size of the cavity is quite variable; in some cases, as in the Amniota, it is almost if not entirely obliterated by the encroachment of the cells of the walls of the somite.

Belonging to the somite, though not apparent on the surface, is a mass of cells which connects, for some time, the somite proper with the lateral plate (Fig. 45). This is known as the intermediate cell-mass or middle plate. Later, the separation of these is effected, the mesial part of the somite being the myotome, the intermediate cell-mass becoming the nephrotome. Each one of these parts contains a cavity, that of the myotome being called the myocœl. From the inner, mesial side of the myotome, embryonic connective-tissue cells (mesenchyme) develop, constituting the sclerotome, or skeletogenous tissue. The sclerotomes, made up of loosely-arranged embryonal connective tissue, grow around the medullary canal and chorda dorsalis, spreading out and fusing with each other. Subsequently this tissue produces the vertebral column and its associated ligamentous and cartilaginous structures. The cuter part of the myotome, sometimes called the cutis plate, gives rise to the corium of the skin of the trunk or perhaps to muscular tissue. The remaining part of the myotome, that situated dorsolaterally, constitutes the muscle- 
plate or myotome proper ; it gives rise to the voluntary musculature of the trunk.

The segmentation of the body of the embryo is an embryological process of great significance.

The segmented condition is common to the developmental stage of all true vertebrates, and in some invertebrates it persists throughout adult life. The development of the axial skeleton and of the muscular system, it will be seen later, bears an important relation to the process of segmentation, as does also the evolution of the genito-urinary system.

Upon reflection, it will be seen that in the region of the embryo corresponding to the future neck and trunk, the segmentation affects only the dorsal part of the body, while the ventral mesoderm, the so-called lateral plate, which contains the colom, remains unsegmented. On the other hand, in the head-region, the segmentation is both dorsal and ventral, the former being in series with the trunk-segments, while the latter, affecting the ventral mesoderm, and therefore also, in the corresponding region, the coelom, produces segments known as branchiomeres, in connection with which the visceral arches are developed (see Chapter VII.).

The relation of the primitive segments to the differentiation of the skeleton and of the musculature of the trunk, and also of the visceral arches to the muscles of the jaws, will be considered in subsequent chapters. 


\section{CHA PTER V.}

\section{THE FORMATION OF THE BODY=WALL, OF THE INTESTINAL CANAL, AND OF THE FETAL MEMBRANES.}

THE formation of the fetal membranes occurs coincidentally with the production of the external form of the body of the embryo. These changes mark the division of the hollow sphere or vesicle of which the germ consists up to this stage into two essentially different parts-namely, the embryonic body and the fetal appendages, the latter of which are destined for the nutrition and protection of the growing embryo. Although the several processes by which are produced the different parts of the embryo and its various appendages go on simultaneously, it is necessary, for the sake of clearness, to consider successively the development of each structure from its inception to its completion.

\section{THE FORMATION OF THE BODY=WALL AND OF THE INTESTINAL CANAL OF THE EMBRYO.}

In the stages of development thus far considered, the part of the ovum that is to become the embryo-that is, the embryonic area-is represented by a localized thickening of the wall of the blastodermic vesicle, of the shape and relative size shown in Fig. 33, which presents a surface view of the germ. On each side of the embryonic axis, represented by the notochord, is the paraxial mass of mesoderm, which has undergone partial segmentation to form the somites; on the distal side of the paraxial column, the mesoderm has split into the somatic or parietal, and the splanchnic or visceral lamellæ, between which is the body-cavity or cœlom. The cavity of the germ until the occurrence of the transformations about to be described is one undivided compartment which is bounded by splanchnopleure; and a conspicuous fea- 
ture of the changes under consideration is the division of this cavity into two by the folding-in of the splanchnopleure composing its walls. It will be well to consider first the formation of the body-wall and the accessory structures of the chick, and then to take up the special modifications that are presented by mammals generally and by the human ovum in particular.

The first indication of the foldings that lead to the differentiation of the embryo from the fetal appendages is seen upon the surface of the germ at a very early stage. A surface view of the germ-in the case of the chick on the first day of incubation-shows, at what becomes the head-end of the embryonic area, a transverse crescentic groove, with its concavity looking backward (Fig. 41); a similar groove is seen at the opposite extremity of the area, and also one at each lateral margin. These marginal grooves are depressions in the somatopleure. The elevated outer edges of the grooves form folds of somatopleure, designated respectively the head-fold, the tail-fold, and the lateral folds of the amnion. As these marginal grooves increase in length they meet each other and now constitute one continuous furrow, which encircles the embryonic area ; its outer elevated edge is the amnionfold. This furrow, which may be called an inverted fold composed of splanchnopleure and somatopleure, progressively deepens and at the same time its bottom is carried inward toward a point vertically under the central region of the embryonic area; that is, a fold composed of somatopleure and splanchnopleure grows from all parts of the periphery of the embryonic area toward the point indicated above, a point which corresponds to the site of the future umbilicus. By the ingrowth of the edges of the fold, the cavity of the archenteron is more and more constricted (Plate II., Figs. 2 and 3), until finally, with the completion of the infolding, it becomes divided into two parts of unequal size ; the smaller of these spaces is the gut-tract, or intestinal canal of the embryo, while the larger is the yolk-sac or umbilical vesicle. The constricted canal through which the gut-tract communicates with the yolk-sac is the vitelline duct (Plate II., Figs. 4 and 5).

While the splanchnopleuric layer of the ingrowing fold 
PLATE II.
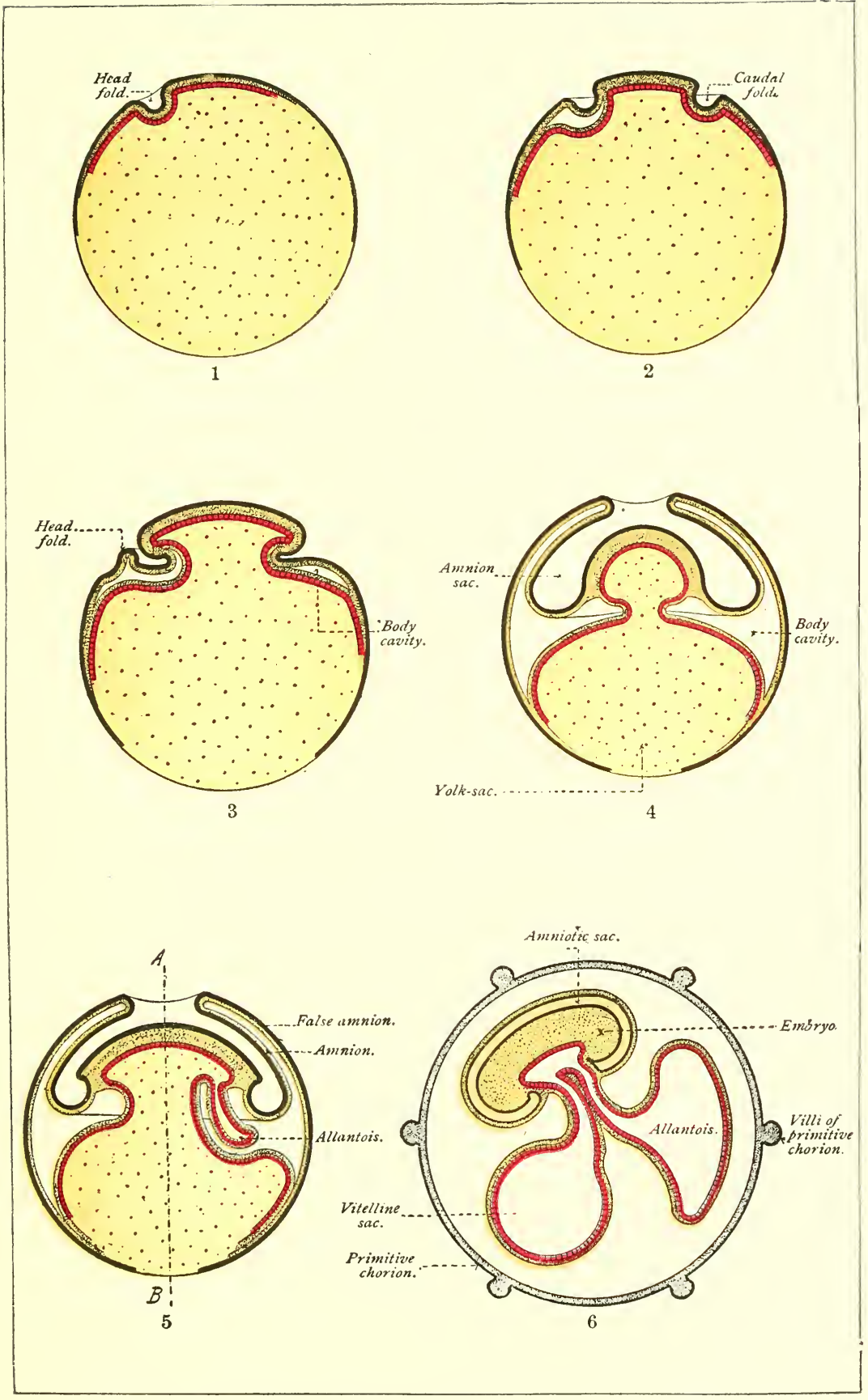

Diagrams illustrating the formation of the fetal membranes (modified from Boule). "The spaces marked 'body-cavity' in Figures 3 and 4 are merely the extra-embryonic portions of the body-cavity. For more recent conceptions as to the formation of these structures, see the text." 

thus outlines and forms the walls of the intestinal canal, the somatopleuric layer, which accompanies it, constitutes the lateral and ventral body-walls of the embryo. During the progress of this infolding of the splanchnopleure and the somatopleure, the part of the latter membrane that forms the outer wall of the groove becomes lifted up to constitute the amnion-fold (Plate II., Fig. 3) ; by the continued upward growth of this amnion-fold and the simultaneous settling down of the embryo upon the yolk-sac, the margins of the fold come to lie above the embryonic body, and, approaching each other, they fuse over its back, in this manner enclosing it in a cavity. It is obvious that the fold just described is a double layer of somatopleure. After the union of its edges, the two layers become completely separated, the inner one constituting the amnion, while the outer layer is the false amnion, or serosa, or chorion (Plate II., Figs. 4-6).

Since the infolding of the splanchnopleure begins at the periphery of the much elongated embryonic area, the resulting gut-tract has the form of a straight tube extending from the head-end to the tail-end of the embryo (Plate III.). "When the caudal and the eephalic portions of the splanchnopleuric fold have advanced but a comparatively short distance, in consequence of which the communication between the gut-tract and the umbilical vesicle is still widely open, as shown in Plate II., Fig. 5, there is a cul-de-sac or pocket formed of splanchnopleure at the head-end of the embryo and a similar one at its tail-end; these recesses are respectively the foregut and the hindgut, the orifices of which are designated the intestinal portals. At this particular stage, therefore, the cavity of the gut-tract is incompletely closed off from that of the umbilical vesicle.

It is evident that the gut-tract, being a tubular cavity enclosed by splanchnopleure, is lined with entodermal cells; this simple straight tube develops subsequently into the adult intestinal canal and its associated glandular apparatus.

It has alrearly been pointed out that the layer of somatopleure which is folded under the embryonic area in company with the splanchnopleure constitutes the lateral and the ven- 
tral walls of the body of the embryo. The fold continues to advance from each side and from each end, and its edges come together and fuse in the median line of the ventral surface of the body. ${ }^{1}$. At one place, however, fusion of the edges of the fold does not occur; this region corresponds to the umbilicus and is often designated the dermal navel. Here the part of the somatopleire that forms the body-wall is continuous with that part of this membrane which constitutes the amnion (Plate II., Fig. 6). By the infolding of the somatopleure the body-cavity or pleuroperitoneal space becomes divided into an intra-embryonic and an extra-embryonic portion, the two communicating for a time through the small annular space that encircles the proximal end of the vitelline duct; this is represented in the accompanying figures.

By this simple process of folding, associated with the unequal growth of different parts, the leaf-like fundament constituted by the embryonic area is differentiated into the body of the embryo; the ventral portion of this body now consists of two tubes, one within the other, of which the smaller, bounded by the splanchnopleure, is the intestinal canal, and the larger, enclosed by the somatopleure, is the body-cavity, the walls of which are the walls of the body of the embryo. In the dorsal region is a third tube, the medullary canal; between it and the dorsal wall of the intestine is the notochord, on each sicle of which are the somites (Fig. 44). The further evolution of this body and the differentiation of its various organs and systems will be described in subsequent sections.

\section{THE AMNION.}

The amnion is a membranous fluid-filled sac, which surrounds the fetus of certain groups of vertebrate animals during a part of their period of development. In man, it is

1 Failure of union of the somatopleuric folds in the median line of the thorax produces the deformity known as cleft sternum; while lack of fusion of the lateral halves of the abdominal wall results in an extra-abdominal position of the intestines, or, if in lesser degree, in exstrophy of the bladder. 
found as early as the fourteenth day, ${ }^{1}$ before the medullary groove has closed to form the neural canal; it attains its maximum size by the end of the sixth month and persists until the end of gestation. It constitutes a loose envelope for the fetus, being attached to the abdominal wall of the latter at the margins of the umbilicus, and loosely enveloping the umbilical cord (see Plate III., Fig. 2).

An amnion is found in birds, reptiles, and mammals, these groups being classed together as Amniota, while fishes and amphibians, which are without an amnion, constitute the class Anamnia.

The first indication of the growth of the amnion is apparent at a comparatively early stage of development. A surfaceview of the blastodermic vesicle of the first day of incubation in the case of the chick shows a eurved line or marking at the anterior edge of the embryonic area (Fig. 41 ); this is the anterior marginal groove, in front of which is another marking, the head-fold of the amnion. Very soon the lateral and posterior marginal grooves appear at the sides and posterior edge respectively of the embryonic area; the outer elevated edges of these marginal grooves constitute the lateral folds and the tail-fold of the amnion. The grooves and folds increase in length in each direction until they meet, when they form one continuous furrow, which circumscribes the embryonic area, and the outer elevated edge of which is the amnion fold. The groove involves both the somatopleure and the splanchnopleure, constituting the inverted fold of these two structures that grows in to form the body-wall and the wall of the gut-tract, while the amnion fold is composed of somatopleure alone (Plate II.). This separation of the somatopleure and the splanchnopleure enlarges the extraembryonic portion of the borly-cavity. The amnion fold continues to grow upward, and finally its edges meet and fuse over the back of the embryo, the line of union being the amniotic suture; the suture closes first at the hear-end of the embryo and last at the tail-end. After the union of the edges

${ }^{1}$ Recently it has been found complete in an ovum estimated to be four days oli. 
of the fold, its inner layer, consisting of ectoderm and parietal mesoderm, separates from the outer layer to constitute the true amnion, whose enclosed space is the amniotic cavity; the outer layer, which is merely a part of the general somatopleure, is the false amnion or serosa. It is apparent from this description that the amniotic cavity is lined with ectodermal epithelium and that its walls consist of somatopleure — that is, of ectoderm and parietal mesoderm.

While the amnion fold is growing upward, the embryonic area-now undergoing differentiation into the embryonic body-is sinking down upon the yolk-sac. The amnion fold does not grow uniformly in all parts of its periphery. The head-fold is produced first and constitutes a cap or hood covering the head of the embryo, which is forming simultaneously by the ventrad growth of the somatopleure at the bottom of the marginal groove. It is only after the development of the head-fold is well advanced that the lateral, and, later, the caudal, portions of the amnion-fold grow up to meet it. The head-fold is, for a time, destitute of mesodermic tissue, since it corresponds to that region of the wall of the blastodermic vesicle described on page 64 as the proammion.

It has been shown (p. 54) that the amniotic cavity of mammals is produced not by the upgrowth of folds of somatopleure, but by a vacuolation of a portion of the cells of the inner cell-mass (Fig. 28, p. 55). Since the enveloping layer, which forms the roof or vault of the amniotic cavity, constitutes the extra-embryonic ectoderm (p. 55), this eavity in mammals as in birds is lined with ectodermal cells, the floor of the cavity being also ectodermal since it is formed by the cmbryonic disk, the amniotic surface of which constitutes the embryonic ectoderm (Fig. 29). Covering the ectodermal roof is a layer of mesoderm continuous with the mesoderm of the embryonic disk. The embryonic bud or disk, at first concave on its amniotic surface, the future dorsal surface, becomes flat and then convex, its edges curving toward the opposite or ventral surface. It should not be forgotten that the entoderm covers this ventral surface and at the periphery of the bud passes to the deep surface of the enveloping 
layer, and also that the embryonic ectoderm is likewise continuous at the periphery of the embryonic bud with that part of the enveloping layer which forms the vault of the amniotic cavity; hence, after the ventral curvature of the embryonic bud, the periphery of which carries with it toward the ventral surface the amniotic ectoderm and mesoderm, we have practically the same conditions as obtain in the avian embryo as shown in Plate II., Figs. 4 and 5. While in the latter case the amnion is produced by the formation of folds, in the mammalian germ the same result is attained by the vacuolation of the inner cell-mass.

As the curving ventrally of the embryonic bud continues, the originally flat mass of cells composing it is converted into an imperfect tube, the lateral and ventral surfaces of which correspond with the former dorsal surface. This ventral folding of the embryonic bud produces the body of the embryo. As the folding includes the entodermal layer on the ventral surface of the embryonic bud, the blastodermic cavity is divided, as in the bird's germ, into the primitive intestinal canal and the yolk-sac (Fig. 48).

The amnion of man presents an important variation from that of all other Amniota, since the inner layer of the amnion-fold does not entirely sever its connection with the outer layer, but remains attached to it over the caudal pole of the embryo. In consequence of this attachment the true amnion is connected with the false amnion, and since the true amnion is continuous with the body-wall of the embryo, the caudal end of the embryonic body is attached to the false amnion or chorion by a mass of mesodermic tissue called the allantoic stalk or belly-stalk, as seen in Fig. 47. The relation of the belly-stalk to the development of the allantois will be pointed out hereafter.

The space within the amnion-the amniotic cavity-is filled with the amniotic fluid or liquor amnii.

The amnion at first envelops only the sides and dorsum of the embryonic body, occupying the upper part of the cavity enclosed by the chorion, as shown in Plate II., Figs. 5 and 
6 ; the groove, or furrow, however, of which the amnion fold is the peripheral or outer elevated edge, becomes deeper, and the bottom of the groove is carried toward the middle of the future ventral surface of the embryo, its ventrad growth continuing until it reaches the position of the future umbilicus (Plate II. : Fig. 4, transverse section; Figs. 5 and 6, longitudinal section). The layer of somatopleure constituting the inner wall of the groove-that is, on the side toward the embryonic area-becomes the lateral and ventral walls of the body of the embryo, as described above; in this manner is effected the transition from the flattened or layer-like embryonic area to the definite form of the embryonic body. The ventral body-wall is continuous at the margins of the umbilicus with the amnion, since the somatopleure, forming the outer boundary of the original groove, is a part of that membrane. After its completion, therefore, the amnion envelops the body of the embryo on every side, lying closely applied to it, since the amniotic cavity is at first very small. With the progress of development and the increase of the fluid the amnion requires more room, until, in the third month, it fills out the entire space within the chorion, with the inner surface of which it acquires a loose connection. The umbilical vesicle and the allantois have meanwhile undergone regression. The walls of the amniotic sac contain contractile fibers; it is to these that the rhythmical contractions observed in the amnion are due. Its lining is at first a single layer of flattened epithelial cells; at the fourth month the cells are cubical for the most part, but to some extent columnar.

The liquor amnii is a watery fluid having a specific gravity of 1.007 , and containing about 1 per cent. of solids (albumin, urea, and grape sumar). The origin of the fluid is believed to be in the blood of the mother, the liquid portion of which transudes into the amniotic cavity. The amniotic fluid increases in quantity until the sixth month of pregnancy ; from this time until the close of gestation it generally diminishes about one half. A pathological excess of the fluid constitutes the condition of hydramnios.

The function of the amniotic fluid is two-fold; it serves as 
a buffer for the fetus, protecting it from mechanical violence, and it supplies the fetal tissues with water, since portions of it are from time to time swallowed. Evidence that the fetus swallows the fluid is afforded by direct observation of chicken embryos, and by the presence of epidermal cells, hairs, and fatty matter in the fetal alimentary canal. After the development of the bladder, the urine of the fetus is from time to time evacuated into the amniotic cavity.

The epidermis of the child in utero is protected against maceration in the amniotic fluid by the presence of a fatty coating, the vernix caseosa, which is a modified sebaceous secretion.

At the end of pregnancy, the amnion is loosely united with the chorion and the decidux; during birth it ruptures, and its fluid escapes.

\section{THE YOLK=SAC.}

The yolk-sac, or umbilical vesicle, as seen in the higher vertebrates, is a capacious sac attached by a narrow pedicle, the vitelline duct, to the ventral surface of the embryonic intestinal canal, the duct passing through the umbilical aperture (Plate II., Fig. 6).

In order to appreciate more fully the function and the morphological relations of this structure, it is necessary to glance at the conditions that obtain in the several classes of vertebrate animals. In ova that develop outside of the body of the parent organism, a special dower of pabulum is provided for the nutrition of the embryo; this dower is represented by the deutoplasm so abundant in telolecithal ova. In the case of amphibians, whose cleavage, it will be remembered, is holoblastic or total, the cells richest in deutoplasm are accumulated, after segmentation, in the floor of the archenteron; this accumulation produces on the future ventral surface of the embryo a marked bulging, which constitutes the amphibian yolk-sac. As the embryo grows, it draws upon this store for its nutrition, in consequence of which the sac gradually shrinks, its cells being, for the most part, 
liquefied and absorbed, while some of them contribute to the lining of the intestinal canal.

In a higher type, as exemplified in sharks and dog-fishes, the yolk-sac is produced by a folding-in of the splanchnopleure and the somatopleure, the walls of the sac being therefore constituted by both of these layers; this folding-in divides the archenteron into a smaller part, the intestinal canal, lying within the body of the embryo, and a larger cavity, the yolk-sac, situated outside of that body. The splanchnopleuric layer of the yolk-sac is continuous with the wall of the intestinal canal, while its somatopleuric layer is continuous with the body-wall. A system of blood-vessels develops upon the yolk-sac, their function being to convey the nutritive material into the body of the embryo. These blood-vessels constitute the so-called vascular area, which appears, in surface views, as a zone encircling the embryonic area, and, later, the embryo, since the latter reposes upon the proportionately much larger yolk-sac. As the contents of the sac become absorbed, the latter shrinks, the splanchnopleuric layer slipping into the abdomen of the embryo through the umbilical opening, the somatopleuric layer contracting to close that aperture.

In the Amniota (p. 83) the development and structure of the yolk-sac are modified by the presence of the amnion. In these groups the yolk-sac and the gut result from the division of the blastodermic cavity by the folding-in of the splanchnopleure alone, since the somatopleure grows away from the splanchnopleure to form the amnion-fold, and thus only partially invests the yolk-sac (Plate II., Fig. 4).

Since the yolk-sac contains the store of food destined for the nutrition of embryos that develop outside of the maternal body, and since the mammalian embryo, which leads an intrauterine existence, is endowed with a relatively small quantity of such store, the yolk-sac of mammals would seem to indicate the descent of the latter from oviparous ancestors. Further and stronger evidence of such descent is found in the fact that the eggs of the lowest order of mammals, the Monotremata, comprising the echidna and the ornithorhynchus, are "laid" and undergo extra-uterine development. 
In the human embryo the umbilical vesicle is found partially constricted off from the intestinal canal by the end of the second week; by the end of the third week the separation of the two cavities has advanced to such an extent that the vitelline duct is present, the sac attaining its maximum size by about the fourth week.

The function of the umbilical vesicle, as above intimated, is to serve as the organ of nutrition for the embryo during a certain period. The manner in which its blood-vessels develop will be considered in Chapter $\mathrm{X}$. Their growth precedes that of the intra-embryonic portions of the vascular apparatus, the vascular area of the yolk-sac being the seat of the earliest blood-vessel formation. The vessels find their way into the body of the embryo along the vitelline duct, and consist of two vitelline arteries and two vitelline veins.

With the development of the allantois the yolk-sac retrogresses, the allantois succeeding it as the organ of nutrition and respiration. By the end of the sixth week the sac has shrunk to a narrow stalk, which is surrounded by the enlarged amnion, and which terminates in a knob; at birth, the knob lies near the placenta (Plate V., Fig. 2), and the atrophic remnant of the stalk is one of the constituents of the umbilical cord.

THE ALLANTOIS.

The allantois is an embryonic structure which is found in those vertebrates possessing an amnion. Its growth is correlated with the retrogression of the umbilical vesicle, which structure it supplants as the organ of nutrition and respiration for the embryo.

Appearing at first as a little evagination or out-pocketing of the ventral wall of the gut-tract, the allantois finally becomes a pedunculated sac lying in the extra-embryonic part of the colom (Plates II. and III.), its stalk leaving the body-cavity proper through the umbilical opening. Being an outgrowth from the intestinal canal, the walls of the allantois are made up of splanchnopleure-that is, of ento- 
derm and visceral mesoderm. Blood-vessels develop in the mesodermic stratum, the principal trunks, the two allantoic arteries and veins, being connected at their proximal ends with the primitive heart; this system of ressels constitutes the allantoic circulation and is the avenue through which the growing embryo is supplied with nutritive material and oxygen. As the fundus of the allantois increases in size, it spreads itself out upon the inner surface of the false amnion or chorion (Plate III., Fig. 1), into whose villi its vascular tissue penetrates, and with which it becomes intimately blended. The union of the allantois and the false amnion produces the true chorion of some authors.

The human allantois presents a striking peculiarity as compared with that of birds and reptiles; in man, the allantois
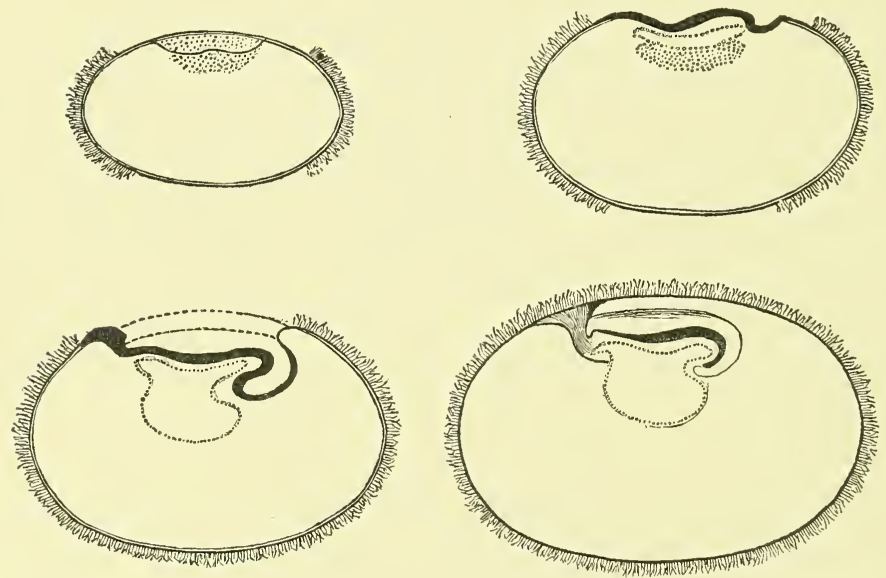

FIG. 47.-Diagrammatic sections representing growth and arrangement of the amnion in the earliest stages of the human embryo (His).

develops not as a free sac projecting into the extra-embryonic body-cavity, but as a mass of splanchnopleuric tissue which contains only a rudimentary cavity and which grows into the abdominal stalk (Fig. 47 and Fig. 58, bst), being guided by that structure to the chorion. Moreover, while the human allantois is in effect an evagination of the ventral wall of the primitive gut-tract, its evagination begins before the gut-tract is constricted-off from the yolk-sac (Fig. 48). 


\section{PlATE III.}
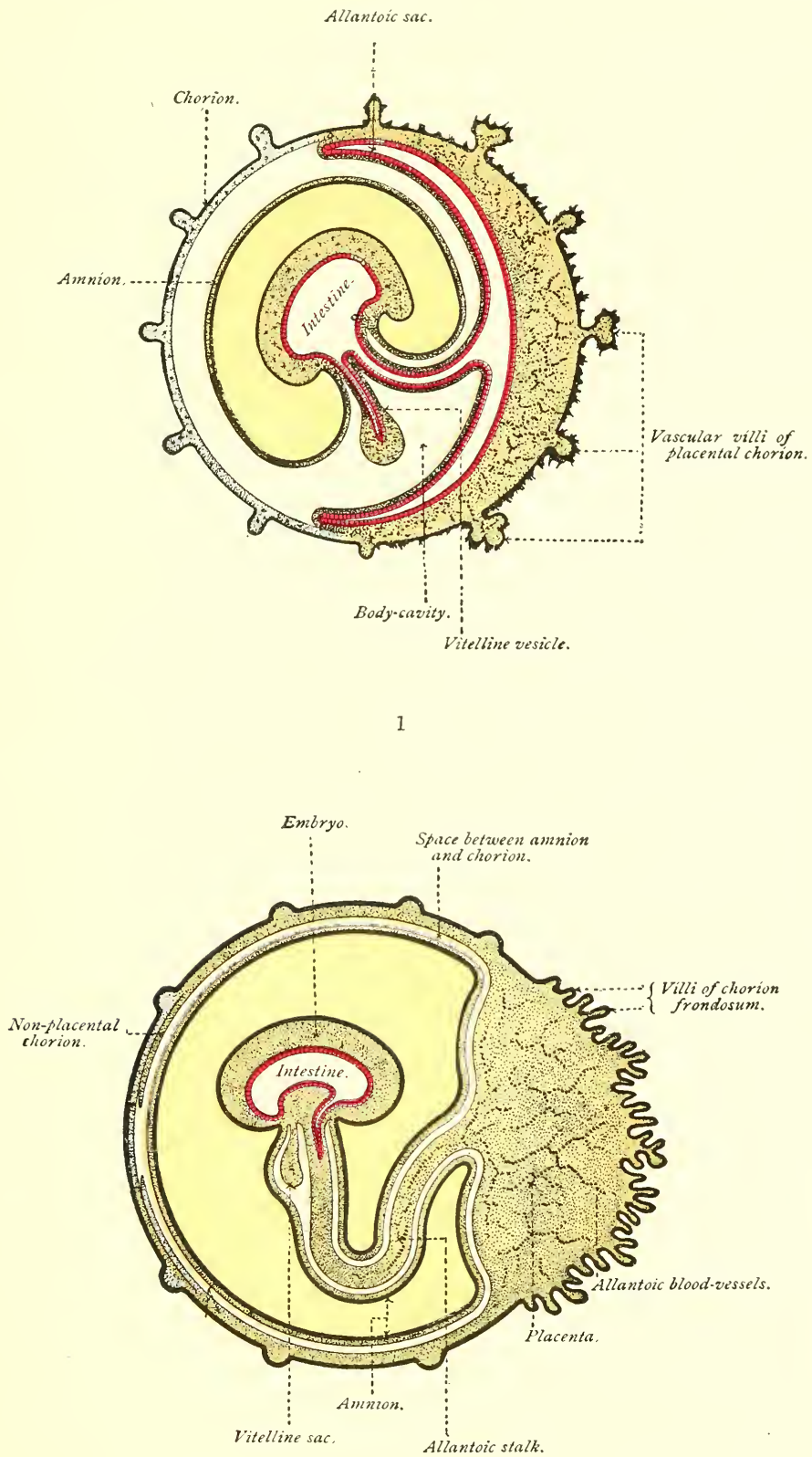

The function of the allantois in egg-laying animals, and possibly in some others, is to serve as a nutritive and respiratory organ and as a receptacle for the fetal urine; in man its cavity is exceedingly minute, and its chief function is to furnish a means of conveying blood-vessels from the embryo to the chorion.

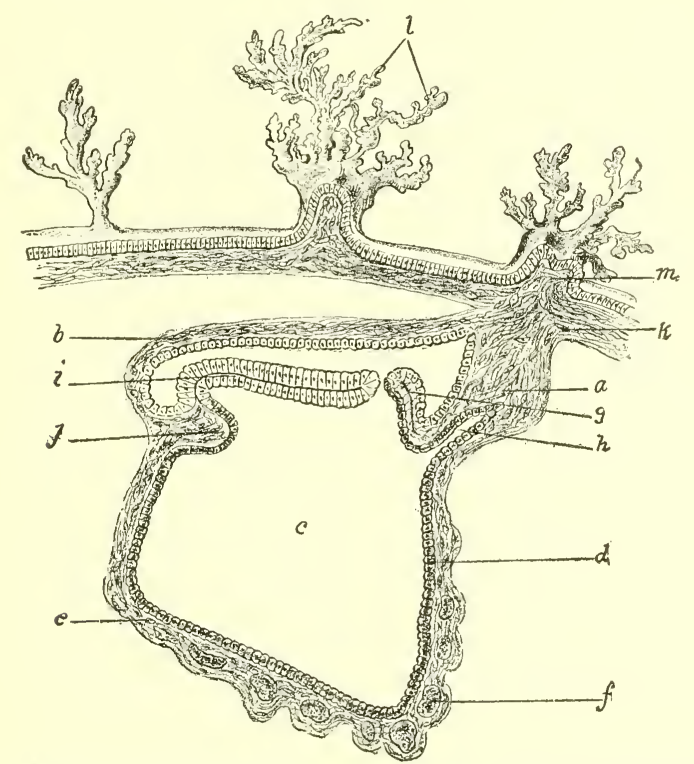

FIG. 48.-Mesial section through an early human ovum (Graf Spee): $a$, Abdominal stalk; $b$, amnion; $c$, yolk-sac ; $d$, hypoblast ; $\epsilon$, mesoblast; $f$, vessels on wall of yolk-sac; $g$, primitive streak; $h$, allantois; $i$, medullary plate; $j$, early heart; $k$, mesoblast of chorion; $l$, early villi; $m$, chorionic mesoblast extending outward into villi.

The part of the allantois contained within the body of the embryo produces three structures of the adult organism : 1 , the urachus, an atrophic cord extending from the summit of the bladder to the umbilicus $;^{1} 2$, the urinary bladder; and 3 , the first part of the urethra of the male, or the entire female urethra. The extra-embryonic portion shrinks after the appearance of the placenta and forms one of the constituents of the umbilical cord, its blood-vessels becoming the umbilical arteries and veins.

${ }^{1}$ If the urachus remains patulous instead of becoming impervious, urine may escape at the umbilicus, and the condition is a variety of urinary fistula. 


\section{THE CHORION.}

At the time when the false amnion is forming, the attenuated zona pellucida still surrounds the embryonic vesicle as the so-called prochorion, which unites with the false amnion, producing the primitive chorion. After the allantois has grown forth from the gut-tract and has spread itself over the inner surface of the primitive chorion, it becomes blended with the latter to constitute the true chorion of some authors. The chorion, according to the above nomenclature, may be defined as the membrane which encloses the germ at the stage following the appearance of the amnion and the false amnion, and which has resulted from the fusion of the allantois with the primitive chorion; or, ignoring the zona pellucida, the chorion results from the fusion of the allantois and the false amnion. Minot, however, defines the chorion as all that part of the extra-embryonic somatopleure which is not used in forming the true amnion, and hereafter in this work the word will be used in this sense. This definition limits the term to the outermost covering of the germ after the formation of the amnion (Plate III., Fig. 1).

The chorion consists of an outer ectodermic layer and an inner lamella of mesodermic tissue. The mesoblastic layer is thin, being composed of from two to four layers of round, oval, or fusiform cells, and is at first devoid of blood-vessels. The latter, in the form of capillaries, make their appearance at some time during the second week, probably as extensions of the blood-vessels of the allantois.

The outer ectodermic or epiblastic cells of the chorion at a very early period, certainly as early as the third day, undergo proliferation to form a layer of tissue called the trophoblast, which is from one to several layers of cells in thickness. The trophoblast layer is thickest at the place of attachment of the ovum to the uterine mucosa. The inner cells of the trophoblast are cubical, and have large, finely granular, round or oval nuclei. In the youngest human ovum as yet examined - that of Peters, estimated to be three or four days old - the trophoblast was found to present many little projections in the form of strands and buds, these 
being the foundations of the future villi of the chorion. The trophoblast layer was not solid, but was honeycombed with little spaces or vacnoles filled with maternal blood, which spaces were partly lined with a nucleated protoplasm, the early syncytium (Plate IV., a). Even at this early stage, therefore, when the trophoblast strands or early villi are as yet devoid of a mesoblastic element, they are bathed with the maternal blood. Very soon the mesoblastic tissue of the chorion grows into the trophoblast strands, thus forming the permanent villus stems; and during the second week capillaries extend into the stems, completing the formation of the fully developed villi of the chorion.

The early development of villi is characteristic of the human chorion (Fig. 49 and Plate II., Fig. 6). At first the

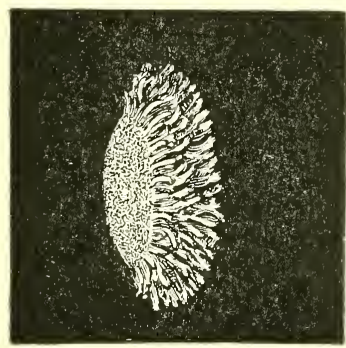

FiG. 49.-Human ovum of about twelve days (Keichert), side view.

In both figures the villi are limited in distribution, leaving the poles free.

villi, either covering the entire surface of the chorion or leaving the two opposite poles free, are of uniform size; in the latter half of the first month, however, there begins to be a differentiation into a region containing smaller, and one having larger and more numerous, projections. The difference between the two areas becoming more marked, the relatively smooth part of the membrane, possessed of rudimentary villi, is designated the chorion leve, while the region with well-developed villous projections is distinguished as the chorion frondosum (Plate III., Figs. 1 and 2); the latter acquires a close relation with the mucous membrane of the uterus and becomes the fetal part of the placenta. 
The vilii in their earlier condition are somewhat club-shaped elevations, which later become branched to form secondary villi. Each fully developed villus consists of a core of mesoblast, covered with ectodermic epithelium and containing blood-vessels (Fig. 54 and Plate $I^{\top}$., a). Their microscopic appearance is so characteristic that they afford a means of positively determining whether a mass discharged from the uterus is or is not a product of conception. The further alterations in the villi as well as in the trophoblast in general, including the syncytium, will be considered in Chapter VI.

A chorion is present, as a rule, in those animals whose embryos develop within the uterus; this would include the entire class Mammalia, with the exception of the monotremes, whose eggs undergo extra-uterine development, and the marsupials, whose embryos, though nourished in the womb, never acquire villi on the serosa, nutriment being absorbed by simple contact of the latter with the uterine mucous membrane. The Mammalia are therefore divided into the Achoria, comprising the monotremes and marsupials, and the Choriata, including all other mammals. 
THE DECIDU $A$ AND THE EMBEDDING OF THE OVUM. THE PLACENTA. THE UMBILICAL CORD.

\section{THE DECIDUAE AND THE EMBEDDING OF THE OVUM.}

THE deciduæ (deciduous or caducous membranes) are the hypertrophied mucosa of the uterus so developed as to form not only a lining for the uterine cavity, but also an envelope enclosing the ovum, and a specially altered part which serves as a bond of connection between the ovum and the womb.

During the four or five days preceding menstruation, the socalled constructive stage of the menstrual cycle, the mucous membrane of the womb becomes much thickened and musually vascular, the purpose of these changes being evidently the preparation of the uterus for the reception of the ovum in the event of impregnation. If impregnation has not occurred, the thickened mucosa, the decidua menstrualis, is in great part cast off as a part of the menstrual discharge; if, on the other hand, conception has taken place, the mucous membrane undergoes still greater hypertrophy. On section, it is seen to consist of a superficial compact stratum and a deeper

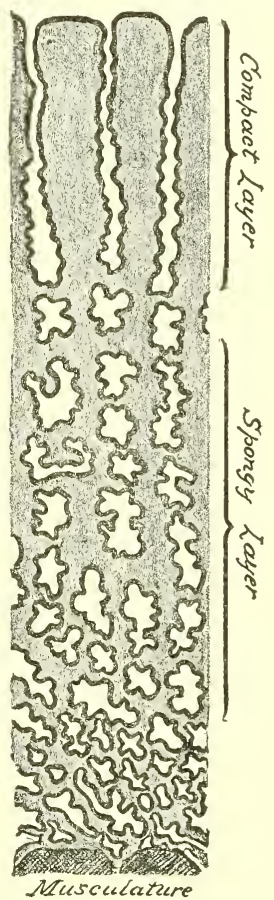

FIG. 51.-Cross section through the mucous membrane of the uterus at the beginning of pregnancy (after Kundrat and Engelmann).

spongy layer reposing directly upon the muscular wall of the uterus. In the compact layer are the necks of the much 
enlarged uterine glands, while in the spongy layer are their greatly branched and often tortuous bodies (Fig. 51). The tortuosity and division of the deeper extremities of the glands produce the characteristic appearance of a section of the spongy stratum.

The alterations necessary to convert the menstrual decidua into the deciduæ of pregnancy take place in part while the ovum is still in the Fallopian tube; when it reaches the uterus it becomes attached to the mucous membrane of the latter, usually along the upper part of the posterior wall. A portion of the mucous membrane eventually comes to enclose the ovum as in a distinct envelope (Plate V., Fig. 1). The part of the uterine mucosa which thus surrounds the ovum is the decidua reflexa; the part still lining the cavity of the womb is the decidua vera; the part that is in contact with the chorion frondosum is the decidua serotina. The decidua serotina afterward becomes the maternal part of the placenta, intimately uniting with the chorion frondosum.

Until recently it was believed that the ovum became implanted upon the surface of the mucosa, and that the latter grew up around and over it to form the decidua reflexa. This theory has been completely set aside by the recent investigations of Peters of Vienna, whose results have been confirmed by Webster of Chicago. Peters' observations were made upon the gravid uterus of a suicide, the ovum being embedded in a triangular prominence on the upper median region of the posterior uterine wall. The ovum measured in three diameters respectively $1.6,0.8$, and 0.9 $\mathrm{mm}$., its estimated age being three or four days.

The embedding of the ovum (Plate $\mathrm{IV}$.), or its sinking into the mucosa, is quickly accomplished by the erosion of the superficial layers of the latter, presumably by the phagocytic action of the trophoblast. Actual erosion is evident from the absence of the surface epithelium at this place. The ovum is thus brought into relation with the deeper layers of the mucosa. The edges of the excaration are undermined, so that the ovum is partly covered by the mueosa, the area not thus covered being occupied by an organized blood clot, 
Plate IV.
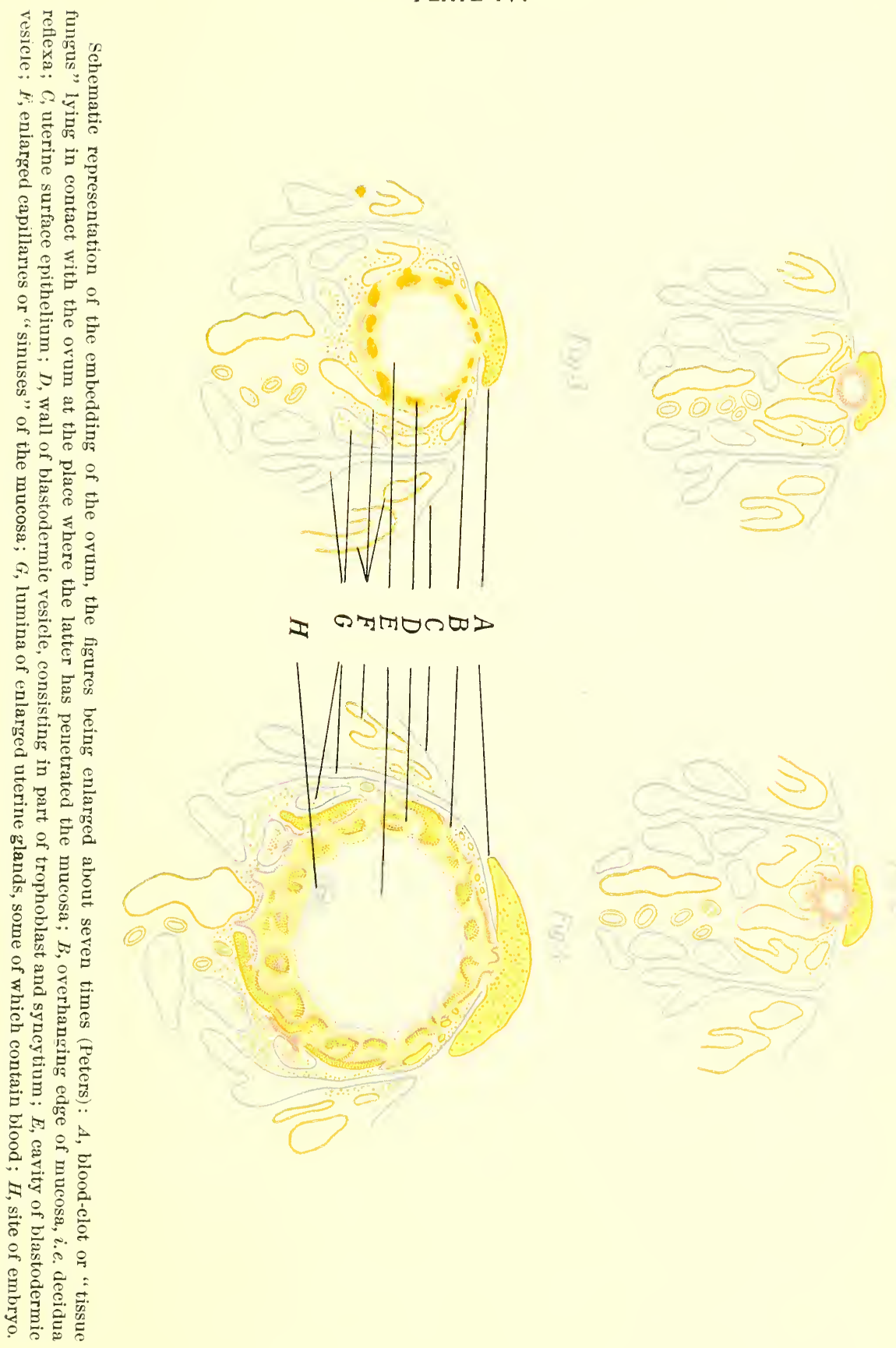

ऽ वसणतण

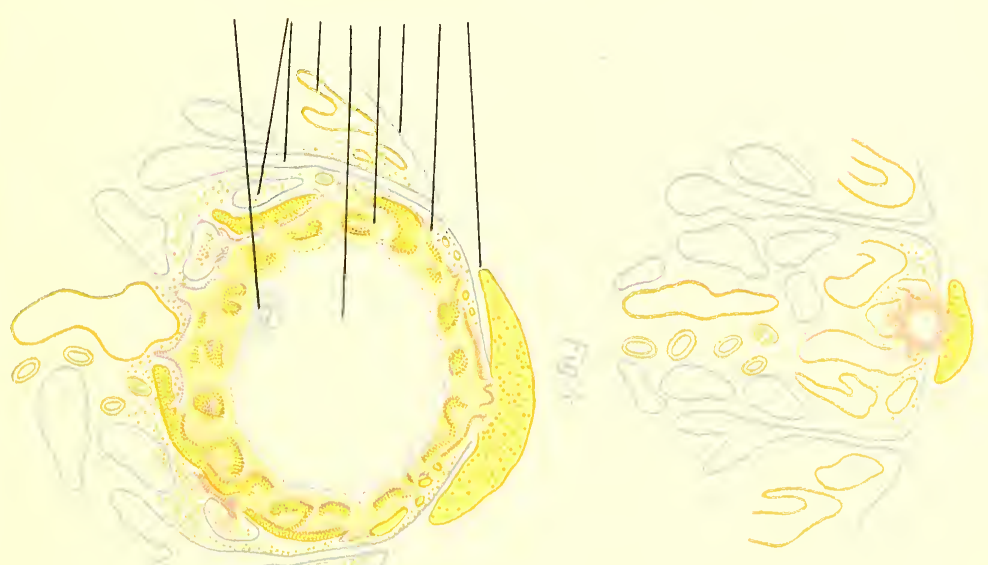



the tissue fungus (Plate IV.). The overhanging edges of the excavation constitute the beginning of the reflexa, which is obviously, therefore, not produced by the upgrowth of a circular fold of mucosa. The trophoblast strands or early villi extend toward and into the serotina, to which some of them become attached. It is thought by Webster that they may absorb fluid and nutriment, and that by phagocytic action they open up the blood-spaces of the serotina, thus bringing them into communication with the lacunæ of the trophoblast.

The blood-lacunæ of the trophoblast form a system of intercommunicating spaces, the beginnings of the later intervillous spaces of the placenta; they are filled with maternal blood from the serotina, and are liner with syncytium (Fig. 53 ), the latter being thin in places and resembling an endothelium. There is no extension, however, of the endothelium of the serotinal vessels, either upon the villi or into the spaces (Peters and Webster).

The syncytium is the more or less irregular layer of nucleated protoplasm which appears upon the surface of the ovum toward the end of the first week, lining the trophoblast lacunæ, and later penetrating as irregular masses into the serotina, where it is found until the end of pregnancy. No traces of it are found on the vera after the sixth week. The origin of the syncytium has long been in dispute. Peters has shown that it results from the transformation of the superficial part of the trophoblast, probably from contact of the latter with maternal blood, which, he thinks, exercises a blending influence upon the trophoblast cells, so that as individual cells they disappear, the result being a non-cellular but nucleated protoplasm. Peters also believes that corpuscles of the maternal blood are appropriated by the syncytium, and that the latter, covering villi and chorion as it does, has something to do with the interchange of nutriment and waste products between the maternal blood and the ovum. What remains of the early trophoblast after the formation of the syncytium is the layer of Langhans.

The ciliated epithelium of the uterine mucous membrane disappears by the end of the first month of pregnancy (Minot); 
somewhat later, that of the uterine glands is also lost. By the end of the fifth month the fetus and its appendages have increased in size to such an extent that they completely fill the cavity of the womb, and the space between the vera and the reflexa is obliterated. After the second month the vera becomes progressively thinner and the reflexa undergoes degenerative changes to such a degree that by the end of pregnancy merely remnants of it are present. ${ }^{1}$ By the sixth month the vera is intimately blended with the chorion.

\section{THE PLACENTA.}

The placenta, in certain groups of mammals, including man, is the organ of nutrition for the fetus during about the latter two-thirds of the period of gestation. In man, it is a discoid structure, attached by one surface to the wall of the womb, and connected on its opposite aspect with the fetus through the medium of the umbilical cord.

The human placenta represents the highest specialization of an apparatus for bringing the fetal blood into intimate relation with the blood of the mother. In eggs that develop outside of the body of the mother, such as those of reptiles, birds, and the lowest mammals, the Monotremata, the growing embryo necessarily acquires no connection with the uterine mucosa, but draws upon its original dower of nutriment, the deutoplasm, until its development is completed, when it breaks through the shell and seeks its own food; in these groups the chorion develops no villi. In the marsupials, a group of mammals higher than the monotremes, the ovum, although developing in the uterus, forms no close connection with it, but obtains its nourishment by simple imbibition from the uterine mucosa. On the other hand, in all mammals higher than monotremes and marsupials, the chorion is distinguished by the presence of villi upon its surface.

Between the human placenta and the non-villous chorion of the marsupials, certain gradations exist; for example, in pigs, whales, and some others, there is no proper placenta,

\footnotetext{
${ }^{1}$ Webster.
} 
the villi being evenly distributed over the surface of the chorion; while in ruminants the villi are grouped into little clusters or tufts called cotyledons, which are easily detachable from the mucous lining of the womb. Owing to this loose connection, the uterine mucosa is non-deciduous-that is, it is not cast off after the birth of the young. The foregoing classes are therefore styled Mammalia indeciduata, in contradistinction to the Mammalia deciduata-including man, rodents, and Insectivora-in which there is a loss of the greater part of the uterine mucous membrane after the

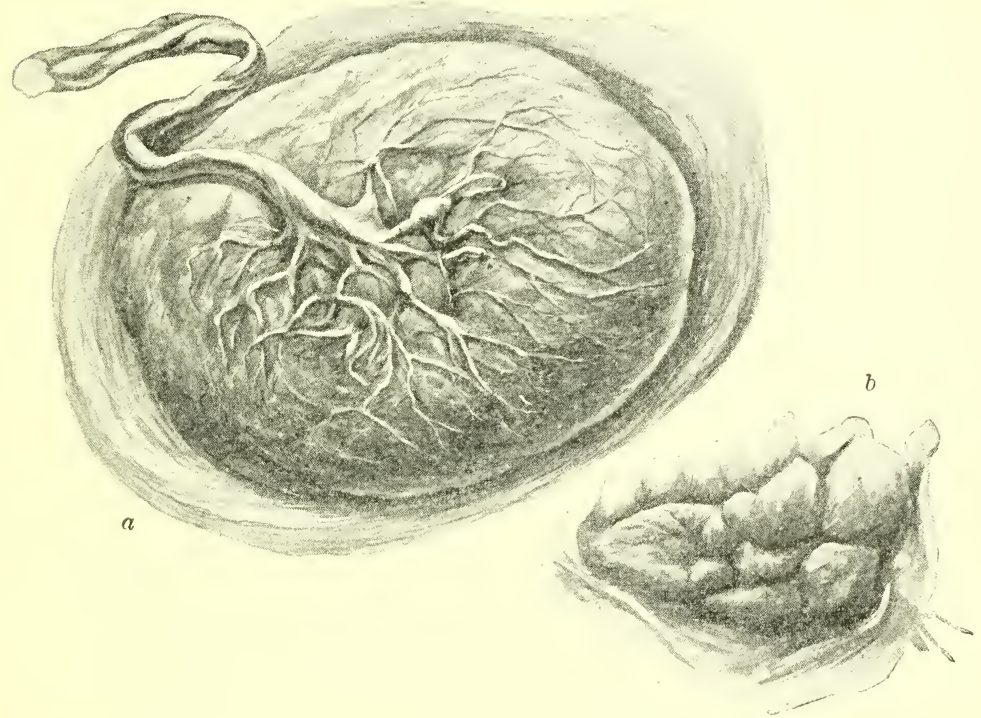

FIG. 52.-Mature placenta: $\alpha$, entire organ, showing fetal surface with membranes attached to the periphery; $b$, a portion of attached surface.

expulsion of the fetus. In the Carnivora the placenta has the form of a zone or ring-placenta zonaria-while in man and certain allied mammals, as apes, rodents, and some others, it is discoid in shape-placenta discoidea.

The human placenta is formed in the third month of pregnancy; since it results from the union of the chorion frondosum with the decidua serotina, it consists of a fetal and a maternal part.

Our conceptions of the development of the placenta must be modified to accord with recent investigations. The em- 
bedding of the ovum in the uterine mucosa and the modifications occurring in the chorion, including the growth of its villi and its differentiation into the chorion frondosum and the chorion leve, have been considered above. It will be recalled that the orum eats its way, as it were, into the mucosa, thus causing the superficial layers of the latter to disappear at the site of implantation. It is possibly this process of erosion upon the part of the fetal trophoblast that opens up the already dilated capillaries of the serotinathe sinuses-and allows the maternal blood access to the blood-lacunæ of the trophoblast, where it thus bathes the primitive villi. Since the entire trophoblast is vacuolated,

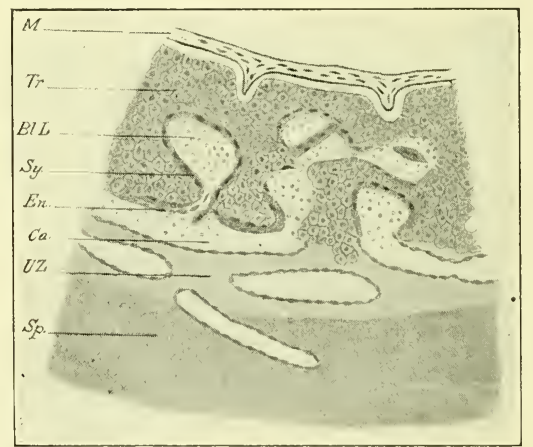

FIG. 53.-Schematic representation of the development of the placenta (after Peters): $M$. ., mesoblast; $T r$., trophoblast ; $B l . L$., blood lacunæ; Sy., syncytium ; $E n$., endothelium; $C a$., maternal capillaries; $U Z$., circumvallation zone; $S p$., spongiosa of serotina.

the maternal blood at this time-the first week-is brought into relation with the whole surface of the chorion. In the latter half of the first month the distinction between chorion frondosum and chorion leve begins to be manifest, the villi of the latter gradually retrograding until, in the sixth week, they are greatly degenerated, many of them being without blood-vessels.

The villi of the chorion frondosum increase in size, number, and complexity, some of them acquiring attachment to the serotina, but not, as a rule, entering the serotinal blood sinuses. The formation of new villi continues throughout pregnancy. The villi acquire capillaries in the second week, these being 
extensions of the allantoic blood-vessels. The syncytium of the chorionic lacunæ, now the intervillous spaces, increases in quantity, and not only lines the spaces, but exists in the form of masses, some of which become attached to the serotina between the villi, while others penetrate into it, many being found at the fourth month in the serotinal connective-tissue spaces.

The decidua serotina (basal decidua) in the first month is edematous and hyperemic, presenting dilated capillaries and blood-spaces, many of which communicate with the intervillous spaces of the chorion. By the sixth week its surface epithelium is entirely lost, and the parts of its glands contained in the compacta are to a great extent obliterated. In

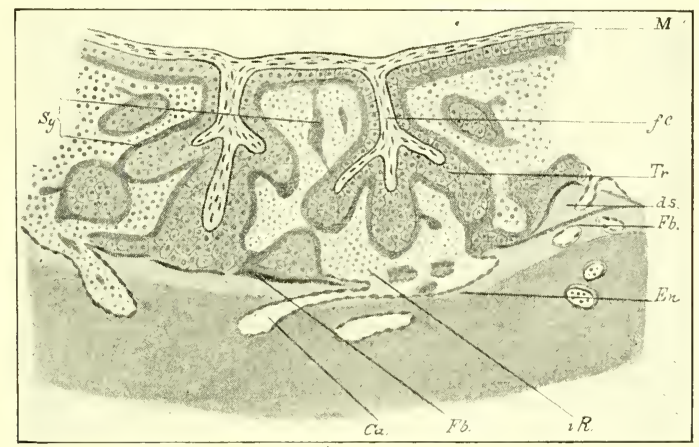

FIG. 54.-Schematic representation of the development of the placenta (after Peters): M., mesoblast; Tr., trophoblast; Sy., syncytium ; En., endothelium ; Ca., maternal capillaries; f.c., fetal capillaries; d.s., decidual septum; F.b., fibrin; i.R., intervillous space.

the fourth month it is thinner, more irregular in thickness, contains less sinuses, and shows degeneration in the compacta, with many masses of syncytium. Toward the end of pregnancy the sinuses increase in size, and the irregularity in thickness and the degeneration are more marked.

The placenta at term is a discoid mass, in situ, but less flattened after its expulsion from the uterus. Its diameter is from 15 to 20 and its thickness from 3 to 4 centimeters. The uterine surface is convex and irregular, and is imperfectly divided into tufts or cotyledons. The somewhat concave fetal surface, rather mottled, is covered by the loosely adherent amnion, and presents, usually near its center, 
the attachment of the umbilical cord. The maternal part of the placenta, the decidua serotina, is of varying thickness, in some places being absent. Its compact layer shows fibrinous degeneration, very few traces of glands, and no epithelium. The blood-spaces, lined with endothelium and representing greatly dilated capillaries, are in communication with the intervillous spaces of the fetal placenta (Plate IV., a). Scattered throughout the serotina are masses of syncytium. In the shed placenta there is very little of the serotina, since separation takes place through the compacta, the spongiosa and a part of the compacta remaining upon the uterine wall.

The fetal part comprises almost the entire thickness of the cast-off placenta. It is made up of villi ${ }^{1}$ of all ages and sizes springing from the chorion (some attached distally to the serotina, others projecting free into the intervillous spaces), and of masses of syncytium attached to both villi and chorion (Plate IV., $\iota$ ). The intervillous spaces are a system of intercommunicating cavities through which the maternal blood circulates and which are in communication with the blood-spaces of the serotina. Elevations of the serotina between the villi constitute the septa placentæ. The so-called marginal sinus at the periphery of the placenta is merely a system of intervillous spaces that intercommunicate more freely because of the relative paucity and small size of the villi in this region.

The site of attachment of the placenta to the uterus is usually the upper part of the posterior wall. Under certain circumstances it may become attached lower down, even extending partly or wholly over the mouth of the womb, constituting then the condition known as placenta prævia.

\section{THE UMBILICAL CORD.}

The blood-ressels through which the fetal blood finds its way from the fetus to the placenta and back again to the fetus, together with the atrophic vestiges of certain structures associated with the development of these vessels, constitute the structure known as the umbilical cord. In considering the growth of the human allantois it was pointed out that the

1 For structure of villi, see pages 93,94 . 


\section{Plate IV $a$.}

1

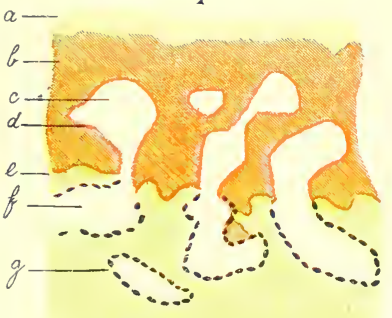

2

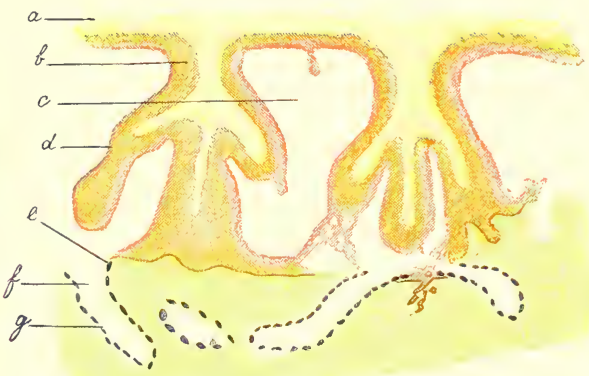

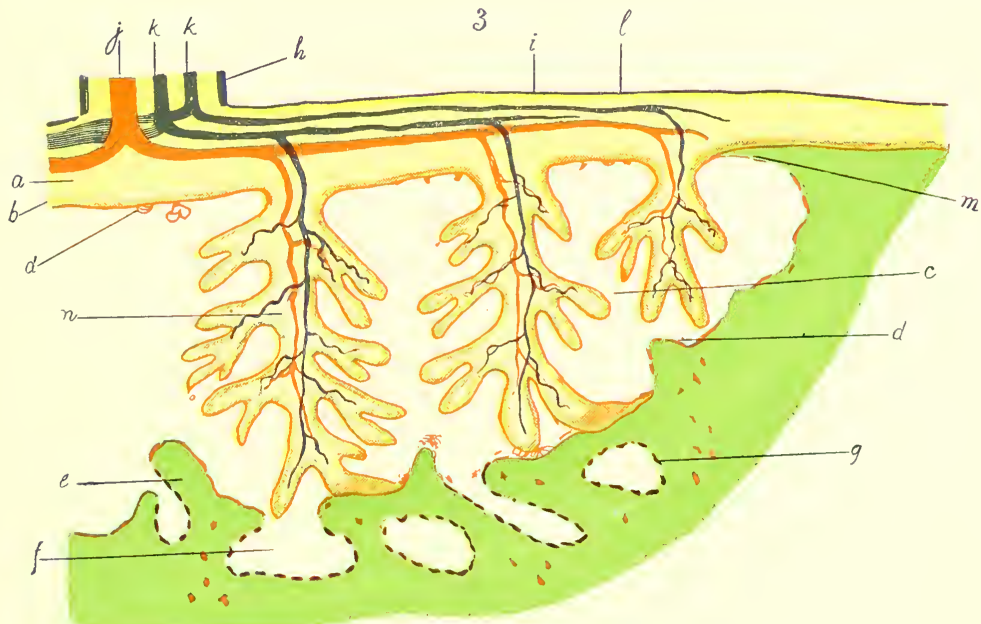

Diagrammatic representation of relationship of ovum to decidua: 1 , in latter half of first week; 2, a few days later; 3, a few months later, when placenta is well defined (Webster): a, fetal mesoblast, showing indications of beginning extension into trophoblast stalks in 1 , actual extension in 2 and $3 ; b$, trophoblast, being reduced in 3 and constituting here the layer of Langhans; $c$, trophoblast lacuna in 1 , enlarged in 2 and 3 as an intervillous space; $d$, syncytium, seen in its earliest stage in 1 ; $e$, decidua; $f$, maternal blood-sinus; $g$, endothelium lining maternal sinus; $h$, epiblastic covering of cord ; $i$, amniotic epiblast; $j$, umbilical vein ; $k$, umbilical artery ; $l$, amniotic mesoblast; $m$, extension of decidua on under surface of chorion at edge of placenta; $n$, large villus-stem. 

latter structure, as it grows from the ventral wall of the guttract into the so-called allantoic or abdominal stalk, becomes the seat of development of the two allantoic arteries and of an equal number of allantoic veins. With the metamorphosis of the chorion frondosum into the fetal placenta, the abdominal stalk becomes more slender and at the same time much elongated, and the allantoic blood-ressels are henceforth the umbilical vessels. The two umbilical veins fuse, so that, at birth and for some time before, there is but one vein, though there are still two arteries. The umbilical vein, entering the body of the fetus through the umbilicus, passes directly to the under surface of the liver, where it unites with the fetal portal vein and gives off a branch of communication, the ductus venosus, to the inferior rena cava, after which it enters the liver through the transverse fissure. The umbilical arteries, whose intra-embryonic portions are called the hypogastric arteries, are the direct continuations of the superior vesical arteries of adult anatomy. They leave the body of the fetus at the umbilicus.

The umbilical cord, while consisting essentially of the three blood-vessels mentioned, contains also the remnant of the allantoic stalk and of the umbilical vesiele, these structures being surrounded and held together by a quantity of embryonic connective tissue, the jelly of Wharton, which makes up the chief part of the mass of the cord; upon the surface is a layer of epithelium, continuous, at the distal end of the cord, with the epithelium of the amnion.

The umbilical cord has an average length of $55 \mathrm{~cm}$., or 22 inches, but varies between the extremes of $15 \mathrm{~cm}$. ( 6 inches) and $160 \mathrm{~cm}$. (64 inches); its thickness is about $1.5 \mathrm{~cm}$. ( $\frac{3}{5}$ inch). The cord presents the appearance of being spirally twisted; it is probable, however, that the appearance of torsion is conferred by the spiral or coiled arrangement of its arteries, due to their excessive growth, rather than by a twist of its entire mass. There may be one or more true knots in the cord, produced by the slipping of the fetus through a loop.

The position of attachment of the cord to the placenta is 
usually near, but seldom exactly in, the center of the fctal surface of that organ; rarely it may be found attached to its edge, and still more rarely to the fetal membranes themselves at some little distance from the edge of the placenta, with which, in the latter case, it is connected by its bloodvessels.

The great length of the human umbilical cord is thought to be due to the relatively large quantity of amniotic fluid present in the human subject.

After birth, the portions of the hypogastric arteries extending from the upper part of the lateral wall of the bladder to the umbilicus undergo atrophy, becoming impervious fibrous cords; the intra-abdominal part of the umbilical vein likewise becomes atrophic and impervious, constituting the so-called round ligament of the liver.

\section{RELATIONS OF THE FETAL MEMBRANES AT BIRTH.}

When the amniotic fluid attains its maximum bulk-at about the end of the sixth month-it requires so much space that it presses the amniotic membrane closely against the chorion, which latter, covered by the remnants of the reflexa, is in turn forced into intimate relation with the vera (Plate V.). At term the vera and chorion have become practically one membrane. The amnion, while adhering to the inner surface of the chorion, is so loosely associated with the latter that it may be peeled off from it. The membrancs, which constitute a fluid-filled sac surrounding the fetus, are ruptured by the contractions of the uterus at some time during parturition. Through this rent the child is forced during birth, the placenta and the membranes remaining behind. After the expulsion of the child, the vera and the placenta detach themselves from the uterine wall, and, with the chorion and the amnion, constitute the afterbirth, which is expelled shortly after the expulsion of the child. The separation of the after-birth takes place in the compact layer respectively of the decidua vera and of the uterine placenta. The spongy layer and what remains of the compacta serve for the regeneration of the uterine mucosa. 


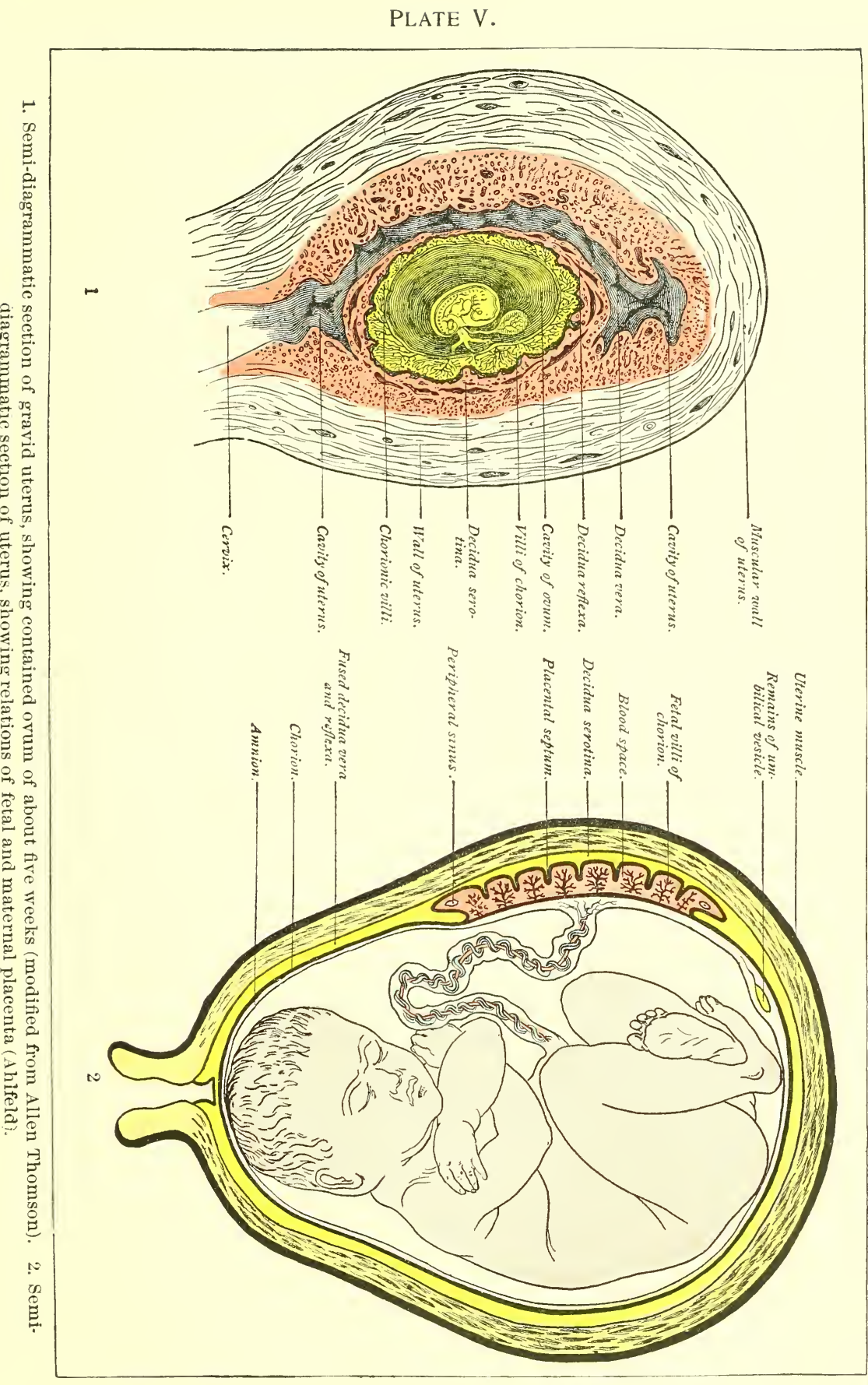





\section{CHA PTER VII.}

\section{THE FURTHER DEVELOPMENT OF THE EXTERNAL FORM OF THE BODY.}

Having traced the growth of the germ to the time when the body of the embryo becomes definitely differentiated from the embryonic appendages or fetal membranes, the development of the individual organs and tissues may be taken up. The discussion of this latter subject, especially of that part of it pertaining to the structures on the exterior of the body, involves a consideration of the external form of the embryo and fetus during the successive stages of growth.

In the preceding chapters it was pointed out that the cells of the segmented ovum arranged themselves in such a manner as to form a hollow vesicle, the blastodermic vesicle (Plate I.); that this vesicle, having at first a single-layered wall, came to consist of two layers of cells, the ectoderm and the entoderm; and that, finally, a third, intervening layer, the mesoderm, made its appearance. It was shown, further, that the thickened portion of the resicle wall, the embryonic area, became more and more differentiated from the remainder, and that, by certain processes of folding, this area was made to assume the definite form of the embryonic body, while from the other parts of the vesicle-walls the fetal membranes were produced (Plate II.). It may be well to remind the reader again that when the body of the embryo has become closed off from the fetal membranes, this body is an irregularly tubular structure whose walls are the somatopleure and whose enclosed space is the body-cavity, and that within it are two other tubes, a larger, the gut-tract, formed by the splanchnopleure, and a smaller ectodermic tube, the neural canal. 
While, as a matter of convenience, the description of the individual organs is taken up after tracing the course of development to this stage, it should be borne in mind that the rudiments of some of them are already distinguishable before the germ-layers become infolded to form the body-wall and the gut-tract. It will facilitate a comprehension of the general principles concerned in the origin of the different parts of the borly to refer to the tabulated statement of the derivatives of the three primary germ-layers as presented in Chapter III.

In considering the external form of the product of conception, one may adopt the classification of His, referred to in the first chapter. This author divides the period of development into three stages, of which the first, the stage of the ovum, or the blastodermic stage, comprises the first and second weeks of intra-uterine growth; the second, the stage of the embryo, extends from the second to the fifth week; and the third, or the fetal stage, includes the time between the fifth week and the end of gestation.

\section{THE STAGE OF THE OVUM.}

During the fortnight allotted to this first stage of development occur the various changes by which the impregnated ovum acquires the form of a hollow sphere, designated the embryonic or blastodermic vesicle. The series of transformations has been described in Chapter II. In this place it will be sufficient to refer to the external characters of the blastodermic vesicle as depicted in Figs. 49, 50, which represent the orum described by Reichert, and Fig. 55, the ovum of Peters. The Peters ovum was a vesicle measuring $1 \mathrm{~mm}$. in diameter, entirely embedded in the thickened uterine mucosa, its wall consisting of a layer of trophoblast lined with mesoderm (the chorion). Upon section it showed an embryonic bud or disk $0.19 \mathrm{~mm}$. in length, in relation by its dorsal surface with the amniotic eavity, while upon its ventral aspect was seen the yolk-sack. The orum of Reichert 
was estimated to be about twelve days old. Its form was that of a sphere somewhat flattened, its short and long diameters measuring respectively $3.3 \mathrm{~mm}$. and $5.5 \mathrm{~mm}$. The flattened surfaces were smooth, while the equatorial zone was

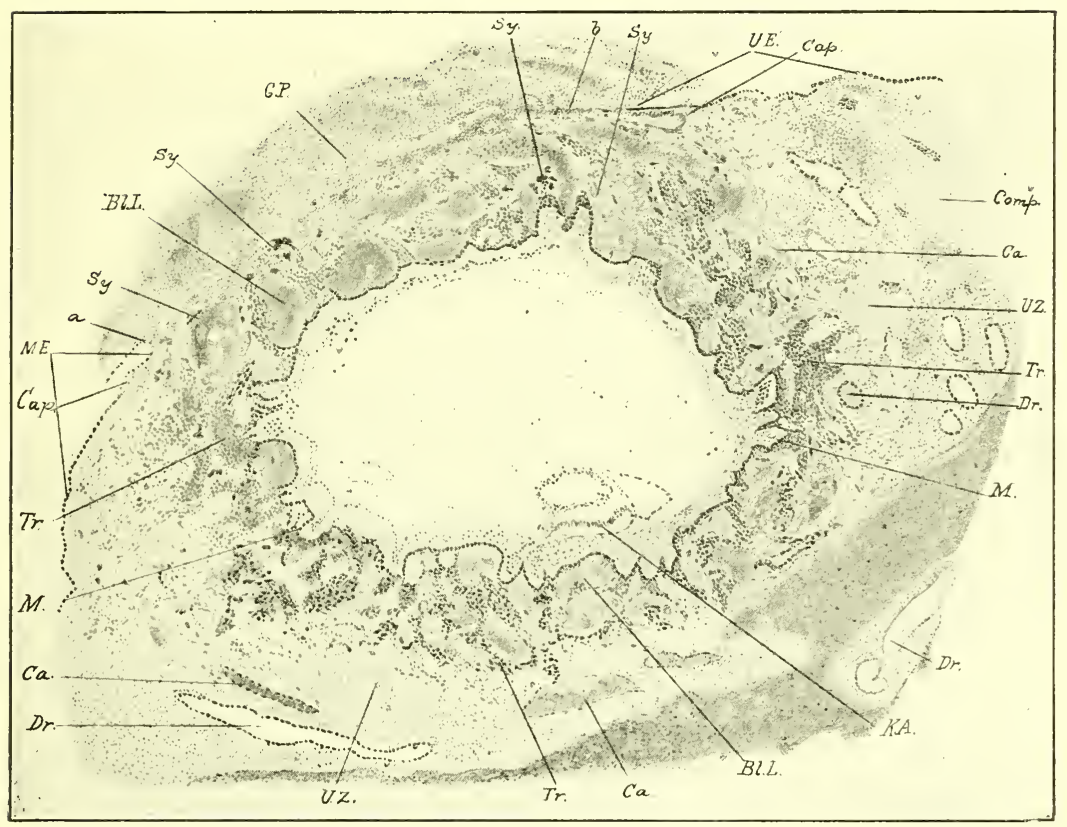

Fig. 55.-Section through orum embedded in the mucosa. Second haif of first week of pregnancy. The largest diameter of the chorionic resicle is sten (H. Peters): G.P, Blood-clot lying on the outer polar portion of the chorionic vesicle; $a, b$, edges of opening in mucosa through which the ovum has excavated; $U . E$, uterine epithelium; Cap, decidua reflexa; $T r$, trophoblast; $C a$, maternal capillary; $D r$, gland of uterine mucosa ; $B$ l.L, lacunæ in the trophoblast, containing maternal blood: $K$.A, site of embryo; Comp, decidua compacta; $\boldsymbol{I}$, fetal mesoblast; $U . Z$, interglandular tissue of mucosa, in which early decidual cells are found.

beset with villi. The early appearance of villi is characteristic of the human orum.

\section{THE STAGE OF THE EMBRYO.}

It is during the early part of the second stage, at about the fourteenth day, that the somatopleuric layer of the blastodermic vesicle becomes folded in to produce the walls of the 
embryonic body. Fig. 57 shows a human embryo of about the fifteenth day, whose form is as yet imperfectly differentiated, the ventral wall of the borly being incomplete, since the gut-tract is still in eommunication with the umbilical vesicle throughout almost the entire length of the embryo. The back and sides of the embryo are enveloped by the amnion, and the dorsal outline is concave. The caudal pole

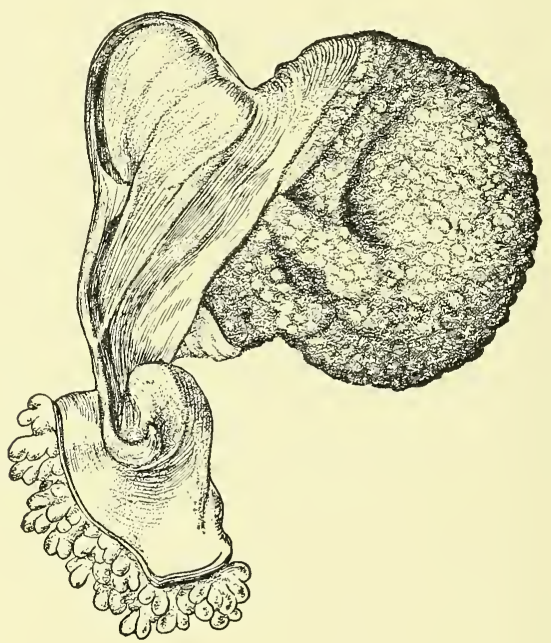

Frg. 56.-Human embryo of about the thirteenth day (His). The caudal pole of the embryo is connected with the blastodermic vesicle by means of the abdominal or allantoic stalk; the amnion already completely encloses the embryo, and the large vitelline sac communicates throughout the greater part of the mitral surface by means of the unclosed gut-tract.

is seen to be connected by means of the allantoic stalk with the primitive chorion, which latter structure, however, is not represented in the figure. The concavity of the dorsal outline is peculiar to the human embryo of this stage. The development of the head is closely associated with the dilatation of the cephalic end of the neural tube and the subsequent division of this dilated extremity into the three primary brain-vesicles, the fore-brain, the mid-brain, and the hindbrain. The oral pit, the first indication of the future mouth, is present in the early part of this stage; it is a depression 
between the prominent fore-brain vesicle and the cardiac dilatation (Fig. 58).

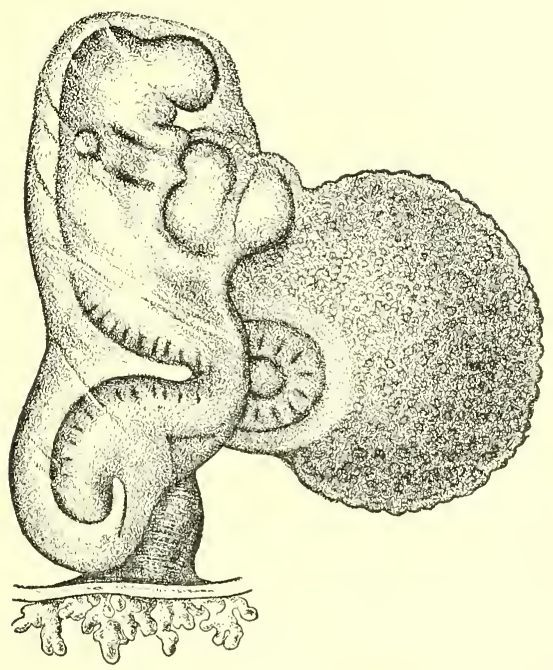

FiG. 57.-Human embryo of about the fifteenth day (His). The embryo is attached to the wall of the blastodermic vesicle by means of the umbilical or allantoic stalk, and is enclosed within the amnion; the large vitelline sac freely communicates with the still widely open gut.

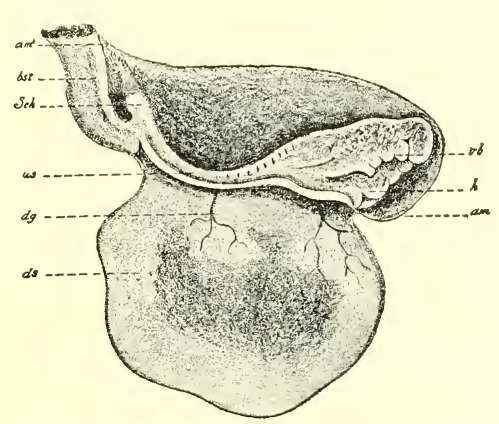

FIG. 58.-Human embryo with yolk-sac, amnion, and belly-stalk of fifteen to eighteen days (after Coste). The chorion is detached at $a m^{\prime}: a m$, amnion; $a m^{\prime}$, the point of attachment of the amnion to the chorion drawn out to a tip; bst, bellystalk; Sch, tail-end; $u s$, primitive segment; $d g$, vitelline blood-ressels; $d s$, yolksac; $h$, heart; $v b$, visceral areh.

Between the fifteenth and the twenty-first day, the lensresicles and the otic vesicles are formed by invaginations of the surface ectoderm (Fig. 59, 7 and 8), these sacs being 

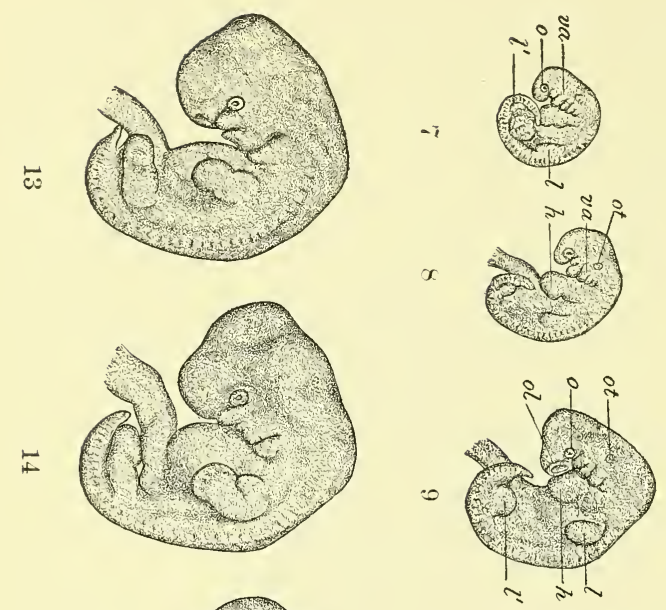

20
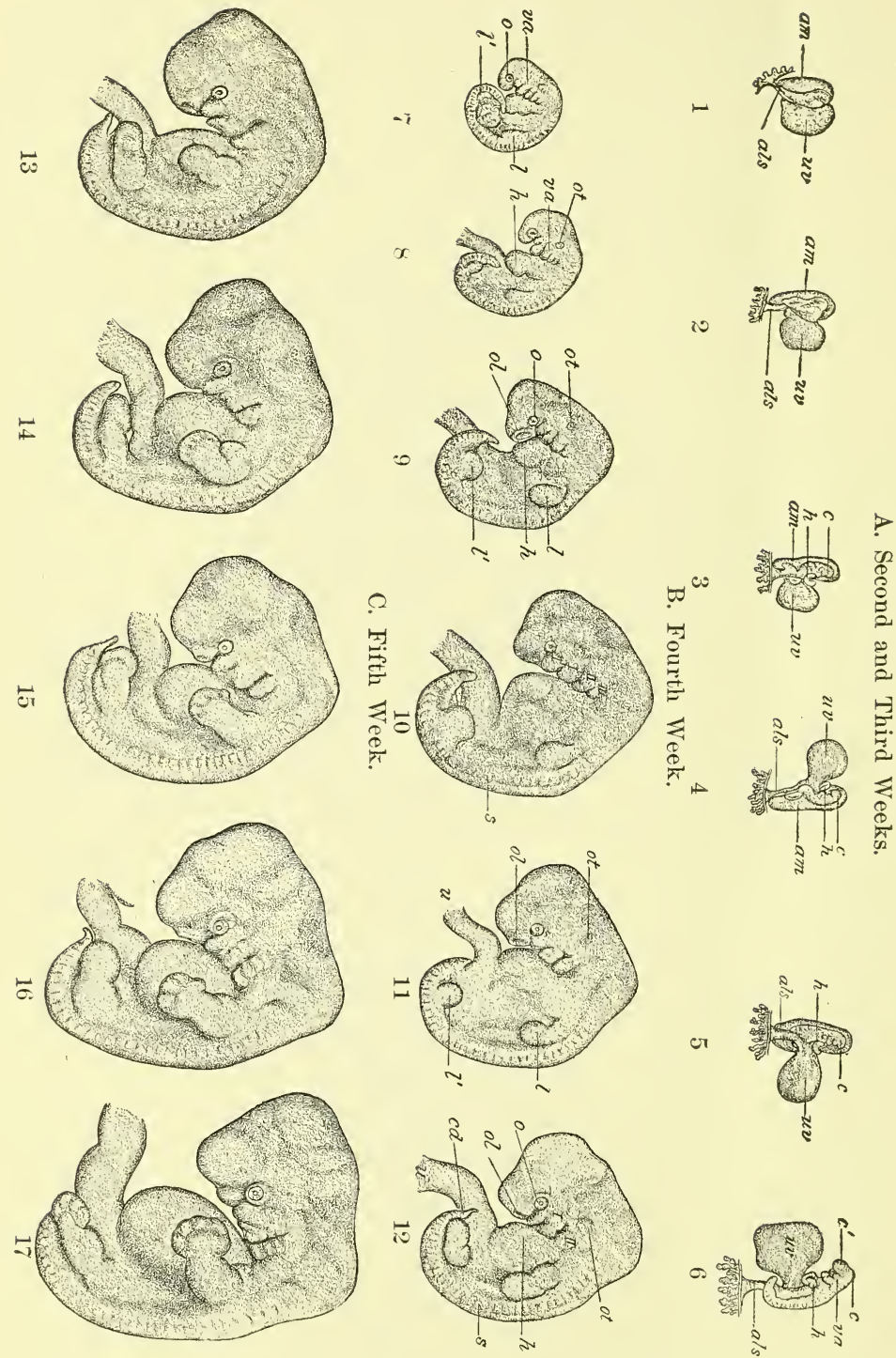

FIG. 59.-Early human embryos, all enlarged about two and a half times (His): 1-4, from twelfth to fifteenth day; 5,6 , from eighteenth to twenty-first day ; 7,8 , from twenty-third to twenty-fifth day; $9-12$, from twenty-seventh to thirtieth day; 13-17, from thirty-first to thirty-fourth day. am, amnion; uv, umbilical or vitelline vesicle; als, allantoic or abdominal stalk; $c, c^{\prime}$, brain-vesicles; $h$, heart ; va, visceral arches; $o$, optic vesicle; ot, otic vesicle; ol, olfactory pit; $l, l^{\prime}$, upper and lower extremities ; $s$, somites ; $c d$, caudal process; $u$, primitive umbilical cord. 
the rudiments respectively of the crystalline lens and of the membranous internal ear; at this time also the visceral arches and clefts first become distinguishable. On the twenty-first day, the rudiments of the limbs appear as little bud-like processes springing from the trunk. The conspicuous projection on the ventral surface between the now almost completed yolk-sac and the cephalic end of the body is produced by the primitive heart (Fig. 59, 10, 11, and 12).

Until the twenty-first day the embryonic body is erect. Between the twenty-first and twenty-third days a marked alteration in the appearance of the germ is brought about by a pronounced bending of the long axis of the embryonic body (Fig. 59). The degree of curvature is such that the caudal and cephalic extremities overlap. The flexion reaches its maximum degree by the twenty-third day. The curved dorsal outline is referable to four well-marked flexions, the position of the most anterior, or cephalic flexure, corresponding to that of the future sella turcica and being indicated by the projection of the mid-brain vesicle (Fig. 62, MII.); at this point the anterior part of the head is bent almost sufficiently to form a right angle with the posterior half. A second or cervical flexure is found in the future neck-region, while further caudad are seen the less pronounced dorsal and coccygeal curves.

The fourth week marks the period of the most active growth of the embryo. After the twenty-third day, the body as a whole uncoils somewhat, although in the latter half of the fourth week the individual flexures noted above become more conspicuous.

The Visceral Arches and Clefts.-The visceral arches, with the intervening visceral clefts, constitute a conspicuous feature of the external appearance of the embryo during this stage. These arches are a series of five approximately parallel ridges appearing upon each side of the future neck-region and extending obliquely downward and forward toward the ventral surface of the embryo (Figs. 60 and 62). The four furrows lying between the five visceral arches are the visceral clefts. A coronal section of the neck- 


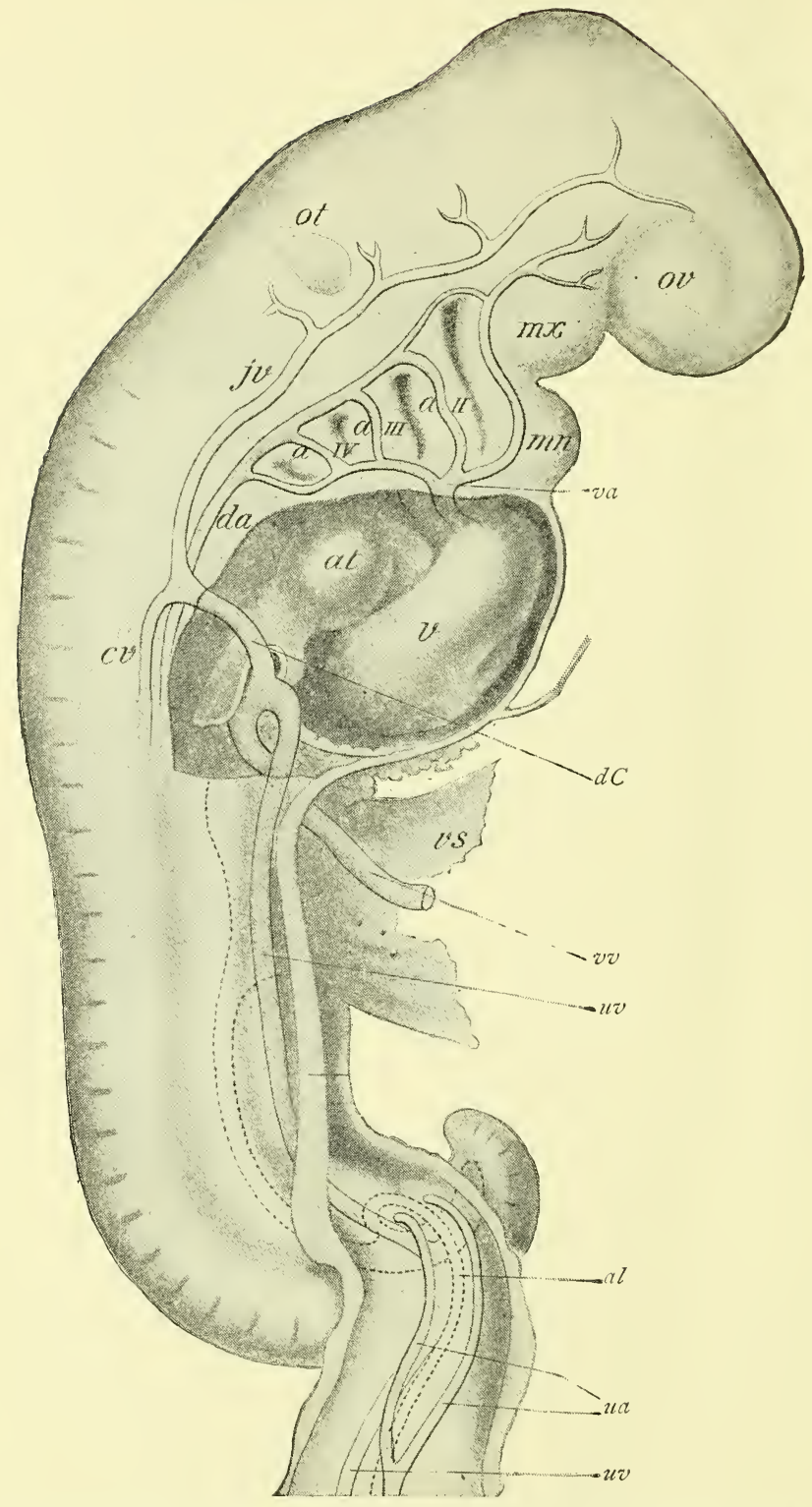

FIG. 60.-Human embryo of about three weeks, showing visceral arches and furrows and their relations to aortie arches (His) : $m x, m n$, maxillary and mandibular processes of first visceral arch ; $a I-a I V$, first to fourth aortic arches ; $j v, c v$, primitive jugular and cardinal veins ; $d C$, duct of Cuvier; $a t, v$, atrium and ventricle of primitive heart; $v s$, vitelline sac; $v a, d a$, ventral and dorsal aortæ; ov, ot, optic and otic vesicles; $u v, u a$, umbilical veins and arteries; $v v$, vitelline vein; al, allantois. 
region (Fig. 61) - a section in a plane parallel with the vent ral surface-shows that the furrows seen on the ectodermic surface correspond in position to a like number of deeper grooves on the inner or entodermic surface. The inner furrows are out-pocketings of the entoderm lining the pharyngeal region of the fore-gut; they are referred to as the pharyngyeal pouches or throat-pockets to distinguish them from the outer clefts. At the bottom of the clefts the ectoderm is in contact with the entoderm, the mesoderm being absent; these two layers constitute the closing membrane. The visceral arches or ridges consist of thickened masses of mesodermic tissue covered outwardly and inwardly respectively
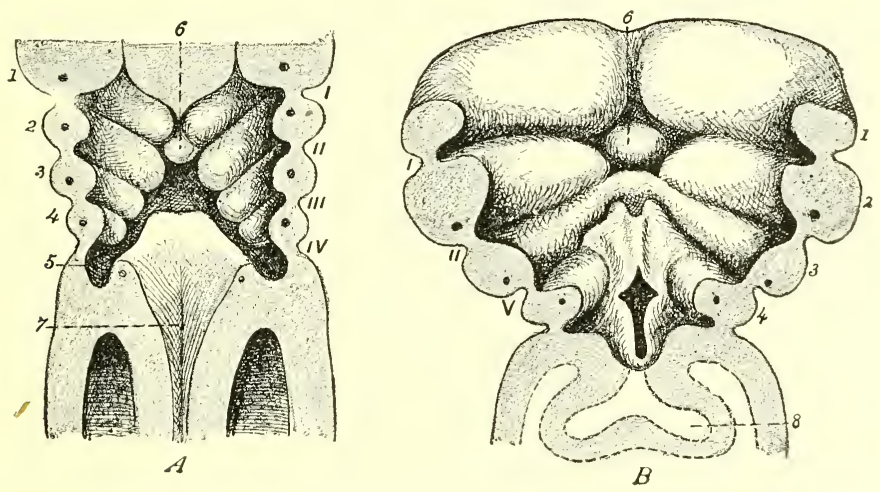

FIG. 61.-Coronal sections of two human embryos, showing ventral wall of pharyngeal end of gut-tract from behind (from Tourneux, after His). A, from embryo of $3.2 \mathrm{~mm}$.; $B$, of $4.25 \mathrm{~mm}$. (about 25 to 30 days). $I, I I, I I I, I \mathrm{l}$, outer visceral furrows; $V$, sinus præcervicalis, comprising third and fourth outer furrows; $1,2,3,4$, visceral arches, each with its visceral-arch vessel ; 6 , tuberculum impar; 7 , orifice of larynx; 8 , pulmonary evagination.

by the ectoderm and the entoderm. Each arch contains an artery, the visceral-arch vessel. These five pairs of visceralarch vessels arise by a common stem, the truncus arteriosus. from the primitive heart. ${ }^{1}$

The morphological significance of the visceral arches and clefts may be appreciated by a comparison of the conditions obtaining in lower types. While in birds and mammals the

${ }^{1}$ For an account of the metamorphosis of the visceral-arch vessels into the adult arteries of the throat and neck the reader is referred to Chapter $\mathrm{X}$. 
number of the clefts is four, in reptiles, amphibians, and bony fishes, five clefts appear, and in some fishes (selachians) the number is six. In all aquatic rertebrates, the thin epithelial closing membranes rupture, thus establishing communications between the alimentary tract and the exterior, through which openings water passes in and ont. The margins of the clefts-except the first or hyomandibular cleftbecome the seat of a rich supply of capillary blood-vessels, the blood of which obtains oxygen from the water and yields to the latter its carbon dioxid; while the risceral arches, excluding the first and second, become known in these classes. as branchial arches from their producing bony arches which support the branchiæ or gills. With the exceptions noted, the visceral arches and clefts with their capillary plexuses therefore functionate in these classes as a respiratory apparatus.

When, in the course of evolution, certain of the vertebrates assume an aerial existence, in consequence of which they acquire a breathing mechanism adapted to such a mode of life, the respiratory function of the clefts or branchiæ ceases, and they either disappear entirely or constitute merely rudimentary structures of the adult. The so-called clefts in man are never actual openings, the closing membrane always being present (His, Kölliker, Piersol, Born). To express the morphology of the visceral clefts briefly, they are permanent structures in fishes and in tailed Amphibia; they are present during the larval stage of other Amphibia, while in birds and mammals they are found only in embryonic life.

The growth of the visceral arches and clefts bears an intimate relation to the differentiation of the head- and the neckregions of the embryo. They first make their appearance at about the twenty-third day and attain their greatest development by the end of the fourth week. Both the arches and the clefts appear earliest and are best developed at the cephalic end of the series, the fifth arch being exceedingly illdefined. During the fifth week the obliteration of the arches and clefts as such begins, since certain of them become metamorphosed into permanent structures while the remainder undergo regression. 
The Metamorphosis of the Visceral Arches and Clefts.The first visceral arch becomes divided into an upper part, the maxillary arch, and a lower portion, the mandibular or jaw-arch (Fig. 62). The maxillary arches or processes of the two sides unite, at their anterior ends, with the intervening nasofrontal process (Fig. 67, and in this way is formed the upper boundary of the mouth-cavity; the mandibular processes become joined with each other anteriorly and constitute the inferior boundary of this cavity. The maxillary processes become the superior maxilla, while the mandibular processes become the lower jaws. 'The mesodermic core of the mass of tissue constituting the mandibular arch divides into three sections, of which the two situated at the proximal end of the arch are quite small and give rise respectively to the incus and the greater part of the malleus; the large distal segment is a slender cartilaginous rod, Meckel's cartilage, whose proximal extremity becomes the processus gracilis of the malleus (see Chapter XVIII.).

The second visceral, or anterior hyoid arch becomes obliterater as such, although a bar of cartilage which it containsReichert's cartilage-gives rise by its proximal extremity to the stapes, ${ }^{1}$ while the remaining portion becomes metamorphosed into the styloid process, the stylohyoid ligament, and the lesser cornu of the hyoid bone.

The third or posterior hyoid arch, which corresponds with the first branchial arch of fishes, likewise loses its identity as a surface marking, while the bar of cartilage it contains becomes the body and greater cornu of the hyoid bone.

The fourth and fifth arches coalesce with the adjacent tissues, producing no special structures.

The first outer cleft, known as the hyomandibular cleft, suffers obliteration except at its dorsal extremity, where the tissues forming its margins produce the external ear. The remaining three outer clefts disappear in the following manner : the fourth outer cleft becomes covered and hidden by the fourth arch, and the third and second clefts are successively

${ }^{1}$ Reichert, Gegenbaur, Hertwig; or to the ring of the stapes according to Salensky, Gradenigo, and Rabl. 
Duried by the growth of the third and second arches. The sinking-in of the lower arches and clefts (Fig. 61) results in

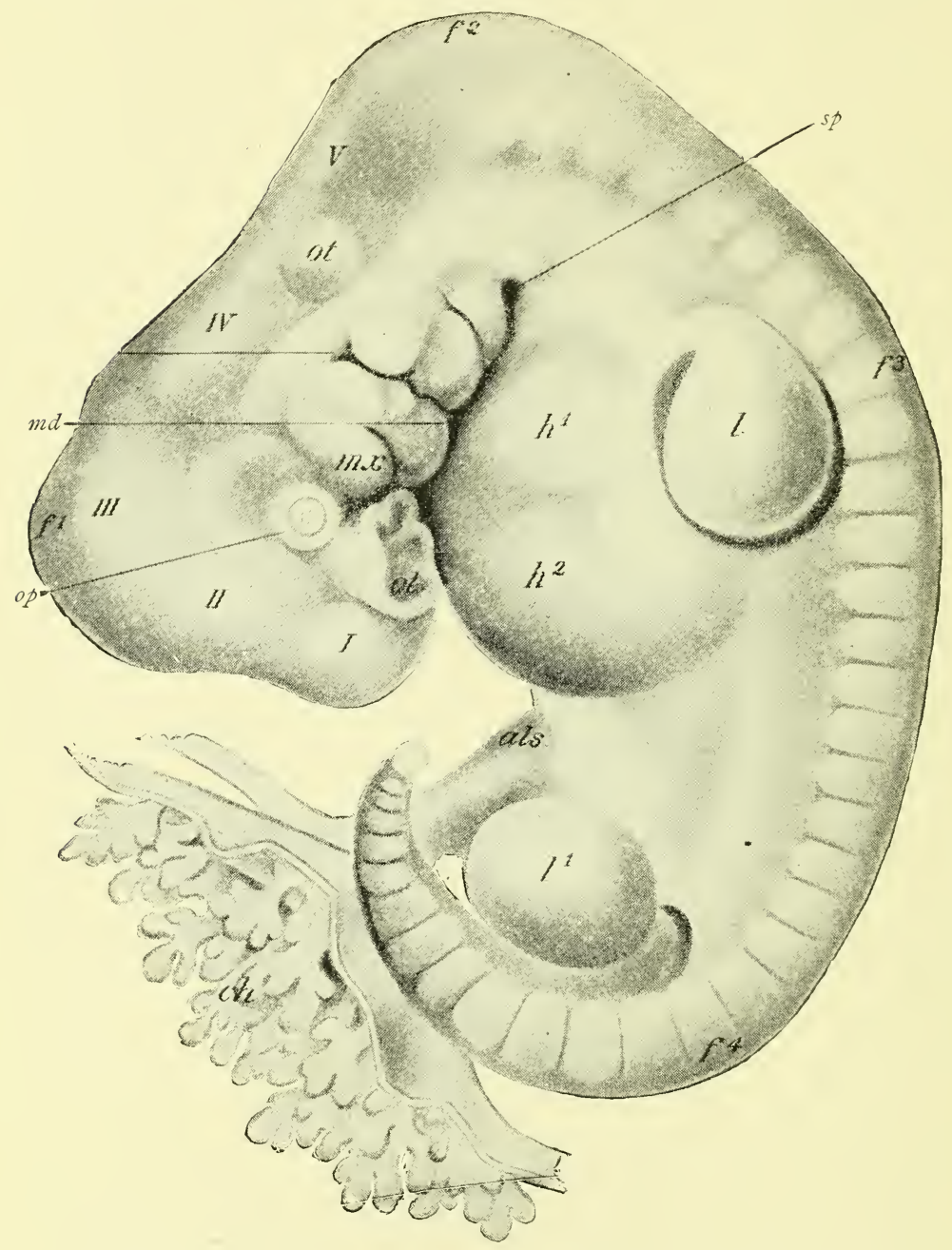

FIG. 62.-Human embryo of about twenty-eight days (His): $I$ - $V$, brain-vesicles ; $f^{1}, f^{2}, f^{3}, f^{4}$, cephalic, cervical, dorsal, and lumbar flexures; op, eye; ot, otic vesicle; ol, olfactory pit; $m x, m d$, maxillary and mandibular processes of first visceral arch ; sp, sinus præcervicalis ; $h^{1}, h^{2}$, heart ; $l, l^{1}$, limbs ; als, allantoic stalk ; $c h$, villous chorion.

the formation of a fossa or fissure on the lateral surface of the neck, the sinus præcervicalis (Fig. $62, s p$ ), which subsequently 
is made to disappear by the coalescence of its edges. Occasionally this sinus, instead of becoming completely obliterated, persists, and the thin layer of tissue forming its bottom ruptures-possibly spontaneously or perhaps more probably as the result of exploratory probing-constituting the anomaly known as cervical fistula. Such a fistula establishes an opening into the esophagns.

The first inner cleft or first pharyngeal pouch becomes metamorphosed into the middle ear and the Eustachian tube, the closing membrane, which separates it from the outer cleft, forming the membrana tympani. The second pharyngeal pouches produce no special structures, but the adjacent tissues give rise to the epithelial parts of the middle lobe of the thyroid body and to the posterior third of the tongue, in the manner more fully indicated on pp. 143 and 226. The third inner cleft produces the thymus body, while from the fourth results the lateral lobes of the thyroid body.

The configuration of the face, depending as it does so largely upon the development of the boundaries of the nose and of the mouth, is closely associated with the growth of the first pair of visceral arches. The earliest indication of the mouth, the oral pit, appears at about the twelfth day as a shallow depression on the rentral surface of the embryonic body between the fore-brain vesicle and the prominence caused by the primitive heart (Fig. 59, 3 to 5). This depression is deepened by the growth of the tissues surrounding it, as also by the flexure of the head, which occurs at the twenty-first day. In the third week, therefore, the oral pit is a five-sided fossa, being bounded above by the nasofrontal process, which has grown down from the elevation of the fore-brain, laterally by the maxillary processes, and below by the mandibular arches (Fig. 67, A). The pharyngeal membrane, which consists of opposed ectoderm and entoderm and which separates the primitive oral cavity from the gut-tract (Fig. $66, r h$ ), ruptures at the time of the appearance of the third branchial arch.

By the end of the third week, the communication between the yolk-sac and the gut-tract has become reduced to the relatively small vitelline duct. At the twenty-fifth day the 
embryo presents a well-dereloped tail. By the termination of the fourth week the yolk-sac has attained its maximum size, and the presence of the somites is indicated by transverse parallel lines on the dorsal surface of the body.

\section{THE STAGE OF THE FETUS.}

This stage comprises the time between the beginning of the second month and the end of pregnaney.

During the second month the rate of growth is far less rapid than in the preceding stage. The marked curvature of the long axis of the body gradually dimishes, the embryo assuming a more erect posture. Owing to the partial disappearance of the cervical flexure, the head becomes raised.

During the fifth week the vitelline duct is seen to be long and slender; the umbilical eord has become longer and more spiral, and may contain a coil of intestine; the abdomen is very prominent, and in the neck-region is a characteristic dorsal concavity. At this time also the nasal pits become conspicuous as depressions situated on either side of the nasofrontal process (Fig. 67). The nasofrontal process mean while undergoes differentiation into the globular processes, which constitute the inner boundaries of the nasal pits, and the lateral frontal processes, which limit these depressions exter-

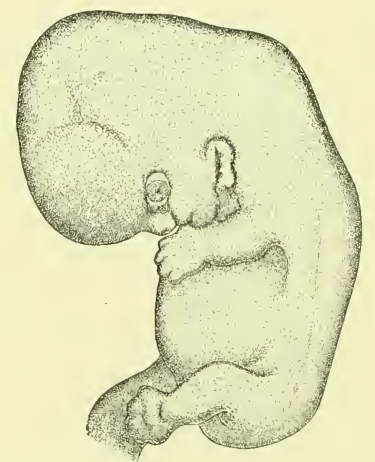

Fig. 63,-Human embryo of about six weeks, enlarged three times (His).

nally and separate them from the depressions for the eyes. The nasal pits are still in communication below with the 
primitive oral cavity. The lacrimal groove is well-marked at this stage, and the external auditory meatus is indicated. The mandibles become united mesially at about the thirtyfourth day. The third and fourth gill-clefts have by this time disappeared in the cervical sinus. The paddle-like limbbuds have lengthened and present, at first, a division into two segments, of which the distal is destined to become the hand or foot, while the proximal portion undergoes segmentation a little later into the arm and forearm or thigh and leg; by the thirty-second day, the hand, now showing differentiation into a thicker proximal and a thinner terminal part, exhibits the first traces of digitation, in the form of parallel longitudinal markings which soon become grooves and, later, clefts. The development of the upper extremities precedes that of the lower by twelve or fourteen days.

During the sixtn week the head assumes more nearly its normal position, and for this reason the apparent length of the fetus is considerably increased, the dorsal concavity in the neck-region being almost obliterated; the rudiments of the eyelids and of the concha become recognizable, and the various parts of the face assume more definite shape. By the fortieth day the oral cavity has become separated from the nasal pits by the union of the nasofrontal process with the maxillary processes, and the external boundaries of the nostrils have become marked out by the meeting of each lateral frontal process with the corresponding maxillary process. As a result of these changes, the nose, although still very broad, begins to assume characteristic form. During this weck also the fingers are seen as separate outgrowths, while in the seventh week the rudiments of their nails become evident.

Toward the end of the second month-about the fiftieth to the fifty-third day - the toes are just beginning to separate, the protrusion of the intestine at the umbilicus is at its maximum, the palpebral conjunctiva separates from the cornea, and the rudimentary tail hegins to disappear.

The eighth week witnesses the total disappearance of the free tail, the formation of the septum that divirles the 
cloaca into the rectum and the genito-urinary passage, and the presence of the projecting genital tubercle with the accompanying genital folds and genital ridges. The external genitals as yet show no distinction of sex. From the end of the second month to the time of birth, fetal growth is, in great measure, merely the further development of organs already mapped out; it is held by many authorities, therefore, that if malformations are ever due to maternal impressions, such impressions could be operative only in the event

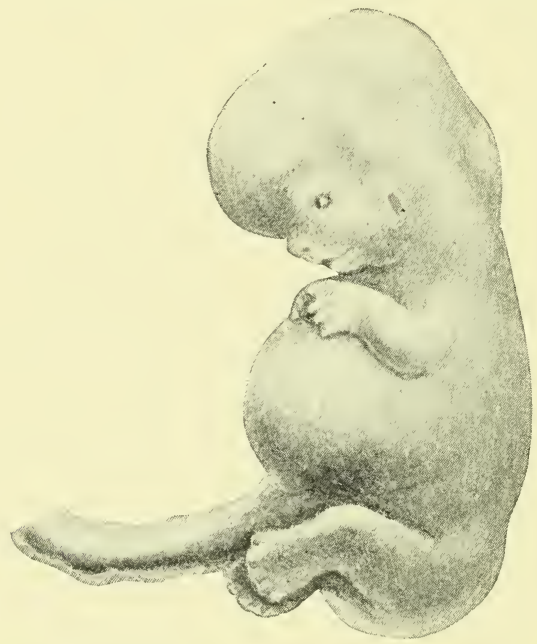

FIG. 64.-Human embryo of about seven weeks, enlarged three times.

of having been received prior to the eighth week of gestation.

During the third month, the face, although definitely formed, still presents thick lips, a pointed chin, and a rather broad and triangular nose. At this time the limbs are wellformed and assume a characteristic attitude, and the fingers and toes are provided with imperfect nails. The external genitals, which, until the close of the second month, preserved the indifferent type, now begin to show sexual distinction.

In the fourth month, a growth of fine hair, the lanugo, appears upon the scalp and some other parts of the borly; 
the anus opens; the intestine recedes within the abdomen; and the external generative organs present well-marked sexual characteristics.

The fifth month marks the inauguration of active fetal movements and the appearance of a more plentiful growth of colorless hair.

In the sixth month the fetal borly becomes coated with the vernix caseosa, a modified sebaceous secretion whose func-

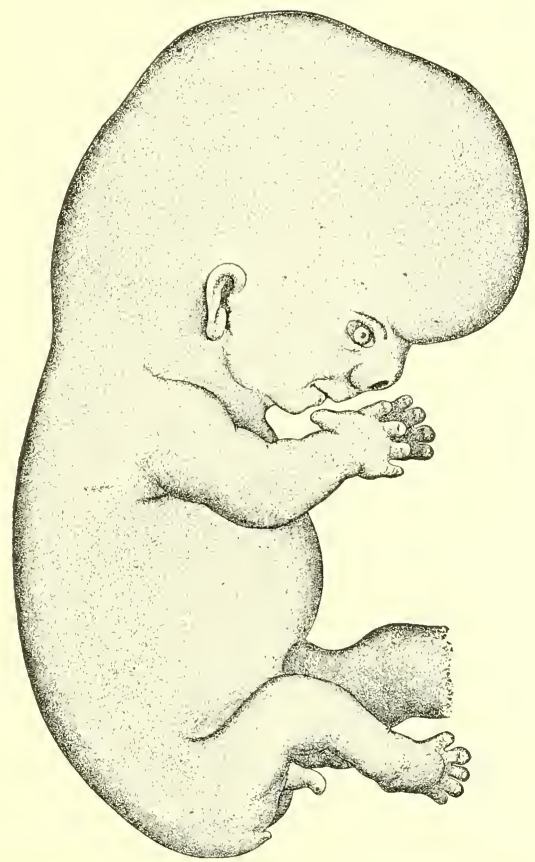

Fig. 65.-Human embryo of about eight and a half weeks, enlarged three times (His).

tion is the protection of the epidermis from maceration in the amniotic fluid. The eyebrows and eyelashes also appear about this time.

The seventh month witnesses the appearance of the lanugo, or embryonal down, upon practically the entire surface of the body; the testes of the male fetus are in the inguinal canal or at the internal abdominal ring; and the nails break through their epidermal covering. Children born at 
the end of the seventh month may survive, but usually they do not.

In the eighth month the lanugo begins to disappear.

In the ninth month the testicles are found in the scrotum, while, in the case of the female, the labia majora are in contact with each other. The contents of the intestinal canal, the meconium, consisting of intestinal and hepatic secretions mingled with epidermal cells and hairs swallowed by the fetus, is now of a dark greenish color. The umbilicus is almost exactly in the middle of the body.

The weight of the fetus at full term is from 3 to 3.5 kilograms (from 6 to 7 pounds), the average weight of the male child being about ten ounces greater than that of the female. While variations from these figures are not uncommon, statements of excessive weight are to be received with reservation, since it has been found, upon careful observation by competent authorities, that the weight of a new-born infant rarely exceeds ten pounds. The weight of the child, besides depending upon the physical condition of both parents, is influenced by the age of the mother, young women having the smallest, and women between the ages of thirty and thirtyfive having the heaviest children ; by the number of previous pregnancies, the weight being greater with each succeeding pregnancy, provided the successive children are of the same sex and are not born at too short intervals; and also by the weight (Gassner) and height (Frankenhaüsen) of the mother, the ratio being a direct one. Minot believes that these various influences operate chiefly by prolonging or abbreviating the period of gestation, and that therefore the variations in weight at birth are referable to two principal causesdifferences in the age at birth, and variations in the rate of intra-uterine growth.

The length of the fetus at the time of birth is about 50 centimeters (20) inches).

The approximate age of an embryo or fetus may be estimated by the characters peculiar to each stage as above noted, and also by employing the rule formulated by Haase. According to Haase, up to the end of the fifth month, the 
square of the age in months equals the length in centimeters, while after the fifth month, the length expressed in centimeters equals the age in months multiplied by five. Thus a fetus of four months would have a length of 16 centimeters; while one of six months would be 30 centimeters long. Hence, the age in months is the square root of the number expressing the length in centimeters; or, if the length exceeds 30 centimeters, the age in months is one-fifth of the length expressed in centimeters.

Reference has been made in Chapter I., page 40, to the relation between conception and menstruation, and to the manner of estimating the age of the product of gestation, based upon this relation. 


\section{CHA P T E R VII.}

\section{THE DEVELOPMENT OF THE CONNECTIVE TISSUES OF THE BODY AND OF THE LYMPHATIC SYSTEM.}

\section{THE CONNECTIVE TISSUES.}

THE variously modified forms of connective tissue distributed throughout the body, including such diversified tissues as the blood and the lymph, areolar tissue, fibrous and elastic tissue, adenoid tissue, tendon, cartilage, bone, and dentine, as well as the connective-tissue stroma of varions organs, all result from alterations affecting the middle germ-layer or mesoderm. As pointed out elsewhere (Chapter III.), the inner and the outer germ-layers are concerned in producing the epithelial structures of the body (with the exception of the epithelium of the greater part of the genital apparatus and of the kidney and ureter), the ectoderm giving rise not only to the epithelium of the surface of the body, but also, by processes of infolding, to such important structures as the central nervous system and the internal ear, while the entoderm differentiates into the epithelial parts of the respiratory and digestive systems with their associated glandular organs.

The proliferation of the cells of the mesoderm goes hand in hand with the differentiations of the inner and onter germlayers, so that even at an early stage of development the middle germ-layer, besides having given rise to the mesothelium of the body-cavity and to the primitive segments, constitutes a lonse aggregation of cells that fill the spaces between the germ-layers and spread about the developing embryonic organs. This primitive relation of the mesodermic tissue foreshadows its future office as the supporting framework not only of the body, but of the functionally 
active epithelial elements of the glands. Thus, the indifferent mesodermic tissue that comes to surround the notochord and the neural canal specializes into the spinal column and the brain-case; while the parts of this tissue into which protrude the epithelial evaginations of the primitive alimentary canal -as, for example, the evaginations which are the beginnings of its glandular organs, the liver and the pancreas-become intimately associated with these epithelial sacs and tubes to constitute the connective-tissue stroma and the vascular apparatus of the completed glands. All organs of the body, therefore, that have a connective-tissue constituent obtain it from the mesoderm. Owing to the varying degree of differentiation of the mesodermic elements in different localities there are formed tissues of widely different character. The most important factor in the production of these modifications is the alteration of the intercellular substance, as to whether it remains soft and homogeneous, whether it acquires a fibrillar or an elastic structure, or whether it becomes dense and hard, as in the case of cartilage and bone. The cells undergo comparatively little change, although, according to the kind of tissue produced, they come to be known respectively as connective-tissue cells, tendon-cells, cartilage-cells, or bone-cells.

The slightest degree of specialization results in the production of mucous tissue. In this case a reticulum is formed by the slender processes which the cells acquire, the spaces of the meshwork being filled with the semifluid or semigelatinous intercellular substance.

A further alteration in the intercellular substance, whereby it acquires greater density and becomes permeated by bundles of fibers, some of which are highly elastic, results in the formation of areolar tissue. Preponderance of the non-elastic fibrous element produces white fibrous tissue, while elastic tissue, such as predominates in the ligamentum nuchæ, is formed if the elastic fibers are in excess. Further increase in the density of the intercellular material, with its accompanying conversion into bundles of non-elastic fibers having a characteristic regularity of arrangement, produces the struct- 
ure of tendon. When the intercellular substance gives rise to a scant amount of fibrous material and the cells become distended with oily or fatty matter, adipose tissue results.

A still greater degree of density of the intercellular substance gives the matrix of cartilage, the cells being enclosed in spaces, the lacunæ, as the cartilage-cells. Partial differentiation into either fibrous or elastic bundles confers the character of either fibrous or elastic cartilage upon the product.

Great condensation of the intercellular substance and its permeation with salts of lime, the cells being fixed in small spaces, results in the production of osseous tissue (see Chapter XVIII.).

Blood and lymph may be looked upon as forms of connective tissue in which the intercellular substance is fluid, constituting the plasma, the cellular elements thus remaining free cells, the blood- or the lymph-corpuscles. The development of both lymph and blood from the mesodermic elements serves to bear out the comparison.

The endothelium of the body is related with the connective tissues genetically as well as anatomically. Reference has been made elsewhere to the changes which occur in the mesodermic cells that bound the body-cavity-the fissure between the two layers into which the parietal plate of the mesoderm splits-to constitute the mesothelium of the bodycavity. These changes consist in the flattening of the cells and their assumption of the characters of endothelium. Similarly, when other smaller clefts are formed in the mesodermic tissue, clefts which may be the beginnings of small lymph-spaces, or of blood-vessels, or of bursal or articular cavities, the bordering cells of these cavities also assume the endothelioid type.

The mode of development of the serous membranes and of the closely allied synovial, bursal, and thecal sacs may be inferred from what has been said about the origin of the endothelium. The connective-tissue stroma of the membrane, upon which the endothelium rests, is simply a condensed and differentiated lamella of connective tissue. 


\section{THE DEVELOPMENT OF THE LYMPHATIC SYSTEM.}

The solid elements of the lymphatic system-the "lymphglands," the lymph-follicles, and the diffuse adenoid tissueas well as the thymus body and the spleen, result from the specialization of mesodermic cells, while the lymph-vessels and the various lymph-spaces of the economy-that is, the serous sacs, joint-cavities, bursal and thecal cavities, subarachnoid and subdural spaces of the brain and spinal cordare developed by vacuolation or hollowing out of the mesoderm.

Definite knowledge is wanting as to many of the details of the genesis of the lymphatic system. The various lymphspaces precede the vessels and the adenoid tissue in development.

The lymph-spaces result from clefts in the mesoderm, the earliest formed and most conspicuous space of this sort being the body-cavity or colom. This large fissure develops, even before the differentiation of the body of the embryo, by the coalescence of numerous small cavities that appear within the middle germ-layer. The body-carity acquires more definite boundaries by the alteration of the mesodermic cells that border it into flattened endothelioid cells, the mesothelium of the body-cavity. When, in the progress of development, the diaphragm and the pericardium are formed, the body-cavity is divided into the peritoneal cavity, the pleural sacs, and the pericardium. At a still later period, a diverticulum of the peritoneum protrules, in the male fetus, through the inguinal canal into the scrotum to constitute the tunica vaginalis testis. The stomata of serous membranes are merely so many apertures of communication between the serous cavities, which are enormous lymph-spaces, and the lymphatic clefts contained within the stroma of the serous membrane, the clefts themselves being the beginnings of lymph-vessels.

The large lymph-sacs surrounding the brain and spinal cord, the subarachnoid and subdural spaces, as well as the spaces within the capsule of Tenon and the sheath of the 
optic nerve, and the perilymphatic spaces of the internal ear similarly develop as racuolations of the mesodermic tissue. The same is true of the joint-cavities, bursal sacs, sheaths of tendons, and the small lymph-clefts found in the areolar tissue and throughout most organs.

The lymphatic vessels first formed, according to O. Schultze, are the subcutaneous vessels, which are present in a human embryo of 2 to $3 \mathrm{~cm} .{ }^{1}$ and at a somewhat later period the deeper vessels appear. From the studies of Sabin ${ }^{2}$ upon pig embryos it appears, howerer, that the larger vessels precede the smaller. This observer found that at the junction of the subclavian and jugular veins of each side a sac or lymph-heart made its appearance, the orifice being guarded by a valve, and from these sacs or hearts branches arose which passed toward the skin, from which branches a general subcutaneous network of vessels arose. From each lymphsac a ressel grows tailward, the vessel on the left side reaching the aorta and dividing there to form two thoracic ducts, which afterward unite into a single duct. Frequently this fetal condition of two thoracic ducts is indicated in the human adult by a double condition of the duct for a greater or less extent, the duct sometimes dividing and reuniting two or three times; sometimes it is double at its termination in the subclavian vein.

The two thoracic ducts before fusion dilate at their caudal extremities, in the region of the kidney, to form respectively two receptacula chyli, and a little farther on unite with the two posterior lymph-hearts or sacs, which have meanwhile developed at the junction of the sciatic veins with the cardinal veins. These latter sacs subsequently lose all connection with the veins from which they grew. Outgrowths from these chief vessels of the lymphatic system serve for its extension into the viscera and the skin. As the primary

${ }^{1}$ O. Schultze: Grundriss der Entwickelungsgeschichte des Menschen und der Säugethiere," Leipzig, 1897.

${ }^{2}$ Florence R. Sabin: "On the Origin of the Lymphatic System from the Veins and the Development of the Lymph-hearts and Thoracic Duct in the Pig," American Journal of Anatomy, i., 1902. 
lymph-sacs increase in length, but fail to correspondingly increase in calibre, they gradually become merged into the vessels.

The lymphoid or adenoid tissue is produced at a later date than the vessels. Observations upon the human lymph nodes seem to have been confined to the inguinal and lumbar nodes. The first indication of an inguinal node is seen in a $3 \mathrm{c.m}$. embryo, in the shape of little aggregations of lymphoid cells that have migrated from the lymphatic cords or networks into a space hollowed out of the mesoderm. This nodule of lymphoid cells is isolated from the surrounding mesodermic elements by a fissure or space except at one point, the future hilum of the node, where strands of embryonal connective tissue connect it with the parent mesoderm. The reticulum of the node appears later, as does also the capsule, the latter of which results from the condensation of the surrounding mesoderm.

The development of the spleen is considered with that of the alimentary system because of its relation to the evolution of the peritoneum, while the account of the development of the thymus will be found in the chapter on the respiratory system. 


\section{CHAPTER IX.}

\section{THE DEVELOPMENT OF THE FACE AND OF THE MOUTH=CAVITY.}

THe evolution of the face depends so largely upon the growth of the parts concerned especially in the production of the mouth and nose that any account of its development must deal for the most part with the development of those structures. In tracing the earliest stages of facial growth, it will be well to consider the face as a whole before proceeding to a detailed description of its several parts. If we seek the principles underlying the conformation of the face, we shall find that its apertures and chief cavities are merely so many provisions for bringing the central nervous system and the alimentary tract into relation with the outside world. It will be seen, for example, that certain small depressions appear upon the surface; that one of these, which is destined to become the mouth and the respiratory part of the nasal cavities, assumes relationship with the alimentary tract and with its offshoot, the respiratory system; that other depressions, which subsequently develop into the olfactory parts of the nasal chambers, come into relation with outgrowths from the brain, the olfactory bulbs; and that still another surface-invagination becomes the lens-vesicle, which likewise meets with an outgrowth from the brain to become a part of a peripheral sense-organ, the eye.

The first step in the differentiation of the face is the formation of the oral plate, the earliest indication of the future mouth. The oral plate appears on the twelfth day, and consists of a small area of ectoderm and entoderm, the mesoderm being absent. It is situated on the ventral surface of 
the head-end of the embryo, which already presents the enlargement of the cerebral vesicles. 'The oral plate becomes relatively depressed by the upgrowth of the surrounding tissues, the fossa thus produced constituting the oral pit or stomodæum (Fig. 57). The oral plate is now the pharyngeal membrane (Fig. 66). Reference to the sagittal section will

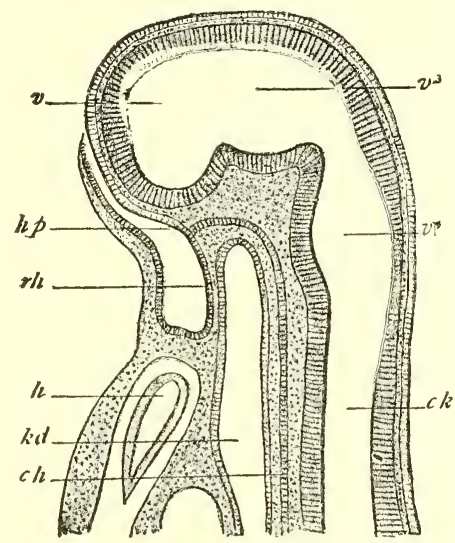

Fig. 66.-Median section through the head of an embryo rabbit $6 \mathrm{~mm}$. long (after Mihalkovies) : $r h$, membrane betwean stomodæum and fore-gut, pharyngeal membrane (Rachenhaut; $h p$, place from which the hypophysis is developed; $h$, heart: $k d$, lumen of fore-gut; $c h$, chorda; $v$, ventricle of the cerebrum; $v^{3}$, third ventricle, that of the between-brain (thalamencephalon); $v^{4}$, fourth ventricle, that of the hind-brain and after-brain (epencephalon and metencephalon, or medulla oblongata); $c k$, central canal of the spinal cord.

show that the oral pit corresponds in position to the headend of the gut-tract. The formation of the pit is, in effect, a pushing-in of the surface ectoderm to meet the alimentary entoderm.

A second important factor in the development of the face is the appearance of the first and second visceral arches, which occurs in the third week. As pointed out in a preceding section, the first visceral arch divides into the mandibular arch and the maxillary process (Fig. 62), the latter being the smaller and appearing to spring from the mandibular arch. Both the maxillary processes and the mandibular arches grow toward the median line of the ventral surface of the body. Owing to the growth of these struct- 
ures and to the sharp flexion of the head and neck that occurs between the twenty-first and the twenty-third day, the oral pit becomes very much deeper and acquires more definite boundaries. During the third week it is a fossa of pentagonal outline. Its upper boundary is formed by the unpaired nasofrontal or nasal process (Fig. 67, A), which is essentially a thickening on the ventral wall of the forebrain vesicle, brought into close relation with the fossa by the flexion above referred to. The lower boundary is formed by the mandibular arches, while the lateral extent of the fossa is limited by the maxillary process of each side.

Soon after the appearance of the oral pit, the future nares are foreshadowed by the development of the two olfactory plates, situated one on each side of the nasofrontal process, widely separated from each other. These epithelial areas, which soon become depressions, the nasal pits, are closely united with the wall of the fore-brain vesicle from the first; they develop subsequently into that part of the nasal mucous membrane which is concerned especially with the sense of smell. This fact becomes very significant when it is remembered that the olfactory bulbs, with which the olfactory epithelium assumes intimate relationship, are outgrowths from the brain.

The nasofrontal process, during the fifth week, becomes much thickened along its lateral margins, forming thus the globular processes (Fig. 67, A), which constitute the inner boundaries of the nasal pits. At the same time, there grow downward and forward from the nasofrontal process two ridges, one on each side, the lateral frontal processes, which form the outer boundaries of the nasal pits (Fig. 67, A). In this manner the pits become much increased in depth. The lateral frontal process projects between the nasal pit and the maxillary process, its line of contact with the latter structure being marked by a groove, the naso-optic furrow or lacrimal groove. This groove later completely disappears; it is of importance, however, as indieating the position of the now developing nasal duct, which will be referred to hereafter. The nasal pits are widely in communication with 
the cavity of the primitive mouth. About the fortieth day, however, the extremities of the maxillary processes have grown so far toward the median line that they have met
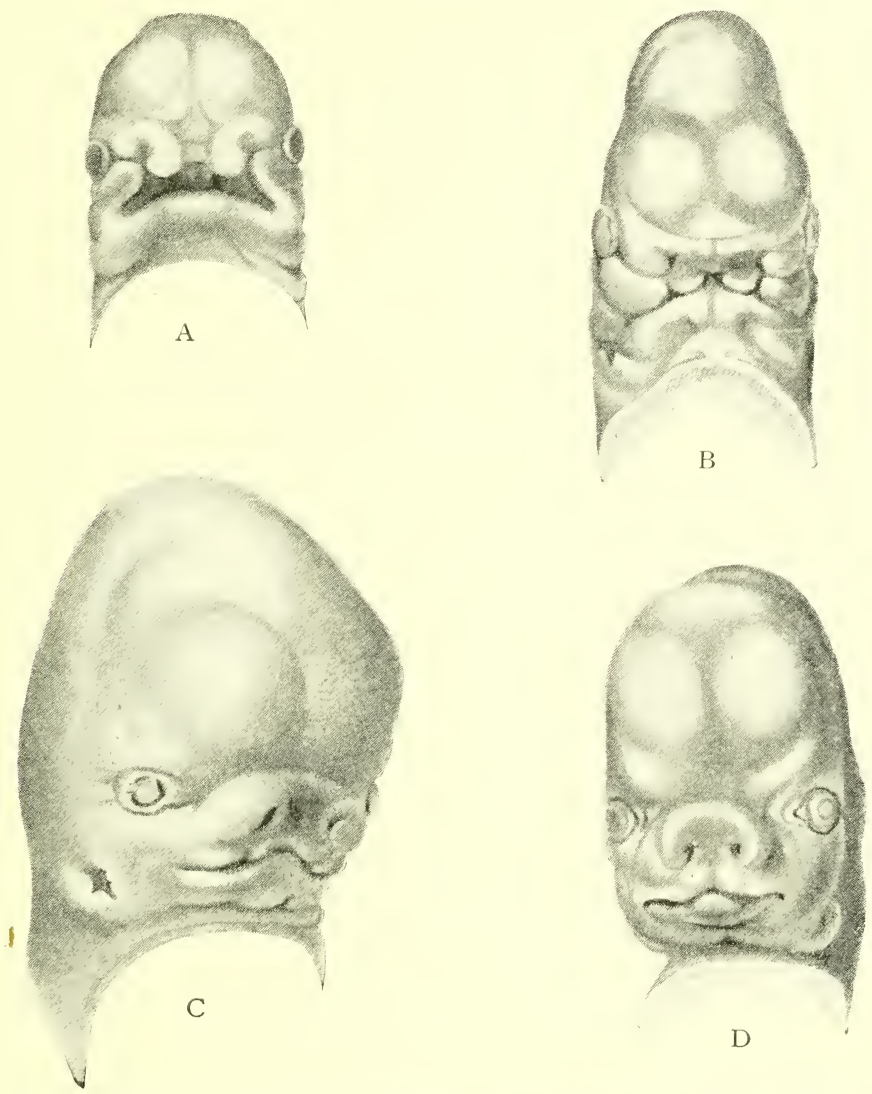

Fig. 67.-Development of the face of the human embryo (His): A, embryo of about twenty-nine days. The nasofrontal plate differentiating into processus globulares, toward which the maxillary processes of first visceral arch are extending. B, embryo of about thirty-four days: the globular, lateral frontal, and maxillary processes are in apposition; the primitive opening is now better defined. C, embryo of about the eighth week: immediate boundaries of mouth are more definite and the nasal orifices are partly formed, external ear appearing. D, embryo at end of second month.

and united with the lateral frontal processes and with the nasofrontal process (Fig. 67, $B$ and $C$ ). In this manner the nasal pits become separated from the oral fossa, each of these openings aequiring more definite boundaries. It is 
apparent from this description that the upper boundary of the primitive oral cavity is not identical with that of the adult mouth. The nasofrontal process is the forerunner of the intermaxillary portion of the upper jaw, including the corresponding part of the upper lip and of the nasal septum and bridge of the nose.' The lateral frontal process becomes the wing of the nose. By the completion of the changes here noted the face acquires more distinctive form. It will be seen that the upper jaw proper results from the metamorphosis of the maxillary processes. The manner in which its sinus, the antrum of Highmore, is added, as well as the ossification of the jaw, will be considered hereafter.

The development of the eye will be described in connection with that of the sense-organs. In so far as the eyes have relation to the external form of the face, it will be sufficient to say that the surface ectoderm is invaginated in the fourth week to form the lens-vesicle, this sac, which gives rise to the crystalline lens, being covered by two little folds of ectoderm, the primitive eyelids; that the organ is situated on the side of the head, in marked contrast to its position in the mature state; and that the naso-optic furrow, previously referred to, passes from the inner angle of the eye toward the wing of the nose. The development of the face having been pointed out in a general way, the individual parts may be considered separately.

THE MOU'TH.

To review briefly, for the sake of convenience and clearness, the earlier history of the development of the mouth, we find the first step to be the appearance, at the twelfth day, of the oral plate. By the enlargement of the anterior end of the neural tube to form the cerebral vesicles, and by

${ }^{1}$ Hare-lip is the deformity resulting from failure of union between the nasofrontal and the maxillary processes. Since the nasofrontal process is an unpaired structure, in which develop the intermaxillary bones, and which unites on either side with the corresponding maxillary process-the latter being the forermner of the upper maxilla proper-we have an explanation of the lateral position of hare-lip. This defect may be, of course, either unilateral or bilateral. 
the development of the visceral arches, this area becomes a depression, the oral pit. The pit is at first bounded caudad by the cardiac prominence and cephalad by the fore-brain vesicle (Fig. 57). In the third week the oral pit becomes a five-sided fossa, owing to the growth of several new struetures. These are the unpaired nasofrontal process, which bounds the fossa above, the mandibular arches, which bound it below, and the maxillary processes, which form the lateral boundaries (Fig. 67). The mandibular arches do not actually unite with each other until the thirty-fifth day. A transverse groove appears on the outer surface of the united mandibular process, the elevation in front of which is the lip ridge, while behind the groove is the chin ridge; these ridges respectively produce the lower lip and the chin. The angle between the maxillary process and the mandibular arch corresponds to the angle of the future mouth. In the sixth week-about the fortieth day-the oral fossa aequires a new upper boundary, which separates it from the nasal pits, by the growth of the maxillary and lateral nasal processes

The primitive oral cavity, as before mentioned, is at first separated from the gut-tract by the pharyngeal membrane (Fig. 66). This structure ruptures at some time during the fourth week, thus bringing the mouth into communication with the upper end of the gut-tract. The exact location of the pharyngeal membrane with reference to the adult pharynx is somewhat difficult to define; it is certain, however, that the primitive mouth includes more than the limits of the adult oral cavity, comprising, in addition to the latter, the anterior part of the adult pharynx. Reference to a sagittal section, as in Fig. 66, shows the relation of the oropharyngeal cavity to the brain-case; in the tissue separating the two the floor of the cranium is subsequently formed. A little evagination from a point ( $h p$, Fig. 66) in the back part of the primitive oral cavity becomes the anterior portion of the pituitary body or hypophysis, the posterior lobe of which develops as an evagination from the floor of the primary fore-brain vesicle. With the development of the floor of the cranium, the hypophysis becomes entirely isolated from the oral cavity. A little pouch or recess usually demonstrable 
in the median line of the roof of the pharynx of the child, though not always present in the adult, is the persistent pharyngeal end of the diverticulum that forms the hypophysis; it is known as the pharyngeal bursa or Rathke's pocket.

Very soon after the formation of the upper jaw in the manner above described, the oral surface of the jaw presents two parallel ridges. Of these, the outer, which is the larger, develops into the upper lip, while the inner smaller ridge becomes the gum. The lip and gum of the lower jaw are produced similarly, at the same time or a little later. So far, the only demarcation between the mouth and the nasal cavity is furnished by the tissue representing the united nasofrontal, lateral nasal, and maxillary processes, the nares opening widely into the cavity of the mouth posterior to this partition.

The formation of the palate, however, effects a separation between the two that gives to each space its permanent limitations. On the inner or oral surface of the upper jaw two shelf-like projections appear, one on each side, which are the rudiments of the future palate. These gradually grow toward each other, the tongue, which has meanwhile been developing, projecting upward between them. In the eighth week, union of these two lateral halves of the palate begins at their anterior extremities. By the ninth week union has taken place as far back as the extent of the future hard palate, and, by the eleventh week, the constituent halves of the soft palate have united also. As these two halves approach each other the tongue recedes from between them, owing to the growth of the lower jaw, so that, when union occurs, that organ occupies its normal position under the palate. Osseous formation within the soft tissue first formed produces the palate processes of the superior maxillæ and of the palate bones, which processes collectively constitute the hard palate of the adult. The intermaxillary bones are formed within the primitive partition between the mouth and the nares. The completion of the palate definitely marks off the nasal chambers from the mouth, thus dividing the early oral cavity into a lower space, the true mouth, and an 
upper region, which is essentially a part of the respiratory system.

The uvula appears during the latter half of the third month as a small protuberance on the posterior edge of the soft palate. ${ }^{1}$

The Teeth.-The teeth, morphologically considered, are calcified papillæ of the skin, eapped by a layer of peculiarly modified and calcified cells of the epidermis. Although in man and the higher mammals the teeth are found only upon the gums, in certain lower types they have a much wider distribution, occurring upon the roof and floor of the mouth and in the pharynx, and also, in selachians, upon the general skin-surface, in which latter case they are so modified as to constitute scales.

The dentine and cementum of the tooth, as well as its pulp, are derived from the mesoderm; the enamel is a direct derivative of the overlying ectodermic epithelium. Mammals are said to be diphyodont, since they develop two sets of teeth; while such groups as sharks, which continue to produce and lose new teeth throughout life, are denominated polyphyodont.

The development of the teeth is inaugurated in the sixth week of embryonic life by the multiplication of the epidermal cells covering the surface of the gums to form a linear ridge. The growth of the ridge is away from the surface, so that the new structure projects into the underlying mesoderm. This horseshoe-shaped ridge, which corresponds in direction and extent to the line of the gum, subdivides into two parallel ridges, of which the outer marks the position of the future groove between the gum and the lip; the inner is the dental shelf or dental ridge, which must be regarded as the earlicst indication of the future teeth. The dental shelf extends into the underlying mesodermic tissue, not directly

${ }^{1}$ Deficiency of union of the halves of the palate, resulting in a median fissure, constitutes the deformity, cleft palate. This deficiency may be limited to the hard or to the soft palate, or it may affect both, or it may be seen in the uvula, either alone-cleft or bifid uvula-or in conjunction with cleft palate. 
downward but in an oblique direction toward the inner or lingual surface of the gum. While the dental shelf is growing, its line of connection with the surface ectoderm is marked by the superficial dental groove, which at one time was looked upon as being the first evidence of tooth-formation.

Upon the side of the dental shelf opposite the free or oral surface, individual protuberances develop, corresponding in number to that of the teeth of the temporary set-ten for each jaw. Each little projection consists of a mass of ectodermic cells, which soon becomes expanded at its deep extremity, becoming thus club-shaped and later flask-shaped, and which is called the enamel-sac or primitive enamel-germ, since the enamel of the tooth is developed from it (Fig. 68).

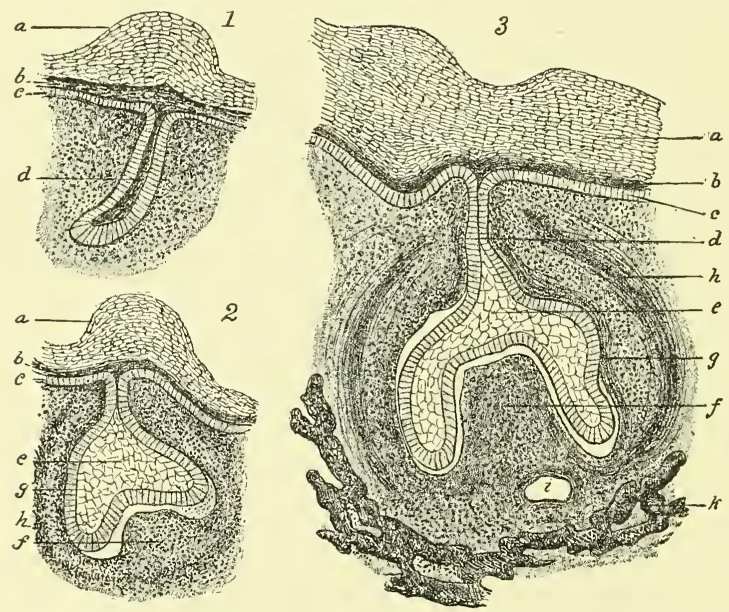

FIG. 68.-Three successive stages in the development of a tooth-germ of a pig embryo (after Frey and Thiersch): $a, b, c$, layers of thickened oral epithelium, showing dental groove on surface in $3 ; e$, enamel organ $; f$, dental papilla; $g, h$, internal and external layers of the follicle wall; $i$, blood-vessel; $k$, maxilla; $d$. epithelial ingrowth, the end of which expands into the enamel-sac.

Meanwhile the continuity of the original dental shelf is broken by the disappearance of the cells in the intervals between the individual enamel-.germs, each germ becoming thereby isolated from its neighbors. The neck of the flask- 
shaped enamel-germ becomes reduced to a slender strand of cells and finally disappears, so that there is no longer any connection between the enamel-sac and the ectodermic cells of the free surface of the gum. While the enamel-sacs for the temporary teeth are growing in this manner, the corresponding structures for the teeth of the permanent dentition bud from the inner side of the dental shelf-that is, the side looking toward the tongue-except those for the three permanent molars, which grow backward toward the articulation of the jaw from the position of the second temporary molar.

As the enamel-germs grow downward into the mesodermic tissue, the latter sends up a number of conical projections, the dental papillæ, one for each enamel-organ. This dental papilla, of mesodermic origin, is the parent of the dentine and of the pulp of the tooth. When the dental papilla and the enamel-sac meet, the sac becomes invaginated, its under surface assuming a concave form. The enamel-sac at this stage therefore is a double-walled cup which caps the dental papilla. It is at about this time that the connection of the enamel-organ with the surface ectoderm is lost.

The further evolution of the enamel-organ consists essentially in the arrangement of its constituent cells into three layers and the formation, by the deepest of these three layers, of the special elements of the fully-developed enamel-the enamel-prisms. The most superficial stratum of the cnamelorgan is composed of low columnar or polyhedral cells; the deepest layer, that nearest the papilla, the so-called membrana adamantina, consists of beautifully regular columnar cells, the ameloblasts or adamantoblasts; between the two is a group of less characteristic epithelial elements. The cells of the deep layer, the enamel-cells, are alone concerned in the production of the enamel. The enamel-organ for a time covers the entire dental papilla. During the course of development, however, the growth of that part of it covering the future root of the tooth aborts, leaving the crown alone covered with the enamel.

The first step in the formation of the enamel-prisms by the enamel-cells is that the protoplasm of the deep extremity 
of each cell becomes homogeneous, and a tuft develops on the end of the cell, projecting toward the papilla. By the

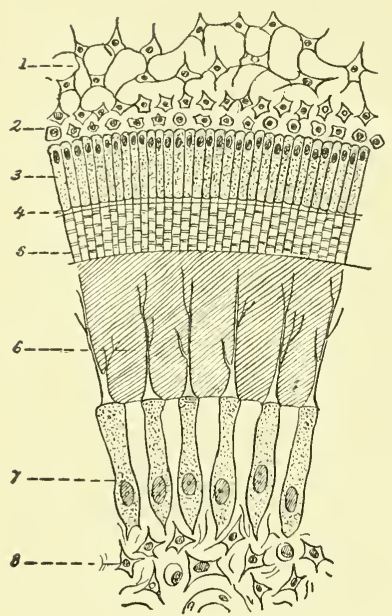

FIG, 69.-Semi-diagrammatic figure showing the several parts of a calcifying enamel-organ (Tourneux): 1 , central cells of enamelorgan; 2 and 3 , cells of inner layer of enamel-organ, 3 being the enamel cells; 4, zone of young enamel; 5, enamel prisms; 6 , young dentine traversed by the dentinal fibers; 7 , odontoblasts ; 8 , central tissue of dental papilla. calcification of this tuft the formation of an enamel-prism is begun (Fig. 69). The process of calcification continues to advance from the deep or papillary aspect of the enamel-organ toward the surface. From this it comes about that the newest enamel is next to the enamel-cells, or, in other words, nearest the surface, and also that the enamel-prisms are arranged in a direction generally vertical to the free surface of the tooth. The formation of the enamel of the milk-teeth begins in the latter part of the fourth month. The middle layer of the enamelorgan becomes greatly altered in constitution, owing to the accumulation of fluid and to the reduction of its cells to the form of thin plates, the appearance being rather that of connective tissue than of an epithelial structure. The superficial layer of cells undergoes atrophy, their exact fate not being known.

The atrophic remnant of the enamel-organ is found upon the free surface of the tooth for a variable time after its eruption, constituting the membrane of Nasmyth.

The dental papilla has been referred to as the structure that gives rise to the dentine. It originates from active multiplication of the mesodermic cells. The number of papille corresponds to the number of enamel-organs. As the papilla grows toward the enamel-organ it early aequires vascularity. The shape of the papilla, whether that of an incisor, of a canine, or of a molar tooth, is determined by the shape which the enamel-organ assumes. The connective- 
tissue cells upon the surface of the papilla assume distinctive character, becoming large and branched, and constitute the so-called odontoblasts (Fig. 69). They are virtually modified osteoblasts. Forming a continuous layer, they have been styled the membrana eboris. Between this layer of odontoblasts and the enamel-organ a layer of intercellular substance appears, the membrana præformativa. The odontoblasts now send out processes toward the enamel-organ, which are known as the dental processes. Calcification begins upon the surface of the papilla and progresses toward its center, but is not complete. Small uncalcified areas, corresponding to the globular spaces of the completed tooth, remain next the enamel. The dental processes likewise fail to become calcified, and these are the adult dentinal fibers occupying the dentinal tubules of the finished dentine. The odontoblasts continue the formation of dentine until the dental papilla is entirely surrounded by it. What remains of the papilla, upon the completion of the tooth, constitutes the pulp, a highly vascular connective-tissue substance supporting upon its surface the odontoblasts. The deposition of dentine begins in the latter part of the fourth month.

During the metamorphosis of the dental papilla the mesodermic tissue immediately surrounding it undergoes slight condensation to form the follicle of the developing tooth. As the enamel-organ recedes from the surface, the follicle increases in extent to such a degree as to envelop the entire rudimentary tooth. Only that part of the follicle which covers the future root of the tooth is of subsequent importance, however; undergoing partial transformation into true bony tissue, it gives rise to the cementum or crusta petrosa, while the unossified external fibrous layer constitutes the lining periosteum of the alveolus (Fig. 68).

The development of the permanent teeth is precisely analogous to that of the milk-teeth. The enamel-germs for the permanent teeth, with the exception of the molars, bud from the lingual side of the dental shelf in the seventeenth week (Fig. 70), the germ for the first permanent molar appearing about a week earlier at the posterior extremity of the dental 
shelf after the manner of a milk-tooth. The germ for the second molar buds from the neck of the first molar in the third month after birth, while that of the third molar, the wisdom tooth, springs from the neck of the second about the third year. At birth, therefore, the gums contain the

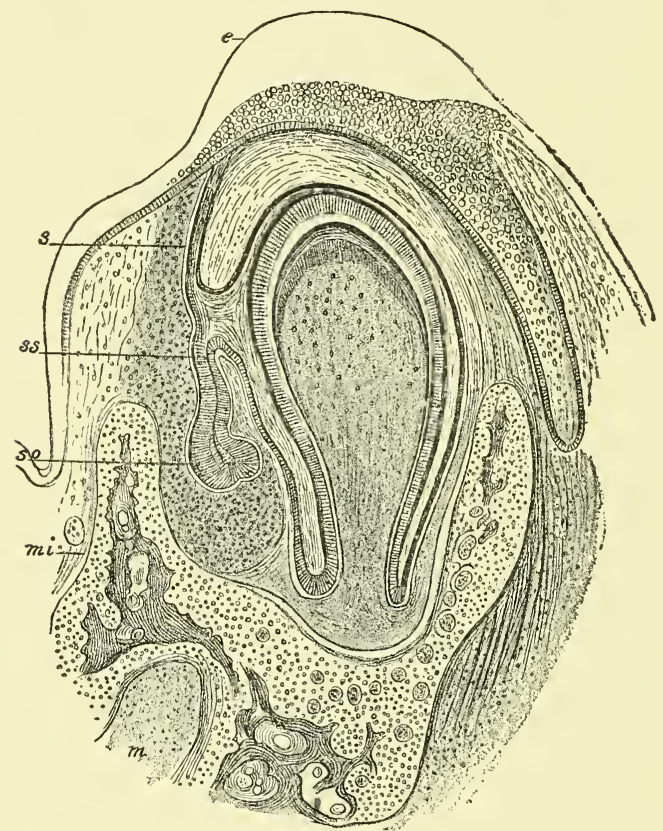

FIG. 70.-Cross-section of the lower jaw of a cat embryo, showing the enamelgerms of a milk-tooth and of a permanent tooth (from Bonnet, after Kölliker): $e$, thickened oral epithelium; so, enamel-organ of permanent tooth, which has grown out at $s s$ from the neck $(s)$ of the enamel-sac of a milk-tooth; mi, lower jaw ; $m$, Meckel's cartilage.

two sets of teeth except the second and third permanent molars.

The eruption of the temporary teeth begins usually at about five and a half months after birth with the appearance of the central incisors, and is complete at from eighteen to thirtysix months, when the second molars are cut. The first teeth of the permanent dentition are the first molars, which are erupted at about the sixth year. The accompanying table shows the time and the order of eruption of the teeth : 
Temporary Dentition.

Central incisors . . . . . . $5 \frac{1}{2}$ to 7 months.

Lateral incisors . . . . . . 7 to 10 months.

First molars. . . . . . . 12 to 14 months.

Canines . . . . . . . . 14 to 20 months.

Second molars. . . . . . . 18 to 36 months.

Permanent Dentition.

First molars . . . . . . . 6 6th year.

Central incisors ...... . . 7th year.

Lateral incisors . . . . . . 8th year.

First premolars . . . . . . . 9th year.

Second premolars . . . . . . 10th year.

Canines. . . . . . . 11th to 12 th year.

Second molars. . . . . . . 12th to 13th year.

Third molars (wisdom teeth) . . . 17th to 21 st year.

The Salivary Glands.-The salivary glands, which in mammals consist of three pairs, the parotid, the submaxillary, and the sublingual, develop as outgrowths of epithelium from the lining mucous membrane of the mouth. The epithelial elements of the glands are therefore of ectodermic origin. The growth of the submaxillary gland begins in the sixth week, that of the parotid in the eighth week. Each epithelial outgrowth is at first a solid cylinder, which undergoes repeated branching and acquires a connective-tissue framework and capsule from the surrounding mesoderm. It is not until the middle of the fifth month that the lumen of the gland appears. This is brought about by the moving apart of the epithelial cells composing the cylinders and their branches. The main duct of the gland first becomes hollow, then its branches, and finally the lumina of the alveoli make their appearance. The respective sites from which the several glands grow correspond in a general way to the positions at which the ducts of the adult glands open into the mouth-cavity.

The Tongue.-Although the tongue originates from tissues belonging really to the walls of the pharynx, its development may be conveniently considered in connection with that of the mouth because of its relations in the mature organism. This organ, composed chiefly of muscular sub- 
stance, is formed from three originally separate parts, an anterior unpaired fundament, and two posterior bilaterally symmetrical segments. The line of union of these three parts is indicated approximately in the adult organ by the V-shaped row of circumvallate papillæ on the dorsun of the tongue. The anterior part of the tongue develops from a small unpaired tuberele, the tuberculum impar, which grows from the median line of the floor or anterior wall of the pharynx between the first, or mandibular, and the second, or hyoid, arch (Fig. 71, 6). The posterior segment of the tongue results
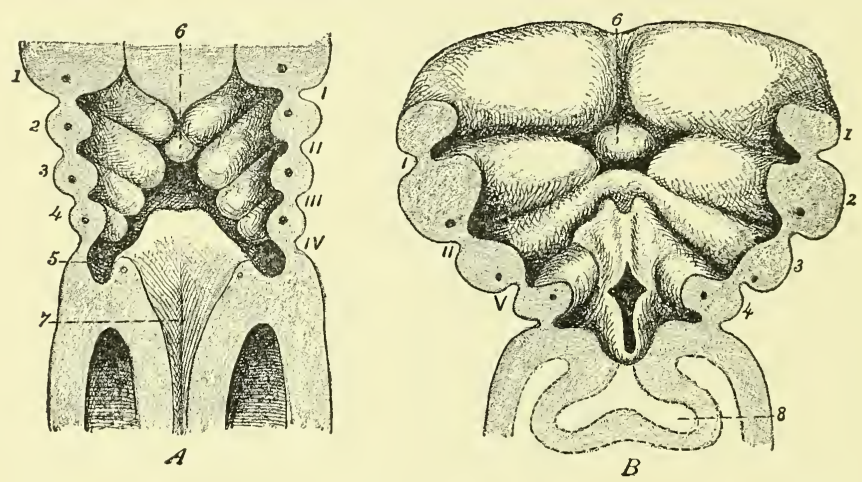

Fig. 71.-Coronal sections of two human embryos, showing ventral wall of pharyngeal end of gut-tract from behind (from Tourneux, after His). $A$, from embryo of $3.2 \mathrm{~mm}$.; $B$, of $4.25 \mathrm{~mm}$. (about 25 to 30 days). $I, I I, I I I, I V$, outer visceral furrows; $V$, sinus præcervicalis, comprising third and fourth outer furrows; $1,2,3,4$, visceral arches, each with its visceral-arch vessel; 6 , tuberculum impar; 7 , orifice of larynx; 8 , pulmonary evagination.

from the growing together of two lateral halves, which develop from the anterolateral walls of the pharynx at the position of the second and third visceral arches, but not from the arches themselves. These ridges are sometimes described as the fused anterior (ventral) extremities of the arches just mentioned. The unpaired tubercle increases in size to such an extent as to constitute the major part of the organ. In the median line of the anterior wall of the pharynx, immediately behind the tuberculum impar, the epithelial lining of this cavity pouches forward and downward to develop later into the middle lobe of the thyroid body. $\Lambda$ s the ridges which are to form the posterior part of the tongue lie laterally and 
posteriorly to this median evagination, they completely enclose it in the process of fusing with each other and with the anterior tubercle. In this manner a canal or duct is formed leading from the surface of the tongue at the angle of junction of its three segments down to the middle lobe of the thyroid body, the latter meanwhile having descended from its original position. This canal is the thyroglossal duct or canal of His. During the further progress of development, the canal suffers obliteration, its only vestige being the orifice, which is known as the foramen cæcum of adult anatomy.

The papillæ of the tongue are found exclusively on the part derived from the tuberculum impar; the line of union between the anterior and posterior parts lies therefore behind the row of circumvallate papillæ. The papillæ begin to make their appearance as early as the beginning of the third month.

Prior to the union of the two lateral halves of the hard palate, by which the primitive oral cavity is divided into the mouth proper and the nasal chambers, the tongue projects upward between the palate-shelves, almost completely filling the primitive mouth. As the palate-shelves approach each other, however, the tongue gradually recedes to its subsequent normal position.

\section{THE DEVELOPMENT OF THE NOSE.}

The nose being an organ of special sense, its devclopment is described in connection with that of the other special-sense organs in Chapter XVI. Owing, however, to its imporiant relation to the other parts of the face, it is desirable to refer to its evolution in this connection. For a more detailed account, the reader is referred to Chapter XVI.

The first indication of the organ of smell is in the form of the two patches of thickened ectoderm, the nasal areas or olfactory plates, which appear on the headward side of the oral fossa in the third week of development. At the end of the fourth week the areas are depressed and constitute the nasal pits (Fig. 67, A). The nasofrontal process, a mass of thickened mesodermic tissue, lies between them. During the 
fifth week the lateral edges of this process become thick and rounded, forming the two globular processes, while growing outward and downward from the sides of its base are the two lateral nasal or lateral frontal processes. Thus the nasal pits, which correspond with the position of the future anterior nares, become bordered on the mesial side by the globular processes and on the outer side by the lateral nasal processes. Below, the pits are continuous with the oral fossa. Owing to the continued growth of these masses the pits gradually become deeper. The lateral nasal process is separated externally from the maxillary process of the first visceral arch by a groove, the naso-optic furrow. The lower extremities of the maxillary and lateral nasal processes soon unite with each other and advance toward the median line below the nasal pit. In the latter part of the sixth week they unite with the nasofrontal process and thus separate the nasal pits from the oral fossa and furnish the basis of the upper lip. The nasal pits are now the anterior nares, and the nose is represented by the irregular masses of tissue surrounding them. While the orifices of the nares are separated from the orifice of the primitive oral cavity, their deeper parts are continuous with the latter, there being as yet no hard or soft palate.

In the eighth week the nose first acquires definite form, owing to the continued growth of the masses of tissue referred to above. The nasofrontal process forms the bridge of the nose with the nasal septum, and also the intermaxillary part of the superior maxillæ and the connective-tissue parts of the upper lip. The lateral frontal process becomes the ala of the nose. The nose is still very broad and flat in the third month, after which time it gradually assumes its characteristic form. 


\section{CH A PTER X.}

\section{THE DEVELOPMENT OF THE VASCULAR SYSTEM.}

THE vascular system, including the blood, the heart, and the blood-vessels, begins its development very early in embryonic life.

While the heart is formed within the body of the embryo, the blood and the earliest blood-vessels have their origin in an extra-embryonic structure, the yolk-sac. It is noteworthy that all parts of the vascular system proceed from mesodermic tissue, the heart and the vessels originating from clefts within this structure, and being lined, therefore, with endothelial cells.

In correspondence with the varying relations which the embryo sustains toward the fetal appendages at different times, its circulatory system is distinguished successively by certain special features. Thus, during the activity of the yolk-sac as an organ of nutrition, the vitelline circulation is present; following and supplanting this is the allantoic circulation, which latter, in turn, gives place to, or, in fact, becomes the placental system of vessels.

\section{THE VITELLINE CIRCULATION AND THE ORIGIN OF THE BLOOD.}

The seat of the first formation of the blood-vessels and of the blood is the wall of the yolk-sac, entirely ontside of the body of the embryo. The wall of the yolk-sac, the reader may be reminded, consists of the extra-embryonic splanchnopleure covered with a part of the somatopleure. The mesodermic layer of the sac exhibits-at the end of the first day in the chick-a network made up of cords of cells, the angioblast (Fig. 72). Interspersed throughout this network are groups of cells, the substance-islands, which lie within the meshes of the „etwork in relation with the cords 
of cells composing it (Fig. 72). Both the cells of the cords and of the substance-islands are mesenchymal cells. The superficial cells of the cell-cords become flattened in each case to constitute a continuous layer which encloses the remaining cells of the cord, and they thus form the endothelial

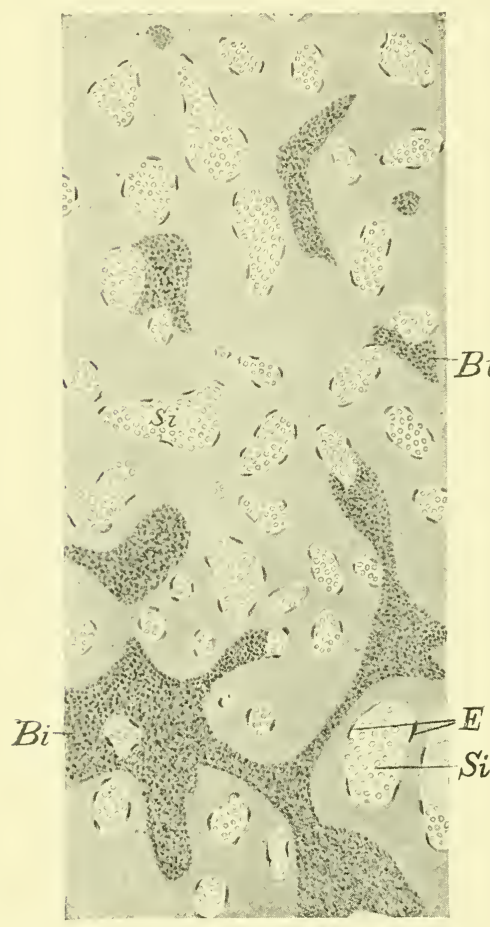

Fig. 72.-Portion of area vasculosa of chick-embryo, showing vascular network, in the vessels of which are seen the bloodisiands, $\mathrm{Bi}$. Abutting against the vessel walls composed of endothelial cells, $E$, are the substance-islands, si, which lie within the meshes of the network. (Disse.) wall of the future bloodvessel. The cells of the substance-islands move apart and acquire prolongations or processes which intercommunicate, while a gelatinous or semifluid intercellular substance is formed, thus producing an embryonal connective tissue in relation with the network of developing ressels. The solid "vessels" thus formed acquire lumina-on the second day of incubation in the chick-by the penetration of fluid from the surrounding mesoderm, this fluid crowding the cells apart, toward the ressel walls. The channels of the ressels are at first quite irregular, being at some points entirely blocked, at others merely encroached upon, by masses of spheroidal cells in connection with the vessel walls.

These cell-masses are the blood-islands, the aggregations from which are derived the fetal red blood-cells (Fig. 72). The cells of the blood-islands multiply by mitotic division and, moving apart, successively become detached and float free in the blood-stream. This process continues until the islands disappear. These cells, the erythroblasts, the first 
corpuscular elements of the fetal blood, are at first colorless, but soon become pale yellow. Their formation goes hand in hand with the formation of new blood-vessels. Their color deepens somewhat, hemoglobin developing within the cytoplasm. Their nuclei are large and reticular. The majority of them acquire small dense nuclei and are then called normoblasts. The erythroblasts continue to undergo mitotic division in the blood-stream just as they did in the blood-islands, division being seen in the embryo chick up to the sixth day. In man, multiplication of erythroblasts occurs quite largely in early fetal life, particularly in regions where the circulation is stow, as in the liver, the spleen, the bone-marrow, and the lymph-nodes; while in later fetal life and after birth it takes place in the red bone-marrow only.

It is especially noteworthy that these early fetal bloodcells are nucleated in contradistinction to the adult nonnucleated red blood-corpuscles; and that the nucleated form is present throughout life in all vertebrates but mammals.

$\mathrm{Up}_{\mathrm{p}}$ to the end of the first month the nucleated red cells are the only corpuscular elements found in the blood. In the second month the non-nucleated red blood-disks, the erythrocytes, make their appearance, and either in the third month or very soon thereafter outnumber the nucleated cells. Differences of opinion obtain as to the mode of origin of the erythrocyte, but the prevailing view is that it results from the normoblast by the loss of the nucleus of the latter. The nucleus becomes globular and more dense, assuming in some cases a dumb-bell shape, and is extruded from the cell, after which it is thought to undergo partial disintegration and then absorption by leukocytes. Some observers maintain that the nuclei are dissolved within the cell. Nuclei in the process of extrusion have been observer in cat-embryos. After extrusion of the nucleus the remaining cytoplasm of the cell assumes the biconcave form of the adult red blood-corpusele or erythrocyte.

The origin of the leukocytes is a somewhat unsettled question. They are found in the blood of chick-cmbryos at the eighth day and in the rabbit-embryo at the ninth day ; in the 
human embryo they are seen in the second month. It is probable that they originate in the lymph-nodes, the bonemarrow, the liver, and the spleen during fetal life, but after birth only in the bone-marrow, the lymph-nodes, and the spleen. Their birthplace would be, therefore, lymphoid tissue and their ultimate origin mesodermic. It has been suggested that they may be derived from young erythroblasts; this is denied by Minot. Beard assigns them an entodermal origin, claiming that they are produced by the entodermal epithelium of the thymus and of the tonsil. From the investigations of Engel and of Florence Sabin it would appear that they are first seen in the blood and the lymph-nodes at the same time.

The blood-platelets have been variously interpreted as small nucleated cells and as fragments of broken-down leukocytes. According to the recent work of Wright they are fragments of the processes of the giant cells (myelocytes) of bone-marrow.

Limiting the first network of vessels on the surface of the yolk-sac is a circular vessel, the sinus terminalis (Plate VI.). Since the yolk-sac is relatively so large that the body of the embryo appears to rest upon it, and since the surrounding somatopleure is translucent, a suriace view of the ovum at this stage shows a vascular zone encircling the embryonic area and the later body of the embryo. This zone is the area vasculosa, or vascular area, the seat of the earliest formation of blood and of blood-ressels of the embryo.

The blood-vessels originate, as shown above, from the angioblastic network of mesenchymal cell-cords of the vascular area, the cords of cells, at first solid, gradually becoming hollowed out to form the vessels. The vaseular network at first formed extends by a process of budding over the walls of the yolk-sac and thence along the vitelline duct into the body of the embryo. The budding consists in the extension of vessel sprouts or cell-cords-probably from proliferation of the terminal cells of the vessels last formed-the sprouts being solid at their ends, since the excavation of a sprout always occurs a little later than its forward extension. 


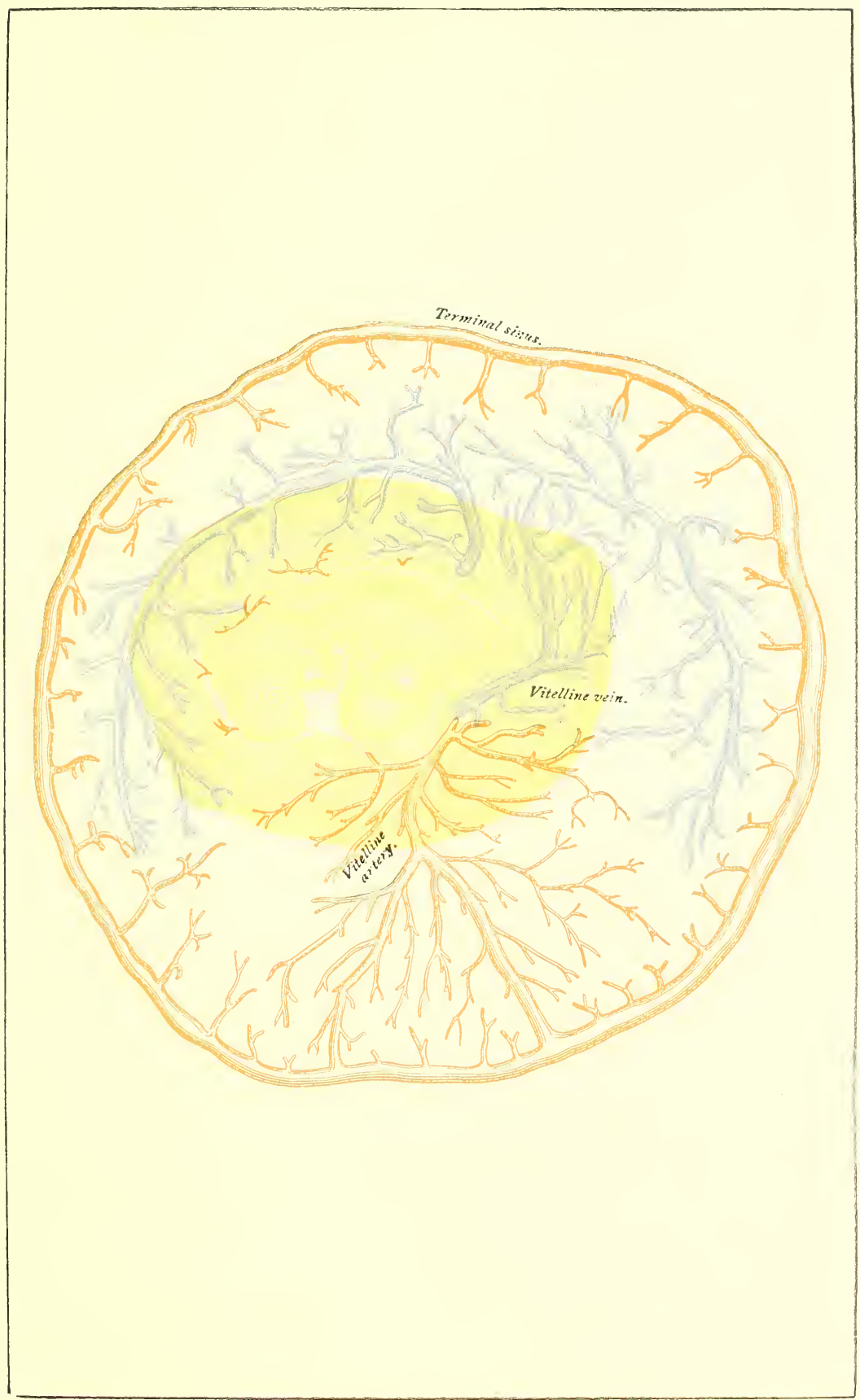

Vaseular area of eleven-day rabbit embryo (E. von Beneden and Julin). The capiliaries are not shown; the terminal sinus is seen to be arterial. 

Neighboring sprouts communicate with each other to a greater or less extent. In a human embryo of about eighteen days the extension of the vessels-appearing macroscopically as fine red threads-along the vitelline duct is well shown. Having reached the body of the embryo, the vessels take their course toward the primitive heart, which has meanwhile been developing. From the anterior and posterior and lateral limits of the vascular area-using these terms with reference to the axis of the embryonic body-four pairs of vitelline veins converge toward the vitelline duct and unite to form the two vitelline or omphalomesenteric veins. These veins, after entering the body of the enbryo, pass headward along the wall of the intestinal tube and empty into the lower or caudal end of the primitive heart. The trunks, which are to constitute the vitelline arteries, after entering the body with the vitelline duct, pass upward along the dorsal body-wall, within the dorsal mesentery, to become continuous with large arterial trunks that have proceeded from the primitive heart.

The large trunks referred to are the visceral-arch vessels, which unite to form the primitive aortre. The visceral-arch vessels (see Fig. 60) are a series of five pairs of arteries that arise by a common stem, the truncus arteriosus, from the upper end of the primitive heart. They pass along the respective visceral arches toward the dorsal surface of the body where all the vessels of one side unite into a common trunk, the primitive aorta. The two primitive aortæ, passing caudalward in the dorsal mesentery, give off, as their largest branches, the two omphalomesenteric or vitelline arteries above referred to. The development and the regression of the visceral-arch vessels correspond with the growth and the decadence respectively of the visceral arches. Not all the vessels are present in a fully-developed condition at any one time, the first pair having begun to atrophy before the fifth pair makes its appearance. The metamorphosis into certain adult vessels of such of them as persist will be considered in a later section.

This system of vessels constitutes the vitelline circulation, the manifest function of which is to convey nutritive mate- 
rial from the yolk-sac to the embryo. While the vitelline circulation is of great importance in any ovum provided with abundant nutritive yolk, such as that of the bird, it is of comparatively slight consequence in man and the other higher mammals, and it must be regarded as a vestige of the avian or reptilian ancestry of the mammalian ovum, or, at least, as a reminder that the mammalian ovum was originally provided with an abundant yolk. It must be borne in mind, however, that the mammalian blastodermic vesicle imbibes from the walls of the uterus a richly nutritive albuminous fluid, which may be taken up later and carried to the cmbryo by the vitelline circulation. This system of yolk-sac vesscls disappears with the regression and disappearance of the yolk-sac-in the human embryo at about the fifth week. The intra-embryonic portion of the right vitelline artery persists, however, to become the superior mesenteric artery of the adult.

To render the comprehension of the later phases of the vascular system more simple, their consideration is deferred until the development of the heart shall have bcen described.

\section{THE DEVELOPMENT OF THE HEART.}

The heart, when studied in the lower-type animals, is seen to be morphologically a dilated and specialized part of a vascular trunk embedded in the ventral mesentery. In man, the first fundament of the heart appears at a very early period-namely, before the splanchnopleure has folded in to form the gut-tract, or, in other words, before the end of the second week. This fundament, in all higher vertebrates, is bilateral, having the form of two tubes produced by vacuolation of the splanchnic mesoderm and lying widely separated, one in each half of the still spread out splanchnopleure (Fig. 73), A). A transverse section through the future neckregion of a sheep- or rabbit-embryo shows the tubes cut across, since their long axes are parallel with that of the body (Fig. 74). With the folding in of the splanchnopleure and the union of the edges of its folds, the tubes are carried toward each other, and subsequently, by the disappearance 
of the tissue intervening between them, their cavities become one (Fig. 73, $B$ and $C$ ). After the formation of the guttract, therefore, and the simultaneous appearance of the ventral body-wall, the heart-fundament is a single straight mesodermic tube, situated in the pharyngeal region, in close relation with the ventral wall of the body, between the latter and the fore-gut. Reference to Fig. 73, C, will show that

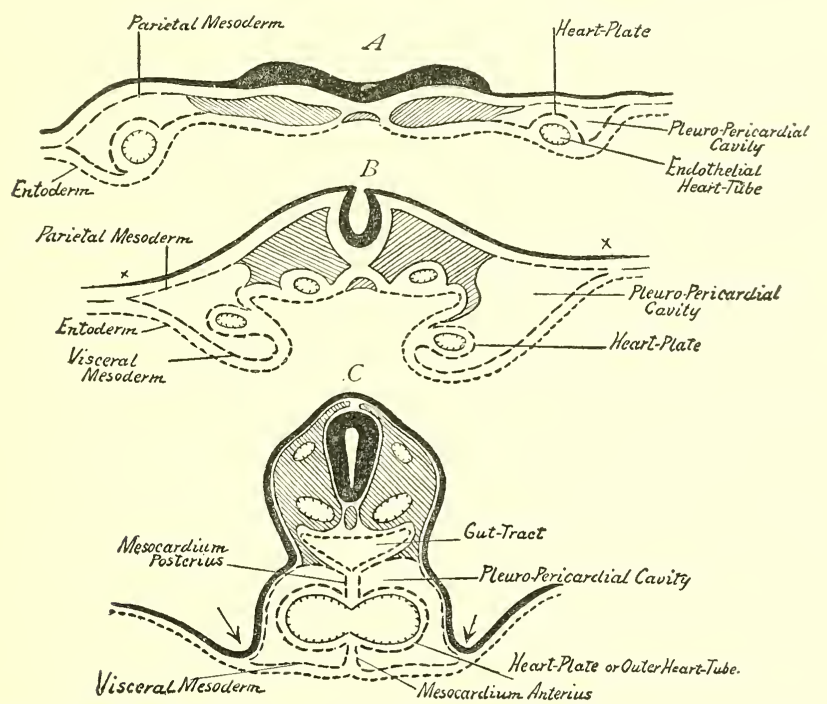

FIG. 73.-Schematic cross-section of rabbit-embryo to show development of heart; $A$, embryonic area with the germ-membranes still spread out; $B$, more advanced stage, the splanchnopleure partly folded in ; $C$, splanchnopleure folded in to form gut-tract, the two heart-tubes fused into one (after Strahl).

the heart-tube is separated from the body-cavity (or cœlom) on each side by a layer of the mesoderm, and that these two layers connect the heart dorsally with the gut-tract and ventrally with the body-wall, forming respectively the mesocardium anterius and the mesocardium posterius. These folds temporarily divide the upper portion of the body-cavity into two lateral parts.

The disappearance of the stratum of mesoderm immediately surrounding the heart-tube and the differentiation of the tissue limiting peripherally the space thus formed, results in the production of a second larger tube enclosing the first. The cells of the outer tube become specialized 
into muscle-cells, which are to constitute the future heartmuscle, while those of the inner cylinder flatten and assume the endothelioid type to become the endocardium. 'The growth of centrally projecting processes from the muscular wall and the outpocketing of the endothelial tube to cover these processes and line the spaces enclosed by them foreshadow the spongy character of the inner surface of the adult heart, with its columnæ carneæ and musculi pectinati. It is significant, as showing the contractility of undifferentiated proto-

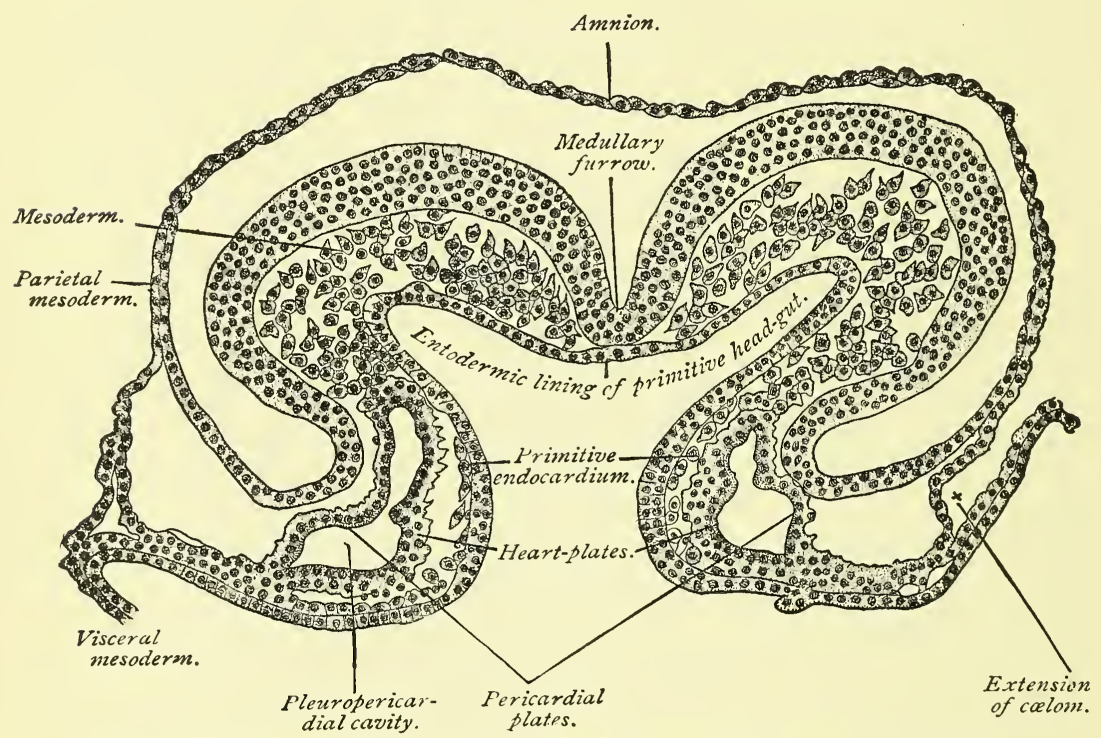

FIG. 74.-Transverse section of a sixteen-and-a-half-day sheep-embryo (Bonnet).

plasmic cells, that the heart begins to pulsate even before the appearance of any muscular tissue in its walls.

The upper end of the heart-tube tapers away into the truncus arteriosus (Fig. 75, 4), a vessel which bifurcates into the first pair of visceral-arch vessels, while its lower extremity receives the vitelline veins above referred to. $\mathrm{Ex}$ cessive growth in length, each end of the tube being more or less fixed in position, necessitates flexion or folding, the form which the heart-tube assumes in consequence being that of the letter S placed obliquely (Fig. 76, A). The venous 
limb of the S lies caudad and toward the left, the arterial segment being directed headward and toward the right, so that
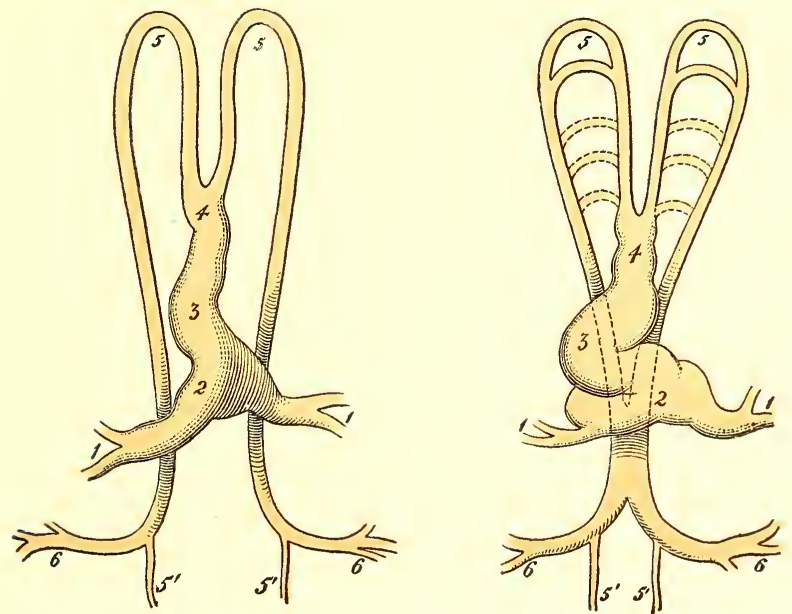

FIG. 75.-Diagrams illustrating arrangement of primitive heart and aortic arches (modified from Allen Thomson): 1, vitelline veins returning blood from vascular area; 2 , venous segment of heart-tube; 3 , primitive ventricle; 4 , truncus arteriosus; 5,5 , upper and lower primitive aortæ; $5^{\prime}, 5^{\prime}$, continuation of double aortæ as vessels to caudal pole of embryo; 6 , vitelline arteries returning blood to vascular area.

A

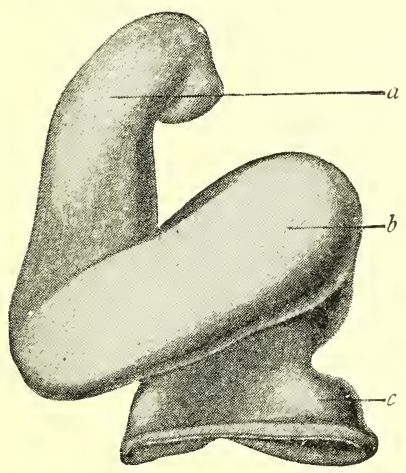

$\mathrm{B}$

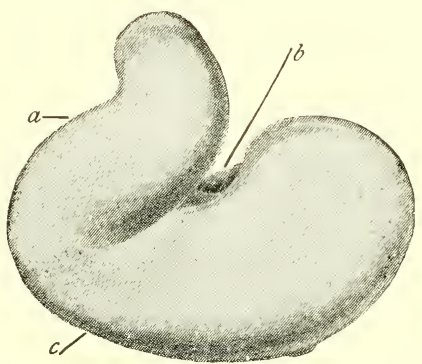

Fig. 76. -A, heart of human embryo of $2.15 \mathrm{~mm}$. (His) : $a$, truncus arteriosus ; $b$, primitive ventricle; $c$, venous segment. B, heart of human embryo of about $3 \mathrm{~mm}$. (His): $a$, truncus arteriosus ; $b$, venous segment (behind); $c$, primitive ventricle (in front).

the two lie almost in the same coronal plane. These relations are soon altered by such a rotation around a longitudinal axis that the venous part of the heart comes to lie nearer 
the dorsal wall of the body, with the arterial portion ventral to it, both being brought at the same time into practically one transverse plane by the headward migration of the venous, and the tailward migration of the arterial, moiety. At this time the heart is relatively so large, and the ventral body-wall covering it so thin, that the organ appears as if situated outside of the embryo's body (Fig. 62, p. 116).

Simultaneously with these alterations in position, the arterial part of the heart is being marked off from the venous segment by a transverse constriction, the former becoming the ventricle, the latter the auricle or atrium (Fig. 76, A). The narrow communication between the two is the auricular or atrioventricular canal, which soon acquires the primitive atrioventricular valves, or endocardial cushions, these being endocardial thickenings on the dorsal and ventral walls of the canal. Growing toward each other, the cushions mect and unite, forming the septum intermedium (Fig. 78, B, $f$ ), which now occupies the middle of the auricular eanal, leaving only its lateral portions patulous. The truncus arteriosus becomes delimited from the ventricle by a circular constriction, the fretum Halleri, the proximal part of the truncus arteriosus dilating somewhat to constitute the bulbus arteriosus. The truncus arteriosus divides into the visceral-arch vessels, as pointed out in the last section.

\section{The Metamorphosis of the Single into the Double} Heart.-The heart with but one ventricle and one auricle or atrium is found not only during the early periods of development in all air-breathing vertebrates, but is the permanent condition in fishes. In the development of the individual, as in the evolution of the higher vertebrate type, the appearance of the lungs, which replace the branchiæ of fishes as an aërating apparatus, is accompanied by a division of the heart into right and left halves for the pulmonary and the general systemic circulation respectively.

The division of the human atrium begins in the fourth week with the growth of a perpendicular ridge from its dorsal and cephalic walls (Fig. 78, B), this being indicated externally by a groove on the outer surface of the corresponding wall of 
the auricle. The ridge, growing downward, becomes the septum primum or auricular septum and fuses with the upper extremity of the septum intermedium of the auricular canal, thus dividing the atrium into the right and left auricles (Fig. 77). The atrioventricular canal, its anterior and posterior cushions having united into the septum intermedium, shares in this division, becoming thereby the right and the left auriculoventricular orifices. The division of the atrium, however, is not as yet complete; a hiatus, the foramen ovale, exists ventral to the free ventral border of the united septum

A

$\mathrm{B}$

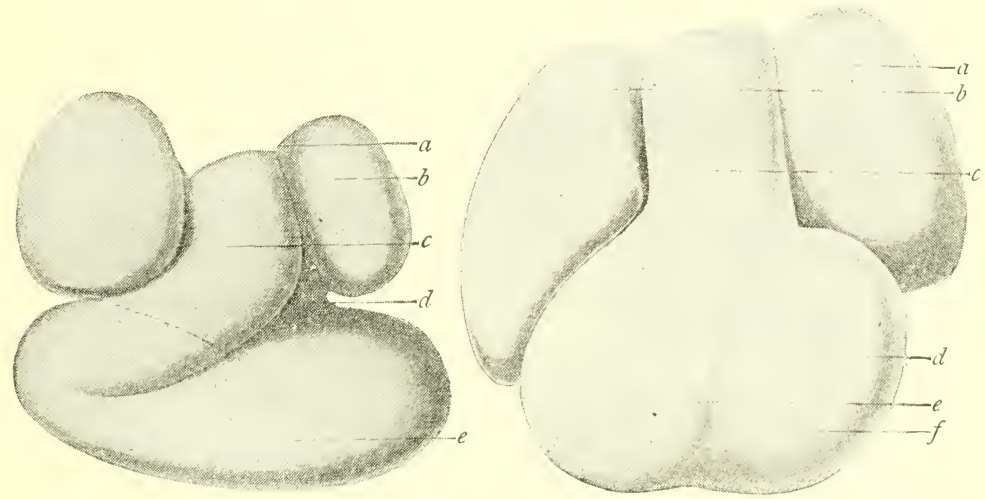

FIG. 77. $-\mathrm{A}$, heart of human embryo of about $4.3 \mathrm{~mm}$. (His) : $a$, atrium; $b$, portion of atrium corresponding with auricular appendage; $c$, truncus arteriosus; $d$, auricular canal; $e$, primitive ventricle. $\mathrm{B}$, heart of human embryo of about the fifth week (His) : $a$, left auricle; $b$, right auricle; $c$, truncus arteriosus; $d$, interventricular groove; $e$, right ventricle; $f$, left ventricle.

primum and septum intermedium. A ridge grows downward from the roof of the atrimm upon the right side of the septum primum and parallel with it; this is the septum secundum (atrial crescent) and is very much thicker than the primary septum. Its downward growth continues in such manner that it comes to bound the foramen ovale ventrally and below, its extremity uniting with the left extremity of the fold which later becomes the Eustachian valve, and thus forming the future annulus ovalis. The part of the primary septum which is thus partially surrounded by the free margin of the septum secundum pouches into the left auricle to con- 
stitute a sort of valve for the prevention of regurgitation. At birth or shortly after, the ventral edge of this valve-like fold unites with the ventral margin of the foramen ovale, thus obliterating the latter, the fold becoming thereby the relatively thin floor of the fossa ovalis of the adult heart. ${ }^{1}$

The division of the ventricle, which follows that of the auricle and which is completed by the seventh week, is first

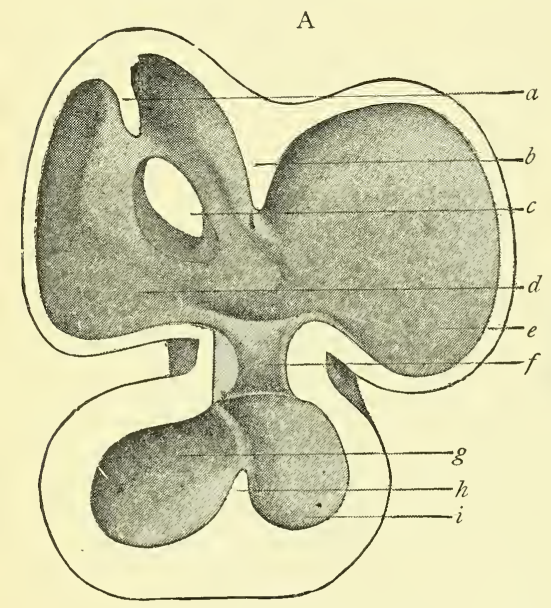

$\mathrm{B}$

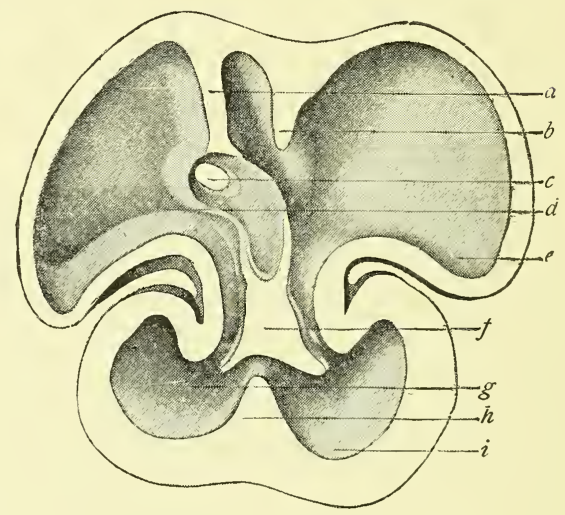

Fig. 78.-A, section of heart of human embryo of $10 \mathrm{~mm}$. (His): $\alpha$, septum spurium ; $b$, interauricular septum; $c$, mouth of sinus reuniens; $d$, right auricle; $e$, left auricle; $f$, auricular canal; $g$, right ventricle; $h$, interventricular septum ; $i$, left ventricle. B, section of heart of human embryo of about the fifth week (His): $a$, septum spurium: $b$, auricular septum; $c$, opening of sinus reuniens (leader passes through foramen ovale); $d$, right atrium; $e$, left atrium; $f$, septum intermedium; $g$, right ventricle ; $h$, ventricular septum; $i$, left ventricle.

indicated by a vertical groove, the sulcus interventricularis, seen on both the dorsal and the ventral surface (Fig. 77). From the internal surface, corresponding to the position of the sulcus, a median centrally projecting ridge appears and develops into a septum (Figs. 78, $h$, and 79, $k s$ ), which, however, is incomplete above and in front. The deficiency thus left, the ostium interventriculare, is obliterated by the downgrowth of the aortic septum (Fig. 79, s), upon the completion

${ }^{1}$ Occasionally the foramen ovale remains patulous for several weeks or months after birth or even throughout life. As this condition allows the venous blood to mingle with the arterial, the surface of the body is bluish or cyanotic, and a child thus affected is said to be a "blue baby." 
of which the original single ventricle is divided into the right and the left ventricles. While the interventricular septum of the completed heart is, for the most part, muscular, that portion of it which is produced by the aortic septum always remains membranous, constituting the pars membranacea septi of the adult heart. If this septum is incomplete, as happens occasionally, there is an abnormal communication between the two ventricles.

The truncus arteriosus, after having become somewhat flattened, is divided by the growth of a vertical septum, or partition (Fig. 79, s), into the aorta and the pulmonary artery. The growth of the partition is initiated by the appearance of two ridges on the opposite walls of the truncus, the ridges growing toward each other and finally uniting to form the aortic septum. Two longitudinal grooves which appear upon the surface of the truncus, following the growth of the ridges and corresponding in position with then', indicate the division of the vessel into the aorta and the pulmonary artery. The septum grows downward to meet and unite with the rentricular septum, as indicated above. Though the three septa referred to develop independently of each other there is such correspondence between them, as to position, that the effect is as if they constituted one continuous structure.

Before the division of the atrium into the auricles, its walls pouch out on each side to form the auricular appendages, one of which belongs to each future auricle (Fig. 77). While it is still a straight tube, the heart receives at its venous extremity the two vitelline veins. Subsequently this particular part of the atrium is distinguished as the sinus venosus or sinus reuniens, this being a short thick trunk into which empty, in addition to the vitelline veins, the ducts of Cuvier and the umbilical veins. The mouth of the sinus venosus is guarded by a valve composed of two leaflets. The right leaflet or fold is continuous above with a ridge on the roof of the atrium, the septum spurium (Fig. 78, a). In the division of the atrium the sinus venosus falls to the right auricle, while emptying into the left auricle is the single pulmonary vein, which is formed by the union of the four pul- 
monary veins. Still later, the sinus venosus is merged into the wall of the right auricle, and hence the venous trunks above mentioned empty by separate orifices into its cavity. The line of demarcation between the sinus venosus and the auricle proper is indicated in the adult heart by the sulcus terminalis, a groove on the outer surface of the auricle which
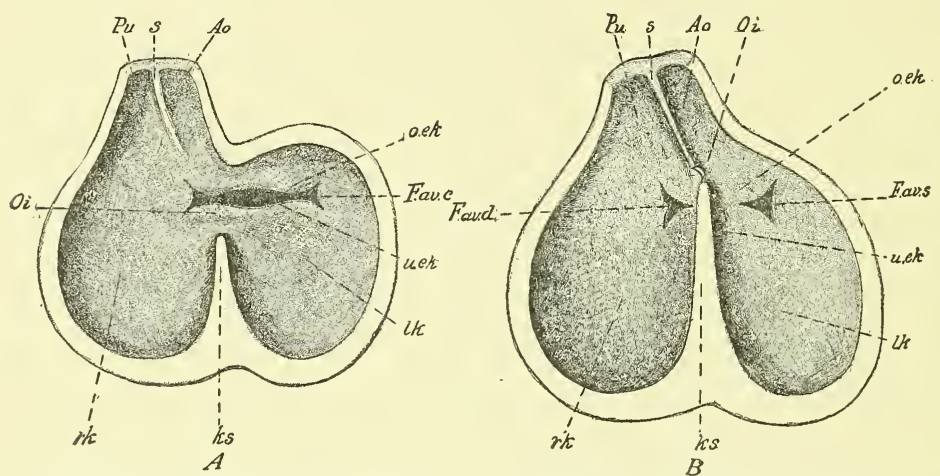

FIG. 79.-Two diagrams (after Born) to elucidate the changes in the mutual relations of the interventricular orifice and the ostium interventriculare as well as the division of the ventricle and large arteries. The ventricles are imagined to have becn divided into halves; one looks into the posterior (dorsal) halves, in which, moreover, the cardiac trabeculæ, etc., have been omitted for the sake of simplifying the view. $A$, heart of an embryo rabbit, in which the head is $3.5-5.8$ $\mathrm{mm}$. long. The ventricle is divided by the ventricular partition $(k s)$ into a left and a right half as far as the interventricular orifice $(O i)$. The right end of the foramen atrioventriculare commune $(F . a v \cdot c)$ extends into the right ventricle; the endocardial cushions $(o . e k, u . e k)$ are developed. $B$, heart of an embryo rabbit, head $7.5 \mathrm{~mm}$. long. The endocardial cushions $(o . e k, u . e k)$ of the foramen atrioventriculare commune are fused, and thereby the foramen atrioventriculare commune is now separated into a foramen atrioventriculare dextrum $(F, a v . d)$ and sinistrum $(F, \alpha v, s)$. The ventricular partition $(k s)$ has likewise fused with the endocardial cushions, and has grown forward as far as the partition ( $s$ ) of the truncus arteriosus. By the closure of the remnant of the ostium interventriculare $(O i)$ the septum membranaceum is formed; $r k$, right, $l k$, left ventricle; $k s$, ventricular partition; $P u$, arteria pulmonalis ; $A o$, aorta ; $s$, partition of the truncus arteriosus ; $O i$, ostium interventriculare; $F$. av. $c$, foramen atrioventriculare commune; $F . a v . d$ and $F . a v . s$, foramen atrioventriculare dextrum and sinistrum; o. $e k, u . e k$, upper and lower endothelial or endocardial cushions.

passes from the front of the superior vena cava to the front of the inferior vena cava.

The left leaflet of the valve at the mouth of the sinus venosus becomes atrophic, as does also the septum spurium; the right divides into two parts, one of which becomes the Eustachian valve at the orifice of the inferior vena cava, 
while the other forms the valve of Thebesius, or the coronary valve, at the opening of the coronary sinus (the latter being the persistent lower end of the left duct of Cuvier). The Eustachian valve serves to direct the blood from the inferior cava through the foramen ovale so long as that aperture is present. The single pulmonary vein is in like manner incorporated in the wall of the left auricle, the four pulmonary veins in consequence acquiring separate openings into that cavity.

The Valves of the Heart.-Before the division of the atrium and the ventricle into right and left halves, the atrioventricular canal has the form of a transverse fissure, each lip of which is thickened into a ridge (Fig. 79, A). These ridges or endocardial cushions are the primitive valves. When the atrial partition grows down and the ventricular septum grows up, their free edges meet and unite with the ridges, each ridge being thereby divided, on its atrial surface by the atrial or interauricular septum, and on its ventricular aspect by the ventricular septum, into a right and a left half (Fig. 79, B). Since the ridges, at their points of union with the septa, fuse likewise with each other, the original orifice is bisected into the right and left auriculoventricular apertures, the only valves of which are the ridges or cushions in question.

To trace the further development of the fully formed valves, it will be necessary to consider the changes which now take place in the walls of the heart. It has been seen that the inner surface of the heart acquires a spongy or trabecular structure at a very early stage by the inward projection of muscular processes from the outer tube and the pouching out of the inner endothelial tube to cover these. The wall of the ventricle in consequence is relatively very thick and is made up largely of a network of fleshy columns, the spaces of which network are lined with the endocardium (Fig. 80, A). While the outer stratum of the ventricular wall now becomes more compact by the thickening of the trabeculæ-and, to some extent, by their coalescence-the trabeculæ in the vicinity of the atrioventricular valves di- 
minish in thickness and lose their muscular character, being replaced by thin connective-tissue cords (Fig. 80, $B$ ). That part of the ventricular wall which surrounds the atrioventricular orifice and to which the endocardial cushions or
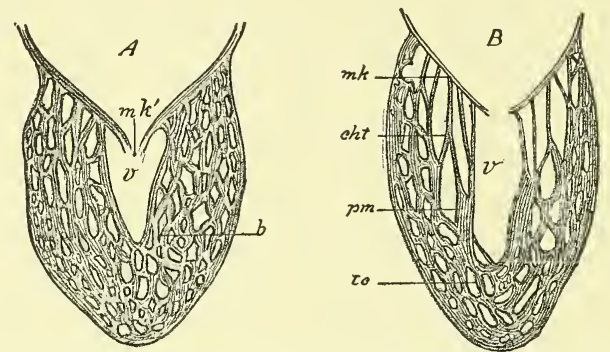

FIg. 80.-Diagrammatic representation of the formation of the atrioventricular valves: $A$, earli $r, B$, later condition (after Gegenbaur): $m k$, membranous valve; $m k^{\prime}$, the primitive part of the same; cht, chordæ tendineæ; $v$, cavity of the ventricle; $b$, trabecular network of cardiac musculature ; $p m$, papillary muscles ; $t c$, trabeculæ carneæ.

primitive valves are attached, likewise becomes deprived of muscle-cells, the remaining connective tissue assuming the form of thin plates. These plates, with the former endocardial cushions attached to their edges, constitute the
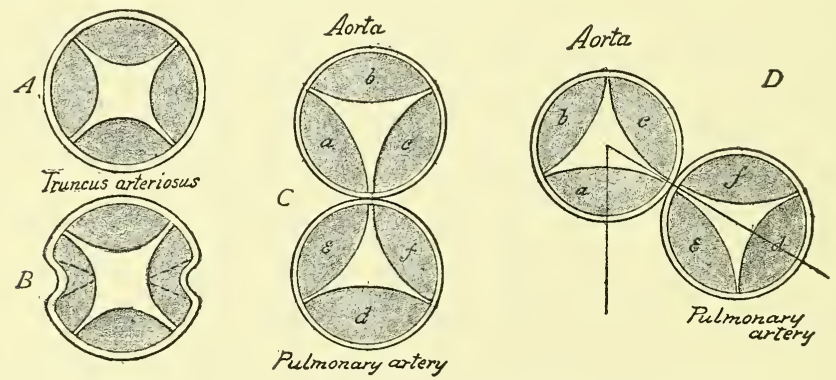

FIG. 81.-Scheme showing division of truncus arteriosus and its valve-leaflets into aorta and pulmonary artery with their leaflets. The division begins in $B$, the lateral leaflets dividing respectively into $a, e$, and $c, f$. Rotation from right to left shown in $D$.

permanent auriculoventricular valve-leaflets. The strands of connective tissue mentioned above as remaining after the degeneration of certain of the muscle-trabecula are the chordæ tendineæ of the adult heart. Attached at one end 
to the valve-leaflets, their other extremity is continuous with trabeculæ that have remained muscular, the adult musculi papillares.

The semilunar valves of the aorta and pulmonary artery appear when the truncus arteriosus divides to form those vessels. The orifice of the truncus arteriosus is provided with a valve having four leaflets (Fig. 81, A). By the division of this vessel into the pulmonary artery and the aorta (Fig. 81, $B$ and $C$ ), the lateral leaflets are bisected, the anterior half of each, with the anterior leaflet, going to the anterior vessel-the pulmonary artery-while each posterior or dorsal half, with the dorsal leaflet, falls within the orifice of the aorta. The resulting disposition of the segments of the aortic and pulmonary valves is such that, in the aorta, two leaflets are situated anteriorly and one posteriorly, while in the case of the pulmonary artery these conditions are reversed (Fig. 81, C). In the fully developed heart, however, it is found that the aorta has two posterior leaflets and one anterior, and that the pulmonary artery presents one posterior and two anterior segments. In the division of the truncus arteriosus, the anterior half, or the pulmonary artery, falls to the right ventricle, and the posterior trunk, the aorta, to the left ventricle, the two ventricles lying side by side. In order, therefore, that the ventricles may acquire the relative positions which they hold in the adult there must be such a rotation that the left ventricle comes to lie behind the right. This rotation of the heart from right to left necessarily alters the relation of the pulmonary artery, causing it to lie not directly in front of the aorta, but in front and to the left. If one conceives of a rotation of the two vessels from right to left through an are of 60 degrees around a vertical axis, the altered relation of the pulmonary and aortic leaflets becomes at once intelligible (Fig. 81, $C$ and $D$ ).

\section{THE allantoic AND THE PLACENTAL CiRCULATION.}

The development of the allantois and its accompanying system of blood-vessels is simultaneous with the decline of the yolk-sac and the vitelline circulation. Since the allan- 
tois is an evagination from the gut-tract (see p. 89), it is a splanchopleuric sae, its walls consisting therefore of an entodermic and a mesodermic layer. Blood-vessels develop within the mesodermic stratum as extensions or branches of previously existing intra-embryonic trunks. These vessels are the allantoic arteries and veins. The two allantoic arteries are branches of the primitive aorta and leave the body of the fetus, in company with the neck of the allantois, at the umbilicus. Having reached the peripheral part of the allantois, they break up into a capillary plexus, the extension of which into the villous processes of the false amnion completes the union of that structure with the allantois to form the true chorion (Plate III.).

The two allantoic veins develop pari passu with the arteries and convey the blood from the chorion to the fetus. Entering the body of the fetus through the still large umbilical aperture, they find their way along the intestinal tube to the septum transversum-which structure may be regarded as the primitive diaphragm-to the region of the heart, where they open into the ducts of Cuvier. Each duct of Cuvier (Fig. 84, A) is formed by the union of the primitive jugular vein with the cardinal vein of its own side, the cardinal and the jugular veins returning the blood respectively from the lower and upper parts of the trunk. This system of blood-vessels constitutes the allantoic circulation; it is of great importance in any ovum that is developed outside of the body of the mother, as in the case of birds, reptiles, and fishes, in which classes the allantois is the organ of nutrition from the time that the yolk-sac ceases to perform that function until development is complete. In man, however, as in all other mammals except the monotremes and marsupials, the allantoic circulation may be looked upon as, in a measure, rudimentary, since it serves to convey nutriment from the chorion to the fetus only until the formation of the placenta.

The placental system of blood-vessels, appearing in the third month with the development of the placenta, includes the principal trunks of the former allantoic system, the allantoic arteries and veins having become the umbilical 
vessels. The two umbilical arteries convey impure blood from the fetus to the placenta, where it circulates through the capillaries of that organ and receives oxygen and nutriment from the blood of the mother. As before stated, there is no intermingling of the fetal and the maternal blood, the two currents being separated by the very thin walls of the capillaries, through which osmosis occurs. The purified blood returns to the fetus through the umbilical veins and reaches the right auricle through the inferior vena cava, a portion of it having passed through the liver. The two umbilical veins which are present for a time fuse subsequently to form a single vein. The complicated details of the arterial and the venous trunks, and the relation of the latter to the development of the liver and its special system of vessels, may be advantageously considered in separate sections.

\section{THE FETAL ARTERIAL SYSTEM.}

The truncus arteriosus, the large artery which arises from the, as yet, undivided ventricle of the heart, bifurcates into two trunks, the first pair of visceral-arch vessels (Fig. 82, 4). These first visceral-arch vessels, also sometimes called the first aortic arches, run from the ventral surface of the body along the first visceral arches, toward the dorsum, where they curve downward and pass caudalward, one on each side of the median line, in front of the primitive vertebral column. Very soon there arise from the truncus arteriosus below the point of origin of the first vessels, four ${ }^{1}$ additional pair's of visceral-arch vessels, which similarly pass dorsad along the corresponding visceral arches, and which unite with the dorsal part of the first pair to form the primitive aorta of each side. Each primitive aorta results, therefore, from the confluence of all the visceral-arch vessels of its own side (Fig. 82). The two aortie afterward beeome merged into a single trunk. At first the principal branches of the aorta are the vitelline arteries. As these latter vessels become

${ }^{1}$ It is sometimes stated that there are six visceral-arch vessels, the fifth of which disappears, so that the vessel here designated the fifth would represent the sixth-arch vessel of the early condition as well as of lower forms. 
inconspicuous, the allantoic or umbilical arteries come into prominence as the chief branches. Indeed, the umbilical arteries may be said to be the continuation of the aorta, since the largest part of the blood-stream is diverted into them. The aorta proper continues in the median line as the caudal aorta, which latter is represented in the adult by the middle sacral artery. A branch from the fifth arch goes to the lungs.

So far the arterial system of the fetus presents an absolutely symmetrical arrangement (Fig. 82). Changes very soon occur, however, which lead to the asymmetrical condition
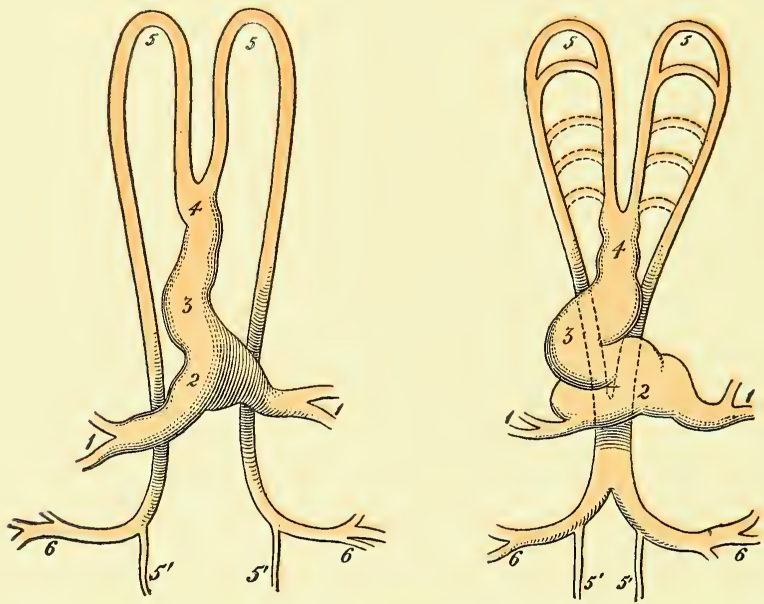

FIG. 82.-Diagrams illustrating arrangement of primitive heart and aortic arches (modified from Allen Thomson): 1, vitelline veins returning blood from vascular area; 2 , venous segment of heart-tube; 3 , primitive ventricle; 4 , truncus arteriosus ; 5, 5, upper and lower primitive aortæ; $5^{\prime}, 5^{\prime}$, continuation of double aortæ as vessels to caudal pole of embryo; 6 , vitelline arteries returning blood to vascular area.

found in the adult. These changes are due to the atrophy of some trunks and the preponderance of others. From the point where the dorsal extremity of the fourth arch joins the fifth, a branch passes 'to the rudimentary arm (Fig. 83). The first and second arches, except their ventral and dorsal limbs, undergo atrophy. The ventral limbs of the first and second arches persist and become the external carotid artery, while their dorsal extremities, with the third visceral-arch vessel, become the internal carotid artery. The ventral stem of the third arch constitutes the common carotid. The right 
fourth-arch vessel becomes the right subclavian, its stream of blood being conveyed to the arm by the branch which has taken its origin from the point of junction of the dorsal ends of the fourth and fifth arches. This latter branch is therefore the continuation of the subclavian. The ventral segment of the right fourth arch would be represented in the

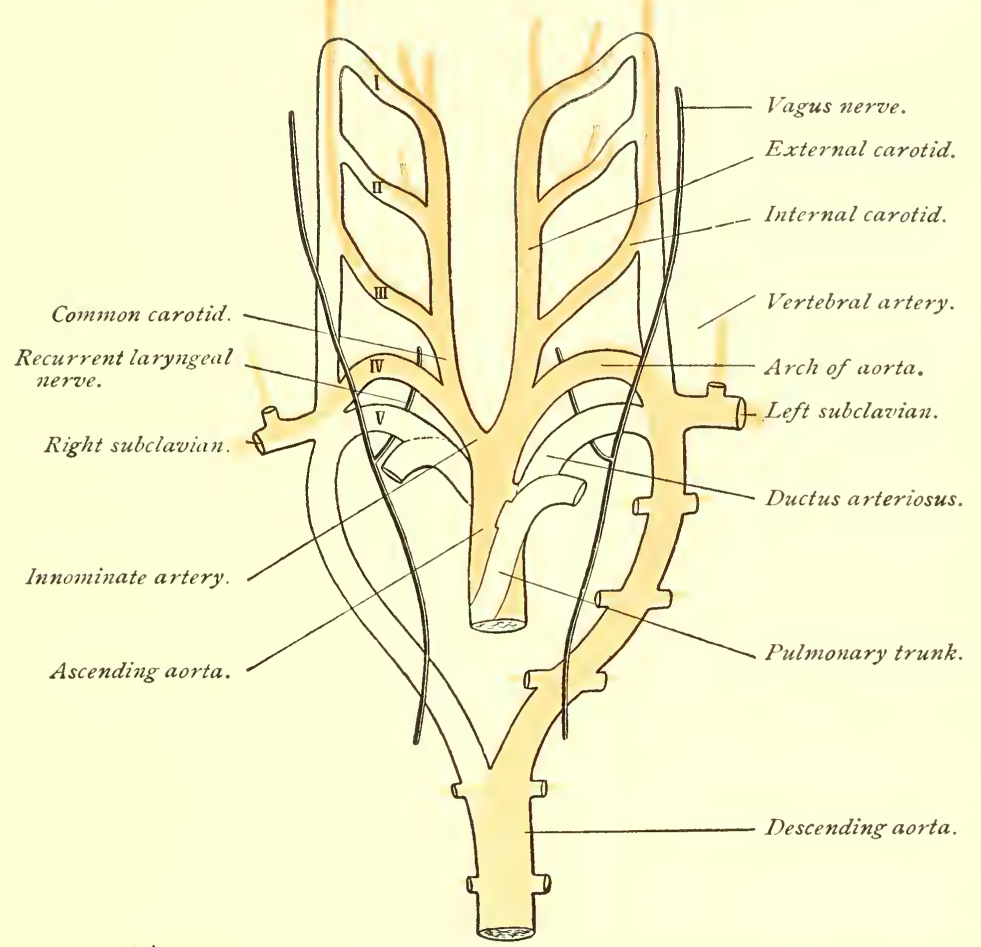

Fig. 83.-Diagram illustrating the fate of the aortic arches in mammals and man (modified from Rathke).

adult by the innominate artery. The fourth arch of the left side assumes a lower position ; sinking into the thorax, it becomes the arch of the aorta. Since the right fifth arch becomes atrophic beyond the point of origin of the right pulmonary artery, the dorsal end of the right fourth-arch vessel-the future right subclavian artery-loses its connection with the primitive aorta, and the latter now appears as the continuation of the left fourth arch. The ventral stem of the left third arch, which becomes the future left common carotid, and 
also the left subclavian, which arises from the posterion or dorsal end of the left fourth arch, are now branches of the arch of the aorta. When the truncus arteriosus becomes divided into the aorta and the pulmonary artery, the left fiftharch vessel and the right pulmonary artery are the only branches of the truncus that fall to the pulmonary artery, all the other risceral-arch ressels being connected with the aorta. The left fifth visceral-arch vessel, therefore, is represented in the adult by the pulmonary artery and the ductus arteriosus. The fetal lungs being impervious, only a very small part of the blood of the pulmonary artery is sent to them. The larger portion of the blood passes from the pulmonary artery to the aorta through a communicating trunk, the ductus arteriosus, which represents the greater part of the left fifth arch and which becomes impervious after birth with the establishment of the proper pulmonary circulation.

These transformations afford an explanation of the different relations of the recurrent laryngeal nerves of the two sides. At first they are symmetrically arranged. The pneumogastric nerve, as it crosses the fourth visceral-arch vessel, gives off the recurrent laryngeal nerve, the latter winding around the artery from before backward on its way to the larynx. When the left fourth arch becomes the arch of the aorta and sinks into the chest, the nerve is carried with it; hence after this time, the left nerve is found winding around the arch of the aorta.

Anomalous arrangements of the branches of the aortic arch, as well as of the arch itself, are referable to anomalous development of the original system of visceral-arch vessels. For example, if the right fourth arch, which usually becomes the right subclavian artery, be suppressed from its origin to the point where the artery for the right upper extremity is given off, the blood must find its way into the latter vessel through the dorsal stem of the fourth arch, and this dorsal stem will then become the right subclavian artery. In such case, the right subclavian of the adult will be found to arise from the left extremity of the areh of the aorta and to pass obliquely upward to the right side of the neck behind the trachea and the esophagus. 


\section{THE FETAL VENOUS SYSTEM.}

The venous system of the embryo presents several successive phases, corresponding in part with the various stages in the evolution of the arterial system. The first trunks to appear are the vitelline veins. These vessels have their origin in the vascular area on the wall of the yolk-sac in the manner already described in connection with the vitelline circulation. The two vitelline or omphalomesenteric veins, which result from the convergence of all the venous trunks of the vascular area, follow the vitelline duct into the body of the embryo through the still widely open umbilical aperture and take their course headward along the intestinal canal to open into the caudal end of the primitive heart-tube (Fig. 82, 1, 1). At a later period they open into the sinus venosus of the heart, and still later, when the sinus venosus becomes a part of the general atrial cavity, into the atrium itself. Near their termination these veins communicate with each other by anastomosing trunks that encircle the future duodenal region of the intestinal tube. As the yolk-sac diminishes in size and importance, the vitelline veins rlecrease in caliber, and the umbilical veins, conveying blood from the allantois and subsequently from the placenta, functionally replace them. The proximal parts of the vitelline veins have an important connection with the circulation of the liver, as will be seen hereafter.

The umbilical veins, which are developed in the mesodermic tissue of the allantois, pass from the placenta along the umbilical cord and, entering the fetal borly at the umbilicus, run at first along the lateral, and later along the ventral, wall of the abdomen toward the heart. Meanwhile there have been established a pair of venous trunks, the primitive jugular or anterior cardinal veins (Fig. 84, A), to return the blood from the head and the upper part of the trunk; and a second pair, the posterior cardinal veins, which bring the blood from the lower part of the trunk, and especially from the primitive kidneys. The primitive jugular vein-which represents the external jugular ${ }^{1}$ of the adult-passing down-

1 According to Salza (observations on guinea-pig) and Mall (observations on human embryo) the external jugular is a secondary vein and the primitive jugular becomes the adult internal jugular vein. 
ward along the dorsal region of the neck, meets the cardinal vein of its own side and unites with it near the heart, the short thick trunk thus formed being the duct of Cuvier. The right and left ducts of Cuvier converge and open together into the sinus venosus (sinus reuniens) of the heart, which also now receives the vitelline veins and the umbilical veins. Upon the development of the upper and the lower limbs, the (posterior) cardinal vein appears as if formed by the confluence of the internal and external iliac veins, while the primitive jugular below the entrance of the subclavian vein is designated, with the duct of Cuvier, the superior vena cava, since, oxving to the preponderance of the jugular over the cardinal vein, the Cuvierian duct appears to be a direct continuation of the jugular. At this time, then, there are two superior venæ cavæ, the terminal parts of which, however, are not exactly symmetrical, since the left passes around the dorsal or posterior wall of the atrium, owing to the rotation of the heart from right to left.

The lower venous trunks likewise present a symmetrical arrangement. The bilateral symmetry of this stage of the venous system, while permanent in fishes, becomes modified in man to produce the familiar asymmetrical condition of the adult venous trunks by two factors principally-first, the development of an unpaired vessel which is to constitute a part of the inferior vena cava, and second, the atrophy of certain vessels and parts of vessels with a consequent diversion of the major part of their blood-stream into other channels. Associated with these alterations is the evolution of a special set of blood-vessels, the portal venous system, for the supply of the developing liver. The development of the portal system, however, may be deferred for separate consideration (see page 177).

When the sinus venosus becomes a part of the atriumconstituting that part of the wall of the adult auricle which is destitute of musculi pectinati-the two ducts of Cuvier, or the superior cavæ, as well as the veins from the abdominal viscera, open by separate orifices into the atrial cavity. An unpaired vessel now develops below the heart in the tissue be- 
tween the primitive kidneys (Fig. 84, 1, 1). This ressel is described as growing downward from the ductus venosus near the point where the latter vessel is joined by the right hepatic veins (p. 180). It is also described (Lewis) as being formed by the enlargement of the right subcardinal vein, the subcardinal veins being themselves produced by longitudinal anastomoses between veins on their way from the mesentery to open into the respective cardinal veins. The vessel in

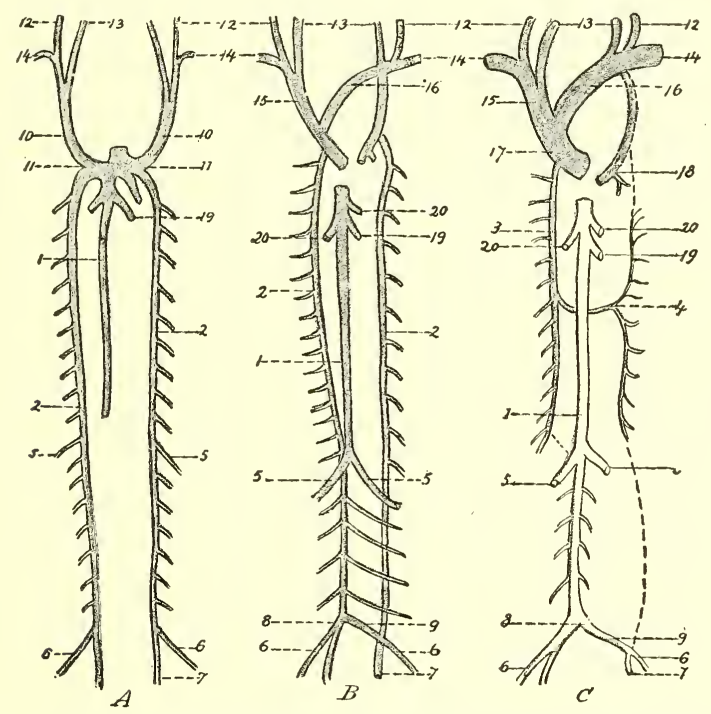

FiG. 84.-Schematic representation of the human venous system, with three successive stages of development (after Hertwig): 1, vena cava inferior; 2, cardinal veins ; 3 , vena azygos major ; 4 , vena azygos minor; 5 , renal veins ; 6 , external iliac vein; 7 , interual iliac vein; 8 and 9 , common iliac veins; 10 , early superior venæ cavæ; 11, ducts of Cuvier; 12, primitive jugular vein ; 13, internal jugular ; 14 , subclavian vein ; 15 and 16 , right and left inmominate veins; 17 , vena cava superior; 18, coronary vein; 19, duct of Arantius ; 20, hepatic veins.

question constitutes the upper or cardiac segment of the inferior vena cava. The lower extremity of this trunk anastomoses by two transverse branches with the right and the left cardinal veins (Fig. 84, B). The cardinal veins of the two sides are further connected by a transverse trunk at their lower extremities and by one that passes across the vertebral column just below the heart. In like manner the two superior venæ cavæ commu- 
nicate with each other by a transverse vessel, the transverse jugular vein, at the upper part of the thorax, above the arch of the aorta. With the exception of the unpaired trunk which is destined to constitute the upper part of the inferior vena cava, the arrangement of the veins at this time is absolutely symmetrical. The apparently meaningless asymmetry of the adult venous trunks is easily accounted for if one notes the alterations in the course of the blood-current which now occur.

The blood-stream of the left superior vena cava gradually becomes entirely diverted into the right cava through the transverse jugular vein, and the part of the left cava below this point, being now functionless, shrivels to an impervious cord (Fig. 84,C). This cord or strand of tissue, the remnant of the left superior cava, is found in postnatal life, in front of the root of the left lung, embedded in a fold of the serous layer of the pericardium, the so-called vestigial fold of Marshall. Since the left superior vena cava receives, near its termination in the auricle, the large coronary vein, which returns the greater part of the blood from the heart-wall, this proximal extremity of the left cava persists as the coronary sinus of the heart. The transverse communicating trunk-the transverse jugular vein-and the part of the left cava above it now constitute the left innominate vein, the course of which from left to right is thus explained. The left superior vena cava of the fetus is represented in the adult, therefore, by the sinus coronarius, by the atrophic impervious cord lying in Marshall's vestigial fold, by the vertical part of the left innominate vein and by a part of the left superior intercostal vein.

The lowest connecting branch between the cardinal veins enlarges and conveys to the right cardinal vein the blood from the left internal and external iliac veins (Fig. 84), in consequence of which the part of the left cardinal vein below the kidney undergoes atrophy and, finally, complete obliteration. The newly-formed transverse trunk is the left common iliac vein. The part of each cardinal vein above the renal region suffers an arrest in growth, in consequence 
of which the blood is diverted from these veins into the transverse anastomosing branches before mentioned as connecting the respective cardinal veins with the lower end of the unpaired caval trunk (Fig. 84, $B$ and $C, 5$ ). As a result, the lower half of the right cardinal vein, now receiving at its distal end the two common iliac veins, becomes directly continuous with the unpaired caval trunk, and with it constitutes the inferior vena cava. The inferior vena cava, therefore, is partly an independently formed structure and is partly the greatly developed lower half of the right cardinal vein. The upper half of the right cardinal vein, conveying now a relatively small part of the blood-stream, becomes the vena azygos major, the termination of which in the superior vena cava is explicable when it is borne in mind that the cardinal and the primitive jugular veins, by their confluence, form the duct of Cuvier.

While no part of the right cardinal vein suffers complete effacement, the left one, in a part of its course, entirely disappears. All the blood of the left external and internal iliac veins being transported to the right side of the body through the lowest transverse trunk-that is, the newly-formed left common iliac vein-the part of the left cardinal vein below the kidney retrogrades and disappears. The part of the left cardinal above the renal region lagging behind in growth, the blood from the left kidney is conveyed to the inferior vena cava by the transverse trunk that connects the carlinal veins in the renal region ; this transverse trunk becomes, therefore, the left renal vein. Since the spermatic veins originally emptied into the cardinal veins, it is found, after these transformations, that the right spermatic opens into the inferior vena cava, while the left spermatic is a tributary of the left renal vein. Some anatomists, indeed, regard the left spermatic vein as the representative of the lower part of the left cardinal vein of the fetus.

As the left renal vein develops into the channel for the major part of the blood from the left kidney, the portion of the left cardinal vein above this point remains an inconspicuous vessel, and that part of it intervening between the 
duct of Cuvier and the cross branch (Fig. 84, C, 4) situated immediately below the heart undergoes total obliteration. The blood ascending through the persisting part of the left cardinal vein must therefore pass across to the upper part of the right cardinal vein, now the vena azygos major; and the pervious portion of the left cardinal vein, with the transverse trunk referred to, constitutes the vena azygos minor.

\section{THE FORMATION OF THE PERICARDIUM, THE PLEURA, AND THE DIAPHRAGM.}

The development of the pericardium is so intimately related with that of the pleuræ and of the diaphragm that an account of it involves a description of the evolution of those structures. By way of facilitating a comprehension of the rather complicated details of the process, the reader is reminder that the tube which constitutes the primitive heart is formed by the coalescence of the two tubes produced within the splanchnic mesoderm, and that this tube and also, for a time, the heart resulting from it, are embedded within the ventral mesentery; and, further, that the part of the ventral mesentery connecting the heart with the ventral
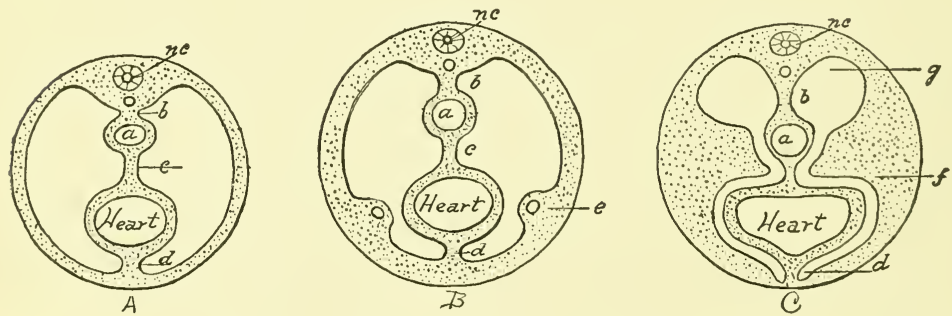

FIG. 85.-Diagrammatic cross-sections of the body of the embryo in the region of the heart at level of future diaphragm: $a$, esophageal segment of gut-tract; $b$, dorsal mesentery; $c$, mesocardium posterius; $d$, mesocardium anterius; $e$, beginning of septum transversum, containing vitelline and allantoic veins; $f$, septum transversum ; $g$, thoracic prolongation of abdominal cavity; $n c$, neural canal.

body-wall is the mesocardium anterius, while the fold passing from the heart to the gut-tract is the mesocardium posterius (Fig. 85, $A$, and Fig. 73, $C$ ). The space between the heart and the body-wall is a part of the body-cavity or coelom (throat-cavity of Kölliker, parietal cavity of $\mathrm{His}$ ). The 
first indication of the separation of this space from the future abdominal cavity is furnished by the appearance of a transverse ridge of tissue growing from the ventral and lateral aspects of the body-wall. This mass is the septum transversum. It bears an important relation to the course of the vitelline and the umbilical veins. As the veins diverge from the body-wall to reach the heart, they carry with them, as it were, the parietal layer of the mesoderm in which they are embedded, forming on each side a fold that projects mesially and dorsally (Fig. 85, $B$ and $C$ ), the two folds approaching and finally meeting with the ventral mesentery in the median plane. The septum transversum thus formed contains in the region nearer the intestine a mass of embryonal connective tissue which is called the liver-ridge or prehepaticus from the fact that the developing liver grows into it. Since the septum transversum, exclusive of the so-called liver-ridge, is the primitive diaphragm, it will be seen that the liver, in the early stages of its growth, is intimately associated with the anlage ${ }^{1}$ of the diaphragm. The septum transversum partially divides the body-cavity into a pericardiothoracic and an abdominal part, as shown in Fig. 85, $B$ and $C$. Near the dorsal wall of the trunk, on each side of the intestine and its mesentery, the septum is wanting, and thus the two spaces communicate with each other by openings that are known as the thoracic prolongations of the abdominal cavity. At this stage, then, the four great serous sacs of the body, the two pleural, the pericardial, and the abdominal, are indicated, but are still in free communication with each other.

The pericardial cavity is the first one of these to be closed off; subsequently the pleural sacs are delimited from the abdominal space. Just as the transverse septum, which partly forms the floor of the thoracic cavity, holds an inportant relation to the course of the vitelline and the umbilical veins on their way to the heart, so is a vertical septum

${ }^{1}$ Anlage, a German word signifying groundwork, or, in embryology, the first crude outline of an organ or part, has come into use in English writings upon the subject because there is no exact English equivalent for it. 
(Fig. 86, $1, b$ ) which separates the pericardial space from the pleural spaces associated with the position of a large vein. This vein, the duct of Cuvier, formed in the upper part of the thorax by the confluence of the cardinal and the jugular veins, lies at first near the dorsal body-wall and then along its lateral aspect. In the latter position it encroaches upon the pleuropericardial space and is covered by the somatic or parietal mesoderm (Fig. 86, A). It is this inwardly project-

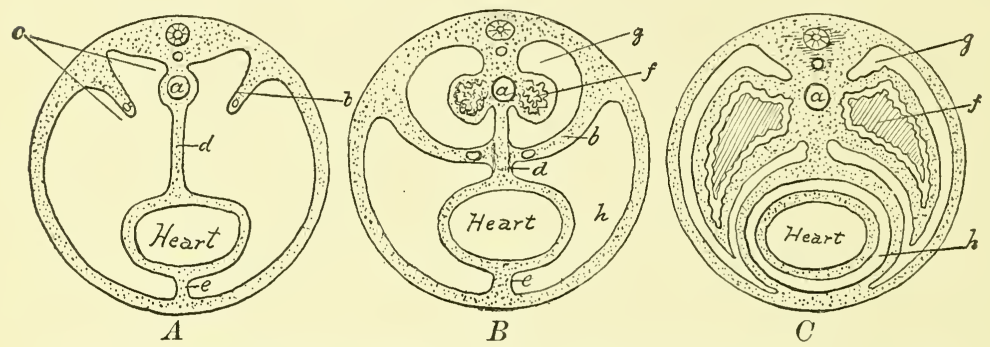

FIG. 86.-Diagrammatic cross-sections of the body of the embryo in the region of the heart entirely above the level of the diaphragm: $a$, esophagus; $b$, pleuropericardial fold containing duct of Cuvier; $c$, pleuropericardial space; $d$, mesocardium posterius ; $e$, mesocardium anterius ; $f$, lung; $g$, pleural cavity; $h$, pericardial cavity.

ing vertical fold of serous membrane containing the duct of Cuvier which constitutes the pleuropericardial fold and the appearance of which initiates the division of the thoracic cavity into two spaces, one for the heart and one for the lungs. The pleuropericardial fold continues to grow toward the median plane of the body until it meets the mesocardium posterius (Fig. 86, B), with which it fuses, thus completing the pericardial sac $(h)$ and isolating it from the pleural space $(g)$.

The heart is still relatively very large and occupies the greater part of the thoracic cavity, leaving only a comparatively small space, situated dorsally, for the accommodation of the developing lungs. This latter space, as previously mentioned, remains for a long time in communication with the abdominal cavity by the two thoracic prolongations of the latter, which lie one on each side of the intestinal tube and its mesentery (Fig. 85, C, and Fig. 86, $1, B$ ). Reference to Fig. 86, $B$, will show that these tube-like spaces are enclosed completely by serous membrane and that they are 
entirely distinct from each other. It is evident also, that the mesial wall of each space is constituted by the mesocardium posterius and the dorsal mesentery. The lungs first appear as two little sacs, connected by a common pedicle, the future trachea, with the upper end of the esophagus. As they grow downward in front of the esophagus and in contact with it, they push the serous membrane before them carrying it away from the esophagus (Fig. 86, B), and thus they acquire an investment of serous membrane, which is the visceral layer of the pleura. The layer of serous membrane in contact with the body-wall is the parietal layer of the pleura. The lower extremities of the lungs at length come into relation with the upper surface of the liver, from which organ they are finally separated by the growth of two folds, the pillars of Uskow, from the dorsolateral region of the body-wall. These folds or ridges project forward and unite with the earlier formed septum transversum to complete the diaphragm. So far, however, the diaphragm is merely connective tissue, the muscular condition being acquired later by the ingrowth of muscular substance from the trunk. Occasionally the dorsal or younger part of the diaphragm fails to unite with the ventral or older fundament on one side of the body, leaving an aperture through which a portion of the intestine may pass into the thoracic cavity. Such a condition constitutes a congenital diaphragmatic hernia.

The heart and its pericardial sac occupy the greater part of the thoracic cavity, while the lungs are merely narrow elongated organs lying in the dorsal part of this space as shown in Fig. 86, $B$. As the lungs increase in diameter, they spread out ventrally and gradually displace the parietal layer of the pericardium (Fig. 86, $B$ ) from the lateral wall of the chest, crowding the pericardium forward and toward the median plane of the body (see Fig. 86, $C$ ) until finally the adult relationship of these structures is established.

\section{THE PORTAL CIRCULATION.}

The circulation of the ardult liver is peculiar in that the organ is supplied not only with arterial blood for its nutrition 
but receives also venous blood larlen with certain products of digestion obtained from the alimentary tract, the spleen, and the pancreats. This renous blood cinters the liver through the portal vein and is designed to supply to the gland the materials for the performance of its special functions.
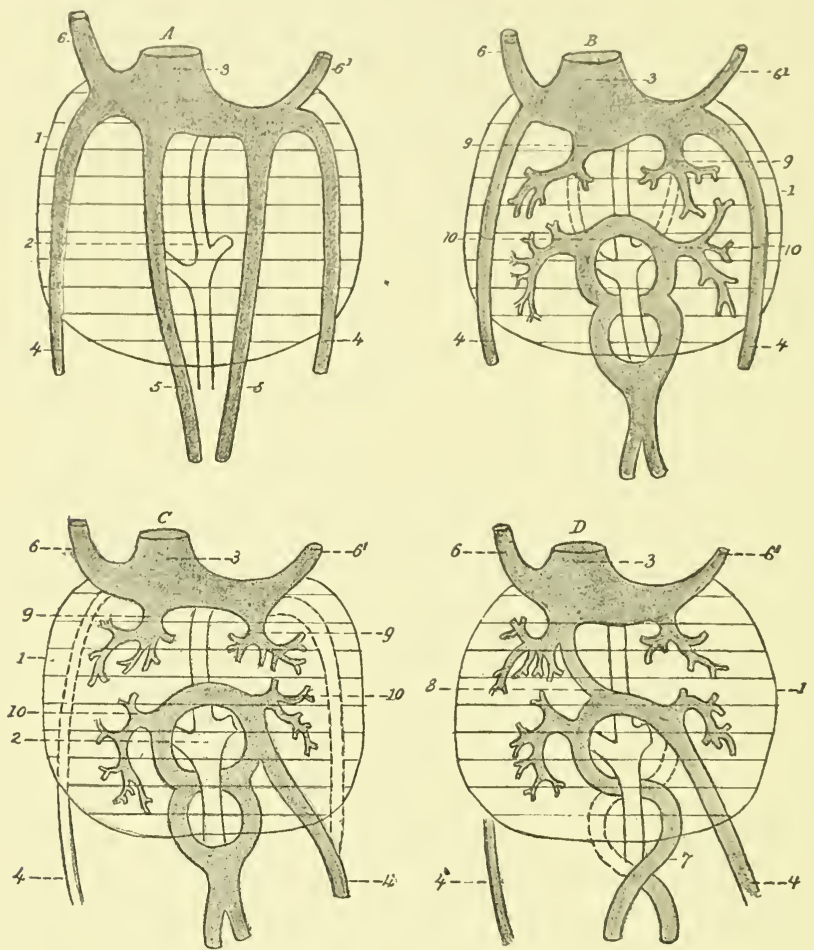

FIG. 87.-Four successive stages in the development of the portal venous system (from Tourneux, after His) : 1, outline of liver; 2 , duodenum ; 3 , sinus venosus; 4,4 , umbilical veins; $5,5, A$, vitelline veins, which in $B$ and $C$ are connected by the annular sinus; 6 , superior vena cava: 6 , coronary vein; 7 , portal vein; 8 , ductus venosus; 9,9 , venæ hepaticæ revehentes; 10 , 10, venæ hepaticie advehentes.

As might be expected from the fact that the liver is an appendage of, and a direct outgrowth from, the intestinal anal, it receives its blood-supply, in the early stages of its development, from the ressels that supply the primitive intestine, that is, from the vitelline veins. These veins, on their way to the heart, pass along the intestinal eanal and are connected with each other in the region of the future 
duodenum by trunks that encircle the bowel, these connecting vessels collectively constituting the annular sinus (Fig. 87, $B$ and $(C)$. The liver originates from a small diverticulum which is evaginated from the ventral wall of the intestinal canal. Growing forward between the folds of the ventral mesentery, this little tubular sac divides and subdivides so as to produce a gland of the componnd tubular type. The developing liver is from the first in close relation with the vitelline veins and their ring-like anastomosing branches, and reeeives its blood-supply from the latter through vessels that are known as the venæ hepaticæ advehentes (Fig. 87, 10, 10). These afferent vessels break up within the liver into a system of capillaries, from which the blood passes through the efferent vessels, the venæ hepaticæ revehentes, into the terminal parts of the vitelline veins. Thus a part of the blood of the vitelline veins is diverted to the liver and, after circulating through that organ, is returned to them further on to be conveyed to the heart. As the liver, with its increasing development, requires more and more blood, the entire blood-stream of the vitelline veins passes to it, and the parts of these veins between the venæe hepatice advehentes and the venæ hepatice revehentes become obliterated (Fig. 87, $B$ and $C$ ). The vitelline veins, therefore, leave the intestinal canal at the duodenal region and traverse the liver on their way to the heart. In this early stage of the development of the liver, then, it receives its nutrition from the yolk-sac, through the vitelline veins.

When the yolk-sack undergoes retrogression, as it does about the fifth week, the liver must draw upon the allantoic and the placental vessels for its nutrition. To do this it must acquire connection with the umbilical veins. The latter vessels pass upward from the umbilicus along the rentral wall of the body and empty into the sinus venosus of the heart above the site of the liver (Fig. 87, 1, 4, 4). The umbilical veins effect communications beneath the liver with the vene hepatice advehentes from the vitelline veins. At about this time the left umbilieal vein begins to predominate over the right one, the latter retrograding until, in the umbilical 
cord, it disappears; the portion of the right vein contained within the abdomen loses its connection with the left at the umbilicus and becomes a vein of the ventral abdominal wall, the left vein henceforth conveying all the blood from the placenta. As the needs of the liver exceed the capacity of the vitelline veins, more and more of the blood of the umbilical vein is sent to it, until finally all the blood of the latter vein passes into the liver, from which it emerges through the venæ hepaticæ revehentes, and reaches the heart through the terminal part of the left vitelline vein. (The left vitelline vein very early begins to predominate orer the right.) The part of the umbilical vein above the liver undergoes atrophy and disappears (Fig. 87). Although, mean while, the yolk-sach as dwindled, the vitelline veins persist, in part, since they receive blood from the walls of the alimentary tract. The liver now, in this second stage of its development, receives blood from two sources, the abdominal viscera and the placenta.

At the time when the right umbilical and right vitelline veins become incorporated in a common trunk, there grows from this trunk, downward and to the left, a vessel which connects with the left vitelline vein beneatl the liver at its point of communication with the (left) umbilical vein. This trunk later becomes the ductus venosus or ductus Arantii. As previously indicated, the proximal half of the inferior vena cava develops as an unpaired vessel connected with the primitive heart. According to the view referred to on p. 171 it results from the enlargement of the right subcardinal vein, which then opens into the upper end of the ductus venosus. As both the right umbilical and vitelline veins are in process of retrogression, and as the enlarged subcardinal, now the upper half of the inferior vena cava, ontstrips the ductus venosus in growth, it soon comes about that the common termination of the right umbilical and vitelline veins is insorporated in the cava, and the latter therefore acquires an opening into the sinus venosus of the heart. The left vitelline vein, until this time a vessel of importance opening into the sinus venosus, must likewise become included at its orifice in 
the cava, whereby its tributaries, the venæ hepaticæ revehentes, come to empty into the cava, the downward growth of the latter carrying downward likewise the terminations of these veins to their normal position as the hepatic veins emerging from the dorsal surface of the liver. Meanwhile the volume of blood flowing through the umbilical vein has increased to such an extent that the liver is no longer able to transmit it to the inferior vena cava, and consequently a part of this blood passes through the ductus venosus, which extends from the portal fissure, along the dorsal surface of the liver. The blood of the umbilical vein is divided, therefore, into two streams-one that enters the inferior vena cava directly through the ductus venosus and one that traverses the liver on its way to the cava.

The portal vein results from the persistence of a part of the vitelline veins. The vitelline veins, as we have seen, anastomose with each other by two ring-like branches that encircle the duodenum. The right half of the lower ring and the left half of the upper one atrophy, so that the blood of the vitelline veins makes its way to the liver through the left half of the lower ring and the right half of the upper one (Fig. 87, D). The left half of the lower ring and the now united portions of the right and left vitelline veins immediately below constitute the superior mesenteric vein, which passes in front of the third part of the duodenum, as in the adult, and which is later joined by the splenic vein; while the anastomosing portion of the loop and the right half of the upper loop become the portal vein. So long as the yolksac is present, the vein receives blood both from it and from the walls of the intestine. After the disappearance of the yolk-sac, the intestinal and the visceral veins are the sole tributaries of the portal vein.

\section{THE FINAL STAGE OF THE FETAL VASCULAR SYSTEM.}

The circulation of the fetus at birth and the changes ensuing immediately thereafter may now be easily understood. The fetal blood being sent to the placenta through the hypogastric or umbilical arteries, receives oxygen there and is 

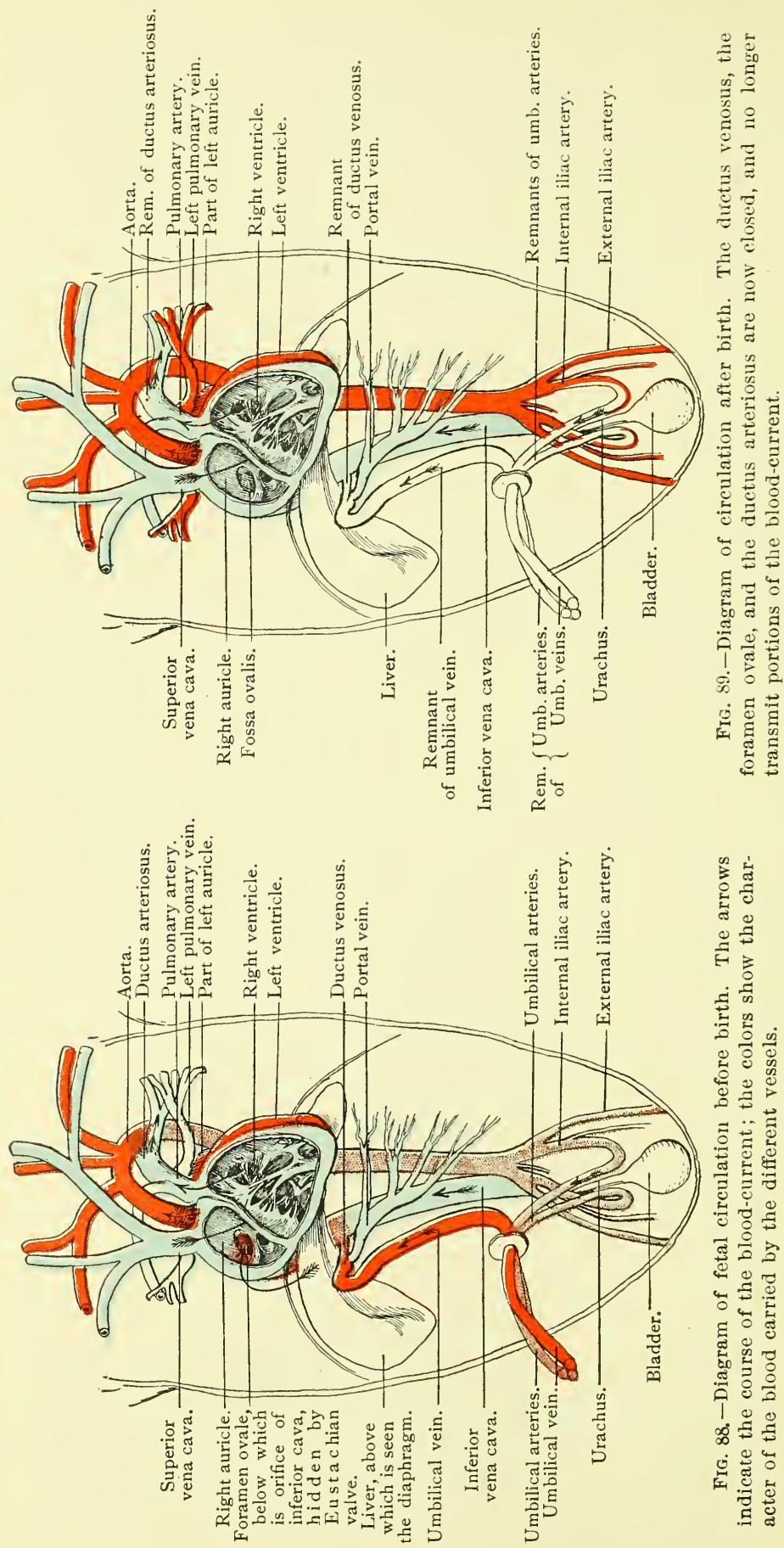

点 ส ๖ $\mathrm{E}$ टं क्ष . 은 $\frac{0}{8}$ \&્ટ 픙 离总总 己

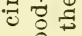
余㝴 ¿ 0 4 द्व 荧 0 ฮั 1. 它造总 
returned to the body of the fetus through the umbilical vein. The latter vessel takes its course upward along the ventral wall of the abdomen to the under surface of the liver, lying here in the anterior part of the longitudinal fissure. In this position the blood-stream of the umbilical vein is divided into two parts, one of which unites with the fetal portal vein to enter the liver, while the other passes through the ductus venosus directly to the inferior vena cava. The blood which enters the liver, after traversing that organ, reaches the inferior vena cava through the hepatic veins. Thus, in the one case directly, in the other case by passing through the liver, all the placental blood reaches the inferior vena cava and passes on to the right auricle of the heart.

From the right auricle the blood passes through the foramen ovale to the left auricle, and thence, through the mitral orifice, to the left ventricle. Being driven from the left ventricle into the aorta, it is conveyed through the branches of the aortic arch to the head and the upper extremities. Finding its way into the veins of these parts, it is returned, through the superior vena cava, to the right auricle, from which cavity it passes, through the tricuspid orifice, into the right ventricle. From the right ventricle it goes into the pulmonary artery. Since the lungs are not as yet pervious, or but very slightly so, the current is deflected almost entirely through the ductus arteriosus to the descending aorta instead of going to the lungs. Some of the blood of the descending aorta is distributed to the various parts of the body below the position of the heart, while some of it is sent through the hypogastric or umbilical arteries to the placenta for aëration. It is evident that no part of the fetal blood, except that in the umbilical vein, is entirely pure, the venous and the arterial blood being always more or less mixed.

With the detachment of the placenta at birth, several marked alterations occur. The circulation through the umbilical vein ceases, that part of this vessel which intervenes between the umbilicus and the portal fissure of the liver becoming, in consequence, an impervious fibrous cord, 
the round ligament of the liver. The ductus renosus likewise suffers obliteration, becoming the ligamentum venosum Arantii. Since the lungs now assume their proper function of respiration, the communication between the right and the left sides of the heart and also that between the pulmonary artery and the aorta cease. Hence, the respective avenues for these communications, the foramen ovale and the ductus arteriosus, become obsolete. There being no further need for the hypogastric (umbilical) arteries, the circulation through them ceases, and they become mere cords of fibrous tissue, whowe presence is evidenced by two ridges in the peritoneum on the inner surface of the anterior wall of the abdomen. The proximal parts of these arteries persist, however, as the superior vesical arteries. 


\section{CHAPTER XI.}

\section{THE DEVELOPMENT OF THE DIGESTIVE SYSTEM.}

THE adult digestive system consists of the mouth with its accessory organs, the teeth, the tongue, and the salivary glands; of the pharynx, the esophagus, the stomach, and the small and the large intestine, including also the important glandular organs, the liver and the pancreas. Notwithstanding the apparent complexity of its structure, the alimentary tract may be regarded as a tube, certain regions of which have become specialized in order to adapt them to the performance of their respective functions, the salivary glands, the liver, and the pancreas being highly differentiated evaginations of its walls. While in man and in the higher vertebrates the tube is thrown into coils by reason of its excessive length, in the lower-type animals it is much more simple in its arrangement. For example, in certain fishes and in some amphibians the alimentary tract has the form of a slightly flexuous tube, the deviations from the simple straight canal being few and insignificant, and the stomach being represented by a local dilatation of the tube.

The simple condition obtaining in the representatives of the animal kingdom referred to above suggests the likewise simple fundamental plan of the human embryonic gut-tract. There is, in fact, a period in development when the gut-tract of the human embryo has the form of a simple straight tube. The processes-incident to the formation of this tube mark the earliest stages of the development of the alimentary system, the tube itself acquiring definite form simultaneously with the production of the body of the embryo.

The first indication of the alimentary eanal appears at a very early period of development, being inaugurated in fact by those important alterations that serve to differentiate the 
blastodermic vesicle into the body of the embryo and the embryonic appendages. It will be remembered that, after the splitting of the parietal plate of the mesoderm into its two lamellæ, and the union of the outer of the layers with the ectoderm and of the inner with the entoderm to form respectively the somatopleure and the splanchnopleure, these two double-layered sheets undergo folding in different directions. Before the folding occurs, the germ is a hollow sphere whose cavity is the archenteron and whose walls are the somatopleure and the splanchnopleure. While the somatopleure in a zone corresponding with the margin of the embryonic area becomes depressed and is carried under that area to form the lateral and ventral body-wall of the enibryo (Plate II., Figs. 2, 3, and 4), and also more distally folds up over the area to produce the amnion and the false amnion, the splanchnopleure, likewise in a line corresponding with the periphery of the embryonic area, is depressed and carried inward from all sides toward the position of the future umbilicus. This folding in of the splanchnopleure effects the division of the archenteron into two parts, a smaller cavity falling within the body of the embryo, which latter is forming at the same time, and a larger extra-embryonic compartment, which is the yolk-sac or umbilical vesicle. The intra-embryonic cavity is the gut-tract. The constricted communication between the two is the vitelline duct. While the vitelline duct is still a rather wide aperture, the anterior and posterior parts of its intestinal orifice are designated respectively the anterior and the posterior intestinal portals.

As the somatopleure closes in around the vitelline duct, it forms the wall of the abdomen, the opening left, which is traversed by the duct, being the umbilical aperture.

It is evident therefore that the lining of the gut-tract is constituted by the innermost germ-layer, the entoderm, and that all its epithelial elements are consequently of entodermic origin. The folding in of the splanchnopleure begins at about the end of the second week, and is so far advanced

${ }^{1}$ Strictly speaking, the somatopleure and the splanchnopleure are not formed before the folding occurs, but the processes go on at the same time. 
A

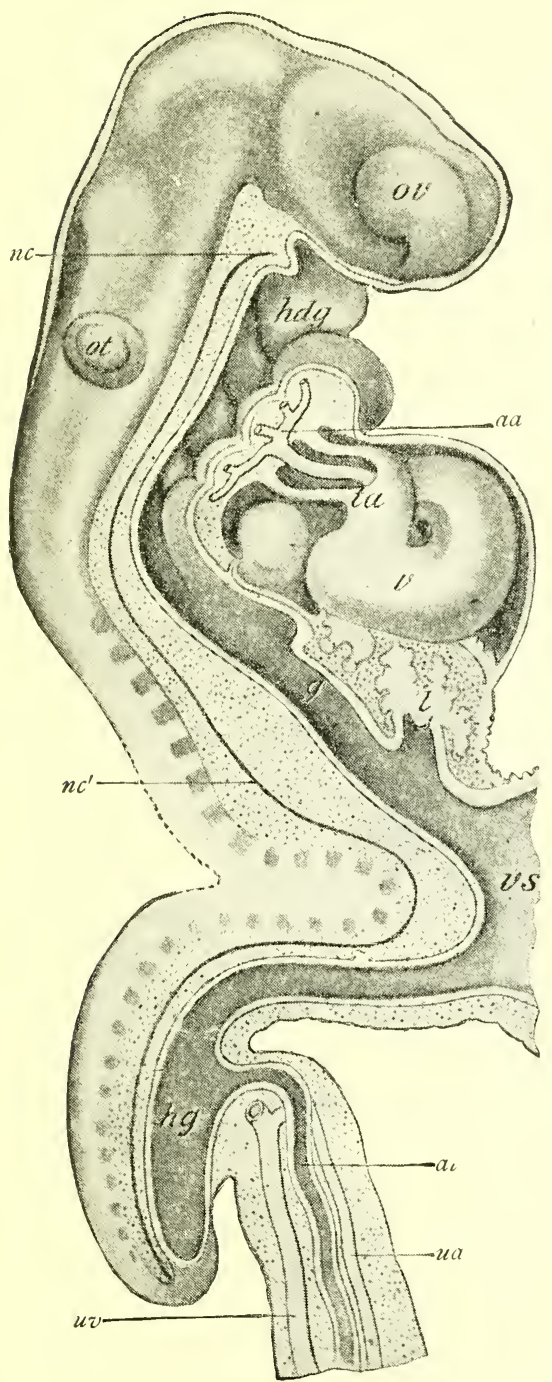

B

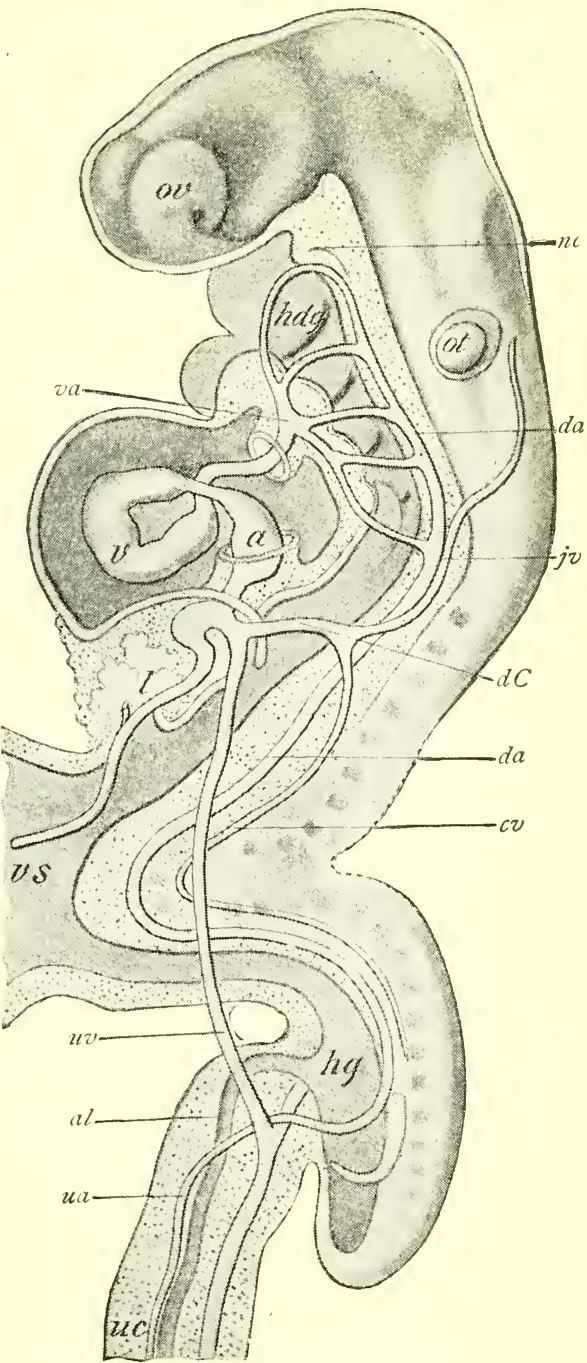

FIG. 90.-Reconstructions of human embryo of about seventeen davs (His) : ov, optic and ot, otic vesicles : $n c, n c^{\prime}$, notochord: $h d g$, head-gut ; $q$, mid-gut ; $h g$, hindgut; $v s$, vitelline sac; $l$, liver: $v$, $t a$, primitive ventricle and truncus arteriosus; $v a, d a$, ventral and dorsal aortæ; $a a$, aortic arches ; $j v$, primitive jugular vein ; $c v$, cardinal vein; $d C$, duct of Cuvier; $u v, u a$, umbilical vein and artery ; $a l$, allantois: $u c$, umbilical cord.

before the end of the third week that the archenteron is definitely divided into the gut-tract and the yolk-sac. 
In its earliest definite form, then, the gut-tract is a tube extending from one end of the embryonic body to the other, which opens widely at the middle of its rentral aspect into the vitelline duct, but which is closed at both ends. It is usual to speak of the primitive gut-tract as consisting morphologically of three parts, the head-gut, which is the region on the headward side of the orifice of the vitelline duct; the hind-gut, which is the part near the tail-end of the embryo; and the mid-gut or intervening third portion (Fig. 90).

The closed hear-end of the gut-tube corresponds with the floor of the primitive mouth-cavity, the two spaces being separated by a thin veil of tissue, which consists of the entoderm and the ectoderm and is called the pharyngeal membrane (Fig. 91). A considerable proportion of the so-

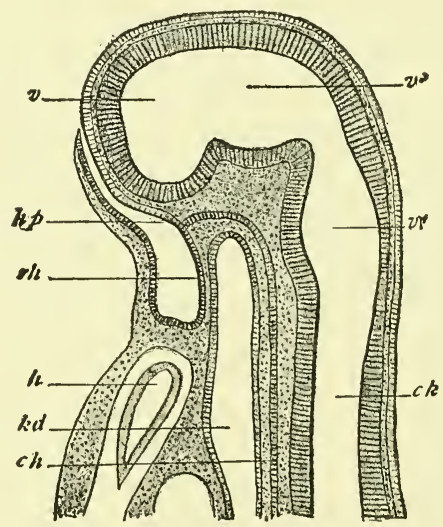

Fig. 91 -Median seetion through the head of an embryo rabbit $6 \mathrm{~mm}$. long (after Mihalkovies): $r h$, membrane between stomodæum and fore-gut, pharyngeal membrane (Rachenhaut); $h p$, place from which the hypophysis is developed; $h$, heart; $k d$, lumen of fore-gut; $c h$, ehorda; $v$, ventriele of the eerebrum; $v^{3}$, third ventricle, that of the between-brain (thalamencephalon); $v^{4}$, fourth ventriele, that of the hind-brain and after-brain (epencephalon and metencephalon, or medulla oblongata); $c k$, ecntral canal of the spinal cord.

called head-gut constitutes the primitive pharynx. This region of the tube has a relatively large caliber, and presents on its lateral and ventral walls the series of recesses or evaginations known as the throat-pockets or pharyngeal pouches (Fig. 71). 
While the inner, entodermic layer of the gut-tube becomes the intestinal mucosa, the outer, mesodermic stratum produces the muscular and the connective-tissue parts of the bowelwall, the most superficial layer of the latter with its mesothelial or endothelial cells forming the visceral layer of the peritoneum. Since the mesodermic layer of the splanchnopleure of each side is continuous with the corresponding mesodermic layer of the somatopleure on either side of the embryonic axis, the primitive intestinal canal has a broad area of attachment with the dorsal wall of the body-cavity (Fig. 92). The ventral wall is likewise connected with the

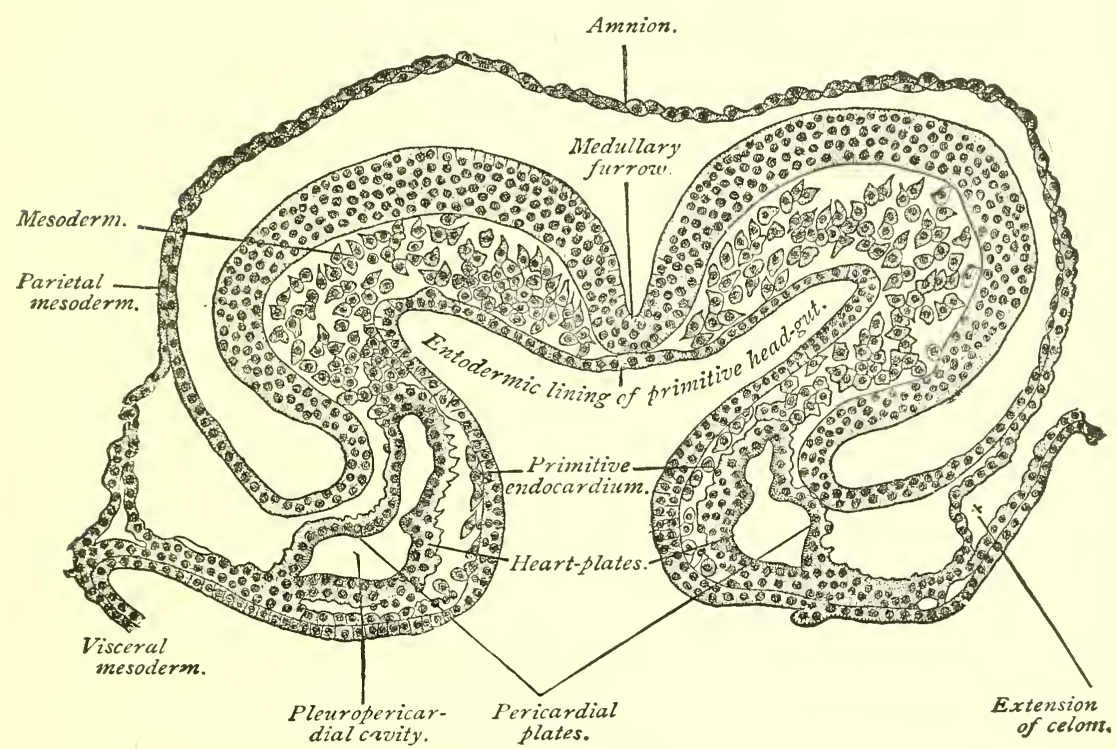

FIG. 92.-Transverse section of a sixteen-and-a-half-day sheep-embryo (Bonnet).

ventral body-wall throughout the anterior or upper part of its extent by the continuity of the splanchnopleuric mesoderm of each side with the somatopleuric mesoderm of the same side. As development advanees, the body-cavity increases in caliber more rapidly than does the intestinal tube, so that the interval between the two is augmented, in consequence of which the masses of connective tissue uniting the dorsal and the ventral surfaces of the gut with the corre- 
sponding walls of the body-cavity become drawn out so as to constitute in each case a median vertical fold consisting of two closely approximated layers of serous membrane with a little connective tissue between them. These folds are the dorsal and the ventral mesenteries (Fig. 93). While the
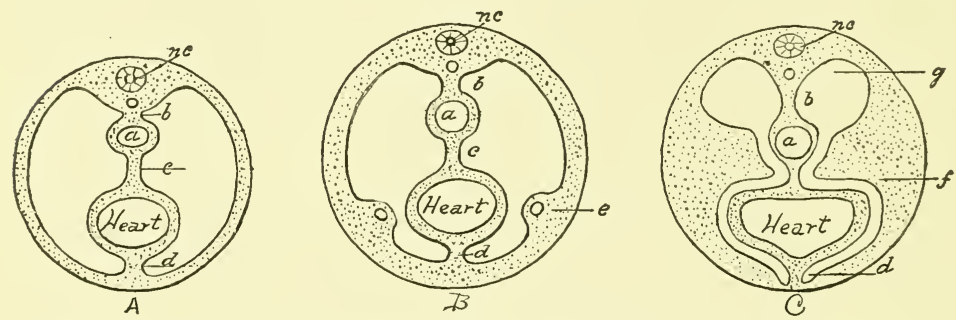

FIG. 93.-Diagrammatic cross-sections of the body of the embryo in the region of the heart at level of future diaphragm : $a$, esophageal segment of gut-tract; $b$, dorsal mesentery; $c$, mesocardium posterius; $d$, mesocardium anterius; $e$, beginning of septum transversum, containing vitelline and allantoic veins; $f$, septum transversum ; $g$, thoracic prolongation of abdominal cavity; $n c$, neural canal.

dorsal mesentery extends throughout the entire length of the canal, the ventral fold is present only at its anterior or upper part, corresponding in the extent of its attachment to the digestive tube to that portion representing the future stomach and upper part of the duodenum (Fig. 94). The ventral mesentery at first is present throughout the entire extent of the canal, but very carly undergoes obliteration except in the situation above noted. Concerning the reason and the method of its disappearance nothing is definitely known.

The intestinal tube, at a comparatively early stage, presents on its ventral surface near the posterior or caudal end a small eragination that enlarges to form the allantois (see p. 89). While a part of the intra-embryonic portion of the allantois dilates and develops into the bladder, the part between this latter and the intestine is known as the urogenital sinus. The part of the gut-tube posterior to, caudad of, the origin of the allantois, is a blind ponch known as the cloaca. The latter is, therefore, the common termination of the urinary and the intestinal tracts.

To repeat, we have now, in the third week of development, the alimentary canal represented by a single straight tube 
(compare Fig. 90), closed at each end, but with mouth-cavity and anus both indicated, the tube lying within a larger tube, the body-cavity, with the walls of which latter it is connected by the dorsal and ventral mesenteries. Along the dorsal wall

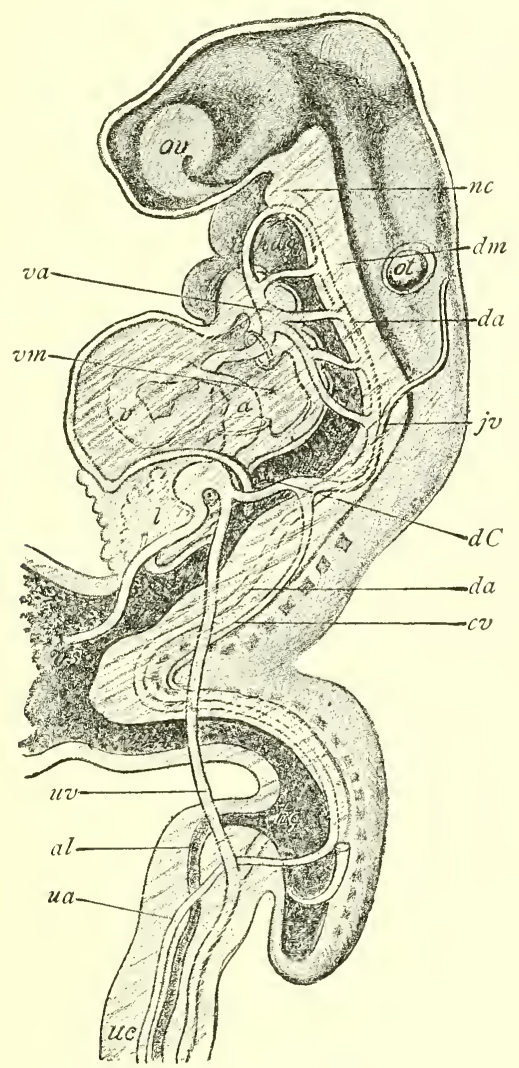

Fig. 94.-Reconstruction of human embryo of about seventeen days (after His) : $o v$, optic and ot, otic vesicles; $n c$, notochord: $h d g$, head-gut; $g$, mid-gut; $h g$, hindgut; $v s$, vitelline sac; $l$, liver; $v$, primitive ventricle; $v a$, $d a$, ventral and dorsal aortæ; $j v$, primitive jugular vein; $c v$, cardinal vein; $d C$, duct of Cuvier; $u v, u a$, umbilical vein and artery ; $a l$, allantois ; $u s$, umbilical cord ; $d m$, dorsal mescntery ; $v m$, ventral mesentery (modified from His).

of the body-cavity, dorsad to the parietal peritoneum, pass the two primitive aortre, and later, the single aorta which results from the fusion of these two. Between the two folds of the dorsal mesentery pass the blood-vessels that nourish the walls of the gut. Within the ventral mesentery are the 
vitelline veins, which bring the blood from the yolk-sac and convey it to the primitive heart. On the ventral wall of the gut is the wide aperture of the vitelline duct. Farther caudad, also on the ventral surface of the bowel, is the orifice of the allantois. These conditions may be better understood by reference to Figs. 90 and 94. Before tracing the further development of the abdominal part of the alimentary system, it will be proper to note certain very important processes pertaining to its anterior or head-extremity, and also to consider the formation of the anus.

\section{THE MOUTH.}

The development of the mouth, the tongue, the teeth, and the salivary glands has been fully described on pages 134143. In this connection, therefore, it will be necessary to call attention to only a few of the salient features of their evolution.

The oral cavity is produced by a folding in of the surfaceectoderm, the fossa thus formed becoming deeper until it meets the head-end of the gut-tract. From the walls of this fossa the salivary glands are developed as evaginations, in the manner already described, while the teeth are specialized growths of its ectodermal lining and of the underlying mesoderm (vide p. 137). The first intimation of this infolding is apparent at the twelfth day in the form of a localized thickening of the surface-cells on the ventral surface of the body of the embryo near the head-end. The thickened area is the oral plate, which speedily becomes depressed, producing the oral pit or fossa. By the third week, the oral fossa or stomodeum is a well-marked pit of pentagonal outline, its boundaries being the nasofrontal process above, the maxillary processes laterally, and the mandibular arches below. The original oral plate, having receded farther and farther from the surface and forming the posterior limit of the mouthcavity, now separates that cavity from the pharyngeal region of the gut-tube and comes into contact with the anterior wall of the latter. It is called the pharyngeal membrane (Fig. 91). Its disappearance occurs at some time during the fourth week, 
by which event the gut-tube is brought into communication with the mouth.

The exact position of the pharyngeal membrane is not easily definable. It is certain, however, that it falls farther back than the posterior limit of the adult oral cavity, since the primitive mouth includes the anterior part of the adult pharynx. For example, the diverticulum that gives rise to the anterior lobe of the pituitary body belongs to the primitive mouth, yet its vestige, the pharyngeal bursal ${ }^{1}$ or Rathké's pocket, is found in the pharynx of the adult. The primitive oral cavity, by the growth of the palate, becomes divided into the adult mouth and the nasal cavities. The hard palate is completed in the ninth week and the soft palate in the eleventh week.

\section{THE PHARYNX.}

The pharynx is represented in the embryo by the expanded cephalic end of the primitive gut-tract. It is of greater relative length in the earlier stages of development than later, including as it does, almost half the length of the gut-tube in the fourth and fifth weeks. The primitive pharyngeal cavity is widest at its anterior or cephalic extremity and narrowest at the opposite end, tapering here into the esophagus. Until the breaking down of the pharyngeal membrane, which takes place in the fourth week, this structure marks the anterior limit of the pharynx and separates it from the oral cavity.

The pharyngeal pouches or throat-pockets have been referred to in connection with the visceral arches on page 111. They are out-pocketings or evaginations of the entodermal lining of the pharynx, there being four furrows on each lateral wall, and they pass from the ventral toward the dorsal wall of the cavity, each pouch lying between two adjacent visceral arches. The entoderm of the pouches comes into close relation with the ectoderm of the outer visceral furrows (Fig. 71). The mesoflermic stratum being

${ }^{1}$ It has been shown recently (Killian) that the pharyngeal bursa is not identical with Rathkés pocket, but is an independently formed evagination. 
absent from the pharyngeal pouches, the ectoderm and the entoderm are in contact, and constitute the closing membrane. As previously mentioned, this closing membrane ruptures in aquatic vertebrates, in consequence of which the pharyngeal cavity in such animals acquires a number of openings. In man, as in other mammals, and in birds, such rupture probably never occurs. Since the visceral arches and clefts are fully considered in Chapter VII., it will be necessary in this connection to refer only to such derivatives of them as pertain directly to the pharynx.

The first inner cleft or pharyngeal pouch becomes closed off from the pharyngeal cavity, its dorsal end giving rise to the tympanic cavity or middle ear, while the remaining part constitutes the Eustachian tube. Hence, the tympanum and the Eustachian tube are to be regarded as differentiated portions of the primitive pharyngeal cavity. The dorsal part of the closing membrane of this cleft persists as the tympanic membrane.

The third pharyngeal pocket or third inner visceral cleft, by an evagination of its entodermal epithelium, gives rise to the epithelial parts of the thymus body, the connectivetissue elements of this "gland" being furnished by the mesodermic cells which surround the epithelial diverticulum and ultimately enclose and isolate its branching processes. In a similar manner, the fourth pocket produces the lateral lobes of the thyroid body.

The ventral wall of the pharynx, between the anterior extremities of the second throat-pockets, evaginates into an entodermic tube which extends caudalward to develop subsequently into the median lobe of the thyroid body.

The tongue (p. 143) also is developed from the walls of the pharynx, the anterior unpaired segment, the tuberculum impar, growing from the median line of the ventral wall, just below the level of the first visceral arch, while the two symmetrical segments that form the posterior third of the organ proceed from the ventrolateral walls at the ventral extremities of the second and third visceral arches.

The tonsil develops as masses of lymphoid tissue about 
an evagination of the lateral wall of the pharynx. In the third month the lateral pharyngeal wall pouches out to form a little fossa (Fig. 95, 1) which is situated between the second and third visceral arches, the fossa being lined with stratified squamous epithelium continnous with that of the pharyngeal cavity. Little solid epithelial buds (Fig. 95) proceed from this diverticulum into the surrounding connective tissue, the buds subsequently becoming hollowed out. Wandering leukocytes from the neighboring blood-vesselsor, according to some author-

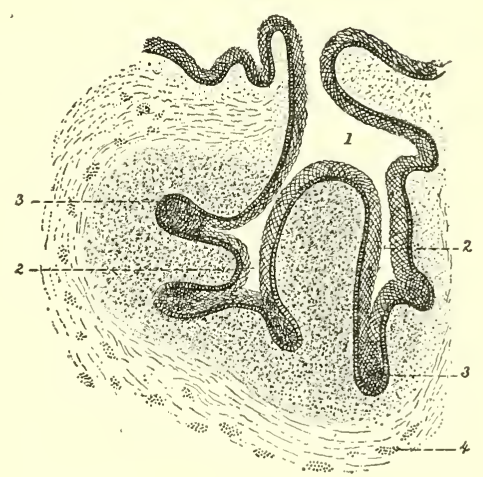

Fig. 95.-Section through anlage of tonsil of a human fetus (Tourneux) : 1 , tonsillar pit, continuous with mouthcavity ; 2 , secondary diverticula; 3 , solid epithelial buds ; 4 , striped muscular fiber. ities, from the mesenchyme cells or from epithelial sourcesinfiltrate the connective tissue around the young erypts, and these cells becoming aggregated into condensed and isolated groups give rise to the lymphoid follicles peculiar to the tonsil. The separate and well-differentiated condition of the follicles is not attained until some months after birth. The place of origin of the tonsil between the second and third visceral arches explains the position of the adult organ between the anterior and posterior palatine arches, since the latter structures represent the deep extremities of the former.

\section{THE ANUS.}

The early stages of the development of the anus are similar to those of the mouth. The so-called anal membrane is produced by the growing together of the ectoderm and the entoderm, the mesoderm being crowded aside. The site of the anal membrane, or anal plate, is in the median line of the dorsal surface of the embryonic body, at its posterior or caudal extremity. It makes its appearance in the third week. Since the tissue immediately in front-that is, headward, of the anal plate projects and develops into the primi- 
tive tail, and since the axis of the body becomes ventrally curved, the anal plate is carried around somewhat toward the ventral aspect of the body. During the following fortnight the anal plate becomes depressed so as to form a small fossa, which is often designated the anal pit or proctodeum. The position of the anal pit does not correspond, in any vertebrate, to the end of the intestine, but to a point short of it ; the gut, therefore, extends beyond the position of the anus. This portion of the bowel is the postanal gut of vertebrate morphology. Ultimately it entirely disappears.

While the anal pit is forming, the allantois is growing forth as a diverticulum from the ventral wall of the gut (Fig. 96). The intra-embryonic part of the allantois is

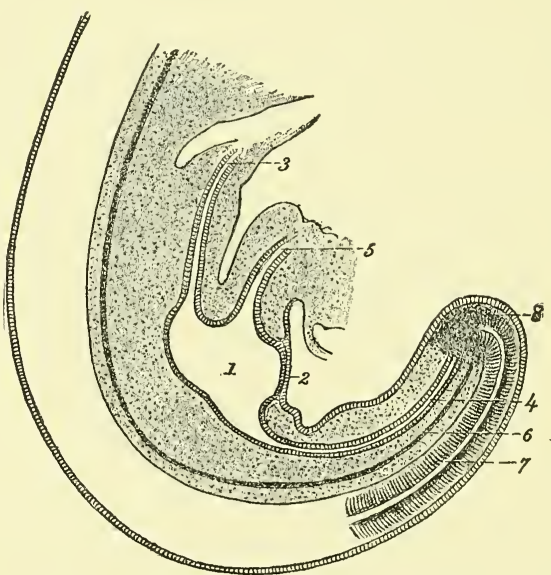

FIG. 96.-Sagittal section of caudal extremity of cat embryo of $6 \mathrm{~mm}$. : 1, cloaca ; 2, cloacal membrane; 3 , intestine; 4 , post-anal gut; 5 , allantoic canal; 6 , chorda dorsalis; 7, medullary canal (Tourneux).

transformed chiefly into the urinary bladder, but it gives rise also, by its proximal extremity, to a short wide duct, the urogenital sinus, which is an avenue of communication with the bowel. The part of the gut on the caudal side of the aperture of the urogenital sinus is the cloaca, which is the common termination, therefore, of the genito-urinary system and of the intestinal canal.

The surface depression referred to above as the anal pit is 
often called the cloacal depression during the time that the cloaca is present. In the lowest mammals, the monotremes, as also in the Amphibia, in reptiles, and in birds, the cloaca is a permanent structure. By the breaking down of the membrane between it and the cloacal depression, it acquires an outlet, through which the feces, the urine, and the genital products find egress. In all higher mammals, however, including man, the cloaca suffers division into an anterior or ventral passage-way, the urogenital sinus, and a posterior canal, the rectum and canal of the anus. This division is effected by the growth of three ridges or folds, of which one grows from the point of union of the urogenital sinus and the gut, while the other two proceed, one from each lateral wall of the cloaca. The three folds coalesce to form a perfect septum. The division is complete at about the end of the second month (or, according to Minot, at the fourteenth week). The cloacal depression or anal pit shares in this division, so that at about the tenth week, it is separated into the anal pit proper, or the proctodeum, and the orifice of the urogenital sinus. The newly-formed septum continues to thicken, especially near the surface of the body, until it constitutes the pyramidal mass of tissue known as the perineal body, or perineum.

The anal pit deepens, the anal membrane being thereby approximated to the end of the bowel, and in the fourth month the anal membrane breaks down and disappears. Persistence of the anal membrane after birth constitutes the anomaly known as imperforate anus.

\section{THE DIFFERENTIATION OF THE ALIMENTARY CANAL. INTO SEPARATE REGIONS.}

The fourth week marks the beginning of certain important changes in the simple straight alimentary tube. 'The reader is again reminded that this tube is connected with the dorsal body-wall by the dorsal mesentery and with the ventral wall, for a part of its extent, by the ventral mesentery; that the canal is, as yet, without communication with the exterior; and also that the vitelline duct and the allan- 
tois are connected with its ventral surface (Fig. 94). The umbilical vesicle having reached the limit of its development in the fourth week and having begun to shrink, the vitelline duct likewise begins to retrograde and very soon becomes an inconspicuous structure.

The dorsal wall of the tube at a point nearer the head-end begins to bulge toward the dorsal body-wall, forming a somewhat spindle-shaped enlargement (Figs. 97, 98). This di-

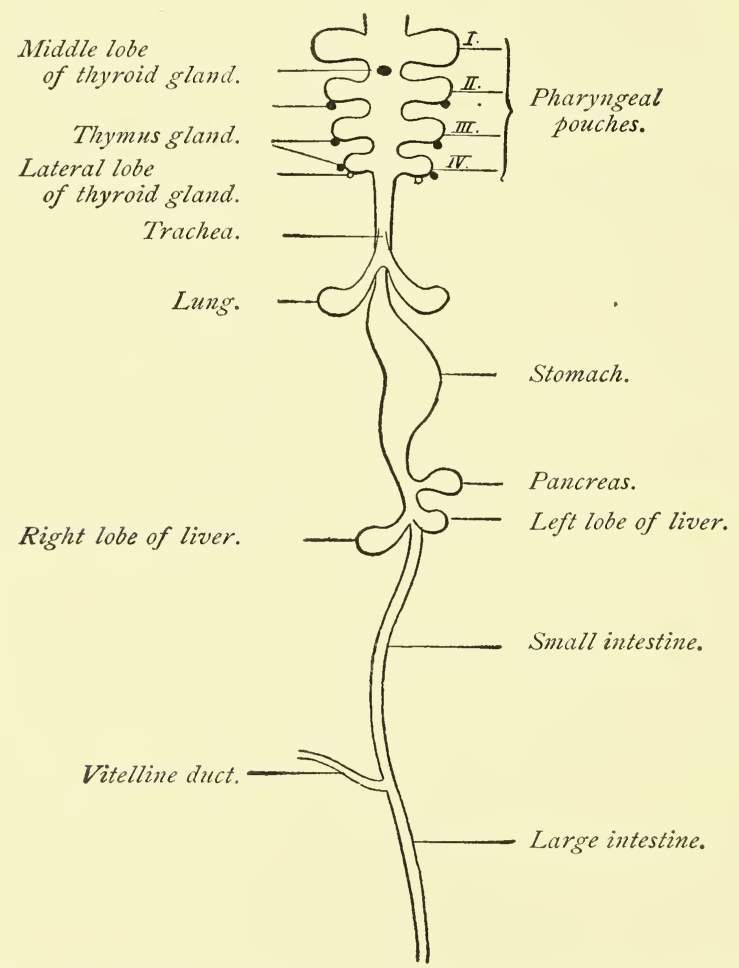

FIG. 97.-Scheme of the alimentary canal and its accessory organs (Bonnet).

latation is the beginning of the future stomach. The part of the canal on the cephalic side of the stomach lags behind somewhat in growth, corresponding in this respect with the relatively smaller size of the adult esophagus. The esophagus begins to lengthen in the fourth week. At this time, also, the beginning of the liver is indicated by a small diver- 
ticulum which ponches out from the ventral wall of the intestine just posterior to (below) the stomach-the future duodenal region therefore-and, which grows into the ventral mesentery. Very soon after the appearance of the hepatic evagination, a similar out-pouching from the dorsal wall of

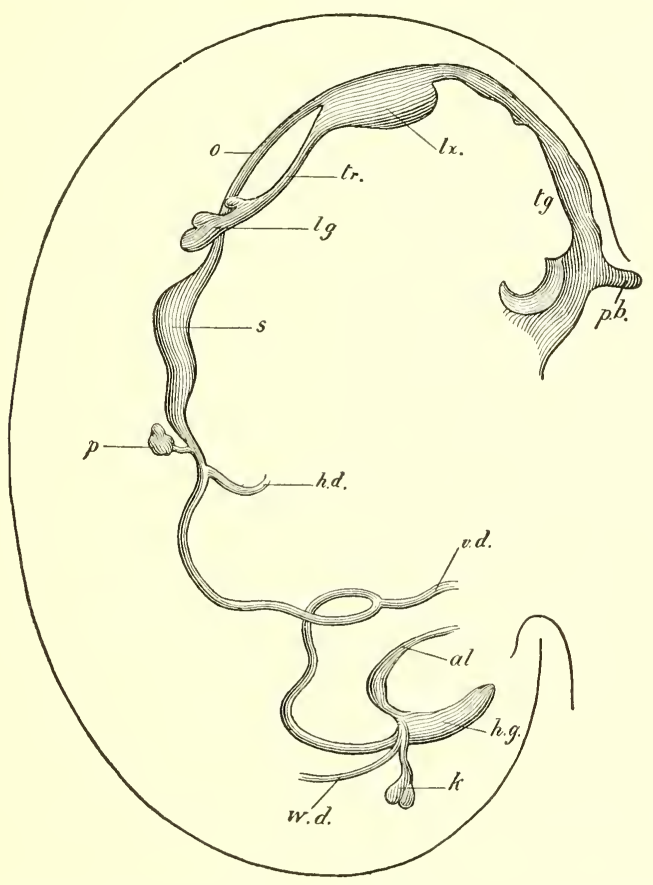

FIG. 98--Outline of alimentary canal of human embryo of twenty-eight days (His) : $p b$, pituitary fossa ; $t g$, tongue ; $l x$, primitive larynx ; $o$, esophagus ; $t r$, trachea ; $l g$, lung; $s$, stomach ; $p$, pancreas ; $h d$, hepatic duct ; $v d$, vitelline duct ; $a l$, allantois ; $h g$, hind-gut; $W d$, Wolffian duct; $k$, kidney.

the future duodenal region of the intestine indicates the beginning of the development of the pancreas.

In the latter part of the third week or in the beginning of the fourth, the esophagus presents a longitudinal groove on the inner face of its ventral wall. This groove increases in depth and caliber and finally becomes constricted off from the esophagus, with which it retains connection only at its pharyngeal end. The tube or tubular sac thus formed is the first step in the development of the lungs and the trachea. 
It may be said then that the gut-tract has now, in the fourth week, reached the stage of differentiation into the pharynx, the esophagus, the stomach, and the intestine, with the liver, the pancreas, the respiratory system, and the allantois fairly begun.

As heretofore pointed out (p. 90), the allantois-which grows directly from the primitive gut-tract, and which con-

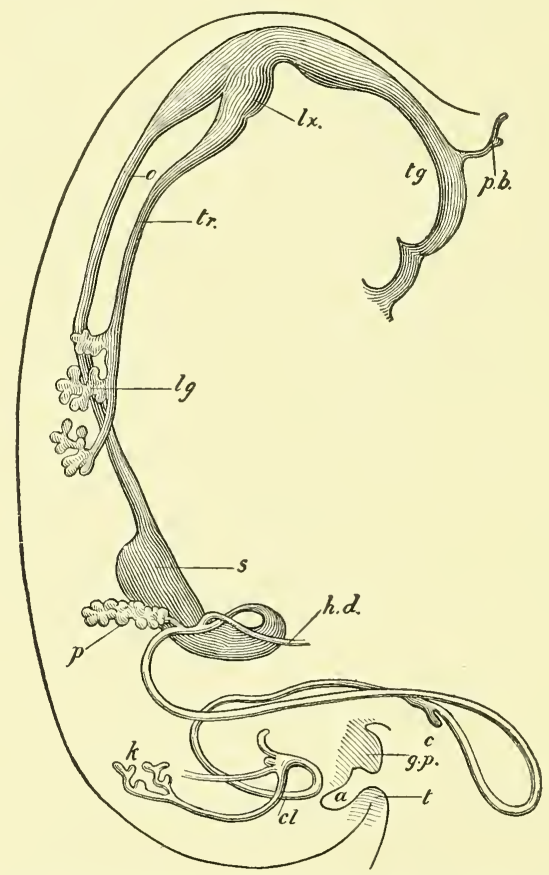

FIG. 99--Outline of alimentary canal of human embryo of thirty-five days (His) : $p b$, pituitary fossa; $t g$, tongue; $l x$, primitive larynx; $o$, esophagus; $t r$, trachea ; lg, lung; $s$, stomach ; $p$, pancreas; $h d$, hepatic duct ; $c$, cæcum ; $c l$, cloaca ; $k$, kidney ; $a$, anus ; $g p$, genital eminence; $t$, caudal process.

sists therefore of the entoderm and the visceral mesodermalthough destined to produce in part the permanent bladder, functionates for a time, after its union with the false amnion to form the chorion, as an organ of respiration; while the permanent respiratory system, as we have seen, likewise develops from the entodermal epithelium of the guttract. The entoderm, therefore, sustains an important re- 
lation to the nutrition of both the embryonic and the adult organism.

Increase in Length and Further Subdivision.The intestinal canal grows in length much more rapidly than does the embryonic body. It is in consequence of this disproportionate growth that the tube becomes bent and thrown into coils or convolutions. During the fifth and sixth weeks a conspicuous flexure appears at some distance below the stomach. Here the bowel assumes the form of a $U$-shaped tube, the closed end of the $U$ projecting toward the ventral body-wall (Fig. 100). In other words, the redundant portion

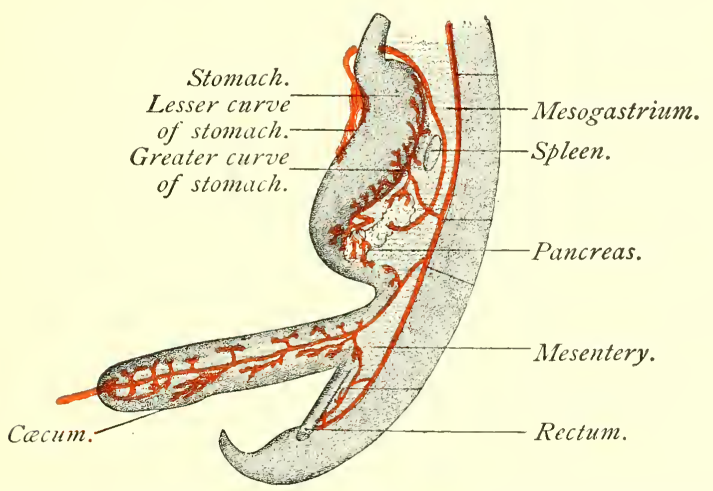

FIG. 100.-Intestinal canal of human embryo of six weeks (Toldt).

of the gut is pulled away, as it were, from the dorsal wall of the body-cavity and, as a consequence, the dorsal mesentery is lengthened in this region to a corresponding extent (Fig. 100). The vitelline duct is attached to the part of the bend nearest the ventral wall (Fig. 98). At a point on the lower limb of the $\mathbf{U}$ the bowel abruptly acquires increased caliber. This dilated part is the beginning of the cæcum or head of the colon, and its appearance initiates the distinction between the large and the small intestine, since the part of the bowel on the distal side of the point in question becomes also of larger caliber and forms the colon.

During the succeeding week or fortnight, the character of the colon and of the creum becomes better established. The remaining part of the lower limb of the U-loop, with all of 
the tube included between the loop and the stomach, is the small intestine, which presents a slight dorsal flexure at its proximal extremity. The stomach meanwhile has increased in size and has almost attained its characteristic shape. By the end of the sixth week, then, the alimentary canal has not only increased in length but has so far differentiated as to have acquired stomach, duodenum, small intestine, cæcum, and rectum.

Alteration in the Relative Position of Parts, and Further Development.-The most important modification of the alimentary tube as it exists at the end of the sixth week is effected by certain changes of position of some of its parts. The stomach and the large intestine are the portions of the tract most conspicuously affected. The lower limb of the U-segment of bowel, which consists chiefly of the rudimentary cæcum and a part of the colon, is lifted, as it were, over the upper limb and comes to oceupy a position above it (Fig. 101, A), the cxeum assuming a position in the right

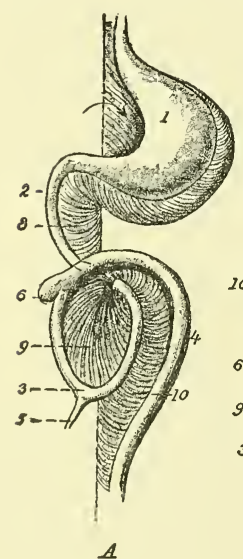

$A$

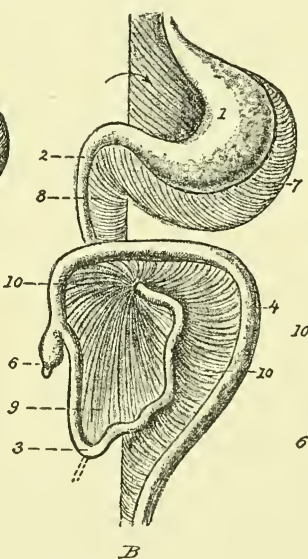

$\not B$

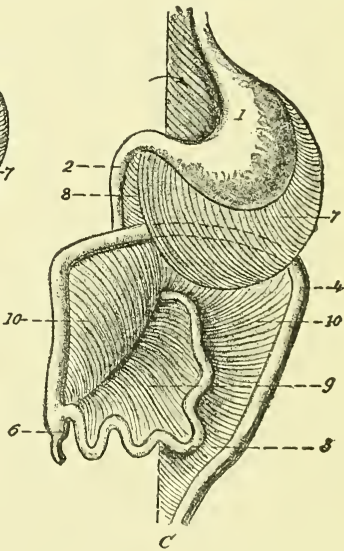

$C^{\prime}$

Fig. 101.-Three successive stages showing the development of the digestive tube and the mesenteries in the human fetus (modified from Tourneux): 1 , stomach ; 2 , duodenum; 3 , small intestine ; 4 , colon ; 5 , vitelline duct; 6 , cæcum ; 7 , great omentum ; 8 , mesoduodenum ; 9 , mesentery ; 10 , mesocolon. The arrow points to the orifice of the omental bursa. The ventral mesentery is not shown.

hypochondriac region, and the colon passing thence transversely across the abdomen ventrad to the duodenum.

This shifting of position on the part of the colon brings 
about important complications in the arrangement of the mesentery, since the part of the dorsal mesentery that pertains to the upper part of the colon correspondingly alters its position and line of attachment, becoming adherent to the peritoneum on the ventral surface of the duodenum. The part of the mesentery in question becomes the transverse mesocolon (Fig. 101, B). The large intestine, after this change of position, presents cæcum, transverse colon, descending colon, and rectum, the ascending colon being still absent.

The vermiform appendix in the third month has already acquired the form of a slender curved tube projecting from the crecum. At the time of its first appearance and for some weeks afterward, the appendix has the same caliber as the crecum. Subsequently the crecum outstrips the appendix in growth, the latter appearing in the adult state as a relatively very small tube attached to the much larger cæcum.

The cæcum soon begins again to change its position, gradually moving downward toward the right iliac fossa (Fig. 101). The downward migration of the cæeum is due to the growth of the colon in the same direction. In this manner the ascending colon is gradually produced, it having developed to such an extent in the seventh month that the crecum lies below the right kidney, while in the eighth month it passes the crest of the ilium. ${ }^{1}$ Corresponding with the growth of the ascending colon, the mesentery shifts its parietal attachment and increases in extent until the ascending mesocolon is produced; and with the descent of the crecum, the terminal part of the small intestine necessarily alters its position to a like degree.

The stomach, up to the third month, is a localized dilatation of the intestinal tube, bulging most in the dorsal direction and having its long axis parallel with that of the body (Fig. 100). In the third month, however, it undergoes an important alteration in position, rotating about two axes. First, it turns about a longitudinal axis, whereby the left side comes to face toward the ventral surface of the body (anteriorly) and the right surface looks toward the spinal column. In addition to the longitudinal rotation, the stom-

${ }^{1}$ According to Treves, the crecum lies under the liver until the fourth month after birth. 
ach also rotates upon a dersoventral (anteroposterior) axis, by which the lower or pyloric extremity moves somewhat upward and to the right, and the cardiac end goes tailward (downward) and to the left (Fig. 101). By this double rotation the stomach is made to assume approximately its adult position. The longitudinal rotation of the stomach, in which the lower portion of the esophagus takes part, explains the relation of the vagus nerves in the adult. The nerves, before the rotation, lie one on each side of the esophagus and stomach, but since the left surfaces of both turn forward and the right surfaces turn backward, the left vagus lies on the anterior surface of the esophagus and of the stomach, while the right nerve is in relation with their posterior surfaces.

The relations of the mesogastrium are influenced in an important manner by the rotation of the stomach. As long as the stomach retains its original position and relations, with its greater curvature facing dorsad (or posteriorly), the mesogastrium is a vertical mesial fold of peritoneum (Fig. 100), while the ventral mesentery similarly connects the future lesser curvature or ventral surface of the stomach with the ventral body-wall. At the very beginning of the process of rotation, the mesogaster becomes somewhat redundant and sags toward the left (Fig. 101, A). As this increases in extent, there is formed, between the stomach and the dorsal body-ivall, a pouch or pocket, the omental bursa, whose opening is toward the right (Fig. 101). In the third and fourth months the original mesogaster, lengthening more and more, and being affected by the increasing torsion of the stomach, projects in the form of a sac considerably below the level of the stomach, in front of (ventral to) the small intestine and the transverse colon. It ultimately becomes the great omentum. The mesogastrium, from having been a vertical mesial fold, is now become a transverse fold, so redundant as to be folded upon itself and to constitute a bag.

In like manner the ventral mesentery (Figs. 94 and 102), which connects the anterior or ventral surface of the stomach with the rentral body-wall, and in which the liver develops, is altered from a mesial fold to a transverse fold by the rotation of the stomach. As the liver migrates to a position 
above the stomach, the part of the ventral mesentery which connects the liver with the body-wall becomes its falciform ligament and coronary ligament, while that portion of this mesentery that connects the originally ventral surface of the stomach, now its lesser curvature, with the liver is the lesser or gastrohepatic omentum. The lesser omentum, therefore, is the anterior or ventral boundary of the orifice of the omental bursa referred to above.

The small intestine begins to exhibit flexures as early as the fifth week, and by the end of the sixth week the duodenum is well indicated as a segment of the gut-tube passing from the pyloric end of the stomach toward the dorsal bodywall. From this time the development of the small intestine, aside from its histological characters, consists chiefly in increase in length with consequent modification of its mesentery. A striking feature of human development is that, with the growth in length of the small bowel, it is gradually extruded from the abdominal cavity into the tissues of the umbilical cord. The extent to which extrusion takes place increases until the tenth week, after which period the intestine is gradually withdrawn into the abdomen. In the fourth month it lies entirely within the abdominal cavity. Failure of complete restoration of the gut to the cavity of the abdomen constitutes congenital umbilical hernia.

The histologica1 alterations incident to the development of the alimentary tube, from the beginning of the esophagus to the end of the rectum, consist in the differentiation of the constituent elements of its walls from the two strata, the entoderm and the visceral mesoderm, which compose the walls of the early gut-tube. As an initial step in the process, the cells of the mesodermic stratum undergo multiplication and arrange themselves in a narrow loose inner zone and a thicker outer lamella. The inner layer subsequently becomes the submucosa of the fully formed state, while the cells of the outer layer undergo differentiation into unstriped muscular tissue, and constitute the muscular coat of the canal. In the case of the esophagus and stomach, at least, this muscular tunic, in the fourth month, exhibits the 
distinction between inner circular, and outer longitudinal, layers. The surface-cells of the mesodermic stratum of the primitive stomach and bowel become the endothelium of the serous coat.

The glands of the entire canal are products of the inner, entodermic stratum, and therefore they are intimately related genetically, as well as histologically, with the mucous membrane.

The glands of the stomach, both the peptic and the pyloric, originate from small cylindrical cell-masses that have been produced by local multiplication and aggregation of entodermal cells. By the hollowing out of the cylinders and the branching of the tubes thereby formed, the two varieties of gastric glands are evolved. Both sets make their appearance in the tenth week. Until the fourth month the peptic glands contain cells of but one type; at this period, however, certain cells of these glands become altered by the gradual accumulation of granules within their protoplasm, by which they are transformed into the characteristic acid or parietal cells of these glands.

The glands and villi of the intestine are likewise products of the entodermal lining of the gut. Their erolution begins in the second month, and they are fairly well formed by the tenth week. As in the case of the gastric glands, the glands of the bowel develop from cylindrical masses of entodermal cells which are at first solid, but which later become hollowed out to form tubular depressions or follicles. In the region corresponding to the upper part of the small intestine many of these follicles branch to give rise to the glands of Brunner, while unbranched, simple, tubular depressions distributed throughout the entire length of the bowel become the glands of Lieberkuihn. While the surface entoderm is thus growing into the underlying mesorlermic tissue to form the glands, it becomes elevated into minute projections between the mouths of the gland-duets, forming the villi of the intestinal mucosa. The connective-tissue core of the villus is derived from the underlying mesodermic tissue, the cells of which, proliferating, grow forth into the entoderm. The villi at first are 
present throughout the large and the small intestine alike, being well developed by the fourth month. While the villi of the small bowel continue their development, those of the large intestine, after the fourth month, begin to retrograde. At the time of birth they are still discernible, but at the end of the first month after birth they are completely obliterated.

Meckel's Diverticulum.-The vitelline duct, it will be remembered, is the avenue of communication between the early gut-tube and the umbilical vesicle. In the sixth week the umbilical vesicle has already begun to retrograde, and the vitelline duct is attached to the ventral extremity of the U-loop of the bowel present at this stage. The vitelline duct in most cases suffers complete obliteration in the later stages of fetal life. In some instances, however, its proximal extremity persists in the form of a small blind tube varying in length from one to several inches, which is known as Meckel's diverticulum. Since the site of attachment of the vitelline duct is not far from the termination of the small intestine, Meckel's diverticulum, when present, is connected with the lower part of the ileum, at a point from one to three feet from its termination. Should this tube remain attached to the umbilical aperture and retain a patulous orifice, there would result a congenital fecal fistula. ${ }^{1}$

\section{THE DEVELOPMENT OF THE LIVER.}

The essential features of the development of the liver will be more easily apprehended if the reader will not lose sight of the fact that the organ is a compound tubular gland, and if, further, he will recall the method by which glands in general are developed-that is, as evaginations of the wall of the cavity or organ to which they pertain.

The first step in the evolution of the liver is the growth of a diverticulum from the ventral wall of the gut-tube at a point corresponding to the region of the future duodenum. This occurs in the third week, since His found the diver-

${ }^{1}$ Meckel's diverticulum is of interest clinically, since by contracting adhesions to adjacent coils of intestine or by entanglement, it may produce acute obstruction of the bowel. 
ticulum in a human embryo of $3 \mathrm{~mm}$. The single diverticulum ${ }^{1}$ very speedily bifureates at its distal extremity (Fig. 97 ). The very short time that elapses between the first appearance of the evagination and its division into two branches explains the statement made in some text-books that two diverticula are present from the first. The hepatic diverticulum is said to grow into the septum transversum (vide Development of the Diaphragm, p. 174). The dorsal part of the septum transversum or primitive diaphragm, the region just ventral to the bowel, contains a mass of young connective tissue, rich in cells and blood-ressels, which has been designated the prehepaticus, and the liver-ridge, by $\mathrm{His}$ and Kölliker respectively (Fig. 90). It is into this vascular

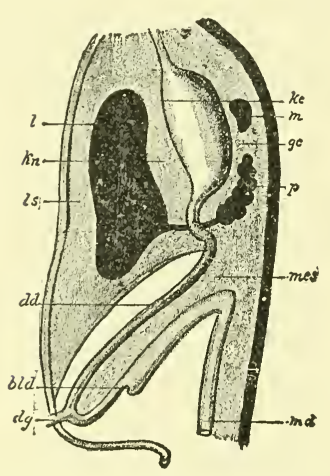

FIg. 102.-Diagram to show the original positions of the liver, stomach, duodenum, pancreas, and spleen, and the ligamentous apparatus pertaining to them. The organs are seen in longitudinal section : $l$, liver; $m$, spleen; $p$, pancreas; $d d$, small intestine; $d g$, vitelline duct ; bld, cæcum; $m d$, rectum; $k c$, lesser curvature; $g c$, greater curvature of the stomach; mes, mesentery; $k n$, lesser omentum (lig. hepatogastricum and hepatoduodenale); $l s$, ligamentum suspensorium hepatis (Hertwig). and cellular mass that the liver diverticulum inserts itself. The septum transversum is united in the median plane of the body with the ventral mesentery, and since the ventral mesentery is connected with theregion of theintestine from which the hepatic diverticulum is evaginated, the latter passes between the two layers of the mesentery to reach the liverridge (Fig. 102). This fact constitutes the key to the topographical relations of the liver and its peritoneal "ligaments," as will appear hereafter.

The two diverticula resulting from the division of the original single evagination embrace between them the two vitelline reins, and by repeated branching produce the right and the left lobes of the liver. Before branching, the diverticula become greatly thickened at their distal extremities by ${ }^{1}$ Single, according to His, Kölliker, Hertwig, Minot, and Piersol. 
abundant cell-proliferation. The numerous branches into which they divide are not tubes, but solid cylinders of cells, the hepatic cylinders. The secondary branches of these cylinders unite with corresponding branches of adjacent systems, producing thereby a network of inoseulating cell-cords, the meshes of which are occupied by young connective-tissue cells and the developing bloodvessels. The connective and vascular tissue of the liverridge, thus surrounding and permeating the epithelial cellcords, produces all the connective-tissue parts of the liver, while the liver parenchyma-the proper hepatic cells-and the epithelium of the bile-ducts originate from the primitive entodermic evagination. The cords of cells are in part hollowed out to form the bile-ducts and bile-capillaries, and in part become the cells of the lobules. The cylinders that are to produce the bile-ducts acquire their lumen by the fourth week.

Until the middle of the fourth month, the right and left lobes of the liver are of equal size, but after this period the right Iobe outstrips the left in growth. The liver grows very rapidly and is relatively of much greater size in the fetus than in the adult, almost filling the body-cavity at the third month. In the later months of pregnancy it reaches almost to the umbilicus, while at birth it makes up oneeighteenth of the body-weight.

The gall-bladder develops as an evagination from the original diverticulum. It is present in the second month. The pedicle of this evagination lengthens somewhat and becomes the cystic duct. The stalk of the hepatic evagination itself becomes the ductus communis choledochus.

The ligaments of the liver, save the round ligament, are simply folds of the peritoneum which connect the organ with the abdominal wall. Falling into the same category, though not usually designated a ligament, is the gastrohepatic omentum, which connects the liver with the stomach. These various peritoneal folds may be looked upon as parts of the ventral mesentery. Since the liver evagination grows between the two layers of the ventral mesentery to reach the 
septum transversum, the liver will be found, in the early stages of its development, embedded between the lamellie of this mesentery, which is a median vertical fold of peritoneum (Fig. 102). The liver is therefore enclosed in the peritoneum and is connected below, by a part of the ventral mesentery, with the lesser curvature of the stomach, which still lies in the median plane of the body, and above and in front, with the diaphragm and the ventral body-wall by the upper and anterior part of the same structure. The latter fold is somewhat modified by the intimate association of the early stage of the liver with the primitive diaphragm, the liver having developed within a portion of the septum transversum, the liver ridge. As development advances, a partial separation of the liver and the diaphragm is effected, the peritoneum, as it were, growing between the two from both the ventral and the dorsal edges of the liver. The region which is not invaded by the peritoneum represents the nonperitoneal surface of the adult liver between the lines of reflection of the two layers of the coronary ligament. Since the peritoneum on the under surface of the diaphragm is reflected from that muscle, both in front of and behind this area of contact, to become continuous with the peritoneum on the convex surface of the liver, there are formerl two transverse, parallel, but separated, folds which constitute the coronary ligament of adult anatomy. The lateral prolongations of these folds to the lateral wall of the abdomen constitute the lateral ligaments of the liver.

The rotation of the stomach to assume its permanent relations alters the position of the fold that connects its lesser curvature with the liver, bringing this fold into a plane parallel, approximately, with the ventral wall of the abdomen. This fold is now the lesser or gastrohepatic omentum.

The round ligament of the adult represents the impervious vestige of the umbilical vein. This ressel, entering the fetal body at the umbilicus and passing to the under surface of the liver, diverges from the abdominal wall to reach that organ and, in doing so, carries with it the parietal peritoneum. The fold thus formed is the falciform or suspensory ligament. 
The special system of blood-vessels belonging to the liver is described in the chapter on the Vascular System, p. 177.

\section{THE DEVELOPMENT OF THE PANCREAS.}

Until recently it was believed that the pancreas developed from an evagination of the dorsal wall of the gut-tube in the region of the future duodenum, opposite the site of the hepatic diverticulum. Later investigations have shown, however (Stoss, Hamburger, Brachet, and others), that three evaginations, one dorsal and two ventral, appear upon the wall of the duodenal region of the gut-tube, the method of development being strikingly similar in mammals, birds, fishes and amphibia.
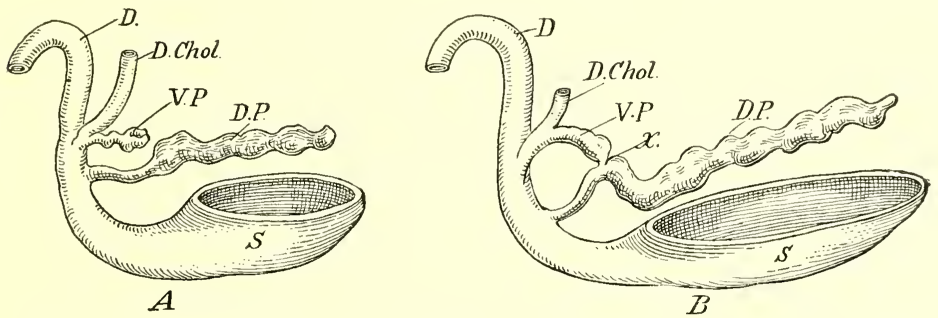

FIG. 103.-Reconstruction of duodenum with pancreatic diverticula (after Hamburger): $A$, Five weeks' embryo ; $B$, six weeks' embryo; $D$, duodenum; $D$.chol., common bile duct; V.P, ventral panereas; D.P, dorsal pancreas; $X$, point of fusion of the two; $S$, stomach.

In the sheep a dorsal evagination appears in a 4-mm. embryo (Stoss), and somewhat later two ventral outpouchings appear in close proximity to, if not in actual connection with, the hepatic diverticulum, the stalk of which latter becomes the common bile-duct. The dorsal diverticulum penctrates between the two layers of the mesogastrium (Fig. 103) and gives off lateral branches, remaining attached to the dorsal wall of the duodenum by its stalk or duct. Eventually this system of branching epithelial tubes, the dorsal pancreas, becomes the body and tail of the adult pancreas.

The right and left ventral evaginations become confluent and form the ventral pancreas. According to some authorities the left diverticulum atrophies, the right alone persisting to form the ventral pancreas. In either ease the stalk or duct 
of this ventral fundament becomes merged into the common bile-duct-if not previously connected with it-so that it is, in effect, a branch of that duct. The ventral pancreas growto the left, in front of the portal vein, this change being farored by the rotation of the duodenum on its long axis, penetrates between the layers of the mesogastrium and fuses with the dorsal pancreas (Fig. 103), becoming the head of the adult organ. This union occurs in the sixth week in man (Hamburger). With the union of the two portions their respective duets-the dorsal duct or duct of Santorini and the ventral or duct of Wirsung-acquire anastomoses with each other, after which event the terminal or intestinal part of the duct of Santorini atrophies and disappears, the duct of Wirsung being heneeforth the avenue by which the later-established secretion enters the duodenum. Occasionally the entire duct of Santorini persists to adult life, entering the duodenum upon its dorsal wall. In the cow and pig the ventral duct atrophies, the duct of Santorini alone persisting, while in the horse and $\operatorname{dog}$ both duets persist.

What has been said above applies to the origin of the epithelial parts of the gland; the connective-tissue and vascular elements are of mesodermic origin.

At six wecks the long axis of the pancreas nearly corresponds with that of the body of the fetis. With the rotation and change of position of the stomach and the alterations in the mesogastrium, it moves to the left, acquiring its permanent relations with the left kidney and the spleen. It continues to be an intraperitoneal organ until the fifth month, when, by the clisappearance of the dorsal part of its investment, it becomes retroperitoneal (Fig. 106, $A$ and $B$ ).

\section{THE DEVELOPMENT OF THE SPLEEN.}

Although the spleen does not belong to the digestive system, it may conveniently be considered here because of its position and relations.

This organ is differentiated from the mesodermic tissue (mesenchyma) found between the liyer's of the mesogastrium in close proximity to the developing pancreas (Fig. 102). 
Primitively, therefore, it is situated behind the stomach. The first step in its development, recognizable at about the end of the second month, is the accumulation of numerous lymphoid cells with large granular nuclei. The origin of these cells has been a matter of dispute. It has been asserted (Maurer, Kupfer) that they come from the epithelium of the gut-tube, but this is denied by most authorities. The findings of Laguesse in fish-embryos, demonstrating the origin of the spleen anlage from mesenchyma in close relation with the branches of the later portal vein, are possibly significant in view of the relationship between the spleen and one of the largest tributaries of the portal vein, namely, the splenic vein. Tonkoff's observations on birds and mammals (1900), confirmed by Hochstetter, reaffirm the mesenchymal origin of the spleen.

The mass of cells is augmented by the addition of cells immediately beneath the peritoneal surfaces of the mesogastrium, which cells elongate until they are spindle-shaped and then become aggregated into fusiform masses. Bloodvessels penetrate the fundament in the third month and become surrounded by cells of the same spindle-shaped type. From both the cells surrounding the blood-vessels and from those of the fusiform aggregations, processes grow out and unite with each other, and from the network thus formed the trabecular framework of the organ is ultimately evolved. Accumulations of small nucleated cells, forming dense masses along the arteries, furnish the chief constituent of the pulp. The delicate intercellular substance which makes up the remainder of the pulp is filled with blood-corpuscles. The Malpighian corpuscles appear before the end of the fourth month. By the sixth month, the spleen attains its characteristic shape and the fibrous capsule is clearly indicated.

The spleen undergoes a change of location coincident with the rotation of the stomach and the alteration of the mesogastrium. The organ being from the first embedded within the mesogastrium, it follows that peritoneal fold to the left side of the abdominal cavity. Here it lies close to the 
cardiac end of the stomach, between the two layers of the mesogastrium, but projecting toward the left. The part of the mesogastrium which intervenes between the spleen and the stomach is the gastrosplenic omentum; whilc the part that passes from the spleen to the posterior wall of the abdomen, representing the parietal attachment of the mesogastrium, constitutes the phrenicosplenic omentum.

\section{THE EVOLUTION OF THE PERITONEUM.}

The arrangement of the peritoneum being subordinate to the position and relations of the viscera contained within the abdomen, the development of this complex membrane can be properly described only by tracing the growth of the digestive system. As the formation of the early gut-tube by the infolding of the splanchnopleure has been pointed out (pp. 186 and 188), we may begin at once with the period when the tract has the form of a straight tube connected with the dorsal and the ventral body-wall respectively by the dorsal and the ventral mesentery (Fig. 104). Covering the tube as a constituent part of its wall, is the splanchnic or visceral layer of the mesoderm, while the somatopleuric or parietal layer of the latter lines the wall of the body. Obviously these two lamellæ of the mesoderm are continuous with each other through the medium of the mesenteries mentioncd above (Fig. 105, $A$ and $B$ ). The space thus enclosed by the mesodermic strata is the body-cavity or coelom or pleuroperitoneal eavity. The surface-cells of both strata flatten and assume the character of mesothelial, the later endothelial, cells. If, at this stage, one begins at any point to trace the mesothelial lining of the body-cavity, that lining is found to form practically one continuous sheet.

This simple arrangement of the primitive peritoneum is transformed into the complicated membrane of the adult, primarily, by the increase in length and consequent tortuosity of the alimentary tube; and, secondarily, by the fact that certain opposed portions of the scrous membrane, which have been brought into contact by the altered relations of the bowel and the stomach, undergo concrescence or fusion 
with each other. Simultaneously with these alterations, the original pleuroperitoneal cavity suffers division into the abdominal or peritoneal cavity and the thoracic part of the body-cavity by the development of the diaphragm. This is described on p. 175.

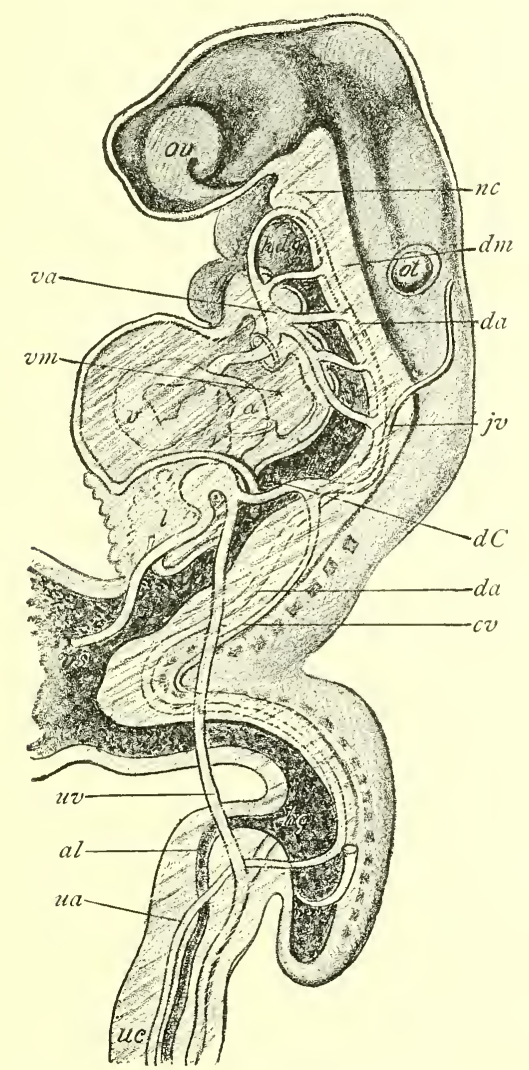

Fif. 104 -Reconstruction of human embryo of about seventeen days (after His): $o v$, optic and ot, otic vesicles; $n c$, notochord: $h d g$, head-gut ; $g$, mid-gut; $h g$, hindgut; $v s$, vitelline sac; $l$, liver; $v$, primitive ventricle; $v a$, da, ventral and dorsal aortæ; $j v$, primitive jugular vein; $c v$, cardinal vein; $d C$, duct of Cuvier; $u v, u \alpha$, umbilical vein and artery ; $a l$, allantois ; $u s$, umbilical cord ; $d m$, dorsal mesentery ; $v m$, ventral mesentery (modified from His).

The first modification of the original arrangement is effected by the development of the stomach as a spindle-shaped dilatation of the gut-tube, differentiating the tube into the stomach and the intestine, and the common dorsal mesentery 
into the mesogastrium and the intestinal mesentery. The drawing out of the U-shaped loop of the intestine from the dorsal body-wall, which is the preliminary step to the distinction between the small intestine and the colon, increases the length of the intestinal mesentery to a corresponding extent (Fig. 10.5, C). As heretofore indicated, the lower limb of the loop presents an enlargement which is the beginning of the development of the large intestine.
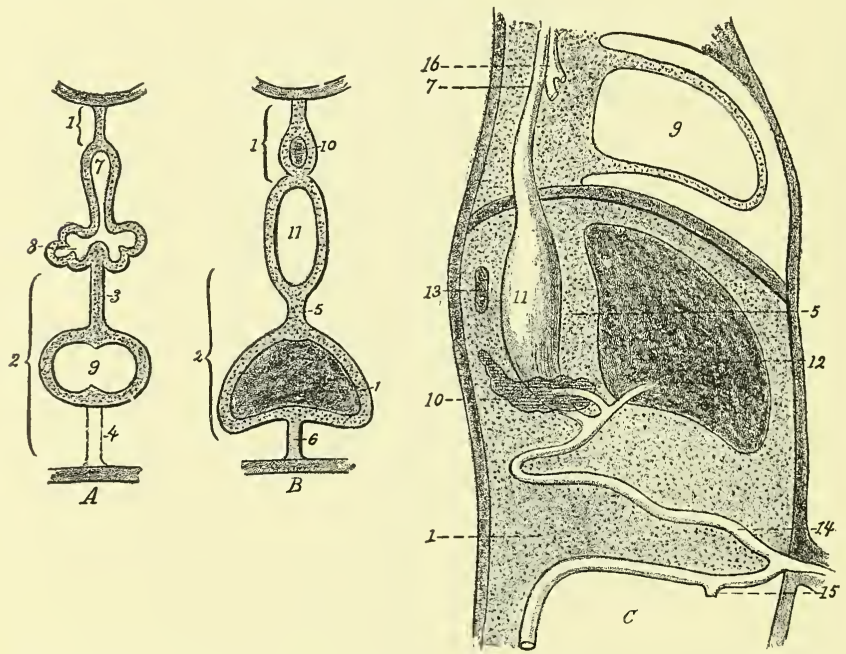

FIG. 105. $-A, B$, two transverse sections, $A$ through thoracic, $B$ through abdominal region; $C$, sagittal section (Tourneux): 1 , dorsal mesentery ; 2 , ventral mesentery ; 3 , mesocardium posterius; 4 , mesocardium anterius; 5 , lesser omentum ; 6 , suspensory ligament of liver; 7 , esophagus; 8 , lungs; 9 , heart; 10 , pancreas; 11 , stomach; 12, liver; 13 , spleen; 14, loop of intestine with vitelline duct; 15 , cæcum; 16 , trachea.

An important stage in the evolution of the peritoneum is marked by the rotation of the stomach and by the migration of the proximal part of the large intestine to a new location. The change of position on the part of the colon may perhaps be best expressed by saying that the U-loop of intestine rotates upon an oblique dorsoventral axis, whereby the lower limb of the loop, in other words, the termination of the small bowel and the beginning of the colon, is carried to a position above, cephalad to, the upper limb (Fig. 101, A). This rotation brings the beginning of the colon into the right hypo- 
chondriac region of the abdomen, from which point the transverse colon passes across the abdominal cavity, ventrad to the proximal end of the small intestine or duodenum. As a consequence of the altered situation of the transverse part of the colon, its mesentery shifts its area of attachment by fusing with the peritoneum of the dorsal wall along a horizontal line and also with that of the ventral surface of the duodenum. The descending colon having meanwhile moved to the left, its mesentery likewise acquires a new area of attachment by concrescence with the parietal peritoneum of the dorsal wall of the abrlomen on the left side. During the progress of these alterations, the small intestine increases in length, and its mesentery becomes correspondingly more voluminous both in the extent of its intestinal border and in length. The convolutions of the small intestine now occupy the space below the transverse colon and its mesentery.

The duodenum, which in the early stage shares with the gastro-intestinal tube in the possession of the common dorsal mesentery, loses its mesenterial connection with the abdominal wall and becomes thereby a fixed part of the intestine. Mention was made above of the fusion of the transverse mesocolon with the peritoneum of the ventral surface of the duodenum. At about the same time, the duodenal mesentery (Fig. 101, A) fuses with the parietal peritoneum of the posterior abdominal wall, the result being that the lower layer of the transverse mesocolon, as it passes downward, is now eontinuous with the parietal peritoneum, there being no longer any serous membrane between the transverse part of the duodenum and the abdominal wall (Fig. 106, B). This part of the duodenum therefore becomes retroperitoneal, there being an investment of serous membrane only on its anterior or ventral surface.

The second modifying factor in the complication of the peritoneum, the rotation of the stomach, initiates alterations in its mesogastrium. The latter membrane, it will be remembered, is a vertical median fold of peritoneum continuous with the mesentery of the duodenum (Fig. 105, C). 
As the stomach moves about its two axes of rotation, the mesogastrium begins to sag toward the left (Fig. 101), so that now it constitutes a pouch or fossa, the omental bursa, situaated between the stomach and the dorsal body-wall, the opening of which looks toward the right side of the body (Figs. 106, $A$ and 107). With the rapidly increasing redundancy of the mesogastrium, the omental bursa becomes

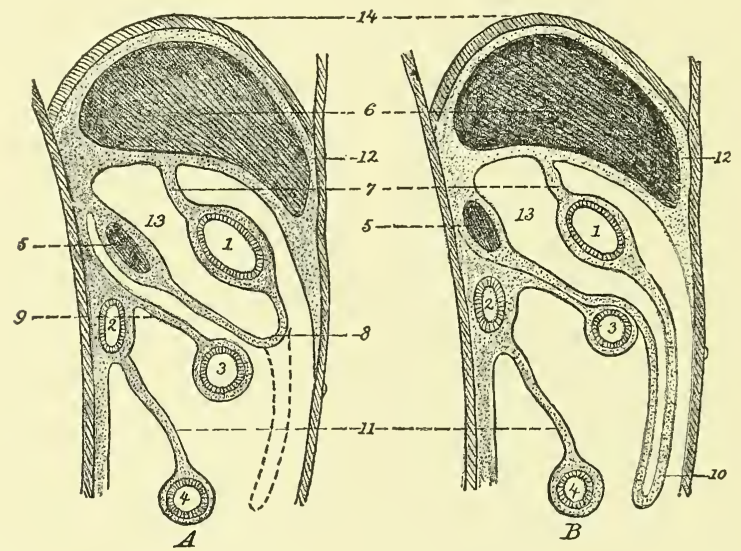

FIG. 106. $-A, B$. Two successive stages of the development of the mesenteries (schematic representation showing sagittal axial section of trunk, after Gegenbaur and Hertwig): 1, stomach ; 2, duodenum; 3, transverse colon; 4, small intestine; 5 , pancreas ; 6 , liver; 7 , lesser omentum ; 8,10 , different stages of great omentum; 9 , transverse mesocolon; 11, mesentery ; 12, suspensory ligament of liver; 13, cavity of omental bursa or lesser peritoneal sac ; 14, diaphragm.

more and more capacious. In correspondence with the progressive rotation of the stomach, what was at first the left surface of the mesogastrium comes into contact with the peritoneum of the dorsal abdominal wall and fuses with it, thus changing its area of parietal attachment from a median vertical line to a transverse one. This change is completed by the time the stomach has attained its normal adult position. The omental bursa now has the position and relations shown in Fig. 106, A, 8. A still further increase in the size of the bursa results in its protrusion downward in front of, ventrad to, the transverse colon and the small intestine. Refence to Fig. 106, $B$ will show that the dependent part of the bursa very nearly corresponds with the fully formed 
great omentum. It will be seen, however, that the deeper layer of the bursa, the layer nearer the intestines, may be traced above the transverse colon and its mesentery to the dorsal wall of the abdomen, where its two lamellæ separate to enclose the pancreas, one lamina passing over the ventral surface of the pancreas to become continuous with the parietal peritoneum, while the other layer passes between the pancreas and the abdominal wall. The latter layer is in continuity here with the parietal peritoneum, which almost immediately leaves the abdominal wall to form the upper layer of the transverse mesocolon.

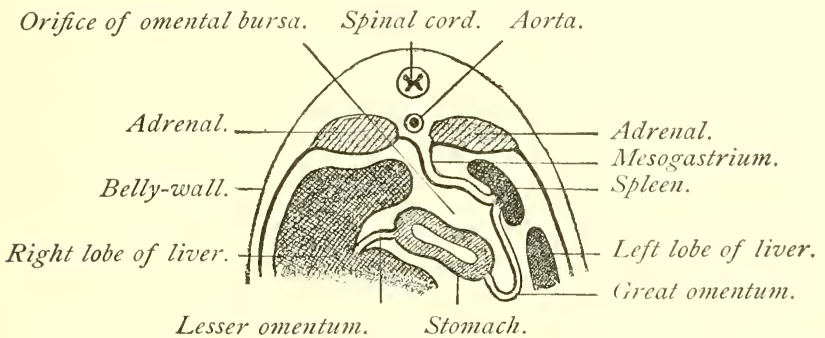

FIG. 107.-Schematic cross-section through body of mammalian embryo in region of stomach, to show development of omental bursa (after Toldt).

The further alterations necessary for the attainment of the completed condition consist in the concrescence of certain opposed peritoneal surfaces. As a conspicuous example of such concrescence, the deeper lamella of the layer of the omental bursa just described fuses with the ventral peritoneal surface of the transverse colon and with the upper layer of the transverse mesocolon (Fig. 106, A), after which event this deeper lamella is practically continuous with the lower layer of the mesocolon, while the superficial lamella is in continuity with the upper layer of the mesocolon (Fig. 106, B). Thus the transverse colon appears as if enclosed between the two lamellæ of the deeper layer of the great omentum, while its mesocolon is constituted by a part of the same structure. In other words, the adult transverse mesocolon includes not only the primitive membrane of that name but also a part of the early mesogastrium. Similarly, the opposed surfaces of peritoneum between the pancreas and 
the dorsal abdominal wall undergo fusion (Fig. 106), the effect of which, after the concrescence of the mesocolon with the deeper layer of the omental bursa, is to make the lower layer of the mesocolon continuous, over the transverse part of the duodenum, with the parietal peritoneum.

The great omentum of descriptive anatomy, resulting from the downwardly projecting process of the omental bursa, consists originally of two layers of membrane, each one having two serous surfaces. At the time of birth these two layers are still separate-the permanent condition in some mammals-but during the first year or two after birth they become adherent, the great omentum thus coming to comprise but a single layer.

It remains to note the metamorphosis of the ventral mesentery, which, prior to the rotation of the stomach, is a vertical median fold connecting the lesser curvature of that viscus with the ventral abdominal wall. Since the evagination of the gut-tube that gives rise to the liver grows between the layers of the ventral mesentery to reach the septum transversum, the liver is not only enclosed by the mesentery, but is connected by it with the stomach and with the ventral wall of the abdomen and also with the primitive diaphragm (Fig. 105). By the rotation of the stomach, the vertical median fold which connects that organ with the liver becomes so altered in position as to lie in a plane approximately parallel with the ventral surface of the body. This fold is now the gastrohepatic or lesser omentum. As reference to Fig. 106 will show, it is the anterior boundary, above the position of the stomach, of the sac described above as the omental bursa.

That part of the ventral mesentery that connects the liver with the abdominal wall and with the diaphragm, while originally occupying the median plane, is modified by the relation of the developing liver to the primitive diaphragm. These organs are intimately united with each other (p. 175) in the early stage of their growth, but with their completion a separation takes place. Upon the two separated surfaces, except in a region near the dorsal wall, the cells assume the 
endothelial type, the opposed surfaces thus acquiring the characters of serous membrane. The peritoneum on the under surface of the diaphragm is continuous with that on the upper surface of the liver, both in front of and behind the non-peritoneal area of contact. Therefore, in the completed condition of the liver and the diaphragm, these two structures are connected by two layers of peritoneum separated from each other by a region containing only areolar tissue. These layers constitute the coronary ligament of the liver. If now Fig. 106 is inspected, it will be seen that the posterior layer of the lesser omentum, and the upper layer of the transverse mesocolon, together with that part of the peritoneum with which they are in direct continuity, enclose a sac which is the so-called lesser bag of the peritoneum or the lesser peritoneal cavity. All other parts of the peritoneum taken together constitute the greater peritoneal cavity. The communication between the two, the foramen of Winslow, situated behind the free right border of the lesser omentum, is the constricted orifice of the early omental bursa.

The position of the kidneys and the ureters as retroperitoneal structures and the relations of the bladder and of the uterus to the peritoneum, encroaching as they do upon the parietal layer of this membrane, and being, therefore, invested by it to a greater or less extent, are easily accounted for when it is recalled that all these organs develop from the somatic or outer layer of the mesoderm.

The peritoneum does not acquire all the characteristic features of a serous membrane until about the third month. The histological alterations begin in the fourth week, from which time until the sixth week the superficial cells, the mesothelium, pass through various phases of transition to reach the condition of somewhat flattened elements. By the eighth week they have acquired the form of true endothelium. It is not, however, until the third month that the subjacent tissue has attained to the condition of a fullyformed basement membrane. 


\section{CHA P'TER XII.}

\section{THE DEVELOPMENT OF THE RESPIRATORY}

SYSTEM.

Alтноugu the nasal chambers and the pharyngeal cavity contribute to the formation of the respiratory system, these

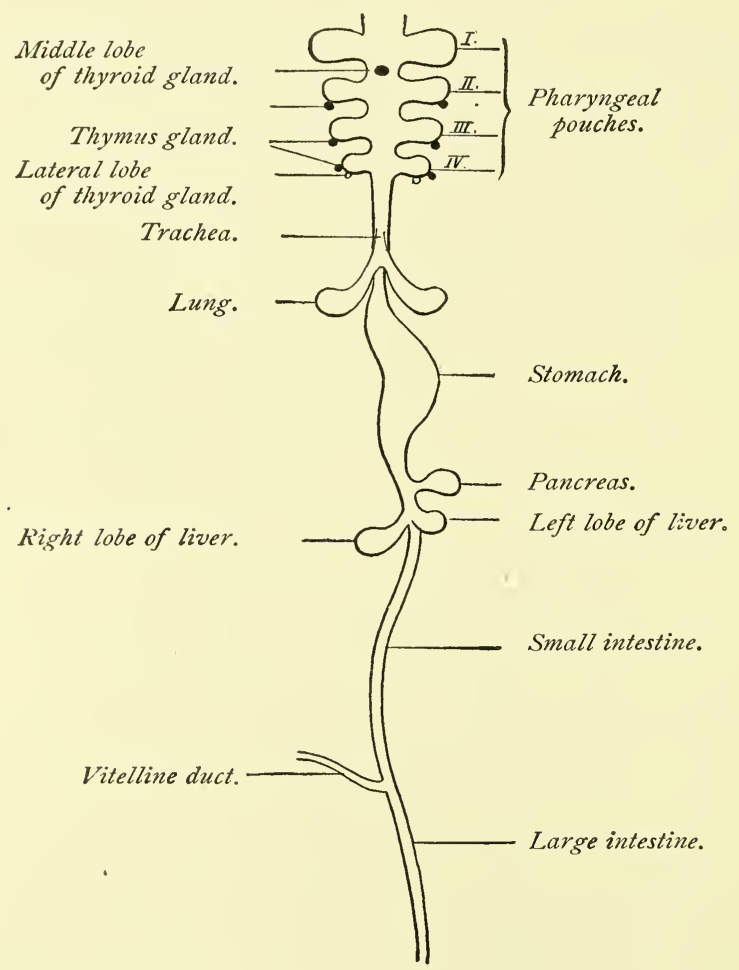

FIG. 108.-Scheme of the alimentary canal and its accessory organs (Bonnet).

parts will not be considered here, since they are described elsewhere. 
Anatomically and according to their mode of development, the lungs might be looked upon as a pair of glands having a common duct, the trachea, which latter, through the medium of its dilated proximal extremity, the larynx, opens into the pharyngeal cavity. In point of fact, these organs are developed as an outgrowth from the entodermal alimentary canal in a manner similar to the development of the liver and the pancreas.

The first step in the development of the lungs is the outpouching of the ventral wall of the esophagus throughout its entire length. The longitudinal median groove thus formed is the pulmonary groove.

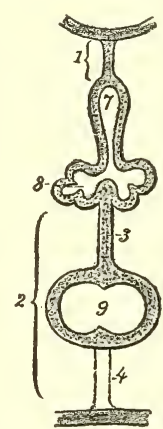

FIG. 109.-Transverse section to show outgrowth of pulmonary anlage from gut-tube (after Tourneux): 1, dorsal mesentery; 2, ventral mesentery including 3 , mesocardium posterius ; 4 , mesocardium anterius ; 7 , esophagus; 8, diverticulum which becomes the lungs, the trachea, and the larynx; 9 , heart.

It makes its appearance when

the embryo has a length of $3.2 \mathrm{~mm}$. (0.128 inch) or probably early in the third week. The groove is more pronounced at its lower or gastric extremity. As the groove deepens, its edges approach and finally meet and fuse with each other. In this manner the groove is converted into a tube, which gradually separates from the esophagus, the separation beginning at the end toward the stomach and progressing toward the pharynx. The separation, however, is not complete, stopping short of the upper end of the groove, so that the tube retains communication with the pharyngeal end of the esophagus. Even before the constricting off of this tube or pulmonary diverticulum is completed, its free end bifurcates. The pulmonary anlage consists, then, at this stage, of two short wide pouches connected by a common pedicle with the primitive pharynx (Figs. 108 and 109), and this condition is present in the fourth week.

Very soon after the end of the first month each of the pouches undergoes division, the right one into three branches, 
the left one into two, while at the same time they increase in size (Fig. 110). The further steps toward the attainment

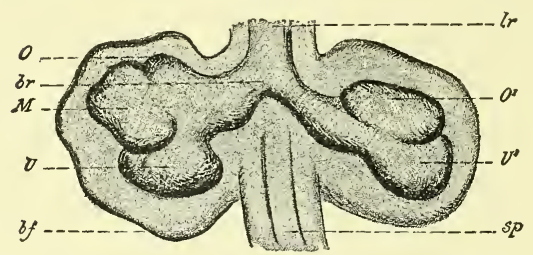

FIG. 110. - View of a reconstruction of the fundament of the lungs of a human embryo (Pr. of His) $10 \mathrm{~mm}$. long, neck measurement (after His) : $l r$, trachea; $b r$, right bronchus; $s p$, esophagus; $b f$, connective-tissue envelope and serous membrane (pleura) into which the epithelial fundament of the lung grows; $O, M, U$, fundaments of the upper, middle, and lower lobes of the right lung; $O^{1}, V^{1}$, fundaments of the upper and lower lobes of the left lung.

of the completed condition consist largely in the continued repetition of this process of dichotomous division (Fig. 111),

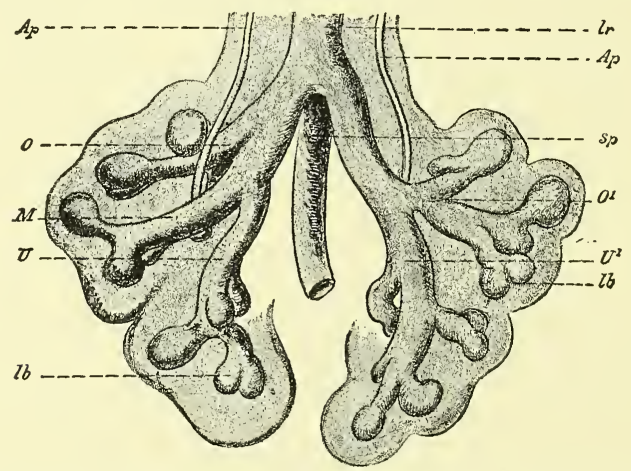

FIg. 111.-View of reconstruction of the fundament of the lungs of a human embryo (N. of His) older than that of Fig. 110 (after His, magnified 50 diameters): $A p$, arteria puimonalis ; $l r$, trachea; $s p$, esophagus : $l b$, pulmonary vesicle in process of division; $O$, upper lobe of the right lung with an eparterial bronchus leading to it; $M, U$, middle and lower lobes of the right lung: $O^{1}$, upper lobe of the left lung with hyparterial bronchus leading to it; $U^{1}$, lower lobe of the left lung.

which latter goes on until the sixth month. The original evagination, consisting of entodermal epithelium, gives rise only to the epithelial parts of the lungs and air-passages. All the other constituents, the connective tissue, the muscular, vascular, and cartilaginous elements, are products of the mesodermic tissue into which the diverticulum grows. Upon their first appearance the "tubes" are always solid 
epithelial cylinders, the lumina being acquired later. At first, the lining entodermal cells of the primitive tubes are tall and cylindrical, the tubes themselves having a relatively small lumen. In the fourth month the cells acquire cilia. From the anatomical standpoint, the lungs now present the characters of compound saccular glands.

From the sixth month to the end of gestation occur the changes which give to the organs their essential characteristics. Upon the dilated extremity of each terminal tube numerous little evaginations develop. These are the air-sacs, or pulmonary alveoli, the terminal tubes from which they are evaginated being the alveolar passages and the infundibula. Their walls remain very thin and their lining epithelium flattens to such a degree as to closely resemble endothelium. The trachea is simply the elongated stalk of the pulmonary diverticulum. Its incomplete cartilaginous rings first appear in the eighth or ninth week.

The larynx is the dilated proximal extremity of the stalk of the pulmonary diverticulum specially modified to serve as an organ of phonation. It is first indicated at the end of the fifth week (or, according to Kallius, in the fourth week). One of the earliest changes is the appearance of two dorso. ventral ridges at the junction of the primitive trachea with the esophagus. They are close together in front, ventrally, but separated dorsally. They are the first indications of the true vocal cords. At this time the pharyngeal aperture of the primitive larynx is at about the level of the fourth visceral furrow, behind the three segments of the developing tongue

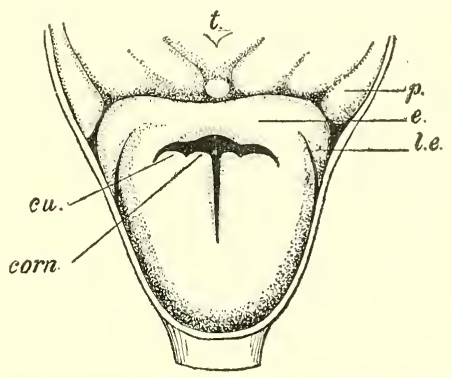

FIG. 112.-Entrance to larynx in a forty- to forty-two-day human embryo (from Kallius): $t$, tubereulum impar; $p$, pharyngo-epiglottic fold; $e$, epiglottic fold; l.e, lateral part of epiglottis; $c u$, cuneiform tubercle; corn, cornicular tuberele.

(p. 144), and is separated from them by the furcula, a horseshoe-shaped ridge which bounds the aperture in front and laterally and which represents apparently the rentral parts 
of the third visceral arches (Fig. 71, A, 3, p. 144). A little later the furcula differentiates into a median elevation, which is the anlage of the epiglottis, and into the two lateral arytenoid ridges, each of which latter presents two little elevations, the cornicular and cuneiform tubercles respectively (Fig. 112). The arytenoid cartilages are thus well indicated by the sixth week. The lateral portions of the furcula also produce the aryteno-epiglottidean folds.

The thyroid cartilage develops in two lateral halves from corresponding masses of mesenchyme which chondrify from two distinct centers for each mass. It is regarded as representing the cartilages of the fourth and fifth branchial arches. The two alæ fuse with each other ventrally as development advances. Failure of cartilaginous union between the two alre constitutes the malformation, foramen thyroideum. The cricoid cartilage is regarded as being an independent cartilaginous formation in series with the rings of the trachea. The chondrification of these various elements of the larynx begins in the eighth or ninth week.

The development of the pleuræ has been described in connection with that of the pericardium and of the diaphragm (p. 175).

\section{THE THYROID, THE PARATHYROID, AND THE THYMUS BODIES.}

These organs may be considered in this connection as a matter of convenience and because of their embryological relationship to the respiratory system, being developed, like the latter, from the epithelium of the gut-tract.

The thyroid body, an organ common to all vertebrates, genetically consists of two parts, a median and two lateral portions, or lateral thyroids.

The median portion originates from an evagination of the ventral wall of the pharynx, in the median line, posterior, caudad, to the tuberculum impar, and between the ventral extremities of the first and second visceral arches. This median diverticulum is present in the human embryo of $5 \mathrm{~mm}$. It soon pouches out on either side, assuming thereby the form of an 
epithelial vesicle connected by the constricted pedicle of the diverticulum with the ventral wall of the pharynx (Fig. 113, 3). From the situation of the original point of evagination behind the tubereulum impar and ventromesial to the two halves of the posterior segment of the tongue, the orifice of the pedicle corresponds to the line of junction of the three parts of the tongue. As a consequence, when these parts

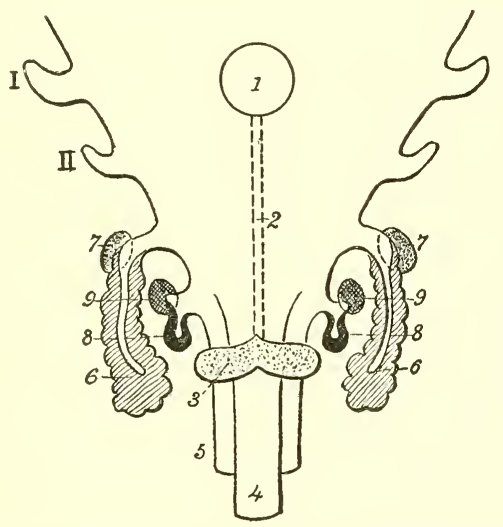

FIG. 113.-Diagrammatic representation of pharynx of human embryo seen from in front (after Tourneux): I, II, first and second pharyngeal pouches ; 1 , tuberculum impar; 2, course of thyroglossal duct leading from 3, median lobe of thyroid gland; 4, laryngotracheal tube: 5 , esophagus; 6 , thymus; 7 , epithelial body [parathyroid]; 8 , lateral thyroid; 9 , postbranchial body [parathyroid?].

unite, the pedicle or duct is prolonged upward and comes to open upon the surface of the tongue. The canal is known as the thyroglossal duct or canal of His. In the fifth week it begins to atrophy, and usually by the eighth week has become obliterated. Occasionally it persists throughout life. The foramen cæcum on the dorsum of the tongue is the vestige of the orifice of the duct. Other vestiges of the thyroglossal duct are sometimes present. For example, the lower part of the duct may persist as a short tube, the thyroid duct, leading upward from the median lobe to the hyoid bone; and again, according to His, isolated persistent segments of the duct constitute the little resicles in the neighborhood of the hyoid bone which are known respectively as the accessory thyroid and the suprahyoid and prehyoid glands. According to some recent observations the 
lower part of what His calls the thyroglossal duct gives rise to the pyramidal process of the thyroid, which extends upward toward the hyoid bone, usually a little to the left of the mid-line. This unpaired median anlage gives rise to the isthmus of the adult organ and also, to a considerable part at least, of each lateral lobe.

The lateral thyroids begin their development somewhat later than does the median portion. In the embryo of 10 $\mathrm{mm}$., the fourth inner visceral furrow or throat-pocket of each side pouches out to form a vesicle (Fig. 113, 8). As the vesicle grows, its pedicle becomes attenuated and finally disappears. After their isolation from the throat-pockets, the
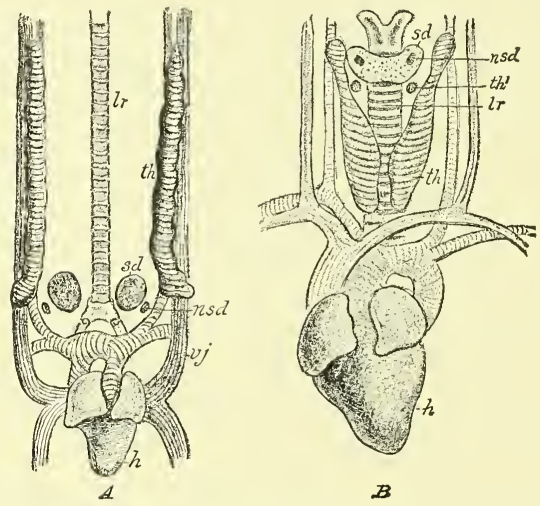

FIG. 114.-Semi-diagrammatic illustrations to show the ultimate position of the thymus, thyroid gland, and postbranchial body on the neck of the chick $(A)$ and the calf $(B)$, after de Meuron: $s d$, thyroid gland; $p$, postbranchial body; th, thymus; $e$, epithelial body [parathyroid]; $l r$, trachea ; $h$, heart ; $v j$, vena jugularis ; $c a$. carotid vein.

vesicles give out small bud-like processes after the usual manner of the development of glands and gradually approach the median lobe (Fig. 114, B), fusing with its posterior surface. The three parts unite probably in the seventh week. In the vertebrates below mammals the lateral parts of the thyroid do not unite with the median segment, and in certain animals they remain widely separated from it as the suprapericardial bodies. According to the older view of His, the lateral thyroids produce all of the lateral lobes of the adult thyroid; later researches have shown that they do not, but 
authorities are not in harmony as to whether they produce a large part or only a small portion of the adult lateral lobes. The more recent view of His is that the adult lateral lobes develop only in part from the lateral anlages. Verdun, the most recent worker in this field, maintains that the entire thyroid body of mammals and man is developed from the median anlage and that the postbranchial bodies (Figs. 113 and 114), by which name he designates the structures referred to above as the lateral thy roids, atrophy.

After the union of the three portions of the gland, the latter consists of a network of cords of cells, the meshes of which reticulum are occupied by embryonal connective tissue. Subsequently the cords of cells become hollowed out and exhibit alternating enlargements and constrictions. By the increase of the constrictions the continuity of the cell-cords is interrupted at short intervals, and so the network is converted into numerous closed follicles lined with epithelium, the formation of follicles beginning in the eighth week. The follicles later undergo considerable increase in size on account of the secretion by their epithelial cells of a peculiar colloid material, characteristic of the thyroid body. The embryonal connective tissue, made up necessarily of mesodermic elements, furnishes the connective-tissue framework and the blood-vessels of the organ, while the epithelium originates in the manner indicated from the entoderm of the gut-tract.

The Parathyroid Bodies.-The parathyroid bodies, usually two in number on each side, were discovered by Sandstrom in 1880. The lower pair lie upon the trachea in close relation with the thyroid body, while the upper pair lie at the level of the lower border of the cricoid cartilage, in relation with the dorsal surface of the lateral lobes of the thyroid body.

Their origin is still somewhat obscure. Apparently they are outpouchings respectively from the third and fourth visceral furrows, being composed, therefore, of entodermal epithelium. These epithelial bodies develop in a manner similar to the development of the thyroid body, but the fact that the cell-groups are not broken up by the invading embry- 
onal connective tissue to the same extent as in the case of the thyroid renders them histologically distinguishable from the latter; moreover, it is stated by Maurer that they never form colloid substance.

The Thymus. - What remains of the thymus after the second year of life is made up chiefly of lymphoid and connective tissue, embedded in which are characteristic little epithelial bodies, the corpuscles of Hassall.

The epithelial parts of the thymus, in all vertebrate animals, are derived from the entodermal lining if the pharyngeal region of the gut-tract. In the lower groups, such as reptiles, amphibians, and bony fishes, the epithelium of all the inner visceral clefts or throat-pouches shares in the development; while in birds, only two or three clefts take part. In mammals, however, including man, the thymus body is derived probably from but one throat-pocket, the third.

The entodermal epithelium of the third inner pouch becomes evaginated (Fig. 113) to form an epithelial sac whose connection with the pharyngeal cavity is subsequently lost. The isolated and elongated sac soon gives out small lateral buds or processes at the distal extremity. While the original sac has from the first a cavity, the bud-like branches are solid masses of epithelium. The branching continues and affects not only the lower or distal extremity of the thymus sac but also the proximal end, the structure now resembling an acinous gland (Fig. 115). While this growth is taking place, the epithelial mass is being invaded by lymphocytes and young connective tissue with developing blood-ressels. (According to some recent studies by E. T. Bell, the lymphoeytes are derived from the epithelium of the original thymus anlage; but this is denied by Stöhr.) The encroachment by these elements continues to such an extent that lymphoid tissue-including leukocytes and erythroblasts-becomes the predominant constituent of the thymus, the epithelial parts suffering reduetion, relatively, and becoming finally broken up into isolated masses which are the corpuscles of Hassall of 
the mature gland. The breaking down of the epithelial cords is probably responsible also for the irregular cavities of the thymus. Not until after birth do the glands of the two sides of the body unite to form a single unpaired structure, and the development of the thymus is not completed until the end of the second year of life. Having attained its full development, the organ begins to retrograde, and at the time of puberty has almost disappeared. Although sometimes persistent throughout life, it is usually represented by an insignificant vestige. (It has recently been said that the thymus increases in size and weight up to puberty, and that it is an active organ until the fortieth year, after which time it atrophies.) While the epithelial parts of the thymus body, represented in the fully developed organ by the corpuscles of Hassall, are derived from the entodermal epithelium of the third inner visceral furrow, all other parts, the lymphoid tissue, connective tissue, and blood-

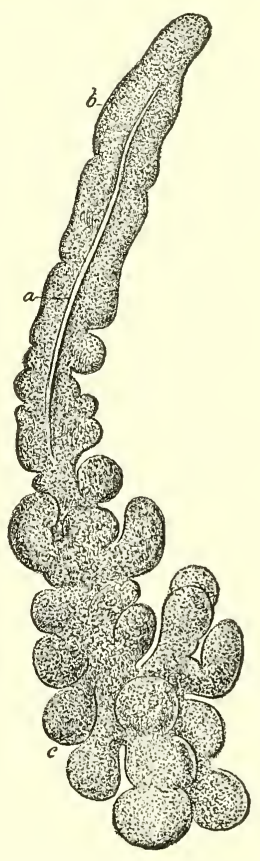

Fif. 115.-Thymus of an embryo rabbit of sixteen davs (after Kölliker), magnified: $a$, canal of the thymus; $b$, upper, $c$, lower end of the organ. vessels, are products of the surrounding mesoderm. 


\section{CHAPTER XIII.}

\section{THE DEVELOPMENT OF THE GENITO=URINARY SYSTEM.}

OwING to the intimate anatomical and functional association of the generative organs with the urinary apparatus, it is necessary to discuss the development of these two systems together.

\section{THE DEVELOPMENT OF THE KIDNEY AND URETER.}

The origin of the kidney and ureter of the higher vertebrates is associated with the development of certain fetal structures, the pronephros and the mesonephros, which represent respectively the kidney of larval amphibians and the permanent kidney of fishes. In man and other allied types, the former structure is of little or no importance functionally, while the latter functionates during a part of fetal life as the organ of urinary excretion, prior to the development of the permanent kidney.

The pronephros or head-kidney constitutes the most primitive vertebrate type of a mechanisin for the excretion of urine. This structure originates in the following manner: When the paraxial mesoderm, which subsequently divides into the somites, is about to separate from the lateral plate of mesoderm, the two parts are connected for a time by an intervening band of tissue, the middle plate or intermediate cell-mass (Fig. 116). The thickening of this intermediate cell-mass produces the Wolffian ridge, which projects into the celom or body-cavity. The mesodermal or mesenchymal elements of the Wolftian ridge become grouped into cords of cells which are in connection at certain points with the mesothelial cells of the colom. The origin of these cell-cords of the Wolffian ridge has long been a matter of dispute, some authorities 
maintaining that they come from the mesothelium of the body-cavity, while other's believe that they are of ectodermic origin. Further changes bring about the hollowing out of the cell-cords so that there results a long tube, the pronephric or segmental duct, which has several sinort transverse tubule:s -in some vertebrates, six; in man, two-opening into it and communicating by their opposite open extremities, the nephridial funnels or nephrostomata, with the cœlom (Fig. 117). In the human embryo the pronephric tubules have been found with open nephridial funnels, but without connection with the pronephric duct. The mesothelium in immediate proximity to the open end of each short tubule is invaginated

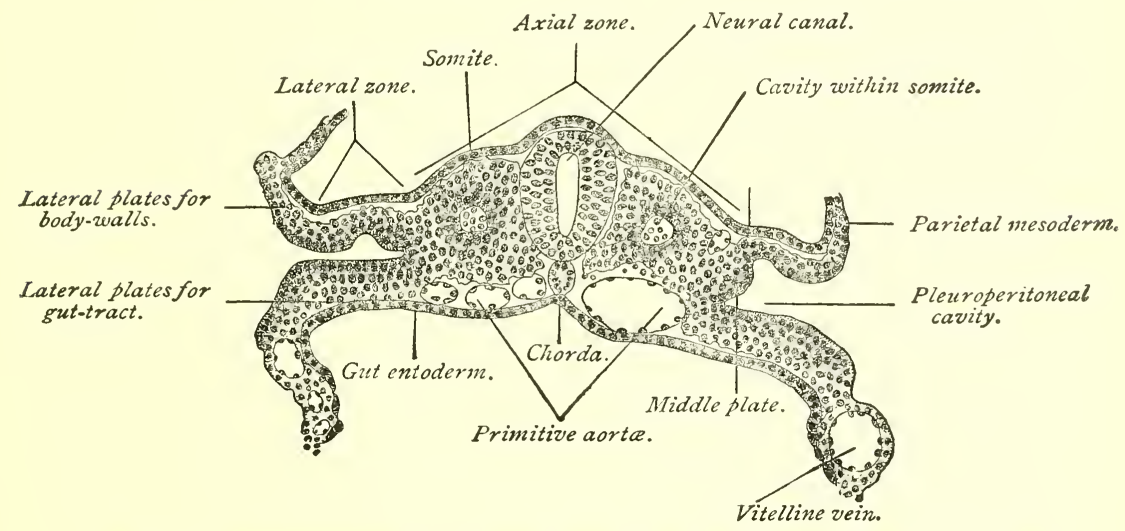

FIG. 116.-Transverse section of a seventeen-and-a-half-day sheep embryo (Bonnet).

by a tuft of capillary blood-vessels from the adjacent primitive aortæ to constitute a glomerulus (Fig. 117, $b b$ ). The pronephric duct passes tailward and opens into the cloaca, a receptacle which receives, in common, the terminal orifice of the primitive bladder and that of the primitive intestine. It is apparent, therefore, that the pronephros or head-kidney is anatomically adapted to the function of removing certain substances from the blood by virtue of the action of the cells surrounding the glomeruli or tufts of capillary blood-vessels, and that these substances may be conveyed away through the duct into the cloaca and thence evacuated from the body. This organ is functionally active, however, only in certain lower 
classes of vertebrates, as in the Amphibia during the larval stage and in bony fishes. In mammals it is exceedingly rudimentary and very soon gives place to a more important organ, the mesonephros.

The Mesonephros or Wolffian Body.-As in the case of the pronephros, the origin of the mesonephros is to be found in the Wolffian ridge. Reference has been made, in treating of the primitive segments, page 77 , to the middle plate (Fig. 116) as a tract of mesodermic tissue connecting the paraxial tract with the parietal plate. When the paraxial mesoderm

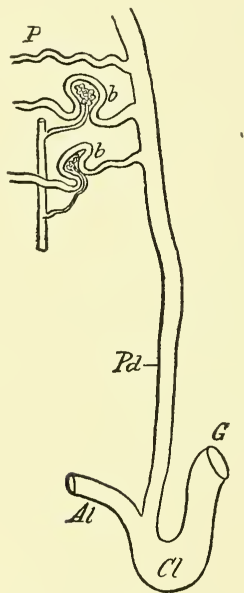

FIG. 117,-Diagram of pronephros $(P)$ and pronephric duct $(P d): A l$, allantois; $G$, gut; $C l$, cloaca; $b b$, glomeruli.

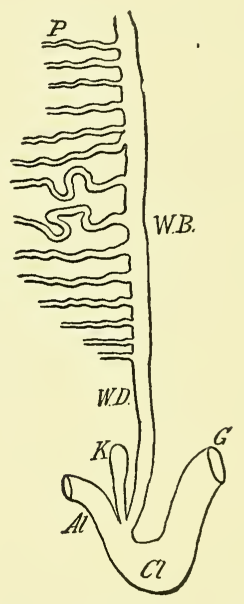

FIG. 118.-Diagram of Wolffian body and duct: $A l$, allantois; $G$. gut; $C l$, cloaca; $K$. kidney evagination.

segments to form the somites the middle plate likewise undergoes segmetation, each segment being designated a nephrotome. Each nephrotome, in the lower vertebrates, contains a cavity which communicates with the general bodycavity and which is, therefore, in effect, an evagination of the mesothelium of this space. In mammals, however, as well as in reptiles and birds, the nephrotome is a solid cord of cells. By the hollowing out of these cell-cords or nephrotomes a series of transversely directed tubules is formed, each nephrotome, in fact, becoming converted into a short 


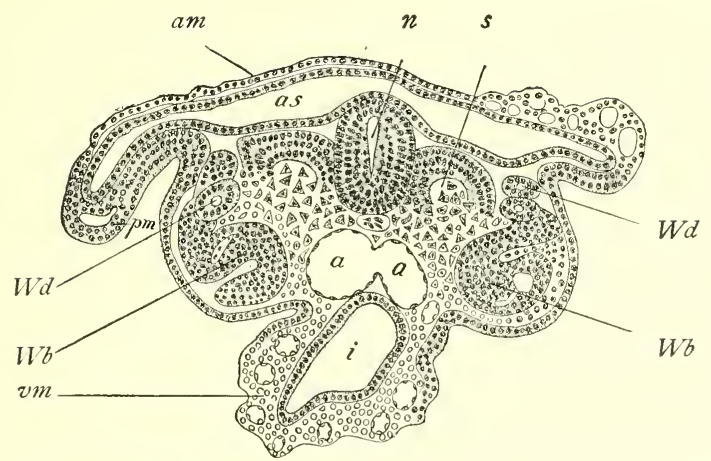

FIG. 119.-Transverse section of seventeen-day sheep embryo (Bonnet): $a m$, amrion; as, amniotic sac; $n$, neural canal; $s$, somite differentiated into muscle. plate; $W d$, Wolffian duct; $W b$, Wolffian body; $p m$, parietal mesoderm; vm, visceral mesoderm; $a, a$, fusing primitive aortæ; $i$, intestine.

canal. These tubes acquire connection by their deeper ends with the previously formed pronephric duct (Fig. 117), which

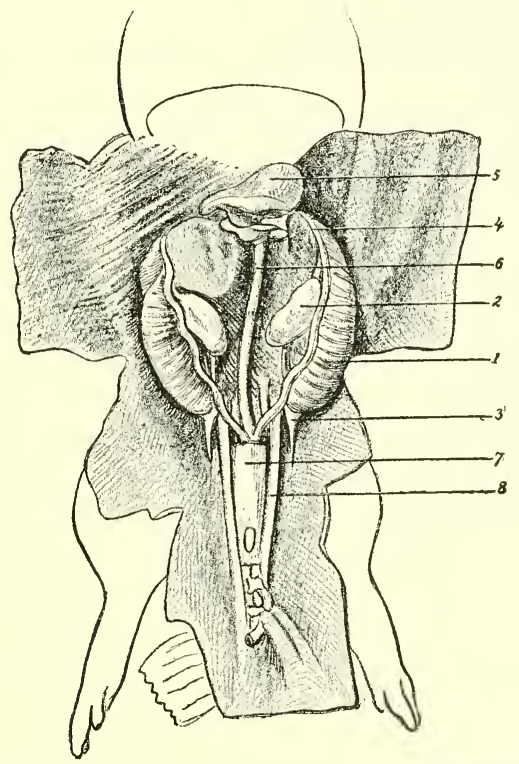

FIg. 120.-Disposition of the genito-urinary organs in the embryo of the hog$5.5 \mathrm{~cm}$ (2.2 in.) long (Tourneux) : 1 , Wolffian body ; 2 , ovary ; 3 , inguinal ligament ; 4 , diaphragmatic ligament; 5 , stomach ; 6 , intestine $; 7$, bladder $; 8$, umbilical artery.

is known hereafter, therefore, as the mesonephric or Wolffian duct (Fig. 118). The latter duct and the short transverse 
tubules which open into it constitute the Wolffian body or mesonephros (Figs. 119 and 120, 1). The tissue of the intermediate cell-mass from which the Wolffian tubules develop is designated, by Sedgewick, the Wolffian blastema, and by Rabl and by Schreiner, the nephrogenic tissue. At this stage of its development the Wolffian body consists of a tube or duct lying behind the parietal layer of the mesoderm, parallel with, and lateral to, the primitive vertebral column, and opening at the caudal end of the embryo into the cloaca; and of a series of transverse Wolffian tubules opening into the duct and abutting by their opposite ends upon the bodycavity. At the head-end of the Wolffian duct the now atrophic pronephric tubes are still in connection with it.

As a further step in the development of an organ adapted to the function of the secretion of urine, each Wolffian tubule becomes somewhat saccular midway between its two extremities, and this dilated part of the tubule is invaginated by the capillary branches of an artery from the aorta. In this manner the cells that line the tubules are brought into relation with the blood of the fetus and acquire at the same time the characters of secreting epithelium. Such an invaginating tuft of capillaries, known as a glomerulus, with its enveloping capsule of Bowman, which latter is the invaginated saccular part of the tubule, constitutes a primitive Malpighian corpuscle, a structure analogous to the Malpighian corpuscle of the permanent kidney. This simple form of the mesonephros is seen as a permanent structure only in some of the lowest vertebrates. In all higher vertebrates it attains to a more complex degree of development, reaching its maximum in man in the seventh week of fetal life. Its complexity is increased by the development of secondary tubules and Malpighian corpuscles connected with those first formed. While at first the number of tubules corresponds with the number of nephrotomes, this correspondence is soon lost by the appearance of the secondary tubules.

The horizontal or transverse tubules of the Wolffian body are divisible into an anterior or upper series, distinguished as the sexual segment, and a lower set of atrophic tubules- 
atrophic for reasons that will appear hereafter. In certain vertebrates that are of higher type than those in which the pronephros functionates, such as adult amphibians and fishes, the Wolffian body persists throughont life as an organ of urinary secretion. In birds and mammals, however, its functional activity is but temporary, since it is supplanted, before the end of fetal life, by the permanent kidney. In man it disappears relatively early, retrogression beginning in the eighth week and the Malpighian bodies having almost disappeared by the fifth month. The presence of the mesonephros as a temporarily functionating organ in birds and mammals, while it is a permanent strusture in certain lower members of the vertebrate series, exemplifies the embryological principle elsewhere referred to, that the higher types pass through stages during their development that are permanent in some of the forms below them in the scale of evolution.

The Metanephros or Permanent Kidney.-While the Wolffian body is temporarily functionating as a kidney, a structure is developing from the lower, caudal end of the Wolffian duct which is to form the permanent organ. It has been stated that the Wolffian duet opens into the cloaca. From the dorsal aspect of this duct, near its cloacal end, a small diverticulum, the kidney evagination (Fig. 118 and Plate VII., 1), grows forth and soon lengthens into a tube which grows headward, dorsomesial to the Wolffian duct, penetrating into the nephrogenic tissue or mesonephric blastema (p. 236).

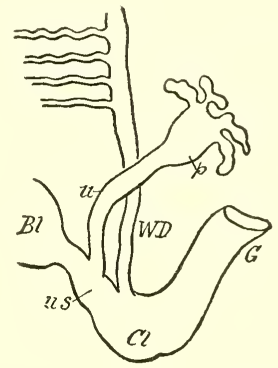

FIG. 121.-Diagram to show extension and branching of kidney evagination and separation of its stalk from the Wolffian duct: $u$, primitive ureter; $p$, pelvis of ureter; $W D$, Wolffian duct ; $B l$, bladder; us, urogenital silus ; $C l$, cloaca; $G$, gut.

The cephalic end of the tube dilates somewhat to form the primary renal pelvis, the anlage of the adult pelvis of the kidney, while the duct itself becomes in time the ureter. From the primary renal pelvis several small diverticula 
pouch out (Fig. 121), while the surrounding blastema becomes condensed and vascular.

The development of the kidney from this stage onward has been for some years a disputed question. According to the older conception, still maintained by Golgi and by Minot, the small tubes which branch from the prinary renal pelvis become the collecting tubules of the kidney and themselves give off branches which, increasing in length and acquiring tortuosity, become the secreting tubules (proximal and distal convoluted tubules, loops of Henle, etc.). The blind end of each convoluted tubule, becoming dilated and saccular, is invaginated by a tuft of capillary blood-vessels, thus being converted into a capsule of Bowman. The invaginating mass of blood-vessels constitutes a glomerulus, and

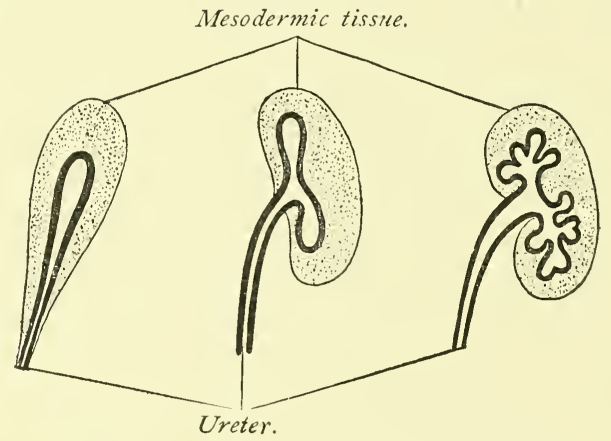

FIG. 122.-Diagrammatic representation of the development of the kidney (after Gegenbaur).

glomerulus and capsule of Bowman together make up a Malpighian corpuscle. Thus the entire system of tubules together with the pelvis and the ureter have a common origin from the caudal end of the Wolffian duet, while the bloodressels and connective tissue, as well as the capsule, originate from the surrounding mesenchyme.

A ceording to the other view-Semper, Sedgewick, Balfour, and others-lately reaffirmed by the researches of Schreiner, whose results have been confirmed for the most part by Huber, the convoluted tubules and the capsule of Bowman originate not as extensions of diverticula from the primitive renal pelvis, but independently from the nephrogenic tissue, 
and in the following manner: The nephrogenic tissue into which the kidney evagination penetrates shows a differentiation into two zones-an inner one, immediately surrounding the primitive renal pelvis, consisting of epithelioid cells, and an outer zone of less differentiated mesenchyme. Soon after the appearance of the small diverticula which evaginate from the primitive renal pelvis and which are designated the primary collecting tubules (which latter correspond with the "primitive renal vesicles" of Haycraft), the nephrogenic tissue breaks up into smaller cell-masses, each such mass surrounding a primary collecting tubule (Fig. 123, $\mathrm{mk}$ ). This part of the nephro-

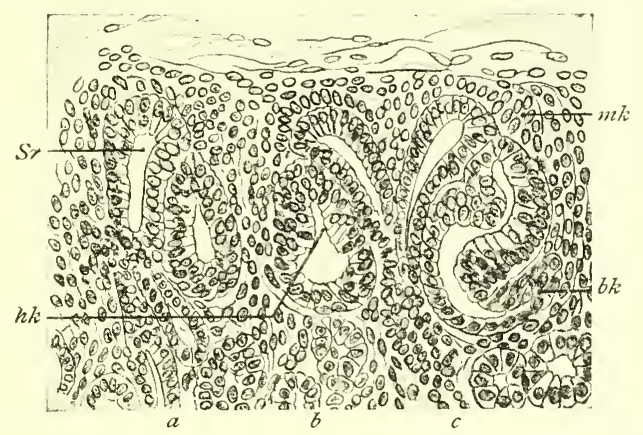

FIG. 123.-Section through the kidney of human fetus of seven montls (from Felix, after Schreiner): $S r$, collecting tubules of which three are shown, each with its cap of metanephrogenic tissue, $m k$; in relation with each is an early uriniferous tubule, the three latter, $a, b, c$, each showing a different stage of development-a, showing beginning of expansion; $b$, evagination at $l k ; c$, S-shaped stage, $b k$ indicating development of Bowman's capsule.

genic tissue Schreiner calls the metanephrogenic tissue by way of distinction from the remaining part of this mesencbymal aggregation which, from its relation to the development of the mesonephros, is called the mesonephrogenic tissue. Each primary collecting tubule, after becoming bulbous at its end, divides into two tubules, each one of which in turn divides into two, this process of division being repeated several times. These tubules become the adult straight collecting tubules. The branching of the primary collecting tubules continues to the time of birth; or until the fifth fetal month, according to Hamburger.

In the development of the secreting tubules the inner zone of nephrogenic or metanephrogenic tissue alone is concerned. 
This tissue presents little bud-like prolongations, each such little bud of eells later acquiring a lumen and separating from the parent tissue (Fig. 123, $a, b, c)$. The buds are now small sacs, the renal vesicles (Emery), of which there are at least two for each collecting tubule. Each vesicle now elongates and assumes an S-shaped form, the concavity of the upper part of the looking toward the collecting tubule,

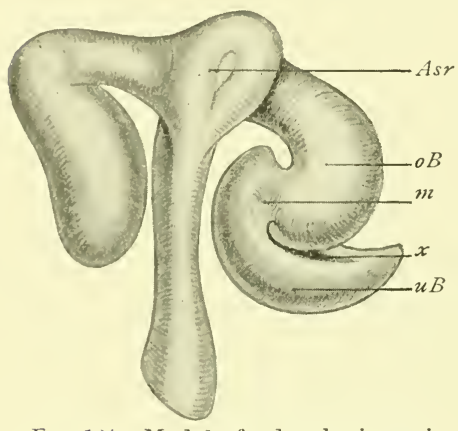

FIG. 124.-Model of a dereloping uriniferous tubule of the human kidney (from Felix, after Stoerk): $A s r$, ampulla of collecting tubule: $o B$, upper limb of $\mathrm{S}$; $u B$, lower limb of S (Bowman's capsule); $x$, position occupied by glomerulus ; $m$, middle-piece of $\mathrm{S}$. the vesicle on the right of the tubule being, therefore, a reversed S (Fig. 124). The lower limb of the $S$, from being simply tubular, becomes expanded, its upper wall being indented (Fig. $124, x)$, so that it acquires the shape of a double-layered saucer, the space between its two layers being continuous with the remaining part of the lumen of the $\mathrm{S}$ tube. In the concavity of the saucer, just beneath the middle piece of the S, a strand of mesenchyme makes its appearance and into this tissue blood-vessels penetrate, so that it finally becomes the glomerulus of the Malpighian corpuscle. The saucer-shaped lower limb of the $S$ becomes the capsule of Bowman; the lumen of the saucer, the space of Bowman. Meanwhile the upper limb of the $S$ acquires continuity with the collecting tubule in close relation with which it has developed, and the two extremes of the $S$ being thus relatively fixed points, the ensuing elongation of the intervening portion necessitates the formation of curvatures. A small part of the lower limb of the $S$, not being concerned in the formation of the saucer-shaped anlage of Bowman's capsule, becomes a part of the proximal convoluted tubule, the remaining portion of the latter, and the succeeding loop of Henle, the distal convoluted tubule and the arched collecting tubule, being developed respectively from the succeding parts of the S.

The outer zone of the metanephrogenic tissue gives rise to 
the capsule of the kidney and the supporting connective tissue, including the columns of Bertini.

The kidney acquires its characteristic features by the end of the second month of fetal life, and it reaches its permanent position by the third month.

\section{THE SUPRARENAL BODIES.}

The development of these structures has been the subject of much discussion. It has been maintained, on the one hand, that the cortex of the organ develops either directly or indirectly from the mesothelium of the body-cavity and that the medulla has its origin in outgrowths from the sympathetic ganglia; on the other hand, that the entire organ is a product of the mesenchyme-indirectly, therefore, of the mesothelium. Thus Minot, Human Embryology, 1892, remarks, "That both the cortex and the medulla of the adult organ are formed in man from the mesenchymal cells, as Gottschau showed was the case in several mammals, is, I think, beyond question"; but in his Laboratory Text-book of Embryology, 1902, p. 267, the same authority, in describing pig-embryos, says: "The sympathetic tissue gives rise to the so-called medulla of the adult organ." Again, Aichel, 1900, from his investigations concluded that both medulla and cortex arose from the mesenchyme. O. Hertwig, in his Lehrbuch, 1906, expresses his conviction of the correctness of Poll's ${ }^{1}$ conclusions as to the double origin of the organ.

The cortex, according to Poll, whose work reaffirms in many particulars the conclusions of some earlier investigators, arises from small bud-like masses of cells that come from the mesothelium of the cœlom in close relation with the genital gland and the mesonephros, but distinct from them. These buds lose their connection with the colomic epithelium by the fifteenth day in the chick. They are situated on each side of the root of the dorsal mesentery and all those of one side unite to form a single organ. This occurs in man by

${ }^{1}$ H. Poll, Die Entwicklung der Nebennieren Systeme, Handbuch der vergleich. und experim. Entwicklungslehre d. Wirbeltiere, Bd. III., Abt. 1, 1905 . 
the twenty-eighth day (Soulié). In the lower vertebrates this organ fails to unite with the anlage which represents the medulla of the higher vertebrate suprarenal body and constitutes the separate interrenal organ of the lower vertebrates. In some cases-e.g., in sharks-the anlages of the two sides unite with each other, forming a single unpaired interrenal organ, which lies between the primitive kidneys. Failure of union of some of the buds, it is believed, is responsible for the accessory suprarenal organs of Marchand which are occasionally found between the layers of the broad ligament of the female or in relation with the epididymis of the male, these having followed the descent of the testis or ovary respectively.

The medulla of the organ, still following Poll's account, originates at a later stage than the cortical anlage from chains of cells that grow forth from the sympathetic ganglia and form groups which for a time retain their connection with the ganglia. The cells show a differentiation into two classes, the sympathoblasts and the phæochromoblasts, the latter gradually becoming the phæochrome cells, so called from their staining darkly by chromium salts. In many vertebrates these cell-masses remain separate from the interrenal anlage and constitute the phæochrome bodies or suprarenal bodies of lower vertebrates. In birds and reptiles the union of the phæochrome and interrenal anlages occurs by a mutual intergrowth of their cells, the result being an irregularly stratified organ; in mammals and man, however, the cells of the sympathetic anlage gradually penetrate in the form of cell-cords into the interior of the interrenal anlage to occupy their adult position as the medulla of the organ. This process of intergrowth continues in man until the time of birth.

In the early stages of fetal life the suprarenal body is relatively much larger than in the adult condition, and is situated chiefly on the ventral surface of the kidney. At about the third month it begins to assume more nearly its normal position.

The account of the development of the bladiler and of the urethra may be deferred until the evolution of the internal sexual system shall have been considered. 


\section{THE DEVELOPMENT OF THE INTERNAL GENERATIVE ORGANS.}

The Indifferent Type.-The internal generative organs of both sexes, in the course of their development, pass through a stage in which there is to be found no distinction of sex. This stage is designated, therefore, the indifferent type of sexual apparatus.

While the Wolffian body is attaining its full development, there appears in its vicinity a tube, the duct of Muiller (Plate VII., Fig. 1), which lies parallel with, and to the outer side of, the Wolffian duct. In non-amniotic vertebrates the duct of Müller arises by fission or longitudinal division of the mesonephric duct. Its exact mode of origin in the amniotic vertebrates is not as yet definitely settled. According to one view its upper or cephalic portion is produced by an evagination of the mesothelium of the body-cavity, while the remaining lower segment results from fission of the mesonephric or Wolffian duct. According to another view the lower or caudal portion is produced by the direct extension of the upper portion in the caudal direction by the proliferation of its own cells. In whatever way the duct may be formed, its lower or caudal end opens into the cloaca, which receptacle receives also the termination of the Wolffian duct. The upper end of the duct maintains a communication with the body-cavity or colom by means of an expanded funnelshaped mouth. Its lower segment is closely associated with its fellow and with the Wolffian ducts, forming thus the genital cord. The function of this canal in lowly organized animals-that of receiving from the body-cavity the female genital products, the ova, and evacuating them from the bodyforeshadows its subsequent metamorphosis in most vertebrates.

While the duct of Müller is forming, the mesothelial cells overlying that part of the free surface of the Wolffian body which looks toward the median plane and somewhat forward, its ventro-mesial aspect, undergo multiplication and thickening (Fig. 125, a), forming an elongated swelling or ridge. This is known as the genital ridge, which produces a projection upon the wall of the body-cavity. 'The genital ridge is still further thickened by the proliferation of 
the mesodermic tissue $(E)$ beneath the mesothelial cells. The genital ridges of the human fetus appear in the fifth week.

Further differentiation of the genital ridge results in its transformation into the so-called indifferent sexual gland (Plate VII., Fig. 1), a structure common to both sexes at this stage. The essential feature of this process is that the thickened mesothelial cells overlying the genital ridge become modified in character and penetrate the ridge in the form of cords or strands of cells. These mesothelial elements were called by

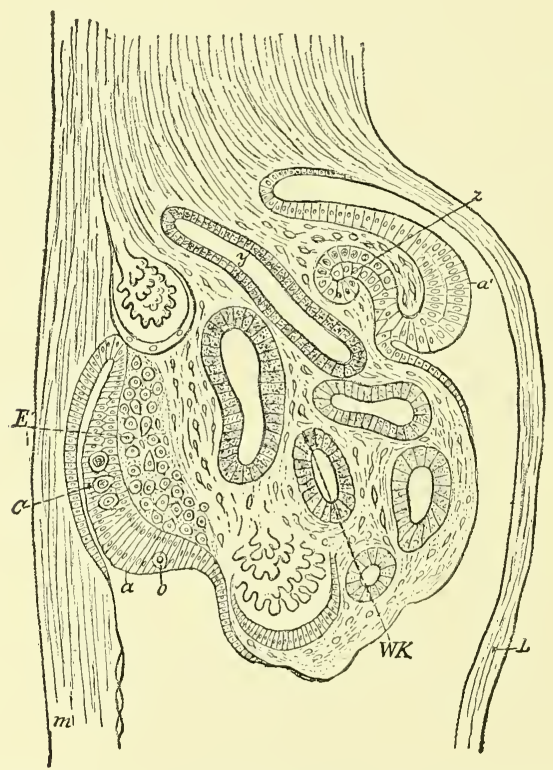

Fig. 125.-Cross-section through the mesonephros, the fundament of the Müllerian duct, and the sexual gland of a chick of the fourth day (after Waldeyer), magnified 100 diameters: $m$, mesentery; $L$, somatopleure; $a^{\prime}$, the region of the germinal epithelium from which the Müllerian duct $(z)$ has been invaginated; $a$, thickened part of the germinal epithelium, in which the primary sexual cells, $C$ and $o$, lie; $E$, modified mesenchyme out of which the stroma of the sexual gland is formed; $W K$, mesonephros; $y$, mesonephric duct.

Waldeyer the germinal epithelium, because, after their extension into the interior of the ridge or gland, they give rise to the germ-cells, namely, the ova or the spermatozoa as the case may be. The cell-cords include two kinds of elements, the smaller mesothelial cells and the primitive sexual cells, which latter are larger and less numerous than the mesothelial cells 
PLATE VII.
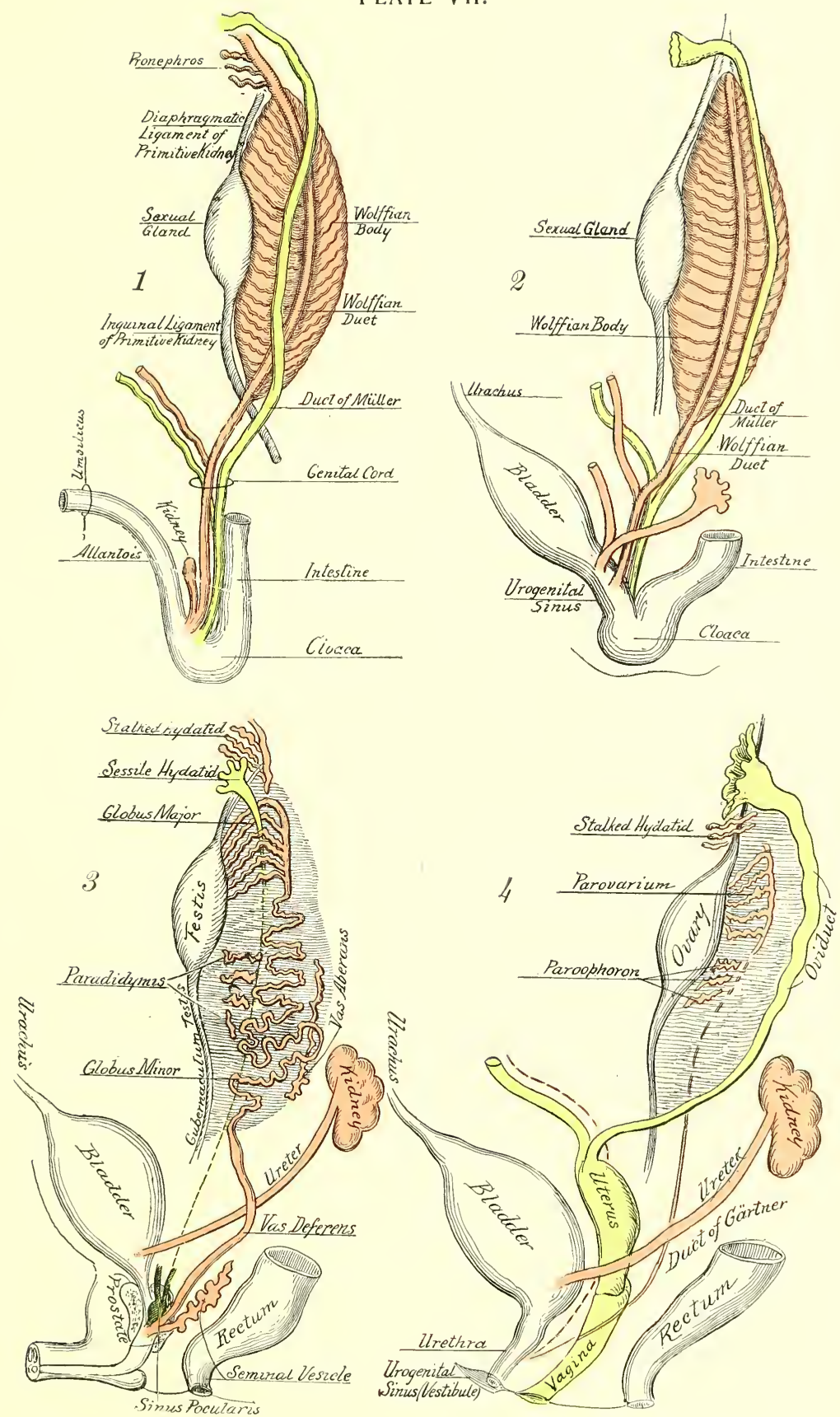

Diagrammatic representation of the development of the genito-urinary system, the Wolffian body and its derivatives being colored red, the Müllerian duct and its derivatives, green: 1 , indifferent type; 2 , indifferent type, later stage, the Wolffian and Müllerian ducts and the primitive ureter now opening into the urogenital sinus: 3 , male type, lower ends of Müllerian ducts fused to form the sinus pocularis; 4 . female type. 

and have large nucleolated nuclei. The primitive sexual or seminal cells, or primitive ova, are so called because it has been assumed that they develop either into the ova or the seminal filaments according to the sex of the embryo. The cell-cords have been seen in the indifferent gland of the human embryo as early as the fifth week. At this time, although there are no gross sexual distinctions recognizable, it is possible to determine from the histological characters of the organ whether it is to be a testis or an ovary, the large sexual cells being far less numerous relatively in the former case than in the latter (Nagel).

The indifferent sexual gland comes into an especially close relation with the upper or sexual series of the mesonephres or Wolffian body (Plate VII., Fig. 1), the significance of which fact will appear later.

The elements of the indifferent stage of the sexual apparatus are, therefore, the indifferent sexual gland, the Wolffian duct, and the duct of Müller (Plate VII., Figs. 1, 2). From this asexual stage, either the male or the female type is produced by the metamorphosis of the indifferent glands into the testicles or the ovaries and the formation of ducts to provide for the escape of the sexual elements, the spermatozoa, or the ova, produced by them.

The Male Type of Sexual System.-The differentiation of the indifferent sexual system into the male type is effected by the further development of some parts and the atrophy or the arrested growth of others.

The testicle has a double origin, since the proper secretory part of the organ is produced by the metamorphosis of the indifferent sexual gland, while its system of efferent ducts is furnished by the Wolffian body. Mention has been made of the cell-cords of the indifferent sexual glands and of their origin from the mesothelium of the borly-cavity, and also of the fact that they consist of the smaller mesothelial cells and the larger and less numerous primitive sexual cells.

The mesothelial cells increase in number and become so grouped as to form cylindrical masses known as sexual cords, each of which includes some of the primitive seminal or 
sexual cells. By the ingrowth of connective tissue from the surrounding mesoderm, the tunica albuginea is formed and the sexual cords are divided into roundish masses, each of which is made up of many of the smaller elements and a less number of the large seminal cells. These follicle-like masses become hollowed out to form the seminal ampullæ, which afterward, undergoing great increase in length, are transformed into the seminiferous tubules. During fetal life, however, and even to the period of puberty the "tubules" remain solid cords of cells. The small mesothelial or epithelial cells give rise to Sertolli's columns, while the primitive seminal cells produce the spermatogonia.

Spermatogenesis, or the development of the spermatozoa from the cells that line the seminiferous tubules of the functionating testicle, has been considered in Chapter I.

While the sexual cords are being transformed into the cylinders that become the seminiferous tubules, the surrounding mesodermic tissue penetrates the genital gland and forms the connective-tissue septa that constitute the stroma of the organ and divide it into lobules. At the same time, also, marked changes occur in the Wolffian body. From certain of the Malpighian corpuscles of this structure, cords of cells, the medullary cords, grow forth and penetrate the genital gland, their ends fusing with the primitive seminiferous tubules. The conversion of these cell-cords into tubes furnishes the initial part of the system of excretory ducts of the testicle, namely, the vasa recta and the rete testis. Somewhat later, in the twelfth week, the rete testis is extended to form the vasa efferentia, and still later, in the fourth and fifth months, the efferent vessels lengthen and become tortuous, producing thereby the coni vasculosi or head of the epididymis (Plate VII., Fig. 3; Fig. 126). The upper part of the Wolffian duct develops into a convoluted tube which constitutes the body and tail of the epididymis, while the lower portion becomes the vas deferens, thus completing the system of canals provided for the escape of the spermatozoa. Near the caudal end of the Wolffian duct a little pouch-like evagination grows from its wall and becomes 
the seminal vesicle, the lower end of the duct, below the orifice of the seminal vesicle, being the ejaculatory duct. Since the Wolffian duct terminates in the cloaca, and since the anterior part of the cloaca corresponds to a portion of the later urethra, the termination of the ejaculatory duet in the prostatic part of the urethra is accounted for. Thus it will be seen that while the secreting part of the testicle results from the transformation of the indifferent genital gland, the secretory cells having their origin in the germinal epithelium, the complicated system

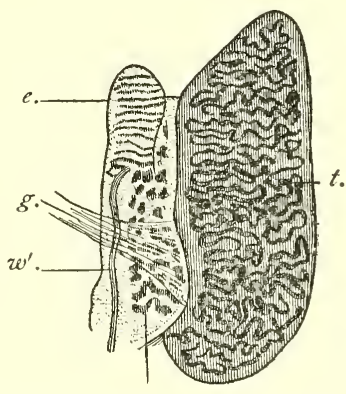

$w$.

FIG. 126,-Internal generative organs of a male fetus of about fourteen weeks (Waldeyer): $t$, testicle; $e$, epididymis; $w^{\prime}$, Wolffian duct; $w$, lower part of Wolffian body ; $g$, gubernaculum testis. of ducts with which it is provided is furnished by the mesonephros or Wolffian body.

The series of tubules connected with the upper extremity of the Wolffian duct, the remnant of the pronephros or headkidney, frequently persists as a little pedunculated sac attached to the upper part of the epididymis; it is known as the stalked hydatid and sometimes also as the hydatid of Morgagni. The posterior or lower set of Wolffian tubules likewise give rise to an atrophic structure, the paradidymis or organ of Giraldès, which consists of a series of short tubes closed at both ends, lying among the convolutions of the tail of the adult epididymis (Plate VII., Fig. 3), while a lateral evagination from that part of the Wolffian duct which forms the tail of the epididymis becomes the vas aberrans.

The duct of Muiller remains atrophic, in the male, throughout its entire extent, and in fact, with the exception of its two extremities, it usually altogether disappears. Its upper extremity persists as a small vesicle, the unstalked or sessile hydatid, attached to the upper aspect of the testicle. The lower extremity of the duct, uniting with its fellow, becomes converted into the sinus pocularis or uterus masculinus of the prostate gland (Plate VII., Fig. 3). If the intervening part 
of the tube persists to post-natal life and remains patulous, it is known as the duct of Rathke.

The change of location which the testicle undergoes is a conspicuous feature of its development. To understand this clearly, it is necessary to recall the relation of the mesonephros and the genital gland to the peritoneum. Since both of these bodies originate from the cells of the outer wall of the body-cavity, or, in other words, from what becomes the parietal peritoneum, necessarily they lie between the body-wall and the parietal peritoneum-that is, behind the peritoneal eavity. With the increase in size of these structures, they project toward the peritoneal cavity, the peritoneum passing over them and forming a "mesentery," which anchors them to the posterior wall of the abdomen. In the case of the testicle, this peritoneal fold or " mesentery" is called the mesorchium; in the case of the ovary, the mesovarium. It is prolonged upward to the diaphragm as the diaphragmatic ligament of the primitive kidney, and downward to the inguinal region as the inguinal ligament of the primitive kidney (Fig. 120), since this latter organ is the largest constituent of the projecting mass. When the primitive kidney has disappeared as such, the inguinal ligament mentioned seems to connect the ovary or testicle with the inguinal region of the abdominal wall.

The inguinal ligament contains between its folds connective tissue and unstriped muscular fibers. These become the gubernaculum testis in the male or the round ligament of the uterus in the female. As the body of the fetus continues to grow while the tissues of the ligament remain stationary or grow less rapidly, the testicle is gradually displaced from its position at the side of the lumbar spine, and by the third month reaches the false pelvis. In the fifth and sixth months it is in contact with the anterior abdominal wall, near the inner abdominal ring. In the eighth month it enters the inguinal canal, and near the end of the ninth month, shortly before birth, it leaves the inguinal canal and enters the scrotum. ${ }^{1}$

${ }^{1}$ Non-descent of the testicles, with consequent emptiness and flabbiness 
Before the testicle leaves the abdominal cavity, the parietal peritoneum pouches through the inguinal canal into the scrotum, the protruded part being the processus vaginalis. Since the testicle is from the first behind the parietal peritoneum, it passes into the serotum behind the vaginal process, the latter then folding around it as a shut sac of two layers. Subsequently the connection of the sac, now the tunica vaginalis testis, with the abdominal peritoneum is reduced to a slender strand of tissue lying in front of the spermatic cord. ${ }^{1}$

The testicle necessarily carries with it, in its descent, its blood-vessels, the spermatic artery and vein; its duct, the vas deferens; as well as its nerves and lymphatic vessels; and these structures collectively constitute the spermatic cord.

The Female Type of Sexual System.-While the indifferent sexual gland, in the development of the male generative system, undergoes metamorphosis into the testicle, it becomes, in the evolution of the female type, so altered as to constitute the ovary; and while the Wolffian tubules and the Wolffian body become in the male the system of excretory ducts of the testicle, they produce in the female merely atrophic structures. On the other hand, the duct of Müller, which gives rise in the male to atrophic appendages, forms in the female type the Fallopian tube and, by fusing with its fellow of the opposite side, the uterus and the vagina.

The ovary results from alterations in the structure of the genital gland analogous to those that occur in the evolution of the testicle. The special features of these changes are better understood, however, than are those of the testicle. As in the case of the development of the testicle, the mesothelial cells on the peritoneal surface of the genital ridge become thickened, these altered cells constituting the germinal

of the scrotum, is designated cryptorchism (hidden testes). The presence of but one testicle in the scrotum is called monorchism.

${ }^{1}$ Occasionally it happens that the funicular process of the tunica vaginalis-that is, the stalk of the sac, remains patulous throughout its entire extent, a condition which allows of the easy and sudden protrusion of a segment of the bowel into the cavity of the tunica vaginalis, constituting the so-called congenital hernia. Or the funicular process may close only at one or the other end, giving rise to other varieties of hernia. 
epithelium (Fig. 125). Coincidentally, the primitive connective tissue-mesodermic tissue-underlying the germinal epithelium proliferates, contributing to the thickness of the genital ridge. By the sixth

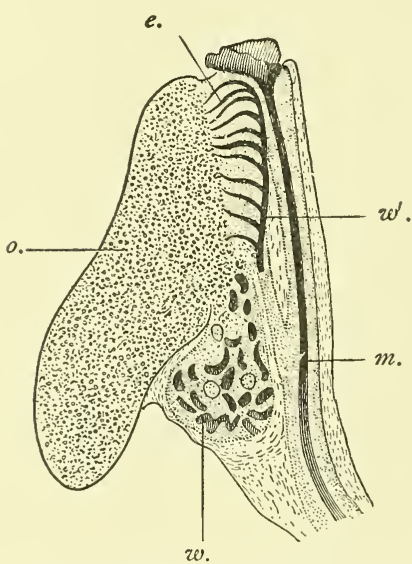

FIG. 127.-Internal organs of a female fetus of about fourteen weeks (Waldeyer): $o$, ovary; $e$, epoöphoron or parovarium ; $w^{\prime}$, Wolffian duct; $m$, Müllerian duct; $w$, lower part of the Wolffian body. or seventh week, the germinal epithelium consists of several strata of cells, groups of which begin to penetrate the underlying mesodermic tissue in the form of cordlike processes (Fig. 128, $e$, sch). The indifferent mesodermic tissue at the same time increases in quantity, in turn penetrating between the groups of advancing cells, so that what takes place might be described as a mutual intergrowth. The presence of the growing connective tissue accentuates the grouping of the cells into cylindrical masses. These latter are the sexual cords or egg-columns (Pflüger's egg-tubes). They contain two special kinds of cells, the large sexual cells or primitive ova (Fig. 128, ue), and the smaller but more numerous mesothelial cells. The connection of the sexual cords with the germinal cpithelium is much more obvious in this case than in the case of the developing testicle, and the primitive sexual cells are much more abundant. The egg-columns, surrounded by young connective tissue, constitute the nucleus of the cortical part of the future ovary. This mass is later sharply marked off from the free or peritoneal aspect of the gland, the region of the germinal epithelium, by a zone of proliferating mesodermic cells which become the tunica albuginea of the ovary.

An important change now takes place in the egg-columns; the primitive ova, or large sexual cells, increase in size, their nuclei becoming especially well developed, while the small 
mesothelial cells become smaller and less conspicuous. It frequently happens that several of the large cells fuse into a single mass of protoplasm, while one of the nuclei outstrips the others in growth and, with the surrounding zone of protoplasm, becomes the ovum. Each egg-column is now broken up into several groups of cells by the penetration of connective tissue, each group (Fig. 128, e, sch') containing a single orum, but many of the smaller cells.

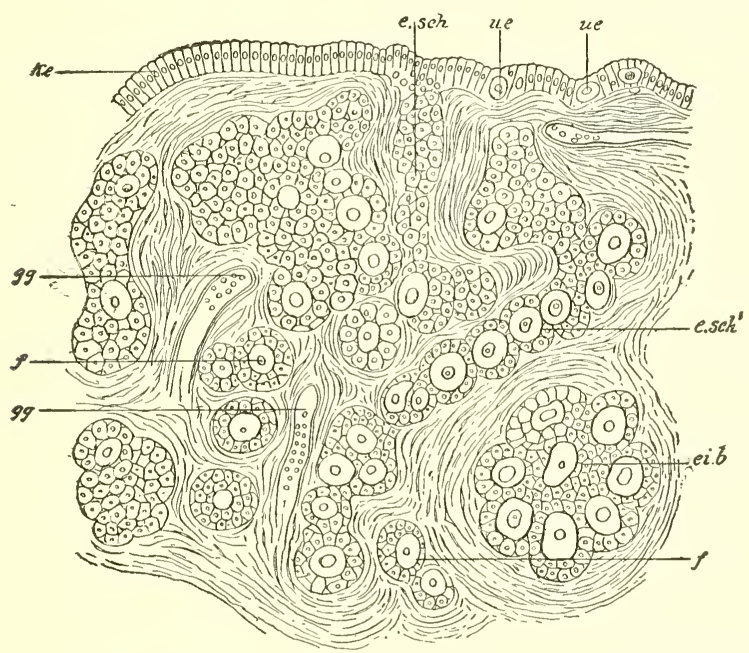

FIG. 128. - Part of sagittal section of an ovary of a child just born (after Waldeyer). Highly magnified: $k e$, germinal epithelium; $e, s c h$, Pflüger's egg-tubes; $u e$, primitive ova lying on the germinal epithelium; $e, s c h$, long Pflüger's tubes, in process of being converted into follicles; $e i, b$, egg-balls (nests), likewise in process of being resolved into follicles; $f$, youngest follicle already isolated: $g g$, bloodvessels. In the tubes and egg-nests the primordial eggs are distinguishable from the smaller epithelial cells, the future follicular epithelium.

These groups are the young Graafian follicles of the ovary $(f)$. The enveloping zone of connective tissue becomes the theca of the follicle, while the single large cell constitutes the ovum, and the smaller cells are the membrana granulosa. At first the granulosa cells surround the ovum as a single layer of flattened cells which gradually assume the columnar type and become so numerous as to form many layers. They secrete a fluid, the liquor folliculi, which crowds the ovum to one side of the follicle where it is enveloped by a special group of granulosa-cells, the discus proligerus (Fig. 129). 
The question of the origin of the follicular cells is still an unsettled one, though it secms probable that they are derived from the cells of the egg-columns, and Minot believes that they are probably descended from the primitive ova.

The formation of new Graafian follicles, and consequently of ova, begins in the deeper part of the ovary and advances toward the surface. The production of ova and follicles is

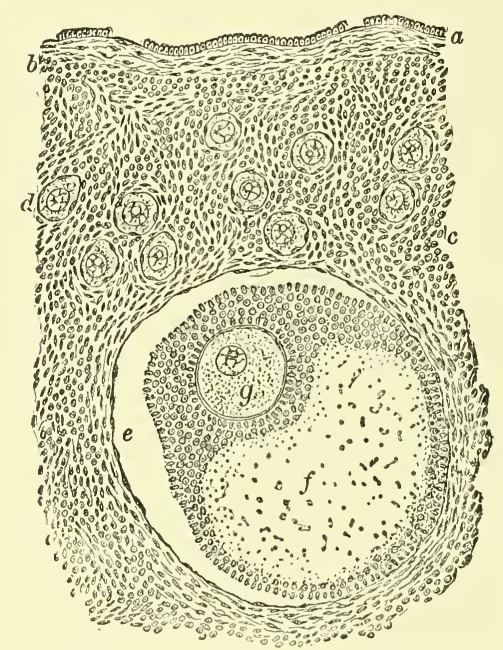

FIG. 129.-Section of human ovary, including cortex : $a$, germinal epithelium of free surface; $b$, tunica albuginea; $c$, peripheral stroma containing immature Graafian follicles $(d)$; $e$, well-advanced follicle from whose wall membrana granulosa has partially separated; $f$, cavity of liquor folliculi; $g$, ovum surrounded by cell-mass constituting discus proligerus (Piersol).

limited to the fetal stage and to the early part of post-natal life, their formation not occurring, according to Waldeyer, after the second year.

What has been said above refers to the development of the cortex of the ovary. The medulla is produced by the growth toward the egg-columns of cord-like processes, the medullary cords, from the epithelial walls of the Malpighian corpuseles of the primitive kidney or Wolffian body, the cords becoming surrounded by connective tissue and forming a network. The fetal medullary cords are represented in both the cortex and the medulla of the mature ovary by the groups of interstitial cells disposed between the bundles of the stroma-tissue. 
The Oviducts, the Uterus, the Vagina.-The system of passage-ways that constitute the outlets for the ova and the means of nourishing them and evacuating the product of gestation from the body in the event of impregnationnamely, the Fallopian tubes, the uterus, and the vagina-result from the metamorphosis of the ducts of Müller. These ducts, as stated above, lie along the dorsal aspect of the body-cavity, separated from it by the parietal peritoneum, and parallel with the primitive spinal column (Plate VII.). The probable method of their formation has been pointed out (p. 243). Near the Iower (caudal) end of the body they approach each other, and finally unite about the second month to form a single duct for the rest of their extent (Plate VII., Fig. 4). The upper, ununited parts of the ducts become the Fallopian tubes or oviducts, while the lower portions, now fused into one, become the uterus and the vagina. The upper end of each single duct expands trumpet-like to form the fimbriated extremity of the Fallopian tube.

Until the fifth month there is no distinction between the vagina and the uterus, the two being represented by a single sac-like structure. The development of a circular ridge in the wall of the sac marks the division between the two organs, the part above the ridge acquiring thick muscular walls, while the part below it, the future vagina, remains thin-walled and more capacious. In the third month the uterus is bifid at its upper extremity, a condition which is permanent in some animals and occasionally in the human subject. ${ }^{1}$

The Wolffian duct, which, in the male, becomes metamorphosed into a part of the epididymis and the vas deferens, remains undeveloped in the female, producing merely atrophic or vestigial structures (Plate VII., Fig. 4). The upper series of Wolffian tubules, the remnant of the pronephros, frequently

${ }^{1}$ The formation of the uterus and of the vagina by the coalescence of two parallel tubes affords an explanation of the uterus bicornis or bifid uterus and of the condition of double uterus sometimes met with, as also of the presence of a median septum in the vagina, since by the failure of union of the two tubes in greater or less degree one or other of these anomalies would result. 
persists, as in the male, in the form of a small pedunculated sac, the stalked hydatid or hydatid of Morgagni. When present, it is to be found in the broad ligament, in the neighborhood of the outer extremity of the ovary. The middle or sexual series of the Wolffian tubules with the adjacent part of the Wolffian duct, which, in the male type, develop into the epididymis, become in the female, an atrophic structure known as the epoöphoron or parovarium, or organ of Rosenmüller (Fig. 127). This structure, which is almost constantly found between the layers of the broad ligament in close proximity to the ovary, eonsists of a larger horizontal tube representing a segment of the Wolffian duct, and of shorter vertical tubes joining this at a right angle and representing the transverse Wolffian tubules. The lower set of small Wolffian tubules, those which, in the male become the paradidymis, give rise in this case to a similar atrophic body, the paroöphoron. This is also situated in the broad ligament, usually to the inner side of the ovary. The Wolffian duct, with the exception of that portion of it that assists in the formation of the parovarium, usually entirely disappears. Occasionally, however, it persists as a small canal traversing the broad ligament close to the uterus and passing on the dorsal side of the upper part of the ragina to be lost upon the wall of the latter or, more rarely, to open near the urinary meatus. When thus persistent, it is known as the duct of Gartner.

The change of position of the ovaries is similar to, though less marked than, that of the testes. The inguinal ligament in the female (Plate VII.) extends from the primitive position of the ovaries in the lumbar region of the abdominal eavity to the groin, where it passes through the abdominal wall, traversing the inguinal canal, to terminate in the labium majus. The upper part of this ligament, containing involuntary muscular substance, firmly unites with the ovary. In the third month the ovary descends to the lower part of the abdominal eavity and is now connected, by the succeeding portion of the inguinal ligament, with the uterus. This connection may be a factor in the final change of position of the 
ovary - that is, its descent into the true pelvis. The part of the inguinal ligament that passes from the ovary to the uterus is the permanent ligament of the ovary, while the remaining portion, which passes from the uterus through the inguinal canal to the labium majus of the vulva, is the round ligament of the uterus. As the inguinal ligament perforates the abdominal wall, a small diverticulum of peritoneum goes with it. Normally this peritoneal pouch subsequently becomes obliterated. Occasionally, however, it persists and then constitutes the canal of Nuck. Should the canal of Nuck be present, the ovary may pass into or through it, thus reaching the labium majus. A patulous canal of Nuck, as in the case of a patulous funicular process of the tunica vaginalis of the male, may permit the sudden occurrence of an inguinal hernia in the female. The mesovarium or "mesentery" of the ovary accompanies the ovary in its descent and constitutes a fold of peritoneum which envelops not only the ovary but also the adjacent part of the duct of Müller and the remnants of the Wolffian body. Upon the uniting of the lower parts of the Müllerian ducts to form the uterus and the vagina these mesovaria unite with each other mesially to become the broad ligament of the uterus. Thus it comes about that the uterus, the ovaries and their ligaments, the epoophoron, and other fetal remnants are contained between the layers of the broad ligament.

The account of the development of the external genital organs will be deferred until after the consideration of the formation of the urinary bladder and of that part of the urethra that originates from the same embryonic structure.

\section{THE BLADDER AND THE PROSTATE GLAND.}

As stated in Chapter V., the urinary bladder and a part of the urethra are derived from the intra-embryonic portion of the allantois. In the same chapter the allantois was described as a sac which developed as a pouching-out of the ventral wall of the gut-tract near its caudal end (Plate II., 5 and 6). The sac protrudes from the still widely open 
abdominal cavity, enters the extra-embryonic part of the body-cavity, and reaches the inner surface of the false amnion, with which structure it intimately unites to form the true chorion (Plate III.). As the walls of the abdomen gradually close, leaving only the umbilical aperture, it is, necessarily, through this aperture that the allantois protrudes.

We have seen (p. 91) what becomes of the extra-abdominal part of the allantois-in what degree it contributes to the formation of the placenta and of the umbilical cord. Obviously, with the severing of the umbilical cord after birth, all this extra-embryonic part of the allantois disappears, giving rise to no adult organ.

Its intra-embryonic portion consists of a tube extending from the caudal end of the intestine to the umbilicus (Plate II., 5 and 6). As early as the second month, the middle segment of this tube dilates and assumes the form of a spindleshaped sac, which becomes the urinary bladder (Plate VII.). The part of the tube connecting the summit of this sac with the umbilicus remains small, gradually loses its lumen, and constitutes in the adult the (usually) impervious cord known as the urachus. Should the cavity of the urachus persist in its entirety, and should there be at the same time an external opening at the umbilicus, the condition would constitute an umbilical urinary fistula. The proximal part of the allantois - that is, the portion intervening between the bladder and the intestine-is designated the sinus urogenitalis, while the caudal end of the intestine, which is, in effect, a ponch in which both the allantois and the intestine terminate, is known as the cloaca (Fig. 96). The urogenital sinus receives the terminations of both the Müllerian and the Wolffian ducts (Plate VII.).

In the sixth week or slightly earlier, there appears upon the surface of the body, in the region corresponding to the position of the cloaca, a depression, the cloacal depression (Fig. 96), which later, except in man and the higher mammals, meets the cloaca, and thus establishes a communication between it and the exterior. In the Amphibia, in reptiles, and in birds, 
as also in the lowest mammals, the monotremes, the cloaca is a permanent structure, and through it, in these groups of animals, not only the fecal matters and the urine, but also the genital products, the spermatozoa and the ova, are evacuated from the body. In all mammals, however, with the exception of the monotremes, the cloaca undergoes division into a posterior part or anal canal and an anterior urogenital aperture. This division is brought about by the growth of three ridges or folds, of which one springs from each side of the cloaca and one from the point of union of the urogenital sinus and the intestine. These folds coalesce about the eighth ${ }^{1}$ week to form a complete septum, which continues to thicken antero-posteriorly up to the time of birth and constitutes the perineum.

It will be remembered that the ureters originally spring from the terminal parts of the Wolffian or mesonephric ducts (Fig. 118), Owing to alterations brought about by processes of unequal growth, the orifices of the ureters subsequently change their position so as to open into the urogenital sinus (Fig. 121), and still later, by the further operation of the same agency, they come to open into the bladder on its dorsal wall, thus gradually assuming their permanent relations (Pl. VII.). After the division of the cloaca the urogenital sinus, as stated above, opens independently upon the surface of the borly. In the female it is transformed into a short tube, the urethra, and an expanded terminal recess or fossa, the vestibule of the vulva (Pl. VII.). In the male it becomes the first or prostatic part of the urethra.

In the twelfth or thirteenth week, the future prostatic urethra acquires very thick muscular walls, and the original epithelial tube pouches out into the muscular tissue in the form of little sacs, the lining cells of which assume the characters of secreting epithelium. In this way is produced the aggregation of muscular and glandular tissue known as the prostate gland. This is a well-developed structure by the fourth or fifth month (Tourneux). The recess in the floor of this part of the urethra, the sinus pocularis or uterus mas-

\footnotetext{
${ }^{1}$ Fourteenth week, according to Minot.
} 
culinus, has been previously referred to as the homologue of the uterus, being the persistent caudal extremities of the ducts of Müller (Plate VII.).

\section{THE EXTERNAL ORGANS OF GENERATION.}

In the early stages of the development of the external genital organs no sexual distinetions are apparent.

Reference has been made to the cloacal depression as a superficial fossa which makes its appearance at the caudal end of the body of the embryo in the sixth week (Fig. 96). At
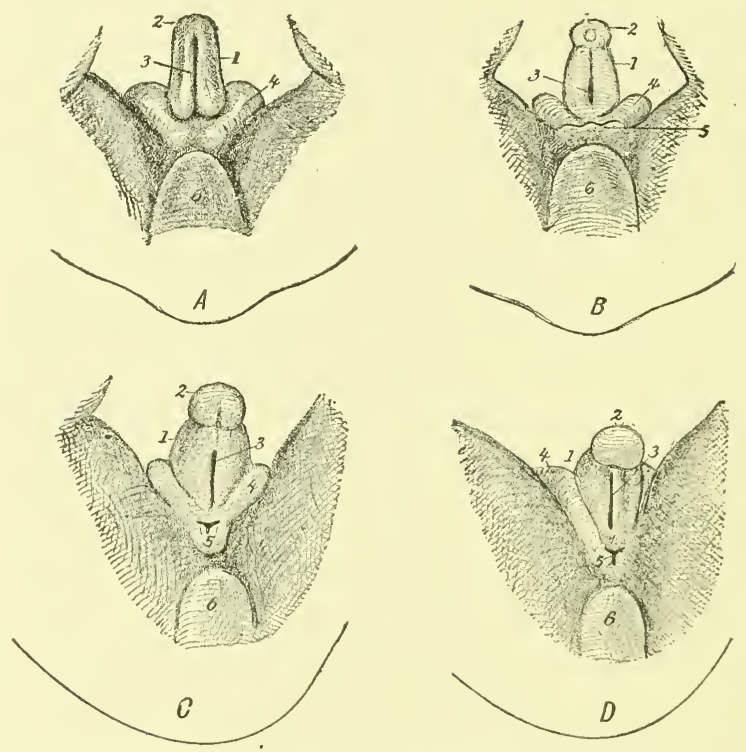

Fig. 130.-Four successive stages of development of the external genital organs (indifferent type) of the human fetus of 24 to $34 \mathrm{~mm}$. (0.95 to 1.35 ineh) (Tourneux): 1, genital eminence or tubercle; 2 , glans; 3 , genital groove; 4 , genital ridge; 5 , cloaeal depression; 6 , coceygeal eminenee.

about the same period an encircling elevation, the genital ridge (Fig. 130, 1, 4), is seen to surround this depression. Within the genital ridge, at the anterior part of the cloacal fossa, a small tubercle, the genital eminence, appears at the same time. On the under aspect of the genital eminence there is soon distinguishable the genital groove (Fig. 130, 3), which appeas as if a continuation of the fissure-like cloacal depression (5), 
and the groove very shortly becomes flanked by two ridges, the genital folds, one on each side.

The genital eminence becomes the penis or the clitoris, according to the sex of the fetus. It very early acquires a knob-like extremity (2) which is the beginning of the glans penis or of the glans clitoridis, as the case may be. Further development of the glans is brought about by the appearance of a partially encircling groove which serves to differentiate it from the body of the organ.

At this stage of development, the rudimentary organs, as described above, are precisely alike in the two sexes. Early in the third month-about the ninth week-sexual distinctions begin to become manifest. Since the female organs exhibit the less degree of deviation from the early indifferent form, they will be first considered.

The External Genital Organs of the Female.-The sexually indifferent genital eminence which, as we have seen, presents even by the end of the second month a rudimentary glans and an indicaton of a prepuce, elongates somewhat and becomes the clitoris. The genital folds bounding the genital groove on the under surface of the genital eminence (Figs. 130 and $131,1)$ never unite with each other as they do in the male, but become prolonged in the direction of the future anus and constitute, by the fourth month, the lateral boundaries of the orifice of the urogenital sinus, or, in other words, of the vestibule of the vagina (Fig. 131, 3). These folds, continuous over the dorsum of the clitoris with its rudimentary prepuce, are the nymphæ or labia minora (Fig. 131, D, 7) of the fullyformed state. The masses of erectile tissue in close relation with each labium minus, the pars intermedialis and the bulbus vestibuli, are the homologues respectively of a lateral half of the male corpus spongiosum and its bulb. 'The genital ridge, which, from the first, encircles the genital eminence and the cloacal depression, and, conseruently, the later clitoris and the aperture of the sinus urogenitalis, increases greatly in thickness. The part of it situated on the ventral side of the clitoris becomes the mons veneris, while the lateral parts of the ridge become the labia 
majora of the vulva. The several parts of the female genitalia develop to such a degree during the fourth month that their sexual characters at this time are well marked.
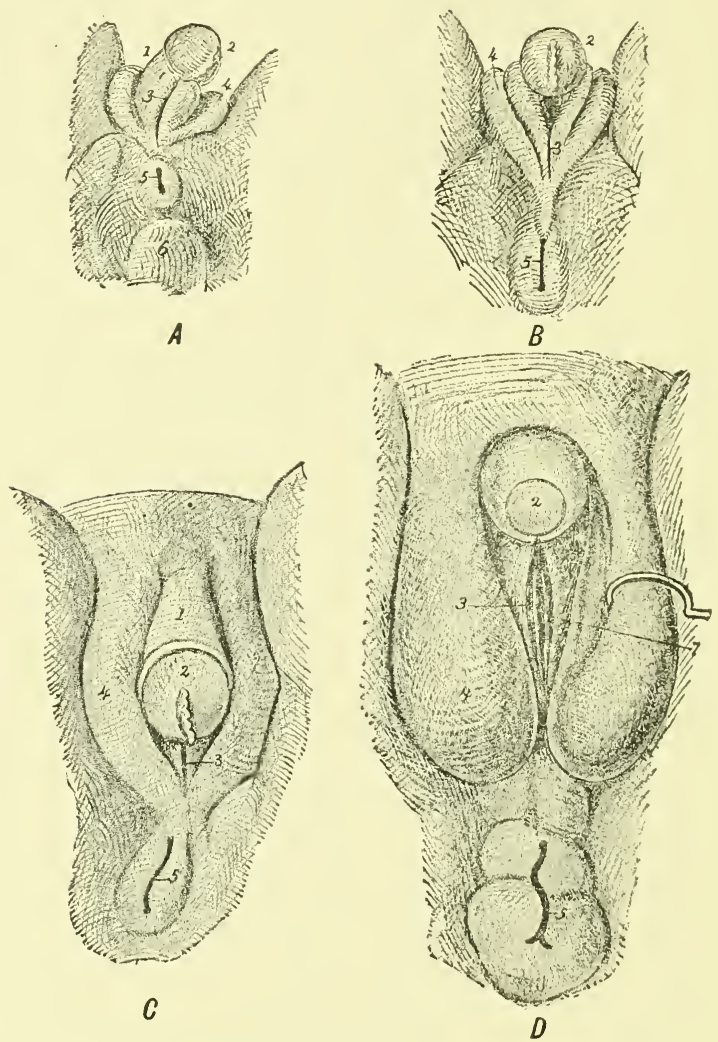

FIG, 131,-Four successive stages of development of the external genital organs of the human female fetus (Tourneux): 1 , clitoris; 2, glans clitoridis; 3 , urinogenital fissure; 4 , labia majora; 5 , anus; 6 , coceygeal eminence; 7 , labia minora.

The reader is again reminded that in the stage when the cloaca is present, the Mïllerian ducts terminate in the sinus urogenitalis (Plate VII.). As previously stated, the sinus urogenitalis becomes the female urethra, its terminal portion expanding into the vestibulum vaginæ. The openings of the Müllerian ducts fall within this latter vestibular region of the sinus. The lower portion of the two ducts by this time, however, have fused to form the uterus and the vagina, and 
hence is established the permanent relationship of these parts -that is, the opening of the vagina and the urethra by separate orifices into the vestibule.

The formation of the hymen begins in the fifth month as a little crescentic fold at the posterior margin of the aperture of the vagina.

The glands of Bartholin develop as evaginations of the wall of the vestibular region of the urogenital sinus.

The Male External Genitals.-The male external organs represent a farther stage of development than the corre-
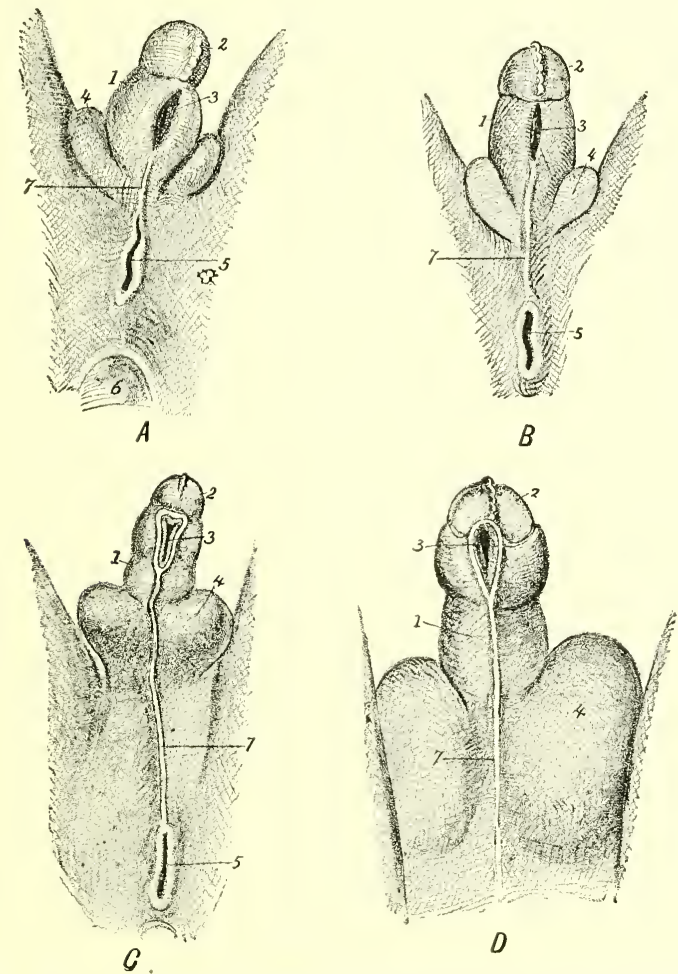

FIG 132.-Four successive stages of development of the human male fetus (Tourneux) : 1 , penis; 2 , glans ; 3 , genital groove; 4 , scrotum ; 5 , anus ; 6 , coccygeal eminence; 7 , perineoscrotal raphe.

sponding female parts. The genital eminence elongates rather rapidly, its length in the tenth week being $1.5 \mathrm{~mm}$. The knob-like extremity becomes better marked and constitutes 
the glans penis, while the integumentary fold that partially encircles the latter assumes more distinctive character as the prepuce. This fold gradually advances over the glans and adheres to it, the adhesion persisting until, or shortly after, birth. All of the rudimentary penis, exclusive of the glans and of the genital folds, becomes the corpora cavernosa of the adult organ. The characteristic structure of the corpora cavernosa is foreshadowed as early as the third month by the appearance in the penis of capillary blood-ressels, which, in the sixth month, undergo marked dilatation.

The groove on the under surface of the penis becomes deeper, and the genital folds, which bound the groove laterally, increase in size. This groove extends from the orifice of the urogenital sinus to the glans penis. The genital folds, which in the female, remain distinct and become the nymphæ, unite with each other in the male and convert the groove into a canal, which latter is practically an extension of the urogenital sinus along the entire length of the penis to the glans. The canal thus formed is the anterior part of the male urethra, or, in other words, it includes all of the urethra except its prostatic portion, which represents the urogenital sinus. The orifice of this newly-formed canal, situated in the glans, is the meatus urinarius. Failure of union of the genital folds, either wholly or in part, results in total or in partial deficiency of the floor of the urethra, this anomaly being known as hypospadias. If the defective closure involves only the glans, the condition is denominated glandular hypospadias.

The genital folds form not only the sides and the floor of the penile urethra, but by an extension of their growth, also its roof, thus completely surrounding it. Upon the acquisition, by the now united genital folds, of blood-vessels and cavernous spaces, they become the corpus spongiosum of the penis, and thus is established the permanent or adult relation of these parts.

The genital ridge becomes differentiated into two prominent folds or pouches placed one on either side of the root of the penis. In the fourth month these unite to form the 
scrotum, the line of union being indicated by the raphe. Failure of union of the two halves of the scrotum is one of the features of certain forms of so-called hermaphroditism.

The glands of Cowper, which correspond to the glands of Bartholin of the female, are developed, like the latter, as . evaginations of the terminal part of the urogenital sinus.

The accompanying tabulation exhibits a comparison of the organs of the two sexes on the basis of their common origin. Male and female parts that develop from the same fetal structure are said to be homologous with each other.

\section{Homologies of the Sexual System.}

\section{Fetal Structure.}

Female Organs.

Indifferent sexual gland. Ovary.

Wolffian body-

Its middle series of tu- Short tubules of par- Vasa efferentia, retetestis bules and ovarium and and coni vasculosi.
Male Organs. 'Testis.

Corresponding part of Horizontal or long tube Tube of epididymis.

Wolffian duct. of parovarium.

Remainder of Wolffian Usually altogether dis- Vas deferens, seminal duct. appears; if persistent, vesicle, and ejaculaGartner's duct. tory duct.

Upper series of short Stalked hydatid of Mor- Stalked hydatid of Mortubules (pronephros). gagni.

Lower series of tubules. Paroöphoron.

Duct of Müller-

Its upper extremity.

Succeeding portion.

Fimbria of oviduct.

Oviduct.

Remaining portion, by Uterus and vagina. fusion with its fellow.

Externat Organs.

Fetal Structure. Genital eminence.

Genital folds.

Genital ridge.

Urogenital sinus.
Female Organs.

Clitoris.

Nymphæ and bulbi ves- Corpus spongiosum, entibuli.

Labia majora.

Urethra and vestibule, Prostatic urethra, memGlands of Bartholin. closing spongy part of urethra.

\section{Scrotum.}

branous urethra, prosgagni.

Paradidymis (organ of Giraldès).

Sessile hydatid.

Usually disappears; if persistent, duct of Rathke.

Uterus masculinus. tate, Cowper's glands. 


\section{SUMMARY.}

1. The male and the female internal generative organs, as well as the kidney and the ureter, originate from the mesothelial lining of the body-carity, being directly produced from the Wolffian body and the duct of Mïller.

2. The bladder and the female urethra, but in the male only the prostatic urethra, result from the metamorphosis of the intra-embryonic part of the allantois, and are therefore to be regarded as of entodermic origin.

3. Before the establishment of the permanent kidney, a temporarily functionating organ, the mesonephros, performs the office of a kidney during a part of fetal life, and this latter is preceded by the pronephros, an organ which, though represented in the higher vertebrates by a vestigial remnant, is functionally active only in larval Amphibia and in bony fishes.

4. The Pronephros.-The mesothelial cells of the outer or parietal wall of the body-cavity become invaginated in a line parallel with the axis of the body, and the cord of cells thus formed becomes hollowed out to constitute the pronephric or segmental duct. At several points this duct retains its connection with the surface-cells by means of cell-cords, which latter become tubes and acquire glomeruli. The long tube and the shorter tubules with their glomeruli constitute the pronephros.

5. The Mesonephros.-The transverse segmentation of the middle plate, which connects the paraxial mesolerm with the parietal plate, results in the formation of a series of cell-masses, the nephrotomes. Each nephrotone becomes a tube and acquires one or more glomeruli. The deeper ends of the tubes become connected with the pronephric or segmental duct, which latter is known henceforth as the mesonephric or Wolffian duct. 'These tubes, with the adjacent part of the Wolffian duct, constitute the mesonephros, which functionates, for a time, even in the human fetus, as an organ of urinary excretion. The entire Wolffian duct, with the pronephric tubules and the mesonephric tubules, constitutes the Wolffian boly. The Wolffian duct opens at its lower or caudal extremity into the cloaca. 
6. The metanephros or permanent kidney develops in part from a small diverticulum that pouches out from the lower or caudal end of the Wolffian duct. The straight collecting tubules and the pelvis of the kidney correspond to the dilated and subdivided fundus of this diverticulum, while the ureter represents its stalk. The secretory tubules of the kidney develop from the inner zone of the metanephrogenic tissue. The surrounding mesodermic tissue furnishes all the component elements of the ureter-walls and of the kidney except the epithelial parts, as noted above.

7. The suprarenal bodies probably are derived, in part, from epithelial outgrowths which proceed from the mesonephros to form the cortical part of the organ, and, in part, from chains of small cells that bud forth from the embryonic sympathetic ganglia to form its medulla. The surrounding mesodermic tissue contributes the connective-tissue parts of the suprarenal body.

8. The sexual apparatus in its earlier stages presents no distinctions of sex. The elements of this early indifferent type are the indifferent genital gland, the Wolffian duct, and the duct of Müller.

9. The indifferent genital gland originates in the mesothelium of the body-cavity. The mesothelial cells overlying the ventromesial aspect of the Wolffian body undergo multiplication in the fifth week and thereby produce an elongated elevation, the genital ridge. Further multiplication of its cells and the addition of other elements bring about the transformation of this ridge into the well-defined genital gland, which now lies in close relation with the Wolffian tubules. The mesothelial cells are the "germinal epithelium" of Waldeyer, the cells that produce the ova or the spermatozoa, according to the future sex.

10. The duct of Müller makes its appearance soon after the Wolffian duct. It lies parallel with and to the outer side of the Wolffian duct and also terminates in the cloaca. It is of mesodermic origin, being produced either by evagination of the mesothelial cells of the body-cavity, or by a splitting off from the Wolffian duct. 
11. The generative systems of both sexes result from the metamorphosis of the three structures making up the early indifferent sexual apparatus-namely, the indifferent sexual gland, the Wolffian body, and the duct of Müller.

12. The male sexual system is produced by the transformation of the indifferent gland into the testicle, and the conversion of the Wolffian tubules and the Wolffian duct into the system of excretory ducts for that gland, the short tubules becoming the vasa efferentia and coni vasculosi, while the Wolffian duct itself furnishes the body and the globus minor of the epididymis, the vas deferens, the vesicula seminalis, and the ejaculatory duct. The duct of Müller remains undeveloped and is represented in the adult by the atrophic sessile hydatid and the uterus masculinus.

13. The female sexual apparatus is brought about by the derelopment of the indifferent gland into the ovary, and by the metamorphosis of the upper segments of the ducts of Müller into the Fallopian tubes, and the fusion of the remaining portions of the two ducts to form the uterus and the vagina. The Wolffian duct and tubules give rise to atrophic structures in the female, the most conspicuous of which is the parovarium or epoophoron.

14. Both the male and the female external genitalia are developed from fetal structures common to the two sexes, the genital eminence, the genital ridge, and the genital folds. The genital eminence is situated at the anterior or ventral part of the cloacal depression. The genital ridge is an elevation surrounding this pit and the genital eminence, while the genital folds are on the under surface of the genital eminence, one on each side of a longitudinal groove.

15. The Wolffian ducts and the ducts of Müller open into the cloaca, but when that aperture becomes differentiated into the anus and the urogenital sinus, as it does at the fourteenth week, these ducts fall to the latter apartment. The orifice of the urogenital sinus being at the base of the genital eminence, the sinus comes into continuity with the groove on the under surface of the eminence.

16. The female external genitalia are produced by the further development of the three structures mentioned above. 
The genital eminence becomes the clitoris. The genital folds on the under surface of the clitoris become somewhat prolonged to constitute the labia minora. The genital ridge becomes, anteriorly, the mons veneris and laterally the labia majora. The orifice of the urogenital sinus is represented by the vestibule, and since the Müllerian ducts near their termination in the urogenital sinus fuse to form the vagina, the latter passage opens in the adult into the restibule. Since, also, the urogenital sinus receives the termination of the allantois, which becomes the female urethra, the latter canal likewise opens into the adult vestibule.

17. The male external genitals represent a further development of the embryonic genital eminence, genital folds, and genital ridge than do the female organs. The genital eminence becomes the penis, the genital folds, uniting with each other so as to surround the groove, producing the corpus spongiosum. The groove itself, being thus converted into a canal which extends the now closed urogenital sinus to the end of the penis, constitutes all of the male urethra except the first or prostatic portion. The prostatic urethra represents the proximal extremity of the allantois. Since the Wolffian ducts open into the urogenital sinus after the division of the cloaca, the terminations of those ducts, represented now by the ejaculatory ducts, open into the prostatic urethra; and since the Müllerian ducts also open into the urogenital sinus, the uterus masculinus, which is the representative in the male of the terminal parts of the Müllerian ducts, is found likewise in the prostatic urethra. The lateral parts of the genital ridge, which, in the female, become the labia majora, fuse with each other in the male to form the scrotum.

18. The condition of so-called hermaphroditism may be produced either by an unusual degree of development of the female external genitals, resulting in a clitoris resembling a penis and in labia majora which simulate a cleft scrotum; or by the arrested development of male organs, whereby the genital folds and the genital ridges fail to unite, the urethra in consequence opening at the base of the penis. 


\section{CHA PTER XIT.}

\section{THE DEVELOPMENT OF THE SKIN AND ITS APPENDAGES.}

THE appendages of the skin include the sebaceous and sweat glands, the mammary glands, the nails, and the hairs.

\section{THE SKIN.}

The skin, consisting of the epidermis or cuticle and of the true skin, or derm, or corium, is derived from two sources,

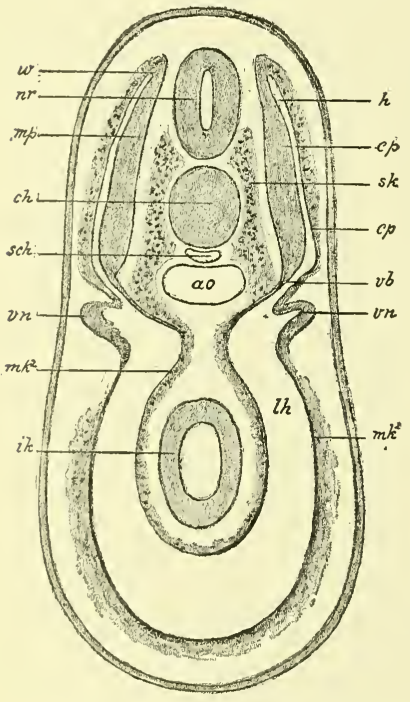

FIG. 133.-Cross-section through the region of the pronephros of a selachian embryo in which the myotomes are in process of being constricted off (Hertwig): $n r$, neural tube; ch, chorda; ao, aorta; $m p$, muscle-plate; $c p$, cutis plate; $v b$, midlle plate; $s{ }^{2}$, skeletogenous tissue; $v n$, pronephros; $m k^{\prime}, m k^{2}$, parietal and visceral mesoderm; $l h$, body-cavity; $i k$, intestinal ectolerm; $h$, cavity of somite. the epithelial epidermis being a product of the ectoderm, and the corium originating from the mesoderm. The nails and hairs are outgrowths of the epithelial layer, while the various glands are derived from infoldings or invaginations of the same stratum.

The corium, the connectivetissue component of the skin, is an outgrowth of the cutis plates of the primitive segments or somites (Fig. 133). It first appears in crude form in the second month as a layer of spindle-cells beneath the ectoderm. In the third month, the more superficial part of this layer acquires more definite and distinctive character, the rather loose aggregation of cells having differentiated into a tissue which is a mesh-work of bundles of 
white fibrous connective tissue with some intermingled elastic and muscular fibers; this constitutes the corium proper. The deeper layer of cells becones a loose, subeutaneous areolar tissue containing a few scattered fat-cells. About a month later the external surface of the primitive corium loses its smooth character and presents numerous little elevations, the villi, which project into the overlying epidermis. The villi, being highly vascular, play an important part in the nutrition of the epidermis and being also freely supplied with nerves they sustain an equally important relation to the sensitiveness of the skin.

From the middle of fetal life onward, the fat-cells in the subcutaneous tissue increase in number to such extent that there is formed a continuous and well-marked subcutaneous layer of fat, the panniculus adiposus.

Certain of the cells of the primitive corium differentiate into unstriated muscular tissue, forming thus the muscles of the hair-follicles, the arrectores pilorum, as well as the subcutaneous muscular tissue of the dartos of the scrotum and penis, and that of the nipple and of the perineum.

The epidermis, consisting of the superficial horny laver and the deeper mucous layer or stratum Malpighii, is entirely an epithelial structure. Its elements are simply the descendants of the early ectodermic cells specially modified to afford the necessary protection to the more sensitive and delicate corium.

The division into the two strata of the epidermis is indicated as early as the latter part of the first month, at which time the cells of the ectoderm have become arranged into two single layers, a superficial layer of rather large flattened cells and an underlying stratum of smaller elements. The cells of the outer layer, or epitrichium, which probably represents the future stratum corneum, successively undergo degeneration and desquamation, the places of those lost being supplied by the formation of new ones from the deeper layer. As time goes on, both layers increase in thickness and the hairs and the glands of the skin are gradually formed. With increased proliferation there is increasingly 
active desquamation of superfieial eells, and as the degenerate and cast-off cells beeome mixed with the products of the sebaceous glands, there is formed a sort of cheesy eoating of the skin, the vernix caseosa or smegma embryonum. 'This is first easily recognizable in the sixth month, and first covers the entire surface of the body in the eighth month. It serves to protect the epidermis of the fetus from maceration in the amniotic fluid.

The completion of the epidermis, aside from the development of its accessory parts, eonsists simply in further increase in thickness and in the modification of the superficial cells to produce the eharacteristic seale-like elements of the corneous layer of the skin, accompanied by the differentiation of the deeper cells into those of the rete mucosum or stratum Malpighii. The extent to which these modifications are carried varies in different regions of the body.

\section{THE DEVELOPMENT OF THE APPENDAGES OF THE SKIN.}

The Nails.-The nails have their beginning in little clawlike projections, the primitive nails, that appear upon the tips of the still imperfect fingers and toes in the seventh week. ${ }^{1} \quad$ These result from localized proliferation of the eells of the epidermis, being entirely epithelial structures. The rudimentary nails project from the tips of the digits, instead of oceupying the dorsal position of the eompleted struetures.

The elaw-like primitive nail, between the ninth and twelfth weeks, becomes surrounded by a groove, which serves to sepa-

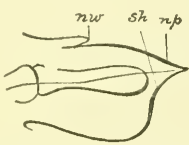

Fif. 134.-Iongitudinal section through the toe of a Cercopithecus ${ }^{2}$ (after Gegenbaur): $n p$, nail-plate; $s$, plantar horn (Sohlenhorn); nw, nail-wall. rate it from the general ectodermic surface. These claw-like rudiments of the human nails are quite similar to the primitive elaws of many mammals, the primitive nail in each ease ineluding a dorsal part, the nailplate, and a portion whieh belongs to the ventral surface of the digit, called the plantar horn (Fig. 134). The striking difference between the nails of the human adult and the claws and

${ }^{1}$ Or ninth week, Minot.

${ }^{2}$ A genus of long-tailed $\Lambda$ frican monkeys. 
hoofs of many animals is due in great measure to the degree of development to which this ventrally situated plantar horn attains. In the hoofed mammals (Ungulata) and the clawed mammals (Unguiculata), the plantar horn undergoes very great development, whereas in man it retrogrades and leaves no trace except the nail-welt, or the narrow line of thickened epidermis where the distal end of the nailbed merges into the ordinary skin. After the atrophy of the plantar horn, the dorsally situated nail-plate being alone present, the rudimentary nail bears a greater resemblance to the adult condition.

As the nail-plate gradually acquires more distinctive character, the deeper layers of the skin specialize into a structure adapted to its nutrition. This is the nail-bed, a highly vascular and sensitive cushion consisting of the corium and of the stratum Malpighii of the epidermis. It is especially from the proximal part of the nail-bed, representing the matrix of the fully-formed condition, that the nail grows. The rate of growth is such that the ends of the nails protrude beyond the tips of the digits in the eighth month.

The tissue of the fully-formed nail corresponds to the stratum lucidum of the typical epidermis, developed to an unusual degree. The epitrichium or future stratum corneum, the most superficial layer of the epidermis, does not form a part of the nail, but constitutes a thin covering, the eponychium; this is lost in the seventh month, with the exception of a small band orer the root of the nail, which persists for a short time as the perionyx.

The nails of the toes are always somewhat behind those of the fingers in development.

To repeat, the claw-like rudimentary nails appear in the seventh week, the nails are perfectly formed about the twelfth week, and break through their epidermal covering in the seventh month, reaching to or beyond the finger-tips in the eighth month.

The Hair.-Each hair consists of the projecting shaft and the emberlied root, with its expanded deep extremity, the hair-bulb, the root being embraced by the hair-follicle. The 
hair is entirely of ectodermic origin, being derived from the epidermal layer of the skin, while the hair-follicle is partly derived from the epidermis and in part is a product of the corium. The hairs are homologous with the feathers and scales of the lower animals.

The development of the hair is initiated in the third fetal month by the appearance of small solid masses of epithelium in the stratum Malpighii of the epidermis. The epithelial plugs or hair-germs grow into the underlying corium and are met by outgrowths or papillæ of the latter, which develop almost simultaneously. The papillæ are very vascular and serve for the nutrition of the developing hair.

The root and the shaft of the rudimentary hair result from the specialization of the axial or central cells of the hairgerm. These cells lengthen in the direction of the long axis of the hair-germ and become hard and corneous, thus constituting the root and the shaft, the cells of the deepest part of the hair-germ forming the bulb. The growth of the hair
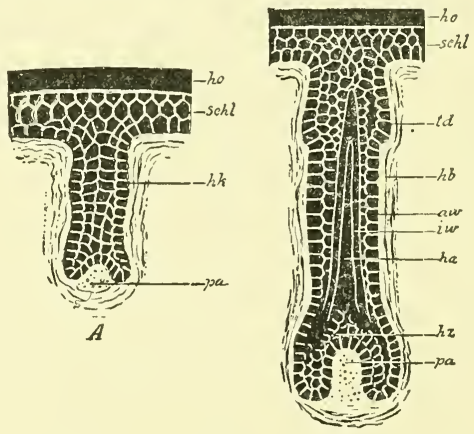

B

FIg. 135.-Two diagrams of the development of the hair (Hertwig): $A$ and $B$, two different stages of the development of the hair in human embryos; ho, corneous layer of the epidermis; schl, mucous layer; $p a$, hair-papilla; $h k$, germ of hair; $h z$, bulb of hair; $h a$, young hair; $a w$, $i w$, outer and inner sheaths of the root of the hair; $h b$, hair-follicle; $t d$, sebaceous gland.

in length is due to the proliferation and specialization of the cells of the bulb. The papilla of the underlying corium indents the deep surface of the hair-bulb, this close relation of the two structures enabling the papilla the better to fulfil its function of providing nourishment to the bulb. 
The hair-follicle, consisting of an outer connective-tissue portion or fibrous layer and an inner epithelial part, the inner and outer root-sheaths, is partly of mesodermic and partly of ectodermic origin. The inner and outer rootsheaths are produced by the peripheral cells of the hairgerm augmented by cells contributed directly by the stratum Malpighii of the epidermis. The outer fibrous constituent of the follicle results from the mesodermic cells of the corium that immediately surround the hair-germ.

Gradually increasing in length by the addition of new cells from the hair-bulb, the primitive hair at length protrudes from the follicle as free hair. This first growth of hair is unpigmented and is extremely fine and soft, being known as the lanugo or embryonal down. This appears upon the scalp and some other parts of the body in the fourth month, gradually extending over the entire surface in the succeeding months. In the eighth month the lanugo begins to disappear, but is not lost as a whole until after birth, when the permanent growth of hair takes its place. Upon the face, in fact, the lanugo persists throughout life.

The development of the secondary hair is still a disputed point. It is claimed by some authorities (Stieda, Feiertag) that they develop from entirely new hair-germs. Most authorities hold, however, that the secondary hair develops from the same papilla that produced the hair just lost. According to this riew, the empty root-sheath of the cast-off hair closes so as to form a cell-cord which represents a hair-germ for the new hair. The cells of this germ in most intimate relation with the underlying papilla produce the new hair in the same manner that hair is produced by the original hair-germs. As the new hair grows toward the surface the old one is gradually crowded out.

The Sebaceus and Sweat-glands.-The sweat-glands, including not only the sweat-glands proper but the ceruminous glands of the external auditory meatus and the glands of Moll of the eyelids, are derived from the ectodermic epithelium. The glands are of the simple tubular type. Each gland develops from a small accumulation of epidermal cells that 
grows, in the fifth month, from the Malpighian or mucous layer of the epidermis into the underlying corium. The solid epithelial plugs become tubes in the seventh month by the degeneration and final disappearance of the central cells. The deeper part of the tube becomes coiled and its lining epithelium takes on the characteristics of secreting cells. Some of the cells of the original epithelial plug undergo specialization into muscular tissue, thus producing the involuntary muscles of the sweat-glands.

The sebaceous glands are developed from solid epithelial processes that originate from the deep layer or rete mucosum of the epidermis in a manner similar to that of the development of the sweat-glands. There is the difference, however, that the epithelial plugs acquire lateral branches and thus usually produce glands of the compound saccular or acinous variety. There is the further difference that the epithelial outgrowths generally develop from the ectodermic cells of the outer sheath of the root of the hair near the orifice of the follicle (Fig. 135, $t d$ ), in consequence of which the ducts of the finished glands usually open into the hair-follicles. In some regions, however-regions devoid of hair, as the prepuce and the glans penis, the labia minora, and the lips-the growth is directly from the stratum Malpighii, as in the case of the sweat-glands.

The Mammary Gland.-The mammary gland represents a number of highly specialized glands of the skin, so associated as to constitute the single adult structure. Its origin, therefore, is to be sought in the cells of the epidermis in common with that of the ordinary glands of the skin.

It is claimed by many authorities, by Gegenbauer especially, that the mammæ are modified sebaceous glands; others assert that they are to be classed with the sweat-glands, Haidenhain having shown that in the development of the milk-glands there is no fatty metamorphosis of the central cells as in the sebaceous glands, and Minot emphasizing the fact that their mode of development closely resembles that of the sweat-glands.

The development of the milk-glands is begun as early as 
the second month. At this time the deep layer of the epidermis, in the sites of the future glands, becomes thickened by the multiplication of its cells, the thickened patch encroaching upon the underlying corium (Fig. 136, A, b). This thickened area enlarges somewhat peripherally and its margins become elevated, owing to which latter circumstance the patch appears relatively depressed $(B)$. The depression is known as the glandular area, and it corresponds with the future areola and nipple. In many mammals the development of the milk-glands is initiated by the appearance of a
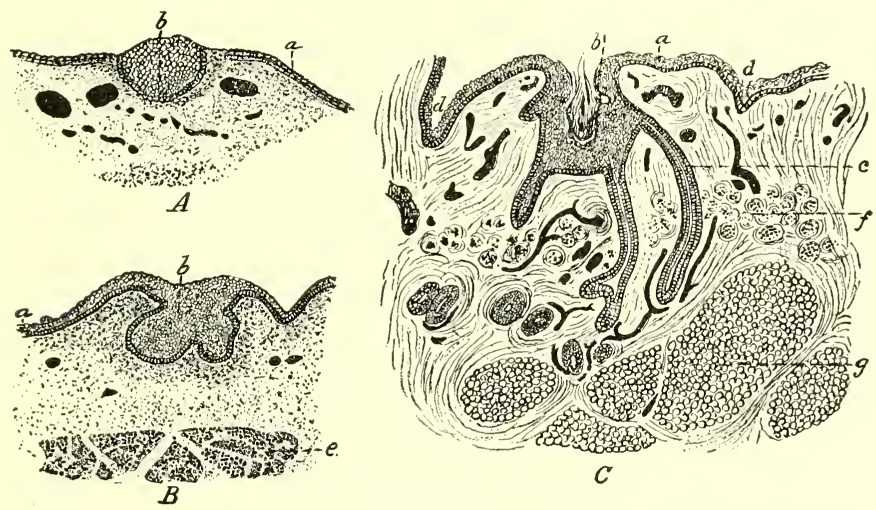

FIG. 136.-Sections representing three successive stages of development of the human mamma (Tourneux): $A$, fetus of $32.40 \mathrm{~mm}$. (1.3 in.); $B$, of $10.16 \mathrm{~cm}$. (4 in.); $C$, of $24.35 \mathrm{~cm}$. (9.6 in.); $a$, epidermis; $b$, aggregation of epidermal cells forming anlage of gland; $c$, galactophorous ducts; $d$, groove limiting glandular area; $e$, great pectoral musele; $f$, unstriated muscular tissue of areola; $g$, subcutaneous adipose tissue.

pair of linear thickenings of the epidermis on the ventrolateral aspect of the body, called the milk-ridges or milklines, from localized thickenings of which the multiple mammary glands of such animals develop. These milklines have also been observed in the human embryo, but the constancy of their occurrence in man has not as yet been established.

From the bottom of the glandular area, numerous small masses or bud-like processes of cells grow down into the corium. Some of the buds acquire lateral branches. By the hollowing out of these cell-buds the latter are transformed 
into tubes $(c)$, which open upon the grlandulararea. The branching of the cords begins in the seventh month and is carried on to such a degree that each original cell-cord gives rise to a tubo-racemose gland. The hollowing out of the solid processes begins shortly before birth, but is not completed until after that event. Each cell-cord becomes, in the strict sense, a complete gland, each such individual structure forming a lobe of the mature organ.

This stage of the human mammary gland-that is, a depressed gland-area upon which open individual glands, the nipple being absent-is the permanent condition in some of the lowest mammals, as in the rchidna, one of the monotremes. In all higher mammals, however, further metamorphoses occur in the tissues of the glandular area, and in the human fetus these tissues become the nipple and the surrounding areola.

The nipple is partly formed before birth, but does not become protuberant until post-fetal life. The depressed glandular area rises to the level of the surrounding parts, and its central region, which includes the orifices of the already formed or just forming ducts, swells out into a little prominence, the nipple. This prominence is a protrusion of the epidermis and includes the terminal extremities of the milk-ducts as well as the blood-vessels and connective-tissue elements which surround the ducts. In the dermal constituent of the rudimentary nipple unstriated muscular tissue develops. The region of the glandular area not concerned in the formation of the nipple becomes the areola.

At birth, as above intimated, the mammary gland is still rudimentary, sinee many of the ducts have not yet acquired their lumina nor their full degree of complexity. Shortly after birth a small quantity of milky secretion, the so-called witches' milk, may he expressed from the glands-in the male and female infant alike. This is true milk according to Rein and Barfruth, but according to Kölliker, it is merely a milky fluid containing the lébris of the degenerated central cells of those rudimentary ducts that were still solid at birth. So far, the milk-glands are alike in the two sexes, but 
while in the male they remain rudimentary structures, they continue to increase both in size and in complexity in the female. The increase affects not only the glandular tissue proper but the connective-tissue stroma as well. At the time of puberty the growth of the glands receives a new impetus, which is very materially augmented upon the occurrence of pregnancy. There may be sair, therefore, to be several distinct phases in the development of the milk-glands, first, the embryonic stage; second, the infantile stage; third, the stage of maturity beginning at the time of puberty; and finally, the stage of full functional maturity consequent upon pregnancy and parturition. 


\section{CHA P'TER X V.}

\section{THE DEVELOPMENT OF THE NERVOUS SYSTEM.}

THE nervous system of the adult, including the cerebrospinal axis and nerves, and the sympathetic system of ganglia and nerves, is made up of the essential neural elements, the neurons, together with the supporting framework or stroma. ${ }^{1}$

The neurons and a part of the stroma result from the specialization of the ectodermic layer of the embryo. The ectodermic origin of the nervous system acquires certain interest in view of the conditions that obtain in some of the lowest and simplest organisms. For example, in the ameba, the single protoplasmic cell which constitutes the entire individual possesses the several fundamental vital properties of protoplasm, such as respiration, metabolism, contractility, motility, etc., in equal degree, no single property being more highly developed than the others, and no particular part of the cell exhibiting greater specialization than the other parts. In other words, the protoplasmic substance of the animal is at once a respiratory mechanism, a nervous apparatus, and an organ for the execution of the various other vital functions.

In somewhat more highly developed creatures, as the infusoria, althongh there is no differentiation into separate tissues and probably not even into separate cells, there is seen some progress toward the specialization of certain parts of the organism for the performance respectively of the different functions of life. For example, the central part of the ani-

1 The neurons are the units of which the nervous system is made up. Each neuron consists of a nerve-cell with everything belonging to it - that is, with its various processes, including the axis-cylinder process or neurit, which becomes the axis-cylinder of a nerve-fiber. 
mal has digestive functions, while it is by the superficial portion alone that the creature is brought into relation with the outside world, the sensitiveness or irritability of the surface, by which the animal is made responsive to external impressions, being the nearest approach to the function of a nervous system that it possesses.

This primitive function of the surface of the organism is suggestive as to the origin of the nervous system of higher type creatures. It will be seen, indeed, that not only is the nervous system proper derived from the ectodermic cells of the embryo but that the peripheral parts of the organs of special sense, as the olfactory epithelium, the organ of Corti, and the retina, have the same origin.

The alteration of those cells of the ectodermic stratum that are to specialize into nervous elements begins prior to the fourteenth day in the human embryo, in the stage of the blastodermic vesicle. The change consists in a gradual modification of the form of the cells, the cells common to the general surface of the germ assuming the columnar type. The process affects the cells of the median line of the embryonic area in advance of the primitive streak, resulting in the production of a thickened longitudinal median zone. This thickened area is the medullary plate (Fig. 41, p. 70). On each side of the plate-which is apparent at the fourteenth day - the adjoining ectodermic cells become heaped up to form the medullary folds, which latter therefore bound the medullary plate laterally. The medullary plate becomes concave on the surface, forming the medullary groove (Fig. 137). By the deepening of the groove, the lateral edges of the plate approach each other (Fig. 138), and finally they meet and unite, thus producing a tube, the neural tube or canal.

Since the medullary folds similarly meet and unite with each other-their union slightly preceding that of the edges of the plate-the neural tube comes to lie entirely beneath the surface-ectoderm and soon loses all connection with it. The closing of the tube and the union of the medullary folds occur first near the anterior end of the embryonic area, in a position that corresponds with the region of the future neck, 
and from this point it proceeds both cephalad and caudad. Since the medullary folds at their caudal extremity embrace

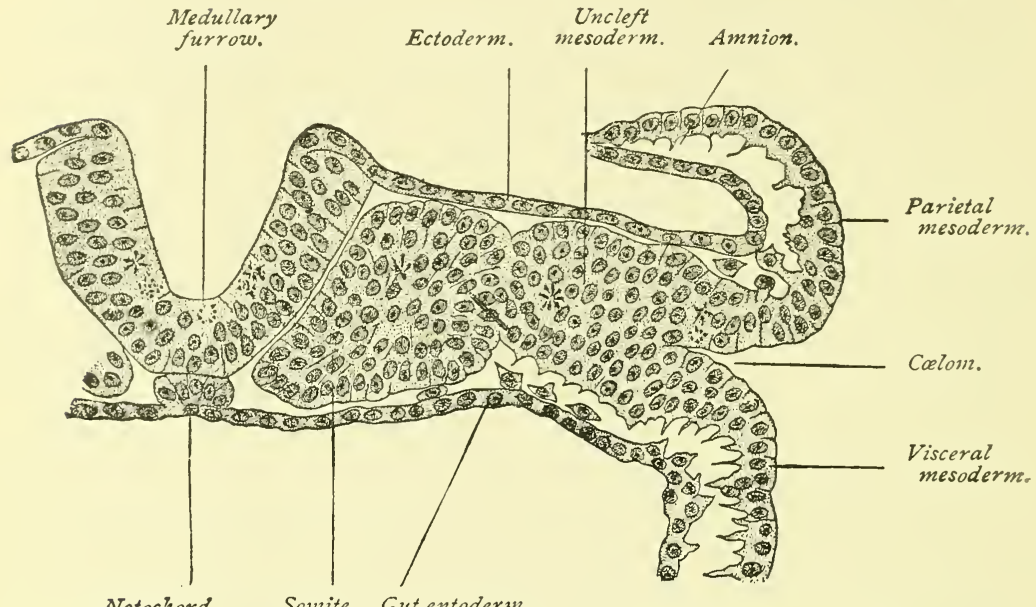

Notochord. Somite. Gut entoderm.

FIG. 137.-Transverse section of a sixteen-and-a-half-day sheep embryo possessing six somites (Bonnet).

the primitive streak (Fig. 41, 1. 70), the latter structure is included within the caudal end of the neural tube by the

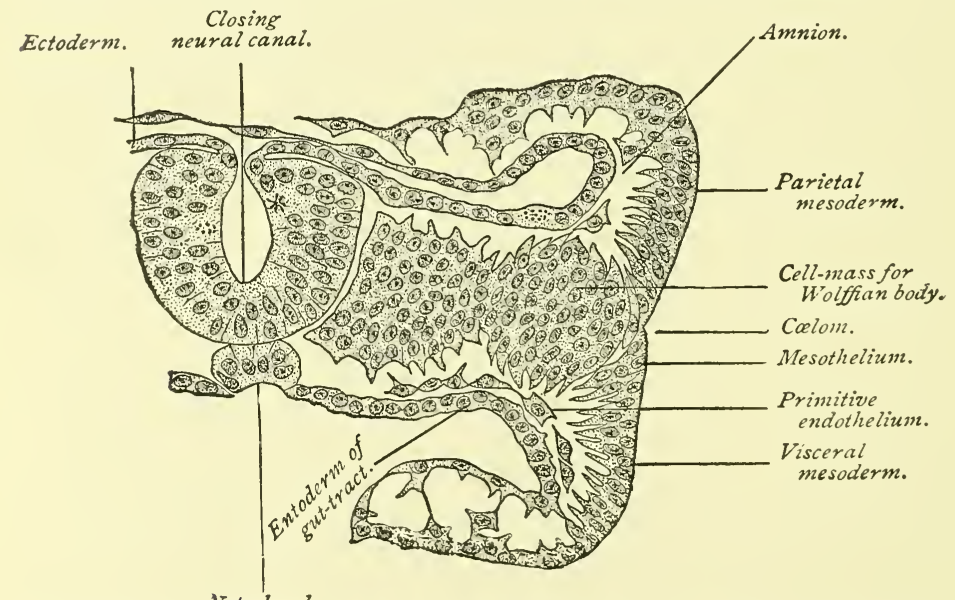

Votochord.

FIG. 138.-Transverse section of a fifteen-and-a-half-day sheep embryo possessing seven somites (Bonnet).

coming together of the folds, and thus the blastopore, which was previously the external aperture of the archenteron, 
comes to constitute the neurenteric canal, or an avenue of communication between the neural canal and the primitive intestine.

The neural canal then is a tube composed of columnar cells, which is formed by the folding in of the ectoderm and which occupies the median longitudinal axis of the embryonic area and consequently of the future embryonic body. From this simple epithelial canal the entive adult nervous system is evolved.

The evolution of the highly complex cerebrospinal axis from such a simple structure as the neural canal is referable both to the principle of unequal grouth-the walls of the tube becoming thickened by the multiplication of the cellsand to the formation of folds.

The portion of the neural canal-approximately one-halfthat is devoted to the formation of the brain is delimited from the part that produces the spinal cord by the dilatation of the anterior or head-end of the tube, and the subsequent division of this dilated sac-like portion into three communicating sacs called respectively the fore-brain, mid-brain, and hind-brain vesicles (Fig. 142). These three vesicles give rise to the brain, while the remaining part of the neural canal forms the spinal cord.

\section{THE DEVELOPMENT OF THE SPINAL CORD.}

In the growth of the spinal cord from the spinal portion of the neural canal we have to consider the evolution of a cylindrical mass of nerve-cells and nerve-fibers with the supporting stroma from a simple epithelial tube.

The wall of the neural tube, although consisting at first of a single layer of epithelial cells, is not of uniform thickness throughout its circumference. While the external outline is oval, the lumen of the tube is a narrow dorsoventral fissure (Fig. 45, p. 73). The cavity is therefore bounded on the sides by thickened lateral columns, while the dorsal and ventral walls, which connect the lateral columns with each other, are thinner and are called respectively the roof-plate and the floor-plate. 
After a short time, the walls of the tube having thickened by the multiplication of the cells, the shape of the lumen

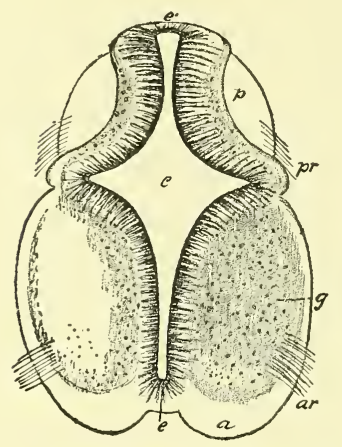

FIG. 139.-Transverse section of the cervical part of the spinal cord of a human embryo of six weeks, $x$ 36 (from Kölliker): c, central canal; $e$, its epithelial lining; at $e^{\prime}$ (superiorly), the original place of closure of the canal; $a$, the white substance of the anterior columns; $g$, gray substance of anterolateral horn; $p$, posterior column; ar, anterior roots; $p r$, posterior roots. alters, two laterally projecting angles being added (Fig. 139). The effect of this change is to partially divide each lateral half into a dorsal and a ventral region. The neural canal at this stage may be said to consist of six columns of cells, the two dorsal zones connected with each other by the roof-plate, and the two ventral zones united by the floor-plate. These regions are also distinguishable, with certain characteristic modifications, in the head-region of the tube. They are important in their bearing upon the further development of the structure, since the dorsal and ventral zones are related respectively to the dorsal or sensory and the ventral or motor roots of the spinal nerves.

The differentiation of the cells of the neural tube into two kinds of elements, one of which gives rise to sustentative tissue or neuroglia while the other produces the nerve-cells, is observed at about the end of the third week. The single layer of columnar cells which at first composes the wall of the tube, the long axes of the cells being radially arranged, soon exhibits near the lumen a row of round cells, probably the first offspring of the columnar cells. The round cells are the germ-cells or germinating cells, from which are developed the neuroblasts or young nerve-cells as well as the neuroglia cells. All the other cells, known as the spongioblasts or ependymal cells, are concerned in producing sustentative tissue.

The stroma of the central nervous system includes two constituents-a connective-tissue element, and a part, the neuroglia, which is of epithelial origin, and which is not to 
be regarded, therefore, as connective tissue. The connectivetissue portion of the stroma is produced by the ingrowth of the pial processes from the pia mater, and is hence of mesodermic origin.

The neuroglia is derived from the spongioblasts, which result from the specialization of the large columnar cells of which the wall of the neural canal is composed. These cells, whose length comprises the entire thickness of the wall of the tube in the earliest stages, undergo partial absorption and disintegration, each cell being transformed into an elongated system of slender processes or trabeculæ, and each such system

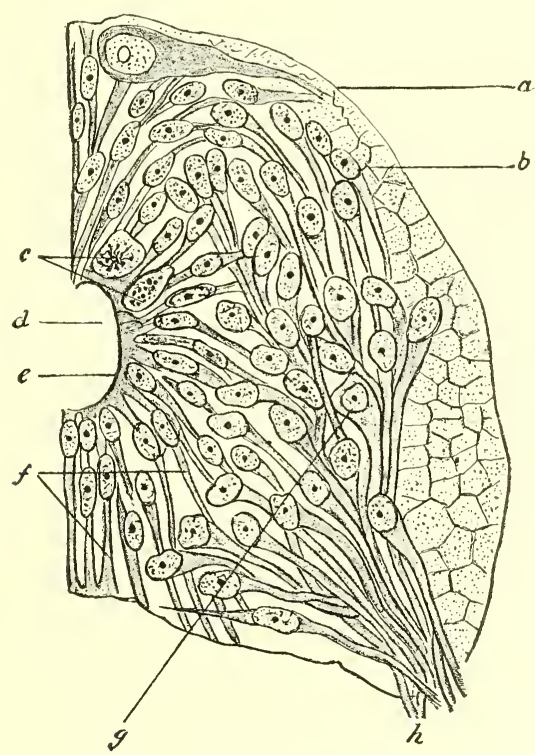

FIG. 140.-Cross-section through the spinal cord of a vertebrate embryo (after His): $a$, outer limiting membrane; $b$, outer neuroglia layer, region of future white matter ; $c$, germ-cells; $d$, central canal ; $e$, inner limiting membrane or ependymal layer; $f$, spongioblasts ; $g$, neuroblasts (mantle layer); $h$, anterior root-fibers.

being a completed spongioblast or ependymal cell (Fig. 140). The inner ends of the spongioblasts coalesce with each other, forming thus the internal limiting membrane, while the peripheral extremities interlace with each other to form a close network, the marginal velum. As the walls of the neural tube increase in thickness, the spongioblasts become more and more broken up to form the delicate neurogliar network 
with interspersed nucleated glia cells, which latter are derived from some of the round cells noted above as lying near the lumen of the neural tube and which have taken a position in the marginal velum. Such of the spongioblasts as border the carity of the neural tube become the cells of the later ependyma of the central canal of the spinal cord and of the ventricles of the brain. The cells of the ependyma become ciliated in the human fetus in the fifth week.

The nerve-cells of the spinal cord-as also of the brainare the specialized descendants of the germ-cells referred to above. The proliferation of the germ-cells produces the neuroblasts, or young nerve-cells (Fig. 140). The latter elements move away from the primitive position of the germcells near the lumen of the tube and, taking up a position between the bodies of the ependymal cells and the periphery of the neural tube, develop into the nerve-cells. The transition is effected by the accumulation of the cell's protoplasm on the distal side of the nucleus and its elongation into a process. This process is a neurit or axon or axis-cylinder process and is the beginning of a nerve-fiber. The dendrites or protoplasmic processes appear considerably later. Some of the fibers thus produced grow out from the neural tube to constitute the efferent fibers of the peripheral nerves, that is, the ventral roots of the spinal nerves, while others contribute to the formation of the fiber-tracts of the cord.

After the appearance of the neuroblasts and developing nerve-cells, the wall of the neural tube is divisible into three layers (Fig. 140): an inner or ependymal layer, next the lumen of the tube; adjoining this, the mantle layer, made up of neuroblasts ; and a peripherally situated neuroglia layer or marginal velum, which occupies the position of the future tracts of white fibers of the cord.

The alterations in the form and size of the spinal cord go hand in hand with the histological changes noted above. While those areas that have been mentioned as the dorsal and ventral zones increase greatly in thickness, the floor-plate and the roof-plate-the rentral and dorsal walls of the neural tube-remain thin (Fig. 141). They are never invaded by the nerve-cells, but consist of thin layers of neuroglia which 
later become penetrated by nerve-fibers that grow from one side to the other. They thus represent the anterior and posterior white commissures of the cord. These plates remain relatively fixed in position because of their failure to expand, while the lateral walls of the tube undergo great expansion, in both the ventral and dorsal directions, as well as laterally. In this way a median longitudinal eleft is produced on the ventral wall of the spinal cord and a similar one on the dorsal wall. These are the anterior and posterior median fissures. Since the so-called posterior median fissure is not a true fissure but merely a septum, it differs from the anterior fissure, and it is held by some authorities that this septum is

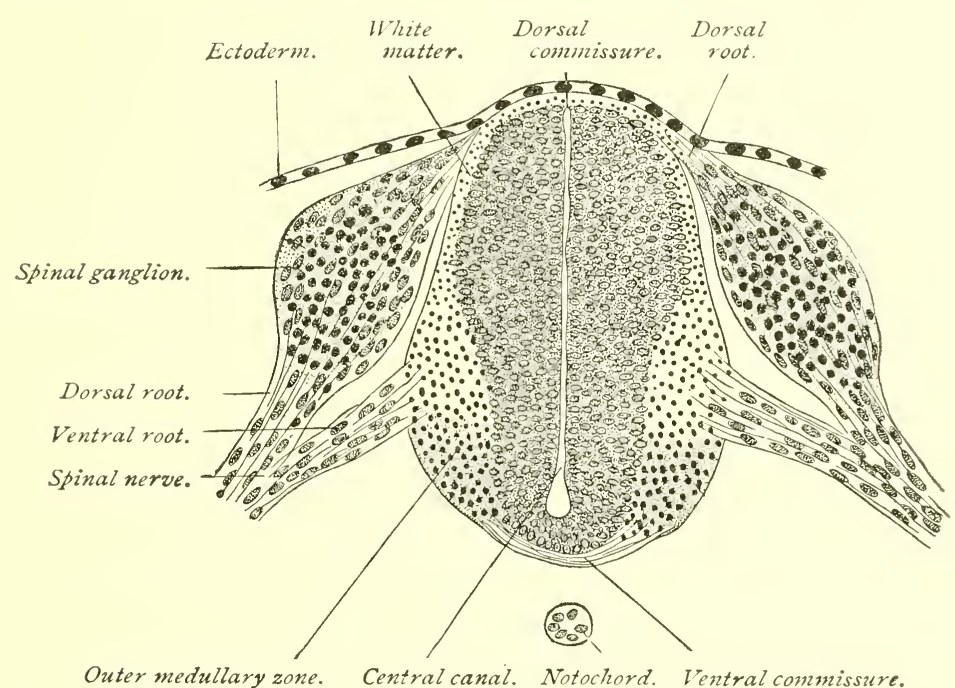

FIG. 141.-Transverse section of developing spinal cord of a twenty-two-day sheepembryo (Bonnet).

formed by the growing together of the walls of the dorsal part of the central canal.

The fiber-tracts or white matter of the spinal cord develop in the outer or neuroglia layer, each fiber being the elongated neurit of a nerve-cell. Some of the fibers originate from the nerve-cells of the cord while others grow into the cord from other sources. As examples of the former method may be cited the direct cerebellar tract, composed of the axons of the cells of the vesicular column of Clark, and the tract of 
Gower, made up of the axons of cells of the dorsal gray horn; while the direct and crossed pyramidal tracts are the axons of cells in the eortex of the cerebrum, and the tracts of Goll and of Burdach are composed largely of the axons of the cells of the spinal ganglia (see p. 318). The development of these fiber-tracts is not complete until the fibers acquire their myelin-sheaths (see p. 414). The myelination of the tracts of Burdach and of Goll occurs in the latter part of the fourth month and in the fifth month; of the direct cerebellar tract, in the seventh month; of the pyramidal tracts, at or soon after birth.

As the walls of the neural canal thicken through the multiplication of the cells, the cavity of the tube is gradually encroached upon almost to obliteration. When development is complete, all that remains of the cavity is the small central canal of the spinal cord.

The length of the spinal cord in the fourth fetal month corresponds with that of the spinal column, its lower termination being opposite the last coccygeal vertebra. From this time forward, however, the cord grows less rapidly than does the spinal column, so that at birth, the cord terminates at the last lumbar vertebra, and in adult life at the second lumbar vertebra. This gradually acquired disproportion in the length of the two structures explains the more oblique direction of the lower spinal nerves as compared with those higher up. In the early condition of the cord, each pair of nerves passes almost horizontally outward to the corresponding intervertebral foramina, but as the spinal column gradually outstrips the cord in growth, the lower nerves necessarily pursue a successively more oblique course to reach their foramina, the lower nerves being almost vertical in direction and constituting, collectively, the cauda equina.

\section{THE DEVELOPMENT OF THE BRAIN.}

The encephalic portion of the neural tube-that part devoted to the production of the brain-after undergoing dilatation, becomes marked off into the three primary brainvesicles, the fore-brain or prosencephalon, the mid-brain or mesencephalon, and the hind-brain or rhombencephalon, by 
constrictions in the lateral walls of the tube (Fig. 142). The constricted part of the hind-brain that adjoins the midbrain is the isthmus. This division occurs at an early stage, before the closure of the tube is everywhere complete. The vesicles communicate with each other by rather wide openings. As in the spinal part of the neural eanal, the walls of the primary brain-vesicles consist of epithelial cells, and it is by the multiplication of these cells in unequal degree in different regions, and by the formation of folds in certain localities, that the various parts of the adult brain are developed from these simple epithelial sacs.

The stage of three vesicles is soon succeeded by the fivevesicle stage, the primary fore-brain vesicle undergoing division into two, the secondary fore-brain (telencephalon) and the inter-brain (thalamencepalon) or diencephalon, and the primary hind-brain vesicle likewise dividing, a little later, into the secondary hind-brain (metencephalon) and the after-brain (myelencephalon).

The division of the primary fore-brain is preceded by the appearance upon each of its lateral walls of a small bulgedout area which soon assumes the form of a distinct diverticulum. This is the optic vesicle, the earliest indication of the development of the eye (Fig. 142). In the further process of growth the base of attachment of the optic vesicle becomes lengthened out into a relatively slender pedicle, which remains in connection with the lower part of the lateral wall of the brain-vesicle.

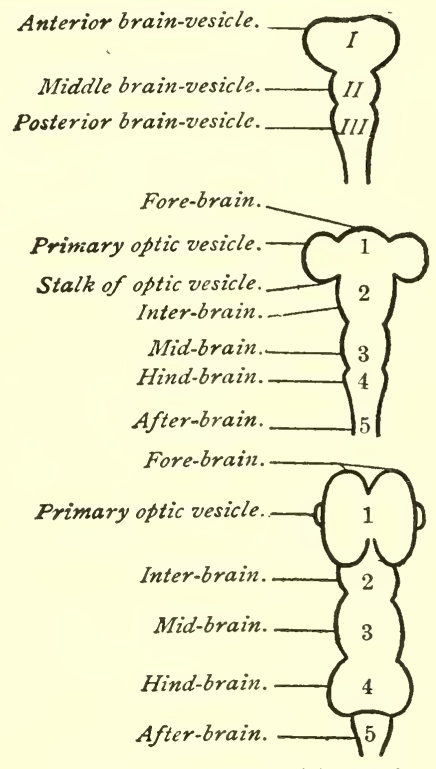

FIG. 142.-Diagrams illustrating the primary and secondary segmentation of the brain-tube (Bonnet).

Following the appearance of the optic vesicle, the anterior wall of the primary fore-brain vesicle projects as a small evagination, which latter is then distinctly marked off from 
the parent vesicle by a groove on either side. This anterior diverticulum is the secondary fore-brain vesicle or the vesicle of the cerebrum, and the original or primary forc-brain vesicle is now the vesicle of the inter-brain.

The division of the primary hind-brain is effected by the development of a constriction of its lateral wall, this resulting in the production of the secondary hind-brain or the vesicle of the cerebellum, and the after-brain or the vesicle of the medulla oblongata.

While the three primary vesicles at first lie in the same straight line, they begin to alter their relative positions shortly before division. The change of position is coincident with the flexures of the body of the embryo that occur at this time. Three well-marked flexures appear, the result being

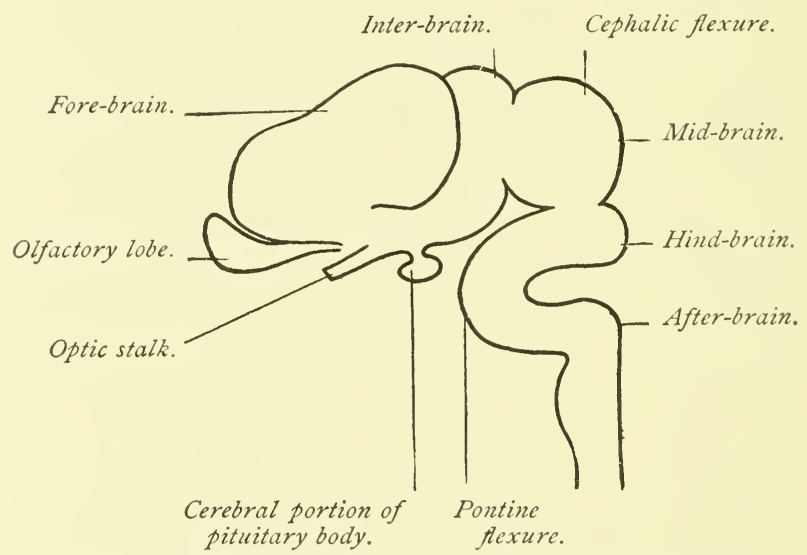

FIG. 143,-Diagram showing relations of brain-vesicles and flexures (Bonnet).

that the fore-brain is bent over ventrad to a marked degree. The most anterior of these flexures, and the first to develop, is the so-called cephalic flexure (Fig. 143), the primary forebrain, in the advanced state of the curvature, being bent around the termination of the chorda dorsalis so as to form a right angle, and later, after its division, an acute angle with the floor of the mid-brain. This curvature makes the mid-brain very prominent as regards the surface of the embryonic body, producing the parietal elevation or the prominence of mid-brain. In the region of the future pons Varolii, on the floor or 
ventral wall of the secondary hind-brain, is a second wellmarked angularity. This is the pontal flexure. Its con-* vexity projects forward.

A third bend, the nuchal flexure, is a less pronounced curvature at the juncture of the after-brain with the spinal part of the neural tube.

The Metamorphosis of the Fifth Brain-vesicle.The fifth brain-vesicle, the caudal division of the primary hind-brain, differentiates into the structures which surround the lower half of the fourth ventricle, these structures con-

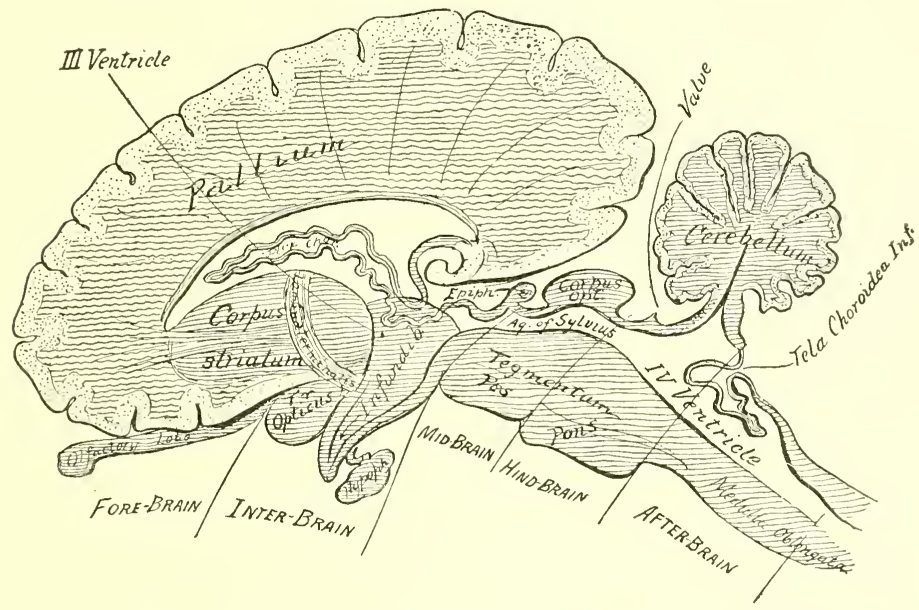

FIG. 144.-Diagram of a sagittal section of the brain of a mammal, showing the type of structure and the parts that develop from the several brain-vesicles (modified from Edinger).

stituting the myelencephalon (Fig. 144). The histological changes correspond essentially with those that occur in the spinal segment of the neural tube, the nerve-cells and fibers and the neuroglia resulting from the differentiation of the original ectodermic epithelium of which the wall of the tube is composed, and the connective-tissue stroma growing into these from the surrounding mesoderm.

There is a marked disproportion between the rate of growth of the tube in different parts of its circumference. The great thickening of the ventral and lateral walls produces the several parts of the medulla oblongata. In the dorsal wall 
growth occurs to such slight extent that the wall in this region remains a thin layer of epithelium. As a consequence, the cavity of the neural tube is not encroached upon on its dorsal side and the central canal of the spinal cord therefore expands in the myelencephalon into a much larger space, the lower half of the future fourth ventricle. This relative expansion of the central canal begins to be apparent in the third week in the human embryo, from which period it continues to increase. A cross-section through the lower part of the developing medulla shows a cavity which is narrow laterally but which has a considerable anteroposterior extent. A section at a higher level discloses a triangular space, the base of the triangle being the dorsal wall of the cavity.

At the time when the cavity of the after-brain acquires a distinctly triangular shape-about the third week-each thickened lateral half of the tube is divisible into a ventral and a dorsal segment, these being known respectively as the basal lamina and the alar lamina (Fig. 145).

The first indication of the longitudinal fiber-tracts of the medulla is presented by two bands of fibers which appear upon the surface of the alar lamina and which

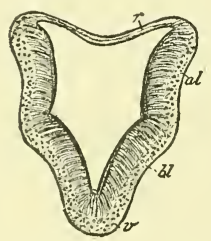

FIG. 145.- S e c tion through upper part (cerebellar region) of the fourth ventricle of an embryo (His): $r$, roof of neural canal: al, alar lamina: bl, basal lamina ; $v$, ventral border. constitute the ascending root of the fifth nerve and the ascending root (funiculus solitarius) of the vagus and glossopharyngeal nerves. These are later covered in by the folding over of the dorsal part of the alar lamina (Fig. 146) and thus come to occupy their permanent position in the interior of the medulla.

The parts of the alar laminæ that are folded over in the manner referred to differentiate for the most part into the restiform bodies or inferior peduncles. These are distinguishable in the third month. The anterior pyramidal tracts develop from the ventral parts of the basal laminie and are recognizable in the fifth month. Coincirlentally with the formation of the fibers, the gray matter of the medulla assumes its permanent form and arrangement. This gray matter, although in part peculiar to the 
medulla, is in great measure but the continuation of the gray matter of the spinal cord rearranged and differently related because of the motor and sensory decussations and of the dorsal expansion of the central canal. A notable feature of this

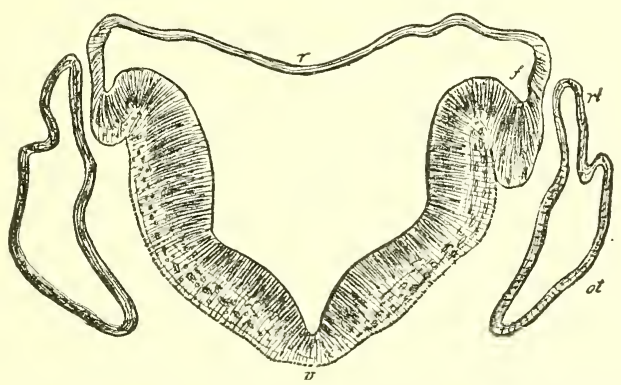

FIG, 146.-Section across the lower half of the fourth ventricle of an embryo, showing gradual opening out of the neural canal, and the commencing folding over of the alar lamina at $f$ (His) : $v$, ventral border; $t$, tenia; ot, otic vesicle; $r l$, recessus labyrinthi.

rearrangement is the presence of masses of gray matter immediately beneath the floor or ventral wall of the now expanded cavity or fourth ventricle.

As stated above, the dorsal wall of the after-brain vesicle remains an extremely thin epithelial lamina, and the cavity in consequence expands toward the dorsal surface. Owing to the excessive delicacy of this dorsal wall of the cavity, it is easily destroyed in dissection, with the effect of disclosing a triangular fossa (Fig. 151) on the dorsal surface of the medulla, which in connection with a similar depression on the dorsal surface of the pons, constitutes the rhomboidal fossa, or the fourth ventricle of the brain.

It is often stated in descriptions of the medulla and fourth ventricle that the latter is produced by the opening out of the central canal of the cord to the dorsal surface. It should be borne in mind, however, that the central canal does not, in reality, open out to the surface, although it may appear to do so because of the attenuated condition of its dorsal boundary. The thin epithelial roof or dorsal wall of the afterbrain becomes adherent to the investing layer of pia mater, thus forming the tela choroidea inferior, which roofs over the lower half of the fourth ventricle (Fig. 144). The pia 
mater invaginates the epithelial layer to form the choroid plexuses of the fourth ventricle. Although apparently within the eavity of the ventricle, the choroid plexuses are excluded from it by the layer of epithelium, the morphological roof of the after-brain, which they have pushed before them.

While, for the most part, the roof of the after-brain consists of the thin epithelial layer referred to above, there are slight linear thickenings, the ligulæ, along its lateral margins, and at its lower angle, the obex. At the upper margin of the roof, at the place of junction with the hind-brain, there is also a thickened area, the inferior medullary velum. These regions of thicker tissue serve to effect the transition from the thin epithelial layer that helps to form the inferior choroidal tela to the more massive boundaries of the rhomboidal fossa.

The Hind-brain Vesicle or Metencephalon.-The metencephalon consists of the pons, the cerebellum with its superior and middle peduncles, and the valve (ralve of Vieussens). These structures are produced by the thickening of the walls of the fourth or hind-brain resicle.

The pons is formed by the thickening of the ventral wall of the vesicle. Its transverse fibers become recognizable during the fourth month.

The cerebellum grows from the posterior part of the roof or dorsal wall of the vesicle (Fig. 14t). The first indication of its development is seen as a thick transverse ridge or fold on the posterior extremity of the dorsal wall (Figs. 147, 148). In the third month the central part of this ridge, now grown larger, presents four deep transverse grooves with the result of dividing the original eminence into five transverse ridges. The grooved part of the ridge is the portion that subsequently becomes the vermiform process or median lobe of the cerebellum, while the smooth lateral portions become the lateral hemispheres. As the vermiform process increases in bulk, two of the ridges come to lie upon its upper surface and three on the inferior aspect. These ridges and furrows persist throughout life as the principal convolutions and fissures of the vermiform process (Figs. 149, 150). 
The lateral parts of the primary ridge increase in size and eventually, in the human brain, outstrip the median lobe in growth. They acquire their chief transverse fissures in the fourth or fifth month, and the smaller sulei later.

The thickened cerebellar ridge on the roof of the hindbrain vesicle being continuous with the lateral walls, the continuity of the cerebellar hemispheres with the pons through the middle and superior cerebellar peduncles and with the medulla by means of the inferior peduncles, is easily

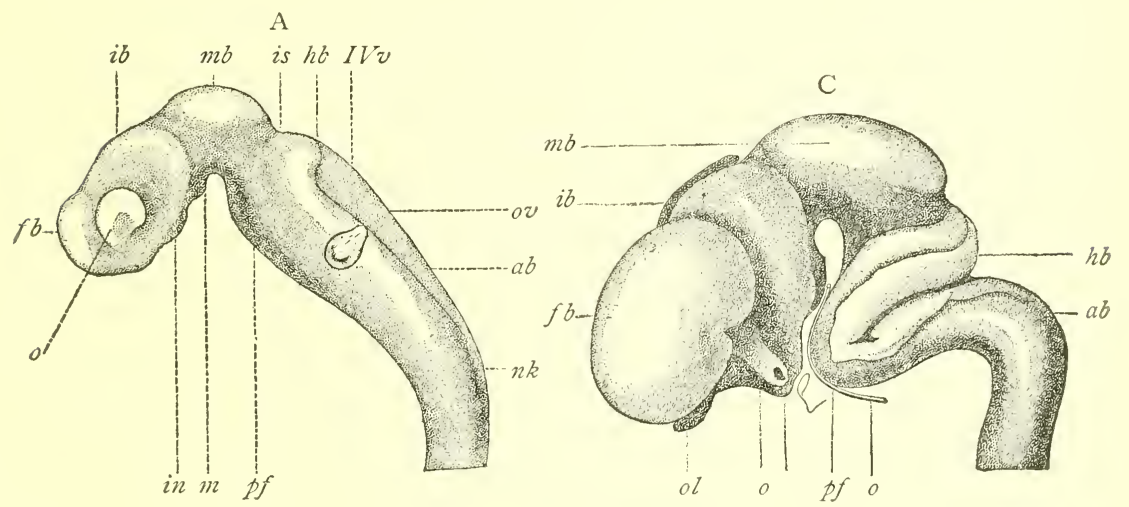

B

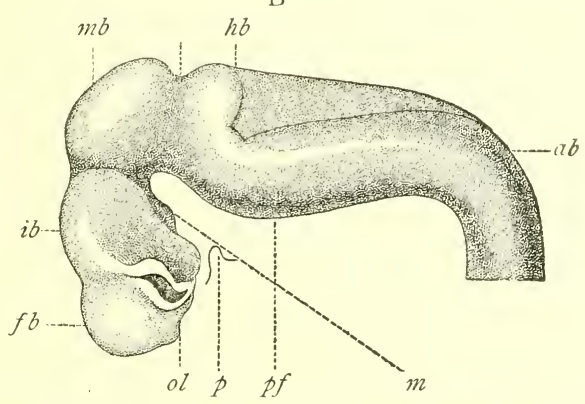

FIG. 147. - Brains of human embryos, from reconstructions by His: A, brain from fifteen-day embryo; $B$, from three-and-a-half-week embryo; $C$, from sevenand-a-half-week fetus: $f b, i b, m b, h b, a b$, fore-, inter-, mid-, hind-, and after-brain vesicles; $o$, optic vesicle; ov, otic vesicle; $i n$, infundibulum ; $m$, mammillary process; $p f$, pontine flexure; $I$ lv, fourth ventricle; $n k$, cervical flexure; ol, olfactory lobe ; $b$, basilar artery ; $p$, pituitary recess.

understood. These bands of fibers become evident, the inferior in the third month, the middle in the fourth month, and the superior in the fifth month.

While the posterior part of the roof of the hind-brain 
thickens and develops into the cerebellum, all the remaining part of this roof remains relatively thin and becomes the anterior medullary velum or the valve of Vieussens (Fig. 144). The relations of this structure in the mature brain, stretching across, as it does, from one superior cerebellar peduncle to the other and being continuous posteriorly with the white matter of the cerebellum, are easily explained in the light of the fact that all these parts are but the specialized dorsal and lateral walls of the hind-brain vesicle. Since the roof of the hind-brain vesicle is continuous with that of the after-brain or fifth vesicle, it will be seen that the cerebellum must be in continuity with the roof of the medullary part of the fourth ventricle. The transition from the cerebellum to the epithelium of the tela choroidea inferior is effected by a pair of thin crescent-shaped bands of white nerve-matter which pass downward from the central white-matter of the cerebellum, and which are collectively known as the inferior or posterior medullary velum. Thus, as the result of unequal growth, there are produced from the continuous dorsal walls of the fourth and fifth vesicles the thin laminar medullary velum or valve, the massive cerebellar lobes, the thin bands known as the inferior medullary velum, and the single layer of epithelium which, with a layer of pia mater, constitutes the inferior choroidal tela.

Although the fourth and fifth brain-vesicles are at first delimited from each other by a constriction, this constriction, as development goes on, disappears, the cavity of the fourth vesicle and that of the fifth together constituting the fourth ventricle of the brain.

The walls of the fourth or hind-brain vesicle then give rise ventrally to the pons, laterally to the superior and middle cerebellar peduncles, and dorsally to the valve and the cerebellum, while its cavity becomes the anterior half of the fourth ventricle.

The Mid-brain Vesicle.-The third brain-vesicle or the vesicle of the mid-brain or mesencephalon gives rise to the structures surrounding the aqueduct of Sylvius, the persistent part of the cavity constituting the aqueduct itself.

The thickening of the ventral wall of the vesicle results in 
the formation of the crura cerebri and the posterior perforated lamina or space included between them. The crura first become apparent in the third month as a pair of rounded longitudinal ridges on the ventral surface of the vesicle. These remain relatively small until the fifth month, when the longitudinal fibers of the pons begin to grow into them. After this occurrence their increase in size is comparatively rapid, their ventral parts or crustæ becoming separated from each other and including between them the posterior perforated lamina.

The roof or dorsal wall of the mid-brain vesicle undergoes considerable thickening (Fig. 147), especially in the Sauropsida (birds, reptiles, fishes). In the fifth week a longitudinal ridge appears upon the dowal wall, which in the third month is replaced by a furrow. The expansion of the wall on each side of the furrow produces a pair of rounded eminences (Figs. 148-151), which, in birds, attain to a much
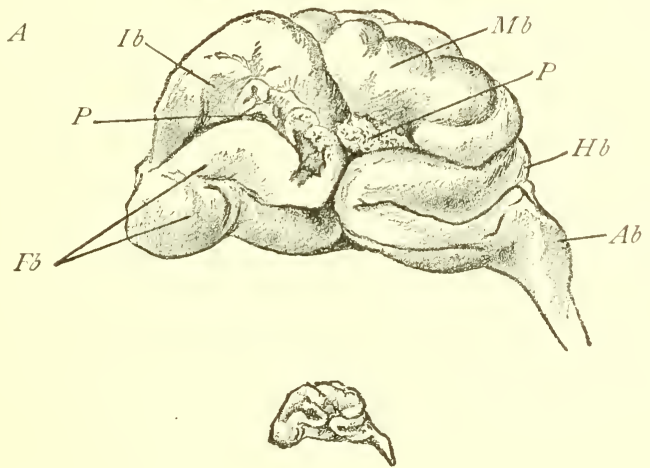

$B$

FIG. 148.-Brain of human fetus of about eight weeks: $A$, enlarged; $B$, actual size; $F b$, fore-brain ; $I b$, inter-brain ; $A b$, mid-brain ; $H b$, hind-brain; $A b$, afterbrain ; $P$, folds of pia mater.

greater development than in mammals and constitute the corpora bigemina or optic lobes. In the human embryo, each of these elevations is divided into two by an oblique groove, and thus are formed the corpora quadrigemina, which are peculiar to man and other mammals.

The part of the dorsal wall of the vesicle that underlies the corpora quadrigemina is the lamina quadrigemina.

The thickening which the walls of the vesicle undergo to 
produce the several parts of the mid-brain encroaches so much upon its eavity that an exceedingly small canal, the aqueduct of Sylvius, remains. It is scarcely necessary to point out that this canal is a part of the rentricular system of the brain, establishing a communication between the fourth ventricle and the third ventricle or cavity of the inter-brain.

The Metamorphosis of the Inter-brain Vesicle.The inter-brain vesicle results from the division of the primary fore-brain vesicle, comprising what is left of the latter after the outgrowth from it of the diverticulum that becomes the secondary fore-brain. The thickening of the walls of the inter-brain vesicle produces the structures which surround the third ventricle in the mature condition, and which constitute collectively the thalamencephalon or inter-brain, the cavity of the vesicle persisting as the adult third ventricle. These structures are the optic thalami, which are formed from the lateral walls; the velum interpositum and the pineal body, which develop from the roof; and the lamina cinerea, the
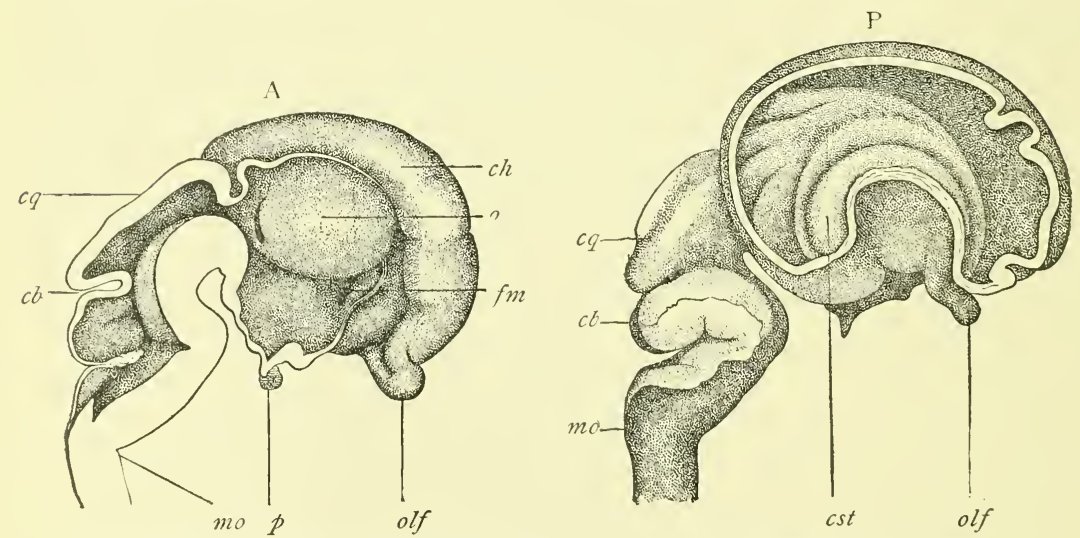

FIG. 149.-A, mesial section through brain of a human fetus of two-and-a-half months (His) : ch, cerebral hemisphere; o, optic thalamus; $f m$, foramen of Monro ; olf, olfactory lobe; $p$, pituitary body ; mo, medulla oblongata ; $c q$. corpora quadrigemina; $c b$, cerebellum. $\mathrm{B}$, brain of human fetus of three months (His): olf, olfactory lobe; $c s t$, corpus striatum; $c q$, corpora quadrigemina; $c b$, cerebellum; mo, medulla oblongata.

tuber cinereum, the infundibulum, the posterior lobe of the pituitary body and the corpora albicantia, which are differentiated from the floor of the vesicle.

The lateral walls of the vesicle undergo the most marked 
thickening. The cell-multiplication here is so rappic that each lateral wall is converted into a large ovoid mass of cells with intermingled bands of fibers, the optic thalamus.

The roof of the inter-brain vesicle, in notable contrast with the lateral walls, remains extremely thin throughout the greater part of its extent (Fig. 144). From the lack part of the roof, at a point immediately in front of the lamina quadrigemina of the mid-brain, a diverticulum grows out and becomes metamorphosed into the pineal body. With this exception, the roof of the vesicle remains a single layer of epithelium, just as in the case of the roof of the afterbrain. This epithelial layer adheres closely to the pia mater, which covers it in common with the other parts of the brain. As the fore-brain expands, it covers the inter-brain, the under surface of the cerebral hemispheres of the former being closely applied to the roof of the latter. As a consequence, the pia mater on the under surface of the fore-

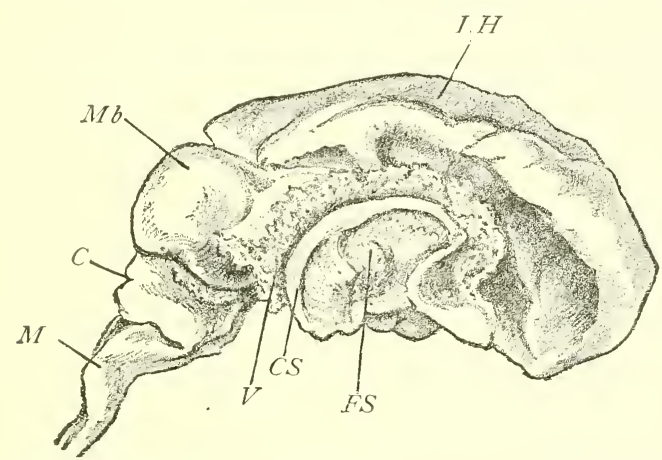

FIG. 150.-Brain of fetus of three months, enlarged. The outer wall of the right hemisphere has been removed; $L H$, left hemisphere; Cs, part of corpus striatum; $F S$, site of fossa of sylvius; $V$, vascular fold of pia mater which has been invaginated through the mesial wall of the hemisphere; $M b$, mid-brain; $C$, cerebellum; $M$, medulla oblongata.

brain is brought into contact with and adheres to the pia covering the roof of the inter-brain. Thus the thin epithelial roof of the inter-brain becomes closely united with the two layers of the pia mater to form the velum interpositum or tela choroidea anterior or superior of adult anatomy. Obviously, the edges of the velum interpositum rest upon the optic thalami, and its piamatral layers are continued into the 
cavities of the lateral ventricles (Fig. 150). The space occupied by the velum is designated the transverse fissure of the brain, and it is often stated that the pia mater is pushed in from behind, between the optic thalami and the cerebral hemispheres. As will be seen from the above description, however, its development really begins in front.

The pineal gland or conarium develops from the back part of the roof of the inter-brain at its point of junction with the mid-brain (Fig. 144). This body is found in all vertebrate animals except the amphioxus, but its form varies greatly in different groups. In all cases it begins as a small pouch-like evagination from the roof of the inter-brain, the diverticulum being directed forward. In the human brain alone the structure is subsequently directed backward, so that it comes to occupy a position just over the corpora quadrigemina. This peculiarity of location is due probably to the greater development of the human corpus callosum, by which the conarium is crowded backward.

In selachians (sharks and dog-fish), the enlarged vesicular end of the diverticulum, which is lined with ciliated columnar cells, lies outside the cranial capsule and is connected with the inter-brain by the long hollow stalk which perforates the roof of the cranium. In many reptiles, the conarium is more highly specialized. In the chameleon, for example, the peripheral extremity has the form of a small closed vesicle which lies outside the roof of the cranium and which is covered by a transparent patch of skin. The stalk in this case is partly a solid cord and partly a hollow canal, which latter opens into the cavity of the inter-brain. The solid portion lies within a foramen in the parietal bone, the parietal foramen. A further modification of the conarium is presented in lizards, blind-worms, and some other reptiles. In these the vesicle undergoes a marked specialization, its peripheral wall being so modified as to become transparent and to resemble the crystalline lens of the eye, while the opposite deeper wall comes to consist of several layers of cells-some of which become pigmented-and acquires a striking resemblance to the retina. The stalk of the body, which perforates the roof of the skull and is attached to the roof of the inter- 
brain, bears a certain likeness to the optic nerve, being solid and composed of fibers and elongated cells. The presence of the transparent epidermal plate which eovers the vesicle serves to complete the similarity of this particular type of pineal body to the eye of vertebrate animals. It is for this reason that it is often. designated the pineal or parietal eye and that it has been looked upon as a third or unpaired organ of vision.

In man and other mammals and in birds the pineal diverticulum does not reach the degree of development that is attained in certain of the Reptilia. The evagination from the roof of the inter-brain begins in the sixth week in the human embryo. The peripheral end of the process enlarges somewhat and small masses of cells project from it into the surrounding mesodermic tissue. These cellular outgrowths, giving off secondary projections, become converted into small closed follicles lined with columnar ciliated cells. The follicles in the case of mammals very soon become solid or nearly so by the aceumulation of cells in their interior. Solid concretions of calcareous matter, the so-called brain-sand (acervulus cerebri) are found in the follieles in the adult. By these alterations the pineal body of birds and mammals aequires a structure resembling that of a glandular organ. Since it is only the end of the diverticulum that becomes thus altered, the remaining part constitutes the relatively slender stalk of the pineal body, the stalk being solid at maturity except at its point of attachment to the inter-brain, where a portion of the cavity persists as the pineal recess of the third ventricle.

The pineal body of man and the higher vertebrates is therefore a rudimentary structure and is the representative of an organ that is much more highly developed in some of the lower members of the same series. Its true significance is still a matter of conjecture. Although resembling the eye in its structure, and although regarded by some on that account as primitively an organ of vision, it is considered probable by others that in its most highly developed condition it is an organ of heat pereeption.

The floor of the inter-brain vesicle presents several interesting 
metamorphoses. The anterior part of the floor remains quite thin and becomes the lamina cinerea of the mature brain (Fig. 144). Immediately posterior to this region, the floor of the vesicle pouches out, this evagination developing into a slender tube, the infundibulum. Behind the point of origin of the infundibulum a second protuberance indicates the beginning of the tuber cinereum. By subsequent alterations, the tuber cinereum enlarging in circumference so as to include the point of origin of the infundibulum, the base of attachment of the infundibulum comes to be the center of the tuber cinereum, so that the eavity of the former is a continuation of the cavity of the latter. The end of the infundibulum becomes the posterior lobe of the pituitary body or hypophysis (Figs. 144 and 149). Posterior to the tuber cinereum a small evagination of the floor of the vesicle appears and becomes divided in the early part of the fourth month into two lateral halves by a median furrow. The two little bodies thus formed become, after further development, the corpora albicantia.

The hypophysis or pituitary body briefly referred to above requires more extended consideration because of its morphological importance. The posterior lobe of this body is the enlarged end of the infundibulum, which is an evagination of the floor of the inter-brain. The cells in the lower end of the infundibulum specialize into nerve-cells, and nervefibers also develop. In some lower vertebrates these elements are retained throughont life, but in man and the higher-type animals the distinctively nervous character of the tissues is soon lost, and the cavity of this part of the infundibulum suffers obliteration. The branched pigmentcells sometimes recognizable in the posterior lobe of the human pituitary body are the only remnant of the early nerve-cells.

The anterior lobe of the hypophysis is essentially different in origin as well as in structure from the posterior lobe. It is produced by an evagination from the posterior wall of the primitive pharynx, but from that region of the pharynx which is anterior to the pharyngeal membrane and which therefore belongs to the primitive mouth-cavity (Fig. 66, p. 131). The 
out-pocketing of the pharyngeal wall begins in the fourth week, shortly after the rupture of the pharyngeal membrane. The little pouch is the pocket of Rathke. The pouch grows upward and backward toward the floor of the inter-brain and meets the end of the infundibulum. As the pharyngeal diverticulum lengthens, its stalk becomes a slender duct, which for some time retains its connection with the pharynx. As the membranous base of the skull becomes cartilaginous, the duct begins to atrophy, and finally entirely disappears. In selachians, however, it is retained permanently, establishing thus a connection between the hypophysis and the pharyngeal cavity. With the disappearance of the duct the enlarged extremity of the diverticulum becomes a closed resicle lying now within the cavity of the brain-case, in contact with the end of the infundibulum. From the wall of the resicle numerous little tubular projections grow out into the enveloping mesodermic tissue, and these, by detachment from the parent vesicle, become closed tubes or follicles. The entire structure becomes converted in this manner into a mass of closed follicles held together by connective tissue, after which event this mass acquires intimate union with the infundibular lobe.

Thus the pituitary body consists of two genetically distinct parts, the anterior lobe being derived from the ectoderm of the primitive pharyngeal or buccal cavity, and the posterior lobe from the ectoderm of the central nervous system. The posterior lobe, developing as it does as an evagination from the floor of the inter-brain, is to be regarded as a small outlying lobe of the brain.

What remains of the cavity of the inter-brain, after its walls have thus developed into the several structures described, is the third ventricle of the adult brain, and the aperture of communication with the secondary fore-brain vesicles becomes the foramen of Monro. Since the lateral walls become the massive optic thalami, while the dorsal and ventral walls give rise to much thinner structures, the cavity of the vesicle is encroached upon to a greater extent on the sides than from above and below, and hence the form of the third ventricle in the mature eondition is that of a narrow vertical fissure between the thalami. 
The Metamorphosis of the Fore-brain Vesicle.The secondary fore-brain vesicle gives rise to the telencephalon, which includes the cerebral hemispheres and the structures belonging directly to them. As above indicated, this vesicle grows from the anterior wall of the primary forebrain vesicle as a diverticulum which is at first single, but which soon becomes divided into two lateral halves by the formation of a cleft in the median plane (Fig. 147, $f b$ ). This cleft or interpallial fissure is the early representative of the longitudinal fissure of the adult cerebrum. The two vesicles remain attached at their bases or stalks with the parent vesicle and communicate by a common orifice with its cavity. The vesicles of the secondary fore-brain grow in an upward and backward direction as well as laterally, and their development is so much more rapid than that of the other vesicles that they soon spread over them and partially hide them from view. It is for this reason that the mass resulting from the fore-brain vesicles, except their basal ganglia, is known in comparative anatomy as the pallium or mantle (Fig. 144).

The relative rate of growth of the cerebral hemispheres is such that in the third month they completely overlie the inter-brain and by the sixth month they have extended so far back as to hide the corpora quadrigemina.

The mesodermic tissue surrounding the developing brain becomes differentiated into the three brain-membranes, which penetrate into the fissure and therefore invest the vesicles on their mesial surfaces as well as elsewhere. The invaginating layers of the dura mater constitute the primitive falx cerebri.

The metamorphosis of this pair of sacs into the cerebral hemispheres is brought about by three important processes: first, the multiplication of the cells which compose its walls to form the masses of nerve-cells and fibers of the hemispheres; second, the formation of folds in the wall whereby are produced the fissures which divide the hemispheres into lobes and convolutions; and third, the development of adhesions within certain areas between the mesial walls of the 
two vesicles, by which the system of commissures of the hemispheres is produced.

The walls of the cerebral vesicles are at first very thin, consisting merely of several layers of spindle-shaped cells. By the rapid multiplication of these cells, the walls are thickened and the cavity of the vesicle is gradually encroached upon until the mature condition of the brain is attained, when the cavity is relatively very much smaller than in the fetus and constitutes the ventricle of the hemisphere or the lateral ventricle. The nerve-cells develop processes or polar prolongations, of which the most conspicuous, the axis-cylinder processes, lengthen out to form the axis eylinders of nerve-fibers. The fibers thus formed are directed away from the surface and make up the white medullary matter of the hemispheres, while the more superficially placed layers of cells constitute the gray matter of the cortex of the brain.

In addition to the cortical or superficial gray matter there are masses of gray matter within the hemisphere, the basal ganglia, which are likewise collections of nerve-cells. Within a limited area on the lateral wall of each cerebral vesicle, near the lower margin, the cells undergo excessive proliferation resulting in the production of a large ganglionic mass, the corpus striatum, and of two smaller aggregations of cells, the claustrum and the nucleus amygdalæ. These basal ganglia are in reality an infolded part of the cortex.

Inasmuch as the cortical matter develops more rapidly, as regards superficial extent, than does the medullary substance, the cortex becomes thrown into folds, forming thus the convolutions and fissures of the hemispheres.

Some of the fissures of the brain are produced by an infolding of the entire thickness of the vesicle-wall so that their presence is indicated by corresponding projections in the walls of the ventricles. Such fissures are distinguished as total fissures. Included in this eategory are the fissure of Sylvius, which is represented in the wall of the lateral ventricle by the corpus striatum; the calcarine fissure, the dentate fissure, and the collateral fissure, which are responsible respectively for the calcar avis, the hippocampus major, and 
the collateral eminence of the lateral ventricle; and the great transverse fissure of the brain, the infolded wall in this case being very thin and consisting merely of the layer of epithelium which covers the choroid plexus.

The fissure of Sylvius is the earliest fissure formed and one of the most important. At an early period in the history of the secondary fore-brain, there is a region in the lower part of the lateral wall of the vesicle where expansion is less rapid than elsewhere, this area, as it were, remaining fixed. As the vesicle-wall immediately surrounding this

A

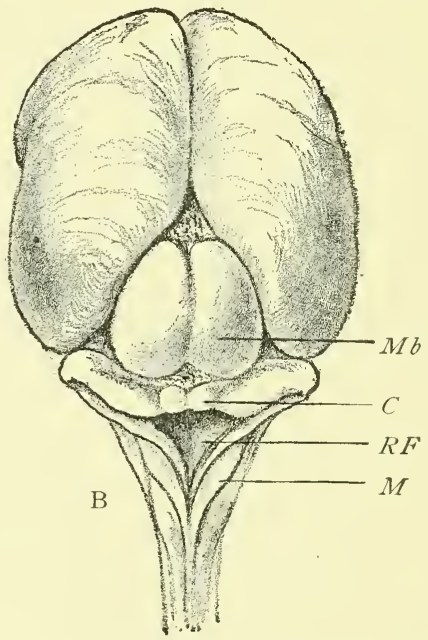

FIG. 151.-Posterior view of brain shown in Fig. 152: A, actual size; B, enlarged; $M b$, mid-brain ; $C$, cerebellum; $R F$, rhomboidal fossa (its dorsal wall having been removed); $M$, medulla oblongata.

spot continues to expand, a dimpling of the wall is produced, the depression being designated the fossa of Sylvius (Fig. 152, $S)$. The part of the vesicle-wall behind the fossa advances forward and downward to form the future temporal lobe, and thus the fossa comes to be surrounded by a convolution having the form of an incomplete ring, open in front-the ring lobe. The floor of the fossa undergoes very considerable thickening to form the basal ganglia-that is, the corpus striatum, the amygdaloid nucleus, and the claustrum. These structures, most conspicuously the corpus striatum, encroach upon the cavity of the vesicle, the nucleus caudatus of the 
corpus striatum bulging into the floor and outer wall of the adult lateral ventricle.

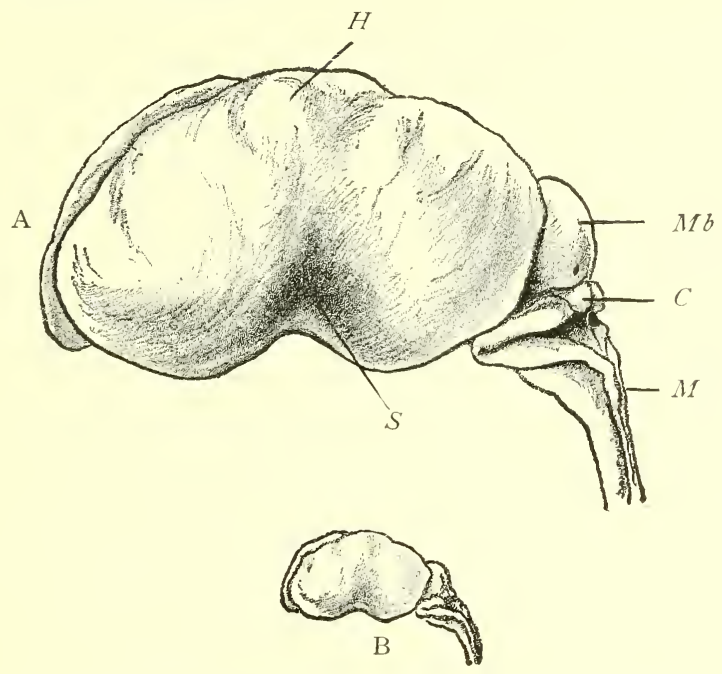

FIf. 152,-Brain of human fetus of approximately three months: A, enlarged; $\mathrm{B}$, actual size; $H$, hemisphere; $M b$, mid-brain; $C$, cerebellum; $M$, medulla oblongata; $S$, fossa of Sylvius.

The cortical matter of the floor of the fossa of Sylvius, being circumscribed by a groove or sulcus, constitutes the

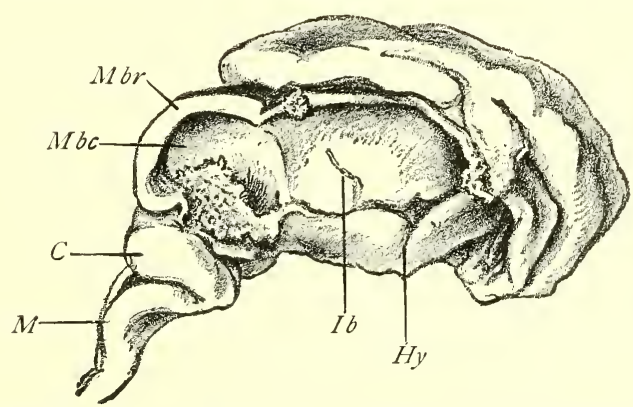

FIG. 153,-Brain of human fetus of three months, with right half of fore-brain, inter-brain, and mid-brain removed: $1 b$, cavity of inter-brain ; $H y$, site of hypophysis ; $M b r$, mid-brain roof; $M b c$, mid-brain cavity ; $C$, cerebellum; $M$, medulla oblongata.

central lobe or island of Reil, which is subsequently broken up, by secondary fissures, into from five to seven small convolutions. 
By the extension of the fossa of Sylvius backward, and by the increased growth of the vesicle-wall above and below it, the fossa is converted into the fissure of Sylvius (Fig. 156, B), and the island of Reil is hidden from view. Subsequently the ascending and anterior limbs are added to the chief or horizontal part of the fissure.

The anterior part of the ring lobe corresponds with the future frontal lobe, the posterior part represents the parietal lobe while the lower part of the ring becomes the temporal lobe. A backward extension of the ring lobe produces the occipital lobe.

The cavity of the vesicle is modified in form and extent coincidentally with the formation of the corpus striatum and the alterations in the ring lobe. Just as the ring lobe partially encircles the fossa of Sylvius, so does the cavity of the ventricle partially encircle the corpus striatum. An anterior prolongation of the cavity extends into the completed frontal lobe as the anterior cornu of the ventricle, and an extension downward and forward into the apex of the temporal lobe constitutes the descending cornu, while the posterior horn is gradually protruded into the occipital lobe as the latter develops. From the earliest stage, therefore, until the completed condition is attained, the cavity of the ventricle conforms in a general way to the shape of the hemisphere. The apertures of communication between the resicles of the cerebrum and the cavity of the inter-brain are the later $Y$-shaped foramen commune anterius or the foramen of Monro.

The mesial surfaces of the hemispheres are much modified in character by the development here of two total fissures, the arcuate fissure and the choroid fissure. These appear in the fifth week while the vesicles are still separate from each other down to their stalks of attachment to the inter-brain, prior to the development, therefore, of the corpus callosum and the fornix. The two fissures lie close together, parallel with each other and with the margin of the ring lobe, their course conforming to that of the cavity of the ventricle. Beginning near the anterior extremity of the brain, above the 
level of the corpus striatum, they pass backward and then downward and afterward forward to terminate near the anterior extrenity of the temporal lobe, thus incompletely encircling the striate body.

The arcuate fissure is the more peripherally placed of the two. Its anterior portion lies just above the region throughout which adhesions subsequently develop between the two hemispheres, or in other words, above the position of the future corpus callosum (Fig. 154, a.f.). This part of the arcu-

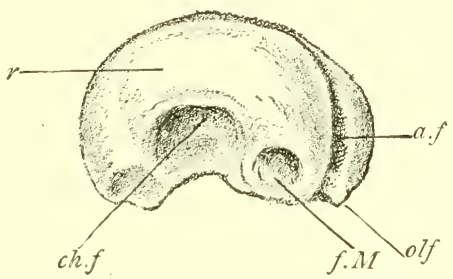

FIG. 154,-Mesial surface of left fore-brain vesicle of brain shown in Fig. $148(F b)$ : $f . M$, foramen of Monro, or opening into inter-brain ; a.f, arcuate fissure : ch.f, choroid fissure ; $r$, "randbogen," corresponding to future corpus callosum and fornix ; olf, olfactory lobe.

ate fissure is the sulcus of the corpus callosum of the mature brain. The posterior segment, that which belongs to the temporal lobe (not present at this stage), is the future hippocampal or dentate fissure. The hippocampal fissure is represented upon the mesial wall of the descending horn of the lateral ventricle by the prominence known as the hippocampus major.

The choroid fissure or fissure of the choroid plexus, forming an incomplete ring within, and parallel with, that described by the arcuate fissure, encircles the corpus striatum more closely (Figs. 154, 155). It begins at the foramen of Monro, and its anterior part lies under the position of the body of the future fornix. It then sweeps around into the temporal lobe and terminates near the anterior part of the latter. The fissure of the choroid plexus, like other total fissures, is an infolding of the wall of the cerebral vesicle. It presents the peculiarity, however, that the infolded part of the wall is extremely thin, consisting of but a single layer of epithelial 
cells. The pia mater, which everywhere closely invests the surface of the brain, is infolded with the vesicle-wall, the infolded part becoming very vascular and constituting the choroid plexus of the lateral ventricle. The choroid plexus, although within the limits of the ventricle, is excluded, strictly speaking, from its cavity by the layer of epithelium which still covers it and which has been simply pushed before it into that cavity. Since the epithelial layer is very thin and easily ruptured, the choroid fissure is apparently an opening into the cavity of the ventricle through which the pia enters ; in the adult it is called the great transverse fissure of the brain.

The calcarine fissure, another of the total fissures, develops in the latter part of the third month as a branch of the arcuate fissure. It bulges into the mesial wall of the posterior horn of the ventricle, producing the elevation known as the calcar avis or hippocampus minor. Since the posterior horn of the ventricle is developed as an extension of the cavity into the backward prolongation of the ring lobe which forms the occipital lobe, the calcarine fissure necessarily is later in appearing than the fissures above described.

The parieto-occipital fissure is added in the fourth month as a branch of the calcarine, effecting the definite demarcation between the parietal and occipital lobes.

The fissure of Rolando develops in the latter part of the fifth month in two parts. The two furrows are at first entirely separated from each other by an intervening area of cortex. Subsequently this part of the cortex sinks beneath the surface, as it were, since it expands less rapidly than the adjacent regions, and in this way the upper and lower limbs of the fissure become continuous. The sunken cortical area is to be found even in the adult brain as a deep annectant gyrus embedded in the Rolandic fissure at the position of its superior genu. The development of the fissure of Rolando effects the division between the frontal and parietal lobes.

The collateral fissure appears in the sixth month as a longitudinal infolding of the mesial wall of the hemisphere 
below and parallel with the hippocampal fissure. Being a total fissure, its presence affects the wall of the eavity of the vesicle, producing the eminentia collateralis. At about the same time the calioso-marginal fissure makes its appearance, and this is morphologically continuous, through the medium of the post-limbic sulcus, with the collateral fissure (Fig. 157). These three fissures constitute the peripheral boundary of a region of the mesial wall which is known in morphology as the falciform or limbic lobe.

The longitudinal fissure in the early stage of the growth of the cerebrum separates the two vesicles from each other except at the place where they are attached to the inter-brain; here the two sacs are united by that part of their common anterior wall which is immediately in front of the apertures of communication with the inter-brain and which is called the lamina terminalis.

The development of adhesions between the mesial surfaces of the hemisphere vesicles throughout certain definite areas marks the beginning of the corpus callosum and the fornix. The fusion of these areas begins in the third month in the region corresponding to the anterior pillars of the fornix, the septum lucidum and the genu of the corpus callosum; in the fifth and sixth months adhesion occurs in the position of the body of the fornix and of the body and splenium of the corpus callosum.

Although the central white medullary matter of the cerebral hemisphere is covered almost universally by the cortical gray matter, there is a limited area of the mesial surface from which the gray matter is absent, leaving the white matter exposed. The area of uncovered white matter has the form of a narrow band, which begins at the base of the hemisphere, in front of the opening into the inter-brain, extends upward along the anterior wall of the inter-brain, then passes backward along its roof and curves downward and outward behind, and then forward under it, to terminate at the front part of the temporal lobe. Thus this white band, which is known as the fimbria, and which represents the lower mesial edge of the hemisphere, almost encircles the inter-brain. The fimbria 
runs between the arcuate fissure and the fissure of the choroid plexus (Fig. 155, $f$ ). It holds such a close relation to the lat-

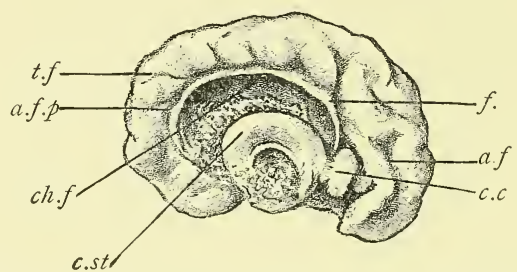

FIG. 155.-Mesial surface of left hemisphere, brain of fetus of three months (enlarged): $f$., fornix ; c.c., beginning of corpus callosum; c.st., part of corpus striatum arching around fossa of Sylvius; a.f., anterior, and a.f.p., posterior parts of arcuate fissure; $c h . f$., choroid fissure, the concarity between which and the corpus striatum accommodates the inter-brain, which has been removed. The fissure is occupied by the pia mater.

ter fissure, being placed on its peripheral side, that it constitutes the edge of the apparent opening into the cavity of the vesicle through which the pia mater, bearing blood-vessels, is reflected into the interior, and which, as pointed out above, is the transverse fissure of the brain. The opening is only apparent, however, since the wall is still unbroken, although reduced to a single layer of epithelium. The pia mater, forming, with its blood-vessels, the choroid plexus of the lateral ventricle, pushes the layer of epithelium before it, and although the plexus is said to be within the cavity of the ventricle, it is still covered by the layer of epithelium, the ependyma, which lines that cavity.

The part of the fimbria that immediately overlies the roof of the inter-brain becomes intimately united, as noted above, with the corresponding part of the fimbria of the other hemisphere, these fused portions of the two fimbriæ forming a flat triangular sheet, the body of the fornix. The anterior and posterior portions of the fimbria, which diverge from the median plane, represent respectively the anterior and posterior limbs of the fornix.

Noting the relation of the anterior part of the fimbria to the aperture of communication between the inter-brain and the cerebral vesicles, it becomes apparent that the anterior pillar of the fornix forms the anterior and upper boundary of 
the foramen of Monro. When, further, one considers the relation of the fimbria to the apparent opening into the ventricle, through which the pia mater is invaginated (the transverse fissure), it is explained why the edge of the fornix appears as a narrow white band, not only as viewed from within the ventricular cavity, but also in a mesial section of the brain (Fig. 156, C).

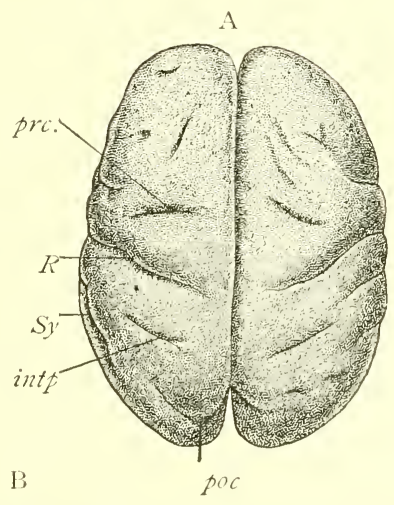

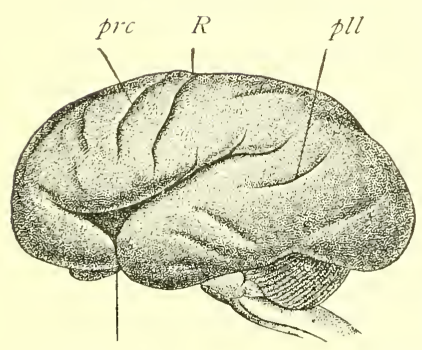

Sy

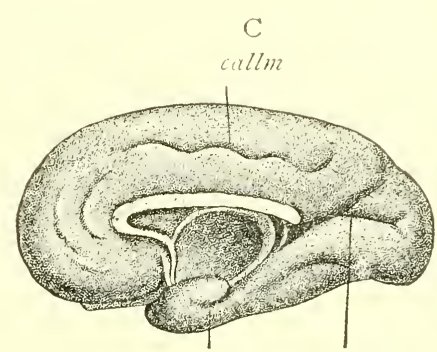

unc calc

FIG. 156.-Fetal brain at the beginning of the eighth month (Mihalkorics): A, superior, B, lateral, $\mathrm{C}$, mesial surface: $R$, fissure of Rolando; pre, precentral fissure ; Sy, Sylvian fissure ; intp, interparietal fissure; $p o c$, parieto-occipital fissure : $p l l$, parallel fissure; callm, callosomarginal fissure; unc, uncus; calc, calcarine fissure.

Another important region of fusion of the opposed mesial surfaces of the hemispheres is that corresponding to the future corpus callosum. Throughout this area the hemispheres closely unite with each other. The line of fusion begins at the bases of the vesicles, some little distance in front of the anterior parts of the fimbrix (Fig. 15.5, c.c.), and after passing upward and forward, curves horizontally backward 
in close relation with the fused portions of the fimbriæ, now the body of the fornix. 'The adhesion begins at the anterior part in the third month, and affects the region of the body and splenium of the future corpus callosum in the fifth and sixth months. Fibers penetrate from one hemisphere to the other throughout this zone of contact, intimately uniting the cerebral hemispheres. The corpus callosum is therefore a great commissure between the two halves of the cerebrum, and is necessarily composed of fibers having a transverse direction.

While the back part of the corpus callosum lies over the body of the fornix and is in close contact with it, the front part of the body of the corpus collusum, as also its genu or curve and its rostrum or ascending part are at some distance from the front parts of the fimbriæ. In other words, while the great longitudinal fissure extends at first to the bases of the cerebral vesicles, this fissure is made relatively less deep by the adhesions which occur between the mesial walls and which result in the development of the corpus callosum; and the space below the anterior part of the corpus callosum, between it and the anterior parts of the fimbriæ (Fig. 156, C), is an isolated part of the great longitudinal fissure. This space is bounded on either side by that part of the wall of the corresponding cerebral vesicle or hemisphere which is limited above and in front by the corpus collusum, and behind by the anterior part of the fimbria or anterior limb of the fornix. The space is the so-called fifth ventricle of the adult brain. The circumscribed parts of the mesial walls of the hemispheres, which form the lateral walls of the space, together constitute the septum lucidum. The parts of the hemisphere walls that become the septum lucidum do not participate in the process of fusion mentioned above. Their surfaces are in contact, however, and do not develop the typical gray cortical matter, such as appears elsewhere upon the surface of the cerebrum. Cortical gray matter is produced here, but only in rudimentary form.

From what has been said, it will be seen that the two layers of the septum lucidum are circumscribed and opposed 
parts of the mesial walls of the hemispheres; that the fifth ventricle is not a true ventricle but an isolated part of the longitudinal fissure having no connection whatever with the system of ventricular cavities; and that this so-called ventricle is not, like the true ventricles of the brain, lined with ependyma, but with atrophic gray cortical matter.

The limbic lobe has been referred to as that part of the mesial surface of the hemisphere which is circumscribed by the calloso-marginal fissure, the post-limbic sulcus, and the collateral fissure. It is limited centrally by the fissure of the corpus callosum and the hippocampal fissure, which are represented in the fetal brain by the single uninterrupted arcuate fissure. Hence the limbic lobe would include the gyrus fornicatus, the isthmus, and the gyrus uncinatus, which constitute morphologically a single ring-like convolution. Schwalbe, however, includes with this so-called limbic lobe all the surface of the mesial wall of the hemisphere included between the arcuate fissure and the fissure of the choroid plexus (Fig. 154), designating it the falciform lobe (Fig. 157).

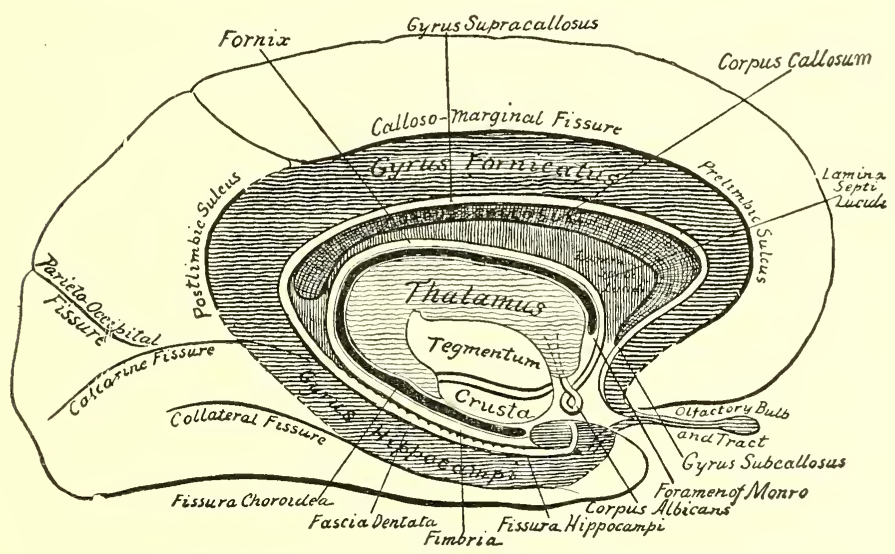

FIG. 157.-Diagram of the limbic lobe (after Quain).

The falciform lobe therefore consists of two ring-like convolutions, one within the other, the two being separated from each other by the arcuate fissure (the adult callosal and dentate fissures) and being limited centrally by the fissure of the choroid plexus (the great transverse fissure of the adult 
brain). While the outer of these concentric convolutionsthe limbic lobe of Broca-develops into the fornicate or callosal, the isthmian, and the uncinate gyri, the inner ring differentiates but slightly, its cortical matter remaining atrophic. The atrophic condition of the cortex here is associated with those adhesions between the mesial walls of the hemispheres that result in the formation of the corpus callosum and the septum lucidum. By these adhesions the continuity of the inmer concentric convolution is broken, and it is therefore represented, after the development of the corpus callosum, by the atrophic gray matter of the septum lucidum, by the gyrus dentatus, and by the lateral longitudinal striæ on the free surface of the corpus callosum, the latter being an atrophic or rudimentary convolution. Since the transverse fissure of the brain is the centric boundary of the ring, the fornix is also a part of the falciform lobe. To sum up, the falciform lobe includes the gyrus fornicatus, the isthmus, the gyrus uncinatus, the lateral longitudinal striæ or tænia tectæ of the corpus callosum, the gyrus dentatus, the laminæ of the septum lucidum and the fornix.

The olfactory lobe or rhinencephalon is an outgrowth from the vesicle of the cerebral hemisphere. Its development begins in the fifth week by the pouching-out of the wall of the vesicle near the anterior part of its floor (Figs. 147 and 149). This diverticulum, which contains a cavity continuous with that of the vesicle, grows forward and soon becomes somewhat club-shaped. In the selachians (sharks and dog-fish) the projection attains a great relative size, the olfactory lobes in these animals being one of the most conspicuous parts of the brain. In all mammals except man it is well developed, and in the horse its cavity persists throughout life. In man the cavity soon becomes obliterated and the lobe itself in part aborts. The protruded portion, becoming more distinctly club-shaped, differentiates into the olfactory bulb and the olfactory tract, the position of the original eavity being indicated by a more or less central mass of neuroglia conspicuous in cross-sections of those structures. The proximal portion of the olfactory lobe is represented in the adult 
human brain by the gray matter of the anterior perforated lamina (or space), and by the trigonum olfactorium and the area of Broca, as well as by the inner and outer roots of the olfactory tract (note olfactory lobe of dog's brain, Fig. 158).

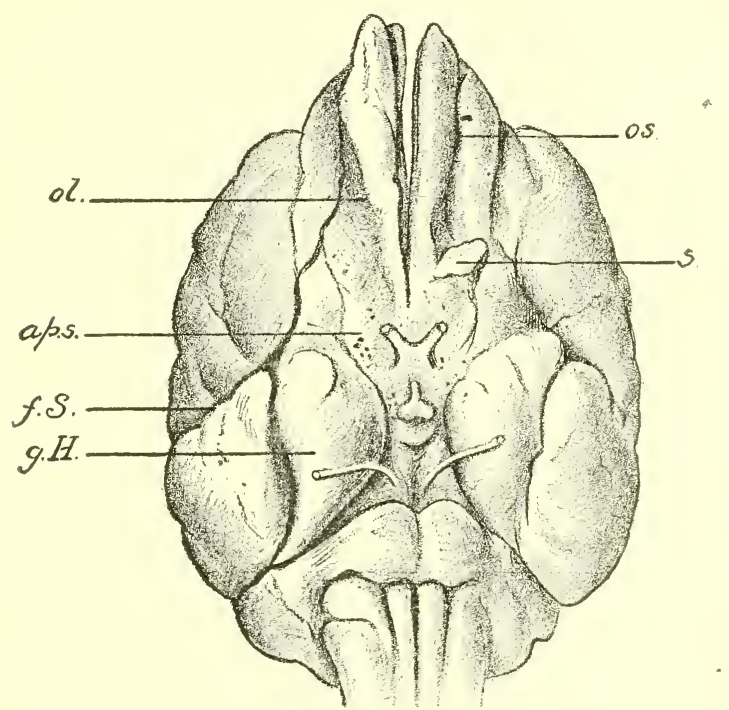

FIG. 158.-Base of dog's brain : ol., olfactory lobe ; a.p.s., region corresponding to anterior perforated space, which is included in the olfactory lobe ; f.S., fissure of Sylvius; g.h., hippocampal gyrus, developed to a greater degree than in human brain; s., sectional surface of olfactory lobe; os., olfactory sulcus.

Because of the relation of the place of evagination of the olfactory lobe to the fossa of Sylvius, it happens that a part of this lobe, the anterior perforated lamina, is situated at the commencement of the fissure of Sylvius and that it is in continuity with both extremities of the ring lobe; hence, the olfactory lobe is connected with both extremities of the falciform lobe. To express it in the language of human anatomy, the outer or lateral root of the olfactory tract is connected with the gyrus uncinatus, while the inner or mesial root may be traced to the fore part of the gyrus fornicatus.

After what has been said, the reader need scarcely be reminded that the olfactory bulb and tract, often erroneously referred to as the olfactory nerve, are parts of a lobe of the 
brain, a lobe which in man is rudimentary but which in all other mammals is well developed.

Tabulated Résumé of the Derivatives of the Brain-vesicles.

\begin{tabular}{|c|c|c|c|c|}
\hline $\begin{array}{c}\text { BRAIN- } \\
\text { VESICLES. }\end{array}$ & FloOR. & ROOF. & LATERAL WALLS. & CAvity. \\
\hline $\begin{array}{l}\text { After- } \\
\text { brain } \\
\text { vesicle. }\end{array}$ & $\begin{array}{l}\text { Medulla } \\
\text { oblongata. }\end{array}$ & $\begin{array}{l}\text { Tela choroidea } \\
\text { inferior. }\end{array}$ & $\begin{array}{l}\text { Inferior pedun- } \\
\text { cles of cerebel- } \\
\text { lum. }\end{array}$ & \multirow{2}{*}{$\begin{array}{l}\text { Fourth } \\
\text { ven- } \\
\text { tricle. }\end{array}$} \\
\hline $\begin{array}{l}\text { Hind- } \\
\text { brain } \\
\text { vesicle. }\end{array}$ & Pons Varolii. & $\begin{array}{l}\text { Posterior medul- } \\
\text { lary velum. } \\
\text { Cerebellum. } \\
\text { Anterior me- } \\
\text { dullary velum. } \\
\text { Posterior part of } \\
\text { tegmentum. }\end{array}$ & $\begin{array}{l}\text { Middle and supe- } \\
\text { rior peduncles } \\
\text { of cerebellum. }\end{array}$ & \\
\hline $\begin{array}{l}\text { Mid-brain } \\
\text { vesicie. }\end{array}$ & $\begin{array}{l}\text { Peduncles of cer- } \\
\text { ebrum. Poste- } \\
\text { rior perforated } \\
\text { space. }\end{array}$ & $\begin{array}{l}\text { Corpora quadri- } \\
\text { gemina. Lam- } \\
\text { ina quadrigem- } \\
\text { ina. }\end{array}$ & $\begin{array}{l}\text { Brachia. Inter- } \\
\text { nal geniculate } \\
\text { bodies. }\end{array}$ & $\begin{array}{l}\text { Aqueduct } \\
\text { of Syl- } \\
\text { vius. }\end{array}$ \\
\hline $\begin{array}{l}\text { Inter- } \\
\text { brain } \\
\text { vesicle. }\end{array}$ & $\begin{array}{l}\text { Corpora albican- } \\
\text { tia. Tuber ci- } \\
\text { nereum, infun- } \\
\text { dibulum, and } \\
\text { part of hypo- } \\
\text { physis. Optic } \\
\text { chiasm. }\end{array}$ & $\begin{array}{l}\text { Pineal body. Pos- } \\
\text { terior commis- } \\
\text { sure. Epithe- } \\
\text { lium of velum } \\
\text { interpositum. }\end{array}$ & Optic thalami. & $\begin{array}{l}\text { Third } \\
\text { ven- } \\
\text { tricle. }\end{array}$ \\
\hline $\begin{array}{l}\text { Secondary } \\
\text { fore- } \\
\text { brain } \\
\text { vesicle. }\end{array}$ & $\begin{array}{l}\text { Anterior perfo- } \\
\text { rated lamina. } \\
\text { Corpus stria- } \\
\text { tum. Island of } \\
\text { Reil. Olfactory } \\
\text { lobe. }\end{array}$ & \multicolumn{2}{|c|}{$\begin{array}{l}\text { Convolutions of cerebral hemi- } \\
\text { spheres. Corpus callosum. For- } \\
\text { nix. Septum lucidum. }\end{array}$} & $\begin{array}{l}\text { Lateral } \\
\text { ven- } \\
\text { tricles. }\end{array}$ \\
\hline
\end{tabular}

\section{THE DEVELOPMENT OF THE PERIPHERAL NERVOUS} SYSTEM.

The development of the peripheral nervous system is still involved in some degree of obscurity. In general terms it may be stated that the peripheral nervous apparatus is derived as an extension of the central cerebro-spinal axis.

In the case of the spinal nerves, each nerve-trunk is composed of both motor and sensory fibers, the former being in continuity with the spinal cord through the medium of the anterior or motor roots, and the latter through the posterior or sensory roots, each sensory root possessing a ganglion. The cranial nerves exhibit a less regular composition. While the trigeminal nerve, for example, arises by two roots, after the manner of a spinal nerve, some others correspond in relative position and in mode of development to the ventral or motor roots of the spinal nerves, and still others are equivalent to the sensory spinal roots. 
The development of the sensory nerve-fibers is dependent upon and is preceded by that of the ganglia of the posterior roots of the spinal nerves, and of several ganglia of the head region which are related to the development of certain of the cranial nerves. Hence the consideration of the genesis of
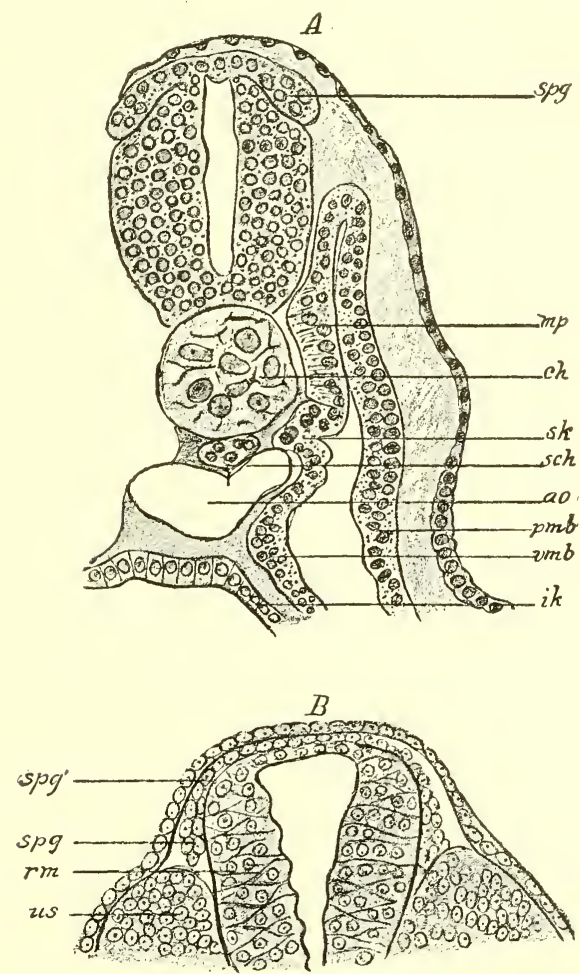

FIG. 159.-A, cross-section through an embryo of Pristiurus (after Rabl). The primitive segments are still connected with the remaining portion of the middle germ-layer. At the region of transition there is to be seen an outfolding ( $s k)$ from which the skeletogenous tissue is developed; $c h$, chorda; spg, spinal ganglion; $m p$, muscle-plate of the primitive segment; sch, subchordal rod; ao, aorta; $i k$, inner germ-layer; $p m b$, parietal, $v m b$, visceral middle layer. $B$, cross-section through a lizard embryo (after Sagemehl) : $r m$, spinal cord; $s p g$, lower thickened part of the neural ridge; $s p g^{\prime}$, its upper attenuated part, which is continuous with the roof of the neural tube; $u s$, primitive segment.

the ganglia must precede the account of the growth of the sensory nerve-fibers.

The origin of the ganglia is connected with the early history of the evolution of the neural tube. Just after the 
sides of the medullary plate (vide p. 279) have united with each other to form the neural tube, there appear two ridges of cells between the tube and the epidermis, one on each side of the raphe or line of union of the sides of the tube. These ridges are the neural crests (Fig. 159). They first appear in the region of the hind-brain and advance from this point both headward and tailward. The ganglia develop from these neural crests. The cells of the neural crest are usually described as growing ont from the neural tube, though according to His it is probable that they originate singly from the ectoderm.

The mass of cells composing the neural crest grows outward and then ventrad along the wall of the neural tube, and very soon undergoes segmentation into a number of cellmasses which are the rudimentary ganglia. In the spinal region the number of segments corresponds to the number of future spinal nerves. In the head region there are four segments. These latter, the cephalic ganglia, will be referred to subsequently.

The segmentation of the neural crest corresponds in the main with the segmentation of the paraxial plate of the mesoderm, whereby the myotomes are produced, and each segment lies upon the inner side of a myotome. The connection of the segments with the neural tube becomes reduced in each case to a slender strand, the point of continuity of which with the tube is shifted farther away from the median line, as development progresses, to correspond with the dorsolateral position of the sensory nerre-roots in the mature condition.

The cells of the ganglia acquire each an axis-cylinder process and a dendrite or protoplasmic process, beeoming thus bipolar cells. The axons or axis-cylinder processes make their way into the spinal cord-in the case of the spinal ganglia-constituting thus the dorsal nerve-roots, and pursue their course within the cord as the columns of Goll and Burdach. The dendrites, constituting the distal portions of the dorsal roots, join the ventral roots on the distal side of the ganglia and become the sensory nerve-fibers of the spinal nerves. Althoigh these two processes grow out from oppo- 
site sides of the cell, the further growth of the cell is such that both processes are connected with it by a common stalk, thus producing the cell with T-shaped process so characteristic of the spinal ganglia. Thus the ganglia are made up of cells which are interpolated in the course of the sensory nerve-fibers, and these cells may be regarded as having migrated from the developing cerebrospinal axis, or, if the view of His be accepted, from the region of the ectoderm from which the tube originates, their connection with the axis being maintained by the gradually lengthening out axis-cylinder process.

The development of the motor nerve-fibers differs from that of the sensory. These fibers, or at least, the axis cylinders of the fibers, are the elongated neurits of nerve-cells of the spinal cord and brain. The neuroblasts of the thickened neural tube, as they become fully differentiated nerve-cells, migrate from their central position into the mantel layer, or superficial stratum (Fig. 140). On the distal side of the nucleus of the cell, the protoplasm first becomes massed and then lengthens out to form an axis-cylinder process or neurit, which in all vertebrate animals grows out from the cerebrospinal axis to form the axis-cylinder of a motor nerve-fiber.

Although, in the case of the spinal nerves, the motor and sensory fibers are separated from each other at their origin from the cord, they soon intermingle to constitute a spinal nerve-trunk. In certain lower types, as eyclostomes and amphioxus, the motor and the sensory fibers permanently pursue separate routes to their peripheral distribution.

The envelopes of the nerve-fiber are acquired at a relatively late period. The appearance of the neurilemma precedes that of the white substance of Schwann. The neurilemma is derived from the mesoderm. The cells of the latter apply themselves to the nerve and, penetrating between the fibers, become arranged as an enveloping layer upon each axis cylinder, ultimately forming a complete sheath, the neurilemma. The persistent nuelei of these cells, scantily surrounded with protoplasm, constitute the nervecorpuscles of the neurilemma. The medulla, or white substance of Schwann, is formed at a considerably later period 
within the neurilemma. The deposit of the medullary sheath varies as to time for different groups of fibers-although the time is constant for each group-and proceeds always in a direction away from the cell from which the fiber originates, or, differently expressed, in the direction in which the fiber conveys impulses. Thus, in the spinal cord, groups of afferent fibers may be distinguished from those that are efferent by observing the direction in which the medullary sheath develops-that is, whether the sheath appears first at the upper end of the fiber or at the lower end.

The cranial nerve-fibers in their development follow in the main the same general principles that govern the growth of the spinal nerves. That is to say, the motor fibers grow out as extensions of the axis-cylinder processes of nerve-cells of the cephalic part of the neural tube and the sensory fibers proceed from the cells of outlying ganglia, or in the case of at least one nerve, the olfactory, from infolded and highly specialized cells of the ectoderm.

The cephalic ganglia, four in number, have been referred to as resulting from the segmentation of the head-region of the neural crest. As previously stated, the neural crest begins to grow first in the region of the hind-brain and extends from this point both forward and backward, oceupying a position upon the roof or dorsal wall of the hind-brain. The part of the neural crest belonging to the head-region then divides into the four masses or head-ganglia which are designated respectively the first or trigeminal, the second or acusticofacial, the third or glossopharyngeal, and the fourth or vagal, ganglia.

The trigeminal ganglion, which is very large, becomes divided into a smaller anterior portion, the ophthalmic or ciliary ganglion, and a larger posterior segment, the trigeminal ganglion proper. These two become widely separated during the progress of development, since they constitute respectively the later ciliary and Gasserian ganglia, the ciliary ganglion belonging to the ophthalmic division of the fifth nerve, while the trigeminal belongs to the superior maxillary division and the sensory part of the inferior maxillary division of the fifth. Their nerve-cells give rise to the sensory 
fibers of these trunks in the same manner that the cells of the spinal ganglia produce the sensory fibers of the spinal nerves.

The acusticofacial ganglion, after its migration from its original position on the dorsum of the hind-brain, lies just in front of the otic vesicle. This ganglion subsequently divides into the facial and the acoustic ganglia. The facial ganglion, the geniculate ganglion or intumescentia ganglioformis of the facial nerve, situated in the facial canal of the temporal bone, although described as a ganglion upon a motor nerve, the facial, is, in reality, connected mainly with the pars intermedia, a bundle of sensory fibers issuing from the nucleus of origin of the glossopharyngeal nerve. It is equivalent therefore to a spinal ganglion.

The acoustic portion of the acusticofacial ganglion divides still further to become the ganglion on the vestibular part of the auditory nerve, and the ganglion spirale of the cochlear division of the auditory, which latter is situated in the spiral canal of the modiolus. It is considered probable that the lateral accessory auditory nucleus, which is connected with the cochlear fibers of the auditory nerve and lies on the outer side of the restiform borly, is also a part of the acoustic ganglion. From the cells of the vestibular ganglion, which is situated in the internal meatus, centrifugal fibers develop to form the vestibular nerve, while other centripetally growing fibers become the ventral or mesial (vestibular) root of the auditory nerve. The cochlear ganglion in the same way gives rise to the cochlear branch of the nerve and to its dorsal or lateral root. Thus the auditory nerve and its ganglia correspond respectively to the sensory root of a spinal nerve and to a spinal ganglion.

The third cephalic ganglion becomes the ganglion of the glossopharyngeal nerve, undergoing segmentation to form the upper or jugular and the lower or petrous ganglia of this nerve, while the axis-cylinder processes of its cells lengthen out to become the sensory fibers.

The fourth cephalic ganglion similarly becomes the two ganglia of the pneumogastric nerve and gives rise to its sensory fibers. 
While the motor fibers of the cranial nerves develop by the outgrowth of the axis-cylinder processes of the motor nerve-cells of the brain, and thus correspond in manner of development with the spinal motor fibers, there is a modification as to their point of emergence from the central axis. Instead of issuing in line with the spinal motor roots, there - are two sets of cranial motor roots, the ventral and the lateral. Both arise from the cells of the ventral zone of the neural tube and thus correspond in point of origin with the spinal motor fibers, but the cells from which proceed the fibers of the ventral roots are situated in the ventral part of this zone, whereas the parent cells of the lateral roots lie near its dorsal edge, close to the deep connections of the sensory fibers. It happens therefore that the lateral roots. emerge in close proximity to the dorsal or sensory bundles, the two apparently constituting one nerve-trunk. The motor fibers of the fifth, seventh, ninth, and tenth nerves have this lateral position, and are so closely identified with the sensory fibers that the two sets form one trunk in each case.

The ventral motor nerve-roots emerge in line with the ventral or motor roots of the spinal nerves. The only cranial nerves which represent persistent ventral motor roots are the abducens and the hypoglossal.

A still further modification in the cranial nerves is presented by their relation to the segmentation of the head. As pointed out above, the segmentation of the spinal part of the neural crest is in correspondence with the segmentation of the trunk, and each spinal nerve therefore may be regarded as belonging to a particular segment of the trunk. In the case of the cranial nerves, however, there is no such regular correspondence, since in some instances, several nerves are referred to one head-segment, while in others, one nerve belongs to several segments. An example of the latter is furnished by the hypoglossal, which arises from the side of the medulla by a series of bundles of fibers which are referable to several segments.

As will be seen later, in the account of the development of the nose and of the eye, the olfactory and optic nerves exhibit certain peculiarities which set them apart from the other cranial nerves. 
From what has been said, it will be apparent that the cranial nerves develop in a far less regular manner than the spinal nerves, and that consequently their trunks consist in some cases of only sensory fibers, in other cases of only motor fibers, and in still others, of both varieties. Typically, each cranial nerve would have a dorsal sensory root with a ganglion, and two motor roots, one lateral and the other ventral. But by the suppression of one or two of these typical roots there will be produced a nerve, for example, representing only the ventral root, as the sixth and twelfth nerves, or a trunk containing sensory and lateral motor fibers, as the vagus, or a nerve consisting solely of sensory fibers, as the auditory.

By way of recapitulation the cranial nerves may be briefly considered seriatim :

First Pair.-The olfactory nerve-filaments grow centripetally from the olfactory epithelium of the nasal mucous membrane.

Second Pair.-The optic nerve is not a true nerve (see Chapter XVI.).

Third Pair.-The oculomotor nerve represents a persistent lateral motor root of the first head-segment (the ophthalmic division of the fifth nerve being the sensory root of the same segment).

Fourth Pair.-The trochlear nerve represents a lateral motor root and belongs to the second head-segment.

Fifth Pair.-The trifacial or trigeminal nerve, containing sensory and motor fibers, represents a persistent lateral motor root and a dorsal sensory root. The ophthalmic portion of the sensory root belongs to the first head-segment, while all the remaining fibers, with the fourth nerve, are assigned to the second segment.

Sixth Pair.-The abducens develops as a ventral motor root and belongs to the third and possibly to the fourth segments.

Seventh and Eighth Pairs. - The acusticofacialis nerve, or the facial and auditory nerves, develop as a single nerve with several roots. The auditory nerve and the sensory fibers of 
the facial-that is, the pars intermedia-correspond to a dorsal sensory root, the division of the acusticofacial gangiion into the several ganglia of the auditory nerve and the geniculate ganglion of the facial accounting for the division of the root into the auditory trunk and the pars intermedia. (The sensory fibers of the facial pass off through the chorda tympani to go to the tongue as special-sense fibers.) The motor fibers of the facial develop as a lateral motor root, originating from cells in the ventral zone. These two nerves, with the sixth, belong to the third and possibly to the fourth headsegments.

Ninth Pair.-The glossopharyngeal nerve, made up largely of sensory fibers, represents a dorsal sensory root and a lateral motor root, the fibers of which latter grow out from cells in the dorsal part of the ventral zone of His, the later nucleus ambiguus. It belongs to the fifth head-segment.

Tenth Pair.-The vagus develops in the same manner as the glossopharyngeal.

Eleventh Pair.-The spinal accessory represents in part motor spinal roots and in part probably the lateral motor and dorsal sensory roots of the cranial nerves.

Twelfth Pair.-The hypoglossal develops as the ventral motor roots of several segments, being identical in mode of origin with the anterior roots of the spinal nerves. This nerve and the vagus belong to the head-segments from the sixth to the tenth inclusive.

\section{THE DEVELOPMENT OF THE SYMPATHETIC SYSTEM.}

There are two views as to the origin of the sympathetic system. One theory, based upon the investigations of Paterson, is that the gangliated cord of the sympathetic is differentiated from mesodermic cells, the cell-cord thus formed acquiring, secondarily, connections with the spinal nerves, and presenting still later the enlargements which constitute the ganglia.

The more generally accepted view, based upon the researches of Balfour and the later work of Onodi and His, is that the sympathetic ganglia develop as offshoots from the 
ventral extremities of the spinal ganglia. Each little mass, which has budded off from a spinal ganglion, moves somewhat toward the ventral surface of the body, its bond of union with the parent spinal ganglion being drawn out to a slender cord, the representative of the future ramus communicans. Each primitive sympathetic ganglion sends out two small processes, one growing tailward from its lower extremity, and one in the opposite direction from its upper end, the approaching processes from each two adjacent ganglia meeting and uniting and thus secondarily establishing the connection between the different ganglia of one side of the body and forming the gangliated cord of the sympathetic. From these ganglia migrating cells probably pass out to develop into the secondary ganglia of certain viscera, as His has shown to be the mode of origin of the ganglia of the heart.

\section{THE CAROTID BODY, THE COCCYGEAL BODY, THE ORGANS OF ZUCKERKANDL.}

In connection with the sympathetic system may be mentioned the "carotid gland," or glomus caroticus, or intercarotid ganglion, found at the bifurcation of the common carotid artery; the coccygeal body or "gland," Luschka's ganglion, found at the lower extremity of the coccyx in relation with branches of the middle sacral artery; and the organs of Zuckerkandl, found in later fetal life and for a short time after birth at the origin of the inferior mesenteric artery.

These structures present features in common with each other in that they are made up of knots of blood-vessels intermingled with collections of cells, among which are numerous chromaffine cells such as are found in the medulla of the adrenal body and in the sympathetic ganglia ; and in the furthor fact that they are penetrated by sympathetic nerve-fibers.

That the cells of the carotid body and of the organs of Zuckerkandl are derived from the adjacent sympathetic ganglia has been established, but whether these bodies are for that reason to be classed as nervous structures is as yet uncertain. 


\section{CHA PTER X VI.}

\section{THE DEVELOPMENT OF THE SENSE ORGANS.}

In the organs of the senses we have to do with peripheral nervous mechanisms of greater or less degrees of complexity, the essential elements of which are elaborately modified or specialized neuro-epithelial cells. These neuro-epithelial structures are specialized cells of the ectoderm, derived from it either directly, by the infolding of patches of ectodermic epithelium, as in the case of the olfactory cells, or indirectly, by growth outward from the central nervous system, as in the case of the retina. The organs of the sense of touch, the tactile corpuscles of the skin and mucous membranes, are distributed somewhat irregularly, while such highly specialized structures as the organs of the special senses of vision, hearing, smell, and taste are provided with special protective and accessory apparatuses.

\section{THE DEVELOPMENT OF THE EYE.}

It will perhaps facilitate the comprehension of the general principles involved in the development of the eye if its function as the organ of vision is kept in mind, and if, therefore, the retina and the optic nerve are recognized as the essential parts of the organ, and the other structures as accessories. The retina and the optic nerve are an outgrowth from the brain, the rod- and cone-visual cells of the former being epithelial cells so specialized as to serve as percipient elements, while the optic nerve-fibers are the conducting medium. To allow of the penetration and refraction of the rays of light, the overlying epidermis differentiates into a transparent and refractive medium, the crystalline lens, and the necessary protection and means of nourishment 
are provided by the other constituents of the eyeball. Further protection is furnished by two folds of modified skin and subcutaneous tissue, the eyelids, and lastly for the lubrication and still further protection of the exposed part of the eyeball, there is formed still another set of accessory organs, the lacrimal apparatus.

The first step in the development of the eye is the growth of a diverticulum from the side of the primary fore-brain vesicle (Fig. 160). These optic evaginations are quite large
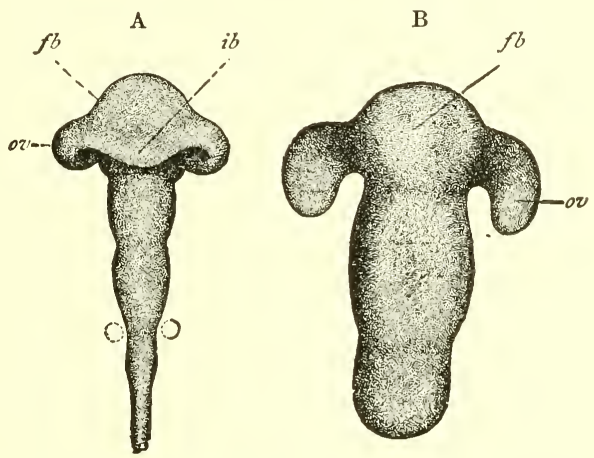

FIG. 160.-A, brain of two-day chick-embryo; B, brain of human embryo of three weeks (His). Shows the development of the optic vesicles and brain-vesicles; $f b$, fore-brain; $i b$, inter-brain; $o v$, optic vesicles.

as compared with the brain-vesicle. They begin to be evident even before the neural tube is completely closed. As the attached part of the diverticulum expands less rapidly than the distal portion, the evagination soon assumes the form of a sac or vesicle, the optic vesicle, connected by a hollow stalk with the primary fore-brain. When the secondary fore-brain vesicles grow out anteriorly from the primary vesicle, the region of the latter that becomes in consequence the inter-brain is the part to which the stalk of the optic vesicle is attached. Hence the optic vesicle is an appendage of the inter-brain or thalamencephalon and its point of attachment to the latter is at the lateral part of the base, in front of the region of the infundibulum (Fig. 147, A and C).

The optic vesicle expands laterally and dorsally until it lies immediately beneath the epidermis, forming a promi- 
nence on the side of the head (Fig. 62). The ectoderm at the point of contact with the optic vesicle becomes thickened and depressed, the differentiation of this lens-area being the starting point of the crystalline lens. The depressed patch of ectoderm, sinking more deeply, is converted into a sac, the lens-vesicle, the connection of which with the surfacecells is soon lost. The distal wall of the optic vesicle, upon coming into contact with the lens-vesicle, undergoes invagination, this wall sinking in until the cavity of the vesicle is almost obliterated. Thus the vesicle is converted into the double-walled optic cup, the opening of which looks laterally toward the surface of the head, and is occupied by the lensvesicle.

The invaginated wall of the vesicle-that is, the layer nearer the center of the cup-becomes the retina, except its pigment-layer, the latter resulting from the outer layer of the cup. The stalk of the cup becomes the optic nerve. The surrounding mesodermic tissue grows into the openings referred to above, and gives rise to the vitreous humor, while the mesodermic cells that closely envelop the optic cup produce the uveal tract and the sclera and cornea.

Having traced briefly the development of the organ, its several parts may now be considered in detail.

The Retina and the Optic Nerve.-These two structures, as stated above, are directly derived from the optic vesicle and its stalk.

To repeat, for the sake of continuity, some points already mentioned, the optic vesicle grows forth as a diverticulum from the side of the primary fore-brain vesicle, its appearance being foreshadowed by a lateral bulging of this vesicle even before the neural canal is completely closed. When the primary fore-brain vesicle divides into the secondary fore-brain vesicles and the vesicle of the inter-brain, the region of origin of the optic vesicle falls to the latter, the point of attachment being at the outer edge of the base of the resicle in front of the infundibular evagination. The optic nerve is to be regarded therefore as springing from the inter-brain or thalamencephalon. 
The outer extremity of the diverticulum expanding more rapidly than its base of attachment assumes the form of a vesicle with a narrow stalk (Fig. 161), the stalked condition

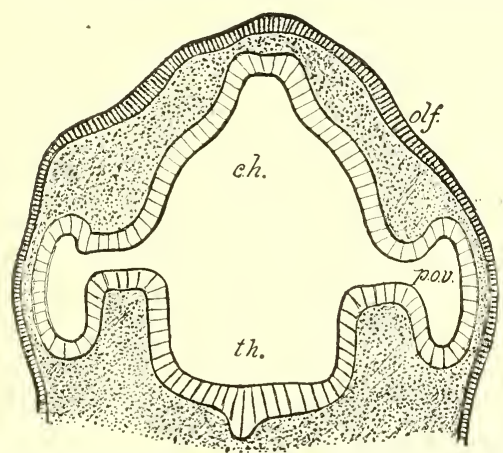

FIG. 161.-Part of a section through the head of an early human embryn, showing the connection of the primary optic vesicles with the fore-brain (His): olf, olfactory area of epiblast; c.h., part of fore-brain which gives rise to cerebral hemispheres; th, thalamencephalon; p.o.v., primary optic vesicles.

being present in the fourth week. The vesicles grow in the outward direction and form a prominence on each side of the head. There being no brain-case at this time, they lie imme-

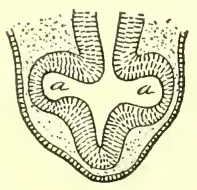

A

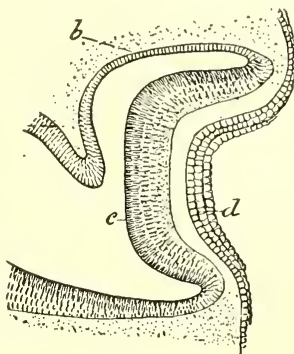

$B$

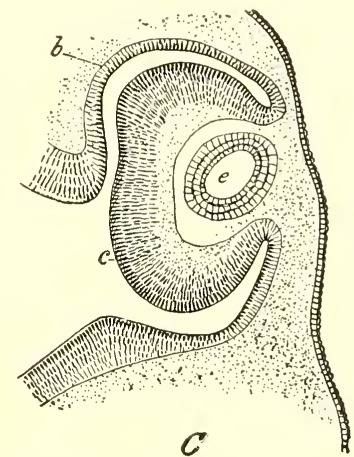

FIG. 162. - Three successive stages of development of the eye, showing formation of secondary optic cup and crystalline lens in human embryos of $4 \mathrm{~mm}$. (A), $6 \mathrm{~mm}$. $(B)$, and $8 \mathrm{~mm} .(C)$, (Tourneux): $a, a$, primitive optic vesicles; $b$, external layer of secondary optic cup (future pigment-layer of retina); $c$, inner layer of cup (retina proper); $d$, lens-pit (thickened and depressed ectoderm); $e$, lens-vesicle.

diately under the epidermis, separated from it by only a thin layer of embryonal connective tissue. This lateral position of the optic vesicles is characteristic of the early stages of development. After the end of the first month the eyes 
gradually move forward and downward toward their permanent position, which is approximately attained probably early in the third month.

Shortly after the fourth week the distal or lateral wall and the under surface of the optic vesicle become invaginated. The invagination begins when the vesicle comes into contact with the lens-vesicle (Fig. 162). When the infolding is complete, the vesicle has become the secondary optic cup, which latter consists therefore of two layers, an inner and an outer. The mouth of the cup, which faces away from the median plane of the head, is occupied by the lens-vesicle. Since the under surface of the vesicle participates in the invaginating process (Fig. 163) there is also in this wall of the cup an aperture, which is known as the choroidal fissure. The invagination likewise affects the under surface of the tubular stalk of the vesicle so that it is converted into an inverted double-layered trough. These invaginations bear an important relation not only to the

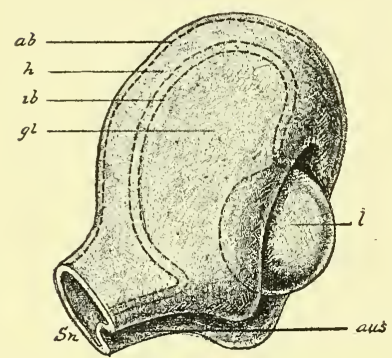

FIG. 163.-Plastic representation of the optic cup with lens and vitreous body (Hertwig): $a b$, outer wall of the eup; $i b$, its inner wall; $h$, cavity between the two walls, which later disappears entirely; Sn, fundament of the optic nerve (stalk of the optic vesicle with a furrow on its lower surface); aus, optic (choroid) fissure; $g l$, vitreous body ; $l$, lens. further metamorphosis of the optic vesicle and its stalk into the retina and the optic nerve, but also to the development of the vitreous body and of the central artery of the retina. Thus, the vitreous body is produced in part at least by the mesodermic tissue that finds access to the cup through the choroidal fissure, and the arteria centralis retince is developed in the vascular mesodermic tissue that invaginates the under surface of the stalk of the vesicle.

The choroidal fissure gradually contracts after the entrance of the mesoderm, and in the last month of fetal life it entirely closes. The mouth of the optic cup embraces the lens, its rim being always on the distal side of, or superficial to, that structure. This opening represents the pupil of later stages. 
The further metamorphosis of the optic cup includes alterations peculiar to each of the two layers and also to the different regions of the cup. The mouth of the cup contracts somewhat by increased growth of the wall, and thus there is a zone bordering this orifice which is anterior to the lens, holding the same relation to the latter body that the future iris holds. A second zone corresponds with the periphery of the lens, while a third region, the fundus of the cup, includes all the remaining part of its wall.

The fundus of the cup undergoes much greater specialization than the other regions. The outer layer of the cup remains thin, consisting of a single layer of cells which assume the cuboidal form and become infiltrated with pigment-granules. This forms the pigment-layer of the retina. The inner lamina of the cup thickens, by the multiplication of its cells, and soon consists of numerous spindle-shaped cells. The thickened fundus is marked off from the zone that surrounds the periphery of the lens by a slight groove which corresponds in position with the future ora serrata. These early spindle-cells give rise to two kinds of elements, the stroma of the retina, or Müller's fibers, and the various nerve-cells, including the highly specialized rod- and cone-visual cells.

The principal sustentacular elements, or Müller's fibers, like the spongioblasts of the neural tube, are radially arranged and extend throughout the entire thickness of the retina. Their inner expanded extremities, in close contact with each other, form the inner limiting membrane, while their outer ends, in the same way, constitute the outer limiting membrane, which latter is in contact with the pigmentlayer. The stroma of the retina receives a small contribution from the mesodermic tissue, which grows into it through the choroidal fissure to furnish the vascular supply.

Of the nerve-cells, those near the pigment-layer undergo great alteration in form and become the sensory epithelium -that is, the rod-and cone-visual cells. At first these lie entirely internal to the external limiting membrane, which separates them from the pigment-layer. After a time, however, processes grow out-that is, away from the center of 
the eyeball-and perforate the external limiting membrane to penetrate between the cells of the pigment-layer. These processes are the rods and cones, and collectively constitute the layer of rods and cones of the adult. 'The bodies of the

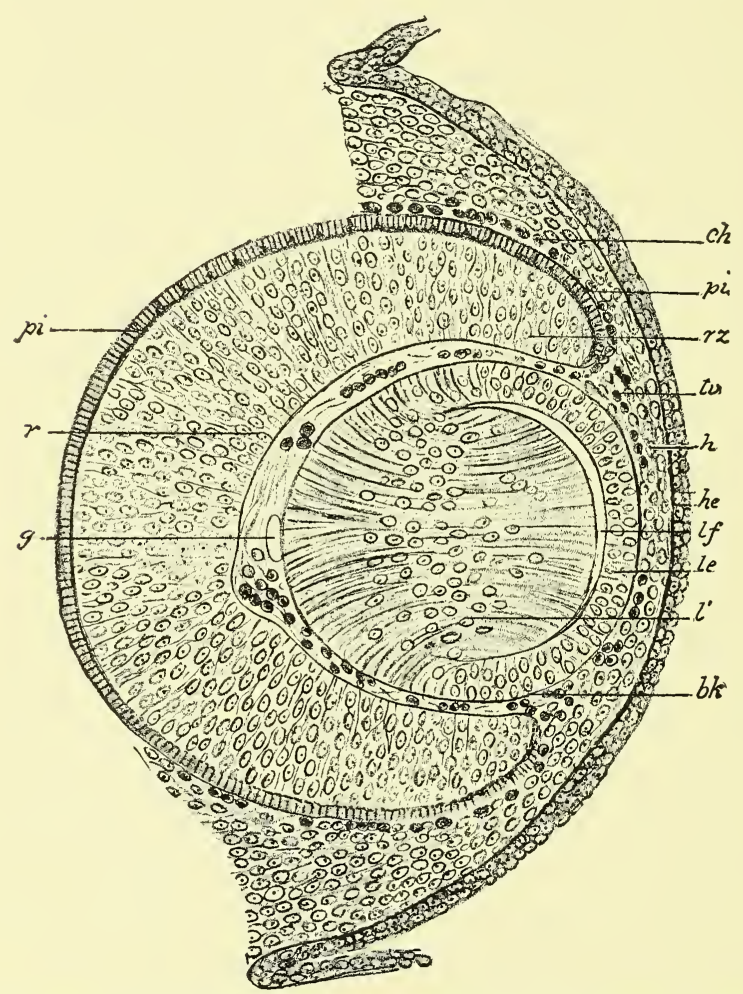

FIG. 164.-Section through the optic fundament of an embryo mouse (after Kessler): $p i$, pigmented epithelium of the eye (outer lamella of the optic cup, or secondary optic vesicle); $r$, retina (inner lamella of the optic cup); $r z$, marginal zone of the optic cup, which forms the pars ciliaris et iridis retinæ; $g$, vitreous body with blood-vessels; $t v$, tunica vasculosa lentis; $b k$, blood-corpuscles; $c h$, choroidea; $l f$, lens-fibers; le, lens-epithelium; $l^{\prime}$, zone of the lens-fiber nuclei; $h$, fundament of the cornea; he, external corneal epithelium.

rod- and cone-visual cells, situated on the inner side of the membrana limitans externa, are elongated into narrow elements, the position of the nuclei being indicated by slight enlargements. They constitute the outer nuclear layer of the mature retina. The outer nuclear layer and the layer of 
rods and cones are to be regarded, therefore, as one layer of highly specialized neuro-epithelium, made up of the rodvisual cells and the cone-visual cells, the inner segments or bodies of the cells being only apparently isolated from the outer segments, the rods and cones respectively, by the fact that the latter project through minute apertures in the external limiting membrane. The axis-cylinder processes of these cells pass toward the center of the eyeball.

The neuro-epithelium of the retina is the last of its elements to develop. In man and in many mammals, it is present at birth. In the eat and the rabbit, the rod- and cone-risual cells develop after birth, and hence the new-born of these species are blind. The macula lutea is developed after birth.

The cells of the inner part of the retina differentiate into the remaining nervous elements, some becoming the bipolar and other cells of the inner nuclear layer-the ganglion retinæ-while others form the large ganglion cells of the ganglion-cell layer. The axis-cylinder processes of the ganglion cells are directed inward to form the nerve-fiber layer, the fibers of this layer converging from all parts of the inner surface of the retina toward the optic disk or papilla. Here they perforate the retina, as well as the choroid and sclera, to pass, as optic nerve-fibers, to the brain.

This part of the optic cup, the fundus, produces then, in the manner described above, the functionating portion of the retina, or the pars optica retinæ, the anterior termination of which is indicated by the orra serrata.

The lenticular zone of the optic cup, which is in relation with the periphery of the lens, undergoes comparatively slight specialization. Its outer lamella is pigmented, as in the fundus of the cup. Its inner layer remains very thin and consists of cells which at first are cuboidal, but which later become cylindrical. At the end of the second month, or the beginning of the third, the two layers of the lenticular zone become plicated, owing to excessive growth in superficial extent. The folds are nearly parallel and are arranged radially with reference to the lens, the margin of which they surround. These folds are the first indication of the ciliary 
processes. The mesodermic tissue immediately external to the optic cup differentiates into the uveal tract, the part corresponding with the lenticular zone of the cup furnishing the ciliary body. The young growing connective tissue penetrates between the folds of the lenticular zone of the cup, acquiring intimate union with the pigment-layer, and thus provides the connective-tissue basis of the ciliary processes. This lenticular zone of the two layers of the optic cup, therefore, constitutes the lining, or internal covering, of the ciliary body, and must necessarily be regarded as the continuation of the retina. It is known as the pars ciliaris retinæ of the fully developed eye.

The marginal zone of the optic cup, or the region bordering its orifice, is also related in its further growth with the uveal tract. Although in the earlier stages of development the lens lies in the mouth of the cup, as time goes on the relation is so altered that the aperture and the zone which borders it occupy a position in front of the lens. In this marginal zone both lamellæ of the cup become pigmented and acquire union with the layer of mesodermic tissue which is differentiating into the iris, and they therefore contribute to the formation of that structure, constituting its pigmentlayer. The pigment-layer of the posterier surface of the iris is, therefore, an extended but rudimentary part of the retina. It is called the pars iridica retinæ.

From what has been said, it will be apparent that the retina forms a complete tunic with an anterior perforation, the pupil, and that it consists of the functionally active part, or retina proper, the pars optica retinæ; of the pars ciliaris retinæ, marked off from the latter by the ora serrata; and of the pars iridica retinæ, which terminates at the margin of the pupil.

The evolution of the optic cup or secondary optic vesicle may be thus summarized:

I. Marginal or most anterior region of cup.

II. Lenticular zone of cup.
The thin atrophic pars iridica retinæ, or pigment-layer of the iris.

Pars ciliaris retinæ, covering inner surface of ciliary body. 
III. Fundus of cup.

A. Outer layer.

B. Inner layer.
Functionating part of retina, or pars optica retinæ, including :

A. Pigment-layer of retina.

B. 1. Neuro-epithelial layer, made up of layer of rods and cones (the processes of the rod- and cone-visual cells); membrana limitans externa; outer nuclear layer (the bodies of the rodand cone-cells).

2. Cerebral layer (representing an interpolated ganglion with connecting fibers), consisting of :

Outer reticular layer;

Inner nuclear layer;

Inner reticular layer;

Ganglion-cell layer;

Nerve-fiber layer.

The optic nerve is the metamorphosed stalk of the optic vesicle. When the distal and under surfaces of the vesicle suffer invagination, the stalk participates in the process, its under surface being marked by a groove which is a prolongation of the choroidal fissure of the optic cup (Fig. 163). By this infolding, the cavity of the stalk is obliterated and the stalk is converted into a double-walled tube enclosing mesodermic tissue which follows the invaginating ventral wall. In this mesodermic tissue is developed the arteria centralis retinæ. In mammals the invagination affects only the distal part of the stalk, the segment included between the eyeball and the point corresponding in the adult to the place of entrance into the nerve of the central artery. It must be apparent that the outer layer of the tube thus formed is directly continuous with the outer layer of the optic cup, while the invaginated lamina is the prolongation of the inner wall of the cup or of the part that becomes the retina proper, since not only the distal wall of the optic vesicle is invaginater, but its under or ventral wall as well.

The primitive optic nerve at this stage consists of layers of spindle-shaped cells, with a central core of vascular connective tissue.

The manner in which the nerve-fibers are developed is 
still a matter of controversy. According to His and Kölliker, the fibers grow out from the ganglion-cells of the optic thalami and the anterior corpora quadrigemina, while Müller and Froriep believe that they are the prolonged axis-cylinder processes of the ganglion-cells of the retina. According to Ramon y Cajal, growth occurs in both directions. In either case, the cells of the optic stalk would furnish only the sustentative tissue of the nerve. 'There is also a contribution of sustentative tissue or stroma from the mesoderm, as in the case of the central nervous system.

The Crystalline Lens.-The lens, exclusive of its capsule, is, like the retina, of ectodermic origin. The first step in its development is the formation of a thickened and depressed patch of the ectoderm on the lateral surface of the head, this area being situated at the place where the optic vesicle is nearest the surface (Fig. 162, $B, d$ ). The depression is the lens-pit. It soon becomes converted into a closed sac, the lens-vesicle, by the gradual approximation and union of its edges. The pit receding from the surface as its lips come together, the completed vesicle lies under the surface ectoderm, with which it is for a time connected by the slender stalk of the invagination. Upon the disappearance of the strand of cells constituting the stalk, the lensvesicle is completely isolated from the outer germ-layer (Fig. 162, C,e).

The lens-vesicle in birds is a hollow epithelial sac several layers thick, but in mammals the central cavity contains a mass of cells, which latter disappear in the later stages of development.

Upon the invagination of the optic vesicle to form the secondary optic cup, the lens-vesicle is embraced by the lips of the cup and still later comes to lie within the cup, near its orifice (Fig. 164).

The further alterations in the vesicle are dependent primarily upon changes in its deep and superficial walls respectively, each of which consists of several layers of cylindrical cells. The cells of the superficial wall alter their form, becoming cuboidal, while the posterior or deeper cells lengthen so as to become fibers. Thus the deeper wall of 
the vesicle thickens at the expense of the central cavity-the central mass of cells at the same time disappearing-while the superficial layer remains thin. The two strata are continuous with each other at the equator of the lens, one form gradually merging into the other at this region, which is a zone of transition (Fig. 164).

The lens at this stage is composed, therefore, of a thin superficial or anterior stratum of cuboidal epithelial cells and a much thicker posterior or deep layer of so-ealled fibers, the latter being simply the greatly elongated cells of the posterior wall of the vesicle. Between the two laminæ is a small remnant of the cavity of the resicle. The epithelial layer persists throughout life as the epithelium of the lens, while the fibrous layer is the basis of the lens-fibers of the mature condition. The cavity sometimes persists as a small space containing a few drops of fluid, the liquor of Morgagni.

The next important stage in the development of the lens is the formation of additional lens-fibers. These result from the proliferation of the cells of the epithelial or anterior layer. The lens-fibers are formed in successive layers, as may be made evident by the maceration of a lens. Each fiber extends from the anterior to the posterior surface of the lens. The ends of the fibers meet each other along regular lines, producing thus the characteristic three-rayed figures or stars of the lens, one of which belongs to each surface. Hence, while the lens-fibers first formed are the elongated cells of the posterior layer of the lens-vesicle, the fibers of later growth originate from the cells of the anterior wall. The epithelial character of the lens-fibers is evinced by the presence of a nucleus in each fiber of a young lens.

The lens-capsule results from the differentiation of the mesodermic tissue which surrounds the lens. It is from this enveloping vascular lamina, the tunica vasculosa lentis, that the growing lens derives its nutrition. The capsule is well marked in the second month. Its blood-vessels are derived from those of the vitreous body. At the end of the seventh month this well-developed, highly vascular membrane begins to undergo retrograde alterations, the final result of which is 
its transformation into the thin, non-vascular, transparent capsule of the mature lens. ${ }^{1}$ The most active growth of the lens itself occurs prior to the degeneration of the tunica vasculosa lentis, so that even before the end of fetal life the lens has nearly attained its full size. Thus the weight of the lens of the new-born child is 123 milligrammes, while that of the adult lens is but 190 milligrammes (Huschke).

Hence the crystalline lens has a double origin, the lens-substance or lens proper being derived from the ectoderm, while the capsule originates from the mesoderm.

The Vitreous Body.-The vitreous body has been regarded usually as a comparatively slightly differentiated form of connective tissue, and as being derived from the middle germ-layer. Recent investigations show, however, that it originates in part at least from ectodermal tissue. According to these observations, processes grow forth from those stromal elements of the optic cup which afterward become Müller's fibers, and these processes, advancing toward the lens-vesicle, interlace to form a network, the primitive vitreous (Kölliker, Froriep). This process continues for a longer time at the marginal zone or mouth of the cup than elsewhere, the protoplasmic fibers which grow from this future ciliary and iridal portion of the cup contributing to the formation of the zonule of Zinn. In mammals the cells of the lens-vesicle, another ectodermal structure, also send forth processes which, according to Lenhossek, bear a prominent part in the development of the vitreous body. The mesodermic tissue, already in the stage of embryonal connective tissue, now gains access to the optic cup through the choroidal fissure (Fig. 163), its ingrowth, in fact, accompanying the invagination of the under surface of the optic vesicle, and constitutes what Kölliker designates the mesodermal vitreous. The intermingling of these two constituent ele-

'It sometimes happens that parts of the fetal lens-capsule persist. The most common example of such persistence is the so-called membrana pupillaris sometimes present at birth, producing congenital atresia of the pupil. This results from the persistence of that part of the fetal capsule which is situated on the anterior surface of the lens, behind the pupil. 
ments produces finally the definitive vitreous. Since the inferior surface of the stalk of the vesicle-the future optic nerve-participates in the invagination of the optic cup, the mass of mesodermic tissue which helps to form the vitreous is continuous with that which invaginates the primitive optic nerve to produce the central artery of the retina. As a consequence, the blood-vessels which soon develop so plentifully in the vitreous body are extensions from the central artery of the retina, the latter itself being continued forward as the hyaloid artery. The terminal branches of the hyaloid artery pass on through the vitreous body to terminate in the vascular capsule of the growing lens, constituting the blood-supply of that structure.

The intercellular substance of the young tissue undergoes but little differentiation, while the cells become gradually reduced to a few stellate elements which ultimately entirely disappear. The peripheral part of the tissue develops into the hyaloid membrane, which anteriorly acquires union with the capsule of the lens.

The blood-vessels of the vitreous disappear during the last two or three months of fetal life. The hyaloid artery persists, although in reduced form, for a longer time than the smaller vessels. Upon its final degeneration it is replaced by a canal, the hyaloid canal, or canal of Stilling, which is present in adult life.

The Middle or Vascular and the Outer or Fibrous Tunics of the Eye.-The outer fibrous coat of the eye, including the sclera and the cornea, and the middle tunic or uveal tract, comprising the choroid, the ciliary body, and the iris, are structures of mesodermic origin, being directly produced by the mesodermic tissue surrounding the optic eup. The richly cellular mesoderm applies itself to the exterior of the cup and differentiates into the two layers in question, the changes involving on the one hand the metamorphosis of the mesodermic cells chiefly into muscular and vascular elements, and on the other hand the evolution of a tissue essentially fibrous in structure. These two tunies are distinguishable in the sixth week. 
The cornea is formed from the thin layer of mesoderm that penetrates between the lens-vesicle and the surface ectoderm. The lens-vesicle lies very near the surface, and the thin stratum of mesoderm that is interposed between the two is the anterior layer of the lens-capsule (Fig. 164). This anterior layer thickens by the immigration of other cells and subsequently splits into two laminæ, a superficial one which produces the cornea (Fig. 164, $h$ ), and a deeper, which is now the proper anterior wall of the lens-capsule. Thus a space filled with fluid appears between the primitive cornea and the lens, which corresponds with the future anterior and posterior chambers of the eye, the division of the space into these two chambers being effected subsequently by the development of the iris. The further development of the cornea consists simply in the differentiation of the mesodermic cells and the intercellular substance into the several characteristic elements of the adult structure.

The uveal tract closely corresponds in extent with the two layers of the optic cup. The choroid is differentiated from that portion of this primitive uveal tract which envelops the pars optica of the retina. In this region the enveloping layer of mesodermic cells develops into the several elements of the choroid, the most conspicuous of which are an inner layer of capillary vessels, the choriocapillaris, and an outer layer of larger vessels, the stroma-layer of the choroid. The development of the choroid bears a certain relation to the choroidal fissure of the optic cup. This fissure has been referred to as a gap in the under surface of the cup corresponding with the line of invagination through which the mesodermic tissue, of which the developing choroid is a part, grows into the cup to produce the vitreous. Although normally this fissure in the retina entirely disappears, its site becomes pigmented later than other regions of the pigmentlayer of the retina, and hence there is, for a time, a clear streak in this part of the retina which has the appearance of a fissure in that membrane. As the pigment-layer of the retina was formerly assigned to the choroid, this streak appeared to be a breach of continuity of the choroid; hence the 
term choroidal fissure. In some cases, however, the choroidal fissure fails to close, and as the development of the choroid is largely dependent upon or is governed by that of the retina there remains a corresponding gap in the choroid. This defect enables the sclera to be seen from the interior in a line extending forward from the optic nerve entrance. It is known as coloboma of the choroid.

The ciliary body is developed immediately in advance of the choroid and from the same layer of mesodermic tissue. The deeper parts of the tissue in this region correspond with the plications of the ciliary part of the retina, sending processes into and between the radial folds of this part of the two layers of the optic cup, with which latter the highly vascular mesodermic tissue acquires firm union. This results in the formation of the ciliary processes. Some of the cells of the more peripheral part of this zone are converted into unstriated muscular tissue, thus producing the ciliary muscle. All the characteristic or important elements of the ciliary body are, therefore, derived from the mesoderm, while the thin layer of tissue on its inner surface, representing an undeveloped part of the optic cup, the pars ciliaris, is of ectodermic origin.

The iris, the most anterior zone of the uveal tunic, is produced from the same mesodermic tract that gives rise to the choroid and to the ciliary body. As stated above, soon after the lens-vesicle becomes constricted off from the surface ectoderm, it is enveloped by a mass of mesodermic cells which constitute its primitive capsule, and the layer of these cells lying between the lens-vesicle and the surface ectoderm splits into an anterior layer, which becomes the cornea, and a posterior stratum which is the anterior wall of the lens-capsule. This produces a space between the lens and the cornea. The lens now recedes farther from the surface, and the margins of the optic cup advance, so that the lens now lies within the cup, the marginal zone of the cup being in front of the lens, between it and the cornea, while its equator is in close relation with the ciliary regions of the cup and of the uveal tract. Thus the space between the lens and the cornea is 
divided into an anterior compartment, the anterior chamber, and a posterior space, the posterior chamber, the orifice of the cup being a means of communication between the two and representing the pupil of a later stage. The marginal zone of the cup furnishes the guiding line for the development of the iris. The mesodermic tissue in relation with

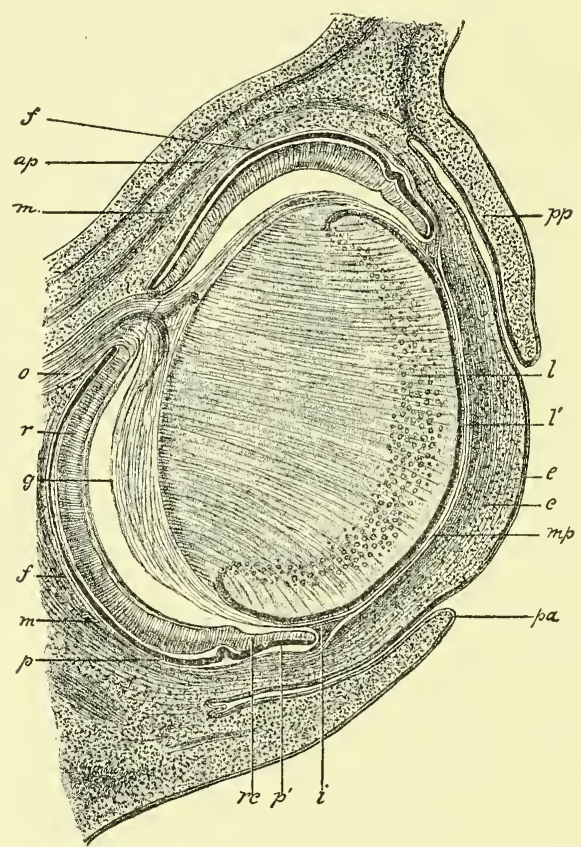

FIG. 165. - Sagittal section through the eye of an embryo rabbit of eighteen days $\times 30$ (Kölliker): $o$, optic nerve; $p$, hexagonal pigment-layer; $r$, retina ; $r c$, ciliary part of the retina; $p^{\prime}$, forepart of the optic cup (rudiment of the iris-pigment); $g$, vitreous, shrunk away from the retina, except where the vessels from the arteria centralis retinæ enter it; $i$, iris ; $m p$, membrana pupillaris; $c$, cornea with epithelium $e$; $p p, p a$, palpebræ; $l$, lens ; $l$ ', lens-epithelium ; $f$, sclerotic ; $m$, recti muscles.

the outer surface of the marginal zone of the cup differentiates into the vascular, muscular, and connective-tissue elements of the iris proper, while its posterior pigment-layer is constituted by the slightly specialized layers of the most anterior part of the optic cup, the part that is known as the pars iridica retinæ. Recent investigations (Nussbaum, Herzog, etc.) indicate that the circular and the radial muscular 
fibers of the iris develop from the outer epithelial layer of the optic cup or, in other words, from the part of the optic cup that becomes the pars iridica retinæ. The circular fibers, sphincter pupillæ, are distinguishable in the fourth month, the radial or dilator fibers, in the seventh month.

Since the anterior and posterior chambers of the eye are spaces hollowed out of the mesoderm, they represent a lymph-space and are, as such, lined with endothelial cells.

The cleft in the inferior wall of the optic cup referred to above as the choroidal fissure necessarily affects the marginal zone of the cup as well as the region posterior to it. If this part of the fissure persists, as it sometimes does, it may be accompanied by a corresponding deficiency in the tissues of the iris proper. Such a congenital defect, appearing as a radial cleft in the lower half of the iris, is known as coloboma of the iris.

The Eyelids and the Iacrimal Apparatus.-The eyelids are developed from folds of the primitive epidermis that form over the superficial part of the developing eyeball (Fig. 165, $p p$ and $p a$ ). After the separation of the lens-vesicle from the surface ectoderm, the latter pouches out into two little transverse folds for the upper and lower lids respectively. Each fold includes a certain quantity of mesodermic tissue, from which are produced the connectivetissue elements of the lids, as the tarsal plates, ete. After the folds attain to a certain degree of development their edges approach each other and become adherent, thus enclosing a space between the primitive lids and the front of the eyeball. The infolded ectodermic layers lining this space acquire the characteristic features of mucous membrane and constitute the epithelium of the conjunctiva, the part of this membrane that covers the cornea adhering closely to that structure as its anterior epithelial layer. The union of the edges of the lids begins in the third month and lasts until near the close of fetal life. A short time before birth the permanent palpebral fissure begins to form by the breaking down of the adhesions.

A part of the mesodermic tissue of the lids undergoes con- 
version into fibrous connective tissue, thus producing the tarsal plates of the upper and lower lids, with the palpebral fasciæ and tarsal ligaments by which the plates are attached to the margins of the orbit.

During the period when the edges of the lids are adherent, the Meibomian glands and the eye-lashes are formed. The glands develop from solid cords of epithelial cells that grow from the deepest or Malpighian layer of the primitive epidermis into the tarsal plates. The cords become hollow tubes by degeneration of their central cells.

In addition to the two principal folds that produce the lids, a third, vertical fold appears at the inner, nasal side of the conjunctival space, beneath the lids. This fold remains quite small in man and forms the plica semilunaris, but in most other vertebrates it attains much greater size as the third eyelid or nictitating membrane. A small part of this third fold develops sebaceous glands and a few hair-follicles and becomes the lacrimal caruncle.

The lacrimal gland is developed in the same manner as the Meibomian glands, by the growth of solid epithelial cords from the conjunctiva. The cords grow into the underlying mesoderm at the outer part of the line of reflection of the conjunctiva from the inner surface of the upper lid to the front of the eyeball. The cords acquire lateral branches and then become hollowed out to form the secreting tubules and efferent ducts of the gland, the connective-tissue stroma of which is contributed by the surrounding mesodermic tissue. The orifices of the adult efferent ducts in the upper outer part of the conjunctival sac correspond with the points from which the primitive cell-cords first grow forth.

The efferent lacrimal apparatus, consisting of the nasal or lacrimal duct and the canaliculi, is related genetically to the growth of the nose and the upper jaw. Soon after the appearance of the nasofrontal process, a lateral projection, the lateral nasal process, grows from its side near the base and advances downward so as to form the outer boundary of the nasal pit and consequently of the future nostril (Fig. 67, A, B). This lateral nasal process is separated from 
the maxillary process of the first visceral arch by an oblique furrow, the naso-optic groove, which extends from the inner angle of the orbit to the outer side of the nostril, or, before the separation of the nasal pit from the primitive mouth, to the upper boundary of the latter orifice. The naso-optic groove indicates the situation of the lacrimal duct. By some authorities-Coste and Kölliker-it is believed that the duct results from the union of the edges of the groove. Later investigations seem to indicate, howerer, that the duct is formed by the hollowing out of a solid cord of epithelial cells that appears at the bottom of the furrow. In either case the epithelial lining of the duct is an ectodermic involution. When the nostrils are separated from the oral aperture by the union of the nasofrontal, the lateral nasal, and the maxillary processes (p. 133), the lower end of the furrow is obliterated, and the partially formed duct is made to terminate in the nasal cavity.

The canaliculi, representing the bifurcated upper extremity of the duct, result, according to one view, from the division of the upper end of the epithelial cord into two limbs, one for each lid, and their subsequent hollowing-out; according to another, from the continuation of the cell-cord into the upper lid and the later addition of a limb for the canaliculus of the lower lid. The lacrimal sac is merely an expanded part of the duct.

\section{THE DEVELOPMENT OF THE ORGAN OF HEARING.}

As in the case of the other sense-organs, the auditory apparatus consists of highly specialized neuro-epithelium, connected by nerve-fibers and interpolated ganglia with the central nervous system, and of protective and auxiliary structures. The neuro-epithelial structures, including the organ of Corti and the cells of the cristæ and maculæ acustice, result from the specialization of certain of the epithelial cells which line the membranous labyrinth. The perilymphatic space, which is a lymph-space, together with its bony walls, the osseous labyrinth, serve for the protection of the delicate neural elements, while the middle ear 
and the external ear act as media for the conduction of sonorous vibrations.

The internal ear being the esssential part of the organ of hearing and being also the part first formed may properly receive first consideration.

The Internal Ear.-The membranous labyrinth of the
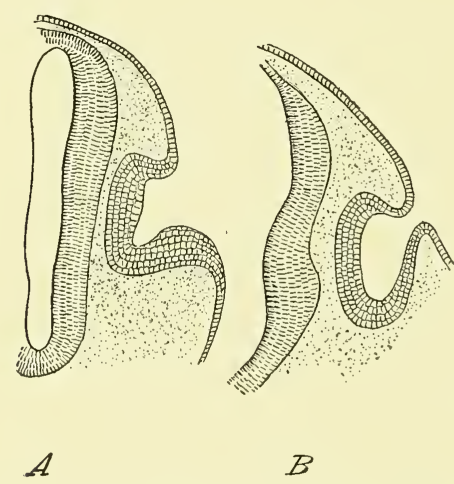

$B$

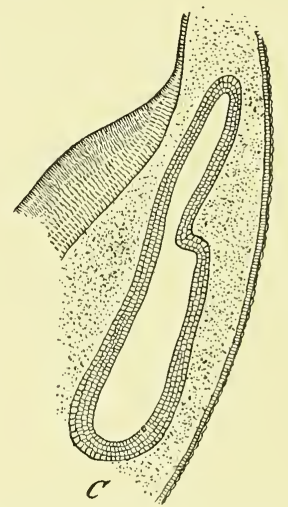

FIG. 166.-Three transverse sections showing development of otic vesicle of human embryo (Tourneux): $A$, from embryo of $3 \mathrm{~mm}$., showing auditory pit; $B$, from embryo of $4 \mathrm{~mm}$., showing the transformation of the pit into the otic vesicle; $C$, from enıbryo of $6 \mathrm{~mm}$., showing otic vesicle detached from surface ectoderm, and presenting a posterior diverticulum, the recessus vestibuli.

internal ear is the oldest part of the organ of hearing. Its origin is from a thickened circular patch of ectoderm on the dorsolateral surface of the head-region of the embryo near the dorsal termination of the first outer visceral furrow. The thickened area sinks below the surface, forming thus the auditory pit, which is present in the third week (Fig. 166, A). The pit becomes deeper, its edges approach each other and finally meet and unite to form the otic vesicle or otocyst. This little epithelial sac gradually recedes from the surface ectoderm. At this stage of development there is no cranial capsule other than the indifferent mesodermic tissue which surrounds the brain-vesicles; hence, the otic vesicle, embedded in this tissue, lies in close proximity to the afterbrain, and comes into relation with the acusticofacial ganglion (p. 321). The vesicle, at first spherical, soon becomes 
pear-shaped owing to the protrusion of its dorsal wall. This dorsal projection, the recessus vestibuli or labyrinthi (Fig. $166,()$, lengthens out into a slender tube, the ductus endolymphaticus (Fig. 168), the slightly dilated end of which, the saccus endolymphaticus, is found in the adult occupying the aqueductus vestibuli of the temporal bone.

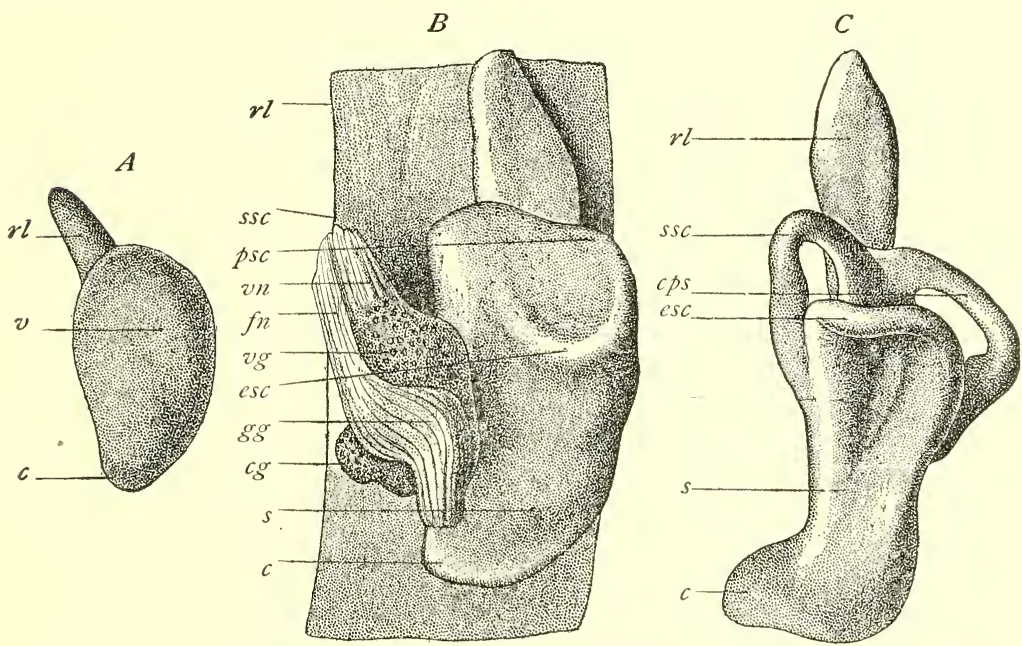

FIG. 167.-Development of the membranous labyrinth of the human ear (W. His, Jr.): $A$, left labyrinth of embryo of about four weeks, outer side; $v c$, vestibular and cochlear portions; $r$, recessus labyrinthi. $B$, left labyrinth with parts of facial and auditory nerves of embryo of about four and a half weeks ; $r l$, recessus labyrinthi ; $s s c, p s c$; $e s c$, superior, posterior, and external semicircular canals; $s$, saccule; $c$, cochlea; $v n, f n$, vestibular and facial nerves; $v g, c g, g g$, vestibular, cochlear, and geniculate ganglia. C, left labyrinth of embryo of about five weeks, from without and below; labelling as in preceding figure.

The opposite, anterior or ventral extremity of the otic vesicle also bulges out into a small evagination, which gradually elongates until it is a tapering tube, slightly curved inward toward the median plane. This lengthens still more and becomes spirally coiled, forming the cochlear duct or scala media of the future cochlea (Fig. 168). The vesicle itself becomes constricted in such manner by an inward projection of its wall as to indicate its division into an upper larger and a lower smaller sac, the terms upper and lower referring respectively to the head-end and the tail-end of the embryonic body. Before the constriction occurs, the wall 
of that part of the vesicle which is to become the future upper or utricular division presents two pouched-out areas (Fig. 167, B). One of these gives rise to the external semicircular canal, while from the other are formed the superior and posterior canals. The pouch that produces the external

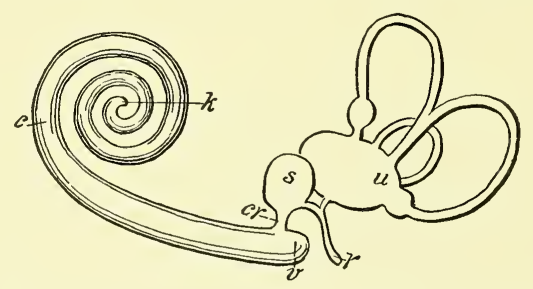

FIG. 168.-Diagram to illustrate the ultimate condition of the membranous labyrinth (after Waldeyer) : $u$, utriculus; $s$, sacculus; $c r$, canalis reuniens; $r$, ductus endolymphaticus; $c$, cochlea; $k$, blind sac of the cupola; $v$, vestibular blind sac of the ductus cochlearis.

canal is semicircular in form and flat, lying in the horizontal plane, its upper and lower walls being in contact with each other. The opposed walls fuse, except at the periphery of the pocket, and hence all that remains of its cavity is a small marginal tube or channel, corresponding with its border and opening at each end into the cavity of the vesicle. Throughout the region of fusion of the walls, the latter become thin and finally disappear, being replaced by connective tissue. Thus a semicircular epithelial tube is formed, which is the horizontal or external semicircular canal. One end of the tube being dilated, the ampulla of the canal is produced.

The superior and posterior semicircular canals are formed in a somewhat similar manner by the other evaginated pouch or pocket, which is irregularly globular. To produce this result, the walls of the pocket contract adhesions throughout two regions, which correspond with the respective spaces enclosed by each of the two future canals in question. The fusion of the walls takes place in such manner as to leave two narrow channels or tubes, one of which almost encircles the inner or mesial aspect of the pocket, while the other bears the same relation to its poste- 
rior wall, the inner limb of the latter semicircle coinciding with the posterior limb of the former. The result of this arrangement is that two vertical semicircular canals are formed with their planes at right angles to each other, the two communicating with the otic vesicle by three openings, one of which is common to both canals. The other two apertures, being dilated, are the ampullated individual orifices of the posterior and superior canals.

The constriction in the otic vesicle referred to above increases until this sac is divided into two parts, a larger, which includes the region from which the semicircular canals have developed and which is now the utricle, and a smaller vesicle, the saccule, comprising the part from which the cochlear duct was evaginated (Fig. 168). The line of division coincides with the middle of the orifice of the ductus endolymphaticus, the proximal end of which participates in the division. Thus the ductus endolymphaticus becomes a Y-shaped tube, and affords the only bond of connection between the saccule and the utricle (Fig. 168).

The beginning of the cochlear duct, failing to keep pace in growth with the other parts, appears as a smaller tube relatively, and is known as the canalis reuniens (Fig. 168, cr).

The structures so far considered-the utricle, the saccule, the semicircular canals, and the cochlear duct-being the product of the ectodermic otic vesicle, represent simply the adult epithelial linings of those cavities. The fibrous layer of the membranous labyrinth, in common with the walls of the bony labyrinth, is a product of the enveloping mesodermic tissue. While the cells of the otic vesicle thus for the most part constitute the walls of the several sacs and canals of the primitive internal ear, some of the cells specialize into neuro-epithelium. The most marked specialization of this sort occurs in the cochlear duct, where most of the cells on that wall of the duct which may be called its floor-the part corresponding to the future membrana basilaris-undergo such profound modification in form as to produce the most highly specialized neuro-epithelial cells anywhere to be found, the elements that constitute the organ of Corti. 
In the utricle and the saccule, as well as in the ampullæ of the semicircular canals, there is a similar but less marked specialization of epithelial cells to produce in the former case the maculæ acusticæ, and in the latter, the cristæ acusticæ of the ampullæ. While, therefore, the cells of the otic vesicle which are to serve as the lining mucous membrane of the membranous labyrinth become flattened polyhedral cells arranged as a single layer, those cells which are to functionate as the peripheral part of the acoustic mechanism become the specially modified columnar cells, many of them with cilium-like appendages, of the maculæ, the eristr, and of the organ of Corti.

From the first the otic vesicle lies in close relation with the acusticofacial ganglion (Fig. 167, B). As pointed out in a preceding chapter (p. 321), this ganglion subsequently divides into two parts, corresponding with the two divisions of the auditory nerve. This division of the ganglion and of the nerve is correlated with the separation of the otic vesicle into a cochlear part, the cochlear duct, and the two vestibular vesicles, the saccule and the utricle. While the cochlear duct is still a short, slightly curved tube, the cochlear part of the ganglion lies in close proximity to the tube, in the concavity on its inner side. As the duct lengthens and becomes more coiled, the ganglion likewise lengthens into a band which follows the spiral course of the duct, lying parallel with the latter and on the side toward the axis about which it is coiled. After the formation of the bony parts of the cochlea, this ganglion occupies the spiral canal of the modiolus and is known as the ganglion spirale. It belongs to the cochlear division of the auditory nerve, which is distributed to the cochlea.

The remaining part of the acoustic ganglion becomes rather widely separated from the spiral ganglion, coming to occupy a position in the internal auditory meatus, and the part of the auditory nerve with which it is connected acquires relation with the macular regions of the utricle and saccule as well as with the cristæ of the ampullæ of the semicircular canals. These nerve-fibers constitute the vestibular division of the 
auditory nerve, while the ganglion is the vestibular ganglion or intumescentia ganglioformis of Scarpa.

The development of the bony labyrinth of the internal ear, as well as of the connective-tissue parts of the membranous labyrinth, is effected solely by the differentiation of the mesodermic tissue which surrounds the epithelial structures above considered. As previously stated, at the time when the otic vesicle is first formed there is no indication of a cranial capsule, the brain-vesicles being surrounded and separated from the ectoderm by indifferent mesodermic cells. During the progress of the alterations in the otic vesicle, this tissue undergoes condensation and alteration to form the membranous primordial cranium, and shortly thereafter the petrous portion of the temporal bone is outlined in cartilage by the further specialization of a portion of this primitive connective tissue. The formation of cartilage does not affect all of the tissue which is afterward represented by the petrosa, the region that borders the semicircular canals, the cochlear duct, the saccule, and the utricle remaining soft embryonal connective tissue. There is thus a cartilaginous ear-capsule produced which is more than large enough to contain the primitive epithelial labyrinth, and the walls of which are separated from the latter by embryonal connective tissne.

The bony semicircular canals are almost exact reproductions, on a larger scale, of the epithelial canals, and they are formed by the ossification of the cartilaginous petrosa. Even before this ossification occurs the soft connective tissue between the cartilage and the epithelial semicircular canals differentiates into three layers. The inner layer, becoming more condensed, is converted into fibrous tissue, and, adhering to the epithelial walls of the canals, furnishes the connective-tissue component of the completed membranous canals. Its blood-vessels serve for the nutrition of the canals. The outer layer also undergoes condensation and forms a fibrovascular membrane, the perichondrium, which later becomes the internal periosteum of the bony canals. The middle layer, on the contrary, becomes softer-by the 
liquefaction of the intercellular substance and the degeneration of the cells-so that gradually increasing, fluid-filled cavities make their appearance, and these latter becoming larger and many of them coalescing, a space is formed around the membranous canals which is filled with fluid, the perilymph. This perilymphatic space is bridged across at intervals by connective-tissue processes that serve for the conveyance of blood-vessels to the membranous canals.

The vestibule of the internal ear is formed in practically the same manner as the bony semicircular canals, the epithelial saccule and utricle acquiring their connective-tissue constituents in the same way. There is the difference, however, that the bony restibule does not conform to the shape of the vestibular parts of the membranous labyrinth, since it is a single undivided cavity enclosing the two little vesicles, the saccule and the utricle.

The bony cochlea, while developed upon the same general plan as the other parts of the bony labyrinth, presents certain conspicuous modifications. The epithelial cochlear duct, as stated above, in its early stage is a short, tapering, and slightly curved tube. While it is still in this condition, chondrification of the petrous bone occurs, whereby the duct acquires its cartilaginous capsule (Fig. 169, kk). This capsule is open at the proximal end of the duct and through this opening the cochlear branches of the auditory nerve gain access to the capsule, being connected with the cochlear division of the auditory ganglion, which, owing to its previously having assumed a position beside the duct, comes to be enclosed by the capsule as the latter is formed (Fig. 169, nc, gsp). It is only after the chondrification that the cochlear duct lengthens out and becomes spirally coiled. The coiling is in such manner that the cochlear nerve is surrounded by the duct-that is, it lies in the axis about which the duct is spirally wound. Within the cartilaginous capsule, filling all the space not occupied by the spirally coiled duct and the cochlear nerve with its lengthened-out ganglion, is the embryonic connective tissue of which formerly the entire cartilaginous petrosa consisted. 
The cochlea consists now of a spirally coiled epithelial tube lying within an elongated cavity in the cartilaginous petrosa, a cavity, the walls of which are, therefore, cartilaginous. The peripheral wall of the coiled tube is in contact with the inner surface of the wall of the cartilaginous capsule (Fig. $169, x)$, a fact which has an important bearing upon the further stages of growth.

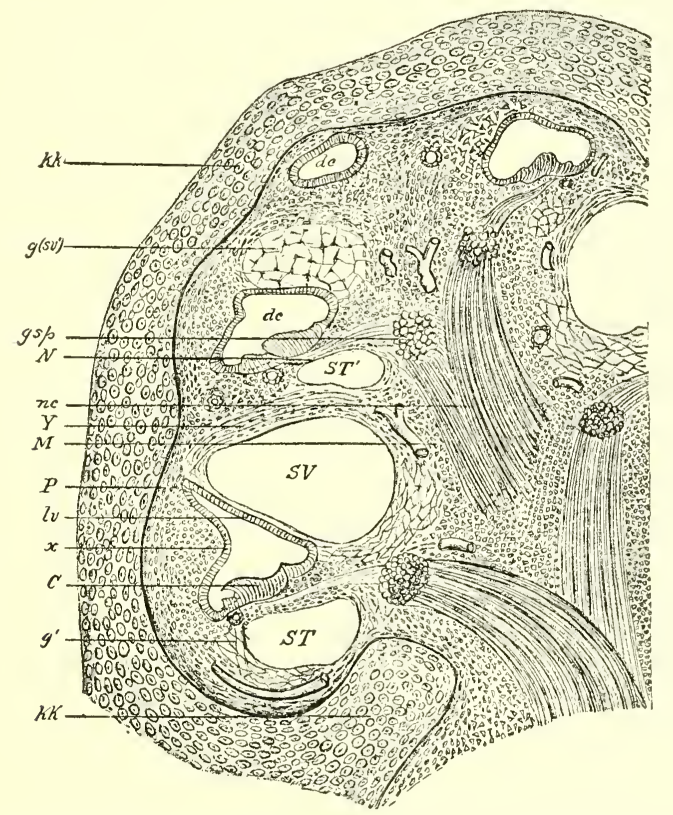

Fig. 169.-Part of a section through the cochlea of an embryo cat, $9 \mathrm{~cm}$. (3.6 in.) long (after Boettcher): $k k$, cartilaginous capsule, in which the cochlear duct describes ascending spiral turns; $d c$, ductus cochlearis; $c$, organ of Corti; $l v$, lamina vestibularis ; $x$, outer wall of the membranous ductus cochlearis with ligamentum spirale; $S \boldsymbol{V}$, scala vestibuli; $S T, S T^{\prime}$, scala tympani; $g$, gelatinous tissue, which still fills the scala vestibuli $\left(s v^{\prime}\right)$ in its last turns; $g^{\prime}$, remnant of the gelatinous tissue, which is not yet liquefied; $M$, firm connective tissue surrounding the cochlear nerve $(n c)$; $g s p$, ganglion spirale; $N$, nerve which runs to Corti's organ in the future lamina spiralis ossea; $Y$, compact connective-tissue layer, which becomes ossified and shares in bounding the bony cochlear duct; $P$, perichondrium.

The embryonal connective tissue within the capsule now undergoes important modifications, which vary greatly in different regions. That portion of this tissue which immediately envelops the cochlear nerve becomes first dense connective 
tissue, which is afterward directly converted into bone, constituting the modiolus, or axis, of the cochlea. The processes of condensation and subsequent ossification extend outward from the modiolus in a spiral line, which corresponds with the intervals between the successive turns of the cochlear duct, until they meet the wall of the original capsule, thus producing the bony cochlea. That is, by the development of this spiral plate and its connection internally with the modiolus and externally with the wall of the capsule, a tube at first partly membranous and partly cartilaginous, and at a later stage osseous, is produced, which encloses the much smaller cochlear duct, and like it is wound spirally around the modiolus. To repeat, the original simple cavity of the cartilaginous capsule is subdivided by the growth of the modiolus and of the spiral shelf in such manner as to become a spirally coiled tube.

The cochlear nerve, enclosed within the coil of the cochlear duct, sends branches (Fig. 169, $N$ ) in a continuous spiral line to the duct, and the soft tissue surrounding and supporting these branches condenses to form a connectivetissue plate which extends outward from the modiolus tc the cochlear duct and which, therefore, has a spiral course about the modiolus, its entire inner edge being attached to that central axis, while its outer border is, throughout its entire extent, in continuity with the inner wall of the duct. At a later stage this spiral plate undergoes direct ossification to form the two lamellæ of the bony lamina spiralis. Thus it is that the ganglion spirale and the successive terminal branches of the cochlear nerve come to be enclosed within the spiral lamina. Recalling the condition of the cochlea before the growth of the spiral lamina, it will be seen that the latter, in connection with the epithelial cochlear duct, divides the tube into two parts (Fig. 169, $S V, S T$ ). It will be evident, too, that the epithelial cochlear duct now holds a relation to the larger tube of the future bony cochlea which is similar in principle to the relation of the membranous semicircular canals to the bony canals, but with the difference that the outer wall of the epithelial duct is in 
close contact with the outer wall of the future bony canal at $x$, and that the inner walls of the two are connected by a spiral plate, the lamina spiralis.

The cochlear duct, then, is surrounded by undifferentiated mesodermic tissue, except on the side farthest from the modiolus, where its wall is in contact with and finally adheres to the wall of the cartilaginous capsule. The lamina spiralis divides this tissue into two parts which respectively occupy the positions of the future scala vestibuli and scala tympani. This soft embryonal tissue, as in the case of the corresponding tissue of the semicircular canals, develops differently in different regions. The innermost stratum, which is in relation with the epithelial cochlear duct, becomes fibrous connective tissue and constitutes the fibrous layer of the adult cochlear duct; that is, on the side of the duct toward the scala tympani, it becomes the connective-tissue layer of the membrana basilaris, while on the side toward the scala vestibuli it forms the fibrous stratum of the membrane of Reissner (Fig. 169). The peripheral zone of indifferent tissue, that in contact with the now cartilaginous wall of the future, bony cochlea, as well as that which lies against the lamina spiralis, also undergoes condensation and forms a fibrous, or fibrovascular, membrane, the internal perichondrium or future periosteum. The tissue intervening between these two layers retrogrades, the cells degenerating and the intercellular substance liquefying, until finally the spaces known as the scala vestibuli and the scala tympani are hollowed out. These channels are lymph-spaces and the fluid they contain is the perilymph. This perilymphatic space is in communication with that of the vestibule. 'Therefore, while the cochlear duct or scala media encloses an epithelium-lined space, as do the saccule, the utricle, and the membranous semicircular canals, and in common with those structures contains the so-called endolymph, the scala vestibuli and the scala tympani are in the same category with the perilymphatic spaces of the other parts of the internal ear.

The Middle and the External Ear.-The middle ear, consisting of the tympanic cavity and the Eustachian tube, 
is developed from the back part or dorsal end of the first inner visceral furrow. The external ear, comprising the external auditory meatus and the auricle, comes from the dorsal extremity of the first outer furrow and the tissue about its margins, the tympanic membrane representing in part the closing membrane which separates the inner furrow from the outer.

The first inner visceral furrow, in common with the other inner furrows, is an evagination of the lateral wall of the primitive pharyngeal cavity, or head-end of the guttract. The ventral end of this groove suffers obliteration, but the dorsal segment, designated the tubotympanic sulcus, becomes converted into a tube by the growing together of its edges. The tube is composed therefore of entodermic epithelial cells. It elongates in the dorsal and outward direction, and its dorsal extremity becomes enlarged to produce the cavity of the tympanum, the remaining part of the canal becoming the epithelial lining of the Eustachian tube. The canal being formed before the development of the cranium, and approximately its posterior half being surrounded by the mesodermic embryonal connective tissue that afterward becomes the petrosa of the temporal bone, the tympanic cavity and a part of the Eustachian tube come to be enclosed within that bone, while the connective tissue encasing the anterior part of the tube differentiates into the curved plate of cartilage that forms the cartilaginous part of the Eustachian tube.

Since the posterior end of the primitive epithelial tube insinuates itself between the otic vesicle and the surface, the tympanum comes to occupy its normal position on the outer side of the internal ear. The tympanum, being derived from the back part of the first visceral cleft, is in close relation with the first and second visceral arches, and the ossicles of the middle ear are derived from the dorsal extremities of the cartilaginous bars of these arches in the manner described in Chapter XVIII. Necessarily the primitive ossicles are exterior to the primitive epithelial tympanic sac, as is also the chorda tympani nerve, which 
passes along its outer side. After the ossification of the temporal bone, these structures are embedded within the abundant soft connective tissue which is between the epithelial sac, now the mucous membrane, and the bony walls of the tympanum. This mass of soft tissue undergoes very considerable diminution, owing to which the mucous membrane comes into contact with the bony walls, and as a result the ossicles and the chorda tympani are enclosed in folds of the mucous membrane and seem to lie within the tympanic cavity. They are exchuded, however, from the true cavity of the tympanum, since they are exterior to the epithelial or mucous-membrane layer.
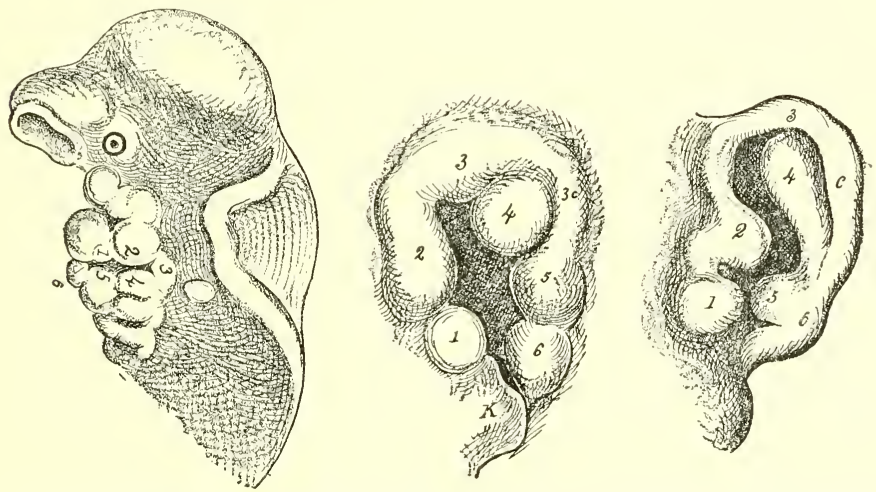

FIG. 170.-Showing the gradual development of the parts of the external ear from prominences upon the mandibular and hyoidean visceral arches (His), variously magnified : 1, 2, prominences on mandibular arch; 3 , prominence between the two arches, prolonged posteriorly in second figure to $3 c ; 4,5$, and 6 , prominences on hyoidean or second visceral arch; $\boldsymbol{k}$, lower jaw. Prominence 1 forms the tragus; $2,3,3 c$, the helix ; 4 , the antihelix ; 5 , the antitragus; 6 , the lobule.

The external auditory meatus is simply the persistent posterior part of the first onter visceral furrow or hyomandibular cleft (see pp. 112, 116), this cleft closing completely everywhere but in this region. The closing plate of the first cleft becomes the tympanic membrane. Hence the outer layer of this membrane is of ectorlermic origin, while the inner layer is entodermic, being continuous with the epithelial tympanic lining, and the middle fibrous layer is derived from the mesoderm. The relation of the malleus to the membrane and of the latter to the bony tympanic plate 
which forms part of the wall of the meatus is dealt with in the chapter on the development of the skeleton.

The auricle is derived from the tissue around the margin of the unclosed back part of the first outer cleft (Fig. 171, C). Six little elevations make their appearance here, the projections being mesodermic tissue covered with ectoderm. The mesodermic component of the elevations differentiates into the cartilaginous and other connective-tissue parts of the auricle. The nodules marked 2 and 3 in Fig. 170 becoming a continuous ridge, produce the helix, while nodule 4 becomes the antihelix. The tragus and antitragus develop respectively from the projections 1 and 5. At the end of the second month, these parts are so far advanced as to be easily distinguishable, and the connective-tissue basis of the ridges and projections and the continuous plate-like mass to which they all are attached begin to undergo chondrification. From the third month onward, this primitive auricle, by continued growth and greater separation from the side of the head, assumes more and more the characters of the fully formed member. The lobule, however, which results from the growth of the little elevation marked 6, lags behind the other parts in development and is rather indistinct until the fifth month, after which time it increases in size and gradually acquires its normal proportions.

\section{THE DEVELOPMENT OF THE NOSE.}

The nose is primarily a special sense-organ, although a part of its cavity serves, in air-breathing vertebrates, as an adjunct to the respiratory system. The evolution of the mature organ of smell may be epitomized by the statement that the olfactory epithelium, the essential part of this senseorgan, is a patch of depressed or infolded ectoderm, the cells of which are highly specialized and are brought into relation with the central nervous system by means of the outgrowth from the latter of a part of its mass, the olfactory lobe.

Very early in intra-uterine life-before the end of the third week-the olfactory plates appear as localized thicken- 
ings of the ectoderm situated just in front of or above the oral fossa. These nasal areas are the forerunners of the future olfactory epithelium. It is worthy of note that the olfactory plates are in very close relation with the primary
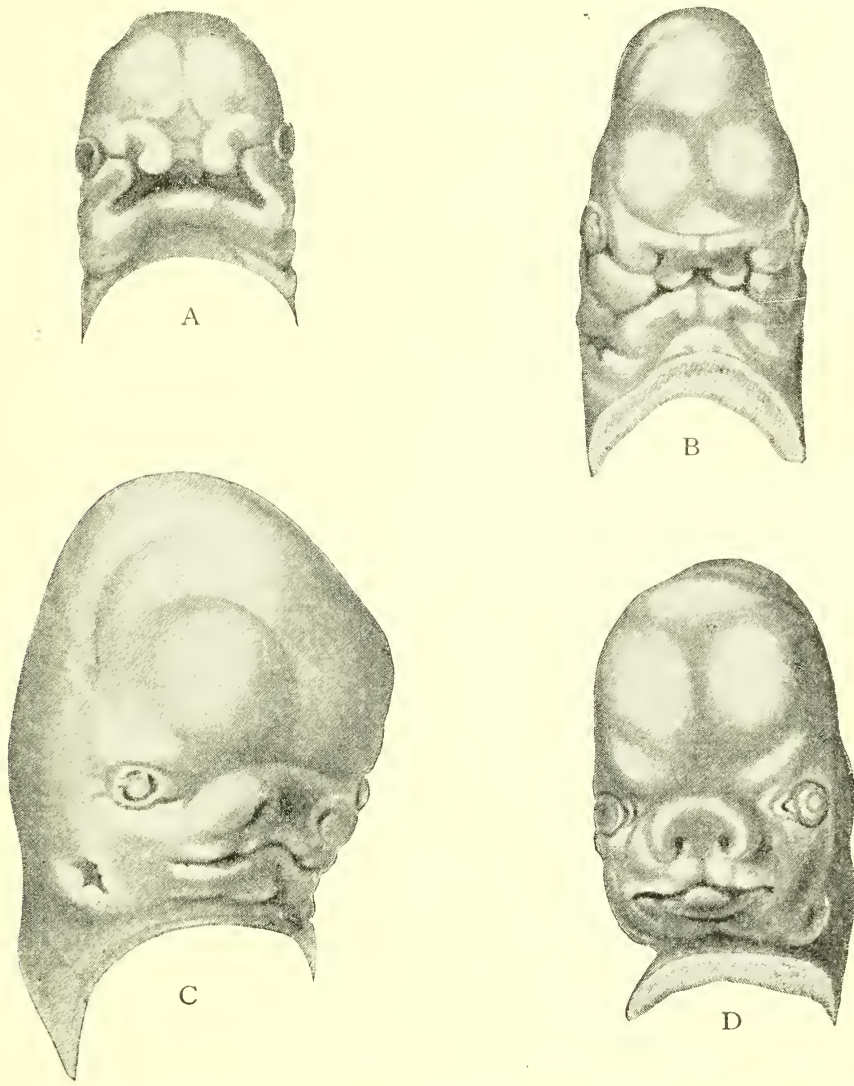

Fig. 171.-Development of the face of the human embryo (His): A, embryo of about twenty-nine days. The nasofrontal plate differentiating into processus globulares, toward which the maxillary processes of first visceral arch are extending. B, embryo of about thirty-four days: the globular, lateral frontal, and maxillary processes are in apposition; the primitive opening is now better defined. C, embryo of about the eighth week: immediate boundaries of moutl are more definite and the nasal orifices are partly formed, external ear appearing. D, embryo at end of second month.

fore-brain vesicle, being, in reality, on the outer surface of the ectodermic covering of its ventral wall.

Owing to the rapid outgrowth of the surrounding tissue, the olfactory plates become relatively depressed, constituting 
now the nasal pits, which are distinguishable at about the twenty-eighth day. The pits are separated from each other by a broad mass of tissue, the nasal or nasofrontal process (Fig. 171), which is, as it were, a localized thickening of the mesodermic tissue on the ventral wall of the primary fore-brain vesicle; and this process makes its appearance in the third week. During the fifth week the nasofrontal process thickens greatly along its lateral margins, the thick edges being known as the globular processes (Fig. 171, A, B). At the same time the lateral nasal processes bud out from the nasofrontal process, one on each side, above the nasal pits, and, growing downward, form the external boundaries of the pits, each of which depressions is bounded on its inner side by the corresponding globular process. The nasal pits, therefore, have well-marked walls on every side except below, where they are directly continuous with the oral fossa.

In the latter end of the sixth week the nasofrontal process, which, it will be remembered, constitutes the upper limit of the oral fossa, is joined on each side by the united maxillary, and lateral nasal, processes. This effects a division between the oral fossa and the nasal pits, and forms, though as yet crudely, the external nose, and the upper lip as well. The definite formation of the external nose may be said to be indicated about the eighth week. The orifices of the nasal pits are now the anterior nares, while the pits themselves have become short canals, opening by their deep orifices, the posterior nares, into the primitive mouth-cavity above the palatal shelves. The nares are separated from each other by the still broad nasofrontal process. That portion of the nasofrontal process that separates the nares gradually becomes thinner and produces the septum of the nose, while its external or superficial part gives rise to the bridge and tip of the organ.

The growth of the palate-shelves (Fig. 172) toward the median line, resulting in their union with each other and with the recently-formed septum, definitely divides the nasal chambers from the cavity of the mouth, the posterior nares now opening into the pharynx. This separation is completed toward the end of the third month. 
The complexity of the adult nasal cavities is produced by the formation of ridges and pouches on the lateral walls of the original nasal pits. Three inwardly projecting horizontal folds of the ectodermic lining of the cavity, the superior, middle, and inferior turbinal folds, appear upon the outer wall of each nasal fossa (Fig. 173). Each fold contains a stratum of mesodermic tissue which devclops into cartilage and subsequently into bone, forming respectively the three turbinated bones. The cartilaginous character of these folds becomes apparent at the end of the second, or the early part of the third, month. An evagination on the lateral wall of each

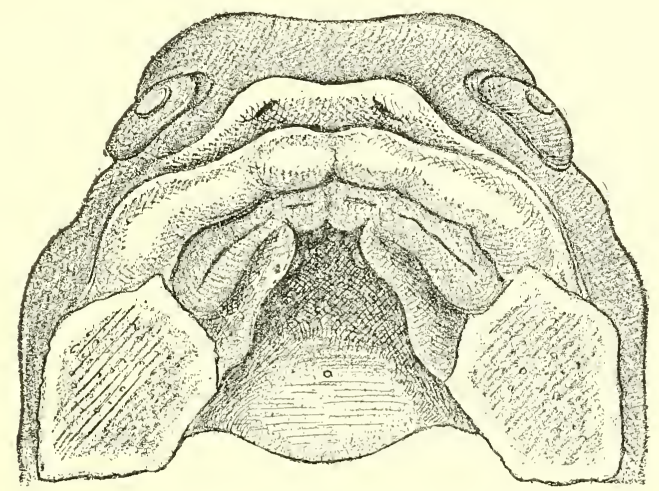

FIG. 172.-Roof of the oral cavity of a human embryo with the fundaments of the palatal processes (after His), $\times 10$.

nasal fossa, between the middle and the inferior turbinal processes, becomes the antrum of Highmore; this is formed in the sixth month. Other evaginations produce the ethmoidal, the frontal, and the sphenoidal sinuses, the last two of which are not completed, however, until after birth. Very early in the development of the nose a small invagination appears on the mesial wall of the nasal pit. In the fourth month of gestation this invagination has become a canal in the septum (Fig. 173, $J$ ), running from before backward and ending in a blind extremity. It is the so-called organ of Jacobson, which, in man, is merely a rudimentary structure, but which, in most other mammals, is more highly developed, being surrounded by a cartilaginous capsule and receiving a special nerve-supply from the olfactory nerve. 
The olfactory plates become separated from the fore-brain vesicle and consequently from the later brain and its outgrowth, the olfactory bulb, by the development of an intervening bony plate, the cribriform lamina of the ethmoid bone. The ectodermic cells of the olfactory plates differentiate into the highly specialized neuro-epithelial elements of the olfactory mucous membrane, the olfactory epithelium, and their associated supporting cells. The axons of the neuroepithelial cells pass upward through the cribriform plate of the ethmoid bone as the olfactory nerve-fibers, and, entering the ventral surface of the olfactory bulb, arborize with the processes of the mitral cells of the bulb, whereby they acquire functional relationship with the olfactory centers in the brain.

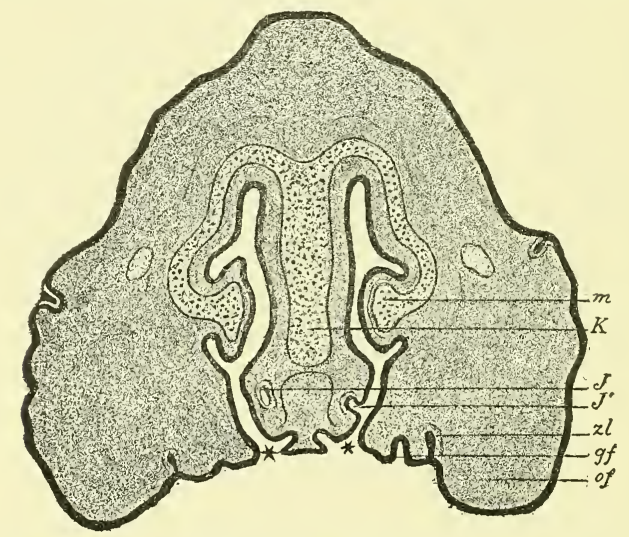

Fig. 173.-Cross-section through the head of an embryo pig $3 \mathrm{~cm}$. (1.2 in.) long, crown-rump measurement. The nasal cavities are seen to be in communication with the oral cavity at the places designated by a*: $K$, cartilage of the nasal septum; $m$, turbinal cartilage; $J$, organ of Jacobson ; $J^{\prime}$, the place where it opens into the nasal cavity; $g f$, palatal process; of, maxillary process; $z l$, dental ridge (Hertwig).

The external nose, as previously stated, first acquires definite form about the eighth week by the union of the distal ends of the lateral nasal processes with the nasofrontal process, the former producing the alæ and the latter the bridge and the tip of the nose. In the third month the organ is unduly flat and broad, but from this time on it gradually assumes the familiar characteristic form. From the third month to the fifth each external naris is closed by a gelatinous plug of epithelial cells. 


\section{CHAPTER X VII.}

\section{THE DEVELOPMENT OF THE MUSCULAR SYSTEM.}

\section{THE STRIATED OR VOLUNTARY MUSCLES.}

THE voluntary muscular system, genetically considered, is divisible into (1) the muscles of the trunk and (2) those of the extremities. The muscles of the trunk include two distinct sets: (a) the muscles of the trunk proper, or the skeletal muscles, and (b) the muscles of the visceral arches or the branchial muscles.

To arrive at a proper comprehension of the evolution of the muscular system it is necessary to revert to an important fundamental embryological process, the segmentation of the body of the embryo, or, as it is sometimes expressed, the segmentation of the colom, or body-cavity. As pointed out in Chapter IV., this process of segmentation occurs in all vertebrate animals and in some invertebrates.

The Muscles of the Trunk Proper.-At a very early stage of development the tracts of mesodermic tissue situated one on each side of the median longitudinal axis of the future embryonic body, the paraxial mesodermic tracts, undergo division or segmentation, in lines transverse to the long axis, into a series of pairs of irregularly cubical masses of mesodermic cells. These masses are the mesoblastic somites or primitive segments, often inappropriately called the protovertebræ. The somite first formed corresponds with the future occipital region, the second one lies immediately in front of the first, while two others, situated still more anteriorly, that is, near the cephalic end of the embryonic area, and seven more, behind the first, are added almost simultaneously. The formation of the primitive segments 
then proceeds tailward until a considerable number have been added. Those in front of the one first formed are denominated the head-segments, while the others are known as the trunk-segments. Each somite is at first triangular in cross-section, the base of the triangle looking toward the chorda dorsalis. Subsequently they assume a more cuboidal shape. In the lower vertebrates-amphibians and fishesthe somite is hollow, its cavity being in these cases a constricted-off portion of the body-cavity (hence the term "seg-

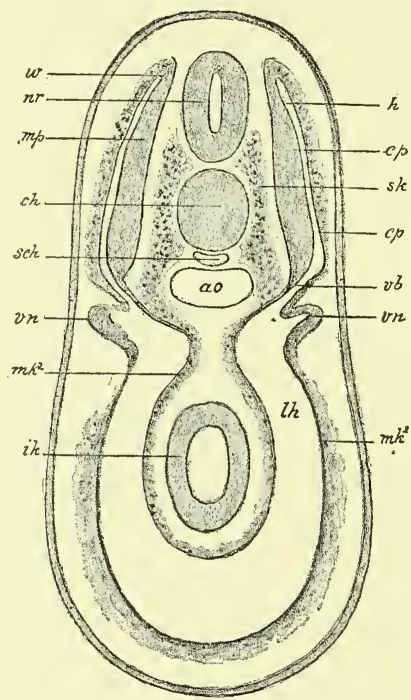

FIG. 174. - Cross-section through the region of the pronephros of a selachian embryo in which the muscle-segments (myotomes) $(m p)$ are in process of being constricted off. Diagram (after Wijhe): $n r$, neural tube; $c h$, chorda; ao, aorta; $s c h$, subnotochordal rod; $m p$, muscle-plate of the primitive segment; $w$, zone of growth where the muscle-plate bends around into the cutis-plate $(c p)$; $v b$, tract connecting the primitive segment with the body-carity, out of which are developed, among other things, the mesonephric tubules; sk, skeletogenous tissue which arises by a proliferation from the median wall of the comnecting tract $v b$; $v n$, pronephros; $m k^{1}, m k^{2}$, parietal and visceral middle layer, from whose walls mesenchyme is developed ; $l h$, body-cavity ; $i k$, entoblast.

mentation of the coelom" to express this process). In the higher vertebrates, however, the cavity is obliterated by the encroachment of the cells of the walls of the somite.

The cells of the somites soon undergo differentiation and rearrangement. It is usually stated that, preparatory to the 
segmentation of the paraxial mesodermic tract, this tract has become separated from the remaining lateral plate of the mesoderm. The separation is not complete, however, and therefore, after the appearance of the primitive segments, each segment is connected with the more laterally placed lateral plate-by the separation of which latter into two lamellæ the colom is formed-by a smaller mass of tissue, the nephrotome, also called the middle plate, or intermediate cell-mass (Fig. 17t, vb). As development progresses the distinction between the primitive segment proper and the nephrotome becomes more sharply expressed, and the former is designated the myotome. The primitive segment on its mesial surface, near the point of union with the nephrotome, sends forth cells which form a mass called the sclerotome (Fig. 174, sk). The sclerotomes spread out and blend with each other, forming a continuous mass of tissue which envelops the chorda and the neural canal, and which also extends laterally between the myotomes, separating them from each other and constituting the ligamenta intermuscularia (vide p. $375)$; this tissue, being concerned in the production of the permanent vertebræ, has no further interest in this connection.

What remains of the primitive segment after the formation of the nephrotome and of the sclerotome is the myotome proper or the muscle-plate. Although, as previously stated, the primitive segments of the higher vertebrates contain no cavity, the myotome and the nephrotome each enclose a space, that belonging to the former being known as the myocœl. The myotomes or muscle-plates are so called because they give rise to the voluntary musculature of the trunk. But not all of the cells of the muscle-plate undergo transformation into muscular tissue. While the cells on the mesial or chordal side of the myocel are going through certain alterations preparatory to their metamorphosis, the cells nearer the body-wall become rearranged to form a characteristic layer which is known as the cutis-plate from the fact that it contributes to the formation of the corium of the skin (Fig. 174, cp). The cutis-plate and the remaining 
part of the muscle-plate are continuous around the myocœl, the transition from one to the other being more or less gradual. To summarize, the primitive segment is differentiated into the nephrotome, the sclerotome, the myotome or muscleplate, and the cutis-plate.

The Metamorphosis of the Muscle-plate.-By the term muscle-plate is meant here the thickened layer of cells on the chordal or mesial side of the myotome proper, which layer constitutes what remains of the myotome after the differentiation of the cutis-plate. These cells having proliferated and increased in size, and having encroached thereby upon the cavity of the myotome, next undergo alteration in shape, becoming cylindrical, with their long axes parallel with that of the body of the embryo. The length of each cylindrical cell equals the thickness of the primitive segment, at least in the Amphibia and probably also in the chick. The next step in the transformation is the acquisition of the transverse striation characteristic of vertebrate voluntary muscle. Soon after this the protoplasm of the cell undergoes longitudinal division into minute fibrillæ-which latter do not necessarily correspond, however, with the primitive fibrillæ of mature muscle-and the cell-nucleus likewise divides. The metamorphosis of the now fibrillated protoplasm into muscular tissue is first completed at the periphery of the fiber, so that a young musclefiber contains a central core of undifferentiated material, including the daughter-nuclei resulting from the division of the original nucleus. Soon after the appearance of striation and the fibrillation of the fiber, the fibers begin to separate from each other, and developing connective tissue with young blood-vessels penetrates between them, the fibers now showing aggregation into bundles. For some time longer the fibers are naked, since the sarcolemma is not acquired until considerably later. The differentiation into muscular tissue gradually extends from the periphery of the fiber to its core, the process being complete in the human embryo at about the end of the fifth month for the muscles of the upper extremities and in the seventh month for those of the lower. 
The embryonic muscle-fibers are smaller than the mature elements and increase in size until the third month.

It is considered highly probable by most embryologists that muscle-fibers undergo multiplication during embryonic life. There are several theories as to the method of this multiplication. The most generally accepted view is that put forth by Weismann, the essential feature of which is that the fibers multiply by longitudinal division or fission. Reference was made above to the repeated division of the nucleus of the cell as one of the initiatory steps in the formation of the muscle-fiber. According to the fission theory, there is one class of fibers in which the nuclei are arranged in a single row, and the fibers of this class do not undergo fission; while there is another class, the fibers of which have their nuclei arranged in several rows. Fibers of the latter type divide longitudinally into as many daughter-fibers as there are rows of nuclei.

Although many of the details of the development of the muscular system are still involved in obscurity, it is a generally accepted fact that each fiber is derived from a single cell, the protoplasm of which develops the function of contractility to the subordination of the remaining vital properties of protoplasm. With this specialization of function there is necessarily a concomitant alteration of structure.

The muscular mass resulting from the transformation of each myotome grows in the ventral direction between the ectoderm and the parietal leaf of the mesoderm, or in other words into the somatopleure, to produce the muscular structures of the ventrolateral body-wall. The off-shoots of the myotomes which thus penetrate the body-wall in the fourth week produce, in the fifth week, a muscle-mass which, for the most part, is non-segmental, and which gives rise to a dorsal and a ventrolateral division; the dorsal division, derived from all the spinal myotomes, being destined for the musculature of the back, while the ventrolateral division, springing from the thoracic myotomes alone, gives rise during the fifth, sixth, and seventh weeks to the muscles of the thoracic and abdominal 
walls (Bardeen and Lewis ${ }^{1}$ ). The dorsal division extends in the dorsal direction, covering and acquiring points of attachment to the vertebral column, which has meanwhile been forming. In addition to the ventral and dorsal extension of the muscle-plates, each one grows both forward and backward-cephalad and caudad-in such manner that overlapping and intermingling result. During the differentiation of the various muscular masses from the myotomes, ventral and dorsal branches of the corresponding spinal nerves grow forth, their final distribution being to muscles developed from the particular myotomes with which the respective nerves correspond. According to Bardeen and Lewis the structures of the body-wall are well differentiated by the end of the sixth week, although their extension to the mid-line is not completed until near the end of the third month.

What has been said above concerning the evolution of the trunk-musculature from the primitive segments refers to those muscles that are developed from the segments of the trunk. As to the evolution of the head-segments comparatively little is definitely known. It is generally accepted that in elasmobranchs-a group including sharks and raysthere are nine primitive segments in the region of the future head. The number present in mammalian embryos has not been clearly worked out. Three occiptal and thirty-five spinal myotomes have been seen in human embryos of the fourth week, at which time the formation of myotomes is said to cease. In the lower vertebrates each segment contains a cavity lined with flattened cells, the mesothelium, the metamorphosis of which into muscular tissue may be inferred to be essentially as already outlined above. The first head-segment, which lies in contact with and partially envelops the optic vesicle, gives rise to the superior rectus, the inferior rectus, and the inferior oblique muscles of the eye-ball (innervated by the third cranial nerve); the second segment produces the superior oblique (innervated by the fourth nerve); and the third, the external rectus (innervated by the sixth nerve). The fourth, fifth, and sixth segments abort and hence produce

1 American Journal of A 1 natomy, vol. i., No. 1. 
no adult structures; while the seventh, the eighth, and the ninth segments become metamorphosed into the muscles that connect the skull with the shoulder-girdle.

From recent studies ${ }^{1}$ it would appear that individual muscles undergo peculiar and significant migrations during their development, and that the origin of the nerve-supply of a muscle indicates the location of the particular myotome or myotomes from which it originated, since the segmental nerves are connected with their respective myotomes and supply the muscles derived from such myotomes. For example, the serratus magnus, being innervated by branches of the cervical nerves, develops from myotomes in the neck region, and subsequently moves down to become attached to the scapula and the ribs.

The Branchia1 Muscles. - This term embraces the muscles of mastication and the various muscles connected with the hyoid bone, with the jaws, and with the ossicles of the middle ear. They result from the metamorphosis of the mesothelium of the visceral arches and acquire connections with structures that have arisen from the so-called mesenchymal cells of these arches or, in other words, from the embryonal connective tissue which makes up the chief part of their bulk. For an account of the growth of the visceral arches the reader is referred to Chapter VII. From this account and from that found in Chapter IV., it will be seen that the formation of the visceral arches and clefts is in reality the segmentation of the ventral mesoderm of the headregion of the embryo, or to express it in another way, it is the segmentation of the ventral colom of that region. It is interesting to note that whereas in the trunk the segmenta"tion of the mesoderm is restricted to the dorsal part of the body, in the head-region the ventral mesoderm also participates in the process. Hence the visceral arches, as might be expected, consist of so many masses of mesodermic tissue, each arch containing a small cavity lined with mesothelium, which cavity is a constricted-off part of the body-cavity or

'See "Development of the Ventral Abdominal Walls in Man," Franklin P. Mall, Johns Hopkins Papers, vol. iii., 1898. 
cnelom. It is these mesothelial cells that produce, by their differentiation, the muscles under consideration. While so much concerning the origin of this group of muscles is practically assured by observations upon the embryos of the lower vertebrates, the details are still obscure. His assumes the origin of the palatoglossus, the styloglossus, and the levator palati from the second or hyoid arch; of the stylopharyngeus, perhaps the palatopharyngeus, the hyoglossus and the superior constrictor of the pharynx from the third arch; and of the midale and inferior pharyngeal constrictors from the fourth arch. Further, it is held by Rabl that the muscles of the face, including those of the scalp and the platysma-the muscles of expression-originate from the mesothelium of the hyoid arch in the form of a thin superficial sheet, which, gradually spreading out from the place of origin, breaks up into the individual muscles.

The Muscles of the Extremities.-The relation of the development of the muscles of the limbs to the myotomes is still a disputed point. Some authorities hold that the limb-muscles of mammals originate from the myotomes, as was shown by Dohrn to be the case with the fin-musculature of Selachians. A fact adduced as a strong argument in favor of their myotomic origin is that the nerve-supply of each limb corresponds with the nerves of the number of myotomic segments in relation with which the limb-bud develops (vide p. 406). On the other hand, it is stated ${ }^{1}$ that the myotomes do not extend into the developing limb-buds, but that the muscles of the limbs are differentiated from the mesenchymal core of the limb-bud, this process following the entrance of the motor nerve-fibers into the member. The muscles of the upper limb are so well advanced in their development by the sixth week as to be individually distinguishable, those of the lower limb reaching a corresponding stage in the seventh week.

${ }^{1}$ Bardeen and Lewis, loc. cit. 
THE INVOLUNTARY OR UNSTRIATED MUSCULAR TISSUE.

This variety of muscular tissue, like that considered above, is of mesodermic origin. But while the voluntary muscles arise from the flattened or mesothelial cells of the primitive segments, involuntary muscle results from the transformation of the embryonal connective-tissue elements, the mesenchymal cells, of the mesoderm. It is for this reason that some authors speak of the voluntary muscles as the mesothelial muscles and designate the involuntary muscular tissue as mesenchymal muscle.

While it is a generally accepted fact that each of the fibercells which make up unstriated muscle is a metamorphosed mesenchymal or connective-tissue cell, the details of the process have not been accurately worked out. One may assume that necessarily the young connective-tissue cell elongates and that its protoplasm must undergo such differentiation as will fit it for the exercise of its future function, contractility.

THE CARDIAC MUSCLE.

The account of the development of the heart-muscle will be found in Chapter $\mathrm{X}$. 


\section{CHA PTER X VIII.}

\section{THE DEVELOPMENT OF THE SKELETON AND OF THE LIMBS.}

Although the skeleton is the framework of the body in the anatomical or mechanical sense, it is not so embryologically, since its development is not begun, at least not to any important extent, until nearly all the principal organs are well differentiated, and its growth is largely subsidiary to that of the structures which, in the mature state, it supports and protects. Morphologists speak of the exoskeleton and the endoskeleton, the former having reference to the hard structures found superficial to the soft parts, for whose protection they serve, such as the carapace of the lobster, and the hard scales of certain fishes; while the latter term signifies the cartilaginous or bony structures found within the bodies of most vertebrate animals. Even in the highest vertebrates, certain bones, such as those of the vault of the cranium, are usually considered by morphologists as being the representatives of part of the exoskeleton of lower types.

The skeleton, using the word in its ordinary sense, consists of the axial skeleton and the appendicular skeleton, or skeleton of the limbs. The former, including the head and the trunk, is common to all vertcbrates; the latter is not found in the lowest members of this class and hence is to be regarded as a later acquisition in the evolution of the skeleton.

In studying the development of the skeleton, as in considering that of other systems and organs, clearer conceptions of the growth of the individual may be obtained by comparing it with the evolution of the type. For example, the simplest form of skeletal apparatus is that of the amphioxus. In this animal the only representative of the skeleton is the 
notochord, a cylindrical rod composed of cellular or gelatinous tissue in which neither chondrification nor ossification ever takes place. Such an animal furnishes an example of the notochordal stage of the skeleton. The surrounding of the chorda with a sheath of embryonal comnective tissue, by which it is strengthened and thereby better fitted to serve as the body-axis, furnishes the membranous type of skeleton, a stage a little farther advanced than the preceding. The next higher type of skeleton is the cartilaginous form. In this case the embryonal connective tissue has undergone transformation into cartilage, at which point development is arrested, the stage of ossification never being attained. The cartilaginous type of skeleton is illustrated by that of the selachian (sharks and $(\log -f i s h)$.

The third and highest type of skeleton is the osseous. This results from the replacement of the cartilaginous tissue by bone. The process of ossification does not, however, affect every part of the cartilaginous skeleton, there being some portions of the latter which remain permanently unossified. As there are, throughout the vertebrate series of animals, various gradations in the degree of differentiation of the skeleton, so in the course of development does the osseous system of every higher vertebrate pass through these stages from the simplest condition, that of the notochordal skeleton, to the highest form of the almost completely ossified skeletal apparatus.

\section{THE AXIAL SKELETON.}

The axial skeleton, as stated above, includes the bones of the trunk and those of the head. Logically the development of the former will first claim attention.

\section{The Development of the Trunk.}

The Stage of the Chorda.--The formation of the chorda dorsalis or notochord is the earliest indication of the axis of the embryonic body and it will be recalled that it is also one of the earliest embryological processes. The mode of development of the chorda from the entodermal epithelium has been described at p. 73. The chorda serves the pur- 
pose, as it were, of an axis about which the permanent vertebral column and a part of the skull are, at a much later date, built up. The anterior or headward termination of the chorda corresponds to the position of the later hypophysis, or pituitary body, and thus the chorda is coextensive, not only with the vertebral column, but also with a portion of the cranium. The cells of the chorda enlarge and become distended with fluid, the protoplasm of each cell being reduced to a thin layer. The peripheral cells, however, constituting a distinct layer, the chordal epithelium, remain small, and it is by their proliferation that the chorda increases in size. In the amphioxus the chorda is the only "skeleton" that is ever acquired, and in this animal it is a permanent structure. In all other vertebrates it becomes surrounded by embryonal connective tissue, mesenchyme, which latter undergoes chondrification, and in the higher types ossification also. While in some of the lower vertebrates, as in certain classes of fishes, the chorda persists as a structure of more or less importance, in the higher members of the series, birds and mammals, it retrogrades as the processes of chondrification and ossification go on, until finally it is represented only by the pulpy centers of the intervertebral disks.

The Membranous Stage.-The notochordal stage of the development of the vertebral column is succeeded by the membranous stage. The transformation is effected by the appearance of an ensheathing mass composed of embryonal connective-tissue cells which surround not only the chorda but also the neural canal or fundament of the nerrous system (Fig. 174, sk). The source of this embryonal connective tissue or mesenchyme bears an important relation to the primitive segments. As the development of the primitive segments was described in the last chapter, and also in Chapter IV., it will suffice to remind the reader that each primitive segment undergoes differentiation into the myotome or muscleplate, the cutis-plate, the nephrotome, and the sclerotome (Fig. 174), the sclerotome occupying the mesial surface of the segment and lying in close proximity to the chorda.

While the myotome originates from the flattened or meso- 
thelial cells of the primitive segment, the sclerotome is made up of cells of the type characteristic of young-growing connective tissue-that is, of the mesenchymal part of the primitive segments as distinguished from their mesothelium. Owing to the rapid multiplication of its cells, each sclerotome spreads out headward and caudalward, and dorsad and ventrad, surrounding both the chorda and the neural canal, until both these structures become enclosed in a common, continuous sheath of embryonal connective tissue. That part of this tissue which surrounds the chorda is often designated the skeletogenous sheath of the chorda and also the membranous primordial vertebral column. The cells of the sclerotomes not only surround the chorda and the neural canal, but they also spread out laterally into the intervals between the muscle-segments to constitute the ligamenta intermuscularia or the bands or strips of connective tissue which separate adjacent muscle-

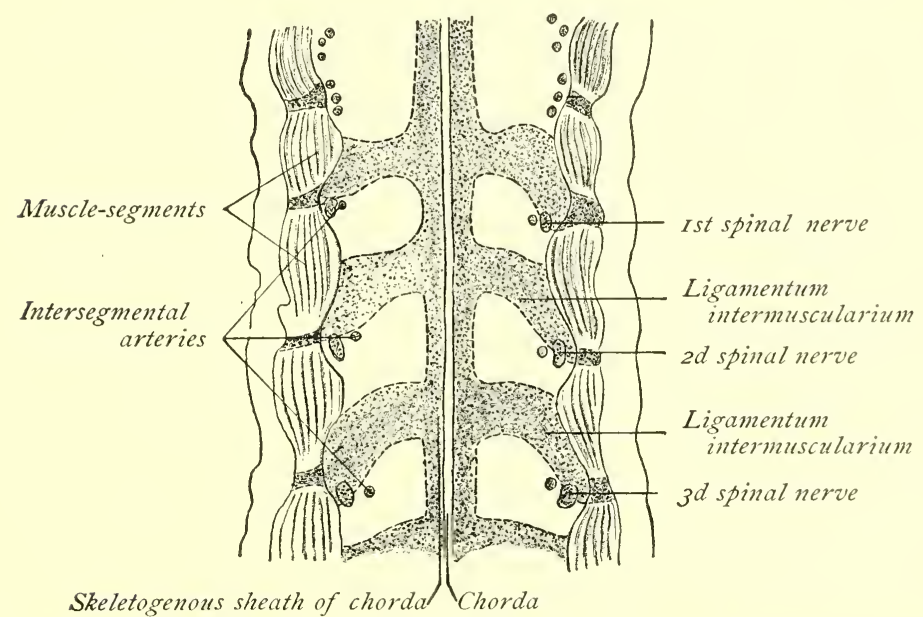

FIs. 175.-Frontal projection from a series of sections through a cow embryo of $8.8 \mathrm{~mm} .(0.35$ in.). (From Bonnet, after Froriep.)

segments from each other (Fig. 175). It is worthy of note that while this skeletogenous sheath of the chorda originates from segmented structures, the somites or primitive segments, and is to that extent related to the segmentation of the body, it now presents no trace of segmentation. 
Very soon, however, this ensheathing membranous tissue exhibits areas of condensation alternating regularly with less dense areas. Each such condensed area has the form of a somewhat obliquely placed bow or half-arch. This halfarch of condensed mesenchymal tissue is called the primitive vertebral bow by Froriep (Figs. 175 and 176), whose investigations upon chick and cow embryos established most of the facts known concerning the development of the vertebræ. ${ }^{1}$ The median portion of the bow is on the ventral side of the chorda and is known as the hypochordal brace. The lateral extremities of the bow abut against the corresponding myotomes, each extremity becoming bifurcated. The dorsal limb of the bifurcation, the neural process, extends gradually over the dorsal surface of the primitive spinal cord, forming the neural arch; while the ventral limb advances ventrad, foreshadowing the hemal arch or costal process of the vertebra, or, as regards the thoracic region of the body, the future rib. ${ }^{2}$ Both dorsal and ventral processes grow into the intervals between adjacent myotomes and hence are intersegmental, that is, they, as well as the vertebral bow from which they spring, correspond to the intervals between the primitive segments of the body. Subsequently these processes of the bow give rise to the various processes of the completed vertebra. The median part of the bow, the hypochordal brace, subsequently becomes cartilaginous and assists in forming the body of the vertebra in birds, but in mammals it remains unchondrified and becomes an inconspicuous and transitory part of the intervertebral ligament-the future intervertebral disk-except in the case of the first cervical vertebra, the atlas, the ventral arch of which it furnishes. The membranous anlage of the cartilaginous body of the vertebra is found in a special condensation of the mesen-

${ }^{1}$ More recently the process has heen studied in the human embryo by Bardeen, American Journal of Anatomy, vol iv., No. 2, 1904.

${ }^{2}$ Morphologically, each vertebra is possessed of a neural arch, for the protection of the spinal cord; and a hemal arch, for the protection of the organs of circulation, respiration, and digestion, the ribs of man and the higher vertebrates being the persistent hemal arches in the region of the thorax. 
chymatous sheath of the chorda on the caudal side of the hypochordal brace. The intervertebral ligament is developed from the perichordal tissue on the dorsal side of the hypochordal brace. Bardeen's primitive disk includes this anlage of the intervertebral ligament plus the hypochordal brace of Froriep, which latter Bardeen regards as a transitory thickening of the ventral edge of the disk.

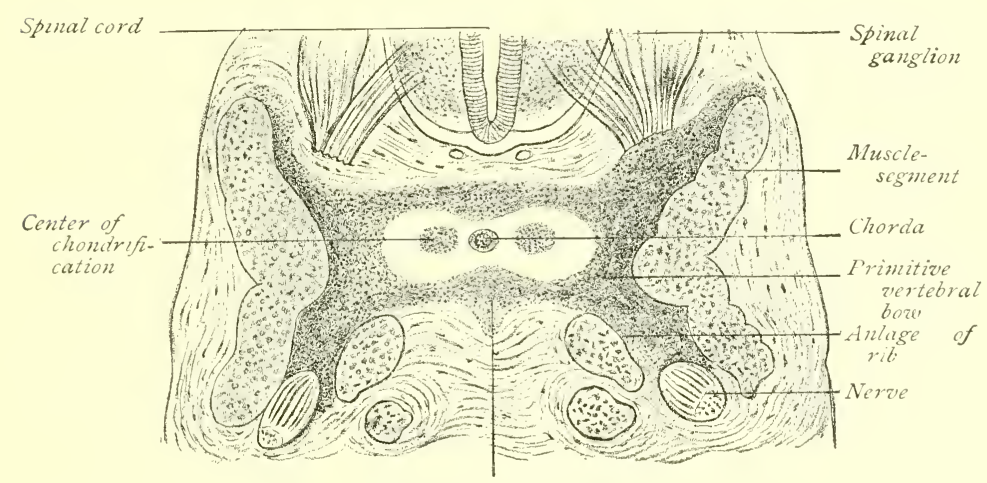

Hypochordal brace

FIG. 176.-Cross-section through the anlage of the third cervical vertebra of a cow embryo of $12 \mathrm{~mm} .(1 / 2$ in.) (Bonnet).

The Cartilaginous Stage.-This stage of the development of the spine is brought about by the metamorphosis of parts of the membranous rerteliral column into the cartilaginous vertebræ. Other and alternating parts of the same structure furnish the intervertebral disks and the ligaments that bind together the individual elements of the spine. 'The histological changes necessary to effect the transformation of the embryonal connective tissue into cartilage are, briefly, the moving apart of the cells and the modification of both the cells and the intercellular substance, the latter acquiring the characteristic qualities of the matrix of cartilage.

For each vertebral body there are two centers of chondrification, one on each side of the chorda within the mass of tissue referred to above (Fig. 176). The formation of cartilage begins in the second month. The two centers are soon connected with each other by a third, which lies on the ventral side of the chorda, the three forming now a cartilaginous half- 
cylinder which is later completed by the development of cartilage on the dorsal side of the chorda (Fig. 177). According to Bardeen, the cartilage of the body grows at the expense of the primitive disk anterior to (above) it. At the time when the chorda is completely encased in cartilage the spinal cord is still ensheathed by merely membranous tissue. Before the end of the second month the neural arches of the vertebræ are indicated by small isolated masses of cartilage which develop in the connective tissue surrounding the spinal cord, the lateral parts of the membranous vertebral bows.

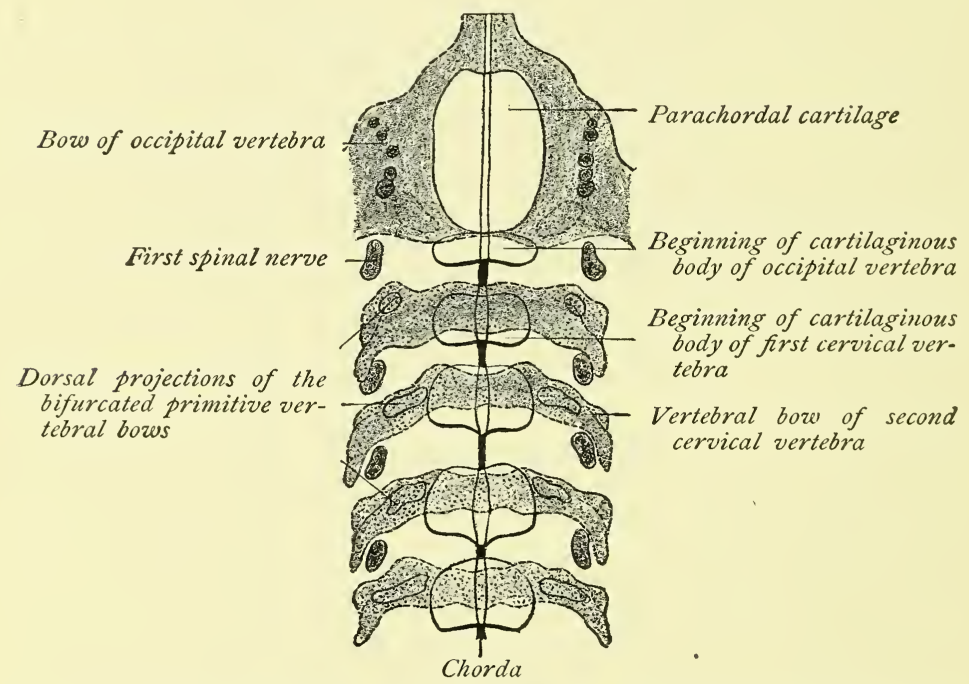

FIG. 177.-Frontal projection from a series of sections through a cow embryo of $17 \mathrm{~mm}$. (0.67 in.), dorsal view (from Bonnet, after Froriep): Connective tissue stippled; cartilage white.

In the eighth week these fuse with the bodies and appear then as projections from them. By the end of the third month the processes, or neural arches, have grown sufficiently to meet with their fellows on the dorsal side of the spinal cord, and in the fourth month the corresponding arches of the two sides become united, thus completing the cartilaginous sheath of the cord.

The masses of connective tissue occupying the intervals 
between the vertebral bodies, originating, as stated above, in condensations of the mesenchymal sheath of the chorda on the dorsal aspect of the hypochordal braces, become the intervertebral ligaments (Bardeen's primitive disks) upon their fusion, in mammals, with the hypochordal braces. Subsequently they become the intervertebral disks. The tissue between the cartilaginous arches becomes differentiated into the ligamenta subflava.

While the unsegmented skeletogenous sheath of the chorda is gradually differentiating into the separate elements of the cartilaginous vertebral column, the chorda itself begins to retrograde. Within the bodies of the vertebræ its development is completely arrested, while those portions of it contained within the intervertebral disks continue to grow. The chorda at this stage consequently shows alternating enlargements and constrictions. In certain fishes it persists as a structure of more or less importance. In vertebrates above cartilaginous fishes, all traces of the parts of the chorda within the vertebral bodies are lost as soon as ossification occurs, while in the intervertebral disks parts of it persist as the soft pulpy cores of the latter.

Thus the cartilaginous vertebral bodies or centra originate in masses of mesenchyme situated between the primitive vertebral bows and are, according to Froriep, segmental, that is, they correspond in position with the muscle-segments, each centrum being developed within the limits of a single segment; while the processes develop from the lateral parts of the vertebral bow and later unite with the body. Bardeen, on the other hand, refers the origin of each vertebral body to two segments, since, according to his observations, the body grows at the expense of the next anterior primitive disk.

The cartilaginous trunk is completed by the chondrification of the ligamenta intermuscularia to form the cartilaginous thorax.

The Osseous Stage.-The process of ossification begins in certain parts of the trunk at the end of the second month, 
before the work of chondrification is entirely completed. As the histological details of bone-formation are to be found in the text-books of histology, it will not be necessary to enter into the subject here. The places in any individual cartilage where ossification begins are called the centers of ossification. The process is one of substitution, the cartilage becoming broken down and absorbed as the formation of bone goes on.

The ossification of each vertebra is begun at three centers, one in the body and one in each arch. The centers for the arches appear in the seventh week. The centers for the bodies appear a little later and are found first in the dorsal vertebræ, appearing successively later in the vertebræ farther up and farther down. The ossified arches unite with each other during the first year of life, but their union with the body of the vertebra takes place between the third and eighth years. At a much later period five accessory centers of ossification are added to each vertebra. Two of these belong to the body and give rise to two annular plates of bone, the epiphyses, one for the upper or cephalic surface and one for the opposite or caudal surface. The remaining three centers belong respectively to the spinous process and the two transverse processes. The epiphyses do not acquire osseous union with the vertebra proper until about the twenty-fifth year.

The so-called transverse process of a cervical vertebra, enclosing a foramen, and consisting of an anterior and a posterior part, includes more than the transverse process proper, since its anterior or ventral portion is the rudiment of a cervical rib. During the time of the fusion of this rudimentary rib with the transverse process, the vertebral artery, which passes between them, is surrounded by the two processes, and thus the adult cervical transverse processes differ from those of the other vertebræ in the possession of a foramen. ${ }^{1}$

The atlas and the axis, being strikingly modified cervical

1 The point is made by some authorities, as Minot, that the bone does not grow around the artery, but that the artery grows through the ossifying tissue. 
vertebræ, require special mention. The atlas contains less and the axis more than an ordinary vertebra, since that which corresponds to the body of the atlas never unites with it but fuses with the body of the axis to constitute its odontoid process.

The atlas presents two centers of ossification for its neural arches-the so-called posterior arch-just as other vertebræ do. Unlike other vertebræ, these centers do not unite with the body but become.joined to each other on the ventral side of the position of the chorda by a piece of cartilage which results from the chondrification of the hypochordal brace, referred to on page 376 . This forms the cartilaginous ventral or anterior arch of the atlas, which, in the first year of life, develops a center of ossification. The arch acquires bony union with the lateral parts between the fifth and sixth years.

The axis or epistropheus develops from the usual centers of ossification and from an additional one for its odontoid process. Bony union of the odontoid process with the proper body of the axis occurs in the seventh year. The odontoid process, in common with every other vertebral body, is traversed in the cartilaginous stage by the notochord.

The transverse processes of the lumbar vertebræ, like those in the cervical region, include not only the transverse process proper but also the rudiment of a rib.

The sacral vertebræ each present the usual ossific centers. Inasmuch as they become articulated firmly with the pelvic bones and undergo fusion to form a single adult bone, the sacrum, their form is much modified during the course of development. 'The transverse processes of each side coalesce to form the lateral mass of the sacrum. Each transverse process consists, as in the cervical and the lumbar vertebræ, of the transverse process proper and a rudimentary rib, the center of ossification for the latter being quite distinct during early stages of development. The intervertebral disks of the sacral vertebræ begin to ossify in the eighteenth year, the process being completed in the twenty-fifth year. 
The coccygeal vertebræ are quite rudimentary. Each one is ossified from a single piece of cartilage, and usually from but a single center of ossification. Occasionally the first piece of the coceyx develops from two ossific centers, the process beginning at birth. Ossification begins in the second vertebra between the fifth and the tenth years; in the third, shortly before puberty; in the fourth, soon after puberty. The lower three pieces fuse into one before middle life, and this unites with the first, and the latter with the sacrum, at variable periods thereafter.

The Development of the Ribs and Sternum.Reference has been made in the preceding pages to the ligamenta intermuscularia as strips or bands of embryonal connective tissue lying between adjacent muscle segments, which have originated, in common with the sheath of the chorda, from the cells of the sclerotomes. The ligamenta intermuscularia become invaded by the costal processes of the primitive vertebral bows, the costal process, which is the ventral division of the tip of the bow, growing ventrad and penetrating the substance of the ligament to constitute a curved rod of connective tissue, the forerunner of the future rib. Thus there are formed connective-tissue represcutatives of the ribs, each of which is embedded in the looser connective tissue of the corresponding intermuscular ligament. It is by the development of cartilage within these curved rods of condensed mescnchyme, the membranous ribs, that the cartilaginous ribs are produced. The process of chondrification commences in the sccond month, but does not involve the proximal ends of the ribs, the tissue here becoming ligamentous and serving to bind together the ribs and the vertebre. Ribs are formed throughout the entire extent of the vertebral column, except in the coccygeal region, but while in the lower vertebrates the entire scries goes on to mature development, in mammals, including man, their growth is arrested in the cervical, lumbar, and sacral regions. In the case of man and mammals only the thoracic ribs persist and become adult structures. 
As the distal (ventral) extremities of the ribs advance toward the ventral median line, the tips of the first five, six, or seven each exhibit an enlargement. These broadened ends soon coalesce, thus forming on either side of the median line a continuous strip of cartilage, the anlages of the sternum. The other ribs remain free at their ends. The sternum is therefore produced from two lateral halves, a circumstance that explains some of its anomalies, as for example, cleft sternum, which is a condition due to arrested development or deficiency of union.

The ossification of the ribs begins in the second month of fetal life and from a single center for each. The process does not involve the entire rib, a portion near the distal extremity remaining cartilaginous and becoming the adult costal cartilage. Accessory centers of ossification for the head and tubercle appear between the eighth and fourteenth years of life.

The ossification of the sternum proceeds from numerous centers. There is one for the manubrium and from six to twelve for the gladiolus. The ensiform acquires a center of ossification in the early years of life, but for the most part remains cartilaginous.

Although, as stated above, the ribs of adult human anatomy are limited to the thoracic region, their rudimentary representatives are found throughout the other regions of the vertebral column. In the cervical, lumbar, and sacral regions each rudimentary rib becomes blended with the transverse process of the corresponding vertebra to form the transverse process of human anatomy. It is from the persistence of the seventh rudimentary cervical rib and its failure to fuse with the corresponding transverse process that the anomaly of a free cervical rib results; while the presence of a thirteenth or lumbar rib, as occasionally met with, is due to the unusual development of the first lumbar rudimentary rib. 


\section{The Development of the Head Skeleton.}

Just as the skeleton of the trunk consists of a dorsally situated bony case for the protection of the spinal cord and a series of ventral or hemal arches for the protection of the organs of circulation and respiration; so does the head skeleton comprise a bony case for the accommodation of the brain with smaller accessory osseous compartments for the organs of special sense, as the orbits and the nasal chambers; and also a ventrally situated apparatus which constitutes both a receptacle for the oral and the pharyngeal parts of the digestive system and a mechanism for the mastication of

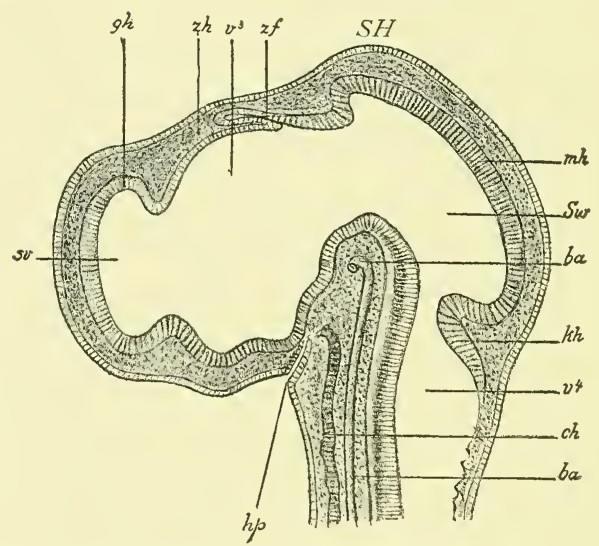

FIG. 178.-Median sagittal section through the head of a chick incubated four and a half days (after Mihalkovies): $S H$, parietal (mid-brain) elevation; $s v$, lateral ventricle of the brain; $v^{3}$, third ventricle; $v^{4}$, fourth ventricle; Sw, aqueductus Sylvii; $g h$, cerebral vesicle; $z h$, between-brain (thalamencephalon); $m h$, midbrain; $h \cdot h$, cerebellum; $z f$, pineal process; $h p$, hypophyseal (or Rathke's) pocket; $c h$, chorda; $b a$, basilar artery.

food. The former part, the cranial capsule, or brain-case, is developed to a great extent from the connective tissue surrounding the head-end of the chorda, its origin thus being similar to that of the spinal column. On the other hand, the ventral parts, as the jaws and the hyoid bone and related structures, constituting the so-called visceral skeleton, develop from the mesodermic tissue of the visceral arches. As in the case of the trunk skeleton, the cranium is first outlined in membranous tissue resulting from the differentiation of the 
embryonal connective tissue which ensheaths the head-end of the chorda, and also of the connective tissue of the visceral arches, this differentiation producing the membranous primor. dial cranium. The metamorphosis of the membranous cranium into cartilage brings about the cartilaginous stage of the cranium, while the replacement of the cartilage by bone is the final step in the process.

Bones that develop from centers of ossification in previously formed masses of cartilage are styled primordial bones, while those that are produced independently of cartilage, either in the skin covering the membranous cranium, or in the mucous membrane lining indentations in its walls, are known as covering or dermal bones. The development of bone is therefore said to be either endochondral or membranous. For the most part, the bones of the base of the skull are of endochondral formation, while those of the vault are developed in membrane. The membranous or dermal bones are similar in point of origin to the exoskeletonplacoid and ganoid scales-of certain fishes.

The Membranous Cranium.-The membranous braincase is differentiated from the mesenchymal tissue which ensheaths the anterior or head-end of the chorda. As previously stated, the anterior end of the chorda is at a point ventrad to the mid-brain vesicle, in the angle formed by the latter with the fore-brain, at a position corresponding with that of the pituitary body (Fig. 178). The skeletogenous sheath of the chorda, in this situation as elsewhere, results from the multiplication of the cells of the sclerotomes, since this region of the body undergoes segmentation in common with the trunk. The number of head-segments is uncertain. According to recent investigations upon shark embryos, there are at least nine primitive segments formed in the headregion.

The skeletogenous sheath of the chorda spreads out dorsad to cover the brain-vesicles. From the terminal point of the chorda, beneath the inter-brain, the sheath advances anteriorly to invest the fore-brain, which latter at this stage is 
bent over ventrad. From the part investing the fore-brain, a protuberant mass, the nasofrontal process, extends toward the primitive mouth-cavity, constituting the anterior or upper boundary of the latter. Meanwhile the mesenchymatic tissue of the visceral arches-that is, that part of the mesodermic tissue of these structures which does not form muscular tissue-is undergoing similar transformation into membranous tissue. The first visceral arch divides into an anterior or upper part, the maxillary process, and a posterior or lower mass, the mandibular arch, these being the membranous jaw arches. The four jaw arches, with the nasofrontal process, form the boundaries of the primitive mouthcavity, the mandibular arches of the two sides having united in the median line to form its lower border, and the maxillary arches having fused with the lateral nasal and the nasofrontal processes to constitute its upper boundary.

The membranous primordial cranium, then, consists of a complete connective-tissue investment for the brain-vesicles, of the membranous jaw arches, and of the hyoid and the branchial arches, and presents in its walls the indications of the cavities for special-sense organs in the shape of the surface invaginations which constitute respectively the otic resicle, the lens-vesicle, and the nasal pits.

The Cartilaginous Cranium.-By the further differentiation of the membranous cranium the cartilaginous stage is attained. The development of cartilage begins in the second month. While the membranous cranium furnishes a complete capsule for the brain, the cartilaginous brain-case is deficient, since the process of chondrification does not affect the regions of the future parietal and frontal bones. This is true at least of man and the higher vertebrates. In those cases where the skeleton remains permanently cartilaginous, as in selachians (sharks, $\log$-fish, etc.), the entire brain-case participates in the chondrifying process. As the skull extends very much farther forward than the end of the chorda - which latter terminates at the position of the future sella turcica-the regions of the primitive skull are designated 
respectively chordal and prechordal (Kölliker), or vertebral and evertebral (Gegenbauer), according as they fall behind or in front of the end of the chorda.

The formation of cartilage begins in the region corresponding to the base of the future skull. On each side of the end of the chorda a mass or bar of cartilage is formed, extending forward and backward, this pair of parallel bars being designated the parachordal cartilages (Fig. 179,1). Farther forward,
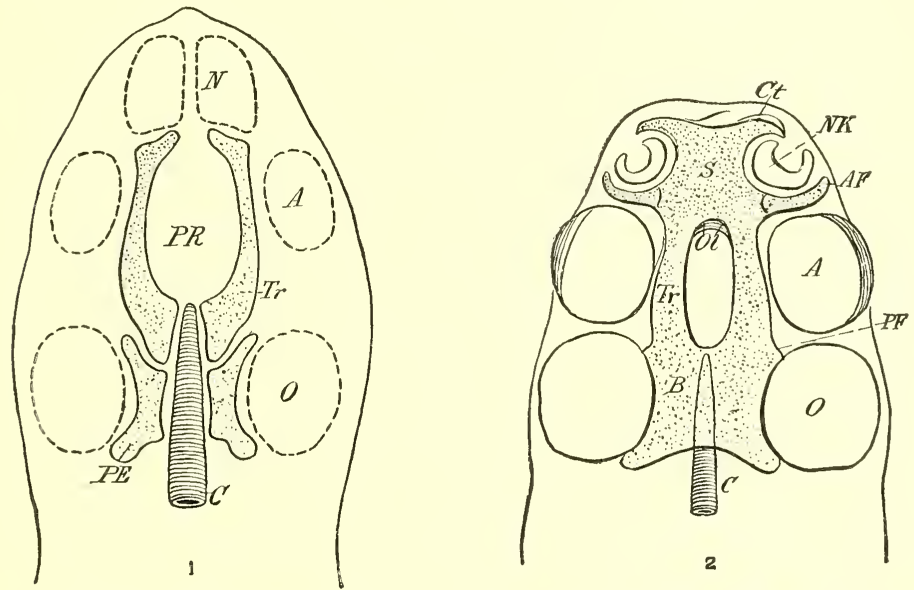

FIG. 179.-First fundament of the cartilaginous primordial cranium (from Wiedersheim): 1. First Stage: $C$, chorda; $P E$, parachordal cartilage; $T r$, Rathke's trabeculæ cranii; $P R$, passage for the hypophysis ; $N, A, O$, nasal pit, optic vesicle, otocyst. 2. Second Stage: $C$, chorda; $B$, basilar plate; $T r$, trabeculæ cranii, which have become united in front to constitute the nasal septum $(S)$ and the ethmoid plate; $C t, A F$, processes of the ethmoid plate enclosing the nasal organ ; $O l$, foramina olfactoria for the passage of the olfactory nerves; $P F$, postorbital process; $N K$, nasal pit; $A, O$, optic and labyrinthine vesicles.

in the prechordal region, another pair of cartilaginous masses is produced, known as the trabeculæ cranii. The latter are not straight bars, but have somewhat the form of a pair of calipers. In a short time the cranial trabeculre unite with each other, but not throughout their entire extent, an aperture being left at the position of the pituitary body. It is through this aperture that the oropharyngeal diverticulum, which forms the anterior lobe of the pituitary body, projects to come into relation with the diverticulum from the inter-brain, which produces the posterior lobe. At a later period ossifi- 
cation occurs here, as elsewhere in the base of the skull, thus completely isolating the pituitary body from the wall of the pharynx. The parachordal cartilages also fuse with each other and with the cranial trabeculæ, the four pieces now forming one mass. The process of chondrification extends to other parts of the membranous cranium so as to produce a cartilaginous brain-case, just as, in the case of the vertebral column, the dorsal extension of cartilage-formation gives rise to a case or canal for the spinal cord. As before stated, however, the chondrifying process does not affect the entire membranous cranium in the higher vertebrates, chondrification occurring around the position of the foramen magnum and in the lateral walls of the cranial capsule, while parts of the vault remain membranous. The anterior extremities of the united cranial trabeculæ become so modified in form as to constitute the plate of the ethmoid and the nasal capsule for the lodgement of the olfactory epithelium. In each lateral region the cartilaginous ear capsule is differentiated.

Meanwhile the cartilaginous visceral skeleton is developing from the membranous structures of the visceral arches. As in the case of the brain-capsule, the chondrifying process does not involve all parts of the membranous risceral skeleton, parts of the latter being replaced later by dermal or covering bones-that is, bones that develop in membrane without having been previously mapped out in cartilage.

In the first visceral arch, the formation of cartilage occurs on!y in the mandibular portion, the maxillary process continuing membranous. The cartilage of the mandibular areh appears in the form of a curved bar running ventrodorsally. This bar divides into a smaller proximal or dorsal piece, the palatoquadratum of comparative anatomy, and a longer distal or ventral segment, Meckel's cartilage. The palato-quadratum subsequently divides into two parts, the cartilaginous anlages respectively of the palato-pterygoid plate and the incus. Meckel's cartilage likewise undergoes division, there being separated from the chief mass a small proximal segment called the articulare, which is the forerunner of the future malleus. Thus the cartilaginous bar of the mandibular arch 
has to do with the formation of certain of the ossicles of the middle ear as well as, to a limited extent, with the development of the mandible.

In the second visceral or anterior hyoid arch, chondrification also occurs, but not throughout its entire extent. A bar of cartilage, the hyoid bar or Reichert's cartilage, is produced in this arch and undergoes division into three segments, of which the proximal or dorsal is the forerunner of the future stapes of the middle ear, while the other two pieces represent respectively the styloid process and the lesser horn of the hyoid bone. The tissue intervening between the position of the styloid process and the lesser hyoid cornu does not chondrify in man but remains membranous and becomes the stylohyoid ligament (see Fig. 185).

In the third visceral arch, or the posterior hyoid arch, a rod of cartilage develops which represents the greater cornu of the future hyoid bone. Ventral to this, there is formed a median unpaired piece of cartilage, the copula, belonging to the arches of the two sides, which later develops into the body of the os hyoides.

To summarize, the head skeleton in the cartilaginous stage of development presents an imperfect cartilaginous brain-case, capsules for the organs of smell, sight, and hearing, and a cartilaginous visceral skeleton, the several parts of which map out the lower jaw, the hyoid bone, the styloid process, and the ossicles of the middle ear.

The Osseous Stage.-The bony condition of the head skeleton is brought about in part by the development of bone from centers of ossification in the cartilages described above, and in part by the growth of covering or dermal bones in the integument covering those areas which are deficient in cartilage; in other words, by both endochondral and membranous ossification. It may be stated in general terms that the bones of the base and of the sides of the skull, including the auditory ossicles, the ethmoid, and the inferior turbinated bone, are produced by ossification in cartilage and are hence called primordial bones; and that the bones of the vault of the cranium, and for the most part of the face, result from the membranous method of 
ossification, and are therefore styled dermal or covering bones. Some of the individual bones, however, are partly of car-

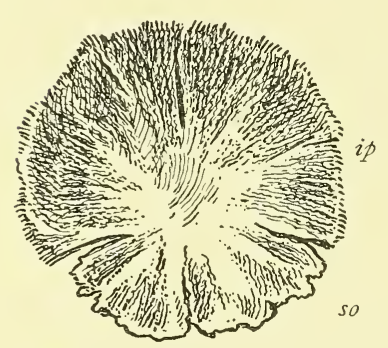

FIG. 180. - Tabular part of occipital bone of about fifth fetal month, inner surface: $i p$, interparietal, which is ossified in membrane; so, supra-occipital, ossified in cartilage. tilaginous and partly of membranous origin, the several portions remaining permanently distinct in certain lower vertebrates, but in man uniting so intimately with each other as to present no trace of their previously separate condition.

The occipital bone consists of two genetically distinct parts, the superior or interparietal portion, which is a dermal bone, and the occipital bone proper, which is of cartilaginous origin. The ossification of the latter occurs from four centers, one on each side of the foramen magnum for the condylar portions, one in front of the foramen for the basilar process, and one posterior to that aperture for all the tabular portion of the bone not belonging to the interparietal segment. Ossification begins in these centers early in the third fetal month and proceeds at such rate that at the time of birth the bone consists of four bony parts which are separated from each other merely by thin layers of cartilage. Since in some animals these parts remain separate throughout life, they are designated by morphologists, respectively, the exoccipitals, the basi-occipital, and the supra-occipital (Fig. 181). The supra-occipital is augmented by the union with it of the interparietal portion, a covering or dermal bone that ossifies from two centers, and that begins to fuse with the supra-occipital near the end of the third month of fetal life. Consisting at birth of four distinct parts, separated by cartilage, the occipital becomes a single bone by the end of the third or fourth year by the bony union of the separate segments. ${ }^{1}$

The temporal bone is made up of three genetically distinct

${ }^{1}$ In some cases the union of the interparietal with the supra-occipital is incomplete, the adult bone then presenting two transverse fissures which pass, one from each lateral angle, toward the median line. 
parts, the squamosal or squamozygomatic, the petrosal or petromastoid or periotic, and the tympanic. At the time of birth these three elements of the bone are still separate from each other, the tympanic being an incomplete ring, and the petro-
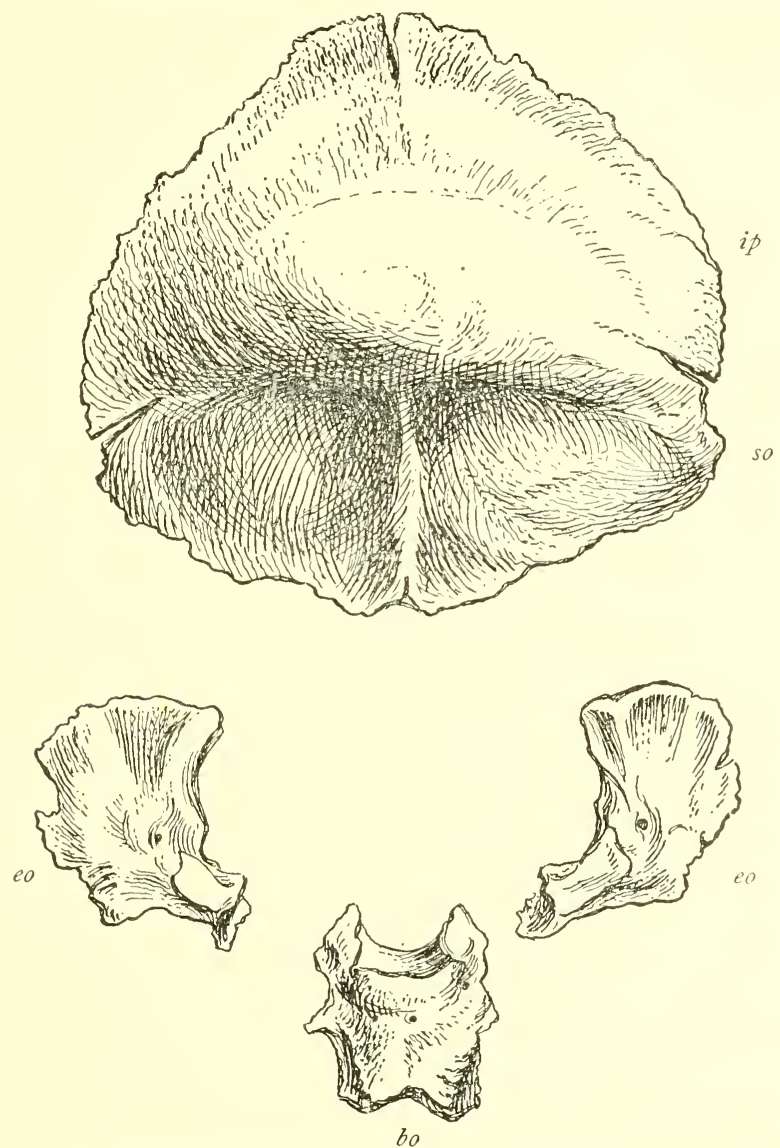

FIG. 181.-Occipital bone at birth, external surface: ip, interparietal; so, supraoccipital ; $\epsilon o$, exoccipitals ; bo, basi-occipital.

mastoid being still without a mastoid process. The petromastoid is the only part of the temporal bone that is outlined in cartilage, the squamozygomatic and the tympanic being represented in the cartilaginous stage of the cranium by membranous tissue.

The squamozygomatic (Fig. 182) is ossified in previonsly 
formed membrane from a single center of ossification, which appears in the lower part of this segment at about the seventh week. The process of bone-formation extends in all direc-
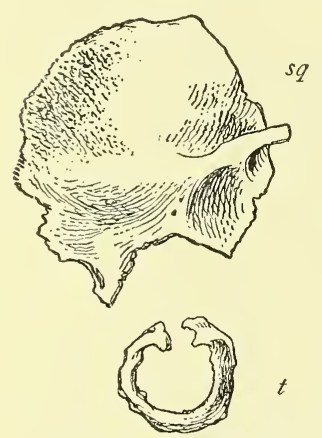

FIG. 182.- Squamozygomatic $(s q)$ and tympanic $(t)$, of temporal bone at wirth. tions from this center, but especially upward into the squamosa and outward and forward into the zygoma.

The periotic or petromastoid results from the ossification of the cartilaginous ear-capsule, which latter constitutes a part of the cartilaginous portion of the early cranium. It should be remembered that the essential part of the organ of hearing, the internal ear, is differentiated from a small pouch of epithelium, the otic vesicle, which is produced by an infolding or invagination of the surface ectoderm, and that it is the cartilaginous tissue enclosing the otic vesicle and its outgrowths, the semicircular canals and the cochlea, that constitutes the cartilaginous ear-capsule.

The ossification of the periotic is usually described as proceeding from three centers. The first of these, the opisthotic, makes its appearance in the latter part of the fifth month on the outer wall of the capsule, at a point corresponding to the position of the promontory, whence the formation of bone spreads in such manner as to produce that part of the petrosa which is below the internal auditory canal. A second center, the pro-otic, appears a little later over the superior semicircular canal and gives rise to that part of the petrosa above the internal auditory meatus, and also to the inner and upper part of the mastoidea. The third nucleus, the epiotic, arises in the neighborhood of the posterior semicircular canal. Ossification proceeds rapidly, the three parts speedily uniting to form one bone, the periotic or petromastoid. The petrous portion of the periotic is the more important and the more constant. The mastoid is of variable size in different animals, and in the human species, at birth, it is flat and devoid of the mastoid process which is so conspicuous in the mature 
condition of the skull. The mastoid process develops during the first two years of life, but its air-cells do not appear until near the age of puberty.

The pars tympanicus, or the tympanic (Fig. 182), which constitutes the bony part of the wall of the external auditory meatus, is ossified in membrane from a single center of ossification. Fhis center appears in the third fetal month in the lower part of the membranous wall of the external eanal, from which point the process of bone-formation extends upward on either side so as to form an incomplete bony ring, open above. This tympanic ring is situated external to both the ear capsule and the ossicles of the middle ear and gives attachment to the periphery of the tympanic membrane. The further growth of the tympanic ring being in the outward direction, it becomes a curved plate or imperfect cylinder of bone which constitutes the bony wall of the external auditory canal. At birth, the pars tympanicus still has the form of the incomplete ring, its further development taking place during the first few years of life. The extremities of the ring unite with the squamozygomatic before birth. The tympanic unites also with the petrosa except in a region adjacent to the proximal end of Meckel's cartilage, where an aperture is left which is the petrotympanic or Glaserian fissure. Since upon the part of Meckel's cartilage which is thus encloser by the union of the two bones is formed the long process of the malleus, the presence of this process in the Glaserian fissure is accounted for.

The styloid process of the temporal bone belongs to the visceral-arch skeleton. It ossifies in two parts in small masses of cartilage that belong to the anterior hyoid arch. One, the tympanohyal, gives rise to the base of the process (Fig. 186); it begins to ossify before birth and soon unites with the temporal. The other segment, the stylohyal, undergoes ossification later and joins with the tympanohyal only after adult age is reached. Sometimes it remains separate throughout life.

The sphenoid bone is for the most part ossified in cartilage. The body of the bone is represented in the fetus by two separate parts, the posterior body, or basisphenoid, or post- 
sphenoid (Fig. 183, bs), which includes all that part of the body of the mature bone which is posterior to the olivary eminence and to which belong the greater wings (alisphenoids); and an anterior body or presphenoid ( $p s)$, situated in front of the olivary eminence, to which belong the lesser wings (orbitosphenoids). The ossification of the basisphenoid proceeds from two centers placed side by side, which
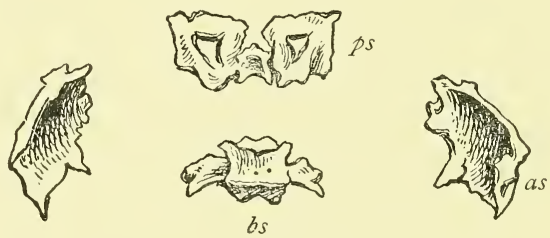

FIG. 183.-Sphenoid bone, fifth or sixth fetal month; seen from above: $p s$, presphenoid or anterior body, with lesser wings; as, greater wings; $b s$, basisphenoid or posterior body.

appear in the eighth week. Two months later two secondary centers appear for the lateral parts of the body. The presphenoid likewise develops from two centers, which are apparent in the ninth week. The union of the presphenoid with the basisphenoid occurs in the seventh or eighth month. Each greater wing develops from a single center of ossification, which is present in the eighth week. The process of ossification spreads from this center to produce not only the greater wing but also the external pterygoid plate. The greater wings remain separate from the body until some time during the first year after birth. Each lesser wing ossifies from a center that appears about the ninth week. The lesser wings unite with the presphenoid in the sixth fetal month.

The internal pterygoid plate differs from the other parts of the sphenoid in that it does not ossify in cartilage but in membrane. It is stated, however, that its hamular process first becomes cartilaginous before it ossifies. It is, therefore, a covering bone. Its center or centers of ossification appear in the fourth month in the connective tissue in the lateral walls of the oropharyngeal cavity. In many animals this plate acquires no connection with the external pterygoid plate, 
but remains throughout life a distinct bone, the pterygoid. In man it fuses with the external plate in the fifth month.

The presphenoid with its attached lesser wings, and the basisphenoid, to which are united the greater wings and the pterygoid plates, remain permanently separate bones in some animals. In man, as noted above, the two parts of the borly of the bone unite shortly before birth, although the greater wings remain separate until some months after that event.

The ethmoid bone and the inferior turbinate are formed in cartilage, resulting from the ossification of the posterior portion of the cartilaginous nasal capsule (Fig. 184, $m$ ). The

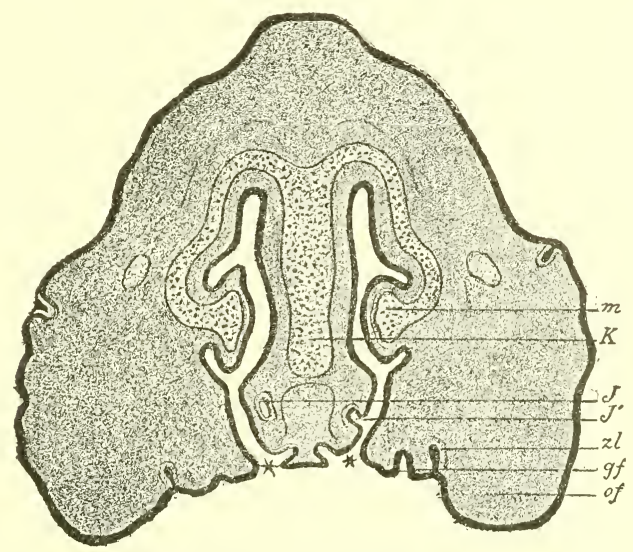

Fif. 181.-Cross-section through the head of an embryo pig $3 \mathrm{~cm}$. (1.2 in.) long, crown-rump measurement. The nasal cavities are seen to be in communication with the oral cavity at the places designated by a *: $K$, cartilage of the nasal septum ; $m$, turbinal cartilage; $J$, organ of Jacobson; $J^{\prime}$, the place where it opens into the nasal cavity; $g f$, palatal process; of, maxiliary process; $z l$, dental ridge (Hertwig).

latter represents the anterior extension of the cartilaginous trabeculæ cranii so modified as to constitute a receptacle for the olfactory epithelium. The anterior part of this capsule remains cartilaginous throughout life as the septal and lateral cartilages of the nose. By the ossification of the posterior part of the nasal capsule the ethmoid and the inferior turbinate bones are produced. Ossification, beginning in the fifth month, involves the lower and the middle turbinals and a part of the lateral masses. The ossification of the superior turbinal, of the vertical plate, of the crista galli, and of the 
remaining parts of the lateral masses is effected after birth. The bony union of the lateral masses with the median plate is completed between the fifth and seventh years.

The frontal bone is a covering or dermal bone, being ossified in membrane from two centers of ossification, one for each lateral half. These centers are situated above the orbital arches and are first apparent in the seventh week. At birth, the two halves of the bone are still separate, their union not occurring until during the first year of life. Sometimes the union fails to take place, the condition of the persistent frontal or metopic suture being known as metopism. Metopism is considerably more common in European skulls than in those of lower type.

The parietal bone is also ossified in membrane. It develops from two nuclei which soon coalesce. Their position corresponds to that of the future parietal eminence.

The bones of the face are for the most part dermal bones. Of these, the upper and the lower maxillæ and the palate bones belong to the visceral-arch skeleton. The others develop in the membranous wall of the cranial capsule.

The nasal and lacrimal bones ossify each from a single center, which appears in the eighth week.

The malar is ossified in membrane from three nuclei, the process beginning in the eighth week.

The palate bone is formed in mucous membrane from a single center which is situated at the junction of the vertical and the horizontal plates.

The vomer develops from two centers of ossification which appear at the back part of the cartilaginous nasal septum. Each center gives rise to a lamina of bone, the two laminæ gradually uniting with each other from behind forward, and embracing between them anteriorly the septal cartilage.

The vomer and the palate bone are examples of the formation of bone in mucous membrane. The centers of ossification first appear in the eighth week in each case.

The skeleton of the visceral arches includes the upper and lower maxillæ, the hyoid bone with a part of the styloid process, the ear ossicles, and the palate bones. The palate bones have been referred to above. These bones of the 
visceral-arch skeleton are partly primordial and partly membranous.

The superior maxilla comprises two parts, the superior maxilla proper and the intermaxillary bone. While these intimately unite in man, in some animals, as the dog, they are permanently distinct, the intermaxillary bone constituting the important and conspicuous premaxilla of the dog. The superior maxilla ossifies in membrane-within the membranous maxillary process of the first visceral arch-from an uncertain number of centers. It seems probable that there are five nuclei of origin, one for the palate process, one for the malar or external part of the bone, one for the portion internal to the infra-orbital foramen and a part of the nasal wall (orbitonasal center), one for the part of the bone between the frontal process and the canine tooth, and one for the premaxilla. The formation of the antrum begins in the fourth month by the development of a recess or fossa on the inner or nasal wall of the bone.

The palate process is formed by the growth, on the inner aspect of the bone, of a shelf-like projection which advances toward the median line until it meets and unites with its fellow of the opposite side (Fig. 172). The horizontal plate of the palate bone develops similarly and very shortly after, and thus is produced the hard palate, which separates the nasal chambers from the mouth. The two halves of the hard palate unite first in front, their union being completed by the twelfth week. If union is incomplete, the anomaly of cleft-palate results. The intermaxillary segment begins its development in the seventh or eighth week upon that part of the nasofrontal process which lies between the nasal apertures. In the fifth month the intermaxillaries fuse with the maxillæ, the line of union being indicated by a suture which is apparent upon the oral surface of the palate processes. The intermaxillaries contain the germs of the four incisor teeth. As previously mentioned, deficiency of union between the maxilla and the intermaxillary results in the deformity of hare-lip. Obviously, the hiatus in hare-lip will be found to be not median, but lateral, corresponding to the position of the line of normal union. 
The lower jaw or mandible is intimately associated in its development with that of the malleus and incus of the middle ear. Inasmuch as these three bones are differentiated from the cartilaginous and membranous visceral skeleton of the first visceral arch it is desirable to consider their development together.

As described above, the membranous jaw-arches form the lateral and lower boundaries of the mouth-cavity, the first visceral arch dividing into the maxillary process and the mandibular arch. There appears in the mandibular arch a bar of cartilage which abuts by its proximal extremity upon the outer wall of the auditory labyrinth. This cartilaginous

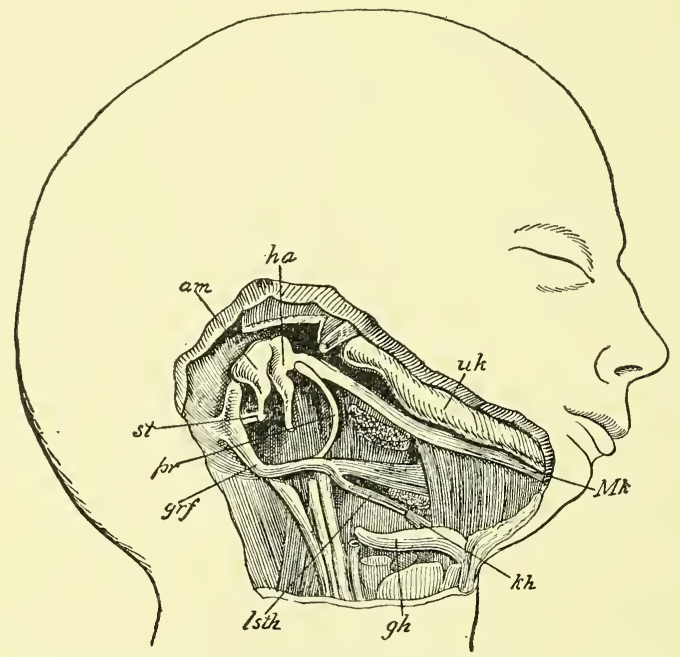

FIg 185. - Head and neck of a human embryo eighteen weeks old with the visceral skeleton exposed (after Kölliker), magnified. The lower jaw somewhat depressed in order to show Meckel's cartilage, which extends to the malleus. The tympanic membrane is removed and the annulus tympanicus is visible: $h a$, malleus, which passes uninterruptedly into Meckel's cartilage, $M k ; u k$, bony lower jaw (dentale), with its condyloid process articulating with the temporal bone; $a m$, incus; st, 'stapes; pr, annulus tympanicus; grf, processus styloideus; lsth, ligamentum stylohyoideum; $k h$, lesser cornu of the hyoid bone; $g h$, its greater cornu.

rod segments into a distal portion, Meckel's cartilage (Fig. $185, M k)$, and a smaller proximal piece, which is called, in comparative anatomy, the palatoquadratum. From the palatoquadratum a process, the palatopterygoid process, 
grows toward the roof of the mouth-cavity and becomes a separate segment. The piece of cartilage remaining, which represents the proximal end of the original bar, undergoes ossification, becoming the incus (Fig. 185, am). The posterior or proximal extremity of Meckel's cartilage, becoming a partly separated cartilage, the articulare, ossifies to produce the malleus (Fig. 185, ha). Though the form of the malleus is recognizable, it is still in direct continuity with Meckel's cartilage. In the opposite direction it is articulated with the incus. As the tympanic ring develops, and the interval below, between this ring and the petrosa, is gradually narrowed to the petrotympanic or Glaserian fissure, the malleus comes to lie within the tympanic cavity, being continuous, through the fissure, with Meckel's cartilage. Upon the separation of the malleus from the cartilage of Meckel, the long process of the malleus represents the former bond of union and therefore occupies, in the mature state, the Glaserian fissure. The joint between the malleus and the incus represents the primitive vertebrate jaw articulation. In the shark, for example, the mandibular joint is between the two pieces into which the cartilaginous bar of the first visceral arch divides-that is, between the palatoquadratum and the representative of Meckel's cartilage, the mandibulare. In mammals, however, the malleus, as we have seen, loses its connection with the mandible, the joint between the latter and the skull, the temporomaxillary articulation, being secondarily acquired in a manner to be pointed out hereafter. While the malleus develops for the most part by ossification in cartilage, its long process develops in membrane as a small covering or dermal bone, the angulare.

The membranous lower jaw with its enclosed bar of eartilage becomes osseous, not by the ossification of the cartilage, but by the development of a casing of bone within the surrounding membrane. In other words, the lower jaw develops chiefly by the intramembranous method of boneformation. Several centers of ossification appear, and from these the process of bone production extends rapidly, forming, by the fourth month, a covering or dermal bone, the 
dentale (Fig. 185, uk), which is situated mainly on the outer side of Meckel's cartilage. A smaller plate appears on the inner side. Thus the cartilage comes to be surrounded by an irregular cylinder of bone. The cartilage of Meckel plays a comparatively unimportant part in the ossification of the lower jaw-bone and begins to degenerate in the sixth fetal month. Its distal extremity, however, undergoes ossification, thus aiding in the formation of a small part of the mandible near the symphysis; while a posterior segment, with the fibrous tissue encasing it, which extends from the temporal bone to the inferior dental foramen, persists as the internal lateral ligament of the lower jaw. With these exceptions, Meckel's cartilage entirely disappears. The angle of the mandible and a small part of the ramus are also ossified in cartilage, which latter is developed independently of Meckel's cartilage. From the posterior part of the dentale the condyloid process develops and becomes articulated with the glenoid fossa of the temporal bone, thus establishing the temporomaxillary articulation. This joint, as previously stated, is a secondary one and replaces in mammals the primitive articulation between the mandibulare and the palatoquadratum of the lower vertebrates.

At birth, the two lateral halves of the inferior maxilla are united at the symphysis by fibrous tissue; bony union occurs during the first or second year after birth.

To summarize, the inferior maxilla develops as a part of the visceral-arch skeleton and is chiefly a covering bone, since, with the exception of the angle, a portion of the ramus, and a small part near the symphysis, which are of cartilaginous origin, it is formed by the membranous method of ossification. The two other products of the mandibular arch, the malleus and the incus, are ossified from cartilage, with the exception of the processus gracilis of the malleus, which is of membranous origin.

The development of the hyoid bone, of the styloid process of the temporal bone, and of the stapes was referred to in considering the cartilaginous visceral-arch skeleton, but for the sake of clearness and completeness it may not be amiss to 
repeat, in this connection, some points previously mentionerl.

The membranous anterior hyoid or second visceral arch, at a certain stage of development, presents, in its interior, the dorsoventral cartilaginous bar known as Reichert's cartilage. This is parallel with Meckel's cartilage, and, like it, is in contact by its dorsal or cranial end with the outer wall of the auditory labyrinth. A shorter bar of cartilage appears in the third visceral arch, which latter is known also as the posterior hyoid arch. Together, these two cartilaginous elements furnish the stapes of the middle ear and the hyoidean apparatus, the latter consisting of the hyoid bone, the stylohyoid ligaments, and the styloid processes. In man the

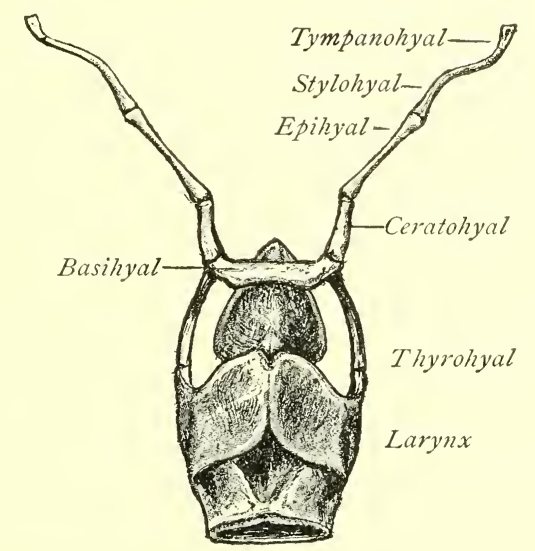

FIG. 186.-Hyoidean apparatus and larynx of dog.

hyoidean apparatus is somewhat rudimentary, but in the dog and many other mammals it is present in its typical form (Fig. 186). In such animals the stylohyoid ligament of human anatomy is represented by a bone, the epihyal, the hyoid bone being, therefore, connected with the skull by a series of small bones articulated with each other. All the elements of the hyoidean apparatus, save the body and the greater cornua of the hyoid bone, are produced by Reichert's cartilage; the hyoid body, known in comparative anatomy as the basihyal, and the greater cornua, or the thyrohyals, ossify 
from the cartilage of the third arch, the cartilage for the body being a median unpaired segment known as the copula.

Reichert's cartilage undergoes division into five segments. The segment at the cranial end, upon ossification, becomes the stapes. ${ }^{1} \quad$ This ossicle, by the closing of the walls of the tympanic cavity, is isolated from the other segments. The second piece, the tympanohyal, ossifies to form the base of the styloid process and ankyloses firmly with the temporal bone at the point of junction of the periotic portion of that bone with its tympanic plate. The third portion, the stylohyal, forms the lower part of the styloid process. It undergoes ossification later than the tympanohyal and does not acquire osseous union with it until the time of adult age. It sometimes remains separate throughout life. The fourth segment, the epihyal, does not even become cartilaginous in man, but remains fibrous, constituting the stylohyoid ligament. In most mammals it ossifies, to form a distinct bone, the epihyal. The ventral extremity of the cartilage of Reichert, the ceratohyal, produces the lesser cornu of the hyoid bone.

\section{THE DEVELOPMENT OF THE APPENDICULAR SKELETON.}

The upper and lower limbs articulate with the trunk through the medium respectively of the pectoral and pelvic girdles, the former being constituted by the scapula and the clavicle, and the latter by the ossa innominata. As in the case of the axial skeleton, the bones of the limbs in their development pass successively through a membranous and a cartilaginous stage.

The general development of the upper and lower extremities is described in a later section. As stated in that account, each limb-bud is to be regarded as an outgrowth from, or as corresponding in position to, several primitive segments, the tissue composing the little bud-like process subsequently differentiating into the muscular, cartilaginous, and connectivetissue elements of the member. The origin of each limb from more than one primitive segment has been established

${ }^{1}$ See foot-note, page 115). 
chiefly by embryological investigations upon the lower vertebrates, and is borne out by the fact that each extremity receives its nerve-supply from a series of spinal nerves instead of from the nerve-trunk of any one segment.

\section{The Development of the Pectoral and the Pelvic} Girdles.-The pectoral or shoulder girdle consists in its earliest stage of a pair of curved bars of cartilage, each of which is made up of a dorsal limb occupying approximately the position of the future spine of the scapula and approaching but not touching the spinal column, and a ventral segment lying near the ventral surface of the trunk. At the angle of union of the dorsal and ventral parts is a shallow depression, an articular surface, which represents the point of articulation with the future humerus.

The scapula is developed, except its coracoid process, from the dorsal part of the primitive shoulder-girdle. This soon acquires a form resembling that of the adult scapula with the infraspinous portion of the bone very much shortened. Ossification begins at the neck of the scapula about the eighth week, and in the third month extends into the spine. The ventral part of the cartilaginous shoulder-girdle extends almost to the median line of the chest-wall. It divides into two diverging bars, the lower one of which undergoes ossification in birds and in some other vertebrates to form the conspicuous coracoid bone. In mammals, however, it aborts and gives rise to a smaller element, the coracoid process of the scapula. At birth the human scapula is but partially ossified, the coracoid process, the acromion, the edges of the spine, the base, the inferior angle and margins of the glenoid cavity being cartilaginous. The coracoid process ossifies from a single center and acquires osseous union with the body of the bone at about the age of puberty. The acromion ossifies from two or three nuclei and joins the spine between the twenty-second and twentyfifth years. 'Still other centers of ossification appear from time to time. Thus there is an accessory center for the base of the coracoid and the adjacent part of the glenoid cavity, 
and one at the inferior angle of the bone, from which latter ossification extends along the vertebral border.

The clavicle does not develop from the prinitive shouldergirdle, but is formed in membrane, for the most part, as a dermal bone. Its ossification begins in the sixth or seventh week, before that of any other bone in the body. Subsequently, cartilaginous epiphyses are added, one at each end. It is by means of the epiphyses that the bone grows in length.

The cartilaginous pelvic girdle consists of a pair of cartilages, which are united with each other by their ventral extremities, and each of which, by its dorsal end, is articulated with the sacral region of the cartilaginous spinal column. At about the middle of each cartilage, on its outer surface, is a depression representing the future acetabular fossa. Anterior to the depression is a large aperture, the thyroid foramen, the upper and lower boundaries of which are respectively the pubic and ischiatic rods or bars, which make up the ventral portion of the cartilage, while posterior to the fossa is the iliac segment, which has a somewhat irregular plate-like form. Ossification begins in the third month, proceeding from three centers, one for each of the three divisions of the innominate bone. At the time of birth a large proportion of the original cartilage is still present, the os pubis, the ischium, and the ilium being separated from each other up to the age of puberty by strips of cartilage. The ischium and the pubes unite first, and later acquire osseous union with the ilium. In addition to the three primary centers of ossification, other and secondary nuclei appear at a later date in the erest of the ilium, the tuberosity of the ischium, and in the various spines and tubereles.

The skeleton of the free portions of each extremity, consisting at first of a continuous mass or rod of partially metamorphosed mesenchymal tissue, undergoes division into segments which represent the skeleton of the arm or of the thigh, of the forearm or of the leg, and of the hand or of the foot. This segmentation corresponds with that of the entire mass 
of the limb, both as to extent and order of appearance (see page 406). Nuclei of chondrification now appear, one in the center of each skeleton-piece, from which cartilage formation extends toward either end. The several cartilaginous elements thus produced present approximately the respective forms of the future bones. The larger cartilages are present in the upper extremity in a six weeks' embryo, but not until somewhat later in the lower limb. All the bones of the extremities are of endochondral origin.

The long bones develop in a fairly uniform manner. The shaft or diaphysis ossifies from a single center, while the two epiphyses each present several centers. The centers for the diaphyses appear at about the eighth week, ossification proceeding at such rate that at birth only the ends of the long bones are cartilaginous. The centers for the epiphyses appear at various times after birth. Osseous union between the diaphysis and the epiphyses does not occur until the growth in length of the bone is completed. As the details concerning the time of appearance and the number of these centers are to be found in the text-books of anatomy, they are omitted here.

Each bone of the carpus and of the tarsus ossifies from a single center, except the os calcis, which has two ossific nuclei. The bones of the carpus are entirely cartilaginous at birth, their ossification beginning in the first year with the appearance of a center in the scaphoid. The pisiform bone is the last of the series to ossify, its ossification beginning in the twelfth year.

The bones of the tarsus begin to ossify earlier than those of the carpus. The os calcis and the astragalus present osseous nuclei in the sixth or seventh fetal month, and the cuboid shortly before birth. With these exceptions the tarsal bones undergo ossification between the first and the fourth years.

The metacarpal and the metatarsal bones and the phalanges present each a center of ossification for the shaft and one epiphyseal center. In the case of the phalanges and of the metacarpal bone of the thumb and of the great toe, the epiphyseal center is at the proximal extremity, while in the 
remaining metatarsal and metacarpal bones it is at the distal end. ${ }^{1}$ The ossification of the shaft begins in the eighth or ninth week of fetal life; of the epiphyses, not until several years after birth. The development of the ungual or distal phalanges-of the hand, at least-is peculiar in that the ossification begins at the distal extremity, instead of in the middle of the shaft.

\section{THE DEVELOPMENT OF THE LIMBS.}

The limbs of vertebrates develop from little bud-like processes (Fig. 62) that spring from two lateral longitudinal ridges, situated one on each side of the body. These ridges are not exactly parallel with the median plane of the body, but converge somewhat toward that plane as they approach the caudal end of the embryo. It results from this circumstance that the posterior limbs are placed closer together than the anterior. In man, the limb-buds appear soon after the third week. Each bud contains a basis of primitive connective tissue contributed by several somites, as well as muscular structure, which is the offshoot from the muscle-plates of a less number of primitive segments.

The assumption of the origin of each limb-bud from more than one primitive segment is borne out by the nerve-supply of the fully-formed limb, each extremity being innervated by a number of spinal nerves (compare page 368). The connective tissue of the limb-bud produces the bony structures of the limb, while the outgrowths from the muscle-plates contribute their musculature. Previous reference has been made (p. 370) to the work of Bardeen and Lewis on the development of the limbs. According to their findings, the myotomes do not extend into the limb-buds, but the limbmusculature develops from the mesenchymal core of the bud. They also state that the bud for the arm is at first opposite the last four cervical and the first thoracic segments, subsequently extending to the level of the third cervical segment, and finally migrating tailward to its adult position; and that

\footnotetext{
1 Quain's Anatomy, 10th edition.
} 
the bud for the leg, at first attached at the region of the lower four lumbar and first sacral myotomes, extends to include the first lumbar and the second and third sacral segments, assuming later a more caudal position.

In the fifth week each limb-bud becomes divided, by a transverse groove, into two segments (Fig. 59, 12, 13), of which the distal part becomes the hand or foot, while the proximal portion very soon afterward divides into the forearm and arm or leg and thigh. Even as early as the thirty-second day, the digitation of the limb-buds-in the case of the upper extremities - is indicated by four longitudinal parallel lines or grooves on the distal extremity of each (Fig. 59, 14). By the conversion of these grooves into clefts, the fingers appear, in the sixth week, as separate outgrowths. The development of the upper extremities precedes that of the lower by twelve or fourteen days, so that, when the fingers are present as distinct projections, the toes are just being marked off in the manner noted above for the fingers. The toes begin to separate, by the deepening of the intervening clefts, from the fiftieth to the fifty-third day. By the end of the eighth week, the fingers are perfectly formed, with the exception of the nails. The nails have their beginning in the seventh or eighth week, in little claw-like masses of epidermal cells, which are attached to the tips of the digits instead of to the dorsal surfaces. Subsequent transformations result in bringing the nail into its normal position on the dorsal surface of the distal phalanx. The nails are well formed by the fifth month, at which time the covering of modified epidermal cells begins to disappear. The extremity of the nail, however, does not break through so as to project beyond the finger-tip until the seventh month. A more complete account of the development of the nails will be found in comnection with the origin of the skin (page 270).

The Position of the Limbs. - The paddle-like limbbuds at first project laterally almost at right angles with the axis of the trunk. At this time the future dorsal surface of each limb looks toward the back of the fetal body (dorsad), the future flexor surface toward its anterior aspect (ventrad), 
while the first digits-the future thumb and great toe-and consequently the radius and tibia, occupy the side of the member that is directed headward or cephalad, the future little finger and fifth toe with the ulna and fibula looking caudad. As the limbs enlarge and differentiate into their respective segments, they apply themselves to the ventral surface of the body, this change in position being facilitated by the occurrence of the future elbow- and knee-flexions, which cause the flexor surfaces of the forearm and leg, respectively, to approach the corresponding surfaces of the upper arm and thigh. At about the same time, the distal segments, the hand and foot, become bent in the opposite direction, producing the condition of the limbs that is permanent in the Amphibia-that is, the condition in which the dorsal surface of the proximal segment of the limb faces in the same direction as the dorsal surface of the trunk, while the middle segment is flexed and the distal is extended. To establish the permanent condition of the human limbs, there occur an outward rotation of the arms and an inward rotation of the lower extremities, on their long axes. The thumb and radius, therefore, instead of looking cephalad, are now directed dorsad-with the forearm in the supine position and the arm outstretched-or laterad, away from the median plane of the body, if the arm hangs by the side in the anatomical position. By the inward rotation of the lower limb, the great toe and the tibia come to lie toward the median plane of the body, causing the extensor surface to look ventrad, the flexor surface, dorsad. 


\section{TABULATED CHRONOLOGY OF DEVELOPMENT.}

\begin{tabular}{|c|c|c|}
\hline \multirow{3}{*}{$\begin{array}{l}\text { Maturation of ovum } \\
\text { in Graafian follicle. } \\
\text { Rupture of follicle. } \\
\text { Entrance of ovum } \\
\text { into oviduct. } \\
\text { Fertilization. } \\
\begin{array}{l}\text { General } \\
\text { Characters. }\end{array}\end{array}$} & \multicolumn{2}{|c|}{ STAGE OF THE OVUM. } \\
\hline & First WeEk. & SECOND WEEK. \\
\hline & $\begin{array}{l}\text { Segmentation of fertilized } \\
\text { ovum to form morula } \\
\text { while passing along ovi- } \\
\text { duct to uterus. } \\
\text { Cleavage-cavity present, } \\
\text { marking stage of blastula. } \\
\text { Great increase in size. } \\
\text { Cells of inner cell-mass re- } \\
\text { arranged to form ento- } \\
\text { derm and ectoderm. } \\
\text { Outer cells become thin- } \\
\text { cells of Rauber. } \\
\text { Embryonal area. } \\
\text { Primitive streak. } \\
\text { Mesoderm. } \\
\text { (Amnion completed at 4th } \\
\text { or 5th day. Peters.) } \\
\text { Trophoblast and early syn- } \\
\text { cytium (3d day). }\end{array}$ & $\begin{array}{l}\text { Ovum in uterus, embedded } \\
\text { in mucosa. } \\
\text { Amnion. } \\
\text { Chorion and its villi (Fig. } \\
\text { 49). Vascularization of } \\
\text { chorion and its villi. } \\
\text { Yolk-sac partly formed. }\end{array}$ \\
\hline $\begin{array}{l}\text { Vascular } \\
\text { System. }\end{array}$ & & $\begin{array}{l}\text { Heart indicated as two tubes } \\
\text { in splanchnic mesoderm. } \\
\text { Vascular system repre- } \\
\text { sented by vascular area } \\
\text { of yolk-sac. }\end{array}$ \\
\hline $\begin{array}{l}\text { Digestive } \\
\text { System. }\end{array}$ & & $\begin{array}{l}\text { Oral pit (12th or 14th day). } \\
\text { Gut-tract partly separated } \\
\text { from yolk-sac. }\end{array}$ \\
\hline \multicolumn{3}{|l|}{$\begin{array}{l}\text { Respiratory } \\
\text { System. }\end{array}$} \\
\hline \multicolumn{3}{|l|}{$\begin{array}{l}\text { Genito-urinary } \\
\text { System. }\end{array}$} \\
\hline \multicolumn{3}{|l|}{ Skin. } \\
\hline $\begin{array}{l}\text { Nervous } \\
\text { System. }\end{array}$ & & Medullary plate (14th day). \\
\hline $\begin{array}{l}\text { Special Sense } \\
\text { Organs. }\end{array}$ & & Nasal areas. \\
\hline \multicolumn{3}{|l|}{$\begin{array}{l}\text { Muscular } \\
\text { System. }\end{array}$} \\
\hline $\begin{array}{l}\text { Skeleton and } \\
\text { Limbs. }\end{array}$ & & \\
\hline
\end{tabular}


Tabulated Chronology of Development (Continued).

\begin{tabular}{|c|c|c|}
\hline & \multicolumn{2}{|c|}{$\begin{array}{l}\text { STAGE OF THE EMBRYo. } \\
\text { ThIRD WEeK. } \\
\text { Fourth WEeK. }\end{array}$} \\
\hline $\begin{array}{l}\text { General } \\
\text { Characters. }\end{array}$ & $\begin{array}{l}\text { Body of embryo indicated. } \\
\text { Dorsal outline concave. } \\
\text { Vitelline duct (21st day). } \\
\text { Segmentation of paraxial } \\
\text { mesoderm begins. } \\
\text { Visceral arches and clefts } \\
\text { begin to appear. } \\
\text { Nasofrontal process. } \\
\text { Allantoic stalk (Fig. } 57 \text { ). } \\
\text { Distinction between chorion } \\
\text { leve and chorion fron- } \\
\text { dosum begins. }\end{array}$ & $\begin{array}{l}\text { Marked flexion of body (21st } \\
\text { to 23d day); gradual un- } \\
\text { coiling after 23d day } \\
\text { Visceral arches and yolk-sac } \\
\text { attain greatest develop- } \\
\text { ment (28th day). } \\
\text { Somites well formed. } \\
\text { Well-marked tail ( } 25 \text { th day) } \\
\text { Lining cells of coelom begin } \\
\text { to flatten. } \\
\text { Increased growth of allan- } \\
\text { tois. } \\
\text { Cephalic flexures. }\end{array}$ \\
\hline $\begin{array}{l}\text { Vascular } \\
\text { System. }\end{array}$ & $\begin{array}{l}\text { Heart with single cavity } \\
\text { present, soon dividing into } \\
\text { atrium and ventricle. } \\
\text { Vitelline circulation begun. } \\
\text { Visceral-arch vessels begin } \\
\text { to appear. }\end{array}$ & $\begin{array}{l}\text { Division of atrium begins. } \\
\text { Completed condition of vitel- } \\
\text { line circulation. } \\
\text { Allantoic vessels developing. }\end{array}$ \\
\hline $\begin{array}{l}\text { Digestive } \\
\text { System. }\end{array}$ & $\begin{array}{l}\text { Gut-tract a straight tube con- } \\
\text { nected with yolk-sac by a } \\
\text { wide aperture. } \\
\text { Liver-evagination present. } \\
\text { Oral pit a five-sided fossa. } \\
\text { Anal plate. }\end{array}$ & $\begin{array}{l}\text { Alimentary canal presents } \\
\text { pharvnx, esophagus, stom- } \\
\text { ach, and intestine. } \\
\text { Pancreas begun. } \\
\text { Liver-diverticulum divides. } \\
\text { Bile-ducts acquire lumina. } \\
\text { Pharyngeal membrane } \\
\text { breaks down. }\end{array}$ \\
\hline $\begin{array}{l}\text { Respiratory } \\
\text { System. }\end{array}$ & $\begin{array}{l}\text { Pulmonary anlage as a longi- } \\
\text { tudinal protrusion of ven- } \\
\text { tral wall of esophagus, } \\
\text { afterward becoming a } \\
\text { stalked sac. }\end{array}$ & $\begin{array}{l}\text { Pulmonary anlage bifur- } \\
\text { cates, the two pouches } \\
\text { being connected by a ped- } \\
\text { icle, the primitive trachea, } \\
\text { with the pharynx. }\end{array}$ \\
\hline $\begin{array}{l}\text { Genito-urinary } \\
\text { System. }\end{array}$ & Wolffian bodies recognizable. & \\
\hline Skin. & $\begin{array}{l}\text { Segmentation of paraxial } \\
\text { mesoderm. }\end{array}$ & $\begin{array}{l}\text { Somites or primitive seg- } \\
\text { ments. } \\
\text { Cutis-plate. }\end{array}$ \\
\hline $\begin{array}{c}\text { Nervous } \\
\text { System. }\end{array}$ & $\begin{array}{l}\text { Neural canal: its cells show } \\
\text { differentiation into spon- } \\
\text { gioblasts and germ-cells. } \\
\text { Fourth ventricle indicated. } \\
\text { Fore-brain, mid-brain, and } \\
\text { hind-brain vesicles, soon } \\
\text { dividing into five vesicles. }\end{array}$ & $\begin{array}{l}\text { Walls of cerebral vesicles } \\
\text { thicken. } \\
\text { Ventral roots of spinal } \\
\text { nerves. } \\
\text { Anterior lobe of hypophysis } \\
\text { begins. }\end{array}$ \\
\hline $\begin{array}{l}\text { Special Sense } \\
\text { Organs. }\end{array}$ & $\begin{array}{l}\text { Auditory pit followed by otic } \\
\text { vesicle. } \\
\text { Olfactory plates. } \\
\text { Optic vesicles begin. } \\
\text { Lens-vesicles. }\end{array}$ & $\begin{array}{l}\text { Otic vesicle with recessus } \\
\text { labyrinthi. } \\
\text { Nasal pits distinct. } \\
\text { Optic vesicle stalked and } \\
\text { transformed into optic cup. }\end{array}$ \\
\hline $\begin{array}{l}\text { Muscular } \\
\text { System. }\end{array}$ & $\begin{array}{l}\text { Segmentation of paraxial } \\
\text { mesoderm. }\end{array}$ & $\begin{array}{l}\text { Somites or primitive seg- } \\
\text { ments. } \\
\text { Myotomes. }\end{array}$ \\
\hline $\begin{array}{l}\text { Skeleton and } \\
\text { Limbs. }\end{array}$ & $\begin{array}{l}\text { Segmentation of paraxial } \\
\text { mesoderm. } \\
\text { Notochord. }\end{array}$ & $\begin{array}{l}\text { Somites or primitive seg- } \\
\text { ments. } \\
\text { Skeletogenous sheath of } \\
\text { chorda. } \\
\text { Limb-budis apparent (about } \\
\text { 21st day). }\end{array}$ \\
\hline
\end{tabular}




\section{Tabulated Chronology of Development (Continued).}

\section{STAGE OF THE FETUS.}

Fifth Week.

SIXTH WEEK.

Body shows dorsal concavity in neckregion.

Globular and lateral nasal processes.

Lacrimal groove.

Third and fourth gill-clefts disappear in sinus præcervicalis.

Umbilical cord longer and more spiral. Umbilical vesicle begins to shrink.

Length of fetus $1 \mathrm{~cm}$. ( $\frac{2}{5}$ inch).

Larynx indicated.

Primitive aorta divides into aorta and pulmonary artery.

The only corpuscular elements of the blood during the first month are the primitive nucleated red blood-cells.

Intestine shows flexures, notably the $\mathrm{U}$-loop, inaugurating the distinction between large and small bowel.

Anal pit.

Right and left bronchi divide into three and two tubes respectively (5th to 7 th week).

Genital ridges appear on wall of bodycavity and soon become the indifferent genital glands.

Ducts of Müller appear.

Epidermis present as two layers of cells.

Olfactory lobe begins.

Arcuate and choroidal fissures on mesial surfaces of fore-brain vesicles.

Cells of central canal of cord ciliated.

Ridge-like thickening of roof of midbrain.

Semicircular canals indicated.

Eyes begin to move forward from side of head.

Mandibles unite (35th day).

Meckel's cartilage.

Limb-buds segment.

Digitation indicated (32d day) for hand.
Nasofrontal, lateral nasal, and maxillary processes unite.

Umbilical vesicle shrunken.

Amnion larger.

Vitelline circulation atrophic and replaced by allantoic circulation.

First indication of teeth in the form of the dental shelf.

Submaxillary gland indicated by epithelial outgrowth.

Duodenum well formed; cæcum; rectum (end of week).

Larynx indicated as dilatation of proximal end of trachea.

Arytenoid cartilages indicated (though not cartilaginous)

Thyroid and thymus bodies begun.

Genital tubercle, genital folds, and genital ridge (external genitals).

Cells of cutis-plate proliferate and gradually spread out beneath epidermis.

Membranes of brain and cord indicated. Pineal body begins.

Dorsal roots of spinal nerves.

Some tracts of spinal cord indicated, and its lumen alters (Fig. 139).

Semicircular canals.

Concha of external ear.

Outer fibrous and middle vascular tunies of eye.

Eyelids

Lower jaw begins to ossify.

Clavicle begins to ossify.

Ribs begin to chondrify.

Bodies of vertebræ are cartilaginous.

Fingers as separate outgrowths. 
Tabulated Chronology of Development (Contiuued).

\begin{tabular}{|c|c|c|}
\hline & $\begin{array}{l}\text { STAGE OF } \\
\text { SEVENTH WEEK. }\end{array}$ & $\begin{array}{l}\text { THE FETUS. } \\
\text { EIGHTH WEEK. }\end{array}$ \\
\hline $\begin{array}{l}\text { General } \\
\text { Characters. }\end{array}$ & $\begin{array}{l}\text { Fetal body and limbs well } \\
\text { defined (Fig. } 64) \text {. } \\
\text { Head less flexed. } \\
\text { No longer any trace of syn- } \\
\text { cytium on decidua vera. }\end{array}$ & $\begin{array}{l}\text { Head more elevated (Fig. } 65 \text { ). } \\
\text { Free tail begins to disappear. } \\
\text { Subcutaneous lymph-vessels } \\
\text { present. } \\
\text { Cells lining the colom are } \\
\text { true endothelium. }\end{array}$ \\
\hline $\begin{array}{l}\text { Vascular } \\
\text { System. }\end{array}$ & $\begin{array}{l}\text { Interventricular septum of } \\
\text { heart completed, the heart } \\
\text { now having four chambers. } \\
\text { Other corpuscular elements } \\
\text { added to blood during sec- } \\
\text { ond month. }\end{array}$ & \\
\hline $\begin{array}{l}\text { Digestive } \\
\text { System. }\end{array}$ & $\begin{array}{l}\text { Transverse colon and de- } \\
\text { scending colon indicated. }\end{array}$ & $\begin{array}{l}\text { Parotid gland begins. } \\
\text { True endothelium lines the } \\
\text { body-cavity. } \\
\text { Gall-bladder present }(2 \mathrm{~d} \\
\text { month). } \\
\text { Anlage of spleen recogniz- } \\
\text { able (2d month). }\end{array}$ \\
\hline $\begin{array}{l}\text { Respiratory } \\
\text { System. }\end{array}$ & $\begin{array}{l}\text { Median and lateral lobes of } \\
\text { thyroid unite. }\end{array}$ & $\begin{array}{l}\text { Larynx begins to chondrify. } \\
\text { Formation of follicles of } \\
\text { thymus. }\end{array}$ \\
\hline $\begin{array}{l}\text { Genito-urinary } \\
\text { System. }\end{array}$ & $\begin{array}{l}\text { Maximum development of } \\
\text { Wolffian body. }\end{array}$ & $\begin{array}{l}\text { Mülleriaul ducts unite with } \\
\text { each other. Genital groove. } \\
\text { Bladder present as spindle- } \\
\text { shaped dilatation of allan- } \\
\text { tois. } \\
\text { Suprarenal bodies recogniz- } \\
\text { able. }\end{array}$ \\
\hline Skin. & $\begin{array}{l}\text { Nails indicated by claw-like } \\
\text { masses of epithelium on } \\
\text { dorsal surfaces of digits. }\end{array}$ & $\begin{array}{l}\text { Corium indicated as a layer } \\
\text { of spindle-cells beneath } \\
\text { epidermis. Development } \\
\text { of mammary glands begun. }\end{array}$ \\
\hline $\begin{array}{l}\text { Nervous } \\
\text { System. }\end{array}$ & $\begin{array}{l}\text { Fore-brain vesicles increase } \\
\text { in size disproportionately. } \\
\text { Cerebellum indicated. }\end{array}$ & $\begin{array}{l}\text { Sympathetic nerves discern- } \\
\text { ible. }\end{array}$ \\
\hline $\begin{array}{l}\text { Special Sense } \\
\text { Organs. }\end{array}$ & & $\begin{array}{l}\text { External nose definitely } \\
\text { formed (Fig. 171). } \\
\text { Lens-capsule. } \\
\text { Palpebral conjunctiva sepa- } \\
\text { rates from cornea. }\end{array}$ \\
\hline $\begin{array}{l}\text { Muscular } \\
\text { System. }\end{array}$ & $\begin{array}{l}\text { Muscles begin to be recog- } \\
\text { nizable, though not having } \\
\text { as yet the characters of } \\
\text { muscular tissue. }\end{array}$ & \\
\hline $\begin{array}{l}\text { Skeleton and } \\
\text { Limbs. }\end{array}$ & $\begin{array}{l}\text { Ossific centers for vertebral } \\
\text { arches and for vertebral } \\
\text { bodies; ossific centers for } \\
\text { frontal bone and for squa- } \\
\text { mosa. } \\
\text { Membranous primordial cra- } \\
\text { nium begins to chondrify. } \\
\text { Claw-like anlages of nails. }\end{array}$ & $\begin{array}{l}\text { Ribs begin to chondrify. Cen- } \\
\text { ters of ossification of basi- } \\
\text { sphenoid, of greater wings, } \\
\text { of nasal and lacrimal } \\
\text { bones, of malar, vomer, pal- } \\
\text { ate, neck of scapula, diaph- } \\
\text { yses of long bones and of } \\
\text { metacarpal bones. Fingers } \\
\text { perfectly formed. Toes be- } \\
\text { gin to separate (53d day). }\end{array}$ \\
\hline
\end{tabular}


Tabulated Chronology of Development (Continued).

\begin{tabular}{|c|c|}
\hline \multicolumn{2}{|c|}{ STAGE OF THE FETUS. } \\
\hline Ninth WeEk. & THIRD Month. \\
\hline $\begin{array}{l}\text { Weight, } 15 \text { to } 20 \text { grams; length, } 25 \text { to } 30 \\
\text { mm. ( } 1 \text { to } 1 \frac{3}{8} \text { inches). } \\
\text { Hard palate completed. } \\
\text { Free tail has disappeared. } \\
\text { Differentiation of lymph-nodes begins } \\
\text { (O. Schultze). Cloaca divided. }\end{array}$ & $\begin{array}{l}\text { Weight (end of month), } 4 \text { ounces; length, } \\
23 \text { inches. } \\
\text { At first chorion leve and chorion fron- } \\
\text { dosum present; later, formation of } \\
\text { placenta (see second frontispiece). }\end{array}$ \\
\hline Pericardium indicated. & $\begin{array}{l}\text { Placental system of vessels. } \\
\text { Blood-vessels penctrate spleen. }\end{array}$ \\
\hline \multirow[t]{2}{*}{$\begin{array}{l}\text { Anal canal formed by division of eloaca. } \\
\text { (Anus opens at end of } 2 d \text { month, ac- } \\
\text { cording to Tourneux.) }\end{array}$} & $\begin{array}{l}\text { Mouth-cavity divided from nose (end of } \\
\text { month). Soft palate completed (11th } \\
\text { week). Papillæ of tongue. Evagina- } \\
\text { tion for tonsil. Intestine begins to re- } \\
\text { cede within abdomen (10th week). Ro- } \\
\text { tation of stomach. Vermiform appen- } \\
\text { dix as a slender tube. Omental bursa. } \\
\text { Gastric glands and giands and villi of } \\
\text { intestine fairly well formed (10th } \\
\text { week). Liver very large. Peritoneum } \\
\text { has its adult histological characters. }\end{array}$ \\
\hline & Epiglottis. \\
\hline \multirow{2}{*}{$\begin{array}{l}\text { External genitals begin to show dis- } \\
\text { tinctions of sex. } \\
\text { Ovary and testis distinguishable from } \\
\text { each other. } \\
\text { Kidney has its characteristic features. } \\
\text { Urogenital sinus acquires its own aper- } \\
\text { ture by division of cloaca. } \\
\end{array}$} & $\begin{array}{l}\text { Union of testis with canals of Wolftian } \\
\text { body complete. } \\
\text { Testes in false pelvis. } \\
\text { Ovaries descend. } \\
\text { Prostate begun (12th week). }\end{array}$ \\
\hline & $\begin{array}{l}\text { Corium proper present as distinct layer. } \\
\text { Nails not quite perfectly formed. } \\
\text { Beginning of development of hair as } \\
\text { solid ingrowths of epithelium. }\end{array}$ \\
\hline $\begin{array}{l}\text { Corpus striatum indicated. } \\
\text { Corpora quadrigemina represented by } \\
\text { two elevations on mid-brain roof. }\end{array}$ & $\begin{array}{l}\text { Cerebrum covers inter-brain. Fornix } \\
\text { and corpus callosum begun. Fissure } \\
\text { of Sylrius. Calcarine fissure. Crura } \\
\text { cerebri. Restiform bodies. Polls. }\end{array}$ \\
\hline $\begin{array}{l}\text { External ear indicated (Fig. 170). } \\
\text { Ciliary processes indicated. }\end{array}$ & $\begin{array}{l}\text { Eyes nearly in normal position. } \\
\text { Eyelids begin to adhere to each other. }\end{array}$ \\
\hline $\begin{array}{l}\text { Centers of ossification of presphenoid, } \\
\text { of lesser wings of sphenoid, and of } \\
\text { shafts of metatarsal bones. }\end{array}$ & $\begin{array}{l}\text { Beginning ossification of occipital bone, } \\
\text { of tympanic, of spine of scapula, of } \\
\text { ossa innominata. } \\
\text { Cartilaginous arches of vertebræ close. } \\
\text { Limbs have definite shape; nails almost } \\
\text { perfectly formed. }\end{array}$ \\
\hline
\end{tabular}


Tabulated Chronology of Development (Continued).

\begin{tabular}{|c|c|c|}
\hline & $\begin{array}{l}\text { STAGE OF 'T } \\
\text { Fourth Month. }\end{array}$ & $\begin{array}{l}\text { THE FETUS. } \\
\text { FIFTH MONTH. }\end{array}$ \\
\hline $\begin{array}{l}\text { General } \\
\text { Characters. }\end{array}$ & $\begin{array}{l}\text { Weight, } 7 \frac{3}{4} \text { ounces; length, } 5 \\
\text { inches. } \\
\text { Head constitutes about one- } \\
\text { quarter of entire body. }\end{array}$ & $\begin{array}{l}\text { Weight, } 1 \mathrm{lb} \text {; length, } 8 \text { in. } \\
\text { Active fetal movements be- } \\
\text { gin. Two layers of decidua } \\
\text { eoalesce, obliterating the } \\
\text { space betweenv vera and re- } \\
\text { flexa. Lymphatic glands } \\
\text { begin to appear. }\end{array}$ \\
\hline $\begin{array}{c}\text { Vascular } \\
\text { System. }\end{array}$ & & Heart very large. \\
\hline $\begin{array}{l}\text { Digestive } \\
\text { System. }\end{array}$ & $\begin{array}{l}\text { Enamel and dentine of milk- } \\
\text { teeth. Germs of permanent } \\
\text { teeth (17th wk); (for 1st mo- } \\
\text { lar, 16th wk). Nuscularis } \\
\text { (longitudinal and circular) } \\
\text { of stomach and esophagus. } \\
\text { Intestine entirely within } \\
\text { abdomen. Acid cells of } \\
\text { peptic glands. Nalpighianl } \\
\text { bodies of spleen. Anal } \\
\text { membrane disappears. }\end{array}$ & $\begin{array}{l}\text { Salivary glands acquire lu- } \\
\text { mina. } \\
\text { Villi of large intestine begin } \\
\text { to disappear. } \\
\text { Liver very large. } \\
\text { Neconium shows traces of } \\
\text { bile (sometimes early in } \\
\text { fourth month). }\end{array}$ \\
\hline $\begin{array}{l}\text { Respiratory } \\
\text { System. }\end{array}$ & $\begin{array}{l}\text { Cells of tracheal and bron- } \\
\text { chial mucous membrane } \\
\text { ciliated. }\end{array}$ & \\
\hline $\begin{array}{l}\text { Genito-urinary } \\
\text { system. }\end{array}$ & $\begin{array}{l}\text { Sexual distinctions of exter- } \\
\text { nal organs well marked. } \\
\text { Closure of genital furrow. } \\
\text { Scrotum. Prepuce. Pros- } \\
\text { tate well formed. }\end{array}$ & $\begin{array}{l}\text { Distinction between uterus } \\
\text { and vagina. } \\
\text { Hymen begins. }\end{array}$ \\
\hline Skin. & $\begin{array}{l}\text { Papillæ of corium. Subcuta- } \\
\text { neous fat first appears. La- } \\
\text { nugo or embryonal down } \\
\text { on scalp and some other } \\
\text { parts. }\end{array}$ & $\begin{array}{l}\text { Panniculus adiposus. } \\
\text { Lanugo more abundant. } \\
\text { Sebaceous and sweat-glands } \\
\text { begin. }\end{array}$ \\
\hline $\begin{array}{l}\text { Nervous } \\
\text { System. }\end{array}$ & $\begin{array}{l}\text { Parieto-occipital fissure. } \\
\text { Corpora albicantia. } \\
\text { Transverse fibers of pons. } \\
\text { Middle peduncles and chief } \\
\text { fissures of cerebellum. } \\
\text { spinal cord ends at end of } \\
\text { coccyx. } \\
\text { Deposit of myelin on fibers } \\
\text { of posterior roots, extend- } \\
\text { ing to Burdach and Goll. }\end{array}$ & $\begin{array}{l}\text { Fissure of Rolando. Body of } \\
\text { fornix and corp. caliosum. } \\
\text { Longitudinal fibers in cru- } \\
\text { ra cerebri. Superior pedun- } \\
\text { cles. Anterior pyramids of } \\
\text { medulla. Chief transverse } \\
\text { fissures of lateral lobes of } \\
\text { cerebellum. Deposit of my- } \\
\text { elin completed for tract of } \\
\text { Goll and later of Burdach, } \\
\text { and for short commissural } \\
\text { fibers (Tourneux). }\end{array}$ \\
\hline $\begin{array}{l}\text { Spocial Sense } \\
\text { Organs. }\end{array}$ & $\begin{array}{l}\text { Eyelids and nostrils closed. } \\
\text { Cartilage of Eustachian tube. }\end{array}$ & Organ of Corti indicated. \\
\hline $\begin{array}{l}\text { Muscular } \\
\text { System. }\end{array}$ & & $\begin{array}{l}\text { Differentiation of museular } \\
\text { tissue of arms. }\end{array}$ \\
\hline $\begin{array}{l}\text { Skeleton and } \\
\text { Limbs. }\end{array}$ & $\begin{array}{l}\text { Osseous center for internal } \\
\text { pterygoid plate. } \\
\text { Antrum of Highmore begins. } \\
\text { Ossification of malleus and } \\
\text { incus. } \\
\text { Tympanic ring. }\end{array}$ & $\begin{array}{l}\text { Ossification of stapes and pe- } \\
\text { trosa. Opisthotic and pro- } \\
\text { otic appear. Ossification } \\
\text { begins in middle and infe- } \\
\text { rior turbinals and lateral } \\
\text { masses of ethmoid. Inter- } \\
\text { nal pterygoid plate fuses } \\
\text { with external. Intermax- } \\
\text { illaries fuse with maxilla. } \\
\text { Legs longer than arms. }\end{array}$ \\
\hline
\end{tabular}




\section{Tabulated Chronology of Development (Continued).}

\begin{tabular}{|c|c|}
\hline \multicolumn{2}{|c|}{ STAGE OF THE FETUS. } \\
\hline Sixth Month. & Seventh Month. \\
\hline $\begin{array}{l}\text { Weight, } 2 \text { pounds; length, } 12 \text { inches. } \\
\text { Vernix caseosa begins to appear. } \\
\text { Amnion reaches maximum size; amni- } \\
\text { otic fluid of maximum quantity. }\end{array}$ & $\begin{array}{l}\text { Weight, } 3 \text { pounds; length, } 14 \text { inches. } \\
\text { Surface less wrinkled owing to increase } \\
\text { of fat. }\end{array}$ \\
\hline $\begin{array}{l}\text { Peyer's patches. } \\
\text { Trypsin in pancreatic secretion (fifth } \\
\text { or sixth month). }\end{array}$ & $\begin{array}{l}\text { Meconium in large intestine. } \\
\text { Ascending colon partly formed. } \\
\text { Cæcum below right kidney. }\end{array}$ \\
\hline Air-vesicles of lungs begin to appear. & \\
\hline Walls of uterus thicken. & $\begin{array}{l}\text { Testes at internal rings or in inguinal } \\
\text { cauals. }\end{array}$ \\
\hline $\begin{array}{l}\text { Vernix caseosa begins to appear. } \\
\text { Eyebrows and eyelashes begin. }\end{array}$ & $\begin{array}{l}\text { Epithelial buds for sebaceous glands ac- } \\
\text { quire lumina. Branching of cords of } \\
\text { milk-glands. Eponychium of nails } \\
\text { lost; nails said to break through. } \\
\text { Lanugo over entire body. }\end{array}$ \\
\hline $\begin{array}{l}\text { Collateral and calloso-marginal fissures. } \\
\text { Body of fornix and corpus callosum } \\
\text { complete. } \\
\text { Hemispheres of cerebrum cover mid- } \\
\text { brain. }\end{array}$ & $\begin{array}{l}\text { Cerebral convolutions more apparent. } \\
\text { Corpora quadrigemina. } \\
\text { Myelination of fibers of direct cerebellar } \\
\text { tracts. (Crossed pyramidal tracts not } \\
\text { until after birth.) }\end{array}$ \\
\hline Lobule of ear more characteristic. & $\begin{array}{l}\text { Lens-capsule begins to acquire trans- } \\
\text { parency. Eyelids permanently open. } \\
\text { Pupillary membrane atrophies. }\end{array}$ \\
\hline & $\begin{array}{l}\text { Differentiation of muscular tissue of } \\
\text { lower extremities. }\end{array}$ \\
\hline $\begin{array}{l}\text { Lesser wings unite with presphenoid. } \\
\text { Meckel's cartilage begins to retrograde. } \\
\text { Ossific nuclei of os calcis and astragalus. }\end{array}$ & $\begin{array}{l}\text { Basisphenoid and presphenoid unite } \\
\text { (7th or 8th month). }\end{array}$ \\
\hline
\end{tabular}


Tabulated Chronology of Development (Concluded).

\begin{tabular}{|c|c|c|}
\hline & $\begin{array}{l}\text { STAGE OF } \\
\text { EIGHTH MoNTh. }\end{array}$ & $\begin{array}{l}\text { THE FETUS. } \\
\text { NINTH MONTH. }\end{array}$ \\
\hline $\begin{array}{l}\text { General } \\
\text { Characters. }\end{array}$ & $\begin{array}{l}\text { Weight, } 4 \text { to } 5 \text { pounds ; length, } \\
16 \text { inches. } \\
\text { Body more plump. }\end{array}$ & $\begin{array}{l}\text { Weight, } 6 \text { to } 7 \text { pounds; length, } \\
20 \text { inches. } \\
\text { Umbilicus almost exactly in } \\
\text { middle of body. }\end{array}$ \\
\hline \multicolumn{3}{|l|}{$\begin{array}{l}\text { Vascular } \\
\text { System. }\end{array}$} \\
\hline $\begin{array}{l}\text { Digestive } \\
\text { System. }\end{array}$ & $\begin{array}{l}\text { Ascending colon longer. } \\
\text { Cæcum below crest of ilium. }\end{array}$ & Meconium dark greenish. \\
\hline \multicolumn{3}{|l|}{$\begin{array}{l}\text { Respiratory } \\
\text { System. }\end{array}$} \\
\hline $\begin{array}{l}\text { Genito-urinary } \\
\text { System. }\end{array}$ & Testes in inguinal canals. & $\begin{array}{l}\text { Testes in scrotum. } \\
\text { Labia majora in contact. }\end{array}$ \\
\hline Skin. & $\begin{array}{l}\text { Vernix caseosa covers entire } \\
\text { body. } \\
\text { Skin brighter color. } \\
\text { Lanugo begins to disappear. } \\
\text { Nails project beyond finger- } \\
\text { tips. } \\
\text { Increase of subcutaneous } \\
\text { fat. }\end{array}$ & $\begin{array}{l}\text { Lanugo almost entirely ab- } \\
\text { sent. } \\
\text { Galactopherous ducts of } \\
\text { milk-glands acquire lu- } \\
\text { mina. }\end{array}$ \\
\hline $\begin{array}{l}\text { Nervous } \\
\text { System. }\end{array}$ & & $\begin{array}{l}\text { Spinal cord ends at last lum- } \\
\text { bar vertebra. }\end{array}$ \\
\hline $\begin{array}{l}\text { Special Sense } \\
\text { Organs. }\end{array}$ & & $\begin{array}{l}\text { Ossification of bony lamina } \\
\text { spiralis and of modiolus. } \\
\text { Neuro-epithelial layer of re- } \\
\text { tina completed ; macula } \\
\text { still absent. } \\
\text { Choroidal fissure closes. }\end{array}$ \\
\hline \multicolumn{3}{|l|}{$\begin{array}{l}\text { Muscular } \\
\text { System. }\end{array}$} \\
\hline $\begin{array}{l}\text { Skeleton and } \\
\text { Limbs. }\end{array}$ & & $\begin{array}{l}\text { Ossification in lower epiph- } \\
\text { ysis of femur, sometimes } \\
\text { also in upper epiphyses of } \\
\text { tibia and humerus. } \\
\text { Tympanohyal begins to os- } \\
\text { sify. } \\
\text { Ossific nuclei for body and } \\
\text { great horn of hyoid bone. }\end{array}$ \\
\hline
\end{tabular}




\section{INDEX.}

Abdominal cavity, development of, 215

Accessory suprarenal organs, 242 thyroid, $2: 2 \pi$

Acetabular fossa, 404

Achoria, 94

Achromatin, 27

Acid cells, formation of, 206

Acoustic ganglion, 3:1

Acusticofacial ganglion, 321

Adamantoblasts, 139

Adenoid tissue, devclopment of, 1:29

Adipose tissue, formation of, 1:6

After-birth, 104

After-brain, 287

Age of fetus, estimation of, 122

Air-chamber of hen's egg, 29

Air-sacs, development of, 2:5

Alæe of nose, development of, 362

Alar lamina, 290

Alecithal ova, 26

Alimentary canal, development of, 185

differentiation into separate regions, 197

histological alterations in, 205

tract, alteration in position of parts, 202

increase in length of, 201

Alisphenoids, 394

Allantoic arteries, 90, 164

circulation, 90

formation of, 163

stalk, 85

veins, 90,161

Allantois, 89, 190, 255

function of, 90

respiratory function of, 200

Alveoli, pulmonary, development of, 225

Ameloblasts, 139

Amnion, 81, 82

false, 81

of man, 85

Amnion-fold, 80, 81, 83

Amniota, 83

Amniotic cavity, 54, 84, 85

fluid, 85,86

function of, 86

suture, 83

Amphibians, blastula of, 51

Amphioxus, blastula of, 50

skeletal apparatus of, 372
Ampullæ of semicircular canals, development of, 348

seminal, 246

Anal canal, 25\%

membrane, 195

plate, 195

Anamnia, 83

Angioblast, 147

Animal pole, 27

Animaleulists, 18

Anlage, 175

merlian, of thyroid body, 228

Annular sinus, 179

Annulus ovalis, 158

Anomalous arrangements of aortic arch, 168

Anterior chamber of eye, 34\% nares, development of, 146,360 pyramidal tracts of medulla, development of, 290

Antitragus, formation of, 358

Antrum of Highmore, development of, 361

Anus, development of, 195

imperforate, 197

Aorta, caudal, 166

development of, $\mathbf{1 5 9}$

primitive, 151, 165

Aortic arch, anomalous arrangements of, 168

arches, 165

septum, 159

Appendages of skin, $2 \pi 0$

Appendicular skeleton, 372

development of, $40 \%$

Aqueduct of Sylvius, development of, 296

Arch, liroid, 115

mandibular, 115

maxillary, 115

of aorta, development of, $\mathbf{1 6 7}$

Arched collecting tubule of kidney, 240

Archenteron, 5:

Arohes, aortic, 165

branchial, 114

mandibular. 135

visceral, 112

Archiblast. 66

Arcuate fissure, 306, 307

Area, embrronal, 59

glandular, 275

opaca, 59 
Area pellucida, 59

vasculosa, $59,88,150$

Areas, nasal, 145, 359

Areola, development of, 276

Areolar tissue, development of, 125

Arrectores pilorum, 269

Arteria centralis retinc, development of, 335

Arterial system, fetal, 165

Arteries, allantoic, 90, 164

umbilical, 103, 165

vitelline, 151

Artery, carotid, common, development of, 166

external, development of, 166

internal, development of, 166

innominate, development of, $16 \tilde{\sigma}$

middle sacral, development of, 166

pulmonary, development of, 168

subclavian, left, development of, 168

right, development of, $16 \tau$

superior vesical, $18 \%$

Aryteno-epiglottidean folds, 2:6

Arytenoid cartilages, development of, $2: 26$

ridges, 2:26

Ascending colon, formation of, 203

mesocolon, formation of, 203

root of fifth nerve, 224

root of vagus, 290

Aster, 45

Atlas, formation of, 381

Atresia of pupil, 338

Atrial crescent, 157

Atrioventricular canal, 156 valves, $156^{\circ}$

Atrophic tubules of Wolffian body, 236

Attraction-sphere, 45

Auditory apparatus, development of, 345

meatus. external, formation of, 357 nerve, formation of, 321

nucleus, lateral accessory, 321

pit, 346

Auricle, development of, 358

Auricles, division into right and left, 157

Auricular appendages, 159 canal, 156

septum, 157

Auriculoventricular apertures, 161 valves, 162

Axial fiber of spermatozoon, 20, 22 skeleton, $3 \% 2$

development of, $3 \approx 3$

Axis, development of, 380

Axis-cylinder process, $284^{\circ}$

BARDEEN's primitive disk, 377, 379

Bartholin, glands of, 261

Basal ganglia, 303, 304

lamina, 290

Basi-occipital bone, 390

Basisphenoid, 394
Belly-stalk, 85

Bifid uterus, 253

Bile-capillaries, formation of, 209

Bile-ducts, formation of, :209

Bladder, development of, 255

Blastema, Wolftiau, :236

Blastodermic vesicle, mammalian, 50 stage of, 49

two-layered stage of, 52

Blastopore, 5?

Blastula stage, 49

Blood, development of, 126, 147

Blood-islands, 148

Blood-lacunæ, 9\%

Blood-platelets, 150

Blood-vessels, 150

"Blue baby," 158

Bodies, polar, 33, 34

Body of vertebra, formation of, 377

Body-cavity, 63, 66, 214

Body-wall, development of muscles of, 367

formation of, 79

Bony cochlea, development of, 352

labyrinth, development of, 351

semicircular canals, 351

Bowman, capsule of, 238, 240

Brain, development of, 286

Brain-case, 354

Brain-membranes, development of, 302

Brain-vesicles, 287

derivatives of, 316

Branchial arches, 114

development of, 369

Branchiomeres, 78

Bridge of nose, development of, 362

Broad ligament of uterus, 255

Brunner, glands of, 206

Bud, embryonic, 54

Bulbus arteriosus, 156 vestibuli, 259

Burdach, tract of, mrelination of, 414

Bursa, omental, 204, 21 pharyngeal, 136

Bursal sacs, development of, 126

Caducous membranes, 195

Cæcum, development of, 202, 203

Calcar avis, 308

Calcarine fissure, 303,308

Callosomarginal fissure, 309

Canal, anal, $25 \%$

atrioventricular, 156

auricular, 156

hyaloid, 339

medullary, 70

neural, 70, 279, 281

neurenteric, 74, 281

of anus, 197

of His, 145,227

of Nuck, 255

of Stilling, 339

Canaliculi, lacrimal, development of, 345 
Canalis reuniens, 349

Cajsule of Bowman, 238, 240

of kidney, 241

Cardinal veins, 164

anterior, 169

posterior, 169

Carotid artery, common, development of, $166^{\circ}$

external, development of, 166

intermal, development of, $166^{\circ}$

body, 325

Carpus, development of bones of, 405

Cartilage, formation of, 126

Meckel's, 115, 398

Reichert's, 115

Cartilage-cells, 126

Cartilaginous capsule of cochlea, 352 cranium, 386

ear-capsule, 351

ribs, 38\%

sheath of spinal cord, 378

stage of skeleton, 373

of trunk skeleton, $3 \pi \%$

vertebral bodies, origin of, 379

processes, origin of, 379

Caudal aorta, 166

Cavity, amniotic, 54, 84

cleavage-, 50

plenroperitoneal, 66

segmentation-, 50

Cell-cords, 150

Cell-mass, inner, 50

intermediate, 7,232

outer, 50

Cells, sexual, 31 mesenchymal, 66

Cementum of tooth, 137 development of, 141

Central canal of cord, formation of, 286

lobe, formation of, 305

Centrolecithal ova, 27

Centrosome, 45

Cephalic flexure, 112, 288 ganglia, development of, 320

Ceratohyal, 402

Cerebellum, development of, 292

Cerebral fissures, development of, 302 vesicles, 287,288

Ceruminous glands, 273

Cervical fistula, 116

flexure, 112

rib, 380,383

Chalazæ, 29

Chambers of eye, 342

Chin ridge, 135

C'horda dorsalis, $\%: 3$

formation of 373

stage of, 373

Chordxe tendinex, 162

Chordal epithelinm, 374

plate, 74

region of primitive skull, 387

Choriata, 91

Choriocapillaris, 340
Chorion, 92

frondosum, 93

leve, 93

primitive, 92

true, 92

Choroid, coloboma of, 341

development of, 340

fissure, $306,30 \tau$

plexus, 308

plexuses of fourth ventricle, 291

Choroidal fissure, 330, 341

Chromaffine cells, 325

Chromatin, 27

Chromosomes, reduction of, 23

Cicatricula, 2o

Ciliary body, development of, 341 ganglion, 320

muscle, development of, 341

processes, development of, 333, 341

Circulation, allantoic, 90, 163

placental, 147

portal, $17 \%$

vitelline, formation of, 147

Claustrum, 30:

Clavicle, development of, 404

Cleavage, kinds of, $4 \tau$

of ovum, 45

partial discoidal, 48

peripheral, 48

total equal, 47

unequal, 47

Cleavage-eavity, 50

Cleavage-nuelens, 43

Cleavage-planes, 46

Cleft palate, formation of, 137 sternum, 383

cause of, 82

uvula, formation of, 137

Clefts, visceral, 112

Climacteric, 38

Clitoris, development of, 259

Cloaca, 190, 196, 256

Cloacal depression, 197,256

Closing membrane, $113,117,196$

Coccygeal body, 325

curve, 112

vertebrr, ossification of, 382

Cochlea, bony, development of, 352

Cochlear duct, formation of, 347

ganglion, 3:1

nerve, 354

Colenteron, 52

Colom, 63, 66, 214

Collateral fissure, 303.308

Collecting tubules of kidney, 237

Coloboma of choroid, 341 of iris, 343

Colon, ascending, formation of, 203 descending, formation of, 201, 203 transverse, formation of, 203

Columnæ carnex. 154

Commissures of hrain, development of, $30 \%$

of cord, white, 285

Conarium, 298 
Conarium, modifications of, 295

Cone-visual cells, 331

Congenital atresia of pupil, $33 \mathrm{~s}$

diaphragmatic hernia, $17 \pi$

fecal fistula, 207

hernia, 249

umbilical hernia, 205

Coni vasculosi, formation of, 246

Connective tissues, development of, 124

Constructive stage of menstrual cycle, 39

Copula of hyoid bone, 339

Coracoid bone, 403 process of scapula, 403

Cord, spinal, development of, 281 umbilical, 10 :

Cords of cells, 147

Corium, development of, 268

Cornea, development of, 340

Cornicular tubercles, 2:26

Corona radiata, 25, 31

Coronary ligament, 210 of liver, 2:21

sinus of heart, $1 \% 2$

valve, 161

Corpora albicantia, 296

bigemina, 295

cavernosa, formation of, 262 quadrigemina, 295

Corpus callosum, formation of, 309, 311 hemorrhagicum, 37

luteum of pregnancy, 37,38

false, 38

of menstruation, 38

true, 38

spongiosum, formation of, 262

striatum, 303

Corpuscle of Hassal, 230

Corti, organ of, 349

Costal process of vertebra, formation of, 376,382

Cotyledons of placenta, 99

Covering bones, 395

Cowper, glands of, 263

Cranial capsule, 394

nerve-fibers, development of, $3: 20$

Cranium, cartilaginous, 336

membranous, 385

osseous, 389

Crescent, atrial, $15 \%$

Cricoid cartilage, 2:26

Cristæ acusticæ, 350

Crossed pyramidal tract, myelination of, 415

Crura cerebri, development of, 295

Crusta petrosa, 141

Cryptorchism, 249

Crystalline lens, development of, 336

Cuneiform tubereles, 226

Cushions, endocardial, 156

Cutis-plate, $77,268,365$

Cuvier, duct of, $164,170,176$

Cystic duct, development of, 209

Cytoblast, 54
DAUGHTER-CELLS, 22

Daughter-wreaths, 45

Decidua menstrualis, 39,95

of pregnancy, 96

reflexa, 96

serotina, 96, 101

vera, 96

Deciduæ, 95

Dendrites, 284

Dental groove, 138 papilla, 139,140

processes, 141

ridge, 137

shelf, $13 \pi$

Dentaie, 400

Dentate fissure, 303, 307

Dentinal fibers, 141 tubules, 141

Dentine, $13 \pi$

Dermal bones, 385 navel, 82

Descending colon, formation of, 203

Descent of testicles, 24s

Destructive stage of menstrual cycle, 39

Deutoplasm of hen's egg, 28 of ovum, 26

Development during eighth month, $12: 2$

during eighth week, 119

during fifth month, 121

during fifth week, 118

during ninth month, 122

during second month, 118

during seventh month, 121

during sixth month, 121

during third month, 120

during third week, 117

length of time necessary for, 18

tabulated chronology of, 409

theories of, 17

Diaphragm, development of, 177

Diaphragmatic hernia, congenital, 178

ligament, 248

Dienceplialon, 287

Digestive srstem, development of, $185,411-416$

Digitation of limb-buds, 40 z

Diphyodont, 137

Direct cerebellar tract, myelination of. 411

Discoirlal cleavage, partial, 48

Discus proligerus, 31,251

Disk. germinative, 28

Distal convoluted tubule of kidney, 240

Diverticula of primary renal pelvis, 237

Dorsal curve, 112

mesenters, 190

nerve-roots of spinal ganglia, 318 pancreas, 211

Double monster, origin of, 58 uterus, 253 
Duct of Cuvier, 164, 170, 176

mesonephric, 234

of Gartuer, 254

of Müller, $243,247,253,265$

of Rathke, 245

of Santorini, 212

of Wirsung, 21:

pronephric, 233

segmental, 233

thyroglossal, 145, 227

thyroid, $2: \%$

vitelline, $80,8 \%, 186$

Wolffian, 23t

Ductus Arantii, 180

arteriosus, 168

communis choledochus, formation of, 209

endolymphaticus, 347

venosus, 103, 180

Duodenum, formation of, 217

EAr, external, development of, 355, 358

internal, development of, 346

middle, development of, 355

Ear-capsule, cartilaginous, 351

Ectoderm, 5\%

derivatives of, 67

Egg, ultimate origin of, 31

Egg-columns, 31, 250

Egg-envelopes, 25

Egg-plasm, 26

Egg-tubes, primary, 31

Eighth month, development during, $12: 416$

pair cranial nerves, development of, 323

week, development during, 119, 412

Ejaculatory duct, formation of, 247

Elastic tissue, formation of, 125

Eleventh pair cranial nerves, 324

Embedding of ovum, 96

Embryo, differentiation of, 69 of eight and a half weeks, 121 of fifteenth dav, 109

of $\operatorname{six}$ weeks, 118

of thirteenth day, 108

of three weeks, 112

of twenty-eight davs, 116

segmentation of body of, 78 stage of, 19,107

Embryology defined, 17

Embryonal area, 58 down, 273

Embryonic bud, 54 crescent, 59

Eminentia collateralis, 309

Enamel of milk teeth, formation of, 140

of teeth. 137

Enamel-cells, 139

Enamel-germ, primitive, 138

Enamel-germs of permanent teeth, 141

Enamel-prisms, 139
Enamel-sac, 138

End-knob of spermatozoon, 21, 2\%

Endocardial cushions, 156

Endocardium, 154

Endochondral bones, 385

Endolymph, 355

Endoskeleton, 37:

Endothelium, formation of, 66,126

End-piece of spermatozoon, 200

Enterocol, 63

Entoderm, 52, 54

derivatives of, 67

Enveloping layer of mammalian blastodermic vesicle, 50

Ependyma, 310

Ependymal cells, 282, 283

layer, 281

Epiblast, 5:

Epidermis, development of, 268, 269

Epididymis, formation of, 246

heal of, 246

Epigenesis, doctrine of, 18

Epiglottis, 2:26

Epihyal, 40\%

Epiotic center of ossification, 392

Epithelial bodies, 2:29

Epithelium, germinal, 29, 31, 244

Epitrichium, 269

Eponychium, 2\%1

Epoöphoron, 254

Erythroblasts. 149

Eryth rocytes, 149

Ethmoid bone, ossification of, 395 cribriform plate of, 388

Ethmoidal sinus, development of, 361

Eustachian tube, development of, 556 formation of, 194 valve, 160

Evagination, kidner, 237

Evertebral region of primitive skull, 387

Exoccipitals, 390

Exoskeleton, 3\%?

Exstrophy of bladder, cause of, 82

External auditory meatus, formation of, $3 \pi \tau$

ear, development of, 355,358

fertilization, 41

genitals, female, 259,266

male, 261,267

organs of generation, 258

Eye, development of. 134,326

Eyelashes, development of, 344

Evelid, third, 344

Eyelids, development of, 343 primitive, 134

FAcE, development of, 117,130

Facial ganglion, 321

Falciform ligament of liver, formation of, 210

lobe, 309,313

Fallopian tubes, development of, 253

False amnion. 81

Falx cerebri, 302 
Fecal fistula, congenital, 207

Female external genitals, 259, 266

internal genital organs, 249

pronucleus, 34

sexual system, 266

Fertilization, 41

artificial, 44

exterual, 42

internal, 42

Fetal arterial system, 165

membranes at birth, 104

vascular system, final stage of, 181 venous system, 169

Fetus, length of, at term, 122

stage of, 20, 118

weight of, at term, 122

Fiber-tracts of cord, development of, 285

myelination of, 414,415

Fibrillæ of muscle, formation of, 366

Fibrous tunic of eye, development of, 339

Fifth brain-vesicle, metamorphosis of, 289

month, development during, 121, 414

pair cranial nerves, development of, 323

ventricle, 312

week, development during, 411

Fimbria, 309

Fingers, development of, 407

First pair cranial nerves, development of, 323

week, development during, 409

Fissure, arcuate, 306, 307

calcarine, 303,308

calloso-marginal, 309

choroid, $306,30 \%$

choroidal, 330

collateral, 303,308

dentate, 303,307

great transverse, 304,308

hippocampal, 307

of choroid plexus, 307

of Rolando, 308

of Sylvius, 303,304

parieto-occipital, 308

Fissures, cerebral, development of, 302,303

mediau, of cord, 285

Fistula, congenital fecal, 207

umbilical urinary, 256

Flexure, cephalic, 112, 288

nuchal, 289

pontal, 289

Floor-plate, 281, 282

Fold, pleuropericardial, 176

Folds, medullary, 72

Folbicle, Graafian, 29 of tooth, 141

Foramen cxeum, 145, 227 commune anterius, 306 of Monro, 301, 306 of Winslow, 221
Foramen ovale, 157

thyroideum, 2:6

Fore-brain, 286, 302

secondary, 287

vesicle, 73

metamorphosis of, 302

Foregut, 81

Formative yolk, 26

Fornix, formation of, 309, 310

Fossa of Sylvius, 304

oral, 192

ovalis, 158

Fourth month, development during, 120,414

pair cranial nerves, development of, 323

ventricle, 291

development of, 290, 294

week, development during, 410

Fretum Halleri, 156

Frontal bone, ossification of, 396

lobe, 306

sinuses, development of, 361

Funiculus solitarius, 290

Furcula, 225

GALL-BLA DDER, development of, 209

Ganglia, cephalic, 320 spinal, $31 \%$

Gangliated cord of the sympathetic, $3: 25$

Ganglion, acoustic, 321

acusticofacial, 321

cephalic, fourth, 321

third, 321

ciliary, 320

cochlear, 321

facial, 321

Gasserian, 320

intercarotid, 325

Luschka's, 325

ophthalmic, 320

spirale, 350

trigeminal, 321

vestibular, 351

Ganglion-cell layer, development of, 333

Gartner, duct of, 251

Gasserian ganglion, 320

Gastral mesoderm, 63

Gastrohepatic omentum, 209, 220 formation of, 205

Gastrosplenic omentum, 214

Gastrula, 52 stage, 52

Generative organs, external, development of, 258

internal, development of, 243

Genital cord, 243

eminence, 259

in male, 261

folds, 259

in female, 259

in male, 262

gland, indifferent, 265 
Genital groove, 258

ridge, 243,258

in female, 259

ridges, 31

Genito-urinary system, development of, $232,409-416$

Germ-cells, :24

Germ-disk, 27

Germ-layers, 52

derivatives of, 67

Germinal epithelium, 29, 31, 244

spot, $25,2 \%$

vesicle, $25,2 \pi$

Germinative disk, 28

Giraldès, organ of, 247

Glands of alimentary tract, formation of, 206

of Bartholin, 261

of Brumner, development of, 206

of Cowper, development of, 263

of intestine, development of, $: 206$

of Lieberkühn, development of, 206

of Moll, 273

of stomach, development of, 206

Glandular area, 275

hypospadias, 26:

Glans clitoridis, formation of, 259 penis, formation of, 259,262

Glaserian fissure, 393, 399

Globular processes, 118, 132, 360

Glomerulus of kidney, $233,235,240$

Glomus caroticus, 325

Goll, tract of, myelination of, 414

Graafian follicle, 29

development of, 251

formation of new, 25 ?

Gray matter of brain, formation of, 303

of medulla, development of, $2: 2 t$

Great omentum, formation of, 204 , $2: 20$

Groove, dental, 140

lacrimal, 119, 132

medullary, 71

naso-optic, 345

primitive, 60

pulmonary, 223

transverse, erescentic, 398

Gubernaculum testis, 218

Gum, development of, 136

Gut, postanal, 196

Gut-tract, 80, 81, 186, 188

Gyrus formicatus, 315

uncinatus, 315

HAIR, development of, 271

Hair-bulb, 2శ1

development of, 272

Hair-follicle, $2 \pi 1$

development of, 272,273

Hair-germs, 27\%

Hard palate, development of, 397

Hare-lip, 134, 397

Hassal, corpuscles of, 230
Head, muscles of, development of, $36 \%$

of epididymis, 246

of spermatozuon, $: 20,22$

Head-fold, 80

of amnion, 80,83

Head-gut, 188

Head-kidney, 232

Head-process of primitive streak, 62, 70

Head-segments, 364

Head-skeleton, development of, 384

Heart, development of, 152

lymph-, 12s

posterior, 128

metamorphosis of single into double 156

valves, development of, 161

Helix, formation of, $35 \mathrm{~s}$

Hemal arch, formation of, 376

Henle's loop, 240

Hen's egg, description of, 27

Hensen's node, 62

Hepatic cylinders, 209

vein, development of, 181

Hermaphroditism, 263, 267

Hernia, congenital, 249 umbilical, 205

Highmore, antrum of, development of, 361

Hilum folliculi, 31

Hind-brain, 286, 292

secondary, 287

vesicle, $73,29:$

Hindgut, 81, 188

Hippocampal fissure, 307

Hippocampus major, 307 minor, $30 \mathrm{~s}$

His, canal of, 145,227

Holoblastic ova, 47

Homogeneous twins, origin of, 59

Homologies of the sexual system, 263

Hyaloid artery, formation of, 339 canal, 339

membrane, formation of, 339

Hydatid of Morgagni, 247

sessile, 247

stalked, 247

unstalked, 247

Hydramnios, 86

Hymen, formation of, 261

Hyoglossus, origin of, 370

Hyoid arch, anterior, 389 posterior, 389

arches, 115

bar. 389

bone. development of, 389,401

Hyoidean apparatus, 401.

Hyomandibular cleft, 115

Hypoblast, 5?

Hypochordal brace, 376

Hypophysis, 300

formation of. 135

Hypospadias, 26? glandular, 262 
ILIAC segment of pelvic girdle, 404 vein, left common, development of, $17: 2$

Imperforate anus, 197

Impressions, maternal, 120

Incus, development of, 388, 399

Indifferent genital gland, 265 sexual gland, 244

Inferior medullary velum, 292 peduncles of brain, 290

Infundibula of lungs, development of, 225

Infundibulum of brain, 296, 300

Inguinal ligament, 248 in female, 254

Inner cell-mass, 50

Innominate artery, development of, 167

Inter-brain, 287, 296

vesicle, metamorphosis of, 296

Intercarotid ganglion, 325

Intermaxillary bones, formation of, 136,397

Intermedial cell-mass, 77, 232, 365

Internal ear, development of, 346 fertilization, $4 \%$ lateral ligament of lower jaw, 400

limiting membrane of spinal cord, 383

Interpallial fissure, 302

Interrenal organ, 242

Intervertebral disks, 377, 379

ligament, development of, 377,379

Intervillous spaces, 97, 102

Intestinal canal, formation of, 79 glands, development of, 206 mesentery, 216

mucosa, formation of, 189

villi, formation of, 206

portals, 81, 186

Intestine, small, development of, 202, 205

Intestino-body cavity, 52

Intumescentia ganglioformis, 351

Involuntary muscle, development of, 371

Iris, coloboma of, 343

development of, 341

Ischiatic rod, 404

Island of Reil, 305

JACOBson's organ, development of, 361

Jaw, upper, development of, 134

Jaw-arch, 115

Jelly of Wharton, 103

Joint-cavities, development of, 128

Jugular vein, primitive, 164, 169 transverse, $17 \%$

KIDNEY, development of, 232

LABIA majora, 260

minora, formation of, 259

Labyrinth, bony, development of, 351
Labyrinth, membranous, development of, 346

Lacrimal bones, ossification of, 396

eanaliculi, 345

caruncle, 344

duct, development of, 344

gland, development of, 344

groove, 119, 132

sac, development of, 345

Lamina cinerea, 296, 299

quadrigemina, 295

spiralis, bony, development of, 354

terminalis, 309

Laughans' layer, 97

Lanugo, 121, 273

Larynx, development of, 225

Latebra, 29

Lateral cartilage of nose, 395

folds of amnion, 80

frontal processes, 118, 132, 134

in formation of nose, 146

ligaments of liver, 210

nasal process, 344,360

plate of mesoderm, 65

plate of somite, 63

ventricle, development of, 303

Length of fetus at term, 122

Lens, crystalline, development of, 336

Lens-area, 328

Lens-capsule, development of, 337

Lens-pit, 336

Lens-vesicle, 110, 134, 328, 336

Lenticular zone of optic cup, 333

Lesser omentum, 220 formation of, 205

Leukocytes, 149

Levator palati, origin of, 370

Lids, unioll of edges of, 343

Lieberkühn, glands of, 206

Ligament of ovary, 255

Ligamenta intermuscularia, 365,375 subflava, 379

Ligaments of liver, formation of, 209

Ligamentum venosum Arantii, 184

Ligulæ, 292

Limb-buds, 119, 406

Limbic lobe, 309, 313

Limb-muscles, development of, 370

Limbs, bones of, development of, 405 development of, 406, 409-416 position of, 407

Limiting membrane, inner, formation of, 331 outer, formation of, 331

Linin, 27

Lip ridge, 135 upper, development of, 136

Liquor amnii, 85, 86 function of, 86

folliculi, 31, 251

of Morgagni, 337

Liver, development of, 207

first rudiment of, 198

ligaments of, formation of, 209 
Liver-ridge, 175, 208

Lobes of liver, 208

Lobule of ear, development of, 358

Longitudinal fiber-tracts of medulla, 290

fissure of brain, $30 \%$

Loop of Henle, 240

Lower jaw, ossification of, 398

Lumbar rib, 383 vertebra, ossification of, 381

Lungs, development of, 223

Luschka's ganglion, 325

Lymph, formation of, 126

Lymph-clefts, development of, 128

Lymph-hearts, 128 posterior, 1:8

Lymph-sacs, development of, 127

Lymph-spaces, development of, 127

Lymphatic system, development of, 127

vessels, development of, 128

Lymphoid follicles of tonsil, 195

tissue, development of, 129

Macula lutea, formation of, 333

Macula acustice, development of, 350

Malar bone, ossification of, 396

Male external genitals, 261, 267 interual genital organs, 245 pronucleus, 42 sexual system, 245,266

Malleus, development of, 388, 399

Malpighian corpuscle, development of, 213,238 primitive, $236^{\circ}$

Mammalia deciduata, 99 indeciduata, 99

Mammals, blastula of, 49

Mammary gland, development of, 274

Mandible, ossification of, 398

Mandibular arch, 115, 135, $386^{\circ}$

Mantle layer, 284

Marginal sinus, 102

velum of spinal cord, 283,284

zone of optic cup, 334

Marshall, vestigial fold of, 172

Maternal impressions, 120

Maturation of ovum, 32

Maxilla, superior, ossification of, 397

Maxillary areh, 115 process, 135,386

Meatus, external auditory, 357 urinarius, male, 262

Meckel's cartilage, $\mathbf{1 1 5}, 388,398$ diverticulum, formation of, 207

Meconium, 122

Median fissures of cord, 285 lobe of cerebellum, 292

Medulla oblongata, development of, 289

Medullary canal, 70

cords, 246,252

folds, 72,279

furrow, $\gamma 1$

groove, 71
Iedullary plate, 70,279

tube, 279

velum, anterior, 294

inferior, 292,294

Meibomian glands, development of, 344

Membrana adamantina, 139

basilaris of cochlea, formation of, 355

eboris, 141

granulosa, 31

formation of, 251

preformativa, 141

Membrane, anal, 193

closing, 113, 117, 194

nuclear, $2 \%$

of Nasmyth, 140

of Reissner, 355

pharyngeal, 117, 131, 188, 192

vitelline, 25,26

tympanic, 194, 357

Membranes, caducous, 95

deciduous, 95

Membranous bones, 385

cranium, 385

labyrinth, development of, 346

ribs, 382

stage of skeleton, 373

of trunk, 374

Menopause, 38

Menstrual cycle, 39

Menstruation, 38

relation of, to ovulation and conception, 40

Meroblastic ova, 48

Mesencephalon, 286, 294

Mesenchymal cells, 66 muscle, 371

Mesenchyme, 66

Mesenteric artery, superior, 152 vein, superior, 181

Mesenteries, 190

Mesentery, intestinal, 216 ventral, 204 development of, 220

Mesoblast, 6:

Mesoblastic somites, 65, 75

Mesocardium anterius, 153, 174 posterius, 153, 174

Mesocolon, ascending, production of, 203

formation of, 203

Mesonephrogenic tissue, 239

Metanephrogenic tissue, 239

Mesoderm, 6:

derivatives of, 68

gastral, 63

paraxial, 65

peristomal. 63

somatic, 66

splanchnic, 66

structures developed from, 124 et seq.

Mesodermal vitreous, 338

Mesogastrium, 204, 216

Mesonephric duct, 235 
Mesonephros, 234, 264

Mesorchium, 248, 255

Mesothelium, 66, 126

Mesovarium, 248

Metacarpal bones, development, 405

Metamorphosis of single into double heart, 156

Metanephros, 237, 265

Metatarsal bones, development, 405

Metencephalon, 287, 292

Metopic suture, 396

Metopism, 396

Micropyle, 25,42

Mid-brain, 2ఠ6, 294

prominence of, 288 vesicle, 73,294

Mid-gut, 188

Middle ear, development of, 194, 355 piece of spermatozoon, 20, 22

plate, $\%$, 232, 365

sacral artery, development of, 166

tunic of eye, development of, 339

Milk-lines, 275

Milk-ridges, 275

Modiolus of cochlea, development, 354

Moll, glands of, 273

Monorchism, '249

Monro, foramen of, 301, 306

Mons veneris, formation of, 259

Morgagni, hydatid of, 247

liquor of, 337

Morula, 45

Mother-cells, 22

Motor nerve-fibers, development, 319

Mouth, development of, 134, 192

Mucous tissue, formation of, 125

Mulberry-mass, 45

Müller, duct of, $243,24 \%, 253,265$

Müller's fibers, 331

Muscle, involuntary, development, $3 \approx 1$

voluntary, development of, 363

Muscle-plate, 78,365

metamorphosis of, 366

Muscles, branchial, development of, 369

of extremities, development of, 370

of trunk, development of, 363

Muscular coat of intestines, formation of, 205

system, development of, 363, 409416

Musculi papillares, 163

pectinati. 154

Myelencephalon, 287,289

Myelin, deposit of, 319

Myoccel, 365

Myotome, $\tau 7,365$

NAIL-BED, 271

Nail-plate, 270

Nails, development of, 270 of toes, 271

Nail-welt, 271

Nares, anterior, formation of, 146 development of, 360
Nasal areas, 145, 359

bones, ossification of, 396

capsule, 388

cavities, development of, 361

pits, $118,132,115,360$

process, 132,360

lateral, 344,360

Nasm yth, membrane of, 140

Nasofrontal process, 115, 118, 132, 134, 360,386

in development of nose, 145

Naso-optic furrow, 132, 134

in formation of nose, 146

groove, 345

Nephridial funnels, 233

Nephrogenic tissue, 236

Nephrostomata, 2:33

Nephrotome, 7\%, 234, 264, 365

Nerve-cells, formation of, 282 of cord, formation of, 284

Nerve-corpuscles of neurilemma, 319

Nerve-fiber, envelopes of, formation of, 319

layer, development of, 333

Nerve-fibers, cranial, development of, 320

motor, development of, 319

sensory, development of, 317

Nerve-trunk, spinal, development of, 319

Nervous system, development of, 278 , 409-416

peripheral, development of, 316

sympathetic, development of, 324

Neural canal, $70,279,281$

crest, segmentation of, 318

crests, 318

process of vertebra, formation of, 376

tube, $2 \gamma 9$

Neurenteric canal, 74, 281

Neurilemma, formation of, 319

Neurit, 278, 284

Neuroblasts, 28:, 284

Neuro-epithelium of retina, development of, 333

Neuroglia, 28:), 283

layer, 284

Neurons, 278

Nictitating membrane, 344

Ninth month, development during, 122,416

pair cranial nerves, development of, 321

week, development during, 413

Nipple, development of, $2 \approx 6$

Node, Hensen's, 62

Normoblasts, 149

Nose, development of, 145,358

Notochord, 73

Notochordal stage of skeleton, 373

Nuchal flexure, 289

Nuck, canal of. 255

Nuclear juice. 27

layer of retina, outer, 332 
Nuclear membrane, 27 spindle, 45

Nucleus amygdalre, 303 cleavage-, 43 of ovum, $2 \tau$ segmentation-, 43

Nutritive yolk, 26

Nymphre, formation of, 259

OBEx, 292

Occipital bone, ossification of, 390 lobe, 306

Odontoblasts, 141

Odontoid process, development of, 381

Olfactory bulb, 314

epithelium, 359,362

lobe, 314

nerve-fibers, 362

plates, $132,145,358$

tract, 314

Omental bursa, 204, 218

Omentum, gastrohepatic, 209, 220 formation of, 205

gastrosplenic, 214

great, formation of, $204,2: 0$

lesser, 2:20

formation of, 205

phrenicosplenic, 214

Omphalomesenteric veins, 151

Ontogeny, 17

Oöcytes, 32

Oögenesis, 29

Oögonia, 32

Ophthalmic ganglion, 320

Opisthotic center of ossification, 392

Optic cup, $3: 8$ secondary, 330

lobes, formation of, 295

nerve, development of, 335

thalami, 296

vesicle, 287,327

Ora serrata, 331

Oral cavity, development of, 192 fossa, 192

pit, 108, 11\%, 131, 135, 192

plate, $130,134,192$

Orbitonasal center, 397

Orbitosphenoids, 394

Organ of Corti, 349

of Giraldès, 247

of Jacobson, development of, 361 of Rosenmüller, 254

Organs of Zuckerkandl, 325

Osseous cranium, 389 stage of trunk skeleton, 379

tissue, formation of, 126

Ossicles of ear, development of, 356

Ossification of ribs, 383

of skull, 389

of sternum, 383

of vertebræ, 380

Ostium interventriculare, 158

Otic vesicle, 109,346

Otocyst, 346
Outer cell-mass, 50

Ova, alecithal, 26

centrolecithal, :2\%

classification of, 26

formation of, 29

holoblastic, 47

meroblastic, 4s

primitive, $31,245,250$

telolecithal, 26

Oraries, change of position of, 254

Ovary, development of, 249

Ovilucts, development of, 253

Ovists, 18

Ovulation, 36 relation of, to menstruation, 40

Ovum, 24, :51

embedding of, 95,96

maturation of, 32

ripening of, 32

segmentation of, 45

stage of, 19, 106

Palate bone, ossification of, 396

formation of, 136 ; process, development of, 397

Palate-shelves, 360

Palatoglossus, origin of, 370

Palatopharyngeus, origin of, 370

Palpebral fascire, 344 fissure, 343

Pancreas, development of, 211

dorsal, 211

first rudiment of, 199

ventral, 211

Pancreatic duct, development of, 211

Pander's nucleus, 28

Panniculus adiposus, 269

Papilla of tongue, formation of, 145

Parablast, 66

Parachordal cartilages, 357

Paradidymis, 217

Parathyroid bodies. 229

Paraxial niesoderm, 65

Parietal bones, ossification of, 396

elevation, 200

eye, 299

foramen, 298

layer of pleura, $17 \%$

lobe, 306

zolle, 76

Parieto-vccipital fissure, 308

Paroöphoron, 254

Parovarium, 254

Pars ciliaris retinge, 334

intermedialis, 259

iridica retinæ, 334

membranacea septi, 159

optica retinæ, 333

Parthenogenetic eggs, 34

Patulous foramen ovale, 157

Pectoral girdle, development of, 403

Pelvic girdle, 404

Pelvis of kidney, primary, 237

Penis, development of, 259

Perforated lamina, anterior, 315 
Perforated space, posterior, 295

Pericardial cavity, 175

Pericardium, development of, 174

Perilymph, 352, 355

Perilymphatic space, 352

Perineal body, 197

Perineum, formation of, 197

Perionyx, 2\%1

Periotic bone, 392

Peripheral cleavage, 48 nervous system, 316

Peristomal mesoderm, 63

Peritoneal cavity, 215

Peritoneum, development of, 214 visceral layer of, 189

Perivitelline space, 25

Permanent kidney, 2:37 teeth, development of, 141 eruption of, 143

Petromastoid bone, 392

Petrotympanic fissure, 399

Pflüger's egg-tubes, 250

Phrochrome bodies, 242 cells, 242

Phalanges, development of, 405

Pharyngeal bursa, 136 constrictors, origin of, 370 membrane, 117, 131, 188, 192 in formation of mouth, 135 pouches, 113, 188, 193

Pharynx, 193

Phrenicosplenic omentum, 214

Phylogeny, 17

Pial processes, 283

Pigment-layer of retina, 331

Pillars of Uskow, 177

Pineal body, 296 or gland, 297, 298

eye, 299

Pit, auditory, 346 oral, 108, 117

Pits, nasal, 360

Pituitary body, 300 formation of, 135

Placenta, 98

at term, 101

discoidea, 99

pravia, 102

zonaria, 99

Placental sinuses, 100

spaces, 102

system of blood-vessels, 164

Placentoblast, 54

Planes of cleavage, 46

Plantar horn, 270

Plasmodoblast, 54

Plate, chordal, 74

medullary, 70

vertebral, 65

Pleura, parietal layer of, $\mathbf{1 7 7}$ visceral layer of, $17 \%$

Pleurx, development of, 174, 175, 226

Pleural sacs, formation of, 174, 175

Pleuropericardial fold, $\mathbf{1 7 6}$

Pleuroperitoneal cavity, 66, 215
Plica semilunaris, 344

Pocket of Rathke, 301

Polar borlies, 33, 34 striation, 45

Polarity of egg, 27

Pole-corpuseles, 33

Polyphyodont, 137

Polyspermia, 42

Pontal flexure, 289

Pons, formation of, 292

Portal circulation, $170,17 \%$

vein, development of, 181

venous system, $\mathbf{1 7 0 , 1 7 7}$

Postanal gut, 196

Postbranchial bodies, 229

Posterior chamber of eye, 342,343 nares, development of, 360

Post-limbic sulcus, 309

Postsphenoid, 393

Preformation theory, 18

Prehepaticus, 175, 208

Prehyoid gland, 227

Premaxilla, 397

Prepuce, formation of, 262

Presphenoid, 394

Primary collecting tubules of kidney, 239

egg-tubes, 31

renal pelvis, 237

Primitive aorta, 151, 165

chorion, 92

disk, $37 \%, 399$

enamel-germ, 138

eyelids, 134

groove, 60

heart-valves, 161

jugular veins, 164, 169

Malpighian corpuscle, 236

nails, 270

ova, $31,245,250$

segment plate, 65

segments, 65, 75

sexual cells, 245

streak, 59

vertebral bow, 376

vitreous, 338

Primordial bones, 385

Proamuion, 64

Process, lateral frontal, 118, 132, 134 nasal, 132, 360

nasofrontal, 115, 118, 132, 134, 360 in formation of nose, 145

Processes, dental, 141

globular, 118, 132, 360

nasal, 360

maxillary, 135

of vertehra, development of, $3 \% 6$

Processus vaginalis, 249

Prochorion, 50, 92

Proctodeum, 196

Pronephric duct, 233

Pronephros, 232, 264

Pronucleus, female, 34

male, 42

Pro-otic center of ossification, 392 
Prosencephalon, 286

Prostate gland, formation of, 257

Prostatic urethra, formation of, 257

Protoplasmic processes, 284

Protovertebra, 63

Proximal convoluted tubule of kidney, 240

Pterygoid plate, internal, development of, 394

Pubic rod, 404

Pulmonary alveoli, development, 225 artery, development of, 159, 168 diverticulum, 223 groove, 223

Pulp of spleen, development of, 213 of teeth, 137

Pupil, 330

congenital atresia of, 338

development of, 342

Pyramidal process of thyroid gland, 228

tracts, anterior development of, 290 crossed, of cord, myelination of, 415

RAmus communicans, 325

Ratlike's pocket, 136, 193, 301

Rauber's layer, 50

Receptacula chyli. 1:8

Receptive prominence, 42

Recessus labyrinthi, 347 vestibuli, 347

Rectum, 197

Recurrent laryngeal nerves, 168

Reduction of chromosomes, 23

Reduction-division, 23

Reichert's cartilage, 115, 389, 401

Reil, island of, 305

Reissner, membrane of, 355

Renal vein, left, 173

vesicles, 240

Reproduction, theories of, 17

Respiratory system, development of, $22: 2,409-416$

Restiform bodies, development of, 290

Rete mucosum, 270

testis, formation of, 246

Retina, development of, 328

Rhinencephalon, 314

Rhombencephalon, 286

Rhomboidal fossa, 291

Rib, cervical, 380,353

lumbar, 383

thirteenth, 383

Ribs, development of, 382

Ridge, genital, 243

terminal, 58

Rinc lobe, formation of, 304

Ripening of ovum, 32

Rod-and-cone layer, formation of, 332

Rod-visual cells, 331

Rolando, fissure of, 308

Roof-plate, 281, 28:

Rotation of stomach, 203,217

Round ligament of liver, 184
Round ligament of liver, formation of, 210

of uterus, $248,25$.

SACCULE, development of, 349

Saccus endolymphaticus, 347

Sacral vertebre, ossification of, 381

Sacrum, formation of, $3>1$

Salivary glands, development of, 143

Santorini, duet of, 212

Sauropsida, blastula of, 51

Scala media of cochlea, development of 347

tympani, development of, 355

vestibuli, development of, 355

Seapula, development of 403

Schwann, white substance of, 319

deposit of, upon fibers of tract of cord, 414,415

Sclerotome, $78,365,375$

Scrotum, development of, 263

Sebaceous glands, development of, 274

Second month, development in, 118

pair cranial nerves, development of, 323

week, development during, 409

Secondary hair, 273

optic cup, 330

Secreting tubules of kidney, 237, 239

Segmental duet, 233

segmentation of body of embryo, 78 of ovum, 45

Segmentation-eavity, 50

Segmentation-nucleus, 43

Semicircular canals, bony, 351

development of, 340

Semilunar valves, development of, 163

Seminal ampullæ, 246

vesicle, formation of, $24 \%$

Seminiferous tubules, formation, 246

Sense organs, development of, 326, 409-416

Sensory epithelium of retina, 331

nerve-fibers, development of, 317 , 318

Septa placentæ, 102

Septal cartilage of nose, 395

Septum, aortic, 159

auricular, $15 \%$

intermedium, $156^{\circ}$

lucidum, formation of, 312

primum, $15 \%$

secundum, 157

spuriulu, 159

transversum, 164, 175

Serosa, 81

Serous membranes, development, 126

Sertoli's columns, 21, 246

Sessile hydatid, 247

Seventh month, development during, 121,415

pair cranial nerves, development of, 323

week, development during, 412

Sexual cells, 31 
Sexual cells, primitive, 245

cords, 31, 245

female, 250

glaud, inditierent, 244

system, female, 249,266

homologies of, 263

indifferent type, 243

male, 245,266

shell of hen's egg, 29

shell-membrane, 29

shoulder girdle, development of, 403

sinus, aunular, 179

poeularis, $247,25 \%$

pracervicalis, 116

reuniens, 159

terminalis, 150

u rogenital, 190, 256

venosus, 159,169

Sixth month, development during, 121,415

pair cranial nerves, development of, 323

week, development during, 119, 411

skeletogenous sheath of chorda dorsalis, 375

tissues, 77

skeleton, appendicular, 373

development of, $402,409-416$

axial, 373

development of, $3 \%$ 2

of head, development of, 384

of trunk, cartilaginous stage, 377

chordal stage of, 373

development of, 373

membranous stage of, 374

visceral, $3-4$

Skin, appendages of, $2 \% 0$

development of, 268, 409-416

Small intestine, development of, 205

Smegma embryonum, 270

Somatic mesodern, 66

Somatopleure, 66, 186

Somites, 63, 75

mesoblastic, 65, \%5

Space, perivitelline, 25

spaces, intervillous, 97,102

Spermatic cord, 249

veins, 173

Spermatids, 22

Spermatoblasts, 22

Spermatogenesis, 21

Spernatogenic cells, 21

Spermatocytes, primary, 22 secondary, 2.2

Spermatogonia, 22

Spermatozooll, 20

power of locomotion of, 21 vitality of, 21

Sphenoid bone, ossification of, 393

Sphenoidal sinus, development of, 361

Spinal cord, development of, 281

Spinous process of vertebra, development of, 380

Splanchnic mesoderm, 66

Splanchnopleure, 66, 186
Spleen, development of, 212

Spongioblasts, 2ऽ2, 283

spot, germinal, 27

Sprouts, vessel, 150

Squamozygomatic bone, 391

Stage of embryo, 19, 107

of f'etus, : 20,118

of ovum, 19, 106

of quiescence of menstrual cycle, 40

of repair of menstrual cycle, 40

Stalked hydatid, 247

Stapes, development of, 389

Stem-zone, 75

Sternum, cleft, 383

development of, 383

Stigma, 31

Stilling, canal of, 339

Stomach, devclopnient of, 203

first rudiment of, 198

glands of, development of, 206

rotation of, 203,217

Stomodrum, 1:31, 19:

straight collecting tubules of kidney, $2: 39$

Stratum Malpighii, 270

Streak, primitive, 59

Striated muscles, development of, 363

Stroma-layer of choroid, development of, 340

Styloglossus, origin of, $3 \% 0$

Stylohyal, $40: 2$

cartilage, 393

Stylohyoid ligament, 389

Styloid process of hyoid, 389

temporal, development of, 393

Styloplaryngeus, origin of, 370

Subclaviain artery, left, development of, 168

right, development of, 167

Submucosa of intestines, formation of, 205

Substance-islands, 147

Subzonal layer of mammalian blastodermic vesicle, 50

Sulcus interventricularis, 158

of corpus callosum. $30 \pi$

terminalis, 160

Superior maxilla, ossification of, 397

Suprahyoid gland, 22:

Supra-occipital bone, 390

suprapericardial bodies, 228

Suprarenal hodies, development of, 241,265

Suspensory ligament of liver, formation of, 210

Sustentacular cells of seminiferous tubule, 21

Suture, amniotic, 83

Sweat-glands, development of, 273

Sylvius, aqueduct of, 296

fissure of, 30:3, 304

fossa of, 304

Sympathetic nervous system, 324

Synestium, 93, 97

Synovial sacs, development of, 126 
TAIL of spermatozoon, 20,22

Tail-fold, so

Tarsal ligameuts, 344 plates, 344

Tarsus, development of bones of, 405

Teeth, development of, 137 permanent, development of, 141 eruption of, 143

temporary, development of, 137 eruption of, 142

Tela choroidea, 297

Telencephalon, 287, 302

Telolecithal ova, 26

Temporal bone, ossification of, 390 lobe, formation of, 304

Temporary teeth, development of, 137 eruption of, 142

Temporomaxillary articulatiou, 400

Tendon, development of, 1:5

Tendon-sheaths, development of, 128

Tenth pair cranial nerves, development of, 324

Terminal filament of spermatozoon, 20,21 ridge, 58

Testicle, development of, 245 descent of, 248

Thalamencephalon, 287, 296

Thebesius, valve of, 161

Theca folliculi, 29

Thecal sacs, development of, 126

Theory of evolution, $1 \tau$ of unfolding, 17

Third evelid, $34 t$ month, development in, 120, 413 pair cranial nerves, development of, 3:3

ventricle, formation of, 296

week, development during, 410

Thirteenth rib, 383

Thoracic prolongations of abdominal cavity, 175

Throat-pockets, 113, 188, 193

Thymus body, 194, 230

Thyroglossal duct, 145,227

Thyroid body, accessory, 227 development of, 194, 226

cartilage, 2:6

duct, 227

foramen, 404

Thyroids, lateral, 226, 228

Tissue fungus, 97

Toes, development of, 407

Tongue, development of, 143, 194

Tonsil, development of, 194

Tonsillar pit, 195

Trabecula cranii, 387

Trachea, development of, 225

rragus, formation of, 358

Transverse colon, formation of, 203

crescentic groove, 80

fissure of brain, 298

processes of vertebræ, 380

Trigeminal ganglion, 320

Trophoblast, 92
True chorion, 92

Truncus arteriosus, 113, 151, 154, 165

Trunk, skeleton of, development of, 373

osseous stage of, 379

Trunk-muscles, development of, 363

Trunk-segments, 364

Tuber cinereum, 296, 300

Tubercles, cornicular, 226

cuneiform, 2:6

Tuberculum impar, 144, 194

Tubotympanic sulcus, 356

Tunica albuginea of ovary, 250 of testicle, 246

fibrosa, 30

propria, 30

vaginalis testis, 249

vaseulosa, 29

lentis, 337

Turbinal folds, 361

Turbinate bone, inferior, ossification of, 395

Turbinated bones, development of, 361

Twelfth pair cranial nerves, development of, 324

Twins, origin of, 59

Tympanic cavity, formation of, 194 membrane, 194

development of, 357

portion of temporal bone, development of, 393

Tympanohyal, 40: cartilage, 393

Tympanum, development of, 356

UMBILICAL aperture, 87, 186

arteries, 103, 165

cord, 102

hernia, congenital, 206

urinary fistula, 256

vein, $103,165,169$

vesicle, $80,8 \%, 186$

function of, 89

human, 89

vessels, 103

Uncinate gyrus, 315

Unstriated muscle, development, $3 \% 1$

Urachus, 91, 256

Ureter, 237

development of, 232

Urethra, female, 258

male, formation of, 262

prostatic, formation of, 257

Urinary fistula, umbilical, 256

Urogenital aperture, $25 \%$

sinus, 190, 196, 256

Uskow, pillars of, 177

Uterus bicornis, 253

development of, 253

double, 253

masculinus, 247, 257

Utricle, development of, 349

Uveal tract, development of, 340

Uvula, formation of, 137 
VAGind, development of, 253 median septum in, 253

Valve, coronary, 161

Eustachiau, 160

of Thebesius, 161

of Vienssens, 294

Valves, atrioventricular, 156 auriculoventricular, 162 of heart, development of, 161 semilunar, development of, 163

Van Beneden's embryonic bud, 54

Vas aberrans, 247

deferens, formation of, 246

Vasa efferentia, 246 recta, formation of, 246

Vascular area, 88 system, development of, 147, 409-416 fetal, final stage of, 18:

tunic of eye, development of, 339

Vegetative pole, 27

Vein, cardinal, 164

hepatic, 181

iliac, left common, development, $17 \%$ portal, development of, 181 renal, left, 173

superior mesenteric, 181

umbilical, 103

Veins, allantoic, 90, 164

cardinal, 164

anterior, 169

posterior, 169

omphalomesenteric, 151

primitive jugular, 169

spermatic, 173

umbilical, 165, 169

vitelline, 151,169

Velum interposituı, 296, 297

Vena azygos major, 173 minor, 174

cava, inferior, 171, 174 superior, 170

Venæ hepaticæ advelientes, 179 revehentes, 179

Venous segment of heart, 156 system of fetus, 169 portal, 170

Vontral meseutery, 190, 204 development of, $2: 0$

pancreas, 211

Ventricles, separation of, 158

Vermiform appendix, development,203 process of cerebellum, 292

Vernix caseosa, 87, 121, 270

Vertebræe, ossification of, 380

Vertebral bow, primitive, 376 column, development of, 373-382 membranous primordial, 375 plate, $6 \overline{5}$ region of primitive skull, 387

Vesicle, blastodermic, stage of, 49 two-layered stage of, 52 germinal, 25, 27

lens-, 110

otic, 109,346

umbilical, 80, 87, 186
Vesicles, cerebral, 287, 288

Vessel sprouts, 150

Vestibular ganglion, 351 nerve, 321

Vestibule of ear, development of, 352 of vagina, 259 of vulva, 257

Vestigial fold of Marshall, 172

Vieussens, valve of, 294

Villi of chorion, 93 of intestine, formation of, 206

Visceral arch, first, function of, 115, 131 arches, 112

metanorphosis of, $\mathbf{1 1 5}$

morphological significance of, 113 clefts, 112

layer of peritoneum, 189 of pleura, 177

skeleton, 384

Visceral-arch vessels, 113, 151, 165

Vitelline arteries, 151

artery, right, $15 \%$

circulation, formation of, 147

duct, $80,8 \%, 186$

membrane, 25, 26

veins, 151, 169

Vitellus, 25, 26

Vitreous body, development of, 338 mesodermal, 338 primitive, 338

Voluntary muscles, development, 363

Vomer, ossification of, 396

Werght of fètus at different stages, $41 \%-416^{2}$

at term, 122

Wharton, jelly of, 103

White commissures of cord, 285

fibrous tissue, formation of, 125

matter of brain, formation of, 303 of cord, development of, 285

of hen's egg, 29

substance of Schwann, development of, $319,409,415$

Winslow, foramen of, 221

Wirsung, duct of, 212

Witches' milk. 276

Wolffian blastema, 236

body, 234

duct, 235

in female. 253

ridge, 232, 234

Wolff' $s$ doctrine of epigenesis, 18

Wreath, 45

YoLK of ovum, 25

Yolk-sac, 80, 87, 186

ZinN, zonule of, 338

Zona pellucida, 25, 31

radiata, 31

Zone, parietal, 76

stem-, 76

Zonule of Zinn, 338

Zuckerkandl, organs of, 325 




\section{SAUNDERS' BOOKS}

\section{on \\ Practice, Pharmacy, \\ Materia Medica, Thera- \\ peutics, Pharmacology, \\ and the Allied Sciences}

\section{W. B. SAUNDERS COMPANY 925 WALNUT STREET \\ PHILADELPHIA}

9, HENRIETTA STREET, COVENT GARDEN, LONDON

SAUNDERS' SUCCESSFUL PUBLISHING

$\mathbf{A}^{\mathrm{S}}$ is well-known, the lists of most publishers contain a number of books that have never paid, and for which the publisher will never get back the money invested. Messrs. W. B. Saunders Company would call attention to the fact that they have no such works on their list. In all the years of their business experience they have never published a book at a loss. This they confidently consider a most remarkable record, and submit the fact to the attention of the profession as an example of what might justly be called "Successful Publishing."

A Complete Catalogive of our Publications will be Sent upon Request 


\title{
AMERICAN EDITION
}

\section{Nothnagel's Practice}

UNDER THE EDITORIAL SUPERVISION OF

\section{ALFRED STENGEL, M.D.}

\author{
Professor of Clinical Medicine in the University of Pennsylvania; Visiting \\ Physician to the Pennsylvania Hospital.
}

It is universally acknowledged that the Germans lead the world in Internal Medicine; and of all the German works on this subject, Nothnagel's "Specielle Pathologie und Therapie"

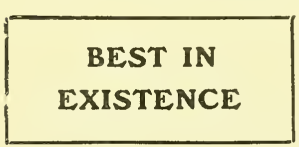
is conceded by scholars to be without question the best Practice of Medicine in existence. So necessary is this book in the study of Internal Medicine that it comes largely to this country in the original German. In view of these facts, Messrs. W. B. Saunders Company have arranged with the publishers of the German edition to issue at once an authorized American edition of this great Practice of Medicine.

For the present a set of 12 volumes, selected with especial thought of the needs of the

FOR THE
PRACTITIONER
practising physician, will be published. These volumes will contain the real essence of the entire work, and the purchaser will therefore obtain, at less than half the cost, the cream of the original. Later the special and more strictly scientific volumes will be offered from time to time.

The work will be translated by men possessing thorough knowledge of both English and German, and each volume will be edited by a prominent specialist. It will thus be brought thoroughly up to date, and the American edition will be more than a mere translation; for,

\section{PROMINENT SPECIALISTS} in addition to the matter contained in the original, it will represent the very latest views of the leading American and English specialists in the various departments of Internal Medicine. Moreover, as each volume will be revised to the date of its publication by the eminent editor, the objection that has heretofore existed to treatises published in a number of volumes will be obviated, since the subscriber will receive the completed work while the earlier volumes are still fresh. The American publication of the entire work is under the editorial supervision of Dr. Alfred STENGEL, who has selected the subjects for the American Edition, and has chosen the editors of the different volumes.

\section{VOLUMES SOLD} SEPARATELY

The usual method of publishers when issuing a publication of this kind has been to require physicians to take the entire work. This seems to us in many cases to be undesirable. Therefore, in purchasing this Practice physicians will be given the opportunity of subscribing for it in entirety; but any single volume or any number of volumes, each complete in itself, may be obtained by those who do not desire the complete series. This latter method offers to the purchaser many advantages which will be appreciated by those who do not care to subscribe for the entire work. Subscription.

\section{SEE NEXT TWO PAGES FOR LIST}




\section{AMERICAN EDITION Nothnagel's Practice \\ Per volume: Cloth, $\$ 5.00$ net Half Morocco, $\$ 6.00$ net}

\section{Typhoid and Typhus Fevers}

By Dr. H. Curschmans, of Leipsic. The entire volume edited, with additions, by William Osler, M. D., F. R. C. P., Regius Professor of Medicine, Oxford University, Oxford, England. Octavo volume of 646 pages, fully illustrated.

\section{Smallpox (including Vaccination), Varicella, Cholera Asiatica, Cholera Nostras, Erysipelas, Erysipeloid, Pertussis, and Hay Fever}

By Dr. H. Immermann, of Basle; Dr. Th. von Jürgensen, of Tübingen ; Dr. C. Liebermeister, of Tübingen; Dr. H. Lenhartz, of Hamburg; and DR. G. STICKER, of Giessen. The entire volume edited, with additions, by Sir J. W. Moore, M.D., F. R. C. P. I., Professor of Practice, Royal College of Surgeons, Ireland. Octavo, 682 pages, illustrated.

\section{Diphtheria, Measles, Scarlet Fever, and Rötheln}

By William P. Northrup, M. D., of New York, and Dr. Th. von JürGENSEN, of Tübingen. The entire volume edited, with additions, by WILLIAM P. Northrup, M. D., Professor of Pediatrics, University and Bellevue Hospital Medical College, New York. Octavo, 672 pages, illustrated, including 24 full-page plates, 3 in colors.

Diseases of the Bronchi, Diseases of the Pleura, and Inflammations of the Lungs

By Dr. F. A. Hoffuann, of Leipsic; Dr. O. Rosenbach, of Berlin ; and Dr. F. Aufrecht, of Magdeburg. The entire volume edited, with additions, by John H. Musser, M. D., Professor of Clinical Medicine, University of Pennsylvania. Octavo, I 029 pages, illustrated, including 7 full-page colored lithographic plates.

\section{Diseases of the Pancreas, Suprarenals, and Liver}

By Dr. L. Oser, of Vienna; Dr. E. Neusser, of Vienna, and Drs. H. Quincke and G. Hoppe-Seyler, of Kiel. The entire volume edited, with additions, by Reginald H. Fritz, A. M., M. D., Hersey Professor of the Theory and Practice of Physic, Harvard University; and Frederick A. Packard, M. D., Late Physician to the Pennsylvania and Children's Hospitals. Octavo of 918 pages, illustrated.

\section{Diseases of the Stomach}

By Dr. F. Riegel, of Giessen. Edited, with additions, by Charles G. Stockton, M. D., Professor of Medicine, University of Buffalo. Octavo of 835 pages, with 29 text-cuts and 6 full-page plates.

\section{Diseases of the Intestines and Peritoneum}

By Dr. Hermann Nothnagel, of Vienna. The entire volume edited, with additions, by H. D. Rolleston, M. D., F. R. C. P., Physician to St. George's Hospital, London. Octavo of 1050 pages, finely illustrated. 


\section{AMERICAN EDITION \\ Nothnagel's PRactice}

VOLUMES NOW READY

Per volume : Cloth, $\$ 5.00$ net Half Morocco, $\$ 6.00$ net

\section{Tuberculosis and Acute General Miliary Tuberculosis}

By Dr. G. Cornet, of Berlin. Edited, with additions, by WAlter B. James, M. D., Professor of the Practice of Medicine, Columbia University, New York. Octavo of 806 pages.

Diseases of the Blood (Anemia, Chlorosis, Leukemia, and Pseudoleukemia)

By Dr. P. Ehrlich, of Frankfort-on-the-Main ; Dr. A. LAzarus, of Charlottenburg; DR. K. VON NoORden, of Frankfort-on-the-Main; and DR. Felix PInkus, of Berlin. The entire volume edited, with additions, by ALFRED Stengel, M. D., Professor of Clinical Medicine, University of Pennsylvania. Octavo of 7 I 4 pages, with text-cuts and I 3 full-page plates, 5 in colors.

\section{Malarial Diseases, Influenza, and Dengue}

By Dr. J. Mannaberg, of Vienna, and Dr. O. Leichtenstern, of Cologne. The entire volume edited, with additions, by Ronald Ross, F. R. C. S. (ENG.), F. R. S., Professor of Tropical Medicine, University of Liverpool ; J. W. W. Stephens, M. D., D. P. H., Walter Myers Lecturer on Tropical Medicine, University of Liverpool ; and Albert S. Grünbaum, F. R. C. P., Professor of Experimental Medicine, University of Liverpool. Octavo of 769 pages, illustrated.

\section{Diseases of Kidneys and Spleen, and Hemorrhagic Diatheses}

By Dr. H. Senator, of Berlin, and Dr. M. LitTen, of Berlin. The entire volume edited, with additions, by JAmes B. Herrick, M. D., Professor of the Practice of Medicine, Rush Medical College. Octuvo of 8 I 5 pages, illust.

\section{Diseases of the Heart}

By Prof. Dr. L. von Schrotter, of Vienna; Prof. Dr. Th. von Jurgensen, of Tübingen; Prof. Dr. L. Krehl, of Greifswald ; and Prof. Dr. H. VIERORDT, of Tübingen. The entire volume edited, with additions, by George Dock, M. D., Professor of Theory and Practice of Medicine and Clinical Medicine, University of Michigan, Ann Arbor. Octavo of about 1000 pages, with 72 text illustrations and 6 colored plates.

\section{SOME PRESS OPINIONS}

London Lancet (Typhoid volume)

"We welcome the translation into English of this excellent practice of medicine. The first volume contains a vast amount of useful information, and the forthcoming volumes are awaited with interest."

Journal American Medical Association( Tuberculosis volume)

"We know of no single treatise covering the subject so thoroughly in all its aspects as this great German work. ... It is one of the most exhaustive, practical, and satisfactory works on the subject of tuberculosis."

Medical News, New York (Liver volume)

"Leaves nothing to be desired in the way of completeness of information, orderly arrangement of the text, thoroughgoing up-to-dateness, handiness for reference, and exhaustive discussion of the subjects treated." 


\section{Anders'}

\section{Practice of Medicine \\ Recently Issued-New (7th) Edition}

A Text=Book of the Practice of Medicine. By James M. Anders, M. D., PH. D., LL. D., Professor of the Practice of Medicine and of Clinical Medicine, Medico-Chirurgical College, Philadelphia. Handsome octavo, I 297 pages, fully illustrated. Cloth, $\$ 5.50$ net; Sheep or Half Morocco, $\$ 6.50$ net.

\section{OVER 27,000 COPIES SOLD}

The success of this work as a text-book and as a practical guide for physicians has been truly phenomenal, it now having reached its seventh edition. This success is no doubt due to the extensive consideration given to Diagnosis and Treatment, Differential Diagnosis being dealt with under separate headings, and the points of distinction of simulating diseases presented in tabular form. Among the new subjects added are Rocky Mountain Spotted Fever, Splanchnoptosis, Cammidge's Test for Glycerose, Myasthenia Gravis, Pseudotuberculosis, Benign Cirrhosis of the Stomach, Intestinal Lithiasia, Intestinal Calculi, Red Light in Variola, Emulsion-albuminuria, and Adams-Stokes' Syndrome. Important additions have also been made to diseases which prevail principally in tropical countries.

\section{PERSONAL OPINIONS}

James C. Wilson, M. D.,

Professor of the Practice of Medicine and of Clinical Medicine, Jefferson Medical College Philadelphia.

"It is an excellent book-concise, comprehensive, thorough, and up-to-date. It is a credit to you; but, more than that, it is a credit to the profession of Philadelphia-to us."

Wm. E. Quine, M. D.

Professor of Medicine and Clinical Medicine, College of Physicians and Surgeons, Chicago.

"I consider Anders' Practice one of the best single-volume works before the profession at this time, and one of the best text-books for medical students."

\section{Bulletin of the Johns Hopkins Hospital}

"The success of this work is well deserved. . . The sections on treatment are excelient and add greatly to the value of this work. Dr. Anders is to be congratulated on the continued success of his text-book." 


\section{Pusey and Caldwell on X-Rays}

\section{in Therapeutics and Diagnosis}

The Practical Application of the Rontgen Rays in Therapeutics and Diagnosis. By William Alleen Pusey, A. M., M. D., Professor of Dermatology in the University of Illinois; and Eugene W. CALDwell, B.S., Director of the Edward N. Gibbs X-Ray Memorial Laboratory of the University and Bellevue Hospital Medical College, New York. Handsome octavo of 625 pages, with 200 illustrations, nearly all clinical. Cloth, $\$ 5.00$ net; Sheep or Half Morocco, $\$ 6.00$ net.

\section{RECENTLY ISSUED - NEW (2d) EDITION, REVISED AND ENLARGED TWO LARGE EDITIONS IN ONE YEAR}

Two large editions of this work within a year testify to its practical value to both the specialist and general practitioner. Throughout the work it has been the aim of the authors to clucidate the practical aspects of the subject, and to this end the text has been beautifully illustrated with clinical pictures, showing the condition before the use of the X-rays, at various stages of their application, and the final therapeutic result obtained. Details are also given regarding the use and management of the apparatus necessary for X-ray work, illustrating the descriptions with instructive photographs and drawings. In making the revision the histories of the cases cited have been brought down to the present time.

\section{OPINIONS OF THE MEDICAL PRESS}

\section{British Journal of Dermatology}

"The most complete and up-to-date contribution on the subject of the therapeutic action of the Röntgen rays which has been published in English."

\section{Boston Medical and Surgical Journal}

"It is indispensable to those who use the X-rays as a therapeutic agent; and its illustrations are so numerous ... that it becomes valuable to every one."

\section{IJew York Medical Journal}

"We have nothing but praise for this volume, the combined work of two authors than whom no one is better fitted by training or experience to write in his individual field." 


\section{Sahli's Diagnostic Methods}

Editors: Francis P. Kinnicutt, M.D., and Nath'l Bowditch Potter, M.D.

A Treatise on Diagnostic Methods of Examination. By Prof. Dr. H. Sahli, of Bern. Edited, with additions, by Francis P. KinniCutt, M. D., Professor of Clinical Medicine, Columbia University, N. Y. ; and Nath'l Bowditch Potter, M. D., Visiting Physician to the City and French Hospitals, N. Y. Octavo of soo8 pages, profusely illustrated. Cloth, \$6.50 net; Half Morocco, \$7.50 net.

\section{JUST READY}

Dr. Sahli's great work, upon its publication in German, was immediately recognized as the most important work in its field. Not only are all methods of examination for the purpose of diagnosis exhaustively considered, but the explanation of clinical phenomena is given and discussed from physiologic as well as pathologic points of view. In the chemical examination methods are described so exactly that it is possible for the clinician to work according to these directions.

Lewellys F. Barker, M.D.

Professor of the Principles and Practice of Medicine, Johns Hopkins University

"I am delighted with it, and it will be a pleasure to recommend it to our students in the Johns Hopkins Medical School.'

\section{Friedenwald and Ruhräh on Diet}

Diet in Health and Disease. By Julius Friedeninald, M. D., Clinical Professor of Diseases of the Stomach, and John RuHraH, M. D., Clinical Professor of Diseases of Children, College of Physicians and Surgeons, Baltimore. Octavo of 728 pages. Cloth, \$4.00 net.

\section{JUST ISSUED-NEW(2nd)EDITION}

This work contains a complete account of food-stuffs, their uses, and chemical composition. Dietetic management in all diseases in which diet plays a part in treatment is carefully considered. The feeding of infants and children, of patients before and after anesthesia and surgical operations, and the latest methods of feeding after gastro-intestinal operations are all taken up in detail.

\section{George Dock, M. D.}

Professor of Theory and Practice and of Clinical Medicine, University of Michigan.

" It seems to me that you have prepared the most valuable work of the kind now available. I am especially glad to see the long list of analyses of different kinds of foods." 


\section{Rolleston on the Liver}

Diseases of the Liver, Gall=bladder, and Bile=ducts. By $\mathrm{H}$. D. Rolleston, M. D. (Сantab), F. R. C. P., Physician to St. George's Hospital, London, England. Octavo volume of 794 pages, fully illustrated, including a number in colors. Cloth, $\$ 6.00$ net.

\section{ENTIRELY NEW-RECENTLY ISSUED}

This work covers the entire field of diseases of the liver, and is the most voluminous work on this subject in English. Dr. Rolleston has for many years past devoted his time exclusively to diseases of the digestive organs, and anything from his pen, therefore, is authoritative and practical. Special attention is given to pathology and treatment, the former being profusely illustrated.

\section{Medical Record, New York}

"The most extensive treatise on diseases of the liver yet published in English. . . . It reflects an unusual degree of experience in a difficult but highly important branch of study."

\section{Boston's Clinical Diagnosis}

Clinical Diagnosis. By L. Napoleon Boston, M. D., Associate in Medicine and Director of the Clinical Laboratories, Medico-Chirurgical College, Philadelphia: Octavo of 563 pages, with 330 illustrations, many in colors. Cloth, $\$ 4.00$ net.

\section{RECENTLY ISSUED-NEW (2d) EDITION TWO EDITIONS IN ONE YEAR}

Dr. Boston here presents a practical manual of the clinical and laboratory examinations which furnish a guide to correct diagnosis, giving only such methods, however, which can be carried out by the busy practitioner in his office as well as by the student in the laboratory. In this new second edition the entire work has been carefully and thoroughly revised, incorporating all the newest advances.

\section{Boston Medical and Surgical Journal}

"He has produced a book which may be regarded eminently as a practical and serviceable guide. ... The illustrations are both numerous and good." 


\section{GET A THE NEW THE REST American STANDARD \\ Illustrated Dictionary}

Just Issued-New (4th) Edition

The American Illustrated Medical Dictionary. A new and complete dictionary of the terms used in Medicine, Surgery, Dentistry, Pharmacy, Chemistry, and kindred branches; with over Ioo new and elaborate tables and many handsome illustrations. By W. A. Newman Dorland, M. D., Editor of "The American Pocket Medical Dictionary." Large octavo, over 800 pages, bound in full flexible leather. Price, $\$ 4.50$ net; with thumb index, $\$ 5.00$ net.

\section{WITH 2000 NEW TERMS}

The immediate success of this work is due to the special features that distinguish it from other books of its kind. It gives a maximum of matter in a minimum space and at the lowest possible cost. Though it is practically unabridged, yet by the use of thin bible paper and flexible morocco binding it is only $13 / 4$ inches thick. In this new edition the book has been thoroughly revised, and upward of two thousand new terms have been added.

Howard A. Kelly, M. D., Professor of Gynecology, Johns Hopkins University, Baltimore.

"Dr. Dorland's dictionary is admirable. It is so well gotten up and of such convenient size. No errors have been found in my use of it."

\section{Goepp's \\ State Board Questions}

State Board Questions and Answers. By R. Max Goepr, M. D., Professor of Clinical Medicine, Philadelphia Polyclinic. Octavo of 800 pages.

\section{READY SOON}

Every graduate who desires to practice medicine must pass a State Board Examination, and to aid him in successfuily passing such an examination this work will be of inestimable value. Dr. Goepp has taken great pains to collect the many questions asked in the past by Boards of the various States, and has arranged and classified them under subjects in such a manner that the prospective applicant can acquire the knowledge on any branch with the least difficulty. 


\section{Stevens' \\ Modern Therapeutics}

A Text-Book of Modern Materia Medica and Therapeutics. By A. A. Stevens, A. M., M. D., Lecturer on Physical Diagnosis in the University of Pennsylvania. Octavo of 670 pages. Cloth, $\$ 3.50$ net.

\section{RECENTLY ISSUED-NEW (4th) EDITION \\ Adapted to the New (1905) Pharmacopeia}

Dr. Stevens, by his extensive teaching experience, has acquired a clear, concise diction that adds greatly to his work's pre-eminence. In this edition new articles have been added on Scopolamin, Ethyl Chlorid, Theocin, Veronal, and Radium, besides much new matter to the section on Radiotherapy. The numerous changes in name or strength of various drugs and preparations, as called for by the new Pharmacopeia, have also been made. The work includes the following sections: Physiologic Action of Drugs; Drugs; Remedial Measures other than Drugs; Applied Therapeutics ; Incompatibility in Prescriptions; Table of Doses; Index of Drugs; and Index of Diseases; the treatment being elucidated by more than two hundred formulæ.

\section{OPINIONS OF THE MEDICAL PRESS}

\section{University Medical Magazine}

"The author has faithfully presented modern therapeutics in a comprehensive work . . . and it will be found a reliable guide and sufficiently comprehensive for the physician in practice."

\section{Bristol Medico-Chirurgical Journal, Bristol}

"This addition to the numerous works on Therapeutics is distinctly a good one. . . It is to be recommended as being systematic, clear, concise, very fairly up to date, and carefully indexed."

\section{Monro's Manual of Medicine}

Recently Issued

Manual of Medicine. By Thomas Kirkpatrick Monro, M. A., M. D.s Fellow of, and Examiner to, the Faculty of Physicians and Surgeons, England; Glasgow Physician to Glasgow Royal Infirmary, Glasgow, etc Octavo volume of 90 I pages, illustrated. Cloth, $\$ 5.00$ net. 


\section{Hatcher and Sollmann's Materia Medica}

A Text=Book of Materia Medica: including Laboratory Exercises in the Histologic and Chemic Examination of Drugs. By Robert A. Hatcher, Ph. G., M. D., of Cornell University Medical School, New York City; and Torald Sollmann, Mi.D., of the Western Reserve University, Cleveland, Ohio. I 2 mo of $4 \mathrm{Ir}$ pages. Flex. leather, \$2.00 net.

\section{RECENTLY ISSUED-A NEW WORK}

This work is a practical text-book, treating the subject by actual experimental demonstrations.

\section{Journal of the American Medical Association}

"The book is well written, the classifications are good, and the book is to be recommended as a practical guide in the laboratory study of materia medica."

\section{Eichhorst's Practice}

A Text=Book of the Practice of Medicine. By Dr. Hermann EichHorst, University of Zurich. Translated and edited by Augustus A. Eshner, M.D., Professor of Clinical Medicine, Philadelphia Polyclinic. Two octavos of 600 pages each, with over I 50 illustrations. Per set: Cloth, $\$ 6.00$ net; Sheep or Half Morocco, $\$ 7.50$ net.

\section{Bulletin of Johns Hopkins Hospital}

"This book is an excellent one of its kind. Its completeness, yet brevity, the clinicai methods, the excellent paragraphs on treatment and watering-places, will make it very desirable."

\section{Bridge on Tuberculosis}

Tuberculosis. By Norman Bridge, A. M., M.D., Emeritus Professor of Medicine in Rush Medical College, in affiliation with the University of Chicago. I $2 \mathrm{mo}$ of 302 pages, illustrated. Cloth, $\$ 1.50$ net.

\section{Medical News, New York} äisease."

" Thoroughly representative of our practical methods of diagnosis and treatment of the 


\section{Thornton's Dose-Book}

Dose $=$ Book and Manual of Prescription=Writing. By E. Q. THoRN Ton, M. D., Assistant Professor of Materia Medica, Jefferson Medical College, Phila. Post-octavo, 392 pages, illustrated. Flexible Leather, $\$ 2.00$ net.

\section{Recently Issued-New (3d) Edition}

D1. Thornton, in making this revision, has brought his book in accord with the new (1905) Pharmacopeia. Throughout the entire work numerous references have been introduced to the newer curative sera, organic extracts, synthetic compounds, and vegetable drugs. To the Appendix, chapters upon Synonyms and Poisons and their antidotes have been added, thus increasing its value as a book of reference.

C. H. Miller, M. D.,

Professor of Pharmacology, Northwestern University Medical School, Chicago.

"I will be able to make considerable use of that part of its contents relating to the correct terminology as used in prescription-writing, and it will afford me much pleasure to recommend the book to my classes, who often fail to find this information in their other text-books."

\section{Lusk on Nutrition}

Elements of the Science of Nutrition. By Graham Lusk, Ph.D., Professor of Physiology in the University and Bellevue Hospital Medical College. Octavo of 325 pages.

Cloth, $\$ 2.50$ net.

\section{JUST READY}

This practical work deals with the subject of nutrition from a scientific standpoint, and will be useful to the dietitian as well as the clinical physician. There are special chapters on the metabolism of diabetes and fever, and on purin metabolism.

Lewellys F. Barker, M.D.,

Professor of the Principles and Practice of Medicine, Johns Hopkins University.

"I shall recommend it highly. It is a comfort to have such a discussion of the subject."

\section{Mathews' How to Succeed in Practice}

How to Succeed in the Practice of Medicine. By Joseph M. Mathews, M.D., LL.D., President American Medical Association, I 898-'99. I 2 mo of 2 I 5 pages, illustrated. Cloth, $\$$ I.5O net. 


\section{Gould and Pyle's Curiosities of Medicine}

Anomalies and Curiosities of Medicine. By George M. Gould, M.D., and Walter L. Pyle, M. D. An encyclopedic collection of rare and extraordinary cases and of the most striking instances of abnormality in all branches of Medicine and Surgery, derived from an exhaustive research of medical literature from its origin to the present day, abstracted, classified, annotated, and indexed. Handsome octavo volume of 968 pages, 295 engravings, and I 2 full-page plates.

\section{Popular Edition : Cloth, $\$ 3.00$ net; Sheep or Half Morocco, $\$ 4.00$ net.}

As a complete and authoritative Book of Reference this work will be of value not only to members of the medical profession, but to all persons interested in general scientific, sociologic, and medicolegal topics; in fact, the absence of any complete work upon the subject makes this volume one of the most important literary innovations of the day.

\section{The Lancet, London}

"The book is a monument of untiring energy, keen discrimination, and erudition. . . We heartily recommend it to the profession."

\section{Saunders' Pocket Formulary}

Recently Issued-New (8th) Edition-Adapted to the New (1905) Pharmacopeia

Saunders' Pocket Medical Formulary. By Wizliam M. Powell, M. D., author of "Essentials of Diseases of Children"; Member of Philadelphia Pathological Society. Containing I 83 I formulas from the best-known authorities. With an Appendix containing Posological Table, Formulas and Loses for Hypodermic Medication, Poisons and their Antidotes, Diameters of the Femaie Pelvis and Fetal Head, Obstetrical Table, Diet-list, Materials and Drugs used in Antiseptic Surgery, Treatment of Asphyxia from Drowning, Surgical Remembrancer, Tables of Incompatibles, Eruptive Fevers, etc., etc. In flexible morocco, with side index, wallet, and flap. \$I.75 net.

\section{Johns Hopkins Hospital Bulletin}

"Arranged in such a way as to make consultation of it as easy as possible. It is remarkable how much information the author has succeeded in getting into so small a book." 


\section{Sollmann's Pharmacology}

Including Therapeutics, Materia Medica, Pharmacy, Prescription-writing, Toxicology, etc.

A Text=Book of Pharmacology. By Torald Sollmann, M.D., Professor of Pharmacology and Materia Medica, Medical Department of Western Reserve University, Cleviland, Ohio. Handsome octavo volume of ro70 pages, fully illustrated. Cloth, \$4.00 net.

\section{RECENTLY ISSUED--NEW (2d) EDITION}

Because of the radical alterations which have been made in the new (1905) Pharmacopeia, it was found necessary to reset this book entirely. The author bases the study of therapeutics on a systematic knowledge of the nature and properties of drugs, and thus brings out forcibly the intimate relation between pharmacology and practical medicine.

\section{J. F. Fotheringham, M. D.}

Prof. of Therapeutics and Theory and Practice of Prescribing Trinity Med. College, Toronto.

"The work certainly occupies ground not covered in so concise, useful, and scientific a manner by any other text I have read on the subjects embraced."

\section{Butler's Materia Medica Therapeutics, and Pharmacology}

A Text=Book of Materia Medica, Therapeutics, and Pharmacology. By George F. Butler, Ph. G., M. D., Associate Professor of Therapeutics, College of Physicians and Surgeons, Chicago. Revised by Smith Ely Jelliffe, M. D., Professor of Pharmacognosy, Columbia University. Octavo of 694 pages, illustrated. Cloth, $\$ 4.00$ net; Half Morocco, \$5.00 net.

\section{RECENTLY ISSUED-NEW (5th) EDITION \\ Adapted to the New (1905) Pharmacopeia}

For this fifth edition Dr. Butler's text-book has been entirely remodeled, rewritten, and reset. All obsolete matter has been eliminated, and special attention has been given to the toxicologic and therapeutic effects of the newer compounds. A classification has been adopted which groups together those drugs the predominant action of which is on one system of organs.

\section{Medical Record, New York}

"Nothing has been omitted by the author which, in his judgment, would add to the completeness of the text, and the student or general reader is given the benefit of latest advices. bearing upon the value of drugs and remedies considered." 


\section{The American Pocket Medical Dictionary. 4th Ed. Recently Issued}

The American Pocket Medical Dictionary. Edited by IV. A. Newman DorLAND, M. D., Assistant Obstetrician to the Hospital of the University of Pennsylvania. Containing the pronunciation and definition of the principal words used in medicine and kindred sciences, with 64 extensive tables. Flexible leather, with gold edges, \$I.0O net; with thumb index, \$I.25 net.

"I can recommend it to our students without reserve."-J. H. Holland. M. D., of the Jefferson Medical College, Philadelphia.

\section{Vierordt's Medical Diagnosis. Fourth Edition, Revised}

Medical Diagnosis. By Dr. Oswald Vierordt, Professor of Medicine, University of Heidelberg. Translated from the fifth enlarged German edition by Francis H. Stuart, A. M., M. D. Octavo, 603 pages, IO4 wood cuts. Cloth, \$4.00 net; Sheep or Half Morocco, $\$ 5.00$ net.

"Has been recognized as a practical work of the highest value. It may be considered indispensable both to students and practitioners."-F. Minot, M. D., late Professor of Theory and Practice in Harvard University.

\section{Cohen and Eshner's Diagnosis. Second Revised Edition}

Essentials of Diagnosis. By S. Solis-Cohen, M. D., Senior Assistant Professor in Clinical Medicine, Jefferson Medical College, Phila.; and A. A. Eshner, M. D., Professor of Clinical Medicine, Philadelphia I'olyclinic. Post-octavo, 382 pages ; 55 illustrations. Cloth, \$I.oo net. In Saunders' Question-Compend Series.

"Concise in the treatment of subject, terse in expression of fact."-American Journal of the Medical Sciences.

\section{Morris' Materia Medica and Therapeutics.}

Recently Issued New (7th) Edition

Essentials of Materia Medica, Therapeutics, and Prescription-Writing. By Henry Morris, M. D., late I lemonstrator of Therapeutics, Jefferson Medical College, Phila. Revised by W. A. Bastedo, M. D., Instructor in Materia Medica and Pharmacology at Columbia University. I 2mo, 30o pages. Cloth, \$1.00 net. In Sounders' Question-Compend Series.

"Cannot fail to impress the mind and instinct in a 'asting manner."-Buffulo Medical Journal.

\section{Williams' Practice of Medicine}

Recently Issued

Essentials of the Practice of Mridicine. By W. R. Willians, M.D., formerly Instructor in Medicine and Lecturer on Hygiene, Cornell University; and Tutor in Therapentics, Columbia University, N. Y. I 2 mo of $45^{6}$ pages, illustrated. In Saunders' Question-Compon.l Series. Double number, $\$ \mathbf{r} .75$ net.

\section{Stoney's Materia Medica for Nurses}

Recently Issued New (3rd) Edition

Materia Medica for Nurses. By Emily M. A. Stoney, superintendent of the Training School for Nurses at the Carney Hospital, South Boston, Mass. Handsome I2mo volume of 300 pages. Cloth, \$I.50 net.

"It contains about everything that a nurse ought to know in regard to drugs."-Journal of the American Medical Association.

\section{Grafstrom's Mechano-therapy}

Recently Issued

A Text-Book of Mechano-Therapy (Massage and Medical Gymnastics). By Axel V. Grafstrom, B. Sc., M. D., Attending Physician to Augustus Adolphus Orphanage, Jamestown, N. Y. I2mo, 200 pages, illustrated. \$I.25 net.

"Certainly fulfills its mission in rendering comprehensible the subjects of massage and medical gymnastics." -New York Medical Journal. 


\section{Jakob and Eshner's Internal Medicine and Diagnosis}

Atlas and Epitome of Internal Medicine and Clinical Diagnosis. By Dr. Chr. JAKoB, of Erlangen. Edited, with additions, by A. A. Eshner, M. D., Professor of Clinical Medicine, Philadelphia Polyclinic. With 182 colored figures on 68 plates, 64 text-illustrations, 259 pages of text. Cloth, \$3.00 net. In Saunders' Hand-Atlas Series.

"Can be recommended unhesitatingly to the practicing physician no less than to the student." Bulletin of Johns Hopkins Hospital.

\section{Lockwood's Practice of Medicine.}

Second Edition, Revised and Enlarged

A Manual of the Practice of Medicine. By Geo. Roe Lockwood, M. D., Attending Physician to the Bellevue Hospital, New York City. Octavo, 847 pages, with 79 illustrations in the text and 22 full-page plates. Cloth, $\$ 4.00$ net.

"A work of positive merit, and one which we gladly welcome."-New York Medical Journal.

\section{Keating's Life Insurance}

How to Examine for Life Insurance. By the late John M. Keating, M. D., Ex-President of the Association of Life Insurance Medical Directors. Royal octavo, 2 I I pages. With numerous illustrations. Cloth, $\$ 2.00$ net.

"This is by far the most useful book which has yet appeared on insurance examination."-Medical Nerws.

\section{Corwin's Physical Diagnosis. Third Edition, Revised}

Essentials of Physical Diagnosis of the Thorax. By A. M. Corwin, A. M., M. D., Professor of Physical Diagnosis, College of Physicians and Surgeons, Chicago. 220 pages, illustrated. Cloth, flexible covers, \$I.25 net.

"A most excellent little work. It arranges orderly and in sequence the various objective phenomena to logical solution of a careful diagnosis."-Journal of Nervous and Mental Diseases.

\section{Barton and Wells' Medical Thesaurus}

A Thesaurus of Medical Words and Phrases. By W. M. Barton, M. D., and W. A. Welis, M. D., of Georgetown University, Washington, D. C. I 2 mo of 535 pages. Flexible leather, $\$ 2.50$ net; thumb indexed, $\$ 3.00$ net.

\section{Jelliffe's Pharmacognosy}

An Introduction to Pharmacognosy. By Smith Ely Jelliffe, Ph. D., M. D., of Columbia University. Octavo, illustrated. Cloth, $\$ 2.50$ net.

\section{Stevens' Practice of Medicine. New (7th) Edition-Recently Issued}

A Manual of the Practice of Medicine. By A. A. Stevens. A. M., M. D., Professor of Pathology, Woman's Medical College, Phila. Specially intended for students preparing for graduation and hospital examinations. Post-octavo, $55^{6}$ pages ; illustrated. Flexibie leather, $\$ 2.50$ net.

"An excellent condensation of the essentials of medical practice for the student, and may be found also an excellent reminder for the busy physician."-Buffulo Medical Journal.

\section{Paul's Materia Medica for Nurses}

Just Ready

Materia Medica for Nurses. By George P. Paul, M.D., Assistant Visiting Physician and Adjunct Radiographer to the Samaritan Hospital, Troy, N. Y. I 2 mo of 240 pages. Cloth, \$1.50 net.

In Dr. Paul's new work the physiologic actions of the drugs are arranged according to the action of the drug and not the organ acted upon. Another important section is that on pretoxic signs, giving the warnings of the full action or the beginning toxic effects of the drug. If these signs be known many cases of drug poisoning may be prevented. 








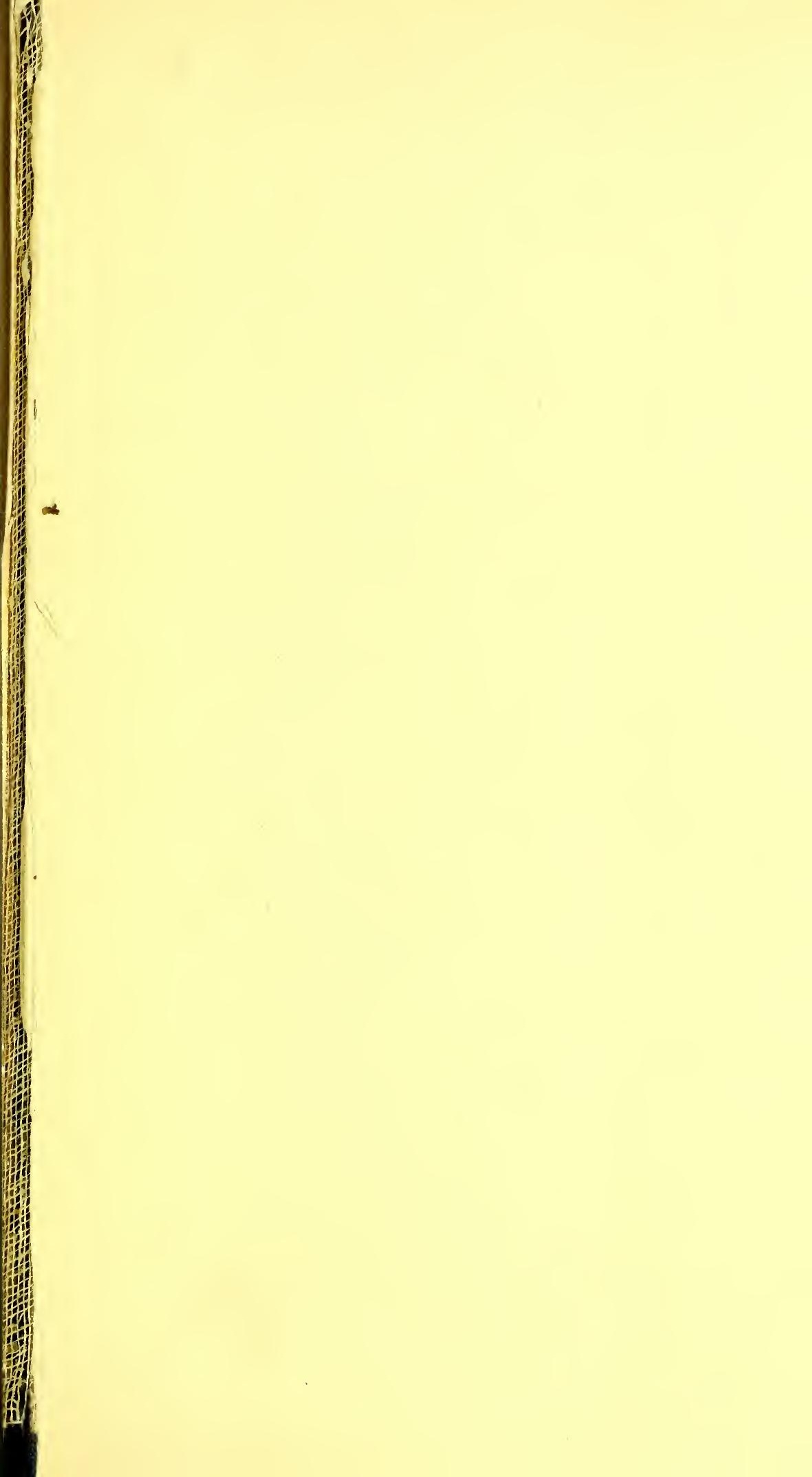


W.

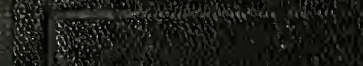

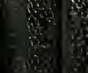

